\title{
DE-EE0000466
}

Final Project Report

\section{Development of Micro-Structural Mitigation Strategies for PEM Fuel Cells: Morphological Simulations and Experimental Approaches}

\section{Submitted to the U.S. Department of Energy \\ 28 June 2013}

By

\author{
Ballard Materials Products \\ Two Industrial Avenue \\ Lowell, MA 0851-5199 \\ Dr. Silvia Wessel (PI) \\ Email: silvia.wessel@ballard.com
}

Prime Contractor: Ballard Materials Products

Project Collaborators: Dr. Jeffery Allen \& Dr. Reza Shahbazian-Yassar - Michigan Technological University (MTU)

Dr. Plamen Atanassov \& Kateryna Artyushkova - University of New Mexico (UNM)

Dr. Rodney Borup \& Dr. Rangachary Mukundan - Los Alamos National Laboratory (LANL)

Dr. Seung Soon Jang - Georgia Institute of Technology (GIT)

Dr. Kunal Karan - University of Calgary

Dr. Jon Pharoah - Queen's University, Canada (Queen's) 
DE-EE0000466

Ballard Material Products Inc.

\section{FOREWORD AND ACKNOWLEDGEMENTS}

This report summarizes and documents the work conducted by Ballard Material Products and its sub-contractors under the DOE Federal Assistance Contract DE-EE0000466 "Development of Micro-Structural Mitigation Strategies for PEM Fuel Cells: Morphological Simulations and Experimental Approaches". The work was started on January 1, 2010 and ended March 31, 2013.

The overall objectives of this project were to improve the understanding of the design space of fuel cell materials and components and to recommend degradation mitigation strategies that facilitate the achievement of the 2020 technical targets. The deliverables from this project to the public, fuel cell community, and DOE are an open-source code fuel cell performance and durability model including a user-guide for dissemination and use within the public domain, and catalyst layer performance and durability correlations with identification of the design spaces relevant to improved durability.

The project was led by Ballard Material Products via a direct subcontract to Ballard Power Systems and included critical contributions from the other subcontractors: Georgia Institute of Technology, Los Alamos National Laboratory, Michigan Technical University, Queen's University/University of Calgary, and the University of New Mexico. Ballard's work, which encompassed the development of the performance and degradation models as well as experimental investigation for model validation and the correlation/durability window development, was carried out at Ballard Power Systems' facility in Burnaby, B.C., Canada.

Ballard recognizes the significant contribution made by each of the project collaborators and their respective team members. As well, Ballard recognizes the support provided from the DOE project managers, Dr. David Peterson and Kathi Epping Martin, and the project technical advisor, Dr. John Kopasz.

The work was funded by the U.S. Department of Energy, Energy Efficiency and Renewable Energy. 
DE-EE0000466

Ballard Material Products Inc.

\section{EXECUTIVE SUMMARY}

The durability of PEM fuel cells is a primary requirement for large scale commercialization of these power systems in transportation and stationary market applications that target operational lifetimes of 5,000 hours and 40,000 hours by 2015 , respectively. Key degradation modes contributing to fuel cell lifetime limitations have been largely associated with the platinum-based cathode catalyst layer. Furthermore, as fuel cells are driven to low cost materials and lower catalyst loadings in order to meet the cost targets for commercialization, the catalyst durability has become even more important. While over the past few years significant progress has been made in identifying the underlying causes of fuel cell degradation and key parameters that greatly influence the degradation rates, many gaps with respect to knowledge of the driving mechanisms still exist; in particular, the acceleration of the mechanisms due to different structural compositions and under different fuel cell conditions remains an area not well understood.

The focus of this project was to address catalyst durability by using a dual path approach that coupled an extensive range of experimental analysis and testing with a multi-scale modeling approach. With this, the major technical areas/issues of catalyst and catalyst layer performance and durability that were addressed are:

1. Catalyst and catalyst layer degradation mechanisms (Pt dissolution, agglomeration, $\mathrm{Pt}$ loss, e.g. Pt in the membrane, carbon oxidation and/or corrosion).

a. Driving force for the different degradation mechanisms.

b. Relationships between MEA performance, catalyst and catalyst layer degradation and operational conditions, catalyst layer composition, and structure.

2. Materials properties

a. Changes in catalyst, catalyst layer, and MEA materials properties due to degradation.

3. Catalyst performance

a. Relationships between catalyst structural changes and performance.

b. Stability of the three-phase boundary and its effect on performance/catalyst degradation.

The key accomplishments of this project are:

- The development of a molecular-dynamics based description of the carbon supported-Pt and ionomer system

- The development of a composition-based, 1D-statistical Unit Cell Performance model

- A modified and improved multi-pathway ORR model

- An extension of the existing micro-structural catalyst model to transient operation

- The coupling of a Pt Dissolution model to the modified ORR pathway model

- The Development A Semi-empirical carbon corrosion model

- The integration and release of an open-source forward predictive MEA performance and degradation model

- Completion of correlations of BOT (beginning of test) and EOT (end of test) performance loss breakdown with cathode catalyst layer composition, morphology, material properties, and operational conditions

- Catalyst layer durability windows and design curves

- A design flow path of interactions from materials properties and catalyst layer effective properties to performance loss breakdown for virgin and degraded catalyst layers 
In order to ensure the best possible user experience we will perform a staged release of the software leading up to the webinar scheduled in October 2013. The release schedule will be as follows (please note that the manual will be released with the beta release as direct support is provided in Stage 1):

- Stage 0 - Internal Ballard Release

- Cross check of compilation and installation to ensure machine independence

- Implement code on portable virtual machine to allow for non-UNIX use (pending)

- $\quad$ Stage 1 - Alpha Release

- The model code will be made available via a GIT, sourceforge, or other repository (under discussion at Ballard) for download and installation by a small pre-selected group of users

- Users will be given three weeks to install, apply, and evaluate features of the code, providing feedback on issues or software bugs that require correction prior to beta release

- $\quad$ Stage 2 - Beta Release

- The model code repository is opened to the general public on a beta release concept, with a mechanism for bug tracking and feedback from a large user group

- Code will be tracked and patched for any discovered bugs or relevant feedback from the user community, upon the completion of three months without a major bug submission the code will be moved to a full version release

- $\quad$ Stage 3 - Full Version Release

$\circ \quad$ Code is version to revision 1.0 and that version is frozen in development/patching 
DE-EE0000466

Ballard Material Products Inc.

\section{REPORT STRUCTURE}

The report is separated into seven separate chapters:

Chapter I gives an introduction to the overall work. It provides the background for the research and describes the high level objectives, the approach, and overall achievements. It also identifies the subcontractors and their responsibility within the project, provides a list the overall project accomplishments, and a list of abbreviations that are used throughout the report. Chapter II to VII are a logical separation of the different work streams and each chapter describes in detail the motivation for the research, the approach, and the results of the technical work.

Chapter II summarizes the MEA investigation that focused on MEA and catalyst layer degradation based on accelerated stress testing. This work was conducted at Ballard.

Chapter III describes the characterization of MEA materials and catalyst layer components. This work was carried out by the following Collaborators: University of New Mexico, Los Alamos National Laboratory, and Michigan Technological University.

Chapter IV summarizes the correlations developed between BOT (beginning of test) and EOT (end of test) performance and cathode catalyst layer structure and composition, catalyst materials and effective properties and operational conditions. The developed catalyst layer durability windows are also part of this chapter.

Chapter V, VI, and VII present the model developments. Chapter V is a report out by Georgia Institute of Technology on the development of the molecular-dynamics based model of the carbon supported-Pt and ionomer system, the Pt dissolution sites of the Pt crystal, and Pt ion transport/diffusion rates in the ionomer.

Chapter VI, covering micro-structural models, describes the model development for both the microstructural PTL model and the microstructural catalyst layer model in detail. The PTL work was done in coordination with Simon Fraser University, while the catalyst model was coordinated with Queen's University.

The development of the Open-source FC-PEM Performance and Durability Model is described in Chapter VII. The work was carried out by Ballard with some fundamental support by both collaborators from Queen's, of which Dr. Karan joined the University of Calgary six months ago.

Due to the extensive technical work and thus resulting large volume of this report, the table of contents at the beginning of the report just provides a high level overview while Chapters II to VII each contain its own details about the structure, list of figures, and list of tables. 


\section{TABLE OF CONTENTS}

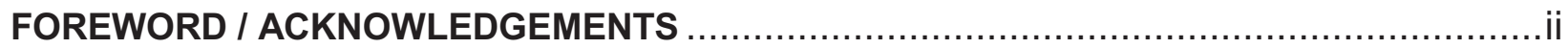

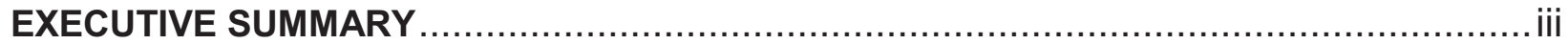

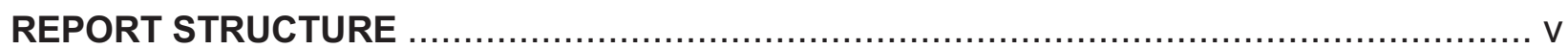

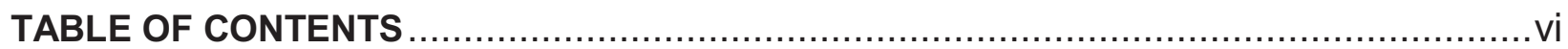

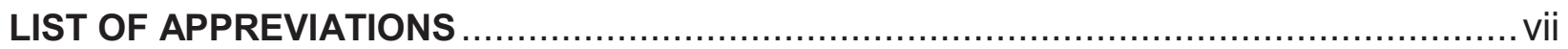

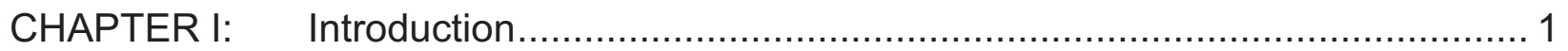

CHAPTER II: Experimental Investigations - Accelerated Stress Testing and Characterization of BOT and Degraded MEAs ......................... 17

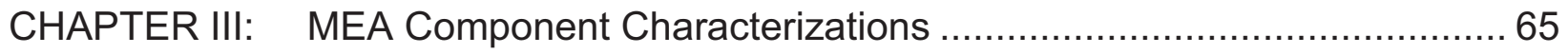

CHAPTER IV: Correlations of Catalyst Layer Structure and Performance/Degradation .................................................... 145

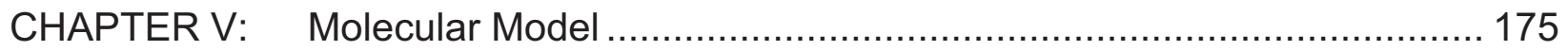

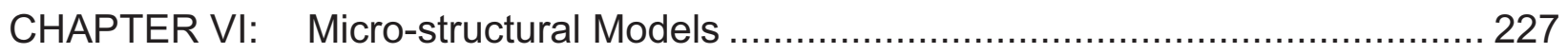

CHAPTER VII: FCPEM Performance and Durability Model..................................... 265 
DE-EE0000466

Ballard Material Products Inc.

\section{LIST OF ABBREVIATIONS}

\begin{tabular}{|c|c|c|c|}
\hline $\mathrm{AA}$ & Atomic Adsorption & HSAC-HT & High Surface Area Carbon - Heat Treated \\
\hline AST & Accelerated Stress Test & LANL & Los Alamos National Laboratory \\
\hline BFCS & Ballard Fuel Cell Systems & LPL & Lower Potential Limit \\
\hline BMP & Ballard Material Products & LSAC & Low Surface Area Carbon \\
\hline $\mathrm{BOL}=\mathrm{BOT}$ & Beginning of Life $=$ Beginning of Test & MD & Molecular Dynamics \\
\hline BPS & Ballard Power Systems & MEA & Membrane Electrode Assembly \\
\hline CB & Carbon Black & MIP & Mercury Intrusion Porosimetry \\
\hline CCL & Cathode Catalyst Layer & $\mathrm{MOL}=\mathrm{MOT}$ & Middle of Life $=$ Middle of Test \\
\hline $\mathrm{CCM}$ & Catalyst Coated membrane & MPL & Micro-porous Layer \\
\hline $\mathrm{CL}$ & Catalyst Layer & MSAC & Mid-range Surface Area Carbon \\
\hline Cond & Conductivity & MTU & Michigan Technologic University \\
\hline CV & Cyclic Voltammetry & NAA & Neutron Activated Analysis \\
\hline DIP & Digital Image Processing & $\mathrm{NI}$ & Neutron Imaging \\
\hline DLC & Double Layer capacitance & OER & Oxygen Evolution Reaction \\
\hline DMA & Dynamic Mechanical Analysis & ORR & Oxygen Reduction Reaction \\
\hline DSC & Differential Scanning Calorimetry & PEM & Polymer Electrolyte Membrane \\
\hline DTF & Density Functional Theory & PEMFC & Polymer Electrolyte Membrane Fuel Cell \\
\hline HOR & Hydrogen Oxidation Reaction & PITM & Pt in the Membrane \\
\hline EDX & Energy Dispersive X-ray Analysis & $\mathrm{Pt} / \mathrm{C}$ & Platinum/Carbon Ratio \\
\hline EIS & Electrode Impedance Spectroscopy & PTL & Porous Transport Layer \\
\hline $\mathrm{EOL}=\mathrm{EOT}$ & End of Life $=$ End of Test & Queen's & Queen's University \\
\hline EPSA & Effective Pt Surface Area (cm2 Pt/cm2 geom) & $\mathrm{RDE}$ & Rotating Disk Electrode \\
\hline EW & Equivalent Weight & $\mathrm{RH}$ & Relative Humidity \\
\hline FA & Failure Analysis & SA & Surface Area \\
\hline FIB-SEM & Focused lon Beam SEM & SD/SU & Shut-down/Start-up \\
\hline NAA & Neutron Activated Analysis & SEM & Scanning Electron Microscopy \\
\hline GIT & Georgia Institute of Technology & UNM & University of New Mexico \\
\hline HFR & High Frequency Resistance & UoC & University of Calgary \\
\hline HOR & Hydrogen Oxidation Reaction & UPL & Upper Potential Limit \\
\hline HREELS & High Resolution Electron Energy Loss Spectroscopy & VLB & Voltage Loss Breakdown \\
\hline HRTEM & High Resolution Transmission Electron Microscopy & XPS & X-ray Photoelectron Spectroscopy \\
\hline HSAC & High Surface Area Carbon & XRD & X-ray diffraction \\
\hline
\end{tabular}


DE-EE0000466

Ballard Material Products Inc. 


\section{DE-EE0000466}

\section{Final Project Report}

Development of Micro-Structural Mitigation Strategies for PEM Fuel Cells: Morphological Simulations and Experimental Approaches

\section{CHAPTER I}

\section{INTRODUCTION}

Project Background and Objectives 


\section{TABLE OF CONTENTS}

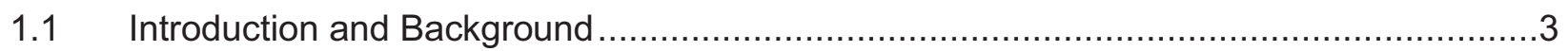

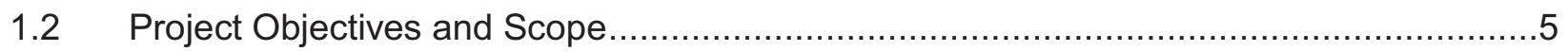

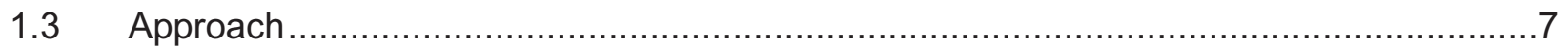

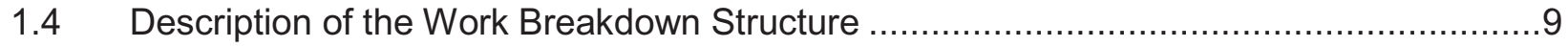

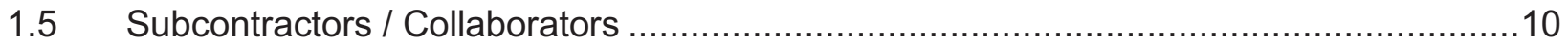

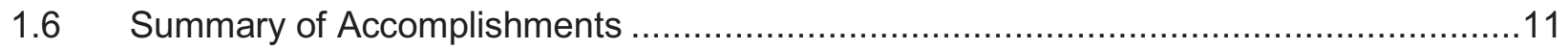

$1.7 \quad$ Publications and Conference Presentations ..................................................

\section{TABLE OF FIGURES}

Figure 1: Representation of the Pt catalyst dissolution and agglomeration mechanisms under fuel cell operation

Figure 2: Depiction of the catalyst carbon support corrosion mechanisms ............................

Figure 3: Open-source unit cell performance and durability model: use and applicability .........6

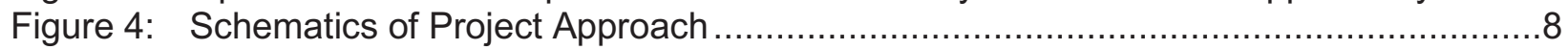

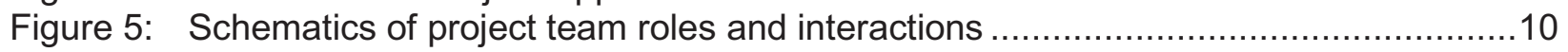


DE-EE0000466

Ballard Material Products Inc.

\subsection{Introduction and Background}

To be commercially viable, automotive and stationary fuel cells must exhibit comparable performance, durability, and cost to incumbent technologies. Of greatest importance is achieving cost reduction, fuel cell performance, and durability to 5000 hours for automotive applications and 60,000 hours for 1 to $10 \mathrm{~kW}$ stationary applications (2020 DOE targets) under realistic operating conditions including stop/start, low power, idling, dynamic operation, and subzero start-up. Although PEMFC technology has progressively advanced towards meaningful commercialization over the past several years and has made substantial advancements towards the DOE targets, further improvements in cost and durability are needed for high volume, full scale adoption of fuel cell products in the transportation and stationary markets. Of particular interest is the durability of the catalyst and catalyst layer which remain to be substantial contributions to fuel cell performance degradation specifically as MEAs are driven to lower Pt loadings in order to meet the commercialization cost targets.

Over the years significant progress has been made in identifying catalyst degradation mechanisms $^{1,2}$ and several key parameters that greatly influence the degradation rates, including electrode potentials, potential cycling, temperature, humidity, and reactant gas composition $^{2,3,4,5}$; however, many gaps with respect to catalyst layer degradation and an understanding of its driving mechanisms still exist. In particular, acceleration of the mechanisms under different fuel cell operating conditions, due to different structural compositions, and as a function of the drive to lower Pt loadings remains an area not well understood. In order to close these gaps, an understanding of the effect of operating conditions and the layer structure and composition on catalyst layer degradation mechanisms and degradation rates is needed.

The degradation mechanisms of the catalyst layer are represented by the cartoons in Figure 1 and Figure 2. These figures show that voltage is a key driver for catalyst degradation. Within the normal range of fuel cell operation bare Pt particles will exist only at low cathode potentials (high current densities) while PtOx films are being formed at potentials of $>0.7$ and possibly lower. Removal and re-forming of the PtOx layer will occur to some extent under dynamic operating condition but specifically during shutdown and start-up of the fuel cell due to the presence of air in the anode compartment. During start-up and shutdown the cathode may experience potential spikes in excess of $1.6 \mathrm{~V}$; this high cathode potential is a powerful driver for carbon corrosion.

1

J. Wu, X.Z. Yuan, J.J. Martin, H. Wang, J. Zhang, J. Shen, S. Wu, W. Merida, A Review of PEM Fuel Cell Durability: Degradation Mechanisms and Mitigation Strategies. Journal of Power Sources 184, 104-119.

2 R. Borup, J.P. Meyers, B. Pivovar, Y.S. Kim, R. Mukundan, N. Garland, D. Myers, M. Wilson, F. Garzon, D. Wood, P. Zelenay, K. More, K. Stroh, T. Zawodinski, J. Boncella, J.E. McGarth, M. Inaba, K. Miyatake, M. Hori, K. Ota, Z. Ogumi, S. Miyata, A. Nishikata, Z. Siroma, Y. Uchimoto, K. Yasuda, K. Kimijima, N. Iwashita, Scientific Aspects of Polymer Electrolyte Fuel Cell Durability and Degradation. Chemical Reviews 2007, 107, 3904-39

3 Y. Shao, G. Yin, Y. Gao, Understanding and Approaches for the Durability Issues of Pt-Based Catalysts for PEM Fuel Cell. Journal of Power Sources 2007, 171, 558-566.

4 M.S. Wilson, F. Garzon, K.E. Sickafus, S. Gottesfeld, Surface Area Loss of Supported Platinum in Polymer Electrolyte Fuel Cells. Journal of the Electrochemical Society 1993, 140, 2872-2876.

5 P.J. Ferreira, G.J. Ia O', Y. Shao-Horn, D. Morgan, R. Makharia, S. Kocha, H. Gasteiger, Instability of Pt/C Electrocatalysts Membrane Fuel Cells A Mechanistic Investigation. Journal of the Electrochemical Society 2005, 152, A2256-A2271. 
The structure of the catalyst can also greatly affect the voltage and water distribution in the catalyst layer as well as the proton and electron conduction facilitating local operating conditions that can greatly shift the onset of the Pt dissolution and/or carbon corrosion mechanisms. Thus, a better understanding of the mechanistic interactions as a function of operating conditions and cathode structure and composition is needed to enable the MEA designer to trade off fuel cell performance, durability, and cost more effectively.

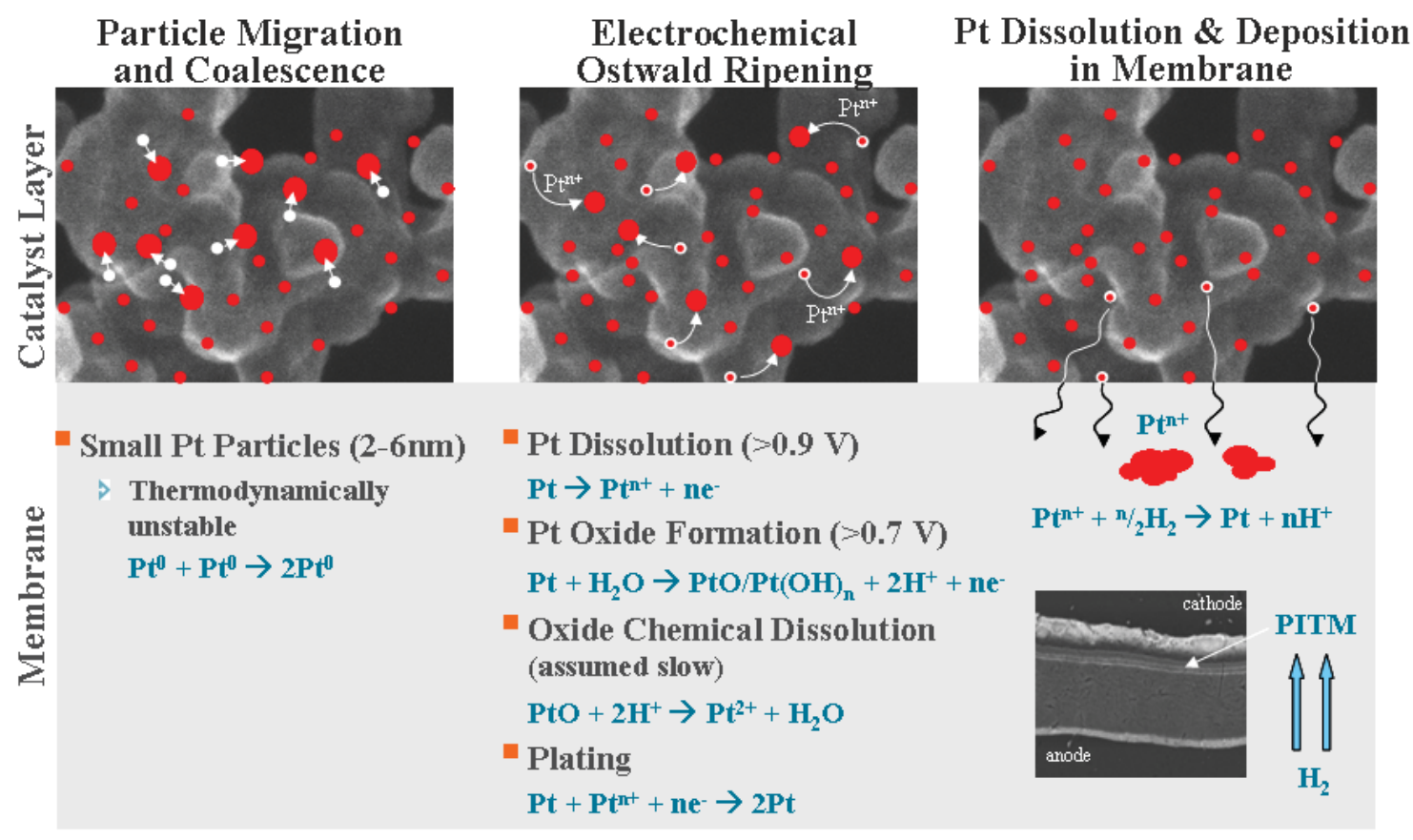

Figure 1: Representation of the Pt catalyst dissolution and agglomeration mechanisms under fuel cell operation

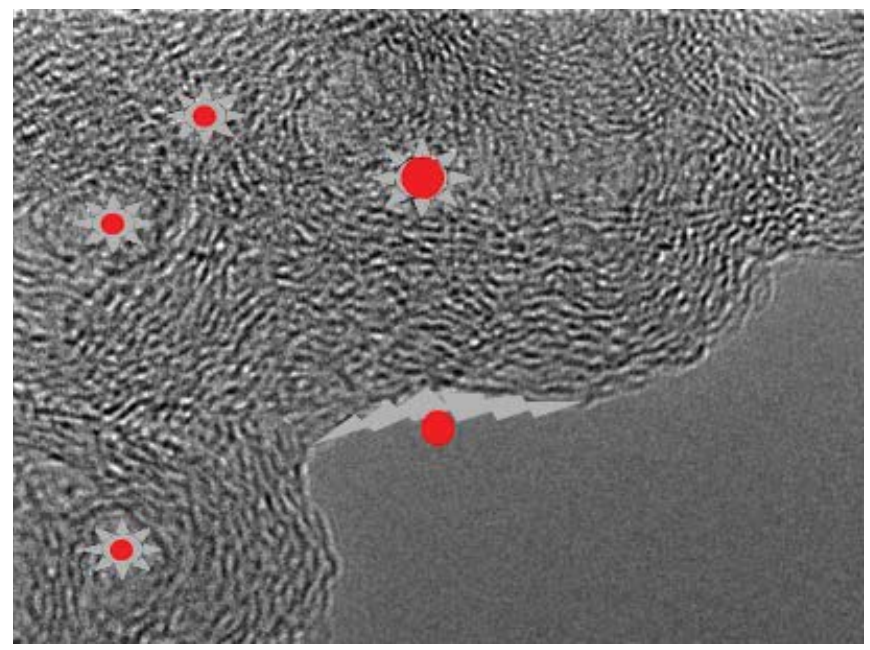

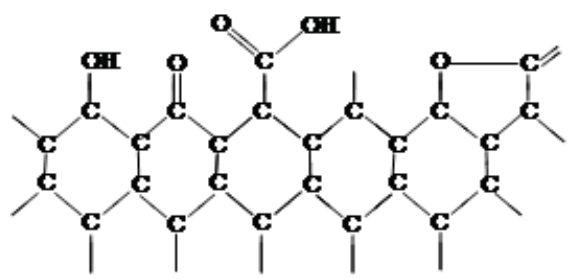

\section{Oxidation Reaction}

$-\mathrm{C}_{x}+\mathrm{yH}_{2} \mathrm{O} \rightarrow-\mathrm{C}_{\mathrm{x}} \mathrm{H}_{(2 \mathrm{y}-2)} \mathrm{O}_{\mathrm{y}}+2 \mathrm{H}^{+}+2 \mathrm{e}^{-}(\mathrm{E}>0.4 \mathrm{~V})$ Corrosion Reaction

$$
\begin{aligned}
2-\mathrm{C}_{x} \mathrm{H}_{y} \mathrm{O}+2 \mathrm{H}_{2} \mathrm{O} & \rightarrow 2 \mathrm{CO}_{2}+2 \mathrm{C}_{x-1} \mathrm{H}_{y}+4 \mathrm{H}^{+}+4 \mathrm{e}^{-} \\
-\mathrm{C}+2 \mathrm{H} 2 \mathrm{O} & \rightarrow \mathrm{CO}_{2}+4 \mathrm{H}++4 \mathrm{e}^{-} \quad(\mathrm{E}>1.2 \mathrm{~V})
\end{aligned}
$$

Figure 2: Depiction of the catalyst carbon support corrosion mechanisms 
DE-EE0000466

Ballard Material Products Inc.

\subsection{Project Objectives and Scope}

The overall goal of this project was to understand the relationships between localized operational conditions, $\mathrm{Pt}$ catalyst layer structures/composition, fundamental materials properties, and their interactions and coupling to the fundamental degradation mechanisms. The motivation was to provide the MEA designer with enhanced tools in the form of a forward predictive unit cell degradation model, correlations of fuel cell performance and durability with operational conditions and catalyst layer structure/composition, and identified durability design windows and mitigation strategies that facilitate the achievement of the 2020 DOE technical targets for stationary and automotive fuel cell applications.

Overall, the project focused on developing forward predictive models and conducted systematic cell degradation studies that enabled the quantification of the cathode catalyst layer degradation mechanisms and rates as well as the development of correlations linking materials properties with key fuel cell operational and catalyst layer structural parameters. The technical work included the following:

1. Identification and verification of catalyst degradation mechanisms:

(a) Pt dissolution, transport, and plating;

(b) Carbon-support oxidation and corrosion; and

(c) Mechanism coupling, feedback, and acceleration.

2. Correlations of compiled data for catalyst performance and structural change as a function of:

(a) Catalyst layer and unit cell operational conditions;

(b) Catalyst layer morphology and composition; and

(c) Gas diffusion layer (GDL) properties.

3. Development of kinetic and material models for catalyst layer aging:

(a) Macro-level unit cell degradation model;

(b) Micro-scale catalyst layer degradation model; and

(c) Molecular dynamics degradation model of the platinum/carbon/ionomer interface.

4. Mitigation strategies for catalyst degradation through modification of:

(a) Operational conditions, and

(b) Component structural morphologies and compositions.

The deliverables of this work are an open source MEA performance and degradation model for use by the industry at large, experimental design curves and correlations linking MEA performance and degradation with cathode catalyst layer structure/composition, and materials properties and operational conditions. A high level schematic of the model applicability and its potential use by the fuel cell industry is shown in Figure 3. 


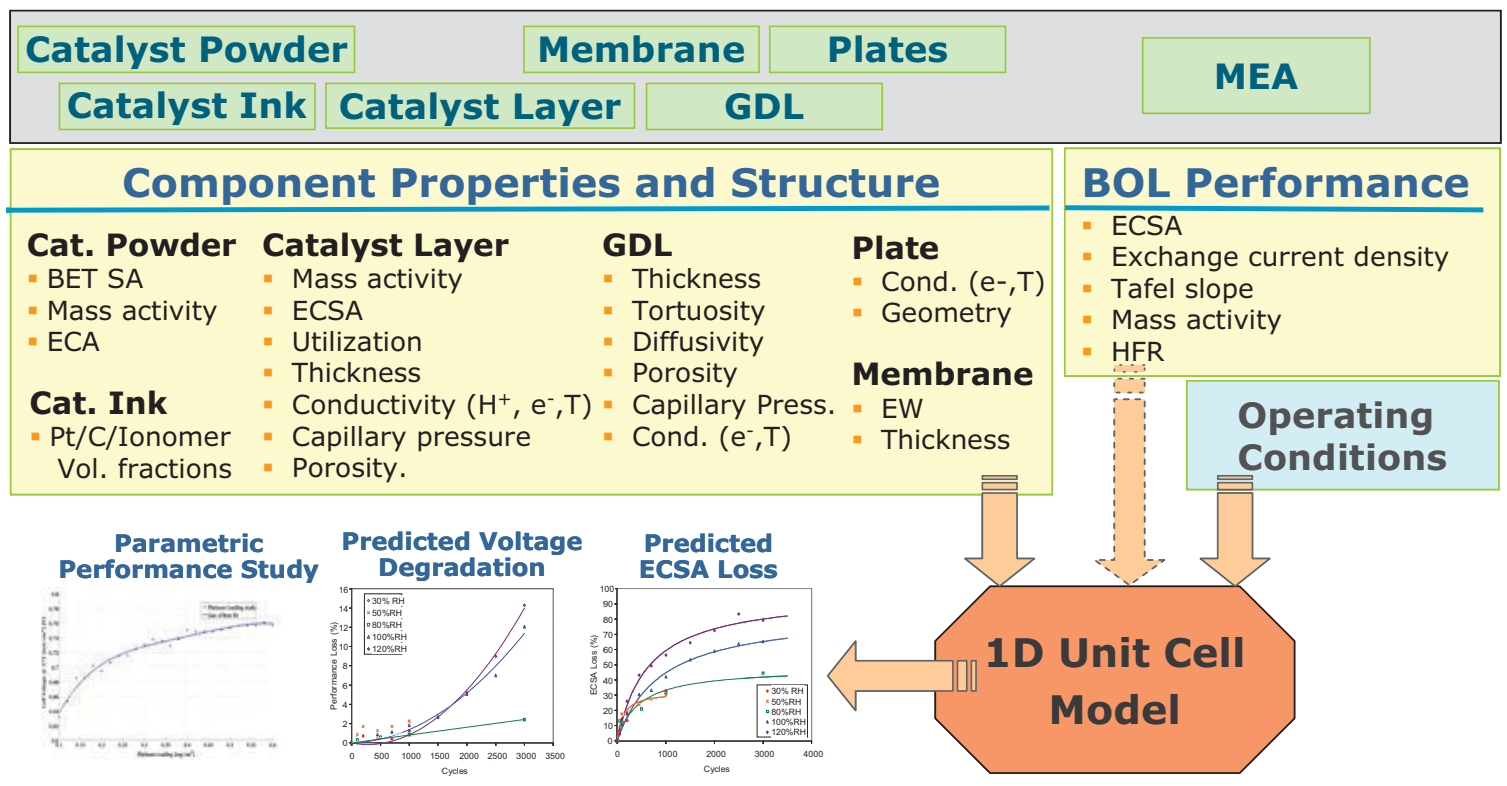

Figure 3: Open-source unit cell performance and durability model: use and applicability

This work addressed the following technical barriers of the DOE Hydrogen, Fuel Cells, and Infrastructure Technology Program Multi-year Research, Development, and Demonstration Plan': (1) Durability of Pt catalysts and Pt catalyst layers (structure/composition and operational conditions), (2) Performance of $\mathrm{Pt}$ catalyst layers (structure/composition), and (3) Cost (indirectly).

The project led by Ballard Power Systems included critical contributions from Georgia Institute of Technology, Los Alamos National Laboratory, Michigan Technical University, Queen's University, and the University of New Mexico. Their roles are discussed in Section 1.5.

6 Hydrogen, Fuel Cells \& Infrastructure Technologies Program: Multi-Year Research, Development and Demonstration Plan, U.S. Department of Energy, Energy Efficiency and Renewable Energy, 2011 revision.

http://www.eere.energy.gov/hydrogenandfuelcells/mypp/ 
DE-EE0000466

Ballard Material Products Inc.

\subsection{Approach}

The work focused on the generation of operational and structural design curves through experimental AST testing, characterization and novel morphological simulations of fuel cell materials and components. Design curves are powerful tools enabling the MEA designer to perform trade-off analyses between performance, cost, and durability. The approach taken in this project was two-fold:

1. Development of performance models at the molecular, micro-structural, and macrohomogeneous scales that include the degradation effects related to platinum dissolution/transport/plating, carbon surface oxidation/corrosion, and ionomer thinning/ conductivity loss. The models will provide the ability to study the effects of composition, the operational window and morphological design on catalyst degradation via Accelerated Stress Testing (AST). The design curves generated in each scale of the modeling work will enable the development of mitigation strategies through trade-off analysis.

2. Development of key operational and catalyst/catalyst layer structural degradation design curves enabling the identification of mitigation strategies and providing experimental validation for various levels of the degradation models above. Accelerated Stress Testing was coupled with 'state of the art' in-situ/ex-situ characterization techniques that allow the correlation of performance loss with structural changes.

The specific project activities included the following:

- Evaluation and improvement in the understanding of the fundamental material degradation mechanisms and the effects of mechanism-mechanism couplings

- Assessment of the impact of microstructure and morphology, as a function of catalyst layer composition, on the performance and durability of the catalyst layer

- Assessment of the stability of the three-phase region within the catalyst layer during degradation processes

- Evaluation of the mechanical and/or chemical stability of the GDL/electrode and catalyst/support

- Development of correlations between catalyst layer durability and operational conditions

- Development of correlations between catalyst layer durability and catalyst layer materials/morphology

- Creation of micro-scale and macro-scale kinetic and materials models of beginning of the life performance and of the aging process, including validation and demonstration of operational and structural mitigation strategies

- Development of durability design windows

- Implementation of the performance and materials aging models into an open source software and release of the software to the general public

The interaction between the various levels of activities is depicted in Figure 4. The cartoon shows the multi-scale modeling approach and the interfaces between the different models, the experimental investigations, and component characterizations. All elements are intimately linked such that simulated or experimental data flows from the lowest simulation scale to the next higher level. 
DE-EE0000466

Ballard Material Products Inc.

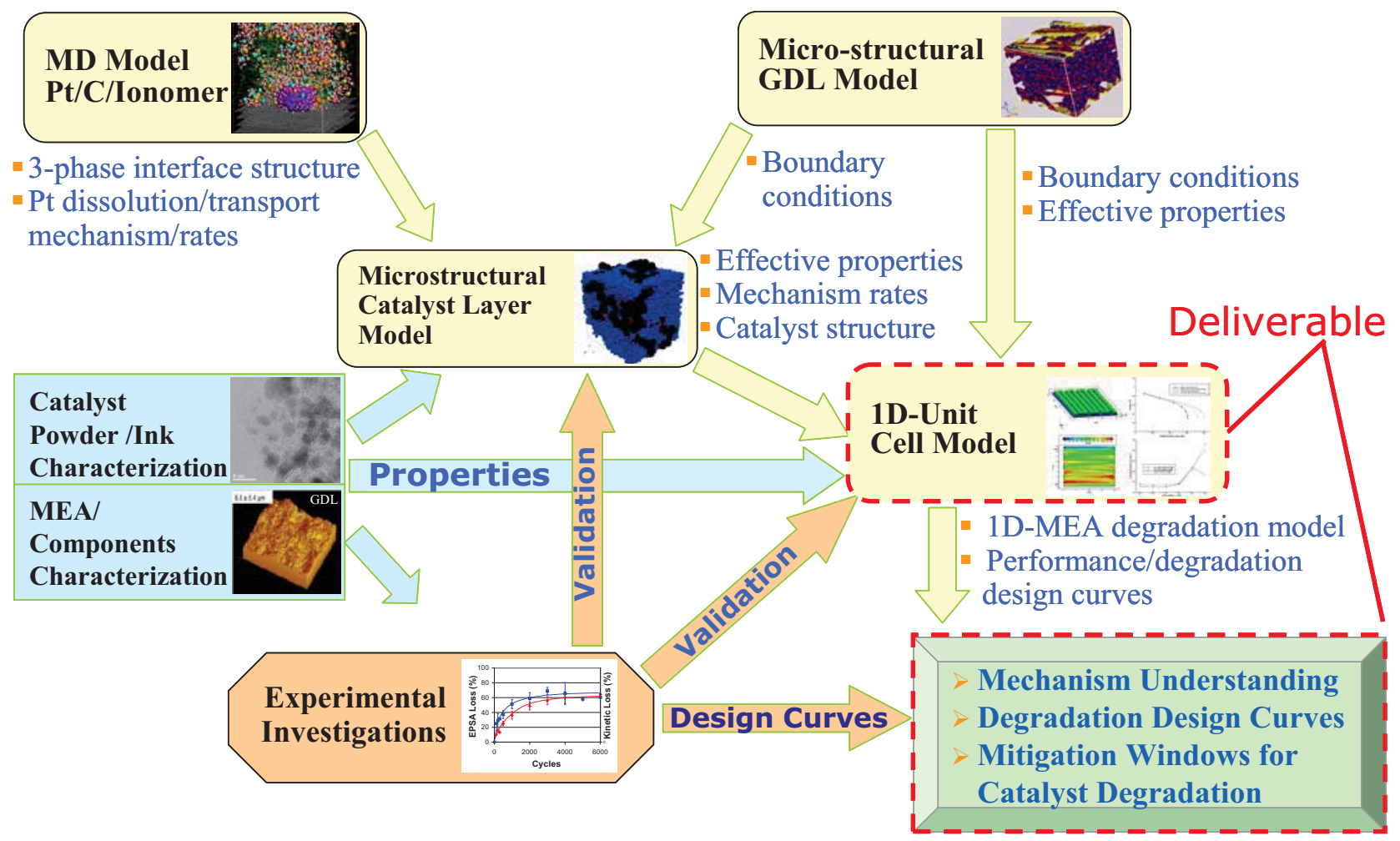

Figure 4: Schematics of Project Approach 
DE-EE0000466

Ballard Material Products Inc.

\subsection{Description of the Work Breakdown Structure}

The project was separated into three separate and parallel running work streams described briefly below. The detailed work is described in Chapters II to VII.

\section{Work Stream 1 - Theoretical Simulation of Fuel Cell Degradation}

The objective of this work was to develop and extend BOL performance models at the molecular, micro-structural, and macro-homogeneous scales to include the degradation effects related to platinum dissolution/transport/plating, carbon surface oxidation/corrosion, and ionomer thinning/conductivity loss. The models will provide the ability to study the effects of composition, the operational window and morphological design on catalyst degradation and are validated via accelerated stress test (AST) cycling. The design curves generated in each scale of the modeling work will enable the development of mitigation strategies through trade-off analysis.

\section{Work Stream 2 - Experimental Investigation of Fuel Cell Degradation}

The objective was to develop key operational and catalyst/catalyst layer structural degradation design curves enabling the identification of mitigation strategies and providing experimental validation for various levels of degradation models. ASTs coupled with 'state of the art' in-situ/ex-situ characterizations techniques that allow the correlation of performance loss with structural changes were used. The ASTs were performed on Ballard test stations and the following experimental variables were measured, during the testing, for both the fuel and oxidant at the inlet/outlet regions: voltage, current, $\mathrm{T}, \mathrm{RH}, \mathrm{P}$, gas flow, coolant flow/temperature, oxidant/fuel composition, and cell resistance.

\section{Work Stream 3 - Materials \& Component Characterization}

The objective was to measure materials changes for the degradation mechanisms; the materials information generated was used to de-convolute degradation AST data and provided model input for model validation. Membrane electrode assembly (MEA) components and MEAs at beginning of life (BOL), after conditioning, and after AST testing were evaluated using different characterization techniques. The following were achieved:

(1) fundamental materials properties measured,

(2) material properties changes with progressive catalyst layer aging identified,

(3) degradation rates for given mechanisms established, and

(4) degradation mechanisms through comparison with theory confirmed. 
DE-EE0000466

Ballard Material Products Inc.

\subsection{Subcontractors / Collaborators}

Ballard, the prime contractor of the work, was supported by experts from five different organizations: the Fuel Cell Research Center of Queen's University (Queen's), Georgia Institute of Technology (GIT), University of New Mexico (UNM), Michigan Technological University (MTU), and Los Alamos National Laboratory (LANL).

The Ballard team, with Dr. Silvia Wessel as the principle investigator, David Harvey leading the model developments, and Dr. Vesna Colbow leading the experimental investigations, were responsible for the overall project deliverables and more specifically the development of the micro-structural catalyst layer and unit cell models and the development of performance and AST based degradation correlations and durability windows. The modeling works as well as some catalyst characterizations were supported by Drs. Pharoah and Karan of Queen's University and University of Calgary, respectively. Dr. Jung of GIT developed the molecular dynamics model of the catalyst 3-phase interface and the molecular scale Pt dissolution and transport mechanisms, while Drs. Atanassov and Artyushkova of UNM investigated materials properties, principle component parameters, and selected effective properties of the catalyst layer. Dr. Allen of MTU developed a catalyst layer capillary pressure tool and characterized the GDL and catalyst layers, and Dr. Shahbazian-Yassar of MTU investigated methodologies to characterize catalyst layer/GDL and membrane interface changes of degraded MEAs. Dr. Borup and his team at LANL characterized materials properties of selected catalyst layers using a range of analytical techniques and conducted specific neutron imaging experiments.

A schematic of the responsibilities and interactions among the different project partners is shown in Figure 5.

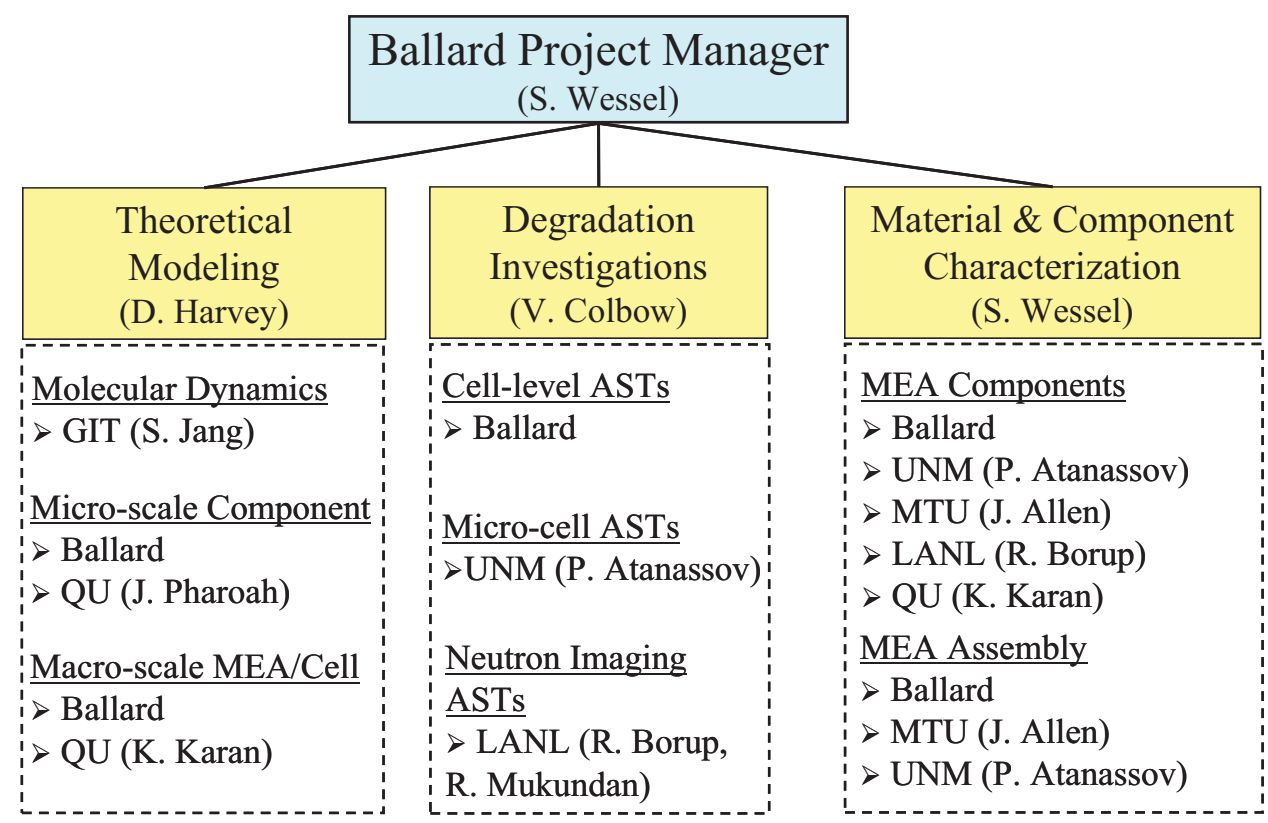

Figure 5: Schematics of project team roles and interactions 
DE-EE0000466

Ballard Material Products Inc.

\subsection{Summary of Accomplishments}

The key and unique accomplishments that have been disseminated in various forms throughout the project duration are:

- Molecular-dynamics based description of the carbon supported-Pt and ionomer system

- Evaluation of the platinum surface coverage of a variety of moieties

- Identified preferred Pt dissolution site on a Pt crystal anchored on a carbon surface

- Extracted $\mathrm{Pt}$ ion transport/diffusion rates in the ionomer.

- Extended validation of the 1D-statistical Unit Cell Performance model to include ranges of composition.

○ $\mathrm{Pt}: \mathrm{C}$ ratio, $20-80 \%$

○ (Pt:C): lonomer ratio, $13-50 \%$

○ Pt loading, $0.05-0.5 \mathrm{mg} / \mathrm{cm}^{2}$

- Transient micro-structural catalyst model predicting local distribution of effective properties.

- Identified oxygen "storage" aspects of the ionomeric films

- Pt dissolution model for mixed Pt oxide formation from water and air for performance and degradation prediction.

o Coupled potential driven metal dissolution models to an improved oxide ORR model

- Semi-empirical carbon corrosion model

- Open-source forward predictive MEA performance and degradation model that predicts accelerated stress test performance, which will be made available for public use.

- Model developed in open-source platform that allows for continued improvement of the model in the public domain

o Developed in common open-source platform that is fully supported by a commercial entity

- Correlations at BOT (beginning of test) and EOT (end of test) based on performance loss breakdown

- Effect of cathode catalyst layer structure and composition

- Effect of catalyst material properties:

$\mathrm{Pt} / \mathrm{C}$ ratio, $\mathrm{Pt}$ loading, carbon type and surface species and operational conditions $(\mathrm{RH}, \mathrm{T})$

- Catalyst layer durability windows and design curves.

- Based on correlations at both BOT and EOT

- A design flow path of interactions from materials properties and catalyst layer effective properties.

- Includes performance loss breakdown for BOT and degraded catalyst layers

This work has provided significant advancements in the ability to model and design durable fuel cell products and in the understanding of the role of catalyst layer morphology on the durability of the MEA. This work will subsequently assist in reducing the iterative design/test cycle process for next generation fuel cell products. 
DE-EE0000466

Ballard Material Products Inc.

\subsection{Publications and Conference Presentations}

\section{Publications}

2010

1. Anant Patel, Kateryna Artyushkova, Plamen Atanassov, Alan Young, Monica Dutta, Zaid Ahmad, Vesna Colbow, Silvia Wessel, and Siyu Ye, "Structural and Morphological Properties of Carbon Supports: Effect on Catalyst Degradation", ECS Transactions 33(1), $425-4312010$.

2011

2. A Patel, K. Artyushkova, P. Atanassov, D. Harvey, M. Dutta, V. Colbow, S. Wessel, "Effect of Graphitic Content on Carbon Supported Catalyst Performance", ECS Transactions 41 (1), 845-852, 2011.

3. S. Knights, R. Bashyam, P. He, M. Lauritzen, C. Startek, V. Colbow, J. Kolodziej, and S. Wessel, "PEMFC MEA and System Design Considerations", ECS Transactions 41 (1), Plenary Session, $39-53,2011$. (Note: Small DOE project content)

4. J. Pharoah, H-W. Choi, C-C Chueh, D. Harvey, "Effective Transport Properties Accounting for Electrochemical Reactions of Proton-Exchange Membrane Fuel Cell Catalyst Layers", ECS Transactions 41 (1), 221-227, 2011.

5. E. F. M'edici, J. S. Allen, "Modeling and Diagnostics of Fuel Cell Porous Media for Improving Water Transport”, ECS Transactions, 41 (1) 165-178 (2011).

2012

6. Patel, K. Artyushkova, P. Atanassov, V. Colbow, M. Dutta, D. Harvey, S. Wessel, "Investigating the Effects of PEMFC Conditions on Carbon Supported Platinum Electrocatalyst Composition and Performance", J. Vac Sci \& Tech,A: Vacuum, Surfaces, and Films 30 (4), 04D107-1 - 04D107-7, 2012.

7. D. Harvey, A. Bellemare-Davis, K. Karan, B. Jayansankar, J. Pharoah, V. Colbow, A. Young, S. Wessel, "Statistical Simulation of the Performance and Degradation of a PEMFC Membrane Electrode Assembly", ECS Transactions, 50 (2) 147-154 (2013).

8. S.A. Stacy and J.S. Allen, "Percolation in a Proton Exchange Membrane Fuel Cell Catalyst Layer", ECS Transactions, 50 (2) 877-884 (2012).

9. G.F. Brunello, J.I. Choi, D. Harvey and S.S Jang, "Multi-scale First-Principles Modeling of Three-Phase System of Polymer Electrolyte Membrane Fuel Cell", ECS Transactions, 50 (2) 155-160 (2012).

10. M. Khakaz-Baboli, D.A. Harvey, J.G.Pharoah, "Investigating the performance of catalyst layer micro-structures with different platinum loadings", ECS Transactions, 50 (2) 765772 (2012).

11. A. P. Young, V. Colbow, D. Harvey, E. Rogers, and S. Wessel, "A Semi-Empirical Two Step Carbon Corrosion Reaction Model in PEM Fuel Cells", Journal of The Electrochemical Society, 160 (4) F381-F388 (2013).

12. J.D. Fairweather, D. Spernjak, A.Z. Weber, D. Harvey, S.Wessel, D.S. Hussey, D.L. Jacobson, K. Artyushkova, R. Mukundan, and R.L. Borup, "Effects of Cathode Corrosion on Through-PlaneWater Transport in Proton Exchange Membrane Fuel Cells", Journal of The Electrochemical Society, 160 (9) F1-F14 (2013). 
DE-EE0000466

Ballard Material Products Inc.

\section{Conference Presentations}

2010

1. Anant Patel, Kateryna Artyushkova, Plamen Atanassov, Alan Young, Monica Dutta, Zaid Ahmad, Vesna Colbow, Silvia Wessel, and Siyu Ye, "Structural and Morphological Properties of Carbon Supports: Effect on Catalyst Degradation", 218 ECS Conference Presentation B7-D3.1 \#899, Las Vegas, 10 - 15 October 2010

2011

2. D. Harvey, A. Bellemare-Davis, J.G. Pharoah, K. Karan: Development of a validated statistical Beginning of Life (BOL) Performance Model, Hydrogen and Fuel Cells 2011 Conference, Vancouver, B.C., 15-18 May 2011

3. D. Harvey, J.G. Pharoah: Development of a full thickness micro structural catalyst model for estimation of effective properties and performance, Hydrogen and Fuel Cells 2011 Conference, Vancouver, B.C., 15-18 May 2011

4. Patel, K. Artyushkova, P. Atanassov, D. Harvey, M. Dutta, V. Colbow, and S. Wessel, "Effect of Graphitic Content on Carbon Supported Catalyst Performance", 220th ECS Meeting \& Electrochemical Energy Summit in Boston, Massachusetts, October 9-14, 2011

5. Jon Pharoah, Hae-Won Choi, Chih-Che Chueh, and David B. Harvey, "Effective Transport Properties Accounting for Electrochemical Reactions of Proton-Exchange Membrane Fuel Cell Catalyst Layers" 220th ECS Meeting \& Electrochemical Energy Summit in Boston, Massachusetts, October 9-14, 2011.

6. S. Knights, R. Bashyam, P. He, M. Lauritzen, C. Startek, V. Colbow, J. Kolodziej, and S. Wessel, "PEMFC MEA and System Design Considerations", 220th ECS Meeting \& Electrochemical Energy Summit in Boston, Massachusetts (October 9-14, 2011) (Note: Small DOE project content)

7. N. N. Parikh and R. Shahbazian-Yassar, "AFM Investigation of MEA Degradation in PEM Fuel Cells", 220th ECS Meeting \& Electrochemical Energy Summit in Boston, Massachusetts (October 9-14, 2011).

8. E. F. M'edici, D. L. Fritz, S. A. Stacy and J. S. Allen, "Modeling and Diagnostics of Fuel Cell Porous Media for Improving Water Transport", 220th ECS Meeting \& Electrochemical Energy Summit in Boston, Massachusetts (October 9-14, 2011).

9. D.B. Harvey, M. Khakbazbaboli, B. Jayasankar, C.C. Chueh, C.A. Bellemare-Davis, J.G. Pharoah, and K. Karan, "Multi-scale Modelling of the PEMFC Catalyst Layer: Coupling Microstructure to Performance", 220th ECS Meeting \& Electrochemical Energy Summit in Boston, Massachusetts (October 9-14, 2011)

10. Silvia Wessel, David Harvey, Vesna Colbow, "Considering thee Role of Component Structure on PEM Fuel Cell Durability", Zing Hydrogen and Fuel Cells Conference 2011, Xcaret Mexico, December 1 to 5, 2011

11. A. Patel, K. Artyushkovaa, P. Atanassov, V. Colbow, M. Duua, D. Harvey, and S. Wessel, "Investigating the Effects of PEMFC Conditions on Carbon Supported Platinum Electrocatalyst Composition and Performance", submitted abstract - AVS 58th International Symposium and Exhibition, Nashville, Tennessee, October 30, 2011 November 04, 2011. 
DE-EE0000466

Ballard Material Products Inc.

\section{2}

12. Silvia Wessel, Vesna Colbow, David Harvey, Shanna Knights, "The Effect of Cathode catalyst structure on PEM Fuel cell Durability" , Grove Fuel Cells Conference 2012, Berlin, April 11-12, 2012

13. G. Brunello, J.I. Choi, S.S Jang, "Multi-scale First Principle Modeling of Three-Phase System of Polymer Electrolyte membrane Fuel cell", submitted abstract $222^{\text {nd }}$ ECS Meeting, Honolulu, Hawai'l, October 7 -12, 2012

14. G. Brunello, J.I. Choi, S.S Jang, "Density Funcional Theory Study and Model development on Pt-Nano-particles", submitted abstract $222^{\text {nd }}$ ECS Meeting, Honolulu, Hawai'l, October $7-12,2012$

15. G. Brunello, J.I. Choi, S.S Jang, "Density Functional Theory Study of Pt Dissolution at Water-Pt Interface", submitted abstract $222^{\text {nd }}$ ECS Meeting, Honolulu, Hawai'l, October $7-12,2012$.

16. V. Colbow, M. Dutta, A. Young, Z. Ahmad, D. Harvey, S. Wessel, "Performance Degradation and Structural Changes with Pt Cathode Catalyst layer Design", submitted abstract $222^{\text {nd }}$ ECS Meeting, Honolulu, Hawai'l, October $7-12,2012$

17. D.Harvey, A. Bellemare-Davis,K. Kunal, B. Jayansankar, J. Pharoah, V. Colbow, A. Young, S. Wessel, "Statistical Simulation of the Performance Degradation of a PEMFC Electrode Assembly" submitted abstract $222^{\text {nd }}$ ECS Meeting, Honolulu, Hawai'l, October $7-12,2012$

18. K. Artyushkova, P. Atanassov, M. Dutta, V. Colbow, S. Wessel, "Ex-situ Characterization of Degradation Mechanism of MEAs by ilmaging XPS and SEM", submitted abstract $222^{\text {nd }}$ ECS Meeting, Honolulu, Hawai'l, October 7-12, 2012

19. S.A. Stacy, J.S. Allen, Percolation in Catalyst Layer of PEMFC", submitted abstract $222^{\text {nd }}$ ECS Meeting, Honolulu, Hawai'l, October 7-12, 2012.

20. R. Shahbazian-Yassar, "Membrane Electrode Assembly Degradation in Proton Exchange Membrane Fuel Cells: A View From Nanoscale", International Conferences of Young Researchers on Advanced Materials, July 1-6, 2012, Singapore.

21. M. Khakaz-Baboli, D.A. Harvey, J.G.Pharoah, "Investigating the performance of catalyst layer micro-structures with different platinum loadings", submitted abstract 222nd ECS Meeting, Honolulu, Hawai'l, October 7-12, 2012.

22. S. Wessel, V.Colbow, M. Dutta, A. Young, D. Harvey, "The impact of Materials Properties and Cathode Catalyst Structure/Composition on MEA Performance and Durability", Hydrogen and Fuel Cells 2013, Vancouver, June 16 - 19, 2013.

23. D. Harvey, A. Bellemare-Davis, B. Jayasankar, K. Karan, J. Pharoah, A. Young, M. Dutta, V. Colbow, S. Wessel, "Simulation of the ORR Multi-pathway and Catalyst Dissolution for a PEMFC: The Effect of Surface Coverage and AST cycling", Hydrogen and Fuel Cells 2013, Van couver, June 16 - 19, 2013.

24. S. Wessel, "Water management in Low Temperature PEM Fuel Cells, Industrieworkshop: Wassermanagement in PEM-Brennstoffzellen, Freiburg (Germany), 31 January 2013.

25. K. Artyushkova, P. Atanassov, M. Dutta, D. Harvey, V. Colbow, and S. Wessel, "Structural Correlations: Design Levers for Performance and Durability of Catalyst Layers" submitted abstract 224th ECS Meeting, San Francisco, CA, October 27 November 1, 2013. 
DE-EE0000466

Ballard Material Products Inc.

\section{Other Technical Presentations}

2009

2010

1. New Fuel cells Project meeting, Washington, October 1, 2009

2. Fuel Cell Tech Team Presentation, Detroit, January 13, 2010

3. 2010 Annual Merit Review Poster Presentation, Washington, June 8, 2010

4. Technical Progress Review, Vancouver, 18 November 2010 (Telecon) 2011

5. Fuel Cell Tech Team Presentation, Washington, 13 February 2011

6. 2011 Annual Merit Review Presentation, Arlington, May 9, 2011

7. Go/No-Go presentation, Vancouver, 25 June 2011. 2012

8. Fuel Cell Team meeting, Detroit, March 14, 2012

9. 2012 Annual Merit Review Presentation, Arlington, May 16, 2011 
DE-EE0000466

Ballard Material Products Inc. 


\section{DE-EE0000466}

\section{Final Project Report}

Development of Micro-Structural Mitigation Strategies for PEM Fuel Cells: Morphological Simulations and Experimental Approaches

\section{CHAPTER II}

\section{EXPERIMENTAL INVESTIGATIONS:}

\section{Accelerated Stress Testing and Characterization of BOT and Degraded MEAs}




\section{TABLE OF CONTENTS}

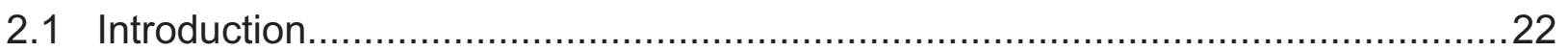

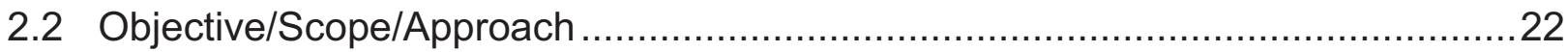

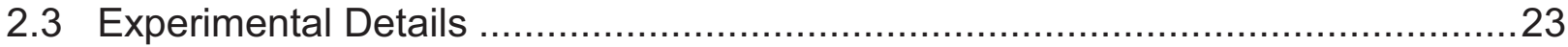

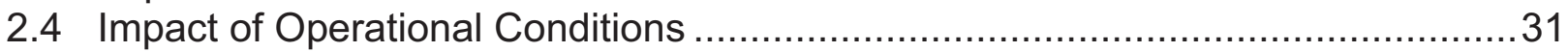

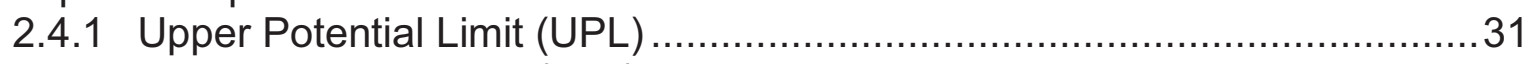

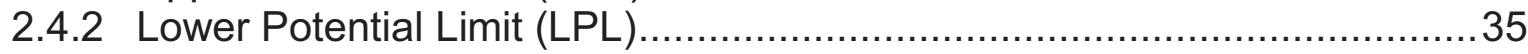

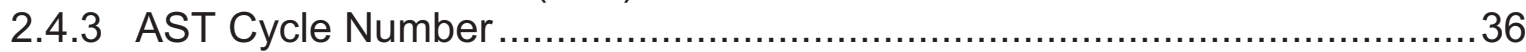

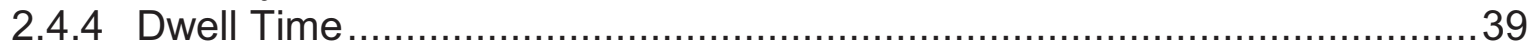

2.4.5 Cathode Relative Humidity …........................................................ 41

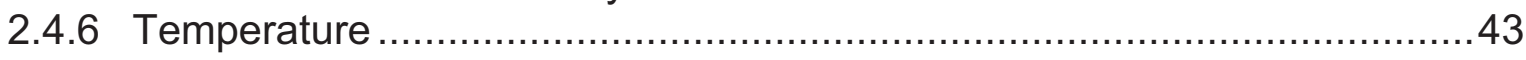

2.4.7 Reactant Composition ................................................................... 45

2.4.8 MEA Water Content at 1.4V UPL - Neutron Imaging Results (LANL) .......47

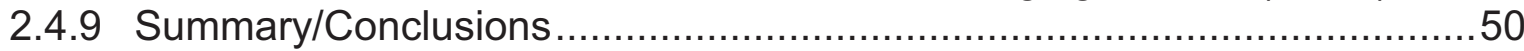

2.5 Impact of Catalyst Layer Structure and Composition ...................................52

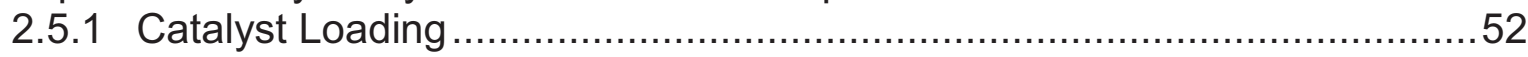

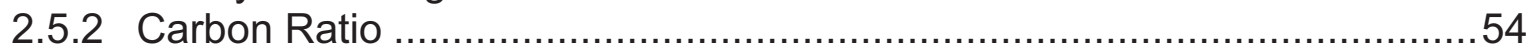

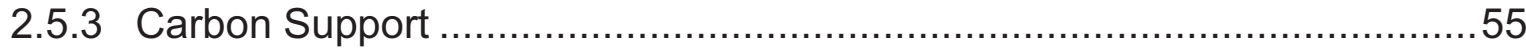

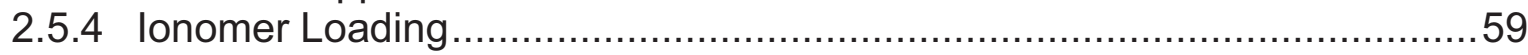

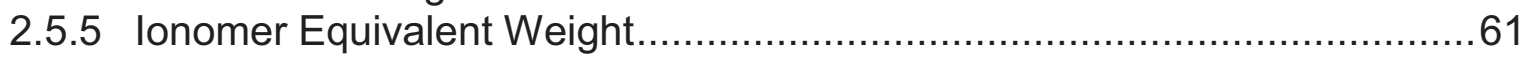

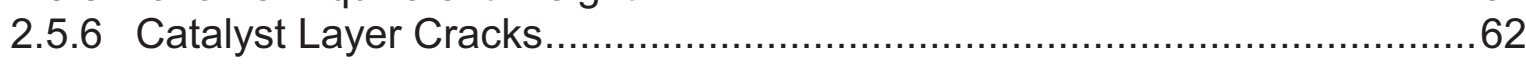

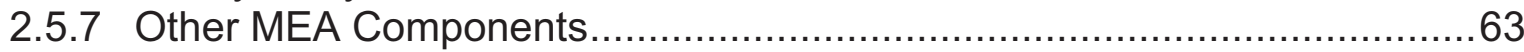

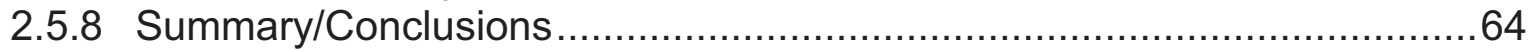


DE-EE0000466

Ballard Material Products Inc

\section{LIST OF FIGURES}

Figure 1: Schematic of experimental approach for developing durability design curves and mitigation strategies for fuel cell durability......

Figure 2: End of test (EOT) data for both the DOE and the Ballard ASTs. (a) Performance diagnostics were done at $65^{\circ} \mathrm{C}, 100 \% \mathrm{RH}, 5$ psig; (b) ECSA obtained using CO-

stripping.....

Figure 3: Ex-situ characterization at end of test (EOT) for the DOE and Ballard ASTs. (a) Quantification of the changes in membrane and the cathode catalyst layer; (b) SEM of MEA cross-section tested using Ballard AST at EOT; (c) SEM of MEA cross-section tested using DOE AST at EOT....

Figure 4: Schematics of AST diagnostics including list of down-selected ex-situ and in-situ measurement techniques.

Figure 5: Voltage cycling profile used to evaluate effect of (a) UPL and (b) shutdown/startup on catalyst degradation .............................................................................

Figure 6: Performance at $0.67 \mathrm{~A} / \mathrm{cm}^{2}$ as a function of AST voltage profiles for Pt catalyst ........32

Figure 7: Cyclic voltammetry at beginning-of-test and end-of-test for Pt catalyst supported

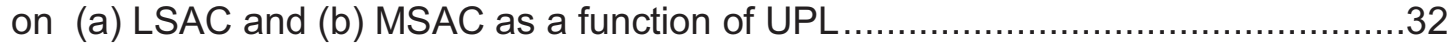

Figure 8: Per cent ECSA loss as a function of cycle number for ASTs cycled to different UPLs for both MSAC and LSAC Pt catalyst

Figure 9: Impact of UPL during cycling on the Pt dissolution mechanism. (a) Pt agglomeration rate and Pt size as a function of UPL; (b) PITM accumulation at end of test (EOT) as a function of UPL

Figure 10: Impact of UPL on the carbon support corrosion. (a) Catalyst layer thickness; (b) Rate of thickness loss as a function of UPL

Figure 11: (A) Percentage of ESCA and performance loss, at $1 \mathrm{~A} / \mathrm{cm}^{2}$, as a function of lower potential limit during AST cycling. (B) Pt size growth as a function of LPL during AST cycling...

Figure 12: Impact of AST cycle number on in-situ and ex-situ characterization techniques.

(a) Air Performance loss and voltage loss breakdown. (b) EPSA loss and catalyst activity loss. (c) Breakdown of ECSA loss into agglomeration, PITM and washout components. (d) Per cent catalyst thinning.

Figure 13: Breakdown of the EPSA Loss in to Agglomeration, PITM and Washout components.

Figure 14: Correlations of in-situ to ex-situ diagnostic data for LSAC-50Pt: (a) Mass activity and performance losses as a function of PITM

Figure 15: Performance loss impact at UPL of 1.4V with $(A)$ time at UPL and (B) carbon loss..39

Figure 16: (a) the ECSA loss as a function of cycle dwell time and cumulative time at a 1.0V UPL, and (b) the ECSA loss normalized by cycle number as a function of cycle dwell time and cumulative time at a 1.0V UPL

Figure 17: (a) \% ECSA loss and (b) Pt in the membrane (PITM) as a function of upper

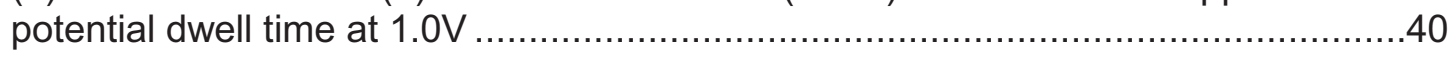

Figure 18: Effect of dwell time at 1.4V UPL on performance ......................................... 41

Figure 19: Effect of oxidant RH on BOT performance ..................................................42

Figure 20: Effect of RH after 4700 AST cycles on (a) Pt dissolution: Pt growth, PITM and ECSA, and (b) performance and ECSA

Figure 21: (A) Effect of time at UPL (corrosion time, $1.4 \mathrm{~V}, 80^{\circ} \mathrm{C}$ ) on ECSA loss for different oxidant relative humidities, (B) Effect of time at UPL (corrosion time, $1.4 \mathrm{~V}, 80^{\circ} \mathrm{C}$ ) on carbon loss for different relative humidities, 
Figure 22: (A) BOT performance sensitivity at $1 \mathrm{~A} / \mathrm{cm}^{2}$ with operating temperature, (B) ECSA loss after AST cycling at 1.2V UPL and different temperatures....

Figure 23: (A) Effect of corrosion time on carbon loss for different temperatures, (B) Arrhenius relationship for oxidation and corrosion rates (1.4V UPL)

Figure 24: Impact of temperature and relative humidity on the carbon corrosion mechanism $(1.4 \mathrm{~V})$

Figure 25: Performance sensitivity with oxygen concentration

Figure 26: \% ECSA loss as a function of AST Cycles $(0.6 \mathrm{~V}(30 \mathrm{sec})$ to $1.0 \mathrm{~V}(60 \mathrm{sec})$.

Figure 27: Effect $\mathrm{H}_{2}$ concentration on performance degradation rate, ECSA loss, and the position of the $\mathrm{Pt}$ in the membrane band after 4700 standard AST cycles (1.2V UPL)

Figure 28: Straight Channel Neutron Imaging Hardware.

Figure 29: Polarization curves after selected numbers of AST cycles at $1.4 \mathrm{~V}$ in NI hardware ...48

Figure 30: ECSA change after 750 cycles at 1.4V UPML (Neutron Imaging Hardware)...........49

Figure 31: Water density image changes at $0.6 \mathrm{~V}$ with progressive cycling with upper potential at $1.4 \mathrm{~V} \mathrm{UPL}$

Figure 32: Water density profiles of the MEA cross section at $0.6 \mathrm{~V}$ for $\mathrm{BOL}$ and after progressive cycling $\left(80^{\circ} \mathrm{C}, 100 \% \mathrm{RH} ; 0.6-1.4 \mathrm{~V}\right.$ cycles in $\mathrm{H} 2 /$ air $)$. (A) Middle of

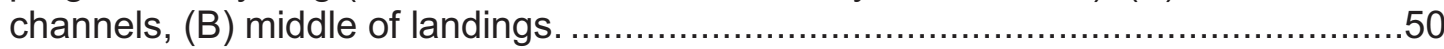

Figure 33: Effect of catalyst loading on BOT catalyst layer thickness and ECSA ...................52

Figure 34: BOT performance with loading at different temperatures, relative humidity, and oxygen concentrations....

Figure 35: (A) Effect of BOT Pt loading on degradation rate, $(B)$ correlation of performance and ECSA

Figure 36: (A) Performance change at various current densities as a function of AST (1.2V UPL) cycle number and (B) voltage loss breakdown at 4700 AST cycles for LSAC catalysts with different $\mathrm{Pt} / \mathrm{C}$ ratios....

Figure 37: (A) ECSA change and (B) Pt crystallite changes as a function of $\mathrm{Pt} / \mathrm{C}$ ratio for PtLSAC catalysts

Figure 38: BOL ECSA for different carbon support structures...

Figure 39: (A) Voltage degradation rate at $1.3 \mathrm{~A} / \mathrm{cm} 2$ and (B) kinetic loss after $4700 \mathrm{AST}$ cycles at an upper potential 1.0V and 1.2V for Pt catalysts supported on different

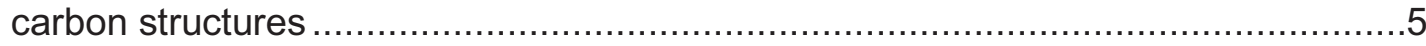

Figure 40: (A) Performance loss due to cathode catalyst ionic changes after 4700 cycles to an UPL of $1.0 \mathrm{~V}$ and $1.2 \mathrm{~V}$. (B) Catalyst layer thickness changes after 4700 cycles at an UPL of 1.2 with respect to catalyst powder ECS and carbon support graphitization

Figure 41: Effect of heat treatment on (a) Pt Particle size, catalyst surface area and metal surface area, and (b) catalyst powder porosity.....

Figure 42: BOL ECSA and performance $\left(\right.$ at $1 \mathrm{~A} / \mathrm{cm}^{2}$ ) for heat treated high surface area carbon catalyst.

Figure 43: ECSA and Performance Loss during AST cycling to 1.2V....

Figure 44: BOT catalyst layer structure and polarization curves for different ionomer loadings

Figure 45: A) EPSA loss with ionomer loadings; B) double layer capacitance change with AST cycling for different ionomer loadings....

Figure 46: BOT and EOT Voltage Loss Breakdown for different ionomer contents ........ 61

Figure 47: Performance characteristics at BOT and after degradation for different ionomer equivalents weights 
Figure 48: Performance and durability of catalyst layers containing an overall cracked area of 1 and $8 \%$.

Figure 49: Performance comparison of MEAs containing two different type of membranes; dense membrane $B$ (baseline MEA) and reinforced membrane A after 4700 AST cycles at $1.2 \mathrm{~V}$ and $1.3 \mathrm{~V}$ UPL

Figure 50: Impact of GDL with and without an MPL layer on CCL degradation; (A) ECSA loss and (B) Performance loss at $0.5 \mathrm{~A} / \mathrm{cm} 2$ as a function of AST cycles (0.6 to $1.3 \mathrm{~V})$

\section{LIST OF TABLES}

Table 1: Summary of DOE and Ballard AST conditions.

Table 2: Effect of different oxidants (air vs. $\mathrm{N}_{2}$ ) on AST results .....

Table 3: List of operating conditions for standard fuel cell operation and the corrosion accelerated stress test.

Table 4: Reproducibility of MEAs (BOT and EOT), including MEA and test station variability 28

Table 5: Effect of Operational Stressors on LSAC Catalyst Degradation............................. 29

Table 6: Operational Stress Test Conditions and Stress Values Evaluated ....................... 30

Table 7: Structural Design Parameters and Parameter Values Evaluated......................... 30

Table 8: Summary table of the operational conditions, their ranges and effects on BOT performance and degradation rates.

Table 9: Impact summary of the different operational stressors on the ECSA and voltage degradation rates calculated at 50\% ECSA I and 15\% performance loss. .............. 51

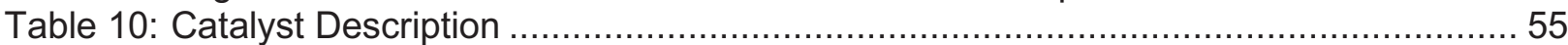

Table 11: Summary of Catalyst Layer Design Studies .............................................. 64 
DE-EE0000466

Ballard Material Products Inc

\subsection{Introduction}

This Chapter is dedicated to the in-situ fuel cell experimental investigations for MEA performance and degradation. It presents the accelerated stress test (AST) based investigations of the impact of operational conditions and catalyst layer structure/composition on the catalyst layer degradation mechanisms and rates. The chapter is divided into five subsections: (i) introduction, (ii) the objective of the different studies, (iii) a description of the experimental details, (iv) the impact of operational conditions, and (v) the impact of catalyst layer structure and composition. Sub-sections (iv) and (v) will also include an overall summary of the learnings. The experimental characterization of the MEA and catalyst layer components through various techniques are discussed in Chapter III and the developed correlations and design windows are presented in Chapter IV.

\subsection{Objective/Scope/Approach}

The overall objective of this work was to develop quantitative design curves and correlations (Chapter IV) that link catalyst layer component properties and catalyst layer effective properties with MEA performance and cathode catalyst layer degradation as a function of operating conditions. The composition, materials properties, and effective properties will serve as validation for the developed models.

The motivation of the experimental work is five-fold: (1) to verify and improve the understanding of known catalyst degradation mechanisms; (2) to identify and evaluate new degradation mechanisms and/or failure modes; (3) to correlate (and compile data) between operational conditions, composition/structure, and structural/performance degradation in the catalyst layer; (4) to recommend mitigation strategies for catalyst degradation; and (5) to generate model validation data sets.

In order to decouple the operational and structural effects on degradation mechanisms and their rates and changes in performance and catalyst layer properties as functions of both operating conditions and structural composition, a set of comprehensive in-situ/ex-situ measurements, ASTs, and a degraded MEA test strategy were applied. This involved the systematic characterization of the catalyst powder, catalyst layer, and MEA at the beginning of test (BOT), at various time intervals (MOT) during the course of the accelerated testing and at the end of the test (EOT). MEA performance and catalyst layer structural changes were analyzed and correlated with operational conditions and the initial structure. The results were compared with literature and theory for mechanism understanding and were used for the development of degradation design windows (Chapter IV) and the identification of mitigation strategies. A flow chart of the approach is shown in Figure 1. 


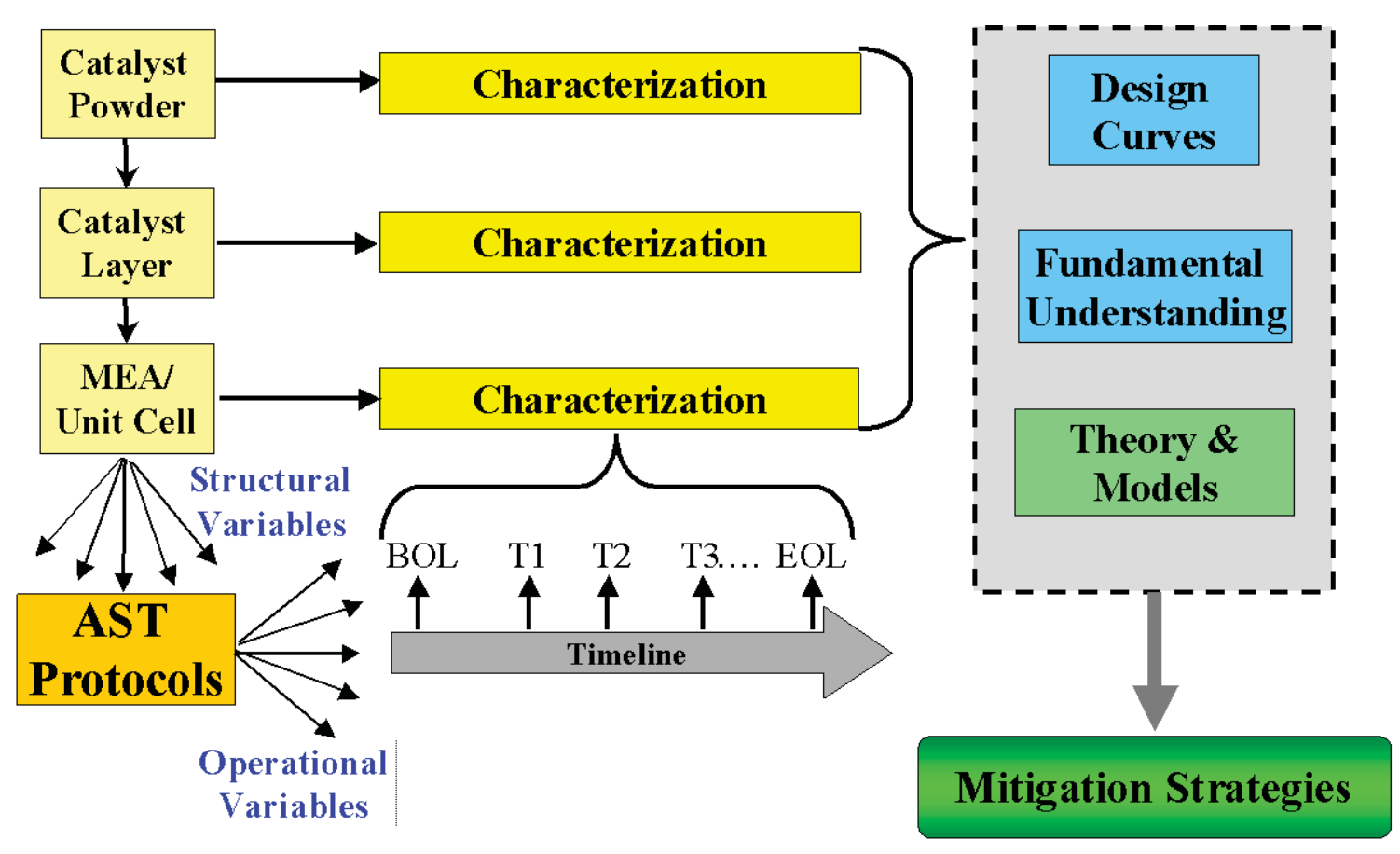

Figure 1: Schematic of experimental approach for developing durability design curves and mitigation strategies for fuel cell durability

\subsection{Experimental Details}

\section{Test Hardware and Reference MEA}

The test hardware used in this project was designed by Ballard to provide quasi-uniform operational conditions with the following features: bladder compression, high flow rates, liquid cooled temperature control, and carbon composite plates with parallel flow fields designed for low pressure and uniform flow, and an active area of $45 \mathrm{~cm}^{2}$. A reference MEA was used made of a catalyst coated membrane (CCM) with a Pt loading of $0.4 / 0.1 \mathrm{mg} / \mathrm{cm}^{2}$ (cathode/anode), $\mathrm{Pt}$ supported on graphitized carbon, a 50:50 Pt/C ratio, and Nafion ${ }^{\circledR}$ ionomer. The CCM was manufactured in-house using the Nafion ${ }^{\circledR}$ NR211 membrane. The gas diffusion layers (GDL) were made by Ballard Material Products (BMP) using a continuous process.

\section{Accelerated Cathode Stress Test Protocol}

A Ballard preferred reference cathode AST protocol for this program used a square voltage cycling profile. As this protocol is substantially different to the DOE recommended electro catalyst AST protocol a comparison between the two protocols was undertaken. The three primary AST protocol differences are: (1) $\mathrm{N}_{2}$ vs. Air, (2) voltage cycling profile, and (3) $1.0 \mathrm{~V}$ vs. $1.2 \mathrm{~V}$ upper potential. The DOE AST was adapted, in terms of inlet pressure and flow rates, to enable operation in the Ballard research hardware. Details of the two ASTs are given in Table 1. 
Table 1: Summary of DOE and Ballard AST conditions

\begin{tabular}{|l|c|c|}
\hline \multicolumn{1}{|c|}{ Attributes } & $\begin{array}{c}\text { DOE AST } \\
\text { Cycle Profile }\end{array}$ & Ballard AST \\
\cline { 2 - 3 } & Triangular Wave & Square Wave \\
\hline \multirow{2}{*}{ Time / Cycle } & $0.6 \mathrm{~V}$ to $1.0 \mathrm{~V}, 50 \mathrm{mV} / \mathrm{s}$ & $0.6 \mathrm{~V}(30 \mathrm{~s})$ to $1.2 \mathrm{~V}(60 \mathrm{~s})$ \\
\hline Number of Cycles & $16 \mathrm{~s}$ & $90 \mathrm{~s}$ \\
\hline Total Cycling Time & 30,000 & 5,000 \\
\hline Temperature & 133 hours & 125 hours \\
\hline RH Anode/Cathode & $80^{\circ} \mathrm{C}$ & $80^{\circ} \mathrm{C}$ \\
\hline Fuel / Oxidant & $100 \% / 100 \%$ & $100 \% / 100 \%$ \\
\hline Pressure & $\mathrm{H}_{2} 4450 \mathrm{sccm}$ & $\mathrm{H}_{2} 4450 \mathrm{sccm}$ \\
\hline
\end{tabular}

The comparison of DOE and Ballard AST results indicates that the performance and ECSA losses are very similar (Figure 2) and consistent with predominately kinetic performance changes for both ASTs. However, there is a significant discrepancy in the degradation mechanisms resulting from the different ASTs.

Figure 3 shows that the DOE AST resulted in larger average Pt crystallite growth compared to the Ballard AST (9.3nm vs. $7.4 \mathrm{~nm})$. This is expected since the DOE AST exhibits Pt accumulation at the cathode/membrane interface resulting in larger $\mathrm{Pt}$ crystallites at the interface, while the Ballard AST resulted in Pt in the membrane (PITM) (Figure 3). The primary operational factor accounting for these differences is the oxidant gas (air vs. $N_{2}$ ) used during the AST.
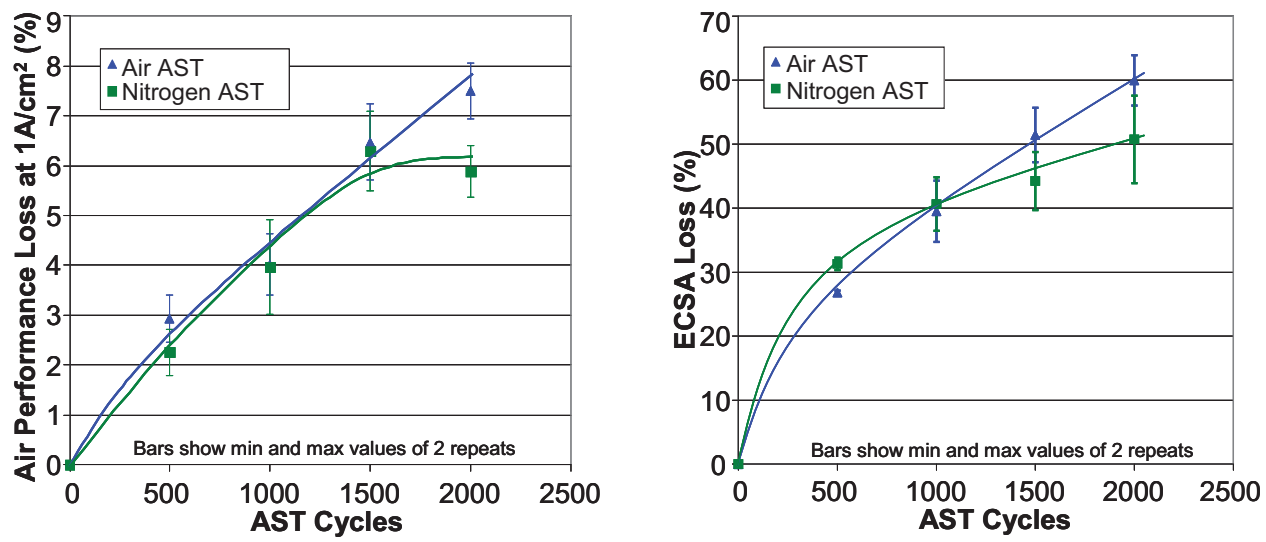

Figure 2: End of test (EOT) data for both the DOE and the Ballard ASTs. (a) Performance diagnostics were done at $65^{\circ} \mathrm{C}, 100 \% \mathrm{RH}, 5$ psig; (b) ECSA obtained using CO-stripping 
(a)

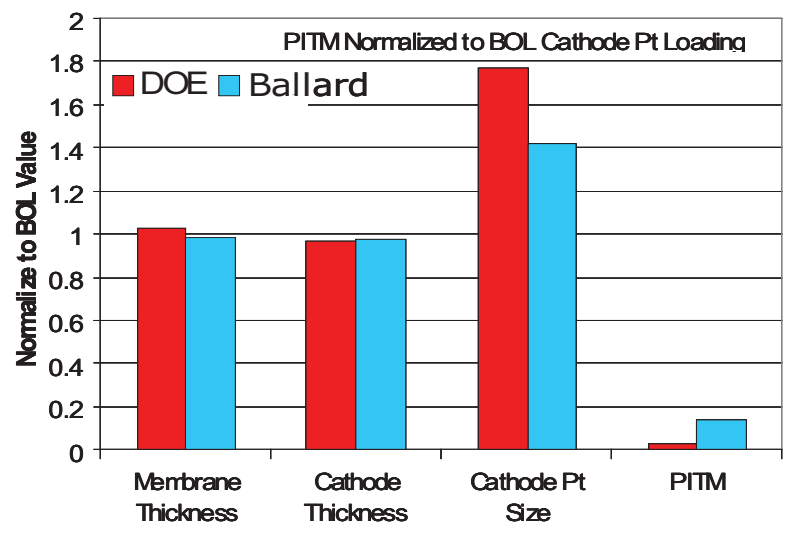

(b)

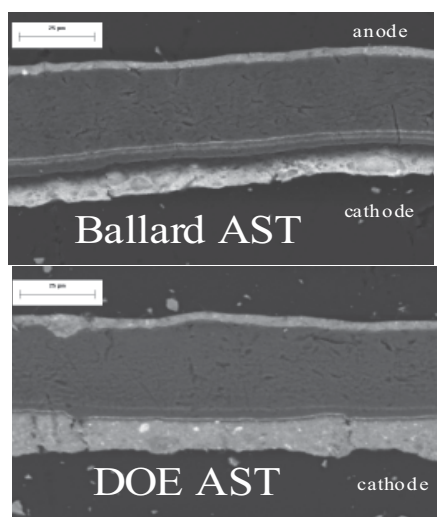

Figure 3: Ex-situ characterization at end of test (EOT) for the DOE and Ballard ASTs. (a) Quantification of the changes in membrane and the cathode catalyst layer; (b) SEM of MEA crosssection tested using Ballard AST at EOT; (c) SEM of MEA cross-section tested using DOE AST at EOT

Table 2 lists the failure modes, advantages, and limitations associated with using either air or $\mathrm{N}_{2}$ during the AST. Based on the findings, it was recommended to use the Ballard reference voltage degradation AST in this program for the following operational features: (1) Square wave potential cycling provides better control of upper and lower potential limits and more accurate control of dwell times resulting in accurate operational design curves; (2) Air operation is more realistic to field data and will enable quantification of PITM; (3) The upper potential limit of $1.2 \mathrm{~V}$ used in conjunction with the Ballard reference MEA enables better comparisons of state-of-the-art catalysts.

Table 2: Effect of different oxidants (air vs. $\mathrm{N}_{2}$ ) on AST results

\begin{tabular}{|c|c|c|c|}
\hline Oxidant & Failure Modes & Advantages & Limitations \\
\hline Nitrogen & $\begin{array}{l}\text { - Pt Agglomeration } \\
\text { - Carbon Surface } \\
\text { Oxidation } \\
\text { - Carbon Corrosion }\end{array}$ & $\begin{array}{l}\text { - Relationships can be } \\
\text { established without } \\
\text { interference of other } \\
\text { degradation modes } \\
\text { - RH can be controlled (No } \\
\text { product water effects) } \\
\end{array}$ & $\begin{array}{l}\text { - Does not simulate PITM } \\
\text { - Does not take into account } \\
\text { possible interference of } \\
\text { membrane degradation bi- } \\
\text { products }\end{array}$ \\
\hline Air & $\begin{array}{l}\text { - Pt Agglomeration } \\
\text { - PITM } \\
\text { - Carbon Surface } \\
\text { Oxidation } \\
\text { - Carbon Corrosion }\end{array}$ & $\begin{array}{l}\text { - Effect of Membrane } \\
\text { Degradation (bi-products) } \\
\text { on voltage degradation } \\
\text { are captured } \\
\text { - Will capture effect of } \\
\text { ionomer degradation } \\
\text { - More realistic to field data }\end{array}$ & $\begin{array}{l}\text { - More difficult to control RH } \\
\text { due to water production } \\
\text { - May be more difficult to } \\
\text { separate failure modes } \\
\text { - More difficult to control/ } \\
\text { set-up equipment } \\
\text { (potentiostat \& loadbank) }\end{array}$ \\
\hline
\end{tabular}




\section{Ex-situ and In-situ Characterization Techniques}

A suite of characterization techniques were down-selected for in-situ and ex-situ MEA/catalyst layer diagnostics of BOL and progressively aged samples. BOT and EOT samples were also supplied to collaborators for analytical analyses. A schematic of the AST diagnostics is shown in Figure 4.

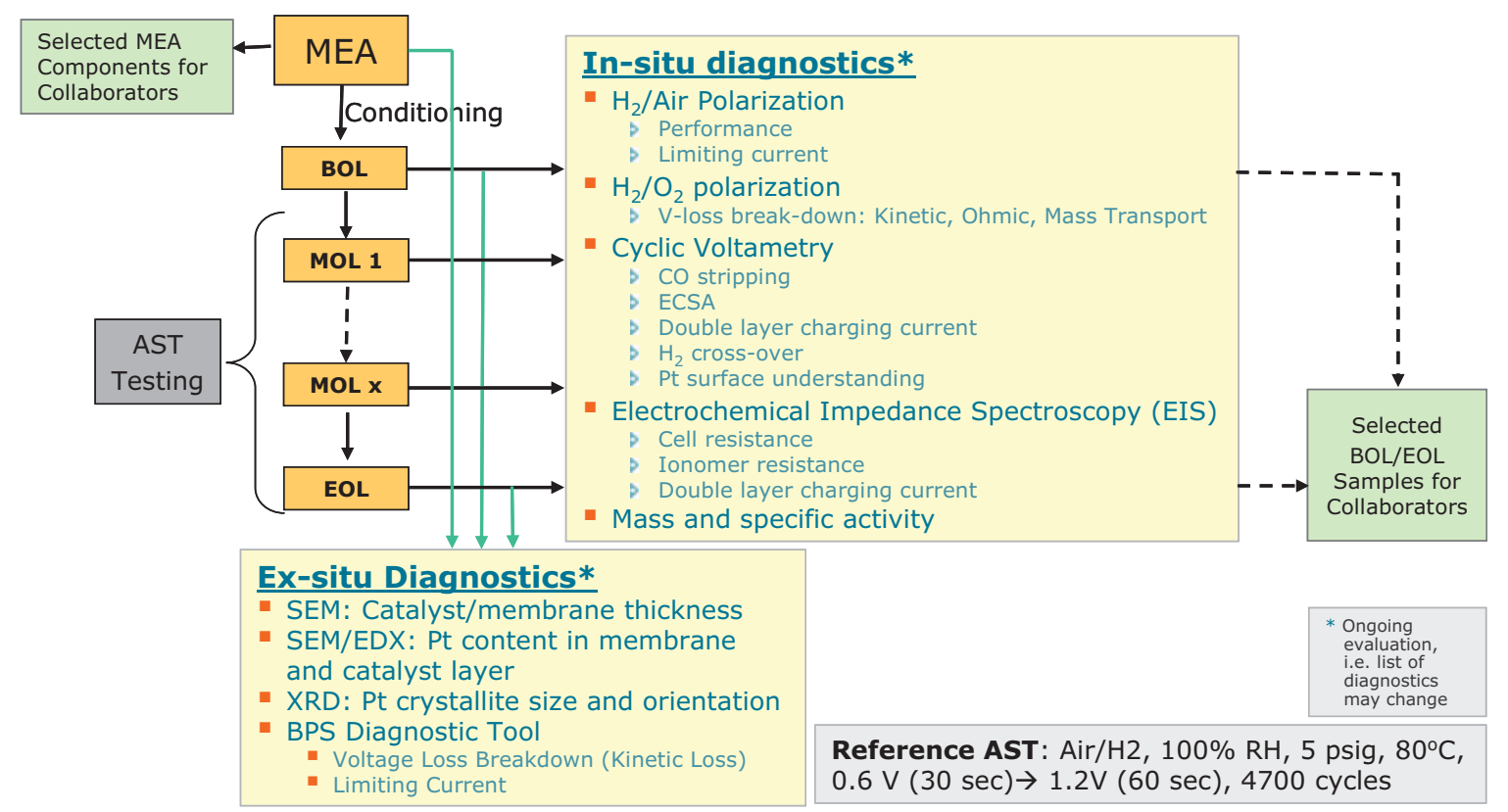

Figure 4: Schematics of AST diagnostics including list of down-selected ex-situ and in-situ measurement techniques

\section{Operating Conditions and Degradation Protocol}

The cell was conditioned at standard steady state operating conditions of $75^{\circ} \mathrm{C}, 100 \% \mathrm{RH}$, 5 psig, $1.3 \mathrm{~A} / \mathrm{cm}^{2}$ as listed in Table 3. The AST conditions (Table 3 ) utilized $100 \%$ gas RH to maximize the amount of degradation ${ }^{1,2}$ and minimize the required test duration. The voltage was cycled in an air (cathode) $/ \mathrm{H}_{2}$ (anode) environment from a lower potential limit (LPL) of $0.6 \mathrm{~V}_{\mathrm{RHE}}$ for $30 \mathrm{sec}$, to an upper potential limit (UPL) that varied between 0.9 to $1.4 \mathrm{~V}_{\mathrm{RHE}}$ for 60 sec. During the AST period, polarization curves, CV and EIS measurements were conducted after $0,50,700,1400,2100$, and 4700 cycles. SEM and XRD analyses were conducted with beginning of test (BOT) and degraded end of test (EOT) MEAs. As discussed, the standard AST held the UPL for $60 \mathrm{sec}$ in order to accelerate the degradation; however, in an operating system the PEMFC would only experience high UPLs for much shorter durations, typically of less than $1 \mathrm{sec}$.

UPL dwell time testing between 5 and $600 \mathrm{sec}$ was conducted to evaluate the impact of dwell time at UPL on the degradation rates. Testing dwell times less than $1 \mathrm{sec}$ could not be

\footnotetext{
1

1 S. Maass, F. Finsterwalder, G. Frank, R. Hartmann, and C. Merten, J. Power Sources, 176, 444 (2008).

2 D. A. Stevens, M. T. Hicks, G. M. Haugen, and J. R. Dahn, J. Electrochem. Soc., 152, A2309 (2005).
} 
reproducibly achieved due to equipment limitations. Temperature and gas $\mathrm{RH}$ dependencies were studied by varying the temperature between 60 to $90^{\circ} \mathrm{C}$, and the reactants gas $\mathrm{RH}$ between 50 to $\geq 100 \% \mathrm{RH}$ (signifies two-phase flow). The carbon loss was calculated by measuring the $\mathrm{CO}$ and $\mathrm{CO}_{2}$ in the cathode and anode gas exhaust using a Fuji Electric ZRH IR $\mathrm{CO}$ and $\mathrm{CO}_{2}$ gas analyzer.

Table 3: List of operating conditions for standard fuel cell operation and the corrosion accelerated stress test

\begin{tabular}{|l|c|c|c|c|c|c|}
\hline $\begin{array}{c}\text { Operating } \\
\text { Conditions }\end{array}$ & $\begin{array}{c}\text { Temperature } \\
\left({ }^{\circ} \mathbf{C}\right)\end{array}$ & $\begin{array}{c}\text { Pressure } \\
(\mathbf{p s i g})\end{array}$ & $\begin{array}{c}\text { Oxidant } \\
\text { Flow Rate } \\
(\mathbf{s} / \mathbf{p m})\end{array}$ & $\begin{array}{c}\text { Fuel Flow } \\
\text { Rate } \\
(\mathbf{s} / \mathbf{p m})\end{array}$ & $\begin{array}{c}\text { Anode \& } \\
\text { Cathode } \\
\text { Gas RH } \\
\%\end{array}$ & $\begin{array}{c}\text { Current } \\
\text { Density } \\
\left(\mathbf{A} / \mathbf{c m}^{2}\right)\end{array}$ \\
\hline Standard & 75 & 5 & $9($ air $)$ & $4.45\left(\mathrm{H}_{2}\right)$ & 100 & 1.3 \\
\hline AST & 80 & 5 & 9 (air) & $4.45\left(\mathrm{H}_{2}\right)$ & 100 & \\
\hline
\end{tabular}

\section{Cyclic Voltammetry (CV)}

CV scans were conducted using a Solatron SI1287 potentiostat to calculate the ECSA, the $\mathrm{H}_{2}$ crossover current, and the double layer capacitance $(\mathrm{Cdl})$. Hydrogen present at the anode acted as the reference electrode as the cathode potential was cycled between $0.1-1.2 \mathrm{~V}_{\mathrm{RHE}}$ at $20 \mathrm{mV} / \mathrm{s}$. The ECSA was determined by CO stripping, assuming a charge density of $420 \mu \mathrm{C} / \mathrm{cm}^{2}$ to break the linear Pt-CO bond. ${ }^{3}$

\section{Electrochemical Impedance Spectroscopy (EIS)}

EIS measurements were taken by applying a $10 \mathrm{mV}$ AC perturbation signal with a $0.45 \mathrm{~V}_{\mathrm{RHE}} \mathrm{DC}$ bias potential. A SI1287 Solatron potentiostat and a 1250 Solatron Hi Frequency Response analyzer were used in a 4-electrode configuration to sweep the frequency between $50 \mathrm{kHz}$ to $0.05 \mathrm{~Hz}$. The bias potential was applied to eliminate any pseudo-capacitive effects that result from hydrogen and oxygen adsorption-desorption. ${ }^{4}$ Z-plot and Z-view software were used to conduct and analyze the EIS spectra to determine the impedance and capacitance. Measurements were taken in a nitrogen and hydrogen atmosphere on the cathode and anode, respectively. The EIS spectra were fit to an equivalent circuit representing a transmission line network of the porous catalyst layer $(\mathrm{CL})$. As discussed elsewhere ${ }^{5}$ in greater detail, the fitted parameters consisted of the high frequency cell resistance, the cathode CL protonic resistance, and the double layer capacitance (Cdl).

\footnotetext{
3

3 T. R. Ralph, G. A. Hards, J. E. Keating, S. A. Campbell, D. P. Wilkinson, M. Davis, J. St-Pierre, and M. C. Johnson, J. Electrochem. Soc., 144, 3845 (1997).

4 E. B. Easton and P. G. Pickup, Electrochimica Acta, 50, 2469 (2005).

5 A. Young, J. Stumper, and E. Gyenge, J. Electrochem. Soc., 156, B913 (2009).
} 
DE-EE0000466

Ballard Material Products Inc

\section{Scanning Electron Microscopy.}

After operation, MEA cross-sections were analyzed with a Philips XL30 SEM to detect changes in the MEA structure due to the Pt dissolution and carbon corrosion. MEA samples were cast into epoxy pucks. Pictures were taken using a backscatter detector at $400 \times$ magnification and a beam energy of $15 \mathrm{kV}$. The membrane and CL thickness was measured. The cathode $\mathrm{CL}$ thickness, in conjunction with a carbon mass balance, was used to calculate the CL porosity in new and degraded samples. The Pt content in the membrane and catalyst layers was measured using an EDS detector.

\section{$\underline{X \text {-ray Diffraction }}$}

Powder samples of the cathode catalyst layer were scraped from the catalyst coated membrane (CCM) and submitted to an external lab for XRD analysis, where the samples were then gently stirred in an alumina mortar and smeared on a zero-background quartz plate with ethanol. Step-scan X-ray powder-diffraction data were collected over the range $6-103^{\circ} 2 \theta$ $\left(0.04^{\circ} 2 \theta\right.$ step, $\left.1.5 \mathrm{~s} / \mathrm{step}\right)$ with CoK $\alpha$ radiation (1.7902 $\AA$ ) on a Siemens (now Bruker AXS) D5000 Bragg-Brentano diffractometer equipped with an Fe monochromator foil, $0.6 \mathrm{~mm}\left(0.3^{\circ}\right)$ divergence slit, incident- and diffracted-beam Soller slits and a VÅNTEC-1 strip detector. The long fine-focus Co X-ray tube was operated at $35 \mathrm{kV}$ and $40 \mathrm{~mA}$, using a take-off angle of $6^{\circ}$. The average platinum crystallite size was determined using the Scherrer Equation,

$$
\mathrm{D}=\mathrm{K} \lambda /(\beta \cos \theta)
$$

where $D$ is the diameter of the crystallite, $\beta$ is the width of the peak at half maximum intensity of a specific phase (hkl) in radians, $\mathrm{K}$ is the Scherrer constant equal to $0.9, \lambda$ is the wavelength of incident $\mathrm{x}$-rays and $\theta$ is the center angle of the peak.

\section{Reproducibility of MEA Results}

The reproducibility of performance and properties of 57 BOT MEAs is summarized in Table 4. The MEA to MEA variability was found to be within a few per cent for the measured parameters with the exception of the catalyst layer ionic resistance. It is believed that the larger error is associated with MEA variability and data fitting variability.

Table 4: Reproducibility of MEAs (BOT and EOT), including MEA and test station variability

\begin{tabular}{|l|c|c|}
\hline \multirow{2}{*}{ Characteristics } & \multicolumn{2}{c|}{ \% Variation (1 Std. Deviation) } \\
\cline { 2 - 3 } OCV & BOT (57 MEAs) & EOT (3 to 5 MEAs) \\
\hline Air Performance, 1.0A/cm ${ }^{2}$ & 1 & 1 \\
\hline Air Kinetic Loss, 1.0A $/ \mathrm{cm}^{2}$ & 3 & 1 \\
\hline ECSA & 4 & 0.2 \\
\hline Double Layer Capacitance & 6 & 4 \\
\hline Catalyst Layer lonomer Resistance & 17 & 12 \\
\hline EOT: 4700 Cycles, $80^{\circ} \mathrm{C}, 100 \% R H, 0.6 \mathrm{~V}(30 \mathrm{~s})$ to $1.2 \mathrm{~V}(60 \mathrm{~s})$, square wave \\
\hline
\end{tabular}




\section{Operational Stressors and Structural/Compositional Variations}

Key operational stressors under consideration were prioritized based on results that were obtained prior to the start of this project. A high level assessment of the operational impact on performance degradation is shown in Table 5. As expected, the effect of the upper potential on degradation was found to be most severe, followed by dwell time at the upper potential, while the impact of temperature and oxidant humidity level was noticeable but not too pronounced. Based on these preliminary results the operational studies focused on the following stressors and values (Table 6). The structural design parameters were also prioritized based on internal results as well as literature reports. These parameters were evaluated for both BOT performance and durability. Table 7 gives the structural design parameters studied and values that were evaluated.

Table 5: Effect of Operational Stressors on LSAC Catalyst Degradation

\begin{tabular}{|c|c|c|c|c|c|c|c|}
\hline \multirow[b]{2}{*}{$\begin{array}{l}\text { Operational } \\
\text { Stressor }\end{array}$} & \multirow[b]{2}{*}{ Range Studied } & \multirow[b]{2}{*}{$\begin{array}{l}\text { Mechanism } \\
\text { Shift }\end{array}$} & \multicolumn{5}{|c|}{ Change in... } \\
\hline & & & \begin{tabular}{|l} 
Performance \\
Degradation Rate @ \\
1A/cm2 (mV/cycle)
\end{tabular} & $\begin{array}{l}\text { EPSA Rate } \\
\text { (/cycle) }\end{array}$ & $\begin{array}{l}\text { Agglomeration } \\
\text { Rate (nm/cycle) }\end{array}$ & $\begin{array}{l}\text { PITM Rate } \\
\text { (PPM/cycle) }\end{array}$ & $\begin{array}{l}\text { Thickness loss } \\
\text { rate (nm/cycle) }\end{array}$ \\
\hline \multirow{3}{*}{ UPL } & $0.8 \gg \gg 1.2 \mathrm{~V}$ & $\geq 1.2 \mathrm{~V}$ & Small Effect & Small Effect & Small Effect & Large Effect & Small Effect \\
\hline & $0.8 \gg>>1.3 \mathrm{~V}$ & $\geq 1.2 \mathrm{~V}$ & Small Effect & Small Effect & Small Effect & Large Effect & Large Effect \\
\hline & $0.8 \gg \gg>1.5 \mathrm{~V}$ & $\geq 1.2 \mathrm{~V}$ & Large Effect & Large Effect & Large Effect & Large Effect & Large Effect \\
\hline $\begin{array}{l}\text { Dwell Time } \\
\text { Cycle \# }\end{array}$ & $\begin{array}{l}30-5000 \text { sec } \\
(10,000-1 \\
\text { cycle })\end{array}$ & & Small Effect & Medium Effect & Small Effect & Medium Effect & Medium Effect \\
\hline $\begin{array}{l}\text { Temp } \\
\text { (UPL 1.3V) }\end{array}$ & $60 \gg \gg 80 \mathrm{C}$ & $\geq 70$ & Small Effect & Small Effect & Negligible Effect & Negligible Effect & Medium Effect \\
\hline $\begin{array}{l}\text { Temp } \\
\text { (UPL 1.2V) }\end{array}$ & $60 \gg \gg 80 \mathrm{C}$ & $\geq 75$ & Small Effect & Small Effect & Small Effect & Small Effect & Negligible Effect \\
\hline $\mathrm{RH}$ & $50 \gg \gg>120 \%$ & $\geq 100 \%$ & Small Effect & Negligible Effect & Small Effect & Small Effect & Small Effect \\
\hline LPL & $0.5 \gg>>0.8 \mathrm{~V}$ & & Small Effect & Negligible Effect & Negligible Effect & Negligible Effect & Small Effect \\
\hline & & & & & \begin{tabular}{|l|} 
\\
Negligib \\
Small Ef \\
Medium \\
Large Ef \\
\end{tabular} & $\begin{array}{l}\text { le Effect: No Sigr } \\
\text { ffect: } 2 \text { to } 5 \text { times } \\
\text { Effect: } 5 \text { to } 10 \text { tim } \\
\text { ffect: }>10 X \text { increa }\end{array}$ & $\begin{array}{l}\text { Legend } \\
\text { nificant Change } \\
\text { increase in rate } \\
\text { nes increase in rate } \\
\text { se in rate }\end{array}$ \\
\hline
\end{tabular}


Table 6: Operational Stress Test Conditions and Stress Values Evaluated

\begin{tabular}{|l|l|}
\hline \multicolumn{1}{|c|}{ Operational Study - Stressors } & \multicolumn{1}{|c|}{ Parameter Values } \\
\hline Upper Potential Limit (UPL) & $0.9 \mathrm{~V}, 1.0 \mathrm{~V}, 1.1 \mathrm{~V}, 1.2 \mathrm{~V}, 1.3 \mathrm{~V}, 1.4 \mathrm{~V}$ \\
\hline Lower Potential Limit (LPL) & $0.1 \mathrm{~V}, 0.4 \mathrm{~V}, 0.6 \mathrm{~V}, 0.8 \mathrm{~V}$ \\
\hline Cycle Number, 1.2V UPL & 0 to 4700 cycles \\
\hline Cycle Number, 1.4V UPL & 0 to 2100 cycles \\
\hline Dwell Time, 1.0V UPL & $20 \mathrm{~s}, 60 \mathrm{~s}, 300 \mathrm{~s}, 600 \mathrm{~s}$ \\
\hline Dwell Time, 1.4V UPL & $5 \mathrm{~s}, 20 \mathrm{~s}, 60 \mathrm{~s}, 300 \mathrm{~s}, 600 \mathrm{~s}$ \\
\hline Relative Humidity, 1.2V UPL & $60 \%, 80 \%, 100 \%, 120 \% \mathrm{RH}$ (oversaturated) \\
\hline Relative Humidity, 1.4V UPL & $50 \%, 75 \%, 90 \%, 100 \%, 120 \%$ RH (oversaturated) \\
\hline Temperature, 100\%RH, 1.4V UPL & $60{ }^{\circ} \mathrm{C}, 70{ }^{\circ} \mathrm{C}, 80^{\circ} \mathrm{C}$, to $90^{\circ} \mathrm{C}$ \\
\hline Temperature, 100\%RH, 1.4V UPL & $70^{\circ} \mathrm{C}, 80^{\circ} \mathrm{C}$, to $90^{\circ} \mathrm{C}$ \\
\hline Oxidant Composition, 1.0V UPL & $5 \%, 10.5 \%, 21 \%, 100 \% \mathrm{O}_{2}$ \\
\hline Hydrogen Composition, 1.2V UPL & $20 \%, 60 \%, 100 \% \mathrm{H}_{2}$ \\
\hline
\end{tabular}

Table 7: Structural Design Parameters and Parameter Values Evaluated

\begin{tabular}{|l|l|}
\hline \multicolumn{1}{|c|}{ Structural Parameters } & \multicolumn{1}{c|}{ Parameter Values } \\
\hline Pt loading study & $0.05,0.1,0.2,0.3,0.4$ and $0.5 \mathrm{mg} / \mathrm{cm}^{2}$ \\
\hline Carbon support type study & $\begin{array}{l}\text { V: Vulcan carbon } \\
\text { LSAC: low surface area carbon } \\
\text { MSAC: medium surface area carbon } \\
\text { HSAC(1): high surface area carbon \#1 } \\
\text { HSAC(2): high surface area carbon \#2 }\end{array}$ \\
\hline Carbon ratio study & $\begin{array}{l}\text { LSAC30, LSAC40, LSAC50, LSAC60 and LSAC80 } \\
\text { (where the number specifies the catalyst Pt content in } \\
\text { percent) }\end{array}$ \\
\hline Catalyst heat treatment study & $\begin{array}{l}\text { HSAC50-HT(1): high surface area carbon \#1, 50\% Pt } \\
\text { heat treated catalyst } \\
\text { HSAC50-HT(2): high surface area carbon \#2, 50\% Pt } \\
\text { heat treated catalyst }\end{array}$ \\
\hline lonomer content study & 12\%, 23\%, 30\%, 38\% and $50 \%$ by weight \\
\hline lonomer EW study & 850EW, 950EW and $1050 E W$ \\
\hline Membrane type study & NR211, Supplier A \\
\hline GDL effect study & BMP GDL, BMP GDL without MPL \\
\hline Catalyst layer processing study & 8\% crack area, 1\% crack area \\
\hline
\end{tabular}


DE-EE0000466

Ballard Material Products Inc

\section{Results and Discussions}

\subsection{Impact of Operational Conditions}

The operational studies focused on the understanding and quantification of the degradation mechanisms and structural and compositional changes of the catalyst layer as a function of key operational stressors. The operational conditions that were investigated, i.e. upper and lower potential limits, cycle number, dwell time, oxidant relative humidity, temperature, and oxidant and fuel compositions are discussed separately below. These studies have all been carried out with the reference MEA and modifications to the standard AST.

\subsubsection{Upper Potential Limit (UPL)}

The impact of the high cathode potential on catalyst degradation has been evaluated extensively in the literature ${ }^{6}$; however these studies are seldom comprehensive or systematic enough to be used as design curves for trade-off between MEA performance, durability and cost. Furthermore, detailed studies of $\mathrm{Pt}$ dissolution rates over a wide range of cathode potentials have generally been conducted with rotating disk electrodes $(\mathrm{RDE})^{7}$, which unfortunately do not compare well with fuel cell tests due to difficulties in preparing catalyst layers that are homogeneous and of uniform thickness.

In order to understand the degradation mechanisms and rates as a function of cathode potential, AST studies were carried out with two different Pt catalysts. The reference MEA (low surface area carbon catalyst support (LSAC)) and a MEA that contained Pt catalyst supported on a medium surface area carbon support (MSAC) were subjected to AST cycling at different upper potential limits (UPL). As well, the MEAs were subjected to a modified AST profile that mimics shutdown/start-up (SD/SU) conditions. The voltage cycling profiles used for the UPL study are shown in Figure 5.

\section{Cycling Profiles}

(a)

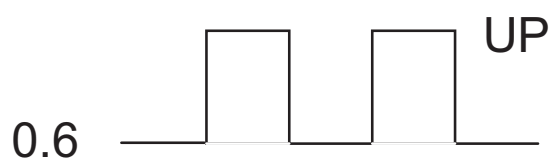

(b)

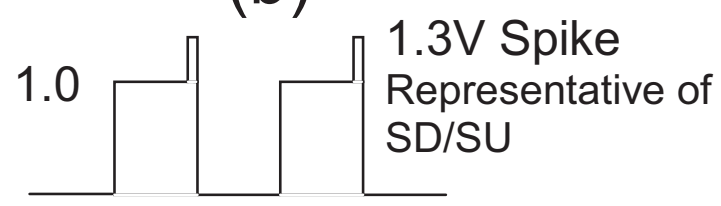

Figure 5: Voltage cycling profile used to evaluate effect of (a) UPL and (b) shutdown/start-up on catalyst degradation

Figure 6 shows the performance loss at 2100 and 4700 AST cycles as a function of UPL. The voltage degradation signature as a function of UPL was found to be similar for the medium and low surface area carbon-supports, i.e. the voltage degradation increases with increasing upper potential and prolonged cycling. For UPLs greater than $1.2 \mathrm{~V}$, the primary degradation mechanism shifts from predominantly Pt dissolution to predominately carbon corrosion. As shown in Figure 6 the SD/SU accelerated stress cycling (1.0V UPL with a superimposed

\footnotetext{
${ }^{6}$ K. Yasuda, A. Taniguchi, T. Akita, T. Ioroi, Z. Siroma, Physical Chemistry Chemical Physics 2006, 8, 746-752.

${ }^{7}$ X. Wang, R. Kumar, D. Myers, Electrochem. Solid-State Lett. 2006, 9, A225-A227.
} 
voltage spike of $1.3 \mathrm{~V}$ ) results in a lower performance loss than observed in the 1.3V UPL test, which confirms that the time at UPL has a significant impact on the degradation rate.

Carbon monoxide stripping cyclic voltammetry scans, shown in Figure 7, reveal distinct differences in the change of the $\mathrm{CO}$ peak shape with increasing degradation for the two $\mathrm{Pt}$ catalyst types. This observation, assumed to be due to differences in the growth of polycrystalline Pt structures ${ }^{8}$ will require further investigation.

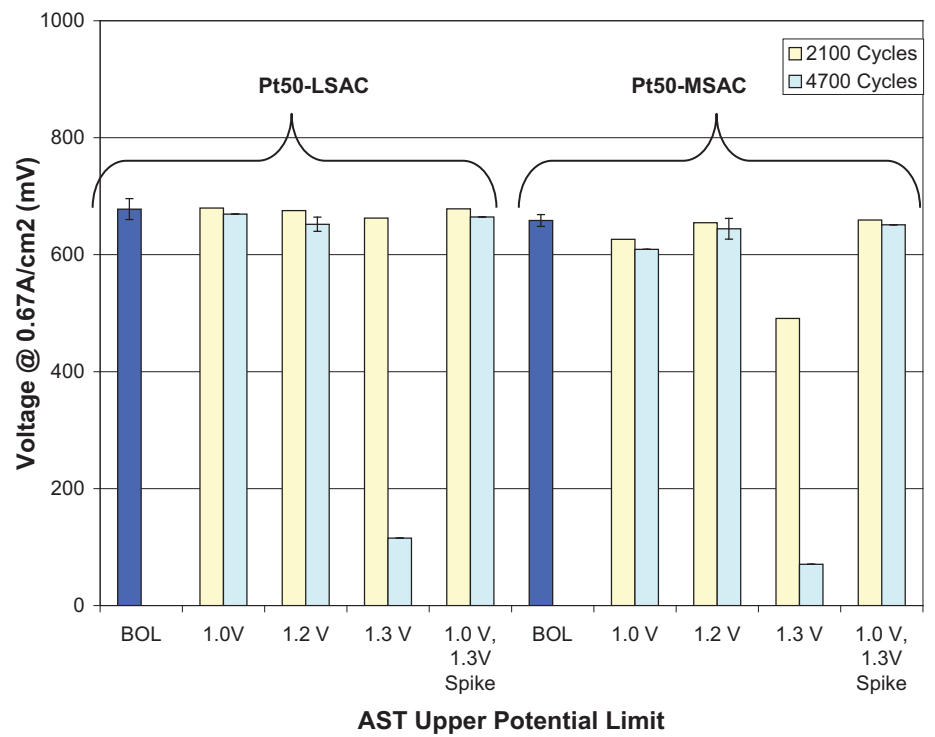

Figure 6: Performance at $0.67 \mathrm{~A} / \mathrm{cm}^{2}$ as a function of AST voltage profiles for $\mathrm{Pt}$ catalyst supported on MSAC and LSAC

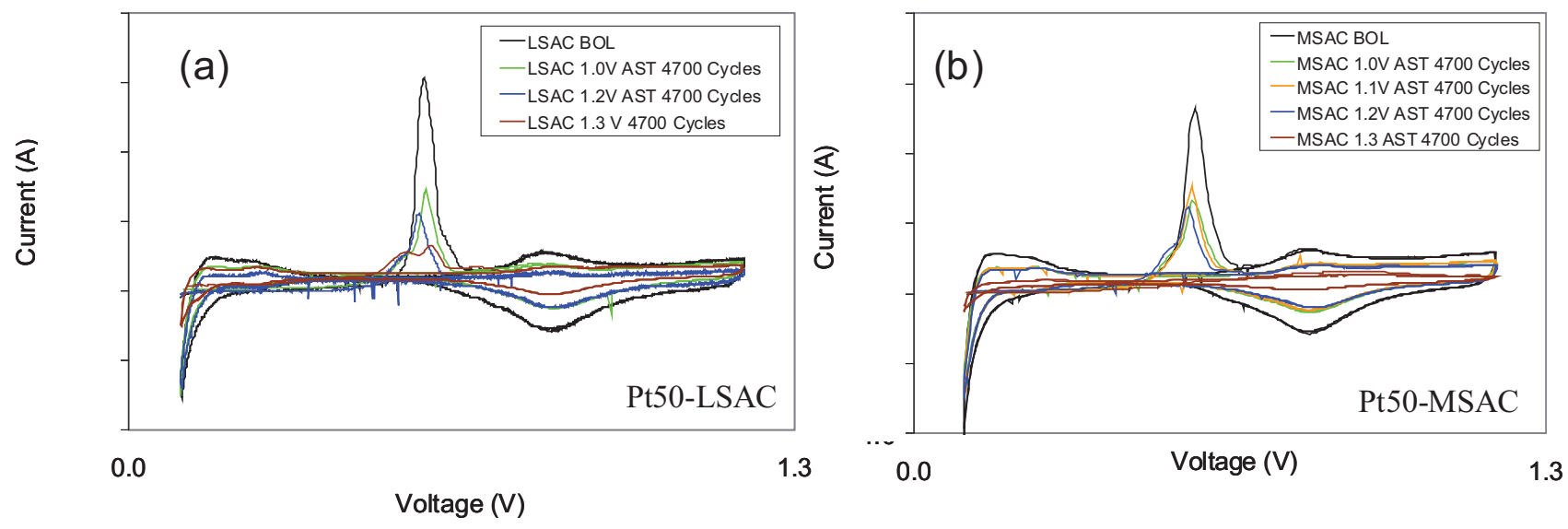

Figure 7: Cyclic voltammetry at beginning-of-test and end-of-test for Pt catalyst supported on (a) LSAC and (b) MSAC as a function of UPL

\footnotetext{
8 A. Cuesta, A. Couto, A. Rincón, M.C. Pérez, A. López-Cudero, C. Gutiérrez, Journal of Electroanalytical Chemistry 2006, 586, 184-195.
} 
DE-EE0000466

Ballard Material Products Inc

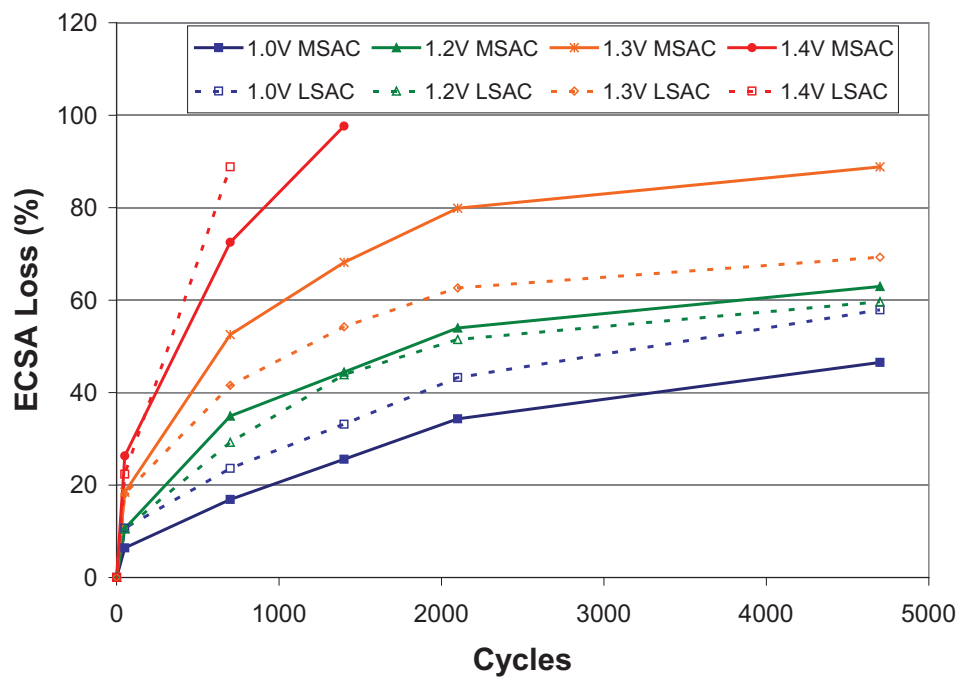

Figure 8: Per cent ECSA loss as a function of cycle number for ASTs cycled to different UPLs for both MSAC and LSAC Pt catalyst

The loss in effective catalyst surface area (ECSA) with progressive AST cycling at various UPLs, shown in Figure 8, reveals severe EPSA loss at the higher potentials which is likely associated with the Pt detachment from the support. Trends in ECSA loss with UPL were found to be similar for the MSAC and LSAC Pt catalysts. ECSA losses were essentially the same for both catalysts after cycling at 1.2V UPL; however, at UPLs above and below 1.2V the per cent ECSA loss as a function of cycle number deviates for the LSAC and MSAC catalysts. At an UPL $<1.2 \mathrm{~V}$, where the primary degradation mechanism is $\mathrm{Pt}$ dissolution, the MSAC based catalyst showed a lower ESCA loss compared to the LSAC based catalyst. The opposite trend (ECSA loss: MSAC>LSAC) has been observed for the AST with a UPL >1.2V, at which point carbon corrosion becomes the dominate degradation mechanism; thus, indicating that the carbon structure has an impact on the degradation of $\mathrm{Pt}$ and carbon. HRTEM observations (see Chapter III) indicate a better Pt particle dispersion on the MSAC support compared to LSAC, which would affect the rate of Pt dissolution and agglomeration at low UPLs.

Figure 9 and Figure 10 summarize the effect of UPL on Pt particle dissolution/agglomeration and support corrosion, respectively. Shown in Figure 9, at an UPL $<1.2 \mathrm{~V}$ the EOL particle size for the MSAC catalyst is smaller compared to the LSAC catalyst; which is consistent with the observed lower ECSA loss shown in Figure 8. At an UPL $>1.3 \mathrm{~V}$, both catalysts show an insignificant change in Pt particle size and similar particle size growth with increasing UPL. As shown in Figure 9(b) the UPL seems to have an impact on the amount of PITM accumulated at the end-of-test with both catalyst types showing similar PITM concentrations. 
DE-EE0000466

Ballard Material Products Inc

(a)

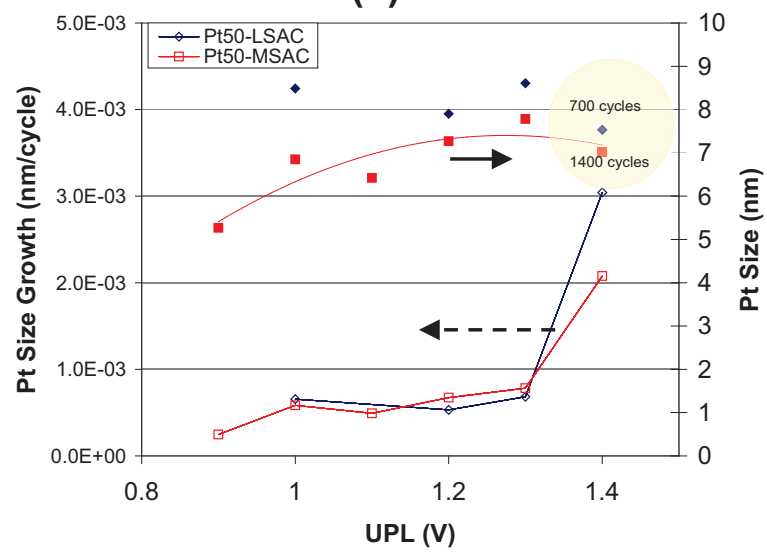

(b)

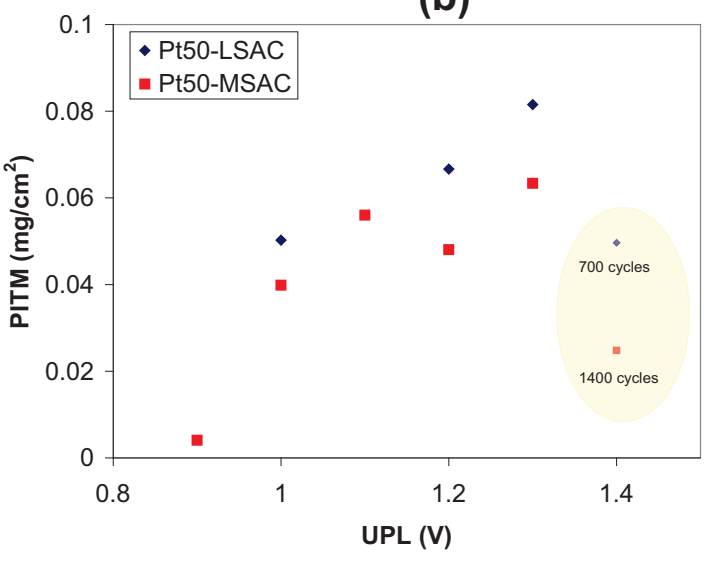

Figure 9: Impact of UPL during cycling on the Pt dissolution mechanism. (a) Pt agglomeration rate and Pt size as a function of UPL; (b) PITM accumulation at end of test (EOT) as a function of UPL

(a)

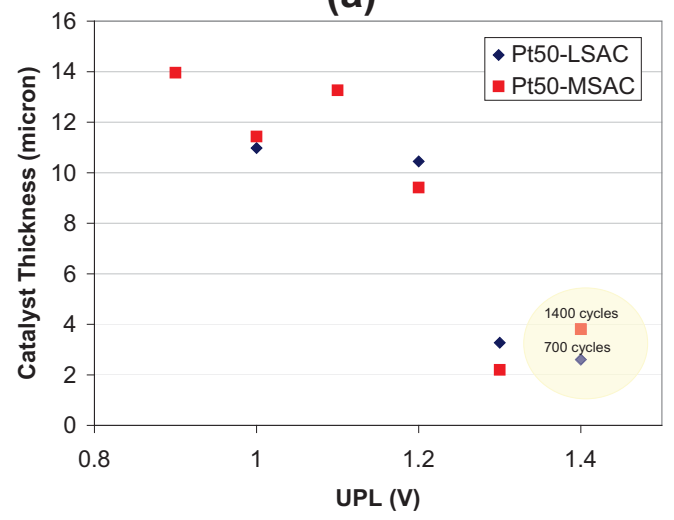

(b)

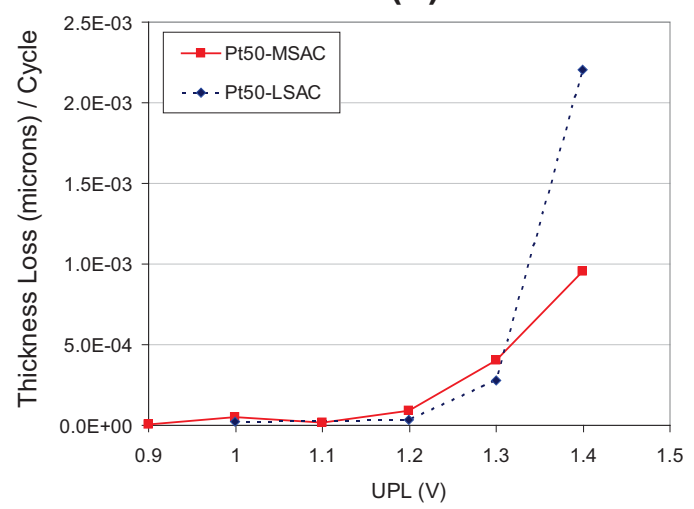

Figure 10: Impact of UPL on the carbon support corrosion. (a) Catalyst layer thickness; (b) Rate of thickness loss as a function of UPL

Figure 10 shows that the rate of cathode layer thinning and thus carbon corrosion increases as a function of UPL and that the carbon supports begin to degrade at potentials between 1.0V to $1.2 \mathrm{~V}$. A comparison of the two catalysts indicates that the graphitized LSAC catalyst is more stable at an UPL of $1.3 \mathrm{~V}$ and less stable at UPL $\geq 1.4 \mathrm{~V}$ compared to the MSAC. It is believed that the graphitization levels and structure of the different supports impact the rate of catalyst thinning at higher potential. As observed from HRTEM analysis (Chapter III) the walls and internal structure of the LSAC and MSAC supports are very different. The highly graphitized surface walls of the LSAC carbon with an amorphous center would suggest that corrosion takes place at the center of the carbon agglomerates causing the walls to collapse after prolonged corrosion. In contrast, the MSAC structure exhibits agglomerates with fewer graphitic walls that are more broken, thus one would expect more uniform thinning of carbon agglomerates.

In summary, Pt catalysts supported on carbon supports of low and medium surface area exhibited the same degradation signature of platinum dissolution and support corrosion with 
generally subtle differences in degradation rates. While the LSAC catalyst seems to be slightly more stable at UPL $\leq 1.3 \mathrm{~V}$, it is notably less stable at a UPL $\geq 1.3 \mathrm{~V}$ than MSAC carbon which is likely associated with the highly graphitized walls and amorphous center of the LSAC support vs. more dispersed graphitic areas observed for the MSAC support.

\subsubsection{Lower Potential Limit (LPL)}

To study the impact of oxide coverage on the Pt dissolution rate, the effect of lower potential limits (LPL) was investigated. Standard MEAs (Pt50-LSAC, cathode Pt loading of $0.4 \mathrm{mg} / \mathrm{cm}^{2}$ ) were subjected to $4700 \mathrm{AST}$ cycles at a LPL ranging from $0.1-0.8 \mathrm{~V}$ and an UPL of $1.0 \mathrm{~V}$. The dwell time at the lower potential was kept at $30 \mathrm{sec}$ and the upper potential at $60 \mathrm{sec}$.

Platinum oxide build-up and subsequent removal on cathode catalyst surfaces is dependent on potential. The cycling profiles evaluated were:

(1) 0.8 to $1.0 \mathrm{~V}$ : The Pt surface will be covered by an oxide film during cycling

(2) 0.6 and 0.4 to 1.0V: A reversible oxide film will be removed in part with each cycle

(3) 0.1 to 1.0V: The irreversible oxide film is completely removed with each cycle ${ }_{9}$

Figure 11 indicates that the $\mathrm{Pt}$ dissolution rates are affected by the oxide removal/formation process. The higher lower potential limit of $0.8 \mathrm{~V}$ exhibits only $\sim 30 \%$ ESCA loss and little growth of the Pt particle compared to the $0.6 \mathrm{~V} \mathrm{LPL}$, where the AST potentials result in the formation and substantial removal of the reversible $\mathrm{Pt}$ oxides. A further reduction in LPL towards $0.1 \mathrm{~V}$, where irreversible Pt oxides are completely removed, shows a slightly higher ESCA loss. The results verify and further support understanding of the $\mathrm{Pt}$ dissolution mechanism in that the cathodic driven dissolution of $\mathrm{Pt}$ oxides affects the $\mathrm{Pt}$ dissolution reaction.
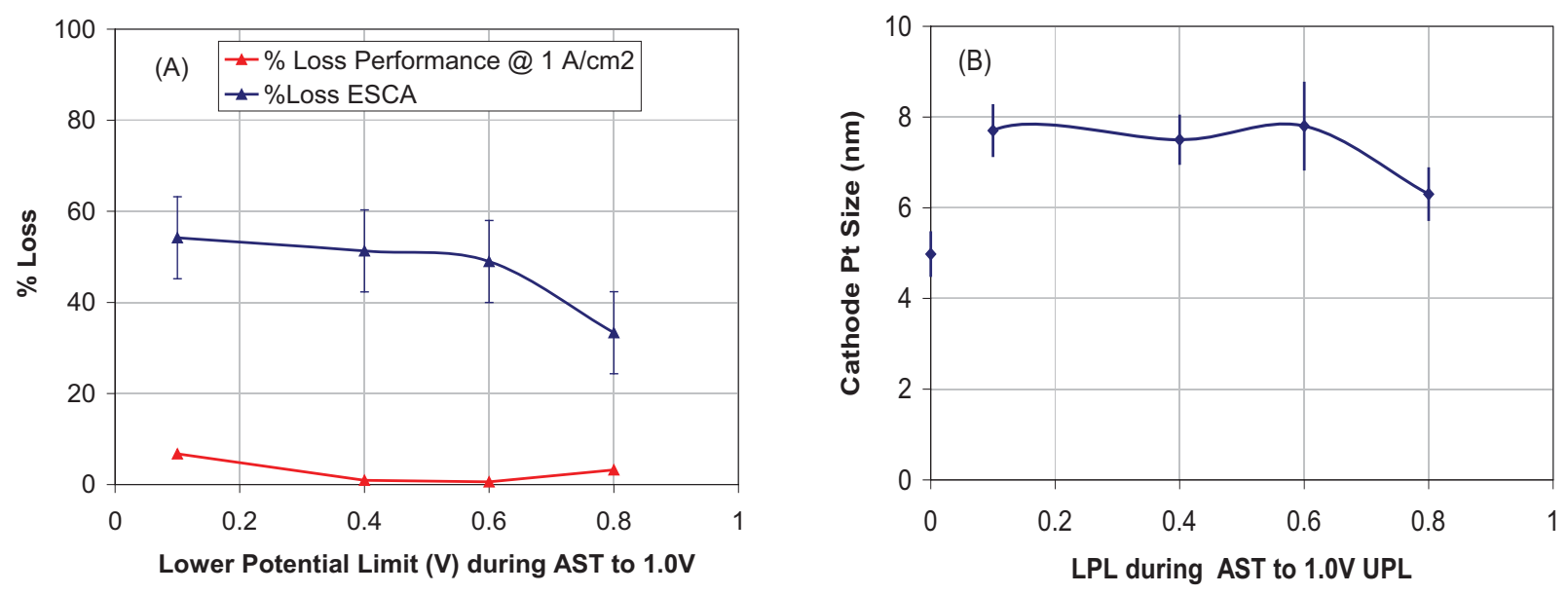

Figure 11: (A) Percentage of ESCA and performance loss, at $1 \mathrm{~A} / \mathrm{cm}^{2}$, as a function of lower potential limit during AST cycling. (B) Pt size growth as a function of LPL during AST cycling

\footnotetext{
${ }^{9}$ B.E. Conway, "Electrochemical Oxide Film Formation at Noble Metals as a Surface-Chemical Process", Progress in Surface Science, Vol. 49, No. 4, pp. 331-452, 1995.
} 
DE-EE0000466

Ballard Material Products Inc

\subsubsection{AST Cycle Number}

Performance degradation was investigated under standard AST conditions ( 0.6 to $1.2 \mathrm{~V}$ cycles) for different number of cycles at an upper potential of $1.2 \mathrm{~V}$ and $1.4 \mathrm{~V}$ to understand the onset of performance loss and catalyst layer changes for the different degradation mechanisms.

\section{(1) Cycle Number Effect at 1.2V UPL}

While the cell voltage loss as a function of cycle number shows a linear response (Figure 12 (a)), the loss breakdown components are non-linear in nature. The kinetic losses increase rapidly at lower cycle numbers and dominate before reaching a plateau at 2100 cycles at which point the catalyst layer ionic losses become more prominent, as observed by its increase with $>2100$ cycles. The EPSA and mass activity losses as function of AST cycling are shown in Figure 12 (b). As expected, both loss parameters follow the same trend.

Quantification of the amounts of the Pt agglomeration, Pt in the membrane (PITM), and Pt washout (i.e. the mass balance of Pt) showed that the loss associated with the mass balance of $\mathrm{Pt}$ is unaccounted for in the catalyst layer or membrane and is believed to result from $\mathrm{Pt}$ loss due to carbon corrosion. Figure 12 (c) shows the EPSA loss due to the amount of Pt Loss and Pt Agglomeration. In Figure 13, the relative amounts of the calculated remaining EPSA in the catalyst layer and EPSA loss are also shown as pie charts for different cycle numbers. The results show that there is a significant increase in EPSA loss in the first 50 cycles predominantly due to $\mathrm{Pt}$ agglomeration. Further cycling shows increases in $\mathrm{Pt}$ agglomeration, PITM, and EPSA losses, which start to plateau after 2100 cycles at which point Pt washout losses are observed. This also coincides with catalyst thinning, shown in Figure 12 (d), and an increase in catalyst layer ionic losses. The catalyst mass activity loss and the air performance loss were found to correlate with the amount of PITM measured (Figure 14) suggesting that the performance loss is due to the Pt dissolution mechanism.

In conclusion, the degradation mechanisms/failure modes monitored as a function of cycle number show that between 0-2100 cycles kinetic losses dominate due to Pt dissolution, resulting in Pt agglomeration and Pt in the membrane (PITM). At greater than 2100 cycles, the catalyst layer (CL) ionic losses become dominant due to an increase in carbon corrosion, resulting in catalyst layer thinning. 
(a)

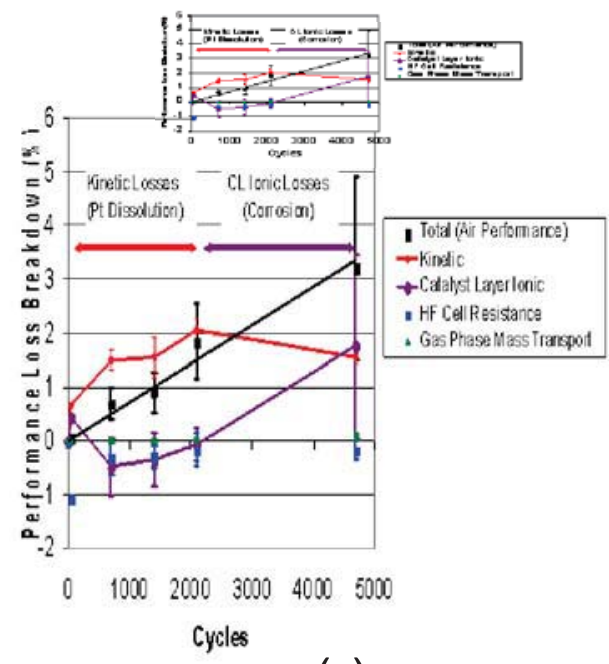

(c)

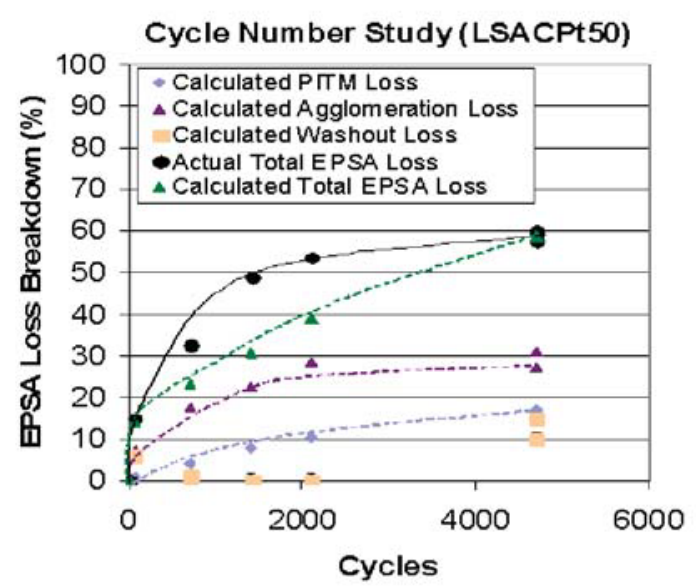

(b)

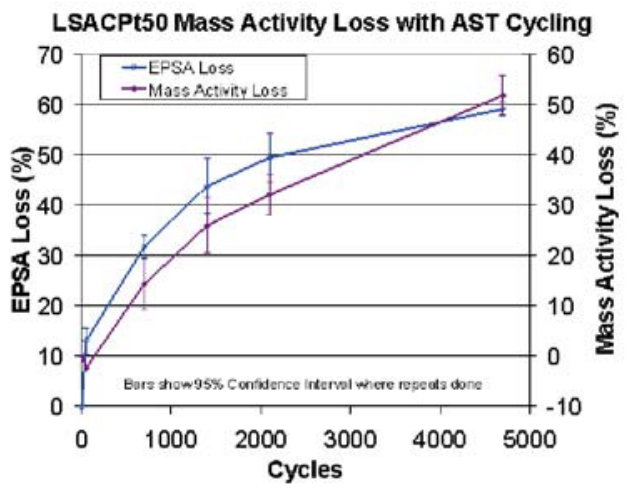

(d).

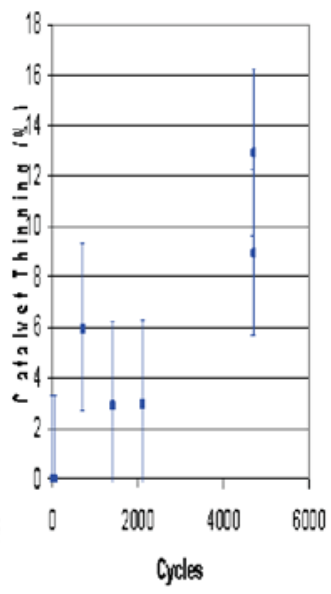

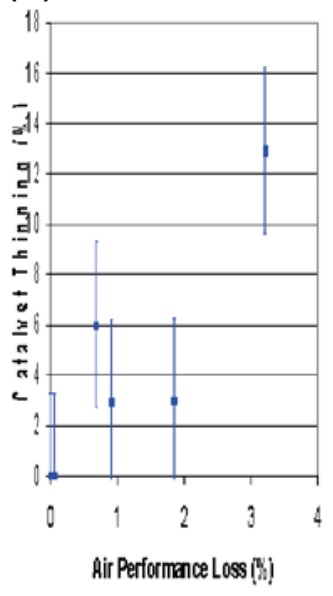

Figure 12: Impact of AST cycle number on in-situ and ex-situ characterization techniques. (a) Air Performance loss and voltage loss breakdown. (b) EPSA loss and catalyst activity loss. (c) Breakdown of ECSA loss into agglomeration, PITM and washout components. (d) Per cent catalyst thinning.

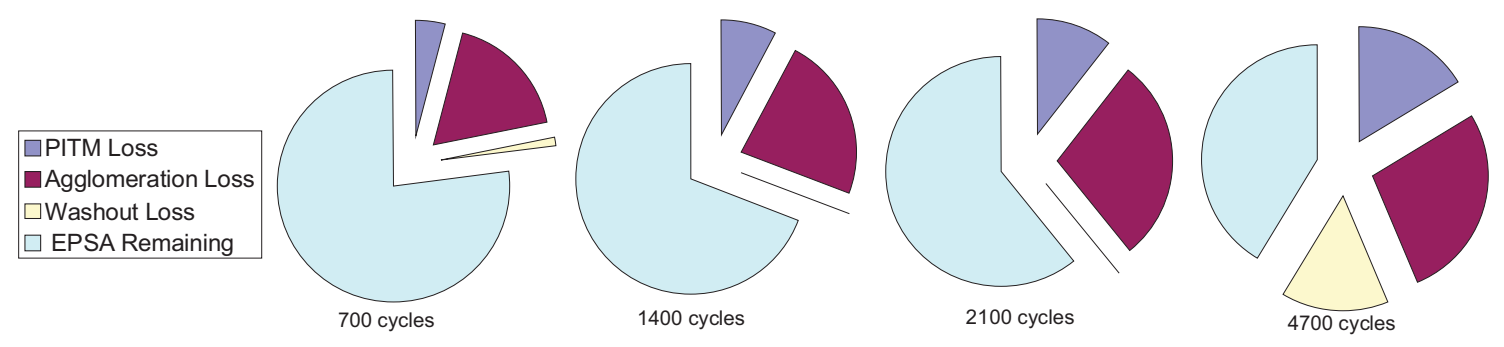

Figure 13: Breakdown of the EPSA Loss in to Agglomeration, PITM and Washout components. 
DE-EE0000466

Ballard Material Products Inc

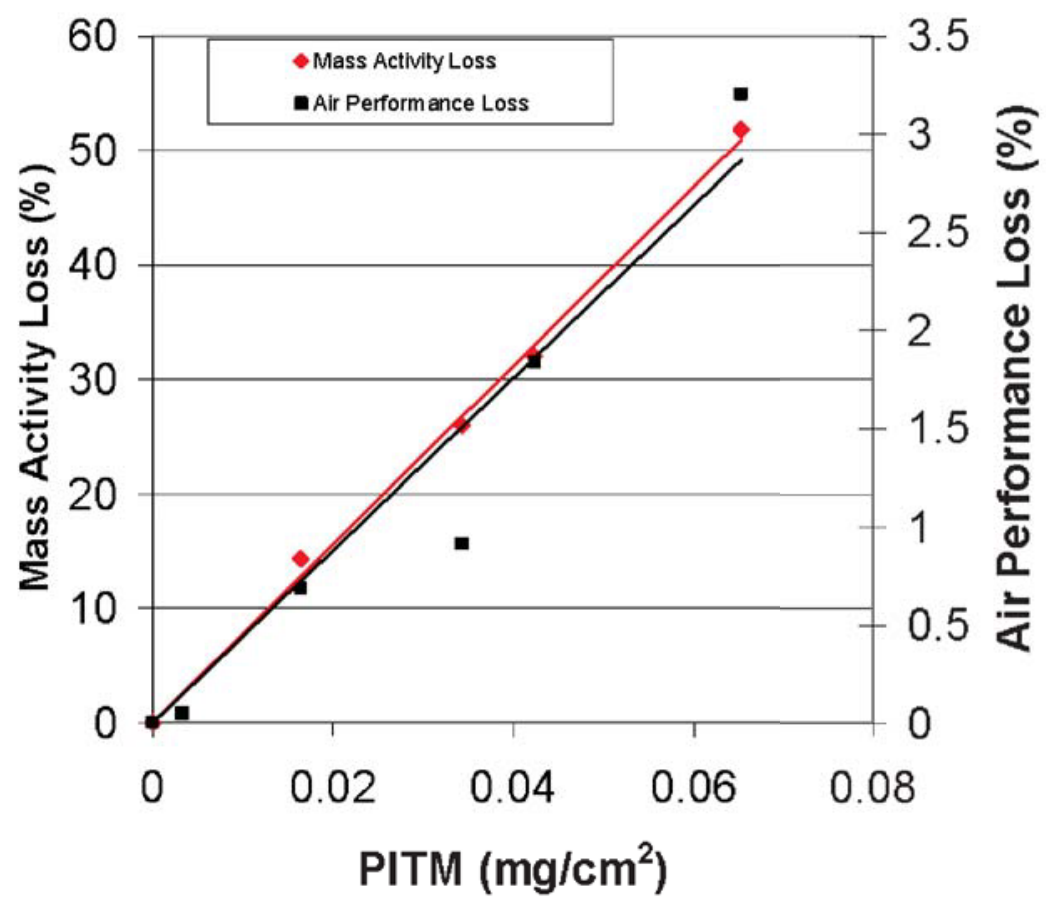

Figure 14: Correlations of in-situ to ex-situ diagnostic data for LSAC-50Pt: (a) Mass activity and performance losses as a function of PITM

\section{(2) Cycle Number Effect at an Upper Potential Limit of 1.4V}

Catalyst carbon support corrosion was investigated using AST cycles at an upper potential of $1.4 \mathrm{~V}$ and a $600 \mathrm{sec}$ dwell time for different cycle numbers. Figure 15 shows that the onset of corrosion (performance loss) is dependent on the total time spent at the upper potential rather than the number of AST cycles. The performance loss onset after $\sim 200$ min at 1.4V UPL was found to be due to kinetic performance loss and an increase in cathode catalyst layer ionic resistance. The onset of performance loss corresponded with a carbon support loss of $40 \%$, evident from the change in cathode catalyst layer thickness and confirmed by $\mathrm{CO}_{2}$ measurement in the cathode exhaust (Figure 15B). 

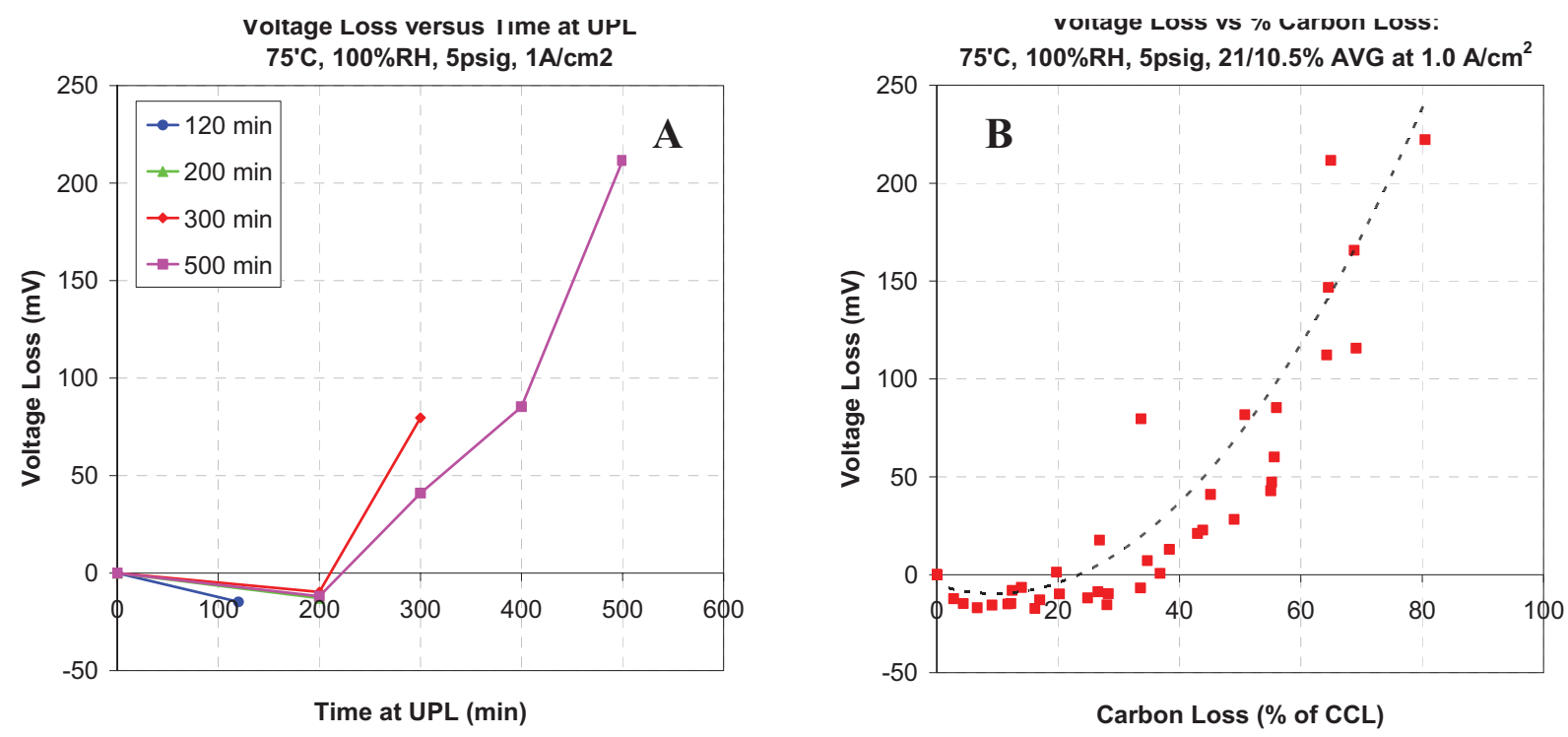

Figure 15: Performance loss impact at UPL of $1.4 \mathrm{~V}$ with $(\mathrm{A})$ time at UPL and $(\mathrm{B})$ carbon loss

\subsubsection{Dwell Time}

The effect of upper potential dwell time on catalyst degradation was investigated at $5,20,60$, 300 , and $600 \mathrm{sec}$ at an upper potential limit of 1.0V (Pt dissolution) to understand its effect on the catalyst layer degradation mechanisms.

\section{(1) Dwell Time Effect at 1.0 V}

Figure 16 shows the ECSA change with total accumulated UPL time, which initially gives the impression that greater dwell times lead to less ECSA loss. Since the number of cycles is a stressor for platinum dissolution the lower ECSA loss at longer dwell times was associated with fewer AST cycles. This becomes evident in Figure 16b where the ECSA normalized by the cycle number clearly shows that longer dwell times resulted in greater platinum loss per cycle. This is further supported by the linear trend of ECSA loss and PITM as a function of dwell time shown in Figure 17.

This result is consistent with literature findings: Matsumoto et al. ${ }^{10}$ conducted an analysis of both cycling and potential hold ASTs and showed a greater Pt dissolution rate with cycling, and an even greater dissolution rate by cycling in the presence of $\mathrm{O}_{2}$ compared to $\mathrm{N}_{2}$. The authors proposed in the presence of $\mathrm{O}_{2}$, a greater place exchange of oxygen into the Pt crystal resulting in high-order Pt oxide formation. Further, it is the cathodic reduction of higher-order Pt oxides which results in faster $\mathrm{Pt}$ dissolution compared to the commonly suspected anodic and/or chemical dissolution process that would be typical under a potential hold situation.

\footnotetext{
${ }^{10}$ M. Matsumoto, T. Miyazaki, and H. Imai, J. Phys. Chem. C, 115, 11163 (2011).
} 
DE-EE0000466

Ballard Material Products Inc
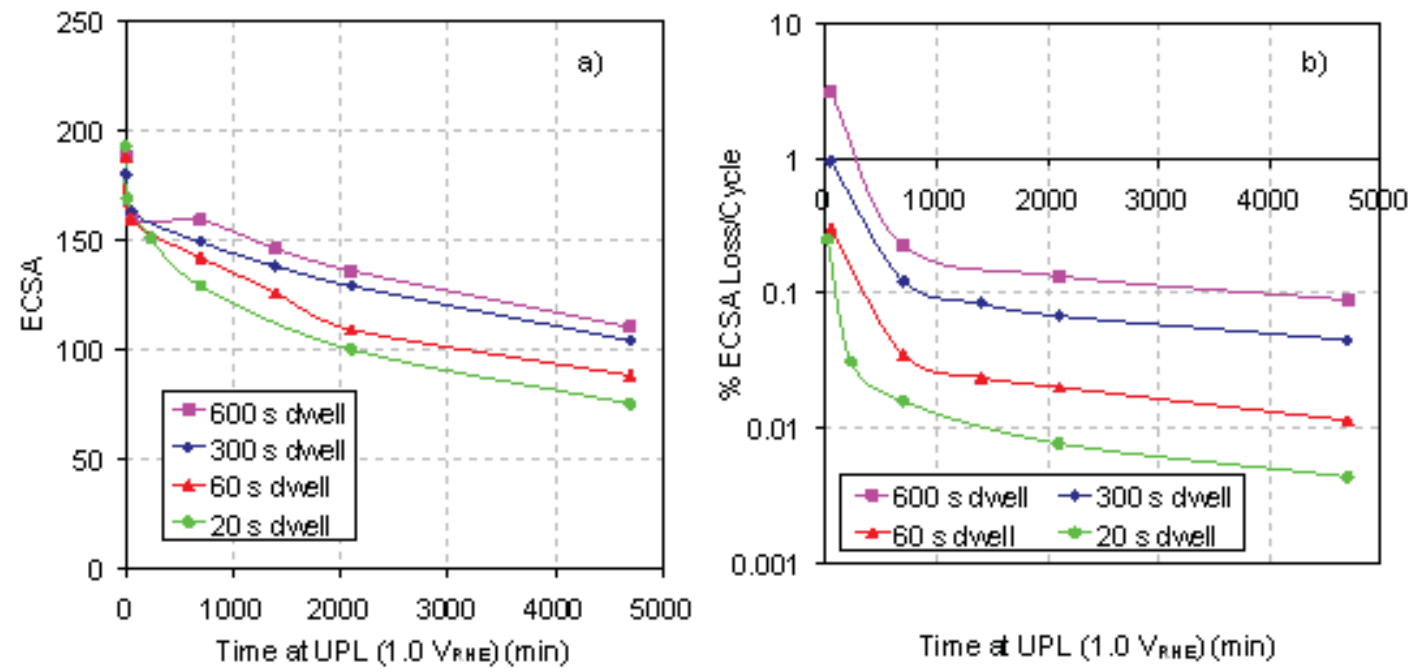

Figure 16: (a) the ECSA loss as a function of cycle dwell time and cumulative time at a 1.0V UPL, and (b) the ECSA loss normalized by cycle number as a function of cycle dwell time and cumulative time at a 1.0V UPL
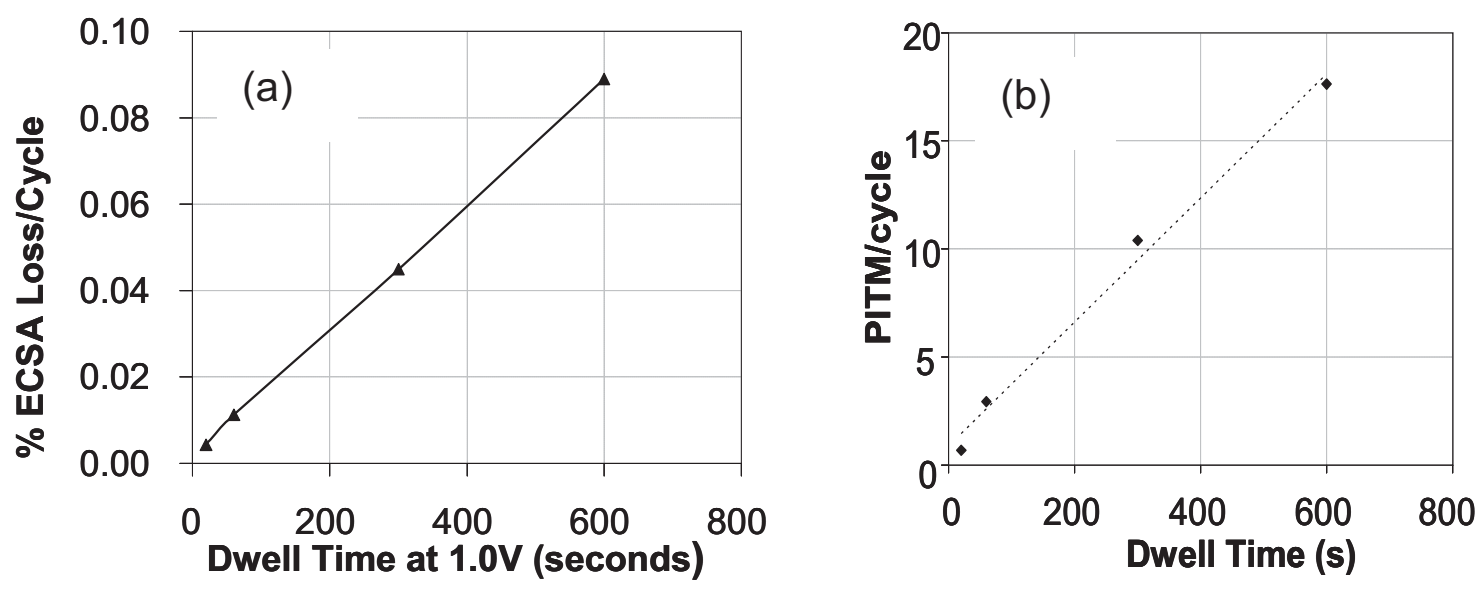

Figure 17: (a) \% ECSA loss and (b) Pt in the membrane (PITM) as a function of upper potential dwell time at $1.0 \mathrm{~V}$

Kongkanand et al. ${ }^{11}$ found similar evidence through XAS measurements. They showed, via an electrochemical quartz crystal microbalance, that operation under $\mathrm{O}_{2}$ or $\mathrm{N}_{2}$ resulted in a similar

${ }^{11}$ A. Kongkanand, and J.M. Ziegelbauer, J. Phys. Chem. C, 116, 3684 (2012). 
total oxide formation. Since the anodic driven oxide formation is similar, this provides further support that the cathodic driven dissolution of higher order Pt oxides is the cause of a faster Pt dissolution rate, and is the reason for the observed greater Pt dissolution when the cathode is cycled in an air environment.

In consideration of the above, it is hypothesized that the greater platinum loss per cycle for longer dwell times seen here, was due to the greater build up and place exchange of higher order Pt oxides that occurred during longer UPL dwell times. Reducing more oxide on the downward cycle caused greater de-stabilization of the platinum structure resulting in surface roughening and greater platinum dissolution. Furthermore, XRD and EDS measurements supported the ECSA trends; an increase in the platinum crystallite size and platinum in the membrane due to the increased Pt dissolution and agglomeration was observed.

\section{(2) Dwell Time Effect at $1.4 \mathrm{~V}$}

The effect of AST cycle dwell time at 1.4V UPL on performance, shown in Figure 18, reveals that the dwell time at $1.4 \mathrm{~V}$ does not affect MEA performance until the total time at the UPL is larger than 200 hours. As observed for the cycle number study (Figure 15) the voltage breakdown analysis indicated that the performance loss observed after 200 hours is associated with carbon support corrosion which causes a loss in kinetic performance and an increase in catalyst layer ionic resistance due to a loss in ECSA and catalyst layer thickness, respectively. While the onset of corrosion (generation of $\mathrm{CO}_{2}$ ) is dependent on the total time spent at the upper potential irrespective of the AST cycle dwell time, this time will vary with the carbon support structure and thus the upper potential limit, as well as the temperature and humidity; temperature and humidity effects are discussed below.

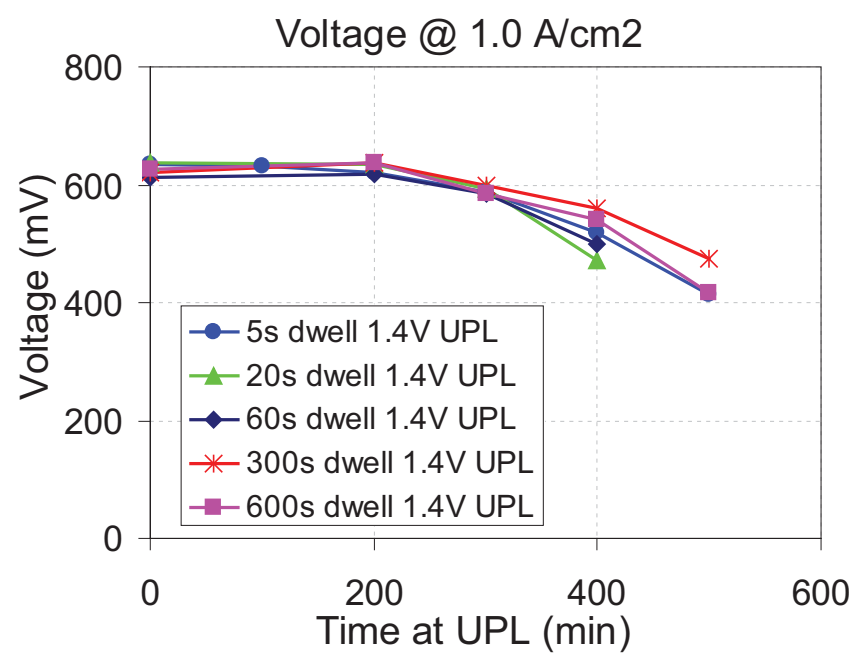

Figure 18: Effect of dwell time at 1.4V UPL on performance

\subsubsection{Cathode Relative Humidity}

The effect of oxidant $\mathrm{RH}$ on BOT performance and degradation was investigated at $1.0 \mathrm{~V}$ and $1.4 \mathrm{~V}$ UPL over a $\mathrm{RH}$ range from $60 \%$ to over-saturated $(120 \%)$ conditions. 


\section{(1) Oxidant RH effect at 1.0V UPL}

Figure 19 shows the BOT performance impact by $\mathrm{RH}$. Not surprisingly, the sensitivity to RH increases with increasing current density as the impact of water management becomes more pronounced. As well, $\mathrm{Pt}$ dissolution increases with increasing humidity in the oxidant gas $\mathrm{RH}$ $(<100 \%)$, thus, resulting in an increase in Pt growth, PITM, and ECSA loss (Figure 20). The relatively small increase in Pt dissolution from $100 \% \mathrm{RH}$ to over-saturated water content $(120 \%$ $\mathrm{RH}$ ) suggests that at $100 \% \mathrm{RH}$ some liquid water may already be present. Furthermore, the performance loss at EOL shows similar trends for ECSA and performance loss with RH in the range from 60 to $100 \%$.

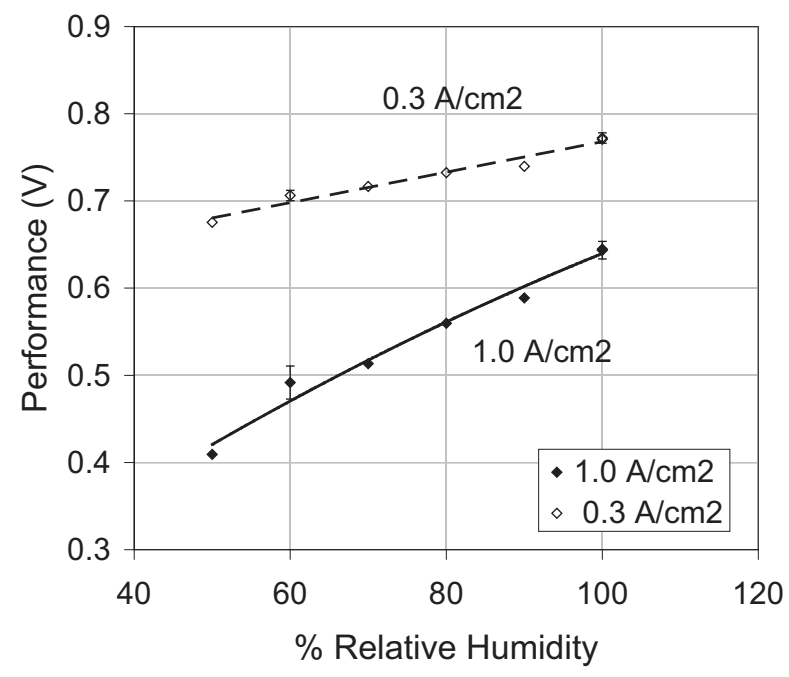

Figure 19: Effect of oxidant RH on BOT performance
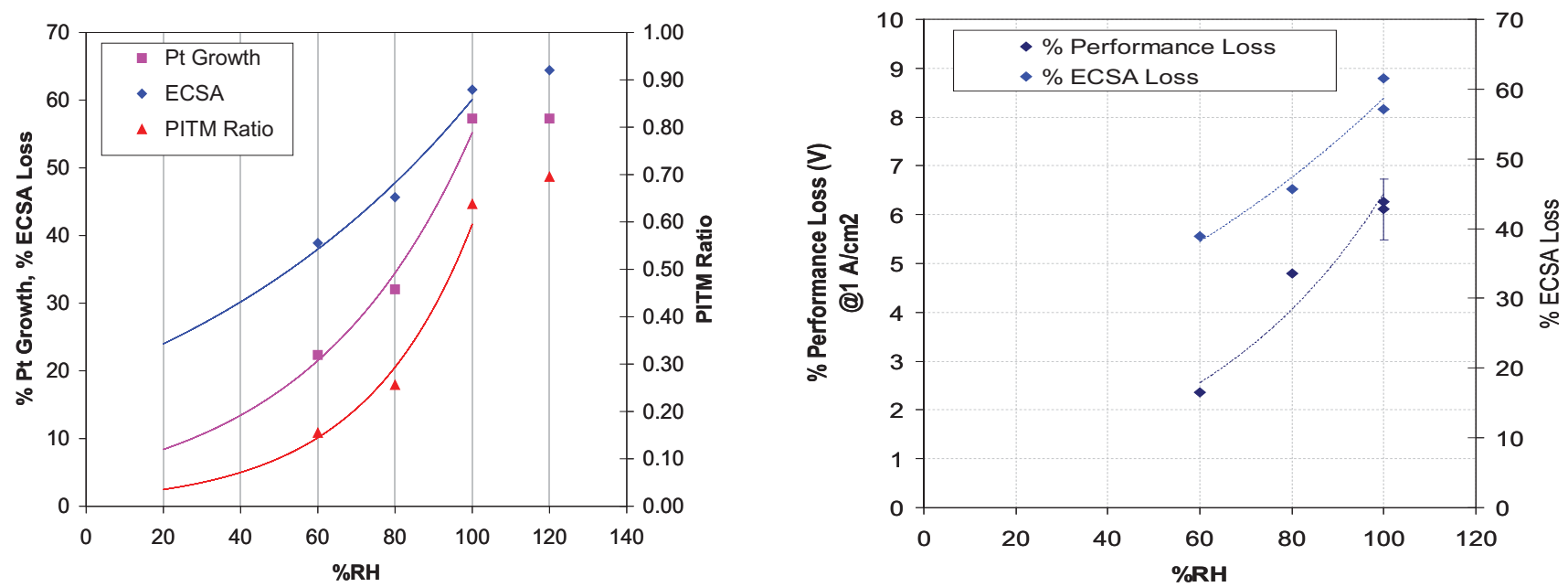

Figure 20: Effect of RH after 4700 AST cycles on (a) Pt dissolution: Pt growth, PITM and ECSA, and (b) performance and ECSA 
DE-EE0000466

Ballard Material Products Inc

\section{(2) Oxidant RH effect at 1.4V UPL}

Figure 21 shows $A$ ) the ECSA loss and B) carbon loss as a function of time at UPL (1.4V, $80^{\circ} \mathrm{C}$ ) for a range of oxidant humidity from $50 \% \mathrm{RH}$ to oversaturated $(120 \% \mathrm{RH})$. The results show that both the $\mathrm{Pt}$ dissolution and carbon corrosion mechanisms are accelerated by increasing $\mathrm{RH}$. There was little difference in ECSA loss and carbon loss from saturated to oversaturated conditions, suggesting that the addition of liquid water has a negligible effect on the degradation rates.
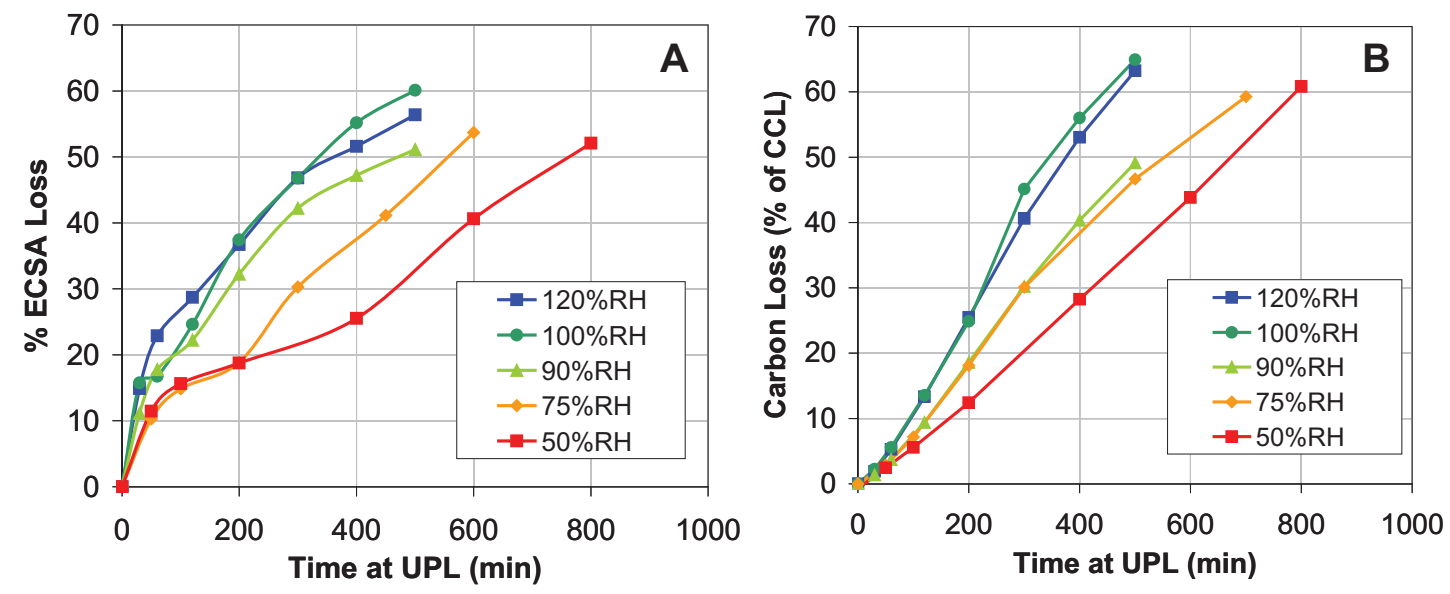

Figure 21: (A) Effect of time at UPL (corrosion time, $1.4 \mathrm{~V}, 80^{\circ} \mathrm{C}$ ) on ECSA loss for different oxidant relative humidities, (B) Effect of time at UPL (corrosion time, $1.4 \mathrm{~V}, 80^{\circ} \mathrm{C}$ ) on carbon loss for different relative humidities

\subsubsection{Temperature}

The impact of temperature on BOT performance and degradation was investigated at $1.2 \mathrm{~V}$ and 1.4V UPL over a range from $60^{\circ} \mathrm{C}$ to $90^{\circ} \mathrm{C}$.

\section{(1) Temperature Effect at BOT and 1.2V UPL}

Figure 22 shows the effect of temperature on BOT performance for different catalyst loadings. While the performance is not very sensitive at higher catalyst loadings, only a small reduction with increasing temperature is observed, the sensitivity to temperature becomes exacerbated at low loadings and results in a pronounced performance drop specifically at the higher temperatures.

The effect of temperature on the Pt dissolution rate (UPL $\leq 1.2 \mathrm{~V}$ ) was also found to be very small, although a linear upward trend with increasing temperature was observed. Thus, it was concluded that the temperature does not have a significant impact on the $\mathrm{Pt}$ dissolution mechanism. 

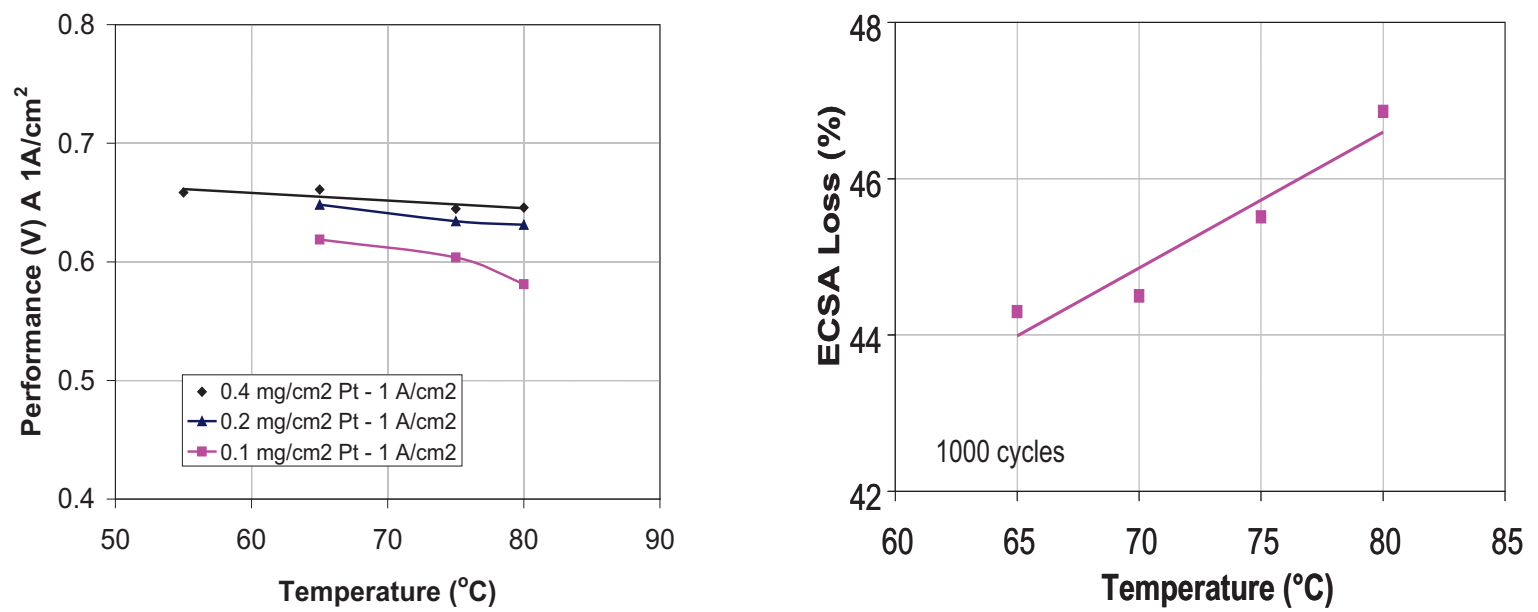

Figure 22: (A) BOT performance sensitivity at $1 \mathrm{~A} / \mathrm{cm}^{2}$ with operating temperature, (B) ECSA loss after AST cycling at 1.2V UPL and different temperatures

\section{(2) Temperature Effect at 1.4V UPL}

As expected, a pronounced dependence on temperature was observed with carbon support corrosion (1.4V UPL), as shown in Figure 23. The carbon corrosion and thus performance loss is accelerated with increasing temperature. The double layer charging current results showed that both the carbon oxidation and carbon corrosion reaction rates increased substantially and follow an Arrhenius type relationship, as shown in Figure 23.
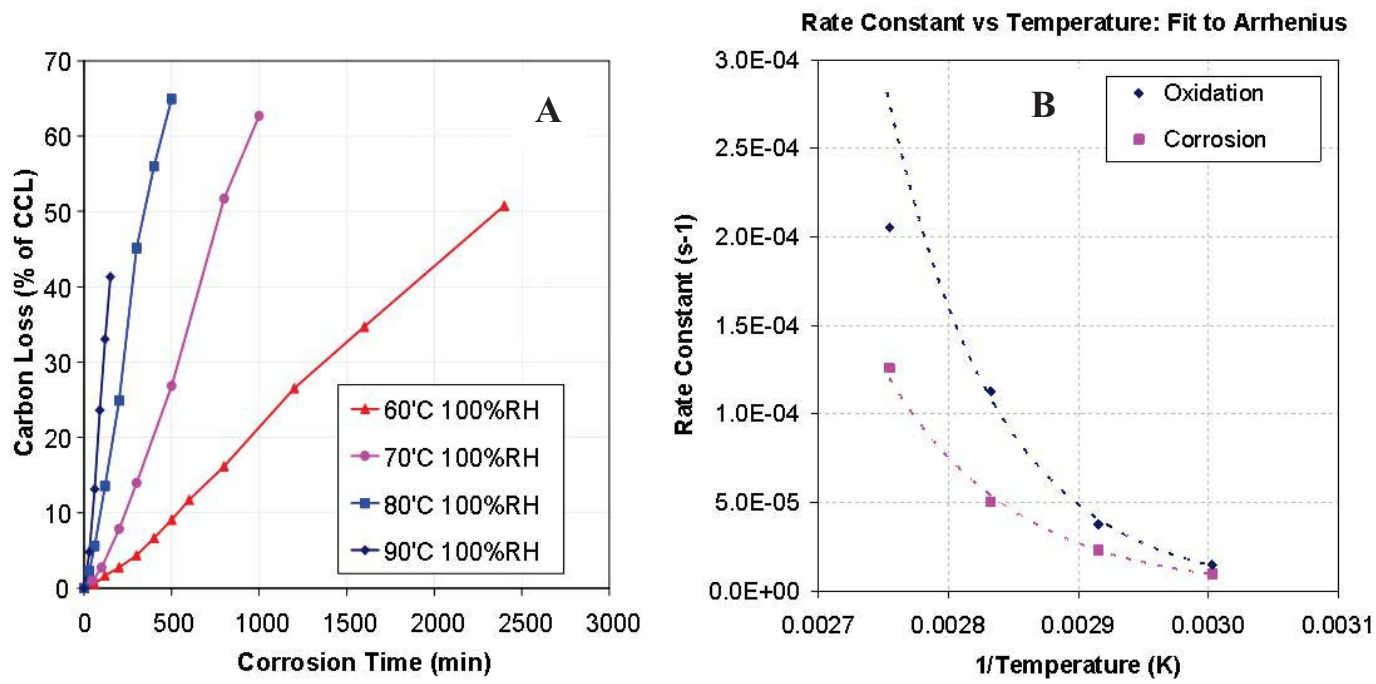

Figure 23: (A) Effect of corrosion time on carbon loss for different temperatures, (B) Arrhenius relationship for oxidation and corrosion rates (1.4V UPL) 

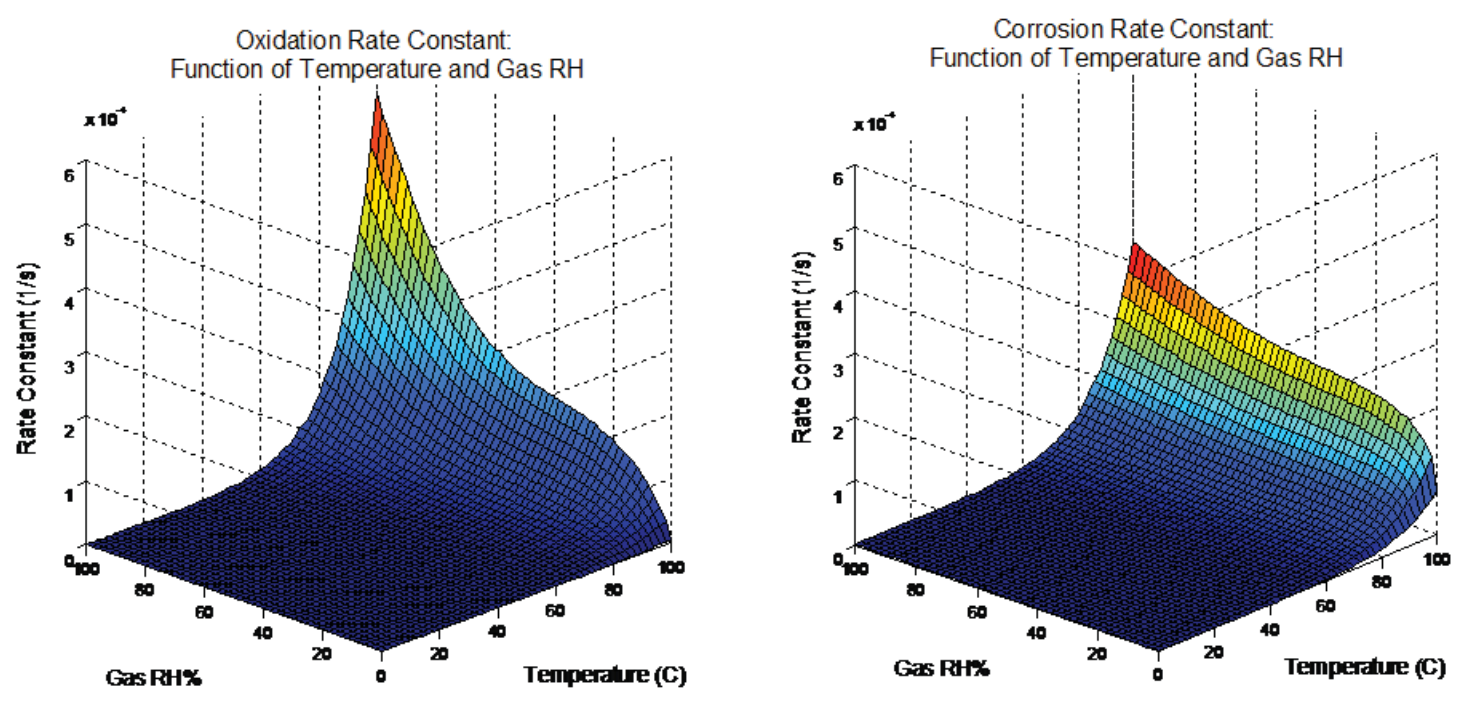

Figure 24: Impact of temperature and relative humidity on the carbon corrosion mechanism (1.4V).

Figure 24, a simulation using the semi-empirical two-step carbon corrosion model developed under this project ${ }^{12}$, shows the effect of temperature and humidity on the carbon oxidation and corrosion rates which indicates the large impact from $\mathrm{RH}$ on both rates and that the carbon oxidation rate accelerates faster than the corrosion rate for both $\mathrm{RH}$ and temperature. The model is described in some detail in Chapter VII.

\subsubsection{Reactant Composition}

The effect of reactant compositions on the degradation mechanisms was investigated using the standard AST protocol (1.2V UPL).

\section{(1) Oxygen Concentration}

The impact of oxygen concentrations ranging from 5 to $100 \%$ on BOT performance shown in Figure 25 reveals the BOT performance sensitivity. Even at $10 \% \mathrm{O}_{2}$ concentration that is often encountered under fuel cell stack conditions, the performance is substantially impacted. However, as shown in Figure 26 degradation rates did not seem to be affected as the EOT losses as a function of $\mathrm{O}_{2}$ concentrations showed very similar responses. Based on current modeling data this may suggest that the oxides derived from water have a greater impact on $\mathrm{Pt}$ dissolution than the oxides from oxygen.

12 A. P. Young, V. Colbow, D. Harvey, E. Rogers, and S. Wessel, "A Semi-Empirical Two Step Carbon Corrosion Reaction Model in PEM Fuel Cells", Journal of The Electrochemical Society, 160 (4) F381F388 (2013). 
DE-EE0000466

Ballard Material Products Inc

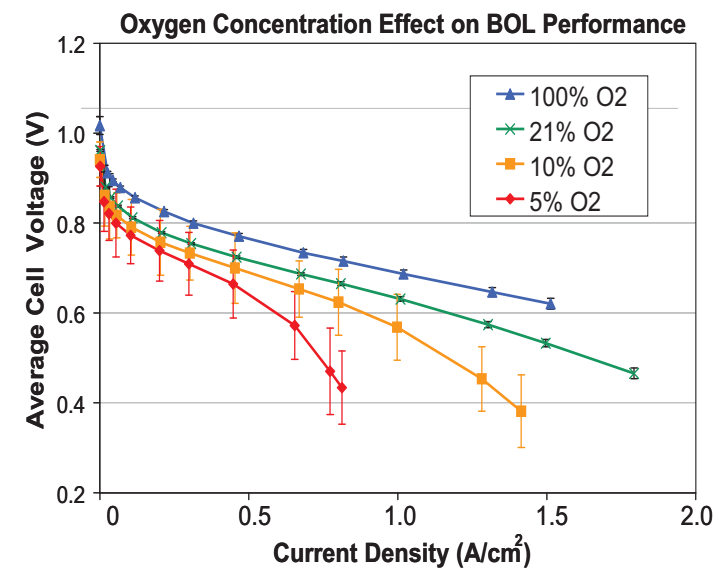

Figure 25: Performance sensitivity with oxygen concentration
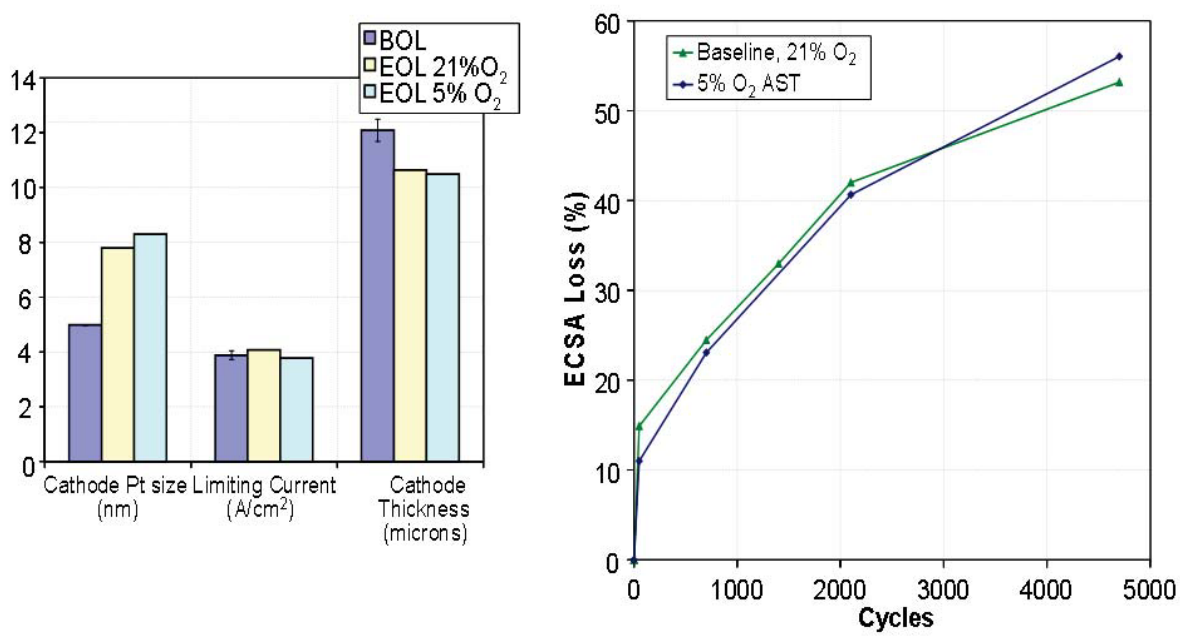

Figure 26: \% ECSA loss as a function of AST Cycles (0.6V (30 sec) to $1.0 \mathrm{~V}(60 \mathrm{sec})$

\section{(2) Hydrogen Concentration ${ }^{13}$}

The hydrogen concentration studies were conducted outside of this project and are reported for the purpose of completeness for the operational investigations. Figure 27 shows that the impact of $\mathrm{H}_{2}$ concentration, i.e. $\mathrm{H}_{2}$ cross-over to the cathode, on the performance degradation rate and ECSA loss is negligible and within experimental uncertainties, although at hydrogen concentrations of $20 \%$ slightly lower degradation rates are observed. Pt agglomeration however shows a trend with $\mathrm{H}_{2}$ cross-over as shown by the increase in Pt crystallite size from $7 \mathrm{~nm}$ for $100 \%$ hydrogen to $\sim 10 \mathrm{~nm}$ for a hydrogen concentration of $20 \%$. This result is linked to

${ }^{13}$ T. Cheng, E. Rogers, A. Young, S. Ye, V. Colbow, S. Wessel, "Effects of crossover hydrogen on platinum dissolution and agglomeration.”, J. Power Sources 196 (2011) 7985-7988. 


\section{DE-EE0000466}

the fact that the crossover hydrogen chemically reduces Pt oxides and subsequently decreases the amount of Pt ions formed via the oxide pathway. Further, the micrographs show the PITM band position is shifted towards the anode for the $20 \%$ hydrogen concentration due to a lower $\mathrm{H}_{2}$ flux.
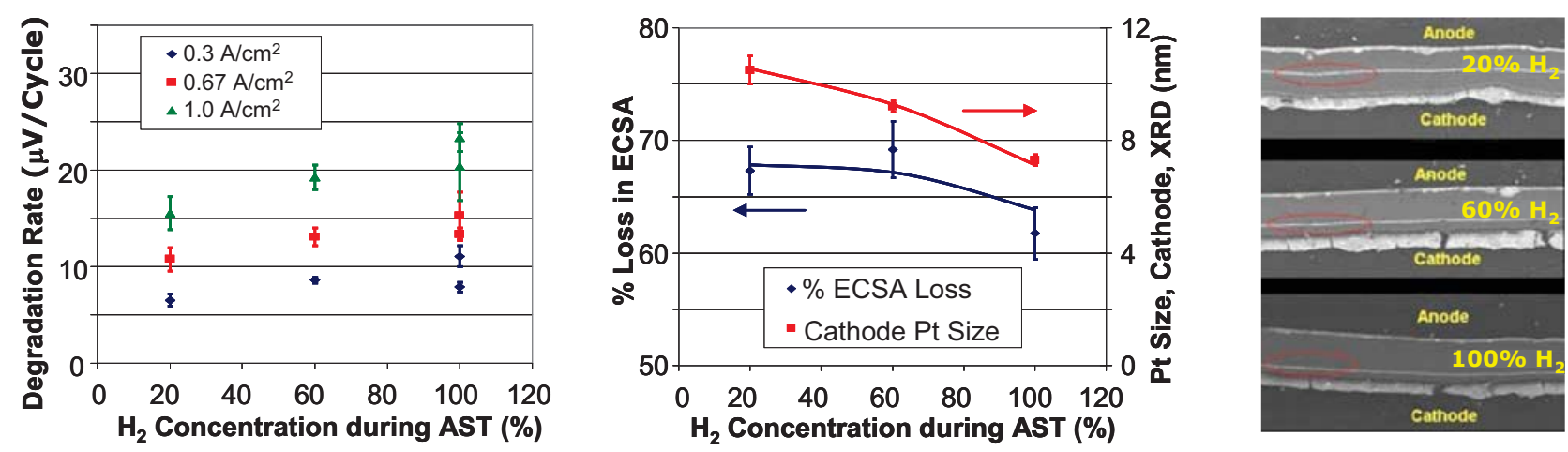

Figure 27: Effect $\mathrm{H}_{2}$ concentration on performance degradation rate, ECSA loss, and the position of the $\mathrm{Pt}$ in the membrane band after 4700 standard AST cycles (1.2V UPL)

\subsubsection{MEA Water Content at 1.4V UPL - Neutron Imaging Results (LANL)}

Water content studies of the reference MEA were conducted at the NIST Neutron Imaging Facility by LANL. For this purpose, a new straight channel neutron imaging cell (shown in Figure 28) was designed to mimic Ballard's AST hardware and characterized for baseline performance and performance sensitivity (temperature, relative humidity, oxygen partial pressure, and inlet gas pressure). The performance and performance sensitivity with the baseline MEA was found to be approximately $35 \mathrm{mV}$ higher at $1 \mathrm{~A} / \mathrm{cm}^{2}$ than Ballard's test hardware due to lower plate resistance, but all sensitivity trends compared very well.

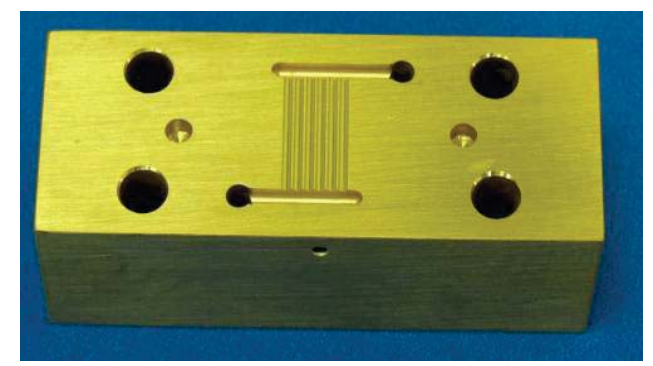

Figure 28: Straight Channel Neutron Imaging Hardware

The baseline MEA was subjected at NIST to AST cycling with an upper potential of $1.4 \mathrm{~V}$ and at predetermined cycle numbers polarization curves, cyclic voltammetry scans and neutron imaging data were obtained. The performance results and ECSA loss with progressive cycling is shown in Figure 29 and Figure 30, respectively. The results in NI hardware compared well with AST cycling results at Ballard, i.e. $300 \mathrm{~mA} / \mathrm{cm}^{2}$ after 700 cycles (Ballard hardware) vs. $320 \mathrm{~mA} / \mathrm{cm}^{2}$ after 750 cycles (NI hardware) at $600 \mathrm{mV}$; the loss in ECSA after 700 cycles was $90 \%$ and $95 \%$ after 750 cycles, respectively. 
The water density images at $0.6 \mathrm{~V}$ for $\mathrm{BOT}$ and various $0.6 \mathrm{~V} \rightarrow 1.4 \mathrm{~V}$ cycles are shown in Figure 31. The images clearly show a higher concentration of water under the landings than the channels at BOT. With progressive cycling, the liquid water under the landings increased up to $\sim 100$ cycles, and then starts to decrease at 250 cycles. At 750 cycles the water content under the landing seems very similar to that under the channels. The water density profiles in Figure 32(A) show very little difference in water content with progressive cycling in the channel area, an indication that the water content in the channels remains constant during the 750 AST cycles. Moreover, the water content was essentially similar on the anode and cathode sides. In contrast, the water content under the landings Figure 32(B) changed dramatically with progressive cycling. Initially the water content on the cathode side increased likely due to a higher current density at $0.6 \mathrm{~V}$ (improved performance) while the anode side remained unchanged. This behaviour is believed to be associated with oxidation of the cathode catalyst carbon support surface thereby losing its hydrophobicity and yielding increased proton conductivity which is reflected by the higher performance shown in Figure 29 . Upon progressive cycling the high water profile on the cathode reduced substantially. This behaviour is likely due to increased support corrosion under the channels as corrosion is substantially accelerated with liquid water content. After $>500$ cycles the water profile was essentially symmetric for the anode and cathode, at which point $\sim 80 \%$ of the average cathode catalyst layer thickness was lost.

This study has provided valuable information in that the water content under the landings is substantially higher than in the channels, thus the onset of carbon support oxidation and corrosion is accelerated under the landing in comparison to the channels.

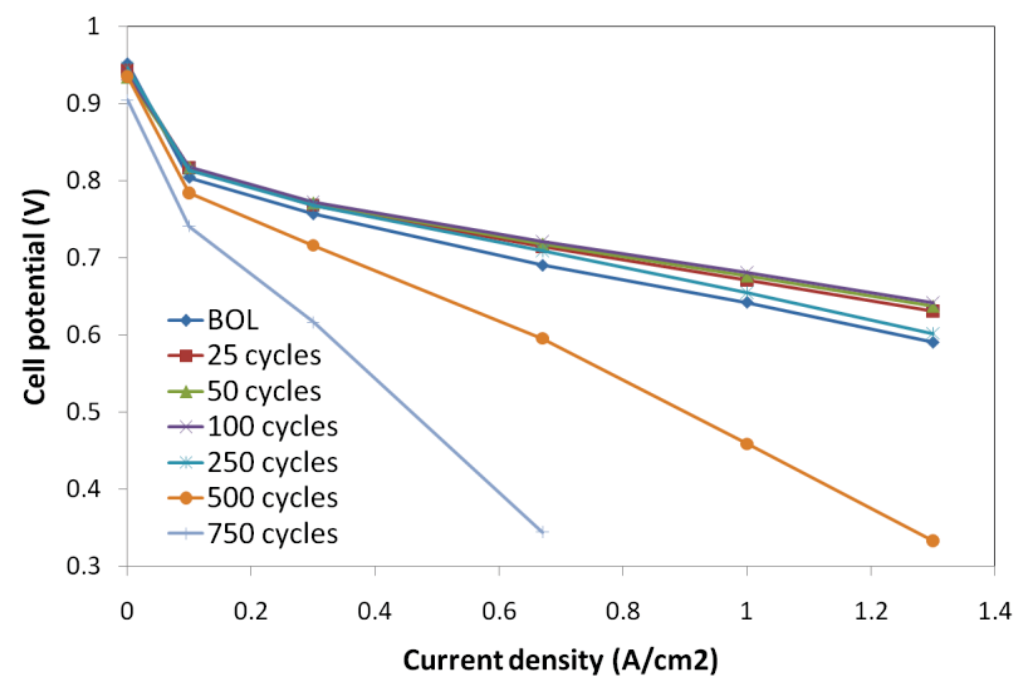

Figure 29: Polarization curves after selected numbers of AST cycles at $1.4 \mathrm{~V}$ in $\mathrm{NI}$ hardware 
DE-EE0000466

Ballard Material Products Inc

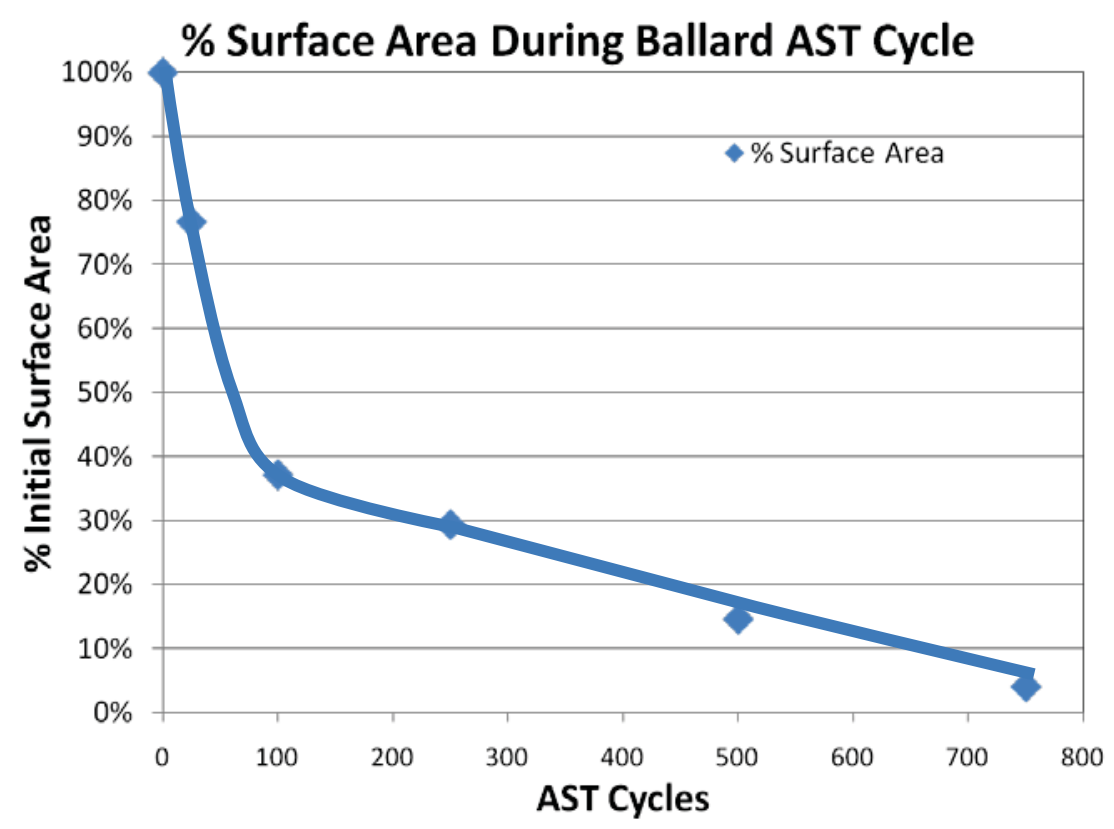

Figure 30: ECSA change after 750 cycles at 1.4V UPML (Neutron Imaging Hardware)

Water density images: $0.6 \mathrm{~V}$ holds, $80^{\circ} \mathrm{C}, 100 \% \mathrm{RH}$; after cycles of $0.6-1.4 \mathrm{~V}$ in $\mathrm{H}_{2}$ /air

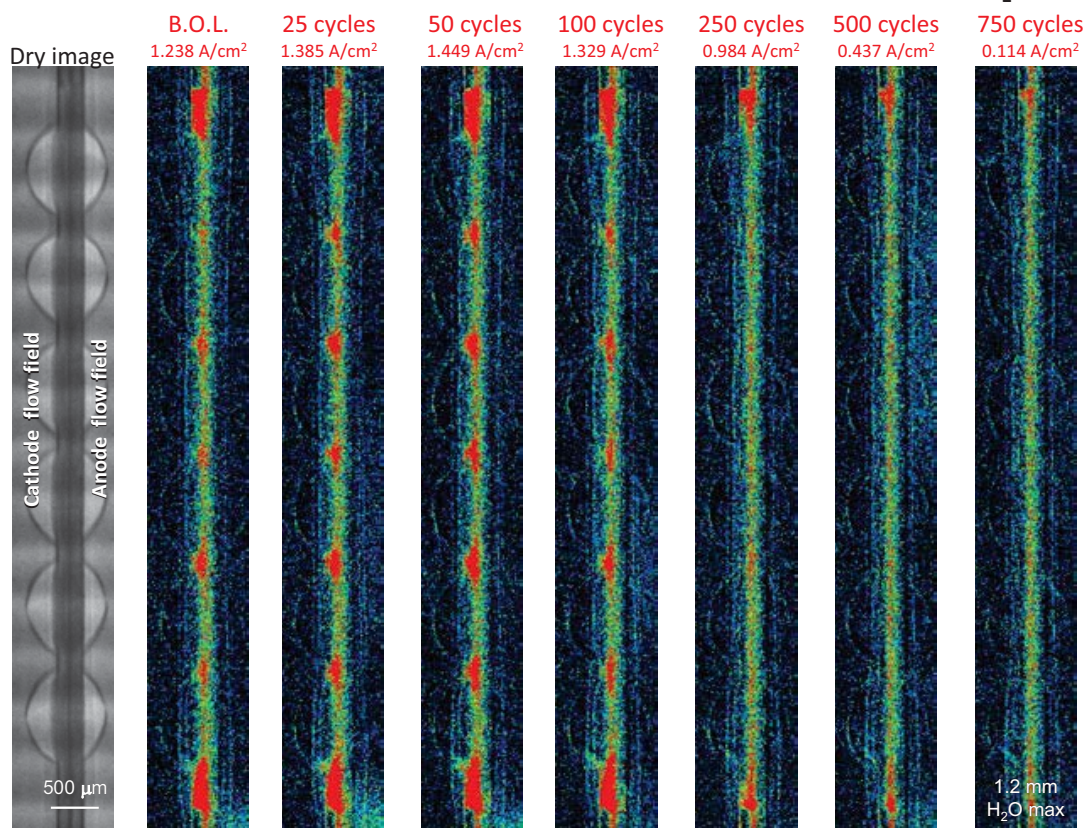

Figure 31: Water density image changes at $0.6 \mathrm{~V}$ with progressive cycling with upper potential at 1.4V UPL 

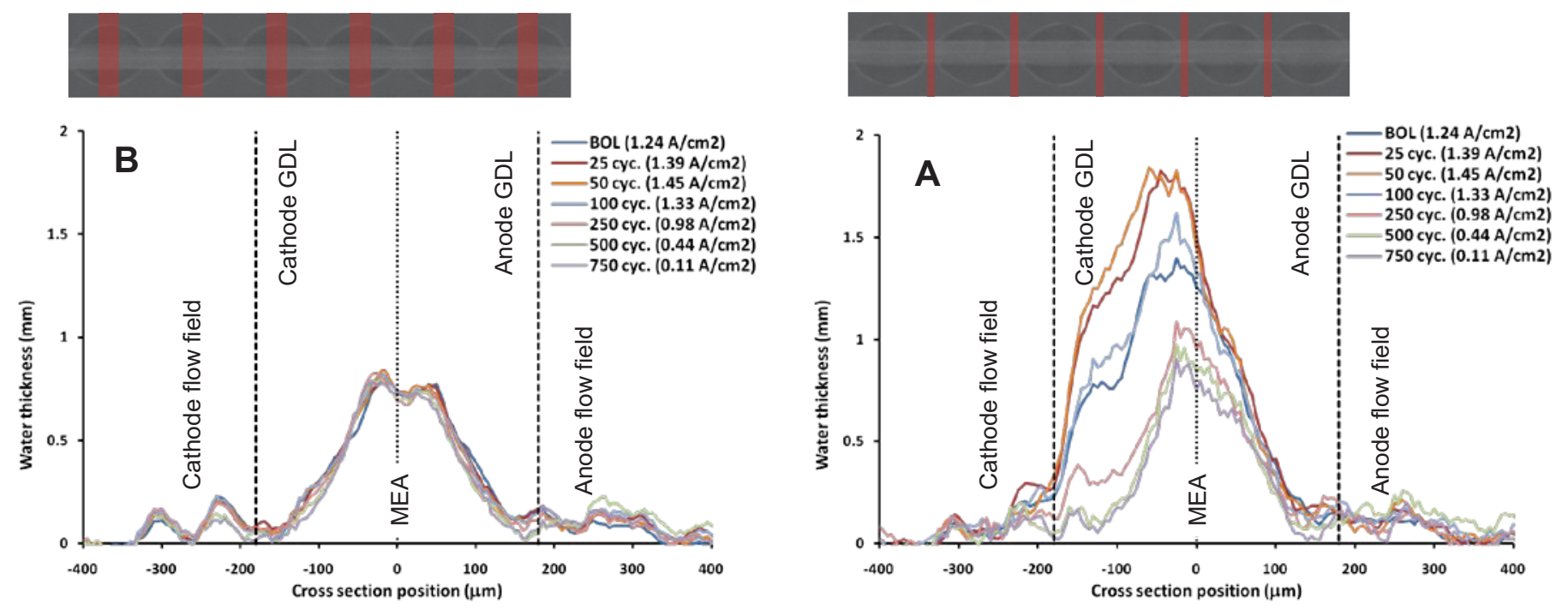

Figure 32: Water density profiles of the MEA cross section at $0.6 \mathrm{~V}$ for $\mathrm{BOL}$ and after progressive cycling $\left(80^{\circ} \mathrm{C}, 100 \% \mathrm{RH} ; 0.6-1.4 \mathrm{~V}\right.$ cycles in $\mathrm{H} 2 /$ air). (A) Middle of channels, (B) middle of landings.

\subsubsection{Summary/Conclusions}

Table 8 provides a summary of the different operational studies. It specifies the parameters and their ranges that were investigated and provides high level information on their impact on BOT performance and the effect on the Pt dissolution and carbon corrosion mechanisms and rates. In terms of the impact of degradation severity the operational parameters follow the following trend:

$$
\text { UPL >> Dwell Time, RH, Cycle number > LPL > T, [O } \left.\mathrm{O}_{2}\right],\left[\mathrm{H}_{2}\right]
$$

Table 9 summarizes the degradation rates of the various operational stressors and thus quantifies the impact of the stressors for $15 \%$ performance loss and $40 \%$ reduction in ECSA. It also identifies that changes with UPL and temperature are exponential in nature, while performance and ECSA losses with RH and dwell time exhibit a linear behaviour. 
Table 8: Summary table of the operational conditions, their ranges and effects on BOT performance and degradation rates

\begin{tabular}{|c|c|c|c|c|}
\hline \multicolumn{5}{|c|}{ Summary of Operational Effect } \\
\hline Stressor Evaluated & Testing Modifications & ВОт Performance & $\begin{array}{c}\text { Mechanism } \\
\text { Investigated }\end{array}$ & Degradation Rate \\
\hline Upper Potential (UPL) & \begin{tabular}{|l|}
$0.9-1.6 \mathrm{~V}(\mathrm{LSAC})$ \\
$0.9-1.4 \mathrm{~V}(\mathrm{MSAC})$ \\
\end{tabular} & Not Applicable & $\begin{array}{l}\text { Pt Dissolution } \\
\text { C-Corrosion }\end{array}$ & Increases with UPL \\
\hline Lower potential Limit & $0.1,0.4,0.6,0.8$ to $1.0 \mathrm{~V}$ & Not Applicable & Pt Dissolution & Lower degradation at LPL $>0.6 \mathrm{~V}$ \\
\hline \multirow{2}{*}{ Cycle Number } & 0 to 4700 Cycles & \multirow{2}{*}{ Not Applicable } & $\begin{array}{l}\text { Pt Dissolution, } \\
\text { C-Corrosion }\end{array}$ & Increases with Cycling \\
\hline & $\begin{array}{l}0 \text { to } 2100 \text { Cycles } \\
1.4 \mathrm{~V}\end{array}$ & & C-Corrosion & Increases with time at UPL \\
\hline \multirow{2}{*}{ Dwell Time } & $\begin{array}{l}5-600 \text { seconds } \\
1.0 \mathrm{~V}\end{array}$ & \multirow{2}{*}{ Not Applicable } & Pt Dissolution & Increases with dwell time \\
\hline & $\begin{array}{l}-600 \text { seconds } \\
14 \mathrm{~V}\end{array}$ & & C-Corrosion & Increases with time at UPL \\
\hline Relative Humidity & $\begin{array}{l}50 \% \text { RH to Oversaturated } \\
1.4 \mathrm{~V}\end{array}$ & Increases with increase RH & \begin{tabular}{|l|} 
Pt Dissolution \\
C-Corrosion \\
\end{tabular} & Increases with RH \\
\hline \multirow{2}{*}{ Temperature } & \multirow{2}{*}{$\begin{array}{l}60-85^{\circ} \mathrm{C} \\
1.4 \mathrm{~V} \\
\end{array}$} & \multirow{2}{*}{$\begin{array}{l}\text { Decreases with temperature } \\
\text { increase }\end{array}$} & Pt Dissolution & Slight increase with $\mathrm{T}$ \\
\hline & & & C-Corrosion & Increaseas with $\mathrm{T}$ \\
\hline \multirow{2}{*}{ Oxidant Composition } & $\begin{array}{l}\text { Air vs. } \mathrm{N}_{2} \\
2100 \text { cycles }\end{array}$ & Not Applicable & Pt Dissolution & $\mathrm{N}_{2}$ (No PITM) < Air (PITM ) \\
\hline & \begin{tabular}{|l|}
$5 \%$ to $100 \%$ \\
$1.0 \mathrm{~V}$ \\
\end{tabular} & Increases with increase $\left[\mathrm{O}_{2}\right]$ & Pt Dissolution & No impact \\
\hline Hydrogen Composition & $20,60,100 \% \mathrm{H}_{2}$ & Not Applicable & Pt Dissolution & $\begin{array}{l}\text { No Impact on rate } \\
\text { Impact on location of PITM band }\end{array}$ \\
\hline
\end{tabular}

Standard AST: Air/ $\mathrm{H}_{2}, 100 \% \mathrm{RH}, 5 \mathrm{psig}, 80^{\circ} \mathrm{C}, 0.6 \mathrm{~V}(30 \mathrm{sec})-->1.2 \mathrm{~V}(60 \mathrm{sec}), 4700 \mathrm{cycles}$

Reference MEA: 50:50 Pt/C, Nafion ${ }^{\circledR}$ ionomer, 0.4/0.1 mg/cm² (Cathode/anode), Ballard CCM, Nafion ${ }^{\circledR}$ NR211, BMP GDLs Ballard Test Cell: 1D, $45 \mathrm{~cm}^{2}$ active area

Table 9: Impact summary of the different operational stressors on the ECSA and voltage degradation rates calculated at 50\% ECSA I and 15\% performance loss.

\begin{tabular}{|c|c|c|c|c|c|c|c|}
\hline \multicolumn{8}{|c|}{$\begin{array}{c}\text { Impact Matrix } \\
\text { Effect of Operational Stressor on CL Degradation }\end{array}$} \\
\hline Operational Stressor & Range Studied & $\begin{array}{c}\text { ECSA Degradation Rate, } \\
\text { evaluated at } 50 \% \text { ECSA LosS } \\
\text { (ECSA units / hour at UPL) }\end{array}$ & $\begin{array}{c}\text { Voltage Degradation Rate, } \\
1.0 \mathrm{~A} / \mathrm{cm}^{2}, \text { evaluated at } 15 \% \\
\text { Performance Loss } \\
\text { (mV loss / hour at UPL) }\end{array}$ & $\begin{array}{c}\text { Voltage Degradation Rate, } \\
1.5 \mathrm{~A} / \mathrm{cm}^{2}, \text { evaluated at } 15 \% \\
\text { Performance Loss } \\
\text { (mV loss / hour at UPL) }\end{array}$ & \multicolumn{2}{|l|}{ AST Details } & $\begin{array}{l}\text { Diagnostic } \\
\text { Conditions }\end{array}$ \\
\hline $\begin{array}{l}\text { Upper Potential Limit } \\
\text { (UPL) }\end{array}$ & $\begin{array}{c}\uparrow \\
1.0 \mathrm{~V} \text { to } 1.4 \mathrm{~V}\end{array}$ & $\begin{array}{c}\uparrow \\
1.6 \text { to } 25.9\end{array}$ & $\begin{array}{c}\uparrow \\
0.4 \text { to } 13\end{array}$ & $\begin{array}{c}\uparrow \\
0.6 \text { to } 13\end{array}$ & \multicolumn{2}{|c|}{$\begin{array}{l}0.6 \mathrm{~V}(30 \mathrm{sec}) \text { to UPL }(60 \mathrm{sec}) \\
4700 \text { cycles, } 100 \% \mathrm{RH}, 80^{\circ} \mathrm{C}\end{array}$} & \multirow{6}{*}{$\begin{array}{c}\text { Air } / \mathrm{H}_{2}, 100 \% \text { RH, } \\
5 \mathrm{psig}, 75^{\circ} \mathrm{C}\end{array}$} \\
\hline $\begin{array}{c}\text { Temperature, during } \\
\text { AST } \\
(1.4 \mathrm{~V} \text { UPL, } 100 \% \mathrm{RH})\end{array}$ & $\begin{array}{c}\uparrow \\
70^{\circ} \mathrm{C} \text { to } 90^{\circ} \mathrm{C}\end{array}$ & $\begin{array}{c}\uparrow \\
7 \text { to } 58\end{array}$ & $\begin{array}{c}\uparrow \\
9 \text { to } 38\end{array}$ & $\begin{array}{c}\uparrow \\
9 \text { to } 41\end{array}$ & \multicolumn{2}{|c|}{$\begin{array}{l}0.6 \mathrm{~V}(30 \mathrm{sec}) \text { to } 1.4 \mathrm{~V}(600 \mathrm{sec}) \\
4700 \mathrm{cycles}, 100 \% \mathrm{RH}, x^{\circ} \mathrm{C}\end{array}$} & \\
\hline $\begin{array}{c}\text { Temperature, during } \\
\text { AST } \\
\text { (1.4V UPL, } 50 \% \text { RH) } \\
\end{array}$ & $\begin{array}{c}\uparrow \\
70^{\circ} \mathrm{C} \text { to } 90^{\circ} \mathrm{C}\end{array}$ & $\begin{array}{c}\uparrow \\
3 \text { to } 12 \\
\end{array}$ & $\begin{array}{c}\uparrow \\
3 \text { to } 19 \\
\end{array}$ & $\begin{array}{c}\uparrow \\
3 \text { to } 18 \\
\end{array}$ & \multicolumn{2}{|c|}{$\begin{array}{l}0.6 \mathrm{~V}(30 \mathrm{sec}) \text { to } 1.4 \mathrm{~V}(600 \mathrm{sec}), x \\
\text { cycles, } 50 \% \mathrm{RH}, \mathrm{x}^{\circ} \mathrm{C}\end{array}$} & \\
\hline $\begin{array}{c}\text { Relative Humidity, } \mathrm{RH} \\
\text { during AST } \\
\text { (1.4V UPL) } \\
\end{array}$ & $\begin{array}{c}\uparrow \\
60 \% \text { to } 100 \% \\
\end{array}$ & $\begin{array}{c}\uparrow \\
8 \text { to } 15\end{array}$ & $\begin{array}{c}\uparrow \\
9 \text { to } 12 \\
\end{array}$ & $\begin{array}{c}\uparrow \\
9 \text { to } 13\end{array}$ & \multicolumn{2}{|c|}{$\begin{array}{l}0.6 \mathrm{~V}(30 \mathrm{sec}) \text { to } 1.4 \mathrm{~V}(600 \mathrm{sec}), \mathrm{x} \\
\quad \text { cycles, } 100 \% \mathrm{RH}, 80^{\circ} \mathrm{C}\end{array}$} & \\
\hline $\begin{array}{c}\text { Relative Humidity, RH } \\
\text { during AST } \\
(1.2 \mathrm{~V} \text { UPL) } \\
\end{array}$ & $\begin{array}{c}\uparrow \\
60 \% \text { to } 100 \%\end{array}$ & $\begin{array}{c}\uparrow \\
0.4 \text { to } 2.8\end{array}$ & $\begin{array}{c}\uparrow \\
0.06 \text { to } 0.5\end{array}$ & $\begin{array}{c}\uparrow \\
0.3 \text { to } 1\end{array}$ & \multicolumn{2}{|c|}{$\begin{array}{l}0.6 \mathrm{~V}(30 \mathrm{sec}) \text { to } 1.2 \mathrm{~V}(60 \mathrm{sec}) \\
4700 \text { cycles, } 100 \% \mathrm{RH}, 80^{\circ} \mathrm{C}\end{array}$} & \\
\hline $\begin{array}{c}\text { Dwell Time at UPL } \\
\quad(1.4 \mathrm{~V} \text { UPL })\end{array}$ & $\begin{array}{c}\uparrow \\
20 \mathrm{~s} \text { to } 600 \mathrm{~s}\end{array}$ & $\begin{array}{c}\downarrow \\
23 \text { to } 15\end{array}$ & $\sim 13$ & $\sim 14$ & \multicolumn{2}{|c|}{$\begin{array}{c}0.6 \mathrm{~V}(30 \mathrm{sec}) \text { to } 1.4 \mathrm{~V}(\mathrm{x} \mathrm{sec}), x \\
\text { cycles, } 500 \mathrm{~min} \text { total UPL time, } \\
100 \% \mathrm{RH}, 80^{\circ} \mathrm{C}\end{array}$} & \\
\hline $\begin{array}{c}\text { Lower Potential Limit } \\
\text { (LPL) } \\
(1.0 \mathrm{~V} \text { UPL) } \\
\end{array}$ & $\begin{array}{c}\uparrow \\
0.1 \mathrm{~V} \text { to } 0.6 \mathrm{~V}\end{array}$ & $\sim 1.3$ & $\sim 0.5$ & $\sim 0.9$ & $\begin{array}{l}\text { LPL }(30 \text { sec }) \text { to } 1.0 \mathrm{~V}( \\
4700 \text { cycles, } 100 \% \text { RH }\end{array}$ & $\mathrm{Neg}$ & Legend \\
\hline & & & & & & \multicolumn{2}{|c|}{ Exponential Effect } \\
\hline
\end{tabular}


DE-EE0000466

Ballard Material Products Inc

\subsection{Impact of Catalyst Layer Structure and Composition}

Variations in catalyst layer structure and composition were investigated to understand the impact on performance and durability. Structural/compositional variations included Pt loading, the $\mathrm{Pt} / \mathrm{C}$ ratio for the LSAC carbon support, carbon support types, and ionomer loading and ionomer equivalent weight. Furthermore, preliminary studies included membrane type, the GDL MPL, and catalyst layer processing. The different studies are discussed below separately.

\subsubsection{Catalyst Loading}

The effect of catalyst loading on performance and degradation was evaluated for a range of $\mathrm{Pt}$ loadings $\left(0.05,0.1,0.2,0.3,0.4\right.$, and $\left.0.5 \mathrm{mg} \mathrm{Pt} / \mathrm{cm}^{2}\right)$ of the reference MEA using the standard cathode AST (1.2V UPL).

Figure 33 shows the effect of catalyst loading on the catalyst layer thickness and ECSA. Both thickness and loading follow the same trend which is essentially linear except for the lower loadings likely due to reduced catalyst utilization.

The effect of Pt loading on BOT performance at different operational conditions is shown in Figure 34. Within the temperature range of 65 to $80^{\circ} \mathrm{C}$ and at low $\left(0.3 \mathrm{~A} / \mathrm{cm}^{2}\right)$ and high current densities $\left(1.0 \mathrm{~A} / \mathrm{cm}^{2}\right.$ ) the BOT performance is essentially unaffected for loadings $>0.2 \mathrm{mg} / \mathrm{cm}^{2}$; however increased temperature sensitivity (reduced performance) becomes apparent at loadings $<0.2 \mathrm{mg} / \mathrm{cm}^{2}$, specifically at the higher current densities. Similarly, the BOT performance is affected at loadings $<0.2 \mathrm{mg} / \mathrm{cm}^{2}$ by $\mathrm{RH}$ and oxygen concentration as shown Figure 34. This sensitivity is likely due to the reduced catalyst layer thickness and increased local water content within the thinner layers. This would also explain the enhanced temperature effect at the higher current density.

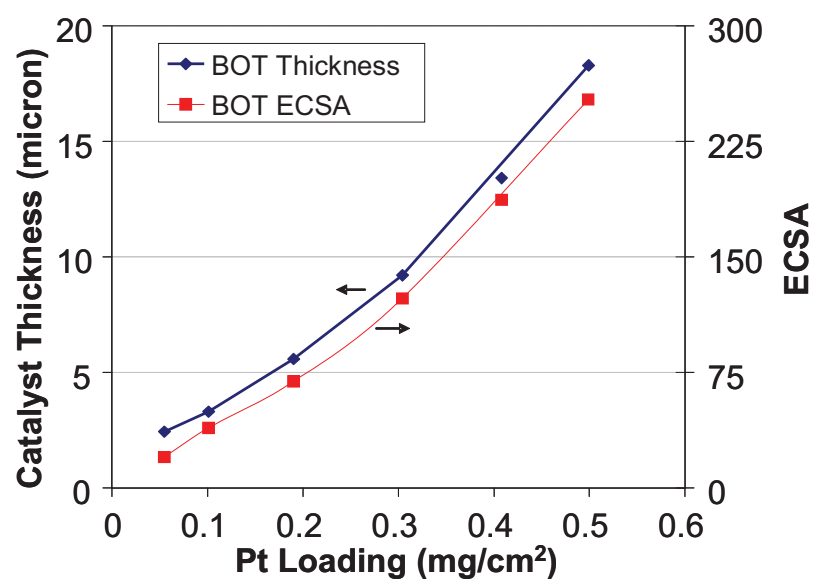

Figure 33: Effect of catalyst loading on BOT catalyst layer thickness and ECSA 

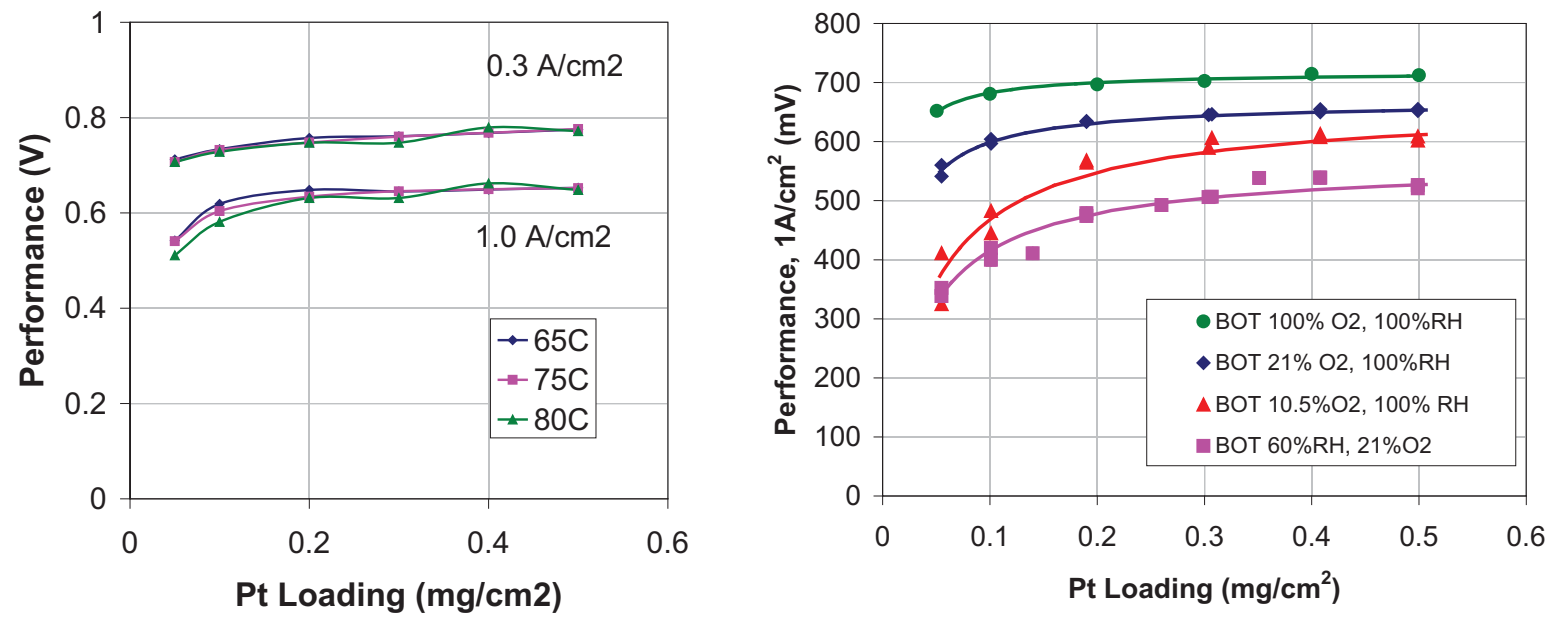

Figure 34: BOT performance with loading at different temperatures, relative humidity, and oxygen concentrations

The effect of Pt loading on degradation is shown in Figure 35. While the degradation rate for $\mathrm{Pt}$ loadings $\geq 0.3 \mathrm{mg} / \mathrm{cm}^{2}$ is essentially constant, an approximately eight-fold increase is observed when the Pt loading is reduced from 0.3 to $0.1 \mathrm{mg} / \mathrm{cm}^{2}$. As observed before, the increased performance degradation correlates well with the ECSA loss caused by Pt dissolution and thus agglomeration and PITM. The increased loss at the lower loadings was found to be associated with carbon corrosion. This relationship between performance and ECSA was found to be the same for BOT and the EOT suggesting that the primary variable affecting performance is ECSA (Figure 35B). Furthermore the performance is only affected once the ECSA drops below $\sim 60$.
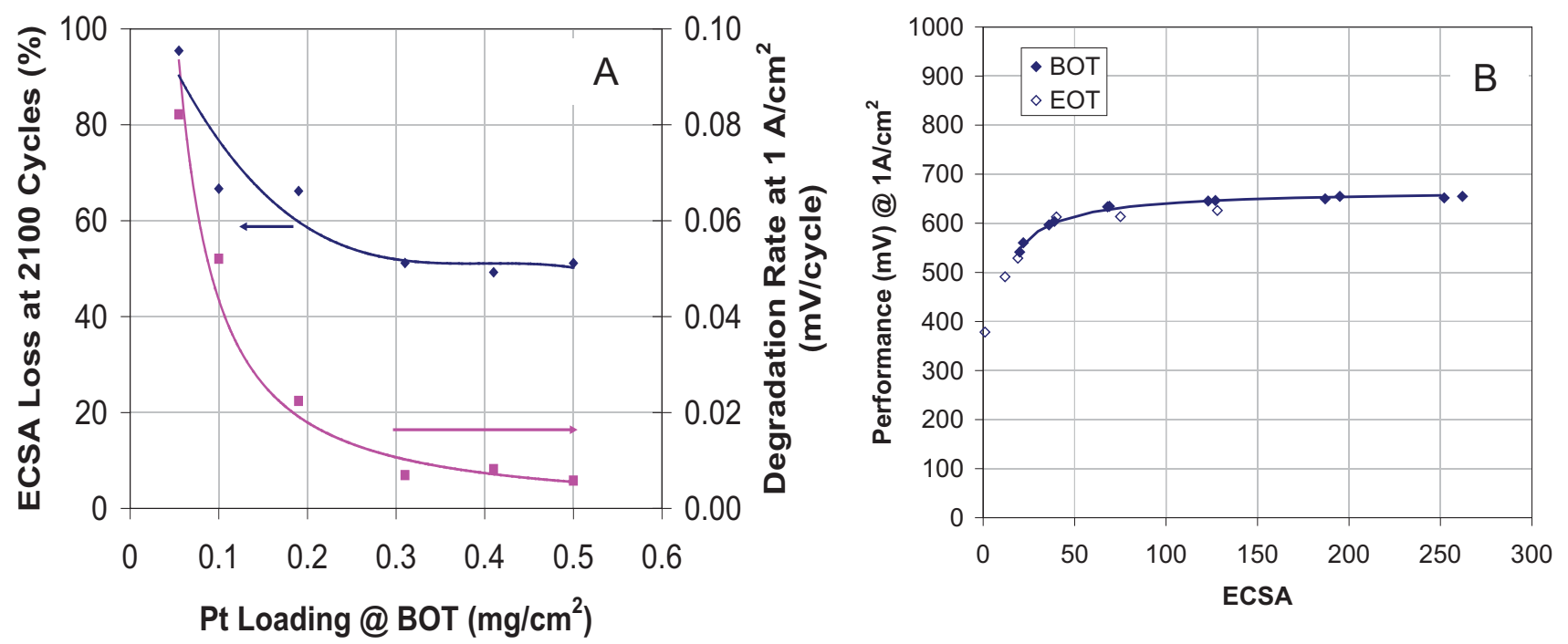

Figure 35: (A) Effect of BOT Pt loading on degradation rate, $(B)$ correlation of performance and ECSA 


\subsubsection{Carbon Ratio}

The effect of $\mathrm{Pt} / \mathrm{C}$ ratio for the LSAC catalyst support on performance and degradation was evaluated for a range of ratios from 30 to $80 \mathrm{wt} \% \mathrm{Pt} / \mathrm{C}$ at $0.4 \mathrm{mg} / \mathrm{cm}^{2}$ using the standard cathode AST (1.2V UPL). Unfortunately, the $80 \% \mathrm{Pt} / \mathrm{C}$ ratio catalyst ink could not be printed with the reference MEA ionomer content, thus degradation results for these MEAs were convoluted and therefore not included.

The impact of the $\mathrm{Pt} / \mathrm{C}$ ratio on performance loss at various current densities is shown in Figure 36. While the BOT performance is very similar for the different $\mathrm{Pt} / \mathrm{C}$ ratios, the performance loss after 4700 cycles at $1.2 \mathrm{~V}$ UPL reveals the lowest degradation for a $\mathrm{Pt} / \mathrm{C}$ ratio of $60 \%$ and the $30 \% \mathrm{Pt} / \mathrm{C}$ ratio MEA exhibited the largest performance loss. Loss analysis of the voltage degradation Figure $36(\mathrm{~B})$ revealed that the voltage degradation was predominantly a result of catalyst layer ionic conductivity changes.
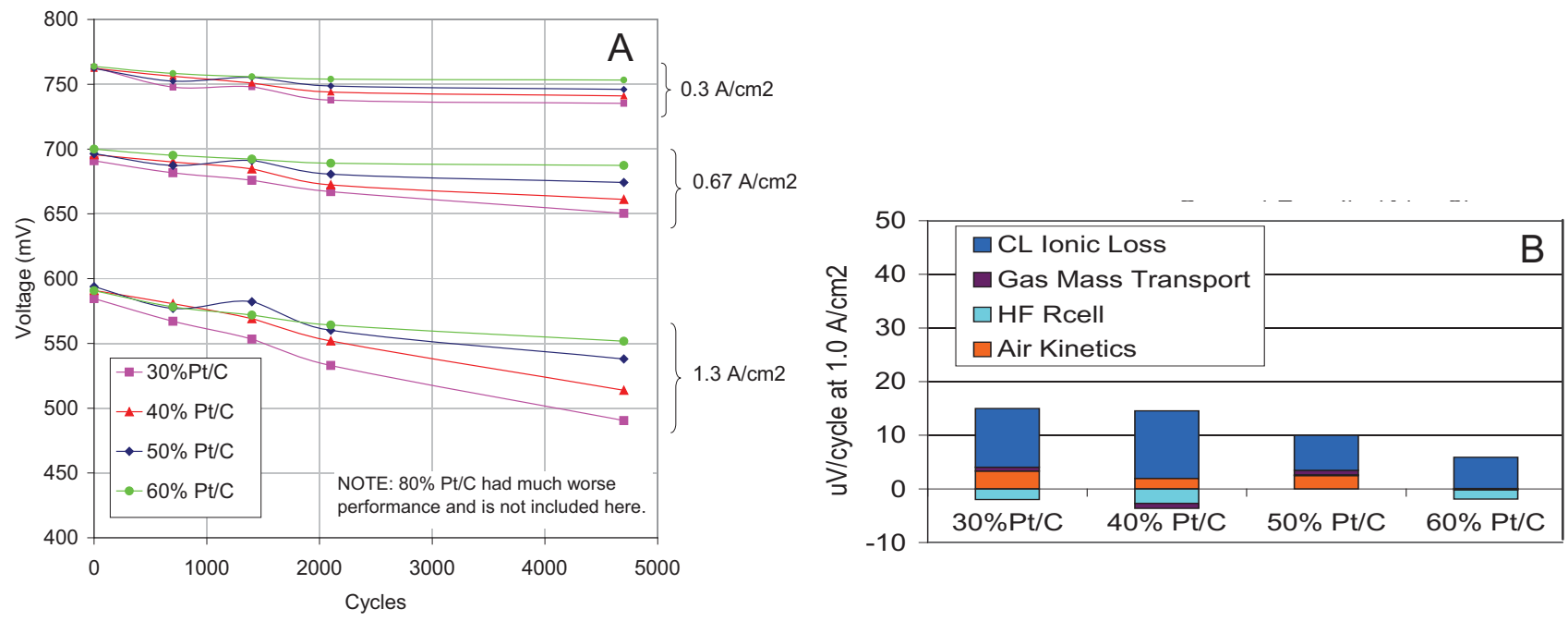

Figure 36: (A) Performance change at various current densities as a function of AST (1.2V UPL) cycle number and (B) voltage loss breakdown at 4700 AST cycles for LSAC catalysts with different $\mathrm{Pt} / \mathrm{C}$ ratios 

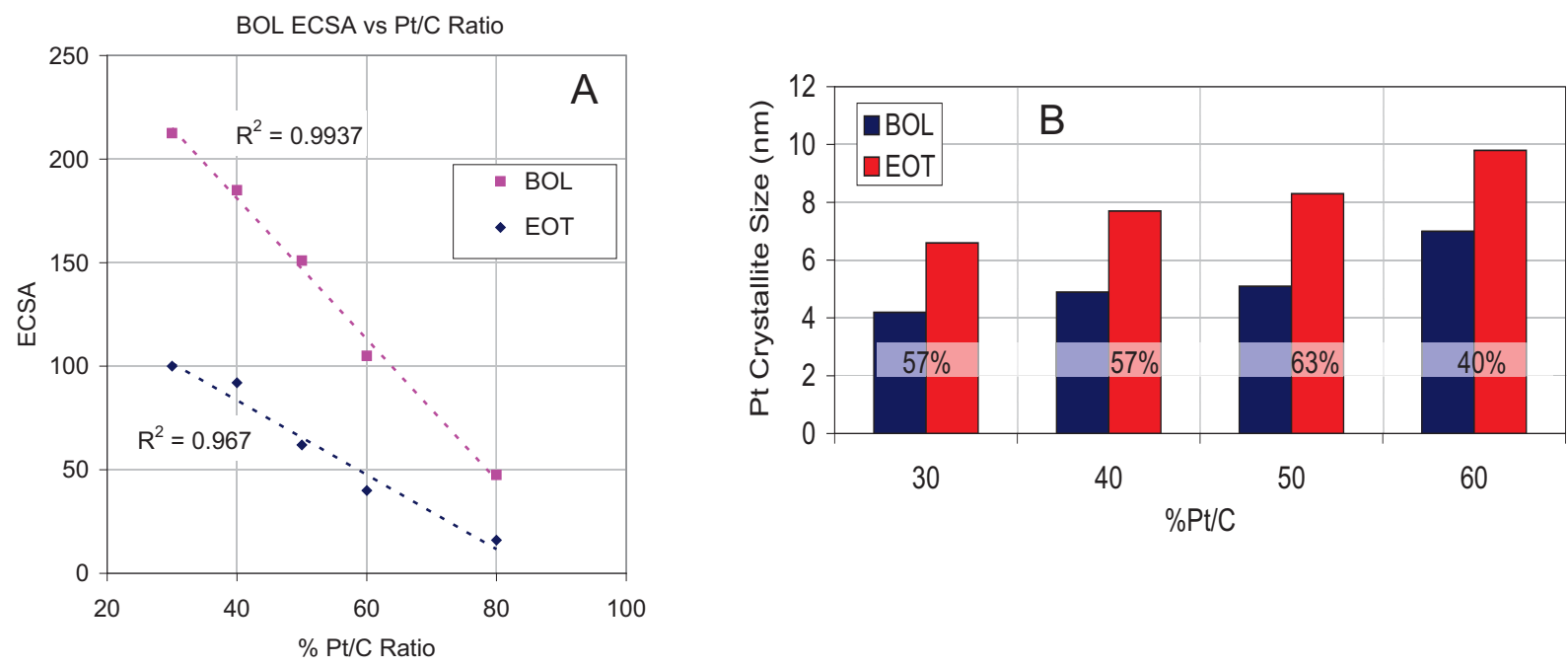

Figure 37: (A) ECSA change and (B) Pt crystallite changes as a function of Pt/C ratio for Pt-LSAC catalysts

The effect of the Pt/C ratio on cathode ECSA is shown in Figure 37. Independent of the Pt/C ratio, the ECSA was reduced by approximately $50 \%$ after AST cycling at $1.2 \mathrm{~V}$, similar to results observed with other catalysts. The low ECSA at the higher Pt/C ratios is due to the presence of substantially larger $\mathrm{Pt}$ crystallites, which consequently affects the $\mathrm{Pt}$ dissolution and agglomeration rate as shown in Figure 37B.

\subsubsection{Carbon Support}

The effect of the carbon support structure on performance and degradation was investigated for catalysts supported on carbon powders of low to high surface area carbons ranging from 160 to $800 \mathrm{~m}^{2} / \mathrm{g}$. MEAs with the different catalysts were subjected to UPLs of $1.0 \mathrm{~V}$ and $1.2 \mathrm{~V}$ to understand their impact on Pt dissolution and corrosion. A description of the different catalysts is provided in Table 10. The carbon and catalyst powder morphologies are discussed under Chapter III.

Table 10: Catalyst Description

\begin{tabular}{|c|c|}
\hline Sample ID & Sample Specifics \\
\hline Pt50-Vulcan & 50/50 Pt/Vulcan \\
\hline Pt50-LSAC & 50/50 Pt/Low Surface Area Carbon \\
\hline Pt50-MSAC & 50/50 Pt/Mid-range Surface Area Carbon \\
\hline Pt50-HSAC \#1 & 50/50 Pt/High Surface Area Carbon \#1 \\
\hline PT50-HSAC\#1 -HT & 50/50 Pt/High Surface Area Carbon \#1 -Heat Treated \\
\hline Pt50-HSAC \#2 & 50/50 Pt/High Surface Area Carbon \#2 \\
\hline Pt50-HSAC \#2 HT & 50/50 Pt/High Surface Area Carbon \#2 - Heat Treated \\
\hline
\end{tabular}


DE-EE0000466

Ballard Material Products Inc

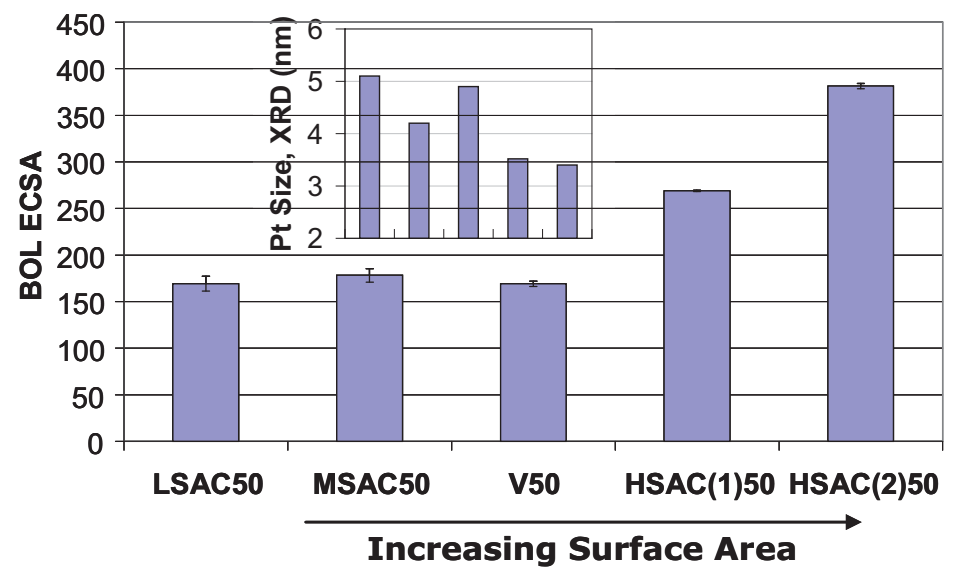

Figure 38: BOL ECSA for different carbon support structures

Figure 38 shows the BOT ESCA for the catalyst supported on the different carbon types. While the ECSA of the different catalysts increases with carbon surface area due to more homogeneous $\mathrm{Pt}$ particle distribution, the BOT performance did not reveal any such trend.

Figure 39 shows the voltage degradation rate at a current density of $1.3 \mathrm{~A} / \mathrm{cm}^{2}$ and kinetic performance loss after 4700AST cycles at an UPL of $1.0 \mathrm{~V}$ and $1.2 \mathrm{~V}$. At $1.0 \mathrm{~V}$ UPL the degradation rates for the different carbons are very low for all catalyst types, while at 1.2V UPL the performance degradation correlates with the surface area of the carbon supports, an indication that at 1.0V UPL, degradation is governed by Pt dissolution for all carbon types, while at $1.2 \mathrm{~V}$ UPL carbon support corrosion becomes evident and dominating for the high surface area carbon. Figure 39 shows that the kinetic losses are very similar at both 1.0 and $1.2 \mathrm{~V} \mathrm{UPL}$ for the different carbons after 4700AST cycles with the exception of the HSAC2 catalyst. This suggests that the kinetic loss is mainly impacted by Pt dissolution until severe corrosion occurs. Thus, based on the fact that the Pt dissolution for both 1.0 and 1.2V UPL is very similar, carbon support corrosion is the predominant mechanism that affects the degradation rate. The noticeable lower kinetic performance loss and voltage degradation at $1.2 \mathrm{~V}$ UPL of the MSAC catalyst has been associated with increased catalyst layer ionic conductivity that is a result of oxidation and increased hydrophilicity of the carbon support.

Figure 40A shows that the response in voltage loss at $1.0 \mathrm{~V}$ and $1.2 \mathrm{~V}$ UPL due to catalyst layer ionic conductivity changes is similar to the trend in voltage degradation rates shown in Figure 39 , an indication that the voltage degradation may predominantly be caused by catalyst layer ionic conductivity changes. 

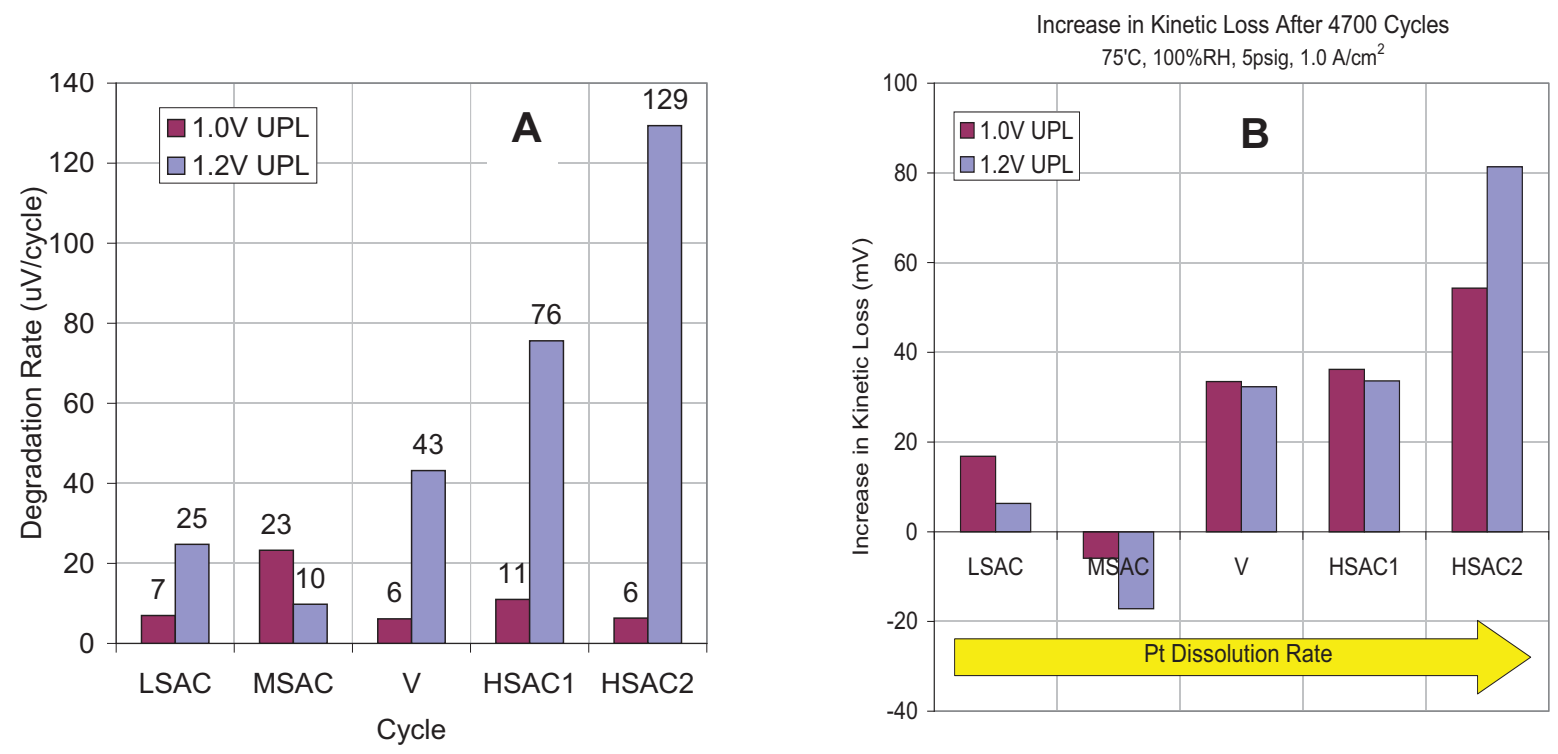

Figure 39: (A) Voltage degradation rate at $1.3 \mathrm{~A} / \mathrm{cm}^{2}$ and (B) kinetic loss after $4700 \mathrm{AST}$ cycles at an upper potential $1.0 \mathrm{~V}$ and $1.2 \mathrm{~V}$ for Pt catalysts supported on different carbon structures

Figure 40B shows the trend in catalyst layer thickness loss after 4700AST cycles at 1.2V UPL with electrochemical surface area of the powder (ECA) and carbon support surface graphitization content. As graphitization affects the number of sites available for corrosion, a low graphitic content results in severe loss in catalyst layer thickness. As well, greater Pt surface area and Pt distribution results in higher corrosion due to the fact that Pt promotes the carbon corrosion reaction.

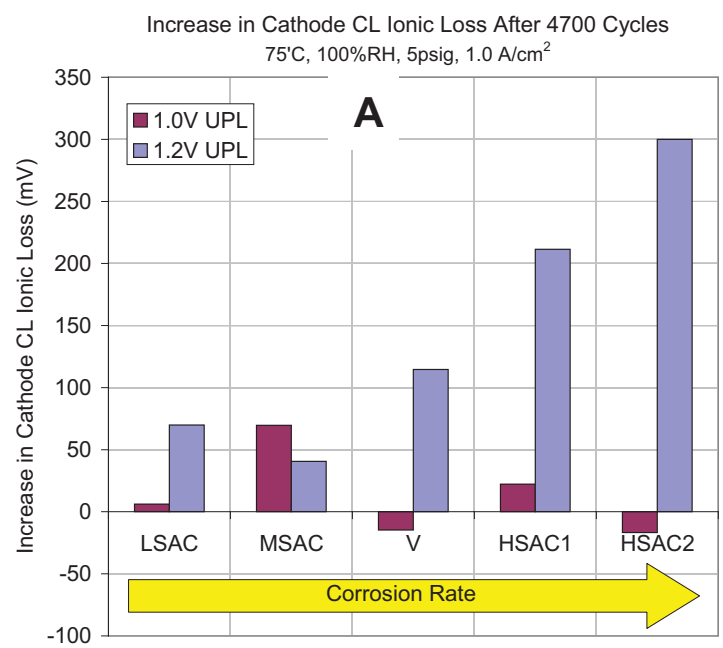

B
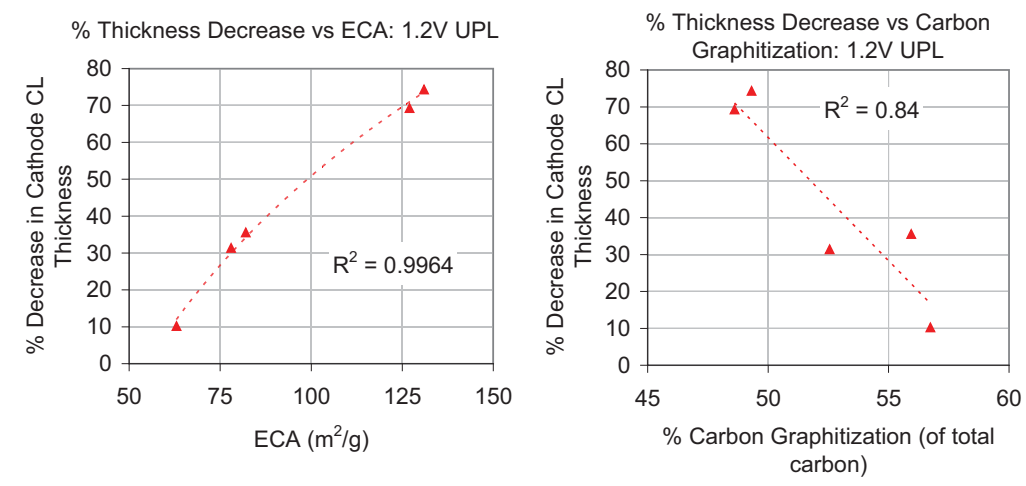

Figure 40: (A) Performance loss due to cathode catalyst ionic changes after 4700 cycles to an UPL of $1.0 \mathrm{~V}$ and 1.2V. (B) Catalyst layer thickness changes after 4700 cycles at an UPL of 1.2 with respect to catalyst powder ECS and carbon support graphitization 


\section{Heat treated catalysts}

The performance and durability of two different heat treated high surface area carbon catalysts were investigated to further understand if these catalysts which generally show high BOT performance would exhibit improved durability. As shown in Figure 41, the heat treatment process results in an increase in the Pt crystallite size and a decrease in the metal BET surface area as well in a change in catalyst powder porosity.

As shown in Figure 42 the ECSA and performance at BOT do not show a clear trend with heat treatment; i.e. HSAC50\#1 BOT performance and ECSA does not change with heat treatment, while the HSAC50\#2 catalyst reveals significantly lower performance and ECSA upon heat treatment. The Pt crystallite size may account for the small losses in ECSA with heat treatment; however other catalyst layer properties are impacting the performance.

Figure 43 shows the ECSA and performance loss with AST cycling at 1.2V UPL. As expected, the heat treatment of high surface area carbons reduced the loss in both performance and ECSA for both carbon support types which is expected due to the increase in graphitic carbon content upon heat treatment.
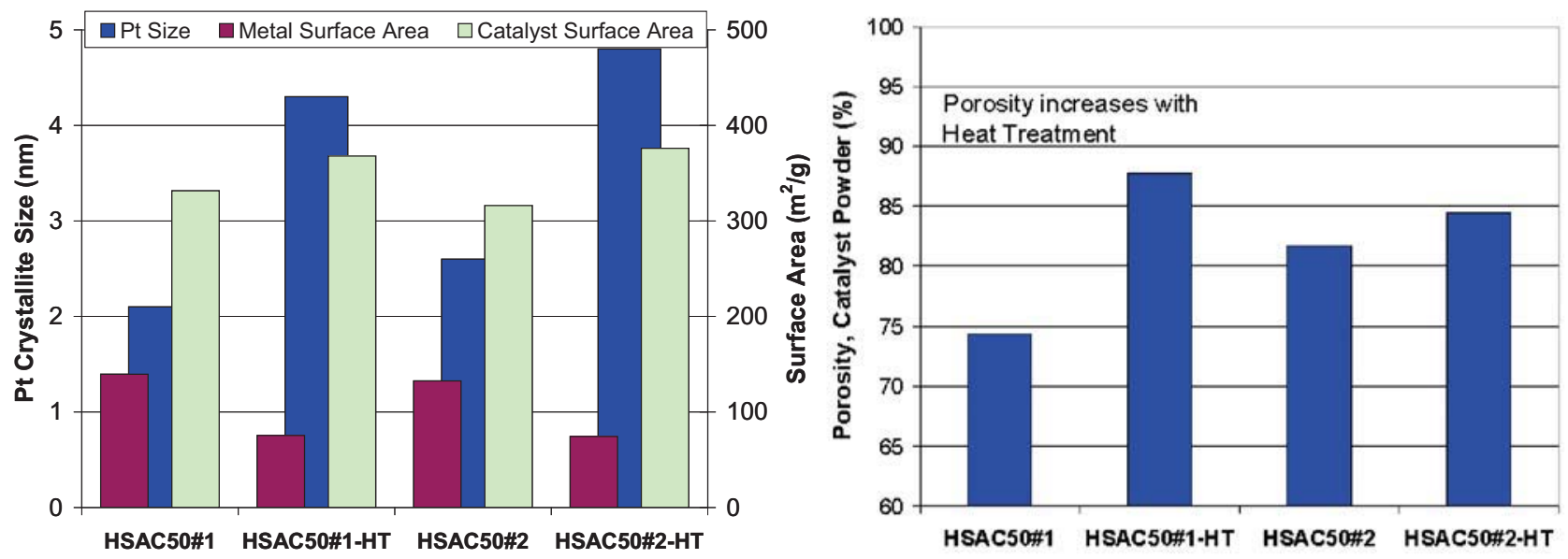

Figure 41: Effect of heat treatment on (a) Pt Particle size, catalyst surface area and metal surface area, and (b) catalyst powder porosity 


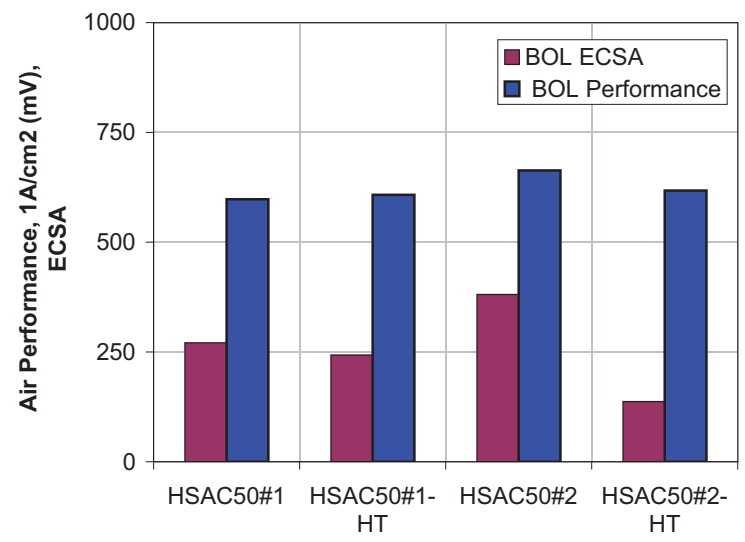

Figure 42: $\mathrm{BOL}$ ECSA and performance (at $1 \mathrm{~A} / \mathrm{cm}^{2}$ ) for heat treated high surface area carbon catalyst
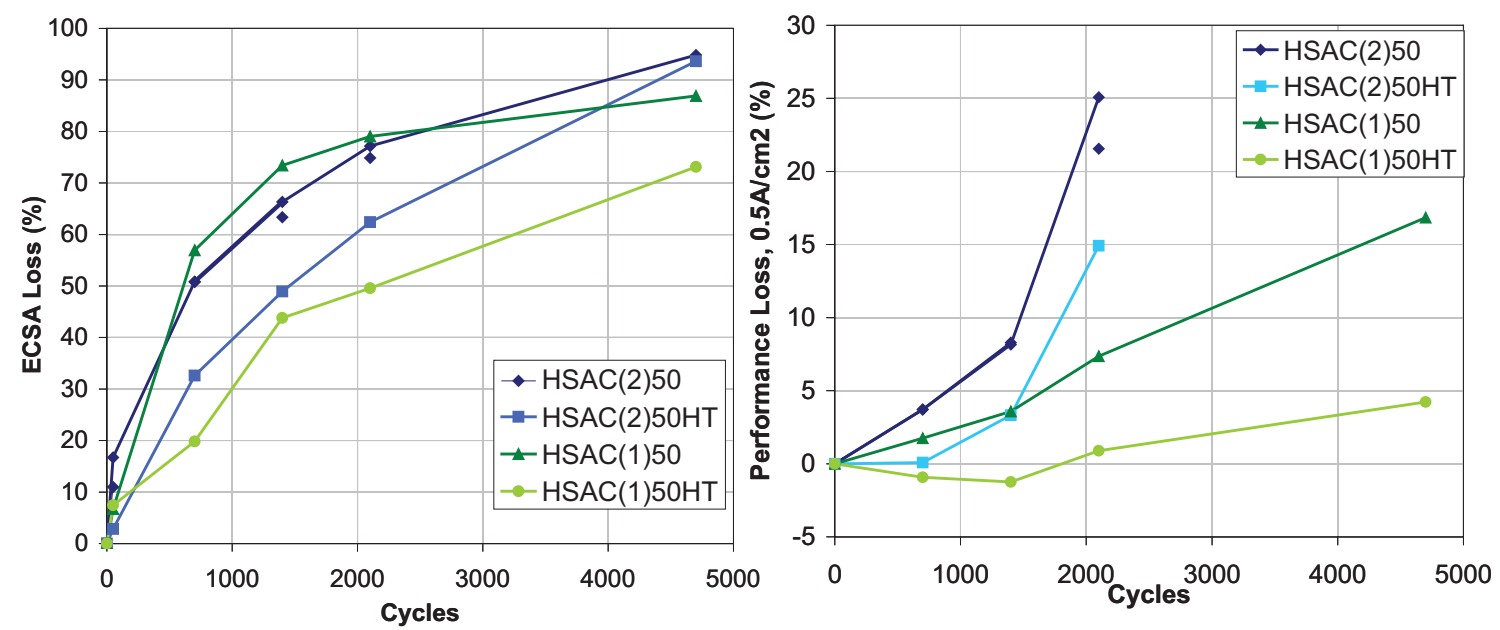

Figure 43: ECSA and Performance Loss during AST cycling to 1.2V.

\subsection{4 lonomer Loading}

The effect of ionomer loading on degradation was investigated for ionomer loadings from 12 to $50 \%$. Previous Ballard studies have shown that the optimal ionomer loading depends strongly on the catalyst type, ionomer type, and equivalent weight. For $20 \%$ Pt on Vulcan catalyst and $1100 \mathrm{EW}$ Nafion ionomer the optimal ionomer loading for maximum performance was approximately $33 w t \%{ }^{14}$. In this study, a Pt supported on a graphitized carbon (LSAC-Pt50) with $1100 E W$ Nafion in both anode $\left(0.1 \mathrm{mg} / \mathrm{cm}^{2}\right)$ and cathode $\left(0.4 \mathrm{mg} / \mathrm{cm}^{2}\right)$ was used.

14

E.Passalacqua et al., Electrochimica Acta 46 p.799, (2001) 
Figure 44 shows the effect of ionomer content on the catalyst layer structure and performance. The porosity of the catalyst layer decreases from 80 to $38 \%$ with an increase in ionomer loading from 12 to $50 \%$ and the optimal performance was observed for a cathode catalyst layer (CCL) Nafion loading of $\sim 30 \mathrm{wt} \%$, consistent with external results and internal modelling.
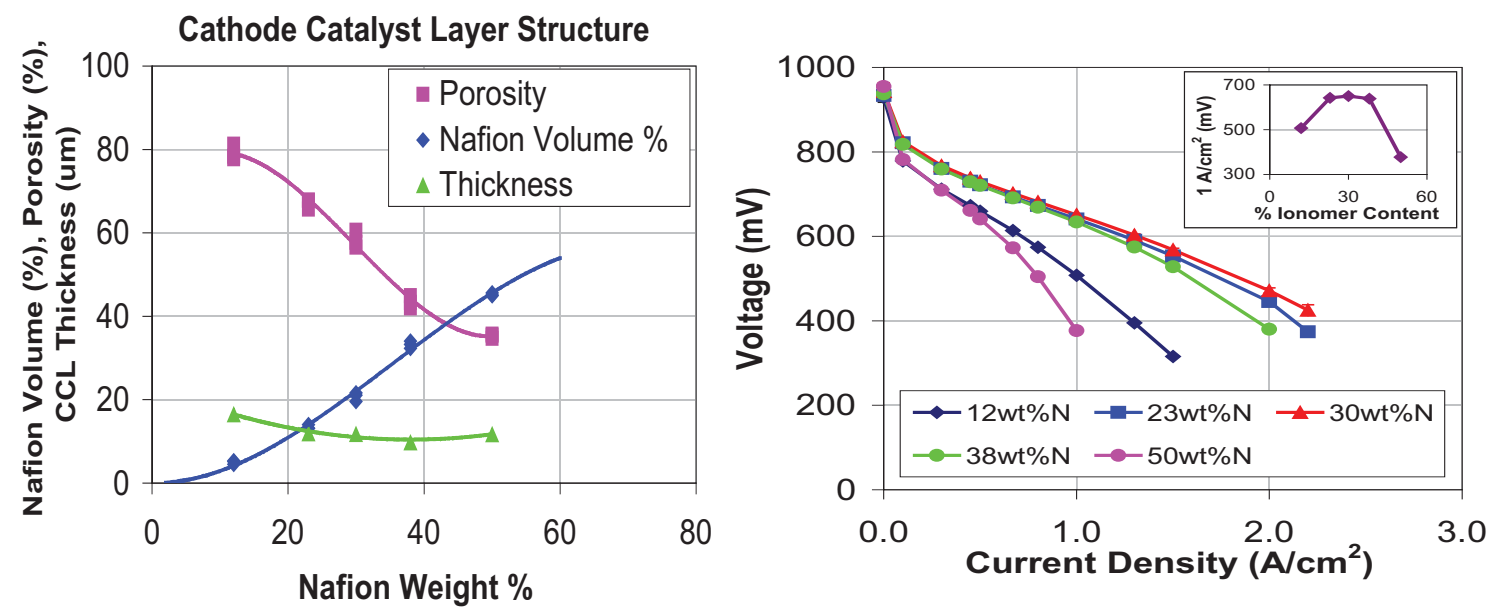

Figure 44: BOT catalyst layer structure and polarization curves for different ionomer loadings

In order to understand the impact of ionomer loading on degradation, the MEAs with different ionomer loadings where subjected to AST cycling (4700 UPL cycles from $0.6 \rightarrow 1.2 \mathrm{~V}$ ). Figure 45A) shows the EPSA loss as a function of ionomer content. It is hypothesized that low ionomer content provided fewer pathways for Pt ion diffusion; therefore, less Pt loss. This is corroborated by the slightly lower platinum crystallite size for the $12 \mathrm{wt} \%$ Nafion MEA. The slightly smaller ECSA loss at very high ionomer content is due to greater agglomeration; therefore, more Pt remains active. The dotted polynomial fit is included to show how well the data can be fitted and extrapolated; however this may not be valid in predicting the values beyond the data range presented.
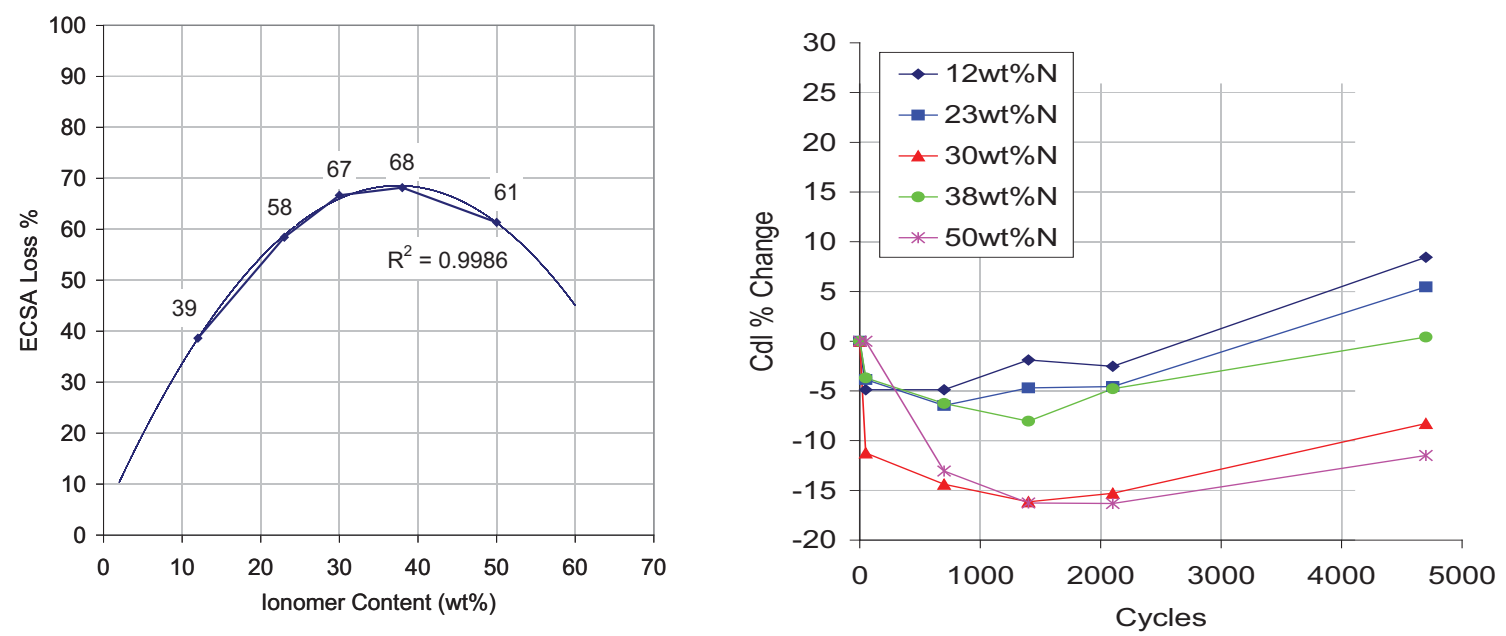

Figure 45: A) EPSA loss with ionomer loadings; B) double layer capacitance change with AST cycling for different ionomer loadings 
The double layer capacitance $(\mathrm{Cdl})$ corresponds to the ionic-electric interface between the Pt/C catalyst and the Nafion ionomer. The surface species that exist at this interface and the interfacial area will determine the capacitance behaviour observed. It is well known that as carbon starts to corrode, carbon-oxygen surface groups form. These surface groups are hydrophilic and will increase the $\mathrm{Cdl}$. As corrosion proceeds, the carbon fully oxidizes to $\mathrm{CO}_{2}$ gas and leaves the system through the gas exhaust. This reduces the carbon surface area and the Cdl. The relatively small increase shown in Figure 45B) indicates an initial loss in Cdl due to ECSA loss, due to slow oxidation of the carbon support.

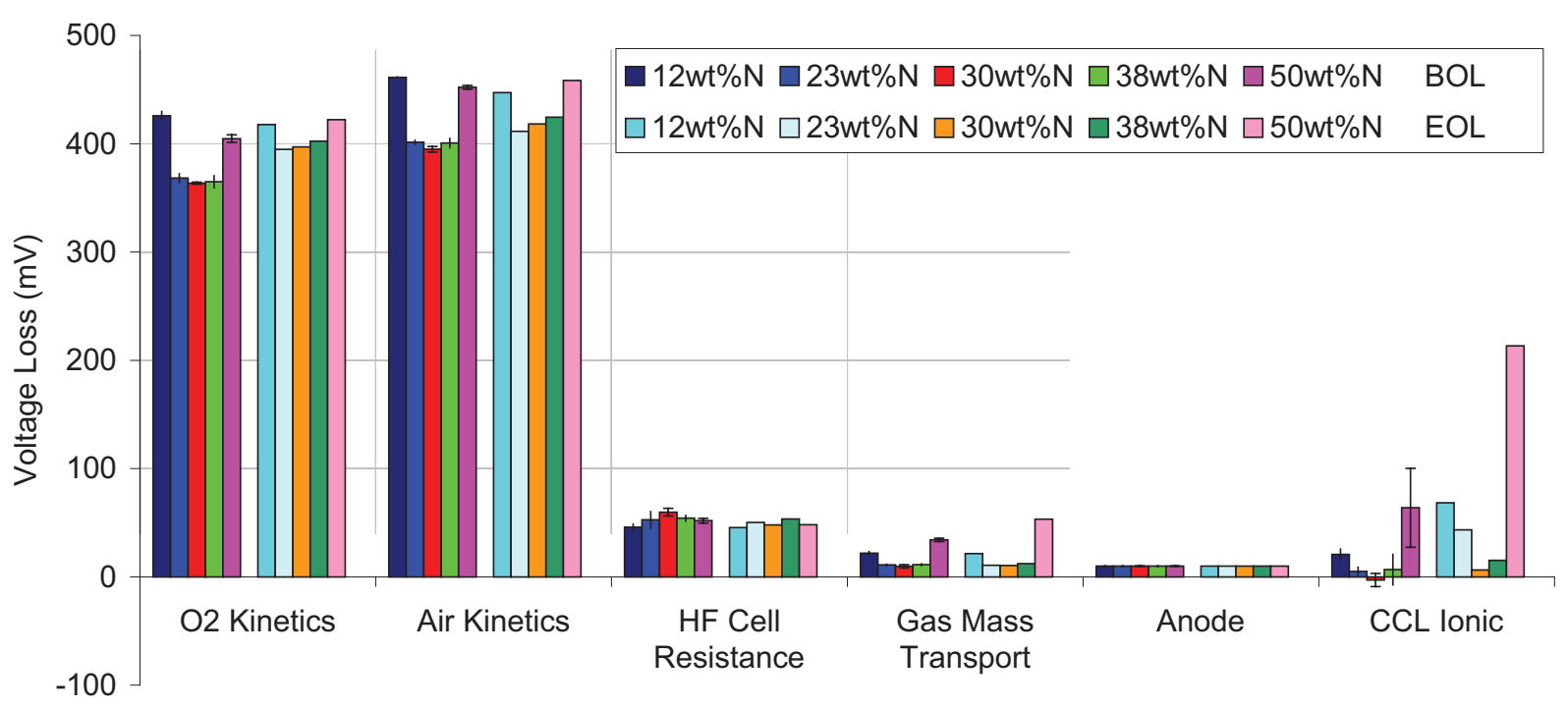

Figure 46: BOT and EOT Voltage Loss Breakdown for different ionomer contents

The end-of-test (EOT) voltage loss analysis shown in Figure 46 shows similar results as BOT; however, smaller kinetic loss with pure oxygen compared to air was observed simply due to the concentration effect, while the trend with ionomer content is the same. The greater voltage loss at low ionomer content is due to the low proton conductivity. The CCL has become so resistive to proton transport with progressive cycling that the ORR is restricted to the CCL-membrane interface; therefore, very little of the EPSA is utilized resulting in a higher platinum current density and thus kinetic losses. The dominant changes are the CCL ionic loss, which is likely caused by the preferential dissolution of Pt at the CCL-membrane interface.

\subsubsection{Ionomer Equivalent Weight}

The effect of ionomer equivalent weight on performance and degradation was investigated for cathode ionomer equivalent weights of 1100 (reference MEA), 850, and 950. The performance at BOT and after degradation was found to be very similar for the three equivalent weights and found to be within experimental uncertainties as shown in Figure 47. As well, changes in ECSA, double layer capacitance, thickness, Pt crystallite size and PITM did not reveal any significant differences for the three different ionomer equivalent weights, an indication that the losses of the catalyst layer was negligible. 

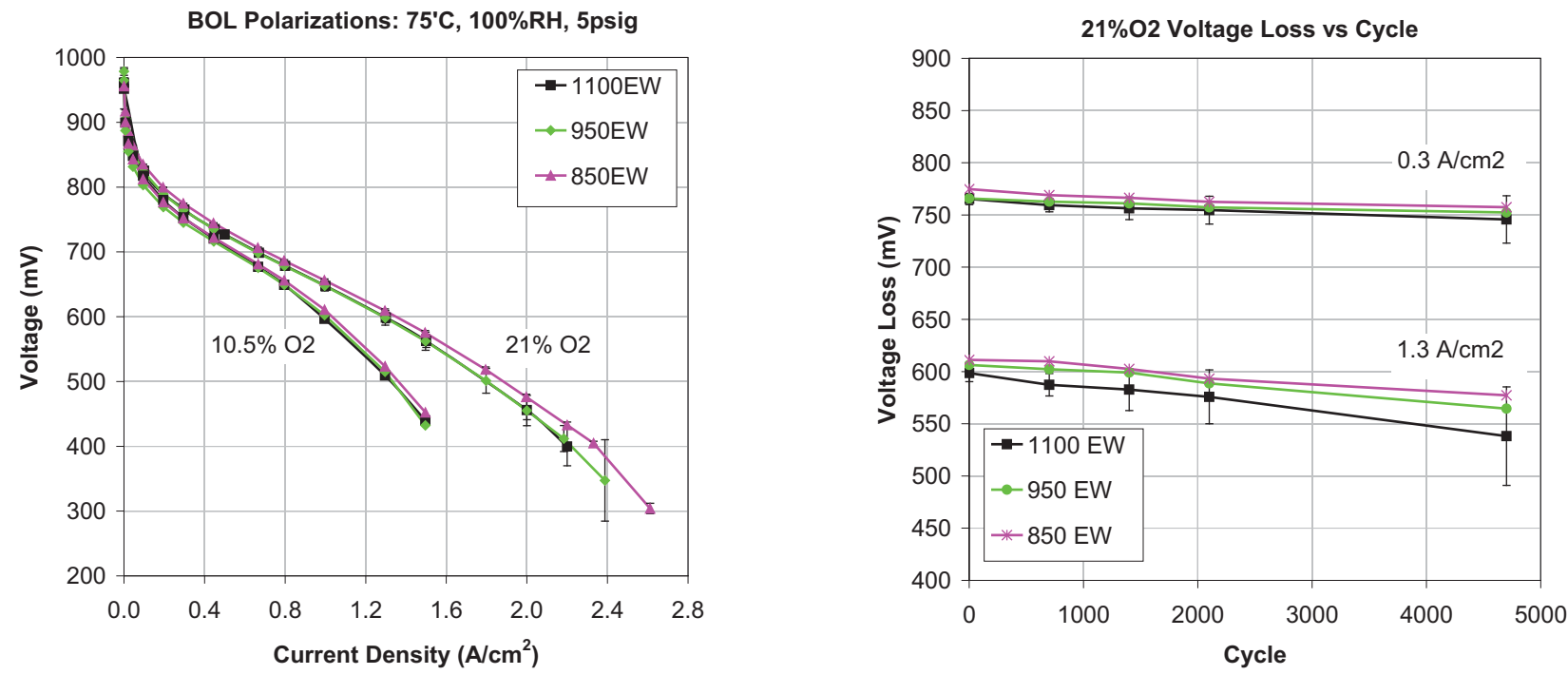

Figure 47: Performance characteristics at BOT and after degradation for different ionomer equivalents weights

\subsubsection{Catalyst Layer Cracks}

Catalyst processing, resulting in cracked layers, were found to affect the MEA performance and degradation. The reference MEA using catalyst layers that exhibited a 1 and $8 \%$ cracked surface area were subjected to AST cycles at an upper potential of 1.2 and $1.3 \mathrm{~V}$. While the BOT performance did not reveal any difference between the two MEAs, as shown in Figure 48, the EOT performance after cycling at a UPL of $1.3 \mathrm{~V}$ showed substantially increased performance loss for the MEA with a crack area of $8 \%$ compared to the $1 \%$ cracked catalyst layer.

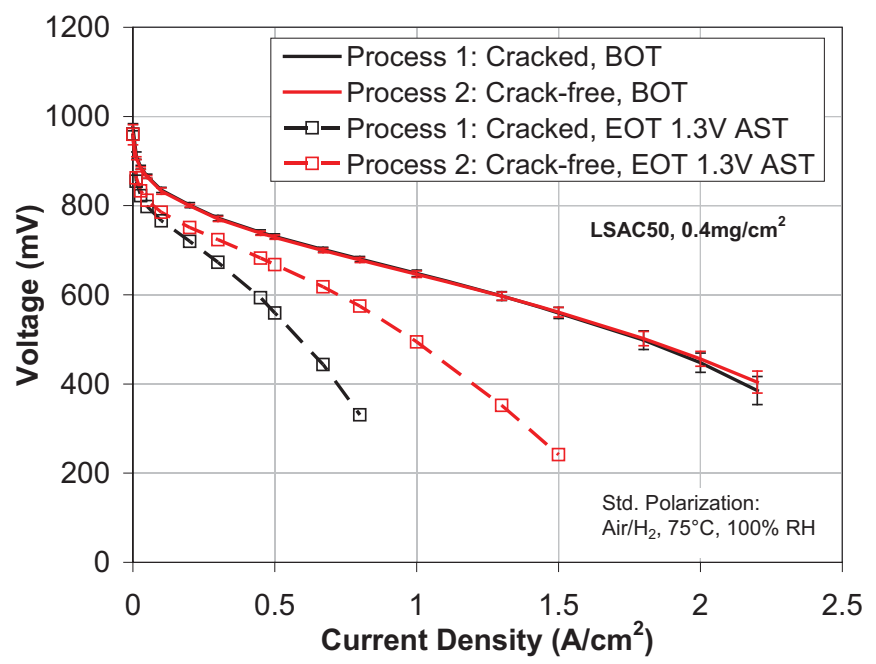

Figure 48: Performance and durability of catalyst layers containing an overall cracked area of 1 and $8 \%$. 
DE-EE0000466

Ballard Material Products Inc

\subsubsection{Other MEA Components}

Preliminary studies were conducted to evaluate other MEA components such as the membrane and GDL that would affect the water management of the MEA and thus may have an impact on catalyst layer degradation.

\section{(1) Membrane Type}

In this preliminary study, the baseline MEA which contains a dense membrane B (NR211) was compared to a MEA made with a reinforced membrane A. Figure 49 shows the performance characteristics of the two MEAs after AST cycling at an upper potential of 1.2 and 1.3V for 4700 cycles. While the performance difference between the 2 MEAs is insignificant after cycling at $1.2 \mathrm{~V} \mathrm{UPL}$, the difference is very pronounced at 1.3V UPL with the baseline MEA showing a lower performance of $\sim 100 \mathrm{mV}$ at a current density of $1 \mathrm{~A} / \mathrm{cm}^{2}$. The higher performance of the reinforced membrane MEA was associated with substantially higher ECSA loss and an earlier onset of carbon corrosion for the baseline MEA. The lower EOT ECSA of 27 for the baseline vs. 57 for the reinforced membrane MEA caused the ORR regime being pushed farther into the catalyst layer resulting in increased catalyst layer ionic resistance for the baseline MEA. In addition, the baseline MEA revealed an earlier onset of corrosion ( 2000 cycles) evident from double layer capacitance (Cdl) measurements and a decrease in catalyst layer thickness.
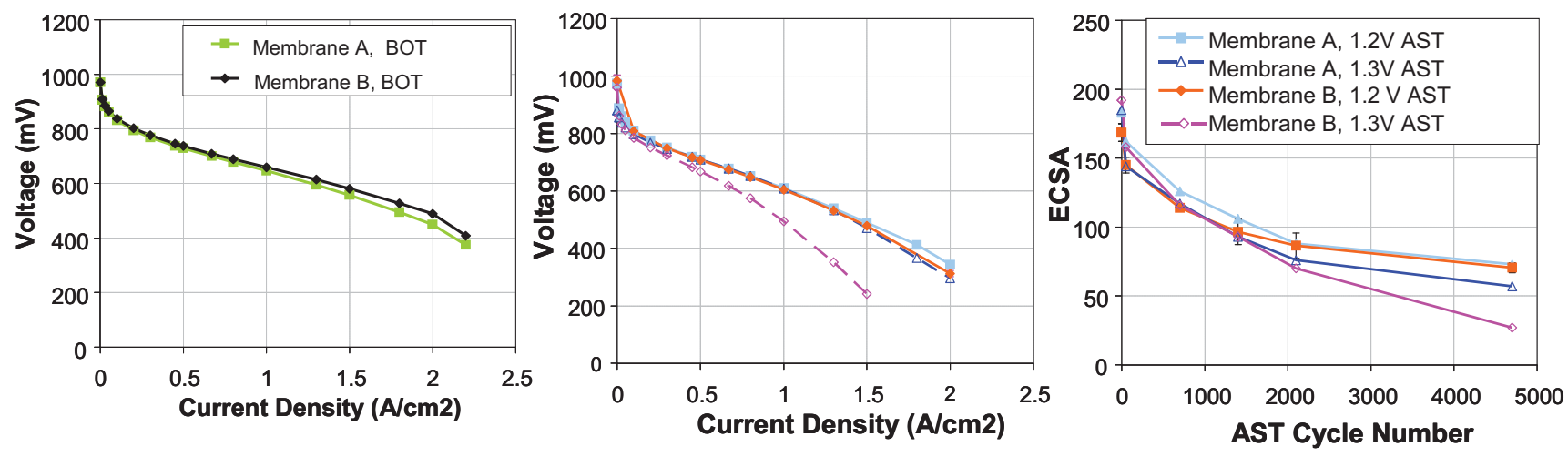

Figure 49: Performance comparison of MEAs containing two different type of membranes; dense membrane $B$ (baseline MEA) and reinforced membrane A after 4700 AST cycles at $1.2 \mathrm{~V}$ and $1.3 \mathrm{~V}$ UPL

\section{(2) GDL-MPL}

In order to understand the effect of GDL on catalyst layer degradation, a MEA with a standard GDL (with micro porous layer (MPL)) is compared to an MEA using a GDL that does not contain a MPL. The MEAs (Pt50-LSAC, cathode Pt loading of $0.4 \mathrm{mg} / \mathrm{cm}^{2}$ ) were subjected to 4700AST cycles from $0.6 \mathrm{~V}$ to $1.3 \mathrm{~V}$. Figure 50 indicates that the presence of the MPL on the GDL does not have a significant impact on the degradation. The initial performance without an MPL layer is slightly reduced as expected. 
DE-EE0000466

Ballard Material Products Inc

A

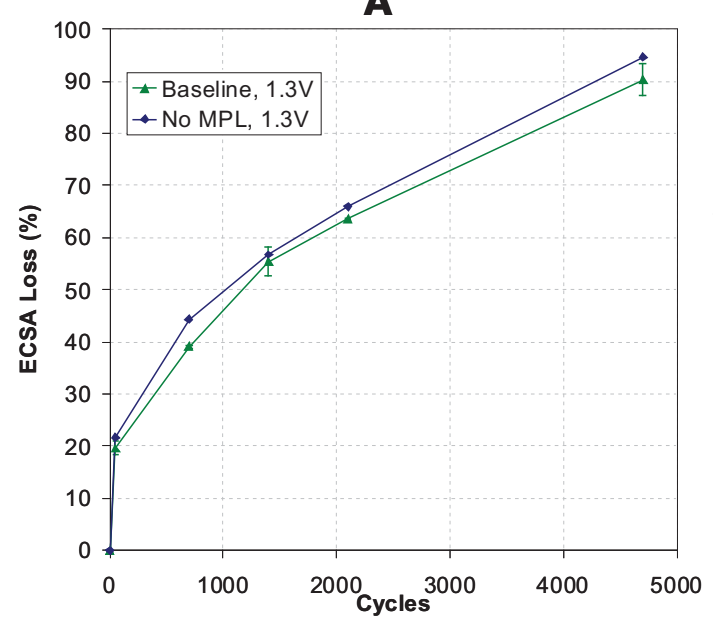

B

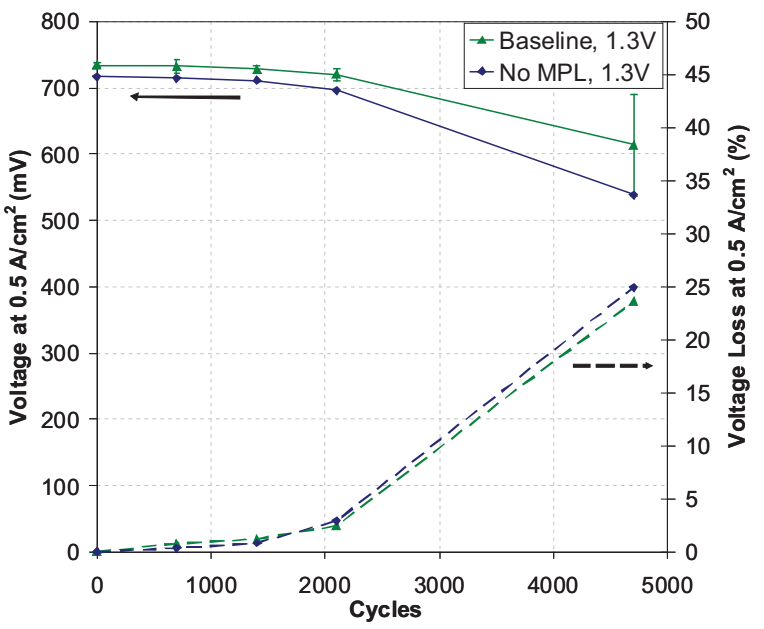

Figure 50: Impact of GDL with and without an MPL layer on CCL degradation; (A) ECSA loss and (B) Performance loss at $0.5 \mathrm{~A} / \mathrm{cm}^{2}$ as a function of AST cycles ( 0.6 to $\left.1.3 \mathrm{~V}\right)$

\subsubsection{Summary/Conclusions}

Table 11 provides a summary of the different studies of the catalyst layer structure and composition, as well as lists of other MEA components. It specifies the compositional parameters and their ranges that were investigated and provides high level information on their impact on BOT performance and the effect on the $\mathrm{Pt}$ dissolution and carbon corrosion mechanisms and rates.

Table 11: Summary of Catalyst Layer Design Studies

\begin{tabular}{|c|c|c|c|c|}
\hline Structure Evaluated & Testing Modifications & ВOT Performance & $\begin{array}{c}\text { Mechanism } \\
\text { Investigated }\end{array}$ & Degradation Rate \\
\hline Catalyst Loading & $\begin{array}{l}\mathbf{0 . 0 5}-\mathbf{0 . 5} \mathbf{~ m g} / \mathbf{c m}^{2} \mathbf{~ P t} \\
\text { BOT: T }(65-85 \mathrm{C}), \mathrm{RH}(60 \% \text { to } \\
>100 \%),\left[\mathrm{O}_{2}\right](10.5-100 \%)\end{array}$ & $\begin{array}{l}\text { Decreases with loading } \\
<0.2 \mathrm{mg} / \mathrm{cm}^{2} \\
\text { Affected by } \mathrm{RH}, \mathrm{T} \text { and [O2] } \\
\text { operation }\end{array}$ & $\begin{array}{l}\text { Pt Dissolution } \\
\text { C-Corrosion }\end{array}$ & $\begin{array}{l}\text { Increases for loadings } \\
<0.2 \mathrm{mg} / \mathrm{cm}^{2} \mathrm{Pt}\end{array}$ \\
\hline Carbon Ratio & $30,40,50,60,80 \mathrm{Pt} / \mathrm{C}$ & $\begin{array}{l}\text { No Impact }(30-60 \mathrm{Pt} / \mathrm{C}) \\
\text { Decreases for } \mathrm{Pt} / \mathrm{C}=80\end{array}$ & $\begin{array}{l}\text { Pt Dissolution } \\
\text { C-Corrosion }\end{array}$ & $\begin{array}{l}\text { Decreases with } \mathrm{Pt} / \mathrm{C} \text { ratio } \\
(30 \text { to } 60)\end{array}$ \\
\hline \multirow[t]{2}{*}{ Carbon Support } & $\begin{array}{l}\text { LSAC50, MSAC50, Vulcan 50, } \\
\text { HSAC50 (1), HSAC50(2) }\end{array}$ & \multirow[t]{2}{*}{$\begin{array}{l}\text { Kinetic Loss: HSAC }<\text { MSAC < } \\
\text { LSAC } \\
\text { Performance: No trend }\end{array}$} & $\begin{array}{l}\text { Pt Dissolution } \\
\text { C-Corrosion }\end{array}$ & HSAC $>$ MSAC $>$ LSAC \\
\hline & $1.0 \mathrm{~V}$ UPL & & Pt Dissolution & No significant Impact \\
\hline $\begin{array}{l}\text { Carbon Support } \\
\text { (Heat Treated) }\end{array}$ & HSAC50-HT(1), HSAC50-HT(2) & Decreases with HT & $\begin{array}{l}\text { Pt Dissolution } \\
\text { C-Corrosion }\end{array}$ & Improves with HT \\
\hline Ionomer Loading & $\begin{array}{l}\text { Nafion Content: } \\
12,23,30,38,50 \% \\
\end{array}$ & Optimal @ 30\% & $\begin{array}{l}\text { Pt Dissolution } \\
\text { C-Corrosion }\end{array}$ & Optimal @ 30\% \\
\hline Ionomer EW & 850-1100 EW & No Significant Impact & $\begin{array}{l}\text { Pt Dissolution } \\
\text { C-Corrosion }\end{array}$ & No significant Impact \\
\hline \multirow{2}{*}{ Impact of Membrane } & \multirow{2}{*}{$\begin{array}{l}\text { Reinforced Membrane } \\
1.2 \mathrm{~V} \& 1.3 \mathrm{AST}\end{array}$} & \multirow{2}{*}{ Similar to baseline } & Pt Dissolution & Similar wrt baseline \\
\hline & & & C-Corrosion & Lower wrt baseline (1.3VAST) \\
\hline \multirow{2}{*}{ Catalyst Layer Process } & \multirow{2}{*}{$\begin{array}{l}1 \text { and } 8 \% \text { crack area } \\
1.2 \mathrm{~V} \& 1.3 \mathrm{AST}\end{array}$} & \multirow{2}{*}{ Similar } & Pt Dissolution & Similar \\
\hline & & & C-Corrosion & $\begin{array}{l}8 \% \text { cracked CCL substantially } \\
\text { higher }(1.3 \mathrm{~V} \text { AST) }\end{array}$ \\
\hline Impact of GDL-MPL & $\begin{array}{l}\text { No MPL } \\
1.3 \mathrm{~V} \text { AST }\end{array}$ & Lower wrt baseline & $\begin{array}{l}\text { Pt Dissolution } \\
\text { C-Corrosion }\end{array}$ & No Impact \\
\hline
\end{tabular}

Standard AST: Air/ $\mathrm{H}_{2}, 100 \% \mathrm{RH}, 5 \mathrm{psig}, 80^{\circ} \mathrm{C}, 0.6 \mathrm{~V}(30 \mathrm{sec})-->1.2 \mathrm{~V}(60 \mathrm{sec}), 4700 \mathrm{cycles}$

Reference MEA: 50:50 Pt/C, Nafion ${ }^{\circledR}$ ionomer, 0.4/0.1 mg/cm² (Cathode/anode), Ballard CCM, Nafion $®$ NR211, BMP GDLs

Ballard Test Cell: 1D, $45 \mathrm{~cm}^{2}$ active area 


\title{
DE-EE0000466
}

Final Project Report

\section{Development of Micro-Structural Mitigation Strategies for PEM Fuel Cells: Morphological Simulations and Experimental Approaches}

\author{
CHAPTER III
}

\section{MEA COMPONENT CHARACTERIZATIONS}

University of New Mexico: XPS, SEM, TEM of Carbon, Catalyst and Catalyst Layers

- P. Atanassov (PI)

- K. Artyushkova

- A. Patel (Ph.D. student)

Los Alamos National Laboratory: BET of Carbon and Catalyst Powders, FTIR of GDLs

- R. Borup (PI),

- R. Mukundan, J. Fairweather, D. Spernjak, J. Davey

Michigan Technological University: Catalyst and GDL Capillary Pressure, GDL Contact Angle, Catalyst Layer Interfaces

- J. Allen (co-PI), R. Shahbazian-Yassar (co-PI)

- E. Medici (Post-Doc)

- N. Parikh, V. Konduru and, S. Stacy (graduate students)

- M. Nettel and C. Smith (undergraduate students) 


\section{TABLE OF CONTENTS}

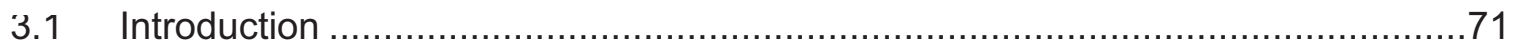

3.2 Morphology/Structure and Surface Analysis of Carbon Powders, Catalyst Powders, and BOT and Degraded Catalyst Layers (UNM) ……......................72

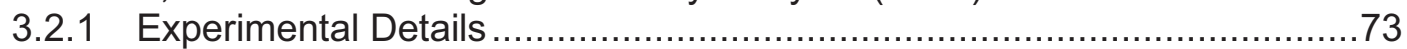

3.2.2 Carbon and Catalyst Powders - Results and Discussion/Conclusion .......74

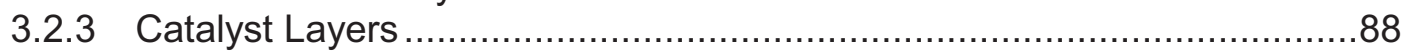

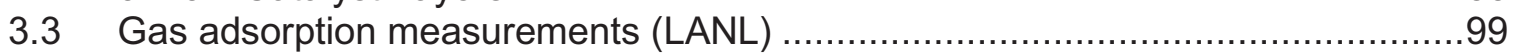

3.4 Catalyst Layer Capillary Pressure (MTU) ……........................................... 100

3.4.1 Development of the Experimental Set-up.......................................100

3.4.2 Capillary Pressure Results..........................................................102

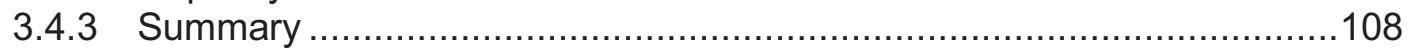

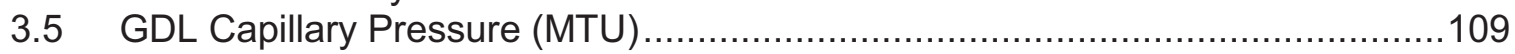

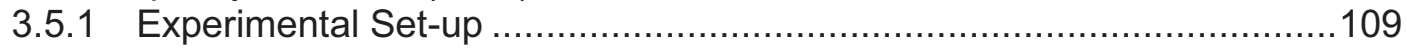

3.5.2 Capillary Pressure Results..............................................................110

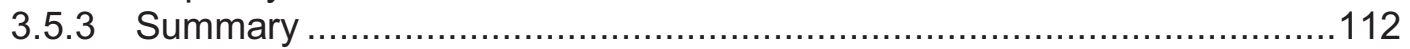

3.6 GDL Wettability - Contact Angle Measurement Technique (MTU) ……............113

3.6.1 Experimental Set-up ………….............................................113

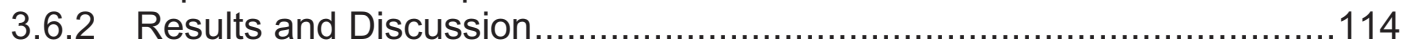

3.7 Wettability Tests after Cyclic Compression on GDL...................................118

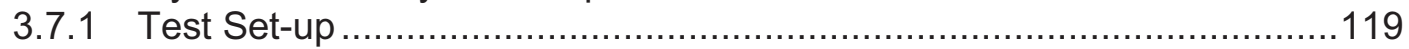

3.7.2 Results and Discussion.........................................................119

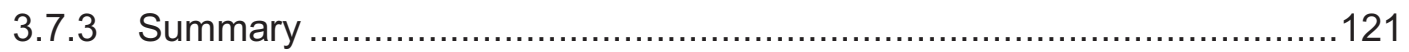

3.8 GDL - MPL Chemical Analysis (LANL) ………...................................122

3.9 Surface Roughness Measurements of GDL and Catalyst Layer (MTU) ............123

3.9.1 Measurement Techniques ………................................................123

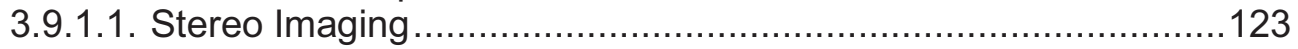

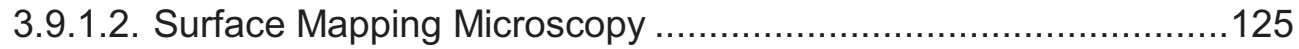

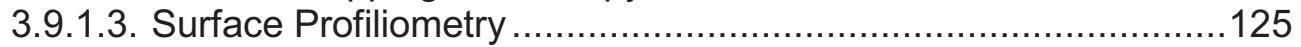

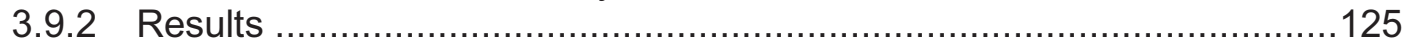

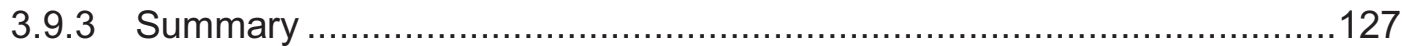

3.10 Catalyst Layer/Membrane Interface Characterizations (MTU) …...................128

3.10.1 SEM-EDS Analysis of Membrane-Catalyst Interface ..........................128

3.10.2 Mechanical Characterization of Membrane-Catalyst Interface ...............131

3.10.3 Mechanical Properties of Membrane-CL Interface for Samples with

Different lonomer Content..............................................................139

3.10.4 Electrical Characterization of Membrane-Catalyst Interface ...................140

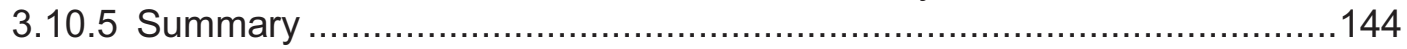




\section{LIST OF FIGURES}

Figure 1: High resolution C1s spectra for: a) LSAC and b) HSAC samples. Larger amount of Pt carbides is detected for HSA carbon ...........................................................74

Figure 2: High resolution Pt 4f spectra for: a) HSAC2 and b) HSAC-HT samples. Increase in metallic content upon heat treatment is detected ..............................................74

Figure 3: LSAC, TEM images at different magnifications …….........................................

Figure 4: LSAC, TEM, and STEM images from the same area .......................................75

Figure 5: Comparison of carbons with different surface areas. TEM images...........................76

Figure 6: LSAC PT50 TEM/STEM results obtained on HF-3300 (same area) ……..................77

Figure 7: LSAC 50 Pt. Typical TEM images .............................................................77

Figure 8: Comparison of SAC-STEM images for 50 Pt loaded samples................................78

Figure 9: PSD calculated from STEM images for BOL samples ........................................79

Figure 10: STEM images, threshold and agglomerates identified. ……...............................79

Figure 11: (A) Distribution of agglomerates with different number of particles in them; (B) Distribution of aspect ratios for agglomerates .................................................. 80

Figure 12: SEM images for carbon blacks a) LSAC, c) MSAC and e) HSAC and Ptdecorated samples b) LSAC50, d) MSAC50, f) HSAC50(1) and g) HSAC50-HT

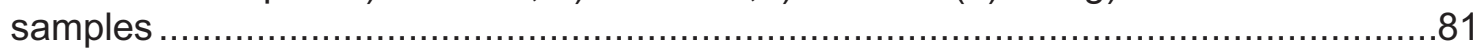

Figure 13: PCA results from morphological parameters for all samples ...................................82

Figure 14: PCA results from XPS data (absolute atomic \%) for catalyst samples ......................84

Figure 15: PCA results from XPS data (absolute atomic \%) and morphological parameters for all samples

Figure 16: PCA results from XPS data (absolute atomic \%) (top) and PCA results from SEM morphological parameters (bottom) for the same loading catalysts sample.

Figure 17: SEM pictures of LSAC PT50 and HSAC2 Pt50 catalysts layers from non-run conditioned and degraded MEAs.

Figure 18: PCA analysis of catalyst layer morphology data for conditioned and AST tested MEA (normal $=$ Pt dissolution, degraded $=$ carbon corrosion)

Figure 19: PCA analysis of LSAC samples subjected to AST testing at various upper

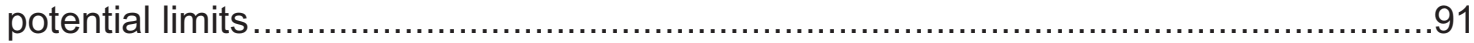

Figure 21: PCA comparing conditioned and degraded samples..............................................93

Figure 22: Change in carbonates, carboxylates and $\mathrm{CF}_{2}$ species for AST vs. conditioned samples

Figure 23: Change in carbonates, carboxylates and $\mathrm{CF}_{2}$ species as a function of AST upper

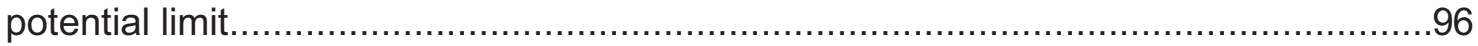

Figure 24: Change in Pt speciation as a function of AST potential ..........................................96

Figure 25: Skewness as a function of AST UPL for overall and different porosity scales .............97

Figure 26: Structure-to-durability correlations ..............................................................98

Figure 27: BET Analysis of various carbon supports and corresponding catalyst powders ..........99

Figure 28: Schematic of final design of the Catalyst Layer Percolation Testing Experimental Set-up. 100

Figure 29: (A) Catalyst Layer experimental setup, (B) new Catalyst Layer Platen without PDMS coating. 101

Figure 30: Degradation to the catalyst layer from the PDMS platens. On the left is the catalyst layer with pieces taken from it and on the right is the PDMS platen with the pieces of catalyst layer stuck to it.

Figure 31: Percolation Pressure Plots of the $50 \%$ ionomer sample against for a flow rate of $37.08 \mathrm{e}^{-4}$ at three different compressions of $41.37,68.95$, and $96.53 \mathrm{kPa} .$. 104 
Figure 32: Percolation Pressure of the $50 \%$ ionomer sample against the Wetted Volume for a flow rate of $37.08 \mathrm{e}^{-4}$ at three different compressions of $41.37,68.95$, and 96.53 $\mathrm{kPa}$.

Figure 33: Summary of the Percolation Pressure testing done on the $50 \%$ ionomer catalyst layer. The plot is showing the difference in Percolation Pressure from BOT to EOT over the testing time from BOT to EOT. The points on the plot represent the test at the compression pressure and flow rate.

Figure 34: Percolation pressure change of MEAs that were degraded at an upper potential limit of (A) 1.0 V and (B) 1.4 V. The samples were tested under compression of $41.37 \mathrm{kPa}$ using a flow rate of $37.08 \mathrm{e}^{-4} \mathrm{~mL} / \mathrm{s}$.

Figure 35: Pressure vs. water saturation for catalyst layers that were subjected to accelerated testing at an upper potential limit of $(A) 1.0 \mathrm{~V}$ and $(B) 1.4 \mathrm{~V}$. The samples were tested under compression of $41.37 \mathrm{kPa}$ using a flow rate of $37.08 \mathrm{e}^{-4}$

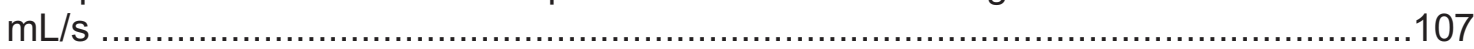

Figure 36: GDL experimental set-up diagram

Figure 37: Showing the Percolation Pressures within the BMP-P-GDL ..............................112

Figure 38: Fit between a sessile drop and the Laplacian curve .......................................113

Figure 39: Schematic of the experimental set-up for contact angle measurement ...................114

Figure 40: Droplet injection apparatus ....................................................................... 114

Figure 41: Static contact angle on the GDLs measured on the substrate side ......................116

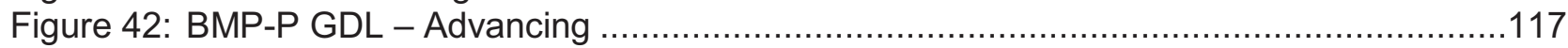

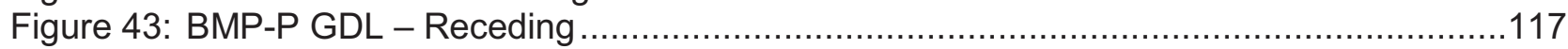

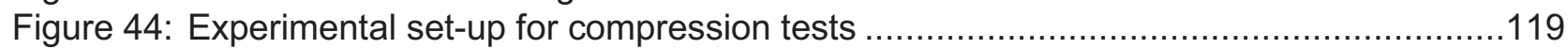

Figure 45: Results for Sample 'A' (Non-Run, P-GDL, B2-MD) .........................................120

Figure 46: Results for Sample 'B' (Non-Run, P-GDL, B2b-MD) .........................................120

Figure 47: Stress vs. Displacement curve for the tested samples for different compression cycles. The vertical bars represent the cut-off point till which the data was collected during the release half of the cycle.

Figure 48: Differential FTIR spectrum of GDLs from MEAs with LSAC Pt50 and MSAC Pt50 cathode catalyst layer (compared to BOL)

Figure 49: Surface roughness measurement of CL and GDL from: (a) Stereo imaging (b) interferometer microscope (c) surface profiliometry ...........................................124

Figure 50: Surface mapping images and cross section curves at magnification of (a) $6.3 x$ (b) $10 x$ (c) $20.3 x$ (d) $63 x$. The red circle indicates the crack in the feature .....................126

Figure 51: SEM images of: (a) BOL Membrane-catalyst interface before and after EDS. (b) $\mathrm{BOL}$ membrane before and after EDS. The accelerating voltage is $20 \mathrm{KeV}$. The blue and green line indicates the loss of particles in membrane after EDS. MMembrane C-Catalyst.

Figure 52: EDS spectra of BOL membrane-catalyst interface at accelerating voltage : (a) 15KV (b) 10KV. C-Carbon, F- Fluorine, Pt- Platinum, S-Sulphur.....

Figure 53: EDS spectra and EDS mapping of BOL membrane-catalyst interface. The accelerating voltage and dwell time are $20 \mathrm{KV}$ and $120 \mathrm{sec}$, respectively.....

Figure 54: EDS spectra and EDS mapping of EOL-1.2V membrane-catalyst interface. The accelerating voltage and dwell time are $20 \mathrm{KV}$ and $120 \mathrm{sec}$, respectively......

Figure 55: EDS spectra and EDS mapping of EOL-1.4V membrane-catalyst interface. The accelerating voltage and dwell time are $20 \mathrm{KV}$ and $120 \mathrm{sec}$, respectively....

Figure 56: Peak-force tapping mode principles (a) plot of the AFM tip force interactions during a single approach-retract cycle. (A) The AFM tip is close to the surface and experiences an attractive field first until it reaches the contact point (B). At 
DE-EE0000466

Ballard Material Products Inc.

this junction it experiences a repulsive field as it starts to indent into the sample.

(C) Represents the maximum peak-force that is used for the feedback loop. In the second half of the cycle, the AFM tip pulls off until it reaches its maximum

adhesion (D) and then returns to its starting position (E). (b) Plot of the force vs. displacement showing how the different material properties are extracted from the force curve. In the peak-force tapping AFM the maximum force ('peak force') is set to be constant for each measurement position

Figure 57: Three-dimensional AFM images of Fresh membrane-catalyst interface: (a) Height (b) Peak force error (c) Modulus (d) Adhesion (e) Deformation. The scan size and scan rate are $10 \times 5 \mu \mathrm{m}$ and $0.5 \mathrm{~Hz}$, respectively

Figure 58: AFM height images of membrane-catalyst interface. Force indentation curves of (b) Membrane (c) Catalyst layer. The indentations were done with $30 \mu \mathrm{N}$ load forces. 5 curves were obtained at different locations on the surface 135

Figure 59: DMT modulus maps of membrane-cathode catalyst interface: (a) Fresh, (b) Aged$1.2 \mathrm{~V}$, and (c) Aged-1.4V. (d) Thickness variation in catalyst layer (e) Average elastic modulus of membrane and catalyst layer. The scan size is $20 \mu \mathrm{m}$. The arrows indicate the region of catalyst layer. The red areas in modulus map represent the modulus range between 0.4-0.6GPa, while yellow-green areas have fairly high modulus in the order of $1 \mathrm{GPa}$. The pink areas represent cluster of particles having high modulus in range of $1.5-2 \mathrm{GPa}$.

Figure 60: Adhesion map and section line of membrane-cathode catalyst interface: (a) Fresh

(b) Aged-1.4V c) Average Adhesion value of membrane and catalyst. The scan size is 20um. (The pink region represents high adhesion areas while green and red areas dominantly in catalyst have low adhesion

Figure 61: DMT modulus image of BOL membrane-catalyst interface: (a) BOL (b) EOL 1.4V ..138

Figure 62: DMT modulus image of membrane-catalyst interface of MEA with different ionomer: (a) 12\% (VD420) (b) 23\% (VD421) (c) 30\% (VD422) (d) 50\% (VD423).....139

Figure 63: Plot of slope of elastic modulus vs. MEA with ionomer 139

Figure 64: (a) Experimental set-up for CAFM. (b) Detail diagram showing the conductive AFM tip and CCM sample. A bias voltage is applied between a conductive tip and CCM sample embedded in epoxy. Silver paint is applied on the AFM stage-CCM cross section interface to make electrical contact between the CCM sample and the stage.

Figure 65: (a) Electrical current images of catalyst at different bias voltage. (b) Percentage of non-conductive area for the catalyst with $30 \%$ Nafion content. Scan size is $5 \mathrm{x}$ $1 \mu \mathrm{m}$. Error bar in the plot represent $95 \%$ confidence intervals

Figure 66: Electrical current images of CCM and CL at 100nA/V current sensitivity: (a) Fresh (b) Aged-1.2V. Current images of Aged-1.4V CCM at current sensitivity of $100 \mathrm{nA} / \mathrm{V} \& 1 \mathrm{nA} / \mathrm{V}$. The bias voltage is $500 \mathrm{mV}$. A, M and $\mathrm{C}$ represent the anode catalyst layer, membrane and the cathode catalyst layer respectively. The white areas represent cluster of $\mathrm{Pt} / \mathrm{C}$ particles having high current regions above $1 \mu \mathrm{A}$, pink areas represent the current in the range of $0.5-1 \mu \mathrm{A}$. The black areas have negligible or no current signal ....

Figure 67: The2D electrical current and 3D overlay of electrical current on topography: (a) Fresh CL (b) Aged-1.2V CL. (c) Current distribution curves 143 


\section{LIST OF TABLES}

Table 1: Summary of the different materials and components properties and associated measurement techniques...............................................................................71

Table 2: List of analysed carbon and catalyst powders ..................................................

Table 3: Roughness and texture parameters extracted from SEM and BS images .................81

Table 4: Chemical Speciation of $\mathrm{C}$ and Pt of Catalyst With and Without Catalyst.....................83

Table 5: XPS elemental composition of catalyst layers after conditioning ………...................90

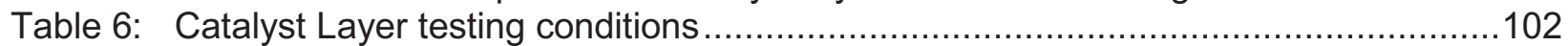

Table 7: List of Non-Run Catalyst Layer samples. The porosity has an uncertainty of $+/-$ $2 \%$, and the thickness $+/-0.5 \mu \mathrm{m}$. .

Table 8: List of Aged and Conditioned Catalyst Layer samples. The porosity has an uncertainty of $+/-2 \%$, and the thickness $+/-0.5 \mu \mathrm{m}$.

Table 9: Summary of a full CCM sample (50\% ionomer loading) Percolation Pressure testing results. The BOT and EOT labelled here are to denote the beginning of the test and end of the test. The slope is taken as the pressure difference over the time difference from BOT to EOT .

Table 10: List of samples that have completed all testing ….........................................110

Table 11: Relationship between capillary numbers and flow rate .....................................110

Table 12: Percolation pressure values in the samples listed, measured in $\mathrm{kPa}$, according to the different flow rates being tested against ..................................................111

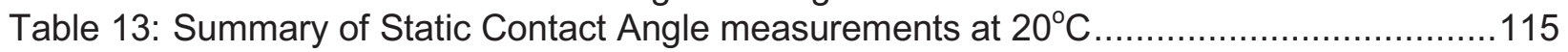

Table 14: Summary of Dynamic Contact Angle measurements ........................................118

Table 15: Contact Angle Measurement on the compressed samples ....................................121

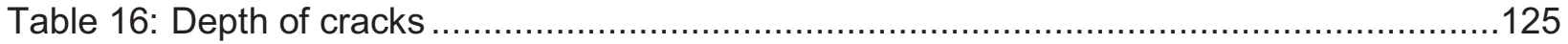


DE-EE0000466

Ballard Material Products Inc.

\subsection{Introduction}

This chapter describes the materials and catalyst layer characterization that were carried out by the University of New Mexico, Los Alamos National Laboratory, and Michigan Technological University. The objective of this work was to complement the AST investigations and modeling activities at Ballard and measure fundamental materials properties, identify material property changes with progressive catalyst layer aging to link to degradation rates for given mechanisms, and further confirm degradation mechanisms by comparison with theory.

The work encompassed characterization of the catalyst powder (Pt structure and support), BOT and degraded catalyst layers, GDL, and the GDL/catalyst layer/membrane interfaces. The materials information generated was used to de-convolute degradation AST data and correlate to the performance and degradation of the catalyst layer and served as model input and for model validation. Table 1 provides a list of measurement techniques and materials properties that were investigated and identifies the rationale for the characterization.

Table 1: Summary of the different materials and components properties and associated measurement techniques.

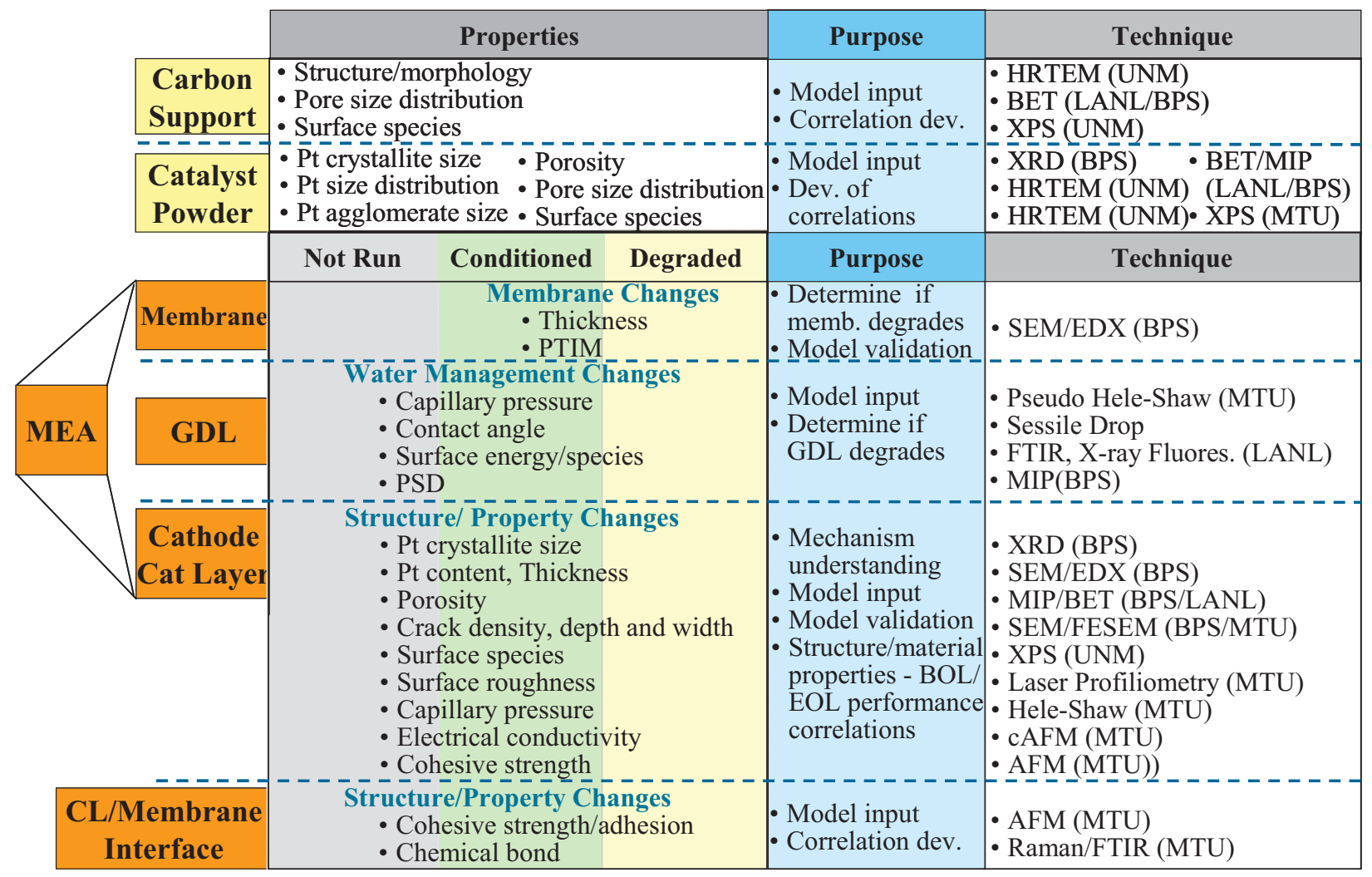




\subsection{Morphology/Structure and Surface Analysis of Carbon Powders, Catalyst Powders, and BOT and Degraded Catalyst Layers (UNM)}

The University of New Mexico (UNM) has been responsible for providing characterization of components of the catalyst layer (CL): from carbons to catalysts to MEAs. Surface characterization using XPS was used to study surface composition for components. Morphological information was obtained from SEM images of components. TEM/EELS was used to study both, carbon supports and catalysts. Statistical structure-to-property correlations were built by means of principal component analysis in which structural, morphological, and performance parameters were combined in one data set. The various carbon catalyst samples that were analysed are listed in Table 2.

Table 2: List of analysed carbon and catalyst powders

\begin{tabular}{|c|c|c|}
\hline \multicolumn{2}{|r|}{ Sample ID } & \multirow[t]{2}{*}{ Sample Specifics } \\
\hline \multirow{5}{*}{ 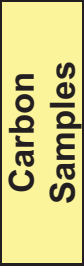 } & Vulcan & \\
\hline & LSAC & Low Surface Area Carbon \\
\hline & MSAC & Mid-range Surface Area Carbon \\
\hline & HSAC \#1 & High Surface Area Carbon \#1 \\
\hline & HASC \#2 & High Surface Area Carbon \#2 \\
\hline \multirow{15}{*}{ 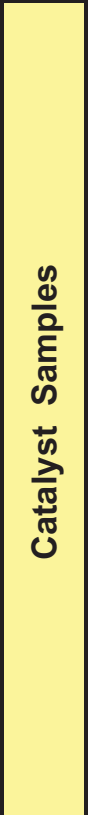 } & Pt50-Vulcan & 50/50 Pt/Vulcan \\
\hline & Pt50-LSAC & 50/50 Pt/Low Surface Area Carbon \\
\hline & Pt50-MSAC & 50/50 Pt/Mid-range Surface Area Carbon \\
\hline & Pt50-HSAC \#1 & 50/50 Pt/High Surface Area Carbon \#1 \\
\hline & PT50-HSAC\#1 -HT & 50/50 Pt/High Surface Area Carbon \#1 -Heat Treated \\
\hline & Pt50-HSAC \#2 & 50/50 Pt/High Surface Area Carbon \#2 \\
\hline & Pt50-HSAC \#2 HT & 50/50 Pt/High Surface Area Carbon \#2 - Heat Treated \\
\hline & Pt30-LSAC & 30/70 Pt /Low surface Area Carbon \\
\hline & Pt40-LSAC & 40/60 Pt /Low surface Area Carbon \\
\hline & Pt60-LSAC & 60/40 Pt /Low surface Area Carbon \\
\hline & Pt80-LSAC & 80/20 Pt /Low surface Area Carbon \\
\hline & Pt30-MSAC & 30/70 Pt /Mid-range surface Area Carbon \\
\hline & Pt40-MSAC & 40/60 Pt /Mid-range surface Area Carbon \\
\hline & Pt60-MSAC & 60/40 Pt /Mid-range surface Area Carbon \\
\hline & Pt80-MSAC & 80/20 Pt /Mid-range surface Area Carbon \\
\hline
\end{tabular}


DE-EE0000466

Ballard Material Products Inc.

\subsubsection{Experimental Details}

\section{Electron Microscopy}

Scanning electron microscopy (SEM) analysis was performed on a Hitachi S-800 instrument. All images were acquired at the same voltage and magnification settings of $2 \mathrm{kV}$ and $50 \mathrm{~K}$ to ensure that intensity variability in images is caused only by the variability in the morphology and not instrumental factors. SEM images from five different areas on the sample were acquired. Multiple locations were chosen per sample, and the locations were chosen randomly in order to prevent user bias from influencing the results. The images were analyzed through Digital Image Processing software to quantify changes to sample morphology due to conditioning and accelerated stress testing.

Aberration Corrected Scanning Transmission Electron Microscopy (STEM) images were acquired at the JOEL 2200FS microscope at the SHaRE user facility at Oakridge National Laboratory.

\section{$\underline{X \text {-ray photoelectron spectroscopy (XPS) }}$}

XPS measurements were performed using Kratos Axis Ultra DLD spectrometer using an Al Ka $X$-ray source, with an emission voltage of $15 \mathrm{kV}$ and emission current of $10 \mathrm{~mA}$. Three large area spectra from $300 \times 700$ micron areas were acquired for each sample analyzed. The base

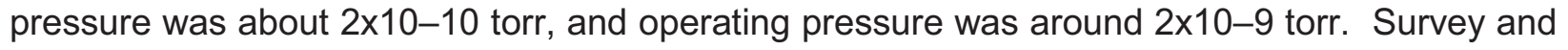
high-resolution spectra were acquired at pass energies of $80 \mathrm{eV}$ and $20 \mathrm{eV}$, respectively. Data analysis and quantification were performed using CasaXPS software. A linear background subtraction was used for quantification of $\mathrm{C} 1 \mathrm{~s}, \mathrm{~F} 1 \mathrm{~s}$ and $\mathrm{O} 1 \mathrm{~s}$ while Shirley background was used for $\mathrm{Pt} 4 \mathrm{f}$ spectra. Sensitivity factors provided by the manufacturer were utilized. Spectra were fitted with a series of $70 \%$ Gaussian / 30\% Lorentzian line shapes. Figure 1 and Figure 2 show an example of the high resolution $\mathrm{C} 1 \mathrm{~s}$ and $\mathrm{Pt} 4 \mathrm{~F}$ spectra and deconvolution of the different surface species.

\section{Electron energy loss spectroscopy (HREELS)}

HREELS measurements were conducted on a JEOL 2010F Transmission Electron Microscope (TEM) using a field emission gun as the source with a voltage of $200 \mathrm{kV}$, and beam energy of $0.1 \mathrm{kV}$. The spectra were acquired at 15 random locations per sample. Finally, highly ordered pyrolytic graphite (HOPG) was used as a reference sample because of its $100 \% \mathrm{sp} 2$ bonding nature.

A range of different catalyst carbon supports of different surface area (ranging from mid-100 to $800 \mathrm{~m}^{2} / \mathrm{g}$ ) and catalyst powders of different Pt to carbon weight ratios (ranging from $30 \%$ to $80 \%$ ) were analyzed. The different carbon and catalyst samples are listed Table 2. 

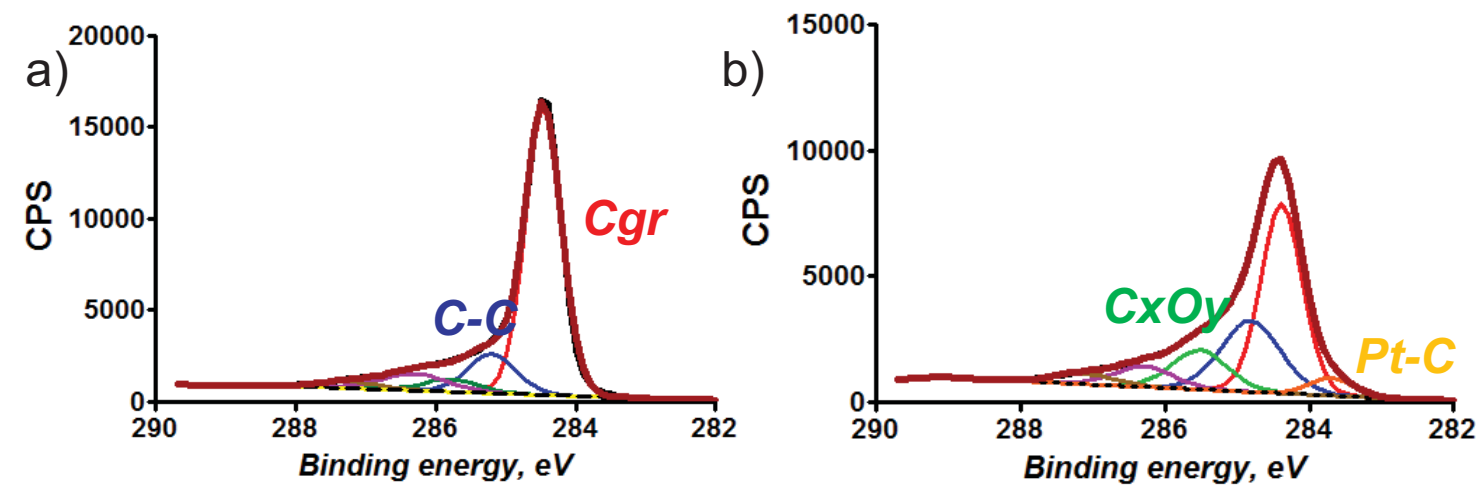

Figure 1: High resolution C1s spectra for: a) LSAC and b) HSAC samples. Larger amount of Pt carbides is detected for HSA carbon
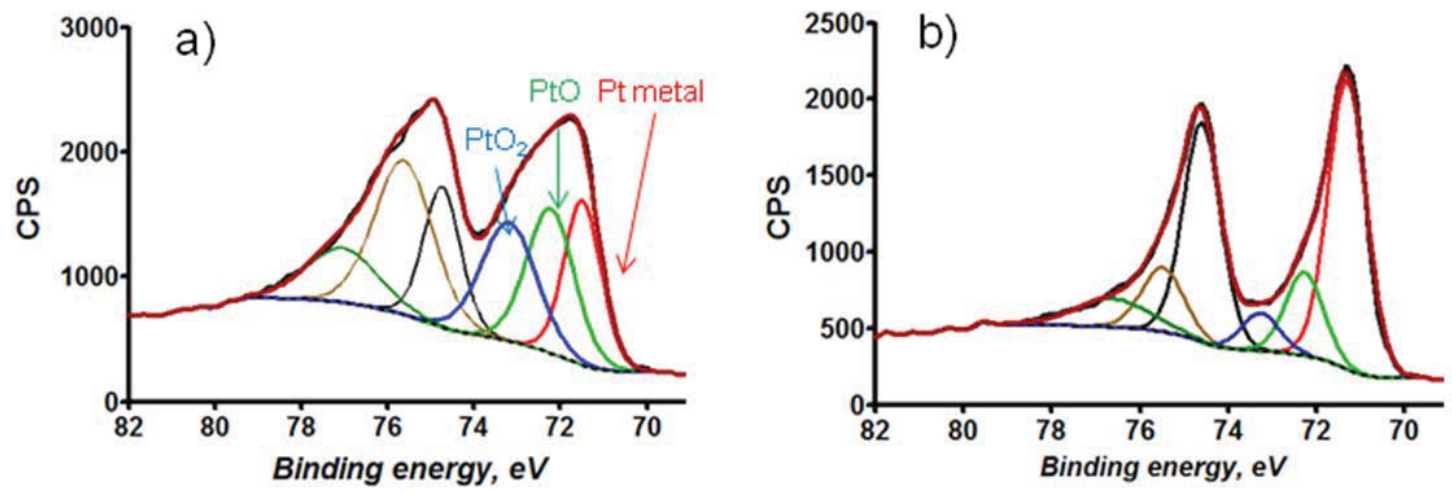

Figure 2: High resolution Pt 4f spectra for: a) HSAC2 and b) HSAC-HT samples. Increase in metallic content upon heat treatment is detected

\subsubsection{Carbon and Catalyst Powders - Results and Discussion/Conclusion}

\subsubsection{Structure - Transmission Electron Microscopic Analysis (TEM)}

TEM analysis of carbon and catalysts, presented in Figure 3 to Figure 8 show the varying structures of the different carbon supports and corresponding Pt catalysts. Figure 3 shows different scales of TEM images for the LSA carbon. Different magnifications were used to identify relative scales; the smallest image was $33.88 \times 33.88 \mathrm{~nm}$ in size. Figure 4 shows TEM and STEM images acquired from the same area for LSAC. Such comparison allows for evaluating whether particles are empty or hollow. 
DE-EE0000466

Ballard Material Products Inc.

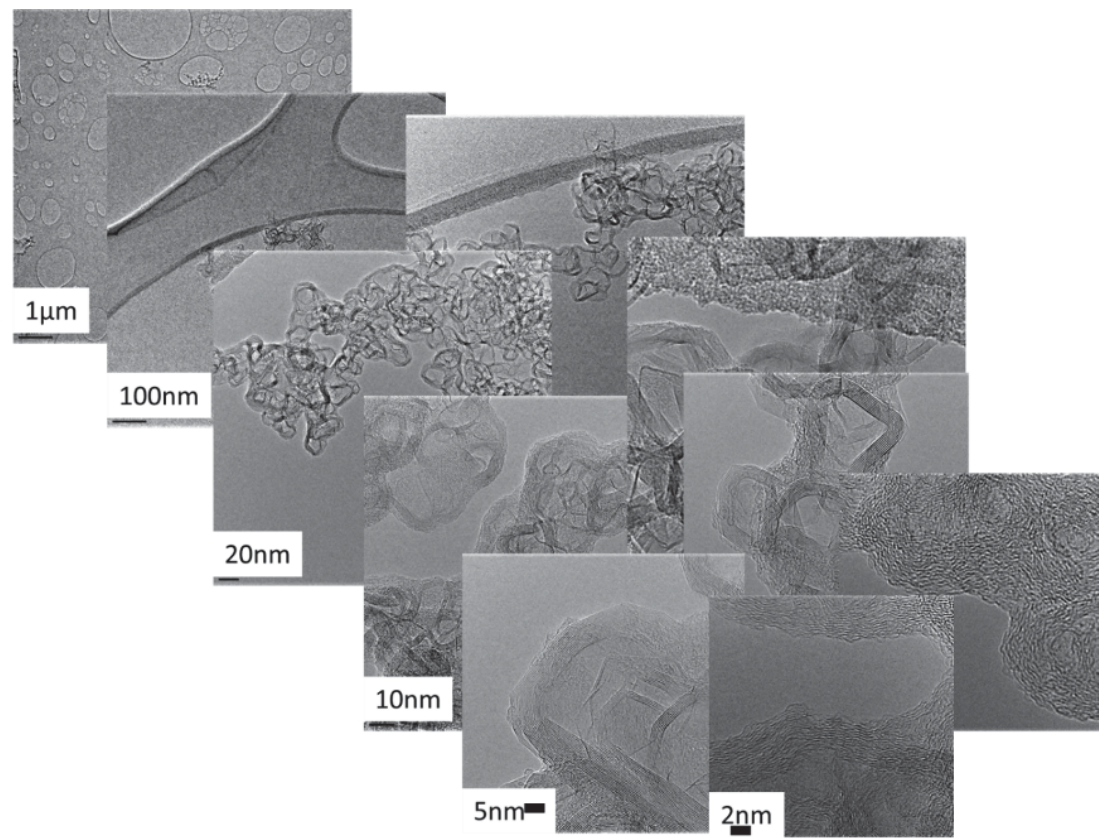

Figure 3: LSAC, TEM images at different magnifications

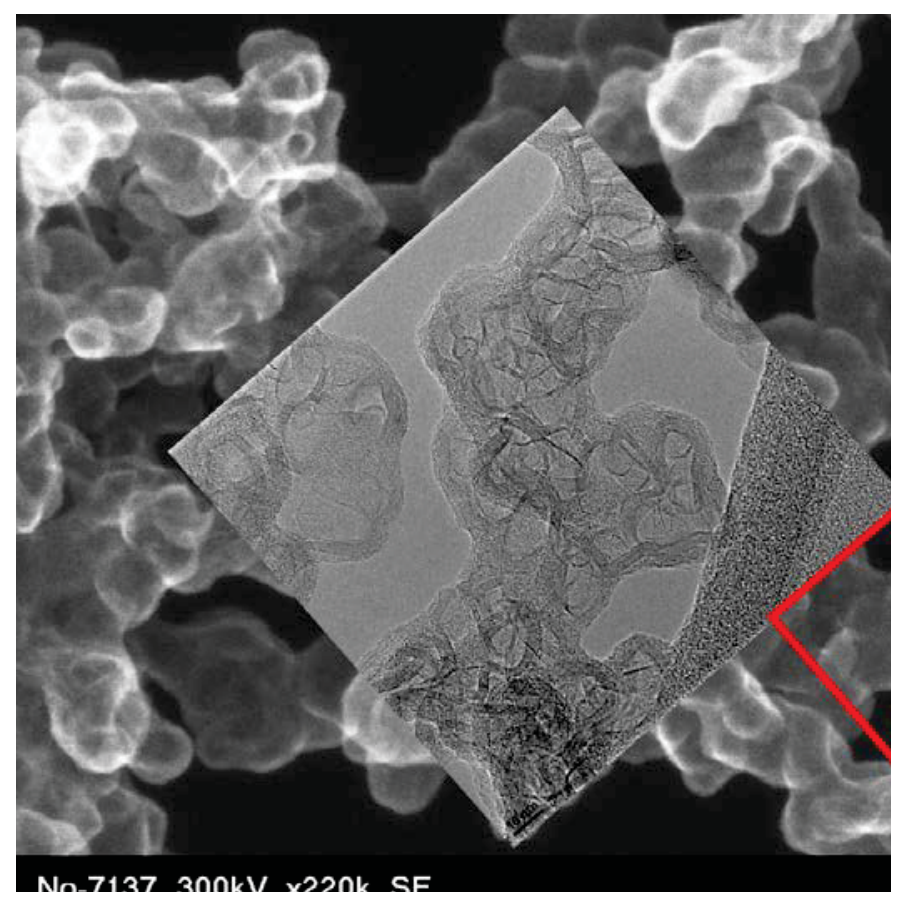

Figure 4: LSAC, TEM, and STEM images from the same area 
DE-EE0000466

Ballard Material Products Inc.

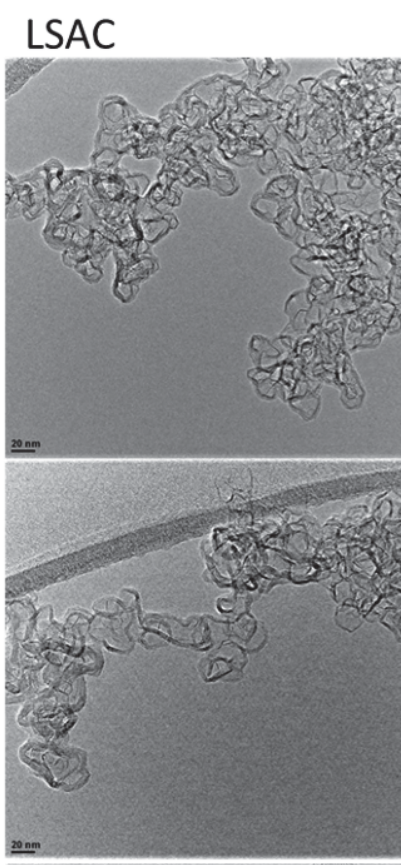

MSAC
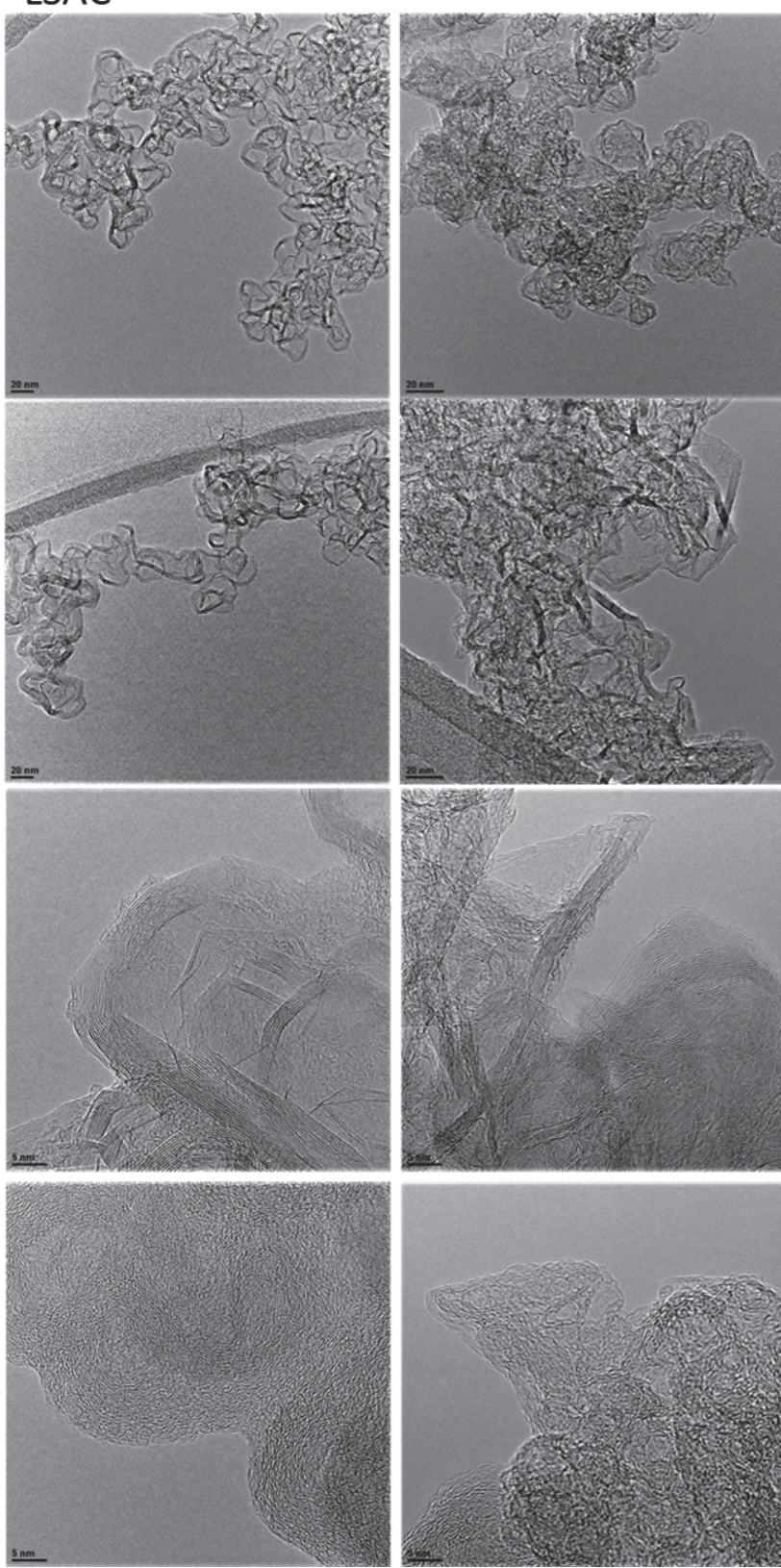

HSAC
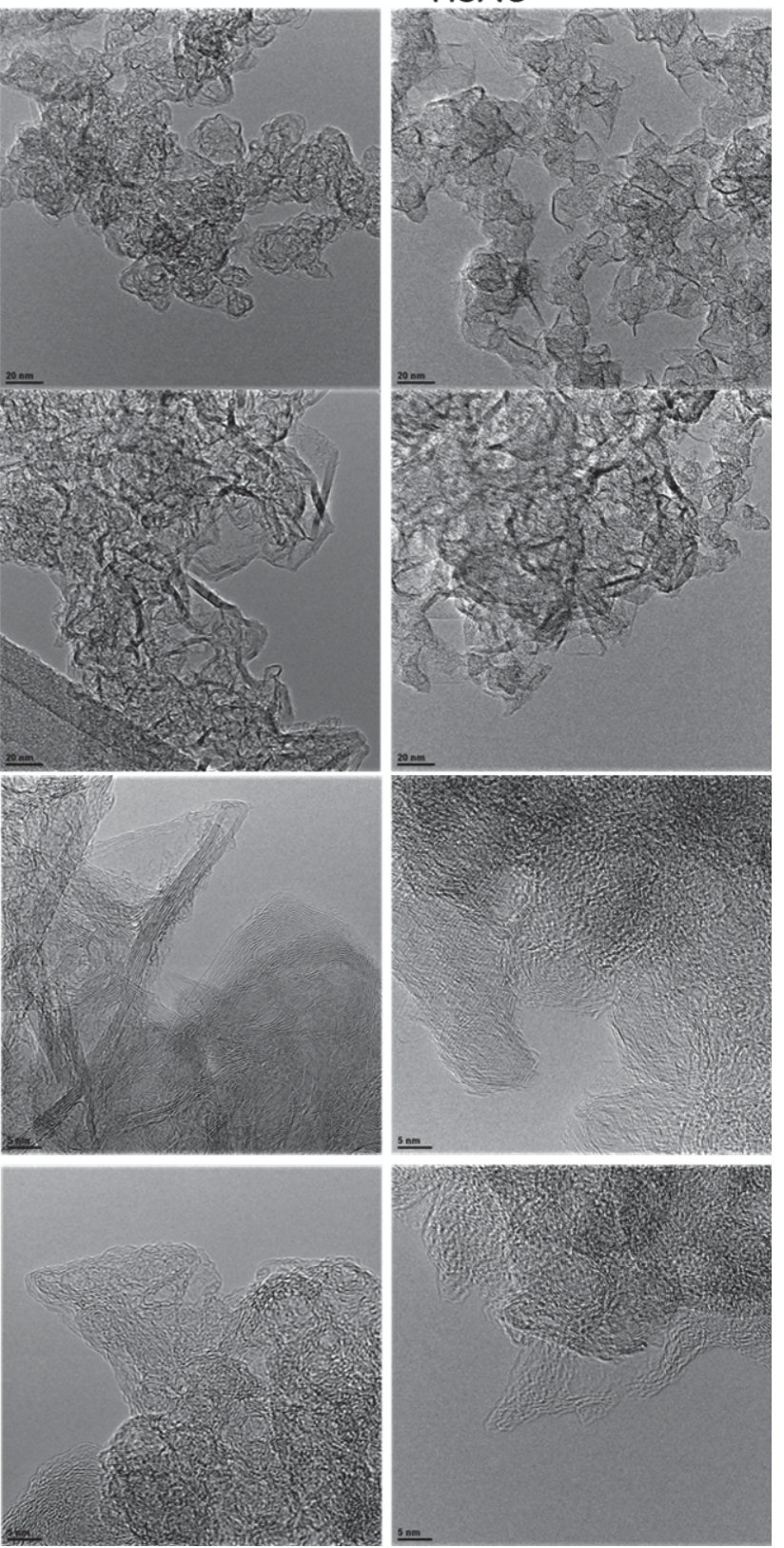

Figure 5: Comparison of carbons with different surface areas. TEM images

Figure 5 compares three types of carbon at two different magnifications. Very different morphologies are found. The LSAC has a high degree of walls with large amounts of graphitic content; and in contrast, HSAC has only a few areas with graphitic walls and the walls of particles are broken with evident amorphous content. MSAC samples shows a morphology somewhere in between the LSAC and HSAC samples. While the walls and internal structure of the three carbons are very different, similar particle agglomeration is observed for the three samples. 
TEM and DF/BF STEM images from the same area of the LSAC Pt50 catalyst sample as shown in Figure 6 and very large amounts of $\mathrm{Pt}$ particles were observed. The particles are highly agglomerated, very rarely were individual particles been observed. Typical TEM images for the LSAC 50 Pt sample (Figure 7) at high magnification identify the Pt particles along edges and at corners where two carbon particles meet.

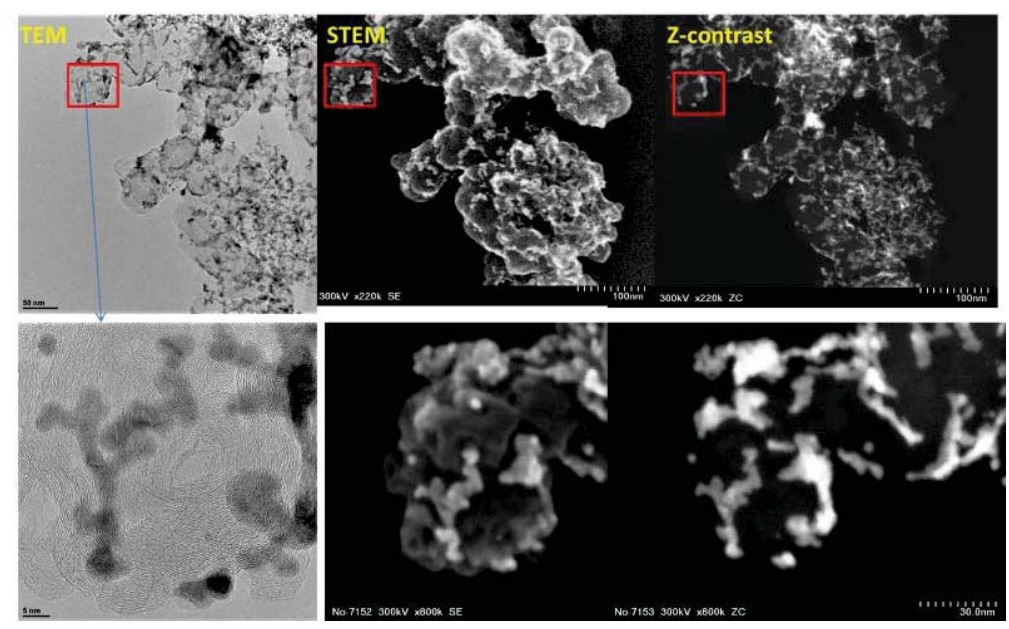

Figure 6: LSAC PT50 TEM/STEM results obtained on HF-3300 (same area)

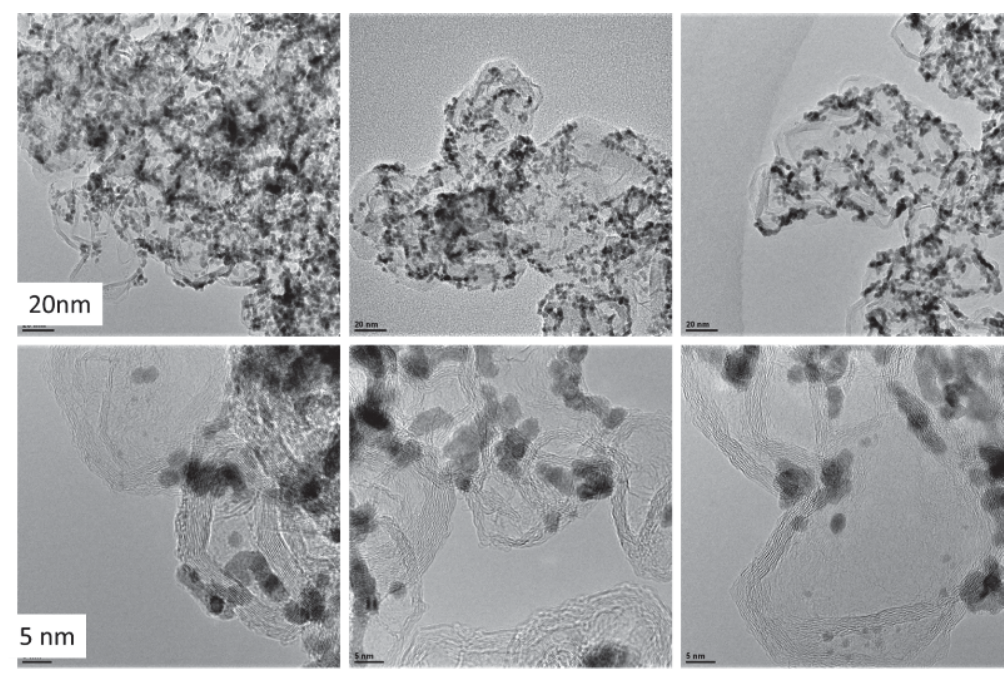

Figure 7: LSAC 50 Pt. Typical TEM images 
Figure 8 shows the SAC-STEM images for four catalysts samples at three different magnifications. Pt particles seem to agglomerate much more for LSAC as already shown Figure 7, while for MSAC and HSAC samples, the dispersion is much better. Detailed particle size analysis was also done, but a visual inspection of images indicates that heat treatment (HSAC HT) causes growth of Pt particle with lattices clearly observed. One also observes that the morphology for the carbon does not change dramatically with Pt deposition.
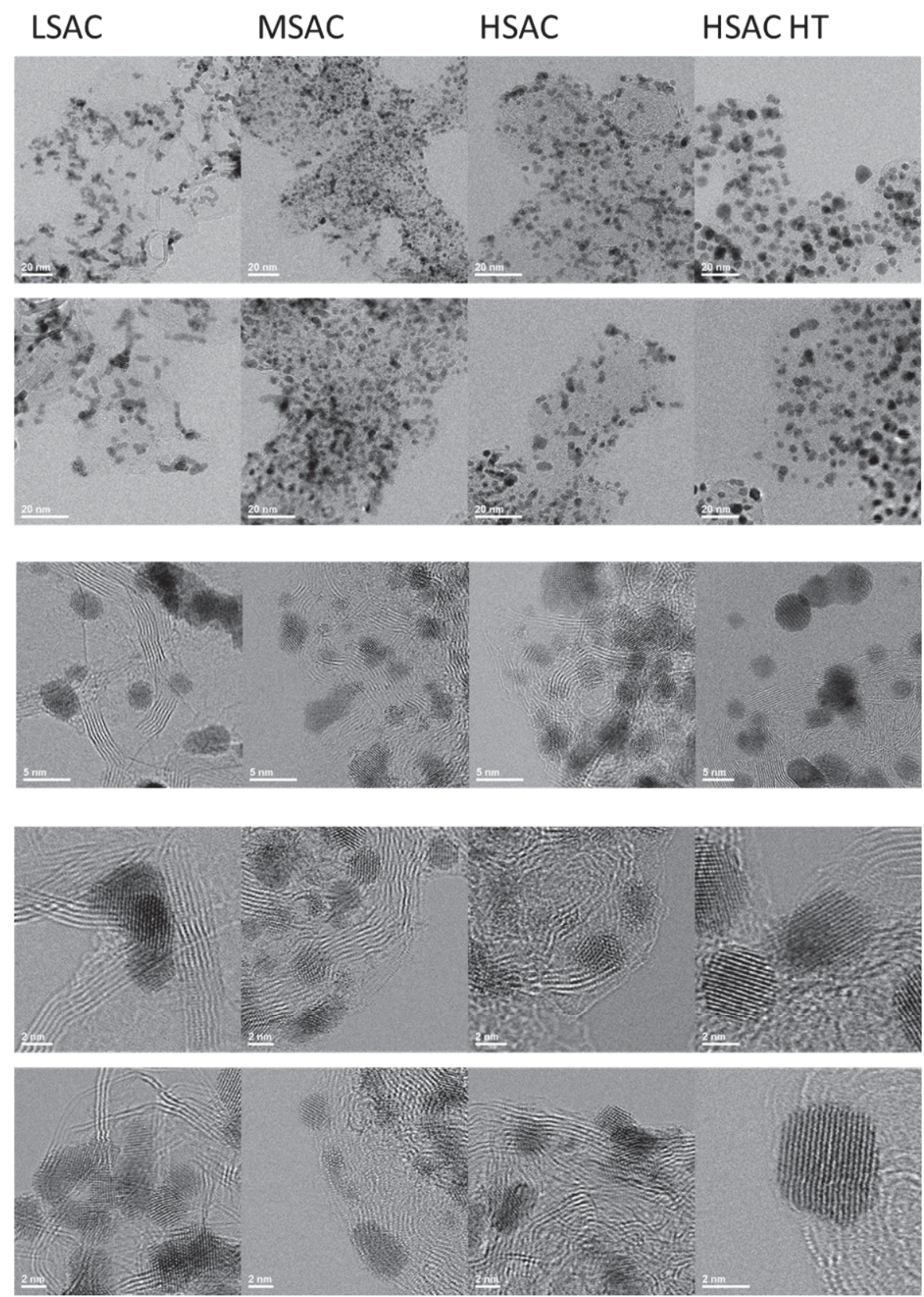

Figure 8: Comparison of SAC-STEM images for $50 \mathrm{Pt}$ loaded samples 
The STEM images acquired for catalyst powders were also used to calculate the Pt particle size distribution (PSD). Only individual clearly separated particles were used for these calculations. Figure 9 showing the PSD for the various catalyst powders indicates that all catalysts except for the heat treated sample have similar PSDs with a mean particle diameter of 2.1 to $2.3 \mathrm{~nm}$. Upon heat treatment, the particle size grew to $3.5 \mathrm{~nm}$ for the HSAC HT sample.

In addition, STEM images were processed to calculate agglomerate size and shape. Figure 10 shows the identification of agglomerates in STEM images. Individual particles are also a part of this calculation. From the agglomerates area and area of individual particles, the number of particles in agglomerates was calculated and normalized to the total number of agglomerates. Figure $11 \mathrm{~A}$ shows that the largest agglomeration is observed for the LSAC Pt catalyst. The aspect ratio (ratio of dimensions in major axis to that of minor axis) calculated for the agglomerates sizes are displayed in Figure 11B. The HSAC-HT catalyst sample has mostly round individual particles. As well, HSAC and MSAC catalysts have significant amounts of $\mathrm{Pt}$ agglomerates that are mostly round, while Pt agglomerates for LSAC tend to form elongated structures.
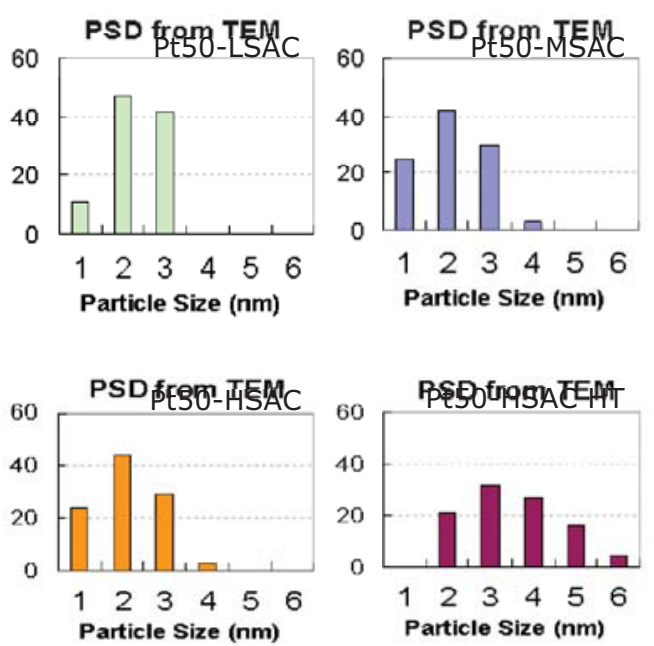

Figure 9: PSD calculated from STEM images for BOL samples
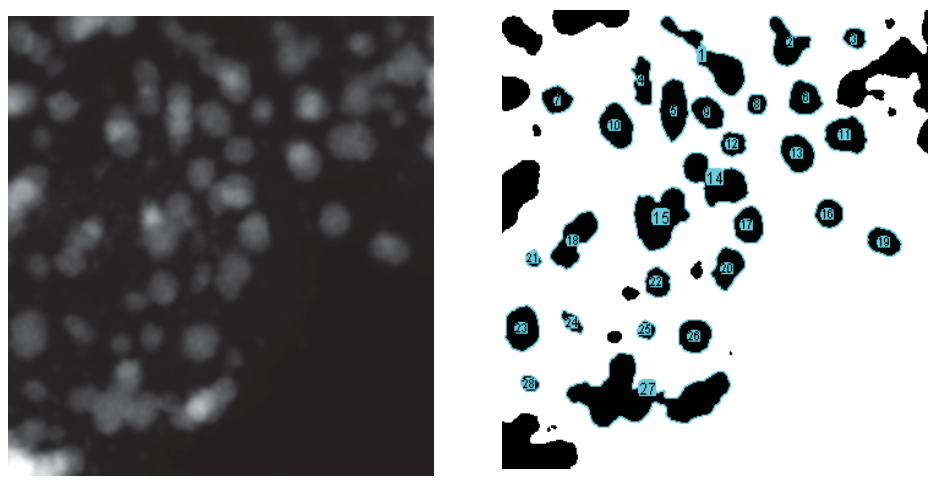

Figure 10: STEM images, threshold and agglomerates identified. 
A

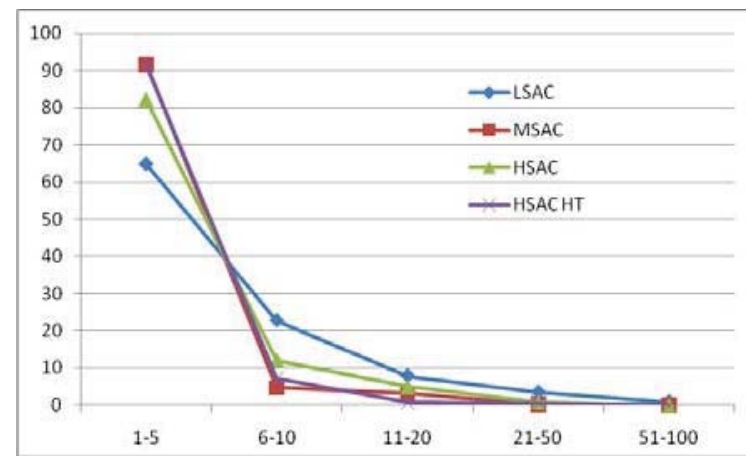

B

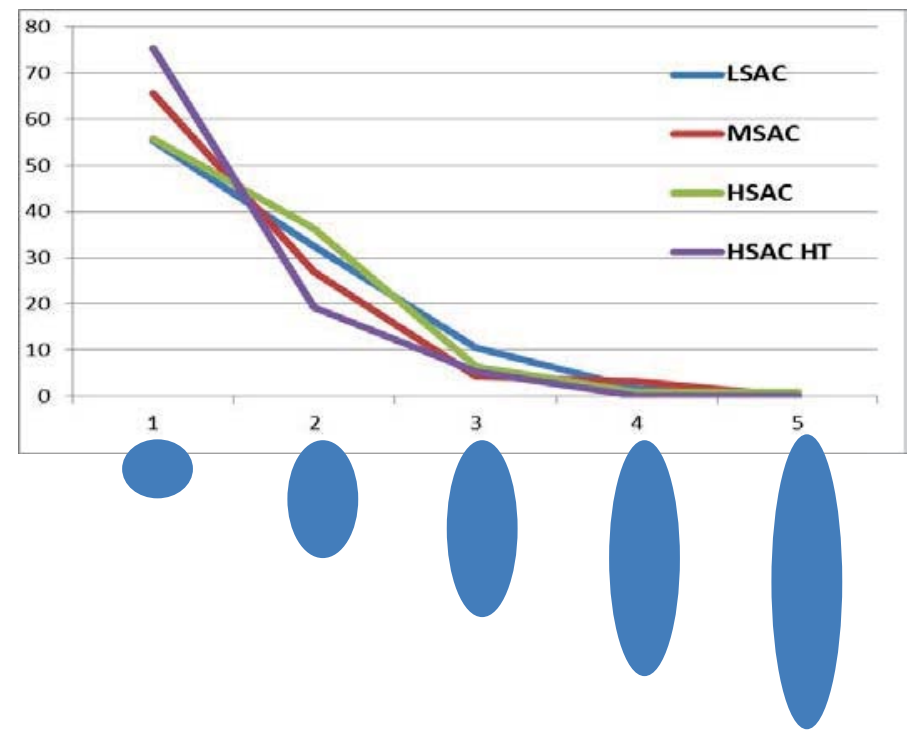

Figure 11: (A) Distribution of agglomerates with different number of particles in them;

(B) Distribution of aspect ratios for agglomerates

\subsubsection{Morphology - Scanning Electron Microscopic Analysis (SEM)}

Scanning Electron Microscopy (SEM) images were acquired to obtain morphological information for carbon black and Pt-catalysts. Representative images of undecorated and Pt decorated carbon blacks can be seen in Figure 12. The backscattered images were taken for all catalysts concurrently with SEM images to study the Pt distribution and the carbon structures. While changes in morphology and porosity were easily observed by visual inspection of the images, quantitative information is important for a clear understanding of the differences between samples and for correlating microscopy results with performance and surface chemistry. Digital image processing (DIP) was applied to find a representation of the intensity distribution of an image and to convert 2-D images into descriptors that are useful for morphology representation. As well, roughness parameters were calculated for SEM images (low and high frequency components individually) and for BS images (overall images). Texture parameters were calculated for both SEM and BS images shown in Figure 12. The majority of catalyst samples exhibit high amounts of pores and an overall homogeneous morphology with high roughness $\mathrm{Pt}$ domains (see Table 3). Lower LSAC Pt loaded samples were found to be most homogeneous showing the largest meso-scale porosity, while HSAC2-HT, LSAC Pt50 and LSAC Pt80 are similar to Vulcan samples which show the largest roughness of the carbon support (Figure 13).

As shown in Figure 14, the carbons have higher amount of pores on both meso and micro scales and are more homogeneous (ordered), while the catalysts have high roughness and higher contrast. The MSAC catalyst revealed less pores than the corresponding carbon since pores are being occupied by small $\mathrm{Pt}$ nano-particles, resulting also in more homogenous images. The samples that exhibit the highest micro-roughness and contrast are the LSAC catalysts, heat treated HSAC, and Vulcan catalysts. 
DE-EE0000466

Ballard Material Products Inc.
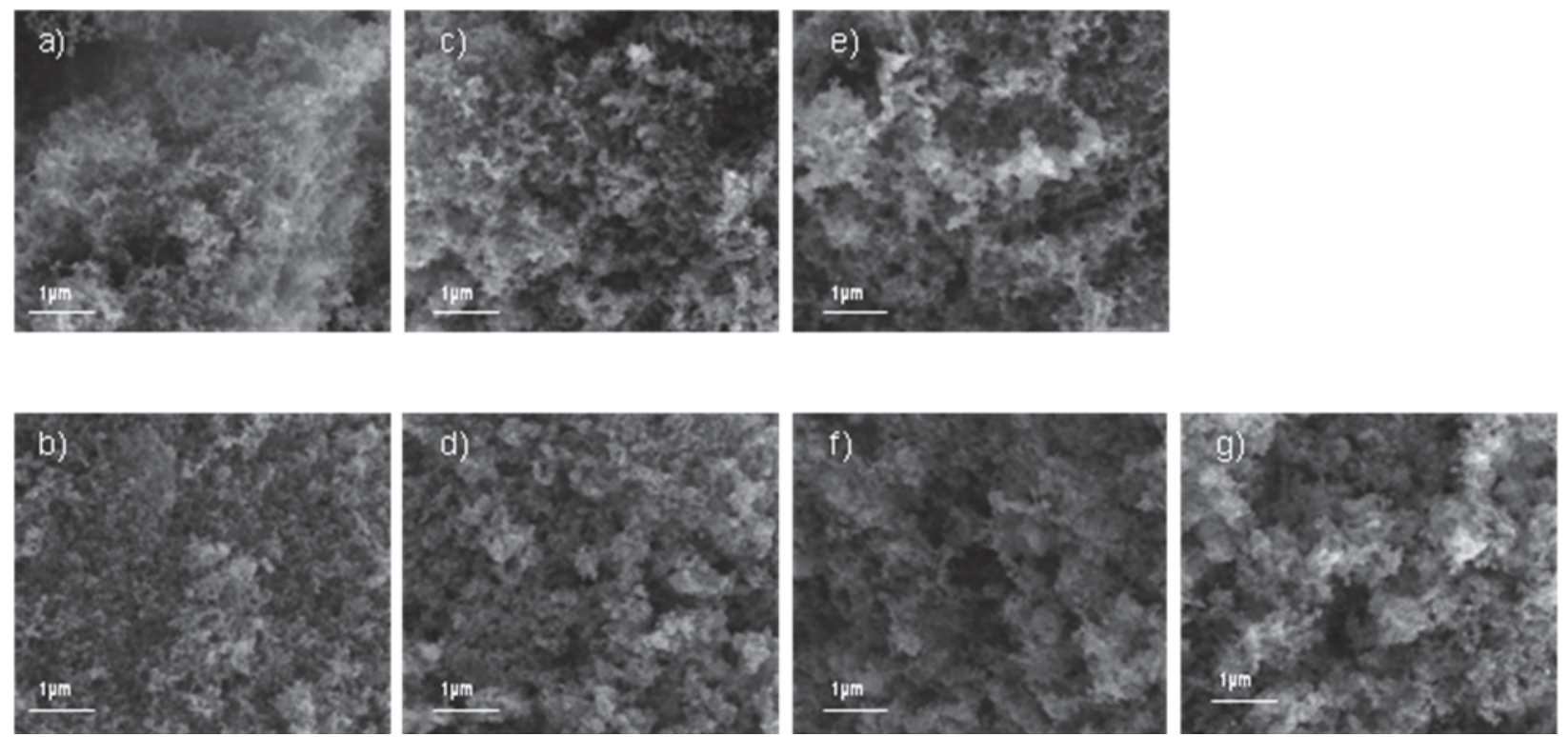

Figure 12: SEM images for carbon blacks a) LSAC, c) MSAC and e) HSAC and Pt-decorated samples b) LSAC50, d) MSAC50, f) HSAC50(1) and g) HSAC50-HT samples

Table 3: Roughness and texture parameters extracted from SEM and BS images

\begin{tabular}{|c|c|c|c|c|c|c|c|c|c|c|c|c|c|c|}
\hline & \multicolumn{8}{|c|}{ SEM images } & \multicolumn{6}{|c|}{ BS images } \\
\hline & \multicolumn{4}{|c|}{ Roughness } & \multicolumn{4}{|c|}{ Texture } & \multicolumn{2}{|c|}{ Roughness } & \multicolumn{4}{|c|}{ Texture } \\
\hline & $\mathrm{RaH}$ & RskH & $\mathrm{RaL}$ & RskL & Contr & Corr & Energy & Homog & Ra BS & Rsk BS & ${ }_{3 \mathrm{~S}}^{\text {Contr }}$ & $\mathrm{BS}^{\text {Corr }}$ & $\mathrm{BS}^{\text {Energ }}$ & $\mathrm{BS}^{\text {Homog }}$ \\
\hline LSAC & 15.1 & 1.210 & 88.7 & 1.103 & 0.351 & 0.797 & 0.162 & 0.837 & & & & & & \\
\hline MSAC & 17.9 & 1.030 & 61.7 & 1.146 & 0.439 & 0.744 & 0.182 & 0.814 & & & & & & \\
\hline HSAC1 & 14.9 & 1.213 & 96.9 & 1.117 & 0.320 & 0.831 & 0.170 & 0.851 & & & & & & \\
\hline HSAC2 & 15.8 & 1.125 & 77.0 & 1.252 & 0.384 & 0.834 & 0.136 & 0.825 & & & & & & \\
\hline Vulcan & 20.4 & 0.811 & 60.9 & 1.212 & 0.521 & 0.784 & 0.135 & 0.792 & & & & & & \\
\hline $\mid \begin{array}{ll}\text { LSAC } & \text { Pt } \\
\text { 50 }\end{array}$ & 23.7 & 0.424 & 91.6 & 1.137 & 0.528 & 0.842 & 0.090 & 0.790 & 98.4 & 1.076 & 0.588 & 0.361 & 0.189 & 0.768 \\
\hline $\begin{array}{ll}\text { MSAC } & \text { Pt } \\
50\end{array}$ & 18.0 & 0.673 & 89.9 & 1.116 & 0.398 & 0.828 & 0.122 & 0.819 & 97.3 & 1.039 & 0.439 & 0.284 & 0.248 & 0.798 \\
\hline $\begin{array}{l}\text { HSAC } \\
50\end{array}$ & 19.7 & 0.473 & 93.1 & 1.129 & 0.412 & 0.854 & 0.111 & 0.817 & 96.9 & 1.045 & 0.386 & 0.394 & 0.258 & 0.821 \\
\hline $\begin{array}{l}\text { HSA A C } \\
\mathbf{5 0}\end{array}$ & 20.5 & 0.496 & 87.9 & 1.151 & 0.454 & 0.844 & 0.116 & 0.813 & 97.9 & 1.047 & 0.421 & 0.375 & 0.241 & 0.806 \\
\hline $\mid \begin{array}{lll}\text { HSAC } & \text { HT } \\
\text { Pt } 50 & \text { 1 }\end{array}$ & 20.2 & 0.553 & 90.1 & 1.154 & 0.395 & 0.865 & 0.110 & 0.824 & 97.6 & 1.047 & 0.425 & 0.365 & 0.249 & 0.807 \\
\hline $\begin{array}{ll}\text { vulcan } & \text { Pt } \\
\mathbf{5 0} & \end{array}$ & 24.6 & 0.430 & 89.5 & 1.090 & 0.594 & 0.786 & 0.093 & 0.771 & 96.1 & 1.102 & 0.630 & 0.402 & 0.196 & 0.767 \\
\hline $\mid \begin{array}{ll}\text { LSAC } \\
30\end{array}$ & 20.1 & 0.547 & 94.0 & 1.138 & 0.405 & 0.861 & 0.110 & 0.820 & 97.6 & 1.055 & 0.570 & 0.266 & 0.208 & 0.765 \\
\hline $\mid \begin{array}{ll}\text { LSAC } \\
40\end{array}$ & 20.6 & 0.597 & 94.4 & 1.173 & 0.447 & 0.868 & 0.106 & 0.811 & 95.9 & 1.047 & 0.501 & 0.273 & 0.222 & 0.778 \\
\hline $\mid \begin{array}{ll}\text { HSAC } & \text { HT } \\
\text { Pt } 502 & \end{array}$ & 27.2 & 0.574 & 90.2 & 1.120 & 0.592 & 0.827 & 0.090 & 0.775 & 96.7 & 1.047 & 0.529 & 0.230 & 0.220 & 0.767 \\
\hline $\begin{array}{l}\text { LSAC } \\
60\end{array}$ & 23.0 & 0.823 & 89.8 & 1.122 & 0.459 & 0.848 & 0.111 & 0.811 & 97.2 & 1.057 & 0.459 & 0.442 & 0.224 & 0.791 \\
\hline $\begin{array}{ll}\text { LSAC } \\
\mathbf{8 0}\end{array}$ & 25.7 & 0.522 & 94.2 & 1.123 & 0.625 & 0.822 & 0.085 & 0.771 & 97.0 & 1.097 & 0.540 & 0.487 & 0.208 & 0.787 \\
\hline
\end{tabular}


DE-EE0000466

Ballard Material Products Inc.

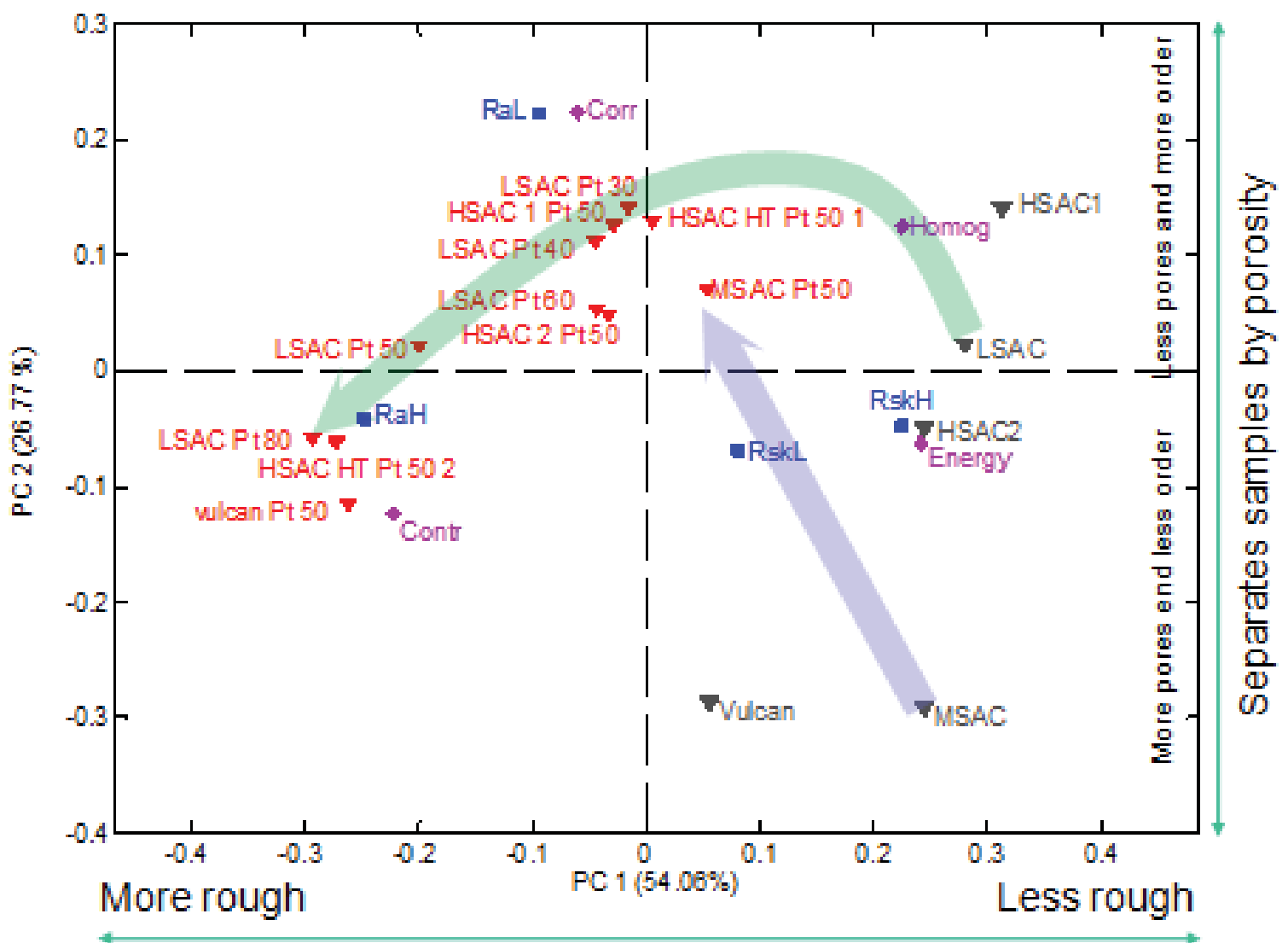

Separates samples by contrast

Separates carbons from majority of catalysts

Figure 13: PCA results from morphological parameters for all samples 


\subsubsection{Surface Chemistry - X-ray Photo-electron Spectroscopy Analysis}

The surface chemistries of the carbon and catalyst samples were analyzed by XPS. The carbon surface species play an important role, not only for the resulting structure and properties ${ }^{1}$ of the carbons and catalysts as observed in the TEM analysis discussed above, but also in the formation of the catalyst and ionomer dispersion within catalyst layer. Improved fundamental understanding of the carbon and catalyst surface chemistry and morphologies and their link to catalyst layer properties is an important driver for future catalyst supplier development.

XPS results for all carbon and catalyst samples tested which were used in the studies described in Chapter II are given in Table 4, which specifies their carbon, oxygen, and platinum speciations. While the carbon speciation was found to be similar for all carbon blacks, various amounts of oxygen were detected for the carbon powders, ranging from $7 \%$ for MSAC to $16 \%$ for LSAC and Vulcan. Moreover, the oxygen content range was even larger for catalysts, ranging from 5 to $21 \%$.

Table 4: Chemical Speciation of $\mathrm{C}$ and Pt of Catalyst With and Without Catalyst

\begin{tabular}{|l|llllll|lll|l|}
\hline & Carb & Cgr & Car & C* & C-O & C=O & Pt & Pt-C & PtO & O\% \\
\hline LSAC & 0.5 & 47.6 & 22.2 & 5.1 & 7.6 & 0.9 & & & & 16.4 \\
MSAC & 0.5 & 51.8 & 22.0 & 3.9 & 11.5 & 2.9 & & & & 7.4 \\
HSAC1 & 1.1 & 43.5 & 25.1 & 6.7 & 9.7 & 3.4 & & & & 10.4 \\
HSAC2 & 2.2 & 42.8 & 22.9 & 10.6 & 6.5 & 1.8 & & & & 13.2 \\
Vulcan & 1.3 & 44.3 & 17.5 & 5.5 & 11.2 & 4.5 & & & & 16.2 \\
LSAC Pt 50 & 1.0 & 48.9 & 21.2 & 5.1 & 4.8 & 2.4 & 1.1 & 1.7 & 0.9 & 12.9 \\
MSAC Pt 50 & 0.4 & 28.2 & 34.0 & 7.2 & 4.9 & 3.7 & 0.8 & 0.7 & 0.7 & 19.4 \\
HSAC 1 Pt 50 & 1.2 & 36.4 & 29.6 & 5.7 & 5.8 & 3.9 & 0.8 & 0.6 & 0.6 & 15.3 \\
HSAC 2 Pt 50 & 1.2 & 33.9 & 32.4 & 3.7 & 7.9 & 5.3 & 0.8 & 0.8 & 0.4 & 13.7 \\
HSAC HT Pt 50 1 & 1.3 & 42.3 & 29.6 & 2.7 & 7.4 & 2.4 & 0.9 & 0.5 & 0.1 & 12.9 \\
vulcan Pt 50 & 0.9 & 43.2 & 15.6 & 5.6 & 4.2 & 4.3 & 1.6 & 2.3 & 1.2 & 21.0 \\
LSAC Pt 30 & 0.2 & 47.7 & 25.5 & 5.5 & 8.7 & 3.0 & 0.4 & 0.8 & 0.1 & 8.2 \\
LSAC Pt 40 & 0.7 & 52.5 & 24.5 & 2.9 & 8.4 & 2.2 & 1.0 & 0.4 & 0.2 & 7.2 \\
HSAC HT Pt 50 2 & 2.1 & 49.1 & 21.7 & 10.0 & 7.6 & 2.1 & 1.6 & 1.1 & 0.2 & 4.6 \\
LSAC Pt 60 & 0.0 & 58.5 & 15.4 & 5.7 & 5.7 & 2.2 & 2.9 & 3.1 & 0.9 & 5.7 \\
LSAC Pt 80 & 1.2 & 59.4 & 18.1 & 6.5 & 6.7 & 1.5 & 1.0 & 0.6 & 0.1 & 4.8 \\
\hline
\end{tabular}

\footnotetext{
${ }^{1}$ X. Yu and S. Ye, Journal of Power Sources 172, 133-144, 2007.
} 
Although, the graphitic and aromatic C content was similar for all carbons ranging from 40-50\% and $\sim 20 \%$, respectively, significant differences were observed for the CxOy species. Upon Pt deposition, the distribution of carbon species became much more variable. The graphitic carbon content of the catalysts ranged from $28 \%$ to $60 \%$ and aromatic carbon ranged from $15 \%$ to $30 \%$. Additionally, XPS analysis revealed three types of Pt, which were identified as metallic $\mathrm{Pt}, \mathrm{Pt}$ bounded to carbon such as organometallic type species and PtO. The largest amounts of Pt species were detected for Vulcan and the LSAC Pt60 catalyst samples.

Performing Principal Component Analysis (PCA), an exploratory tool that converts a set of observations into a set of values of uncorrelated variables called principal components, of the XPS data set (shown in Figure 14), resulted in the identification of four groups:

- Group I has highest amount of Pt: LSAC Pt50, LSAC Pt60, Vulcan

- Group II has catalysts with highest amount of graphitic carbon: heat treated HSAC 1, LSAC Pt80

- Group III has the highest amount of oxygen, particularly $\mathrm{C}=\mathrm{O}$ : MSAC, HSAC1, HSAC2

- Group IV has the largest amount of carbides: HSAC1-HT, LSACPt20, LSACPt30, LSACPt40

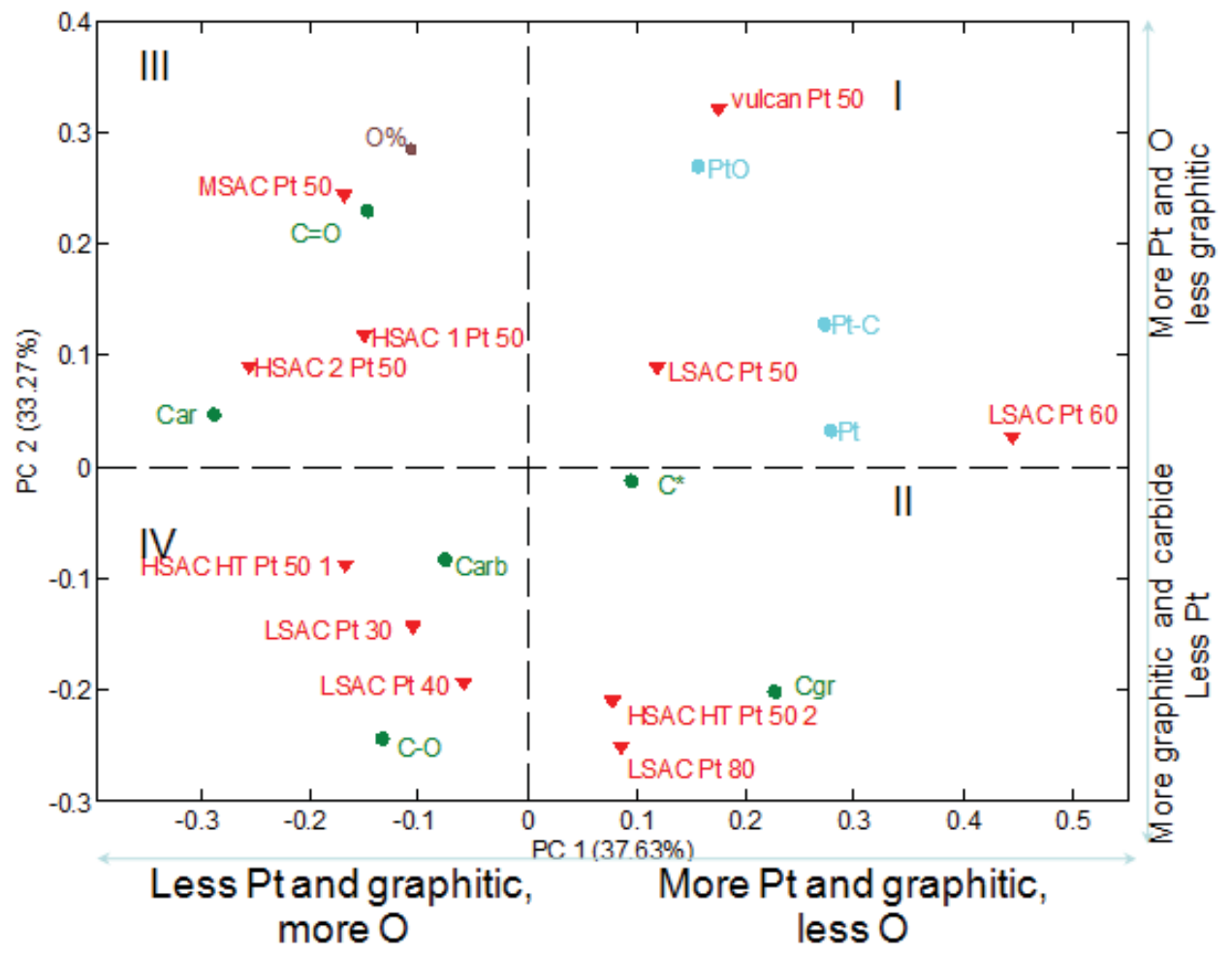

Figure 14: PCA results from XPS data (absolute atomic \%) for catalyst samples

The MSAC and both HSAC catalysts as well as one of the heat treated HSAC-HT catalyst samples showed comparable composition to LSAC with the lower $\mathrm{Pt} / \mathrm{C}$ ratios having more oxygen and more carbides. An increase in the Pt/LSAC ratio and heat treatment of HSAC 2 showed similarity to Vulcan, with higher graphitic and Pt content. 
In addition, catalysts with $\mathrm{Pt} / \mathrm{C}$ ratios of $50 \mathrm{wt} \%$ (except heat treated) revealed more oxygen, $\mathrm{PtO}$, and aromatic carbon species. The LSAC Pt50 catalyst is similar to Vulcan having higher oxygen and PtO content, while MSAC and both HSAC catalysts exhibited more aromatic C.

In summary, the LSAC catalysts speciation is similar to the corresponding carbon powder, while MSAC and HSAC catalyst exhibited higher $\mathrm{O} \%$ and $\mathrm{C}=\mathrm{O}$ content compared to the corresponding carbons. The most dramatic change upon deposition of $\mathrm{Pt}$ was observed for MSAC and HSAC2. Both carbons, Vulcan and HSAC 1 carbons, were found to be similar in composition and revealed only slight changes with Pt deposition.

\subsubsection{Surface Chemistry - Morphology (XPS-SEM) Correlations}

In Figure 15, the $1^{\text {st }}$ Principle Component $(\mathrm{PC})$ separate porosity vs. roughness and oxygen vs. graphitic content. More pores are correlated with more $\mathrm{O}$ and more roughness with more graphitic character. The $2^{\text {nd }} \mathrm{PC}$ is separating heterogeneity vs. homogeneity and oxygen vs. graphitic surface species. For both the carbons and catalysts, more pores are correlated with more $\mathrm{O}$ and larger roughness is correlated with a more graphitic character. As well, a larger heterogeneity is correlated with more graphitic and a larger homogeneity with more oxygen. Two groups of catalysts were identified:

- Catalysts MSAC, both HSAC, HSAC HT 1 and LSAC with the lowest Pt loading are more homogeneous than other catalyst powders and contain more of the oxygen and aromatic carbon on the surface.

- Catalysts Vulcan, HT HSAC \#2 and all other LSAC samples with higher Pt loadings have the highest micro-roughness which correlates with highest amounts of graphitic and carbides.

A direct comparison between the catalyst samples and the surface speciation and the morphological characteristics shown in Figure 16 reveals that more stable samples (LSAC, MSAC catalysts) have more graphitic carbon, secondary carbons, and PtO and have high contrast, high overall roughness and high micro-porosity. Less durable samples (Vulcan HSAC, HSAC HT) have more oxygen, carbide and surface oxides, metallic Pt and more homogeneous images and larger meso-porosity. It is suggested that Quinone groups could lead to better Pt distribution. 
DE-EE0000466

Ballard Material Products Inc.

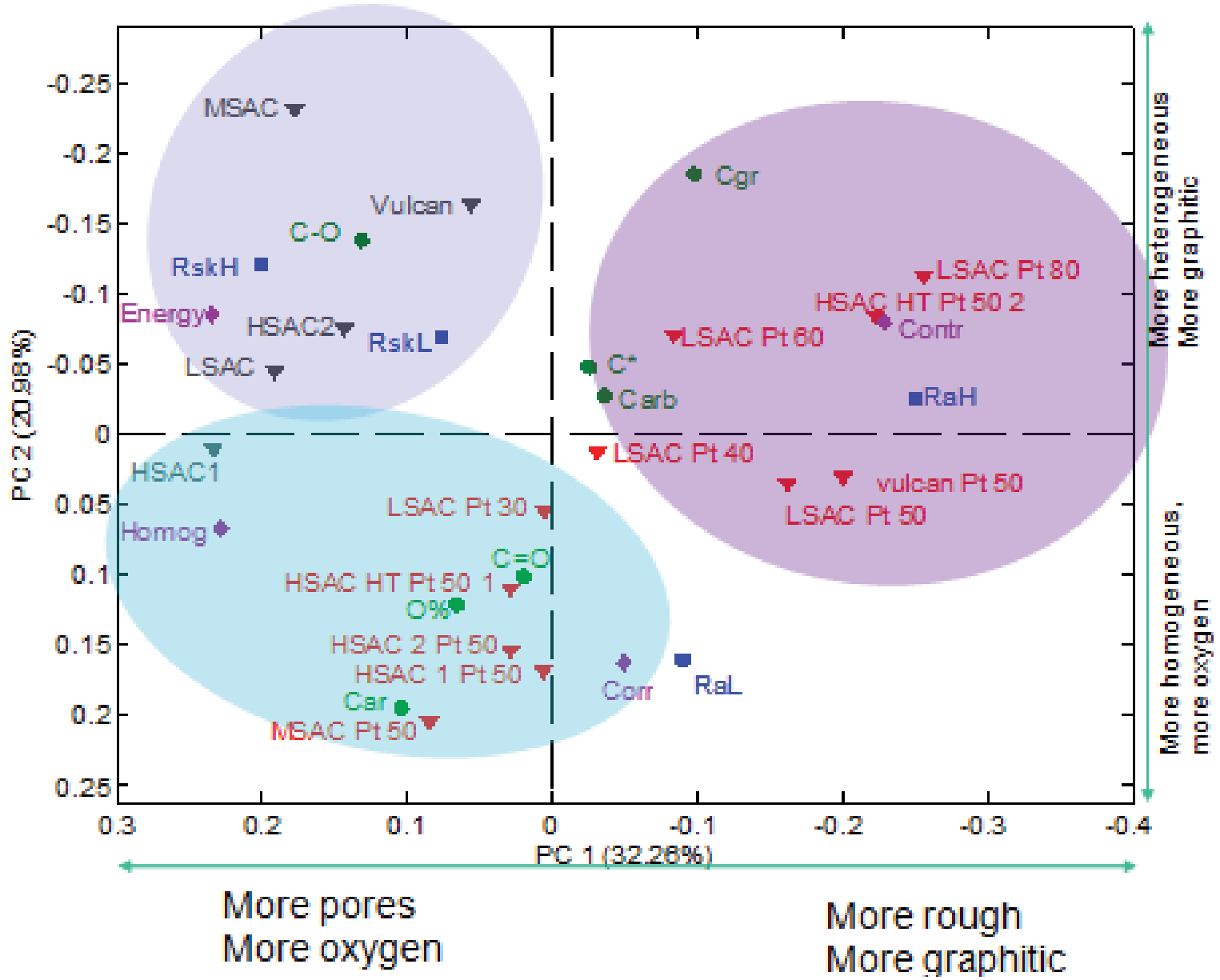

Figure 15: PCA results from XPS data (absolute atomic \%) and morphological parameters for all samples 

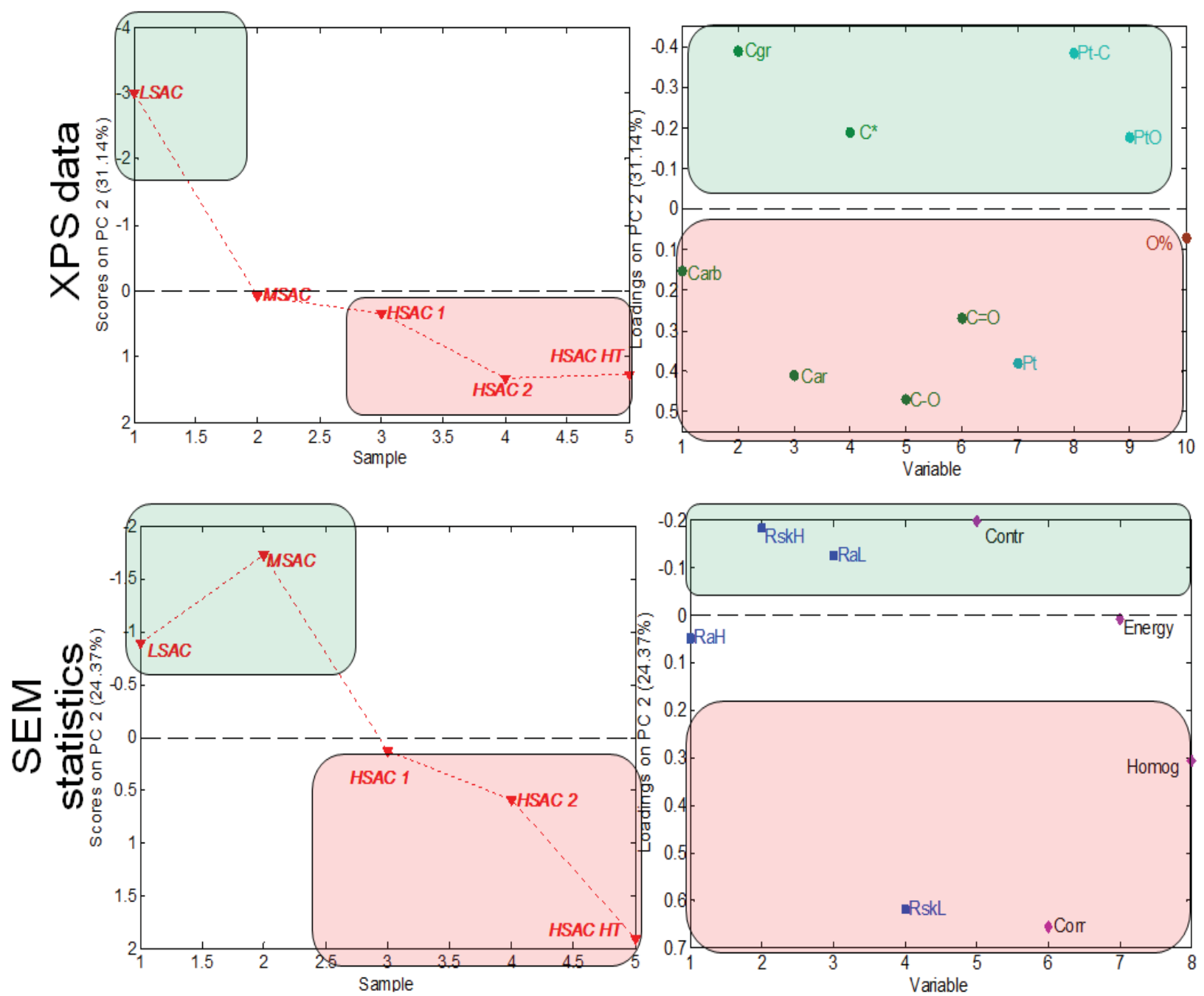

Figure 16: PCA results from XPS data (absolute atomic \%) (top) and PCA results from SEM morphological parameters (bottom) for the same loading catalysts sample.

\subsubsection{Structure-to-performance correlations}

The catalysts surface species and morphology was correlated via PCA to fuel cell performance and degradation. Indirect observations confirmed that more stable samples (resistive to carbon corrosion) contain a higher content of graphitic carbon. They also contain more secondary carbons and PtO, and have high contrast, high overall roughness and high micro-porosity. Less durable samples have more oxygen, carbide and surface oxides, metallic $\mathrm{Pt}$ and more homogeneous images and larger meso-porosity. Direct correlations showed that LSAC Pt50 has the best performance, which was again correlated with graphitic content from both XPS and EELS, with the total amount of $\mathrm{C}$ and $\mathrm{Pt}$ and contrast and total roughness as well as larger size of agglomerates. MSAC Pt50 and HSAC Pt50 samples revealed the largest amount of oxygen present as well as a higher degree of uniformity and more pores. A HSAC HT catalyst sample indicated the largest Pt crystallite size which correlates with largest relative amount of metallic Pt present, more homogeneity and less roughness. 
DE-EE0000466

Ballard Material Products Inc.

\subsubsection{Catalyst Layers}

\subsubsection{Morphology - Scanning Electron Microscopic Analysis (SEM)}

Catalyst layers delaminated from as prepared, conditioned and aged MEAs were analyzed by SEM. The degraded catalysts that were analyzed were from the studies presented in Chapter II. The micrographs were acquired at different magnifications and image processed.

Figure 17 shows the surface texture of the catalyst layer containing Pt supported on low and high surface area carbon, LSAC50 and HSAC(2)50 respectively. Similar morphological changes between the degraded 1.2V UPL LSAC and 1.0V HSAC samples and the 1.3V UPL LSAC and 1.2V HSAC samples are observed due to the fact that the samples pairs underwent the same degradation mechanisms, namely $\mathrm{Pt}$ dissolution (first sample pair) and carbon corrosion (second sample pair). Digital Image Processing (DIP) of the SEM micrographs and XPS data revealed that conditioned samples display high frequency roughness (20nm-1 micron), a higher number of small pores $(20-100 \mathrm{~nm})$ and larger amounts of PtO and PtC than degraded catalyst layers. Aged catalyst layers that did not undergo corrosion exhibited the largest overall roughness, more uniform morphology and large amount of average pore sizes $(0.2-0.6 \mu \mathrm{m})$, while corroded catalyst layers showed the highest amount of surface oxides largest amount of largest pores $(3-20 \mu \mathrm{m})$ and largest level of micro-roughness $(20-100 \mathrm{~nm})$ compared with conditioned catalyst layers.

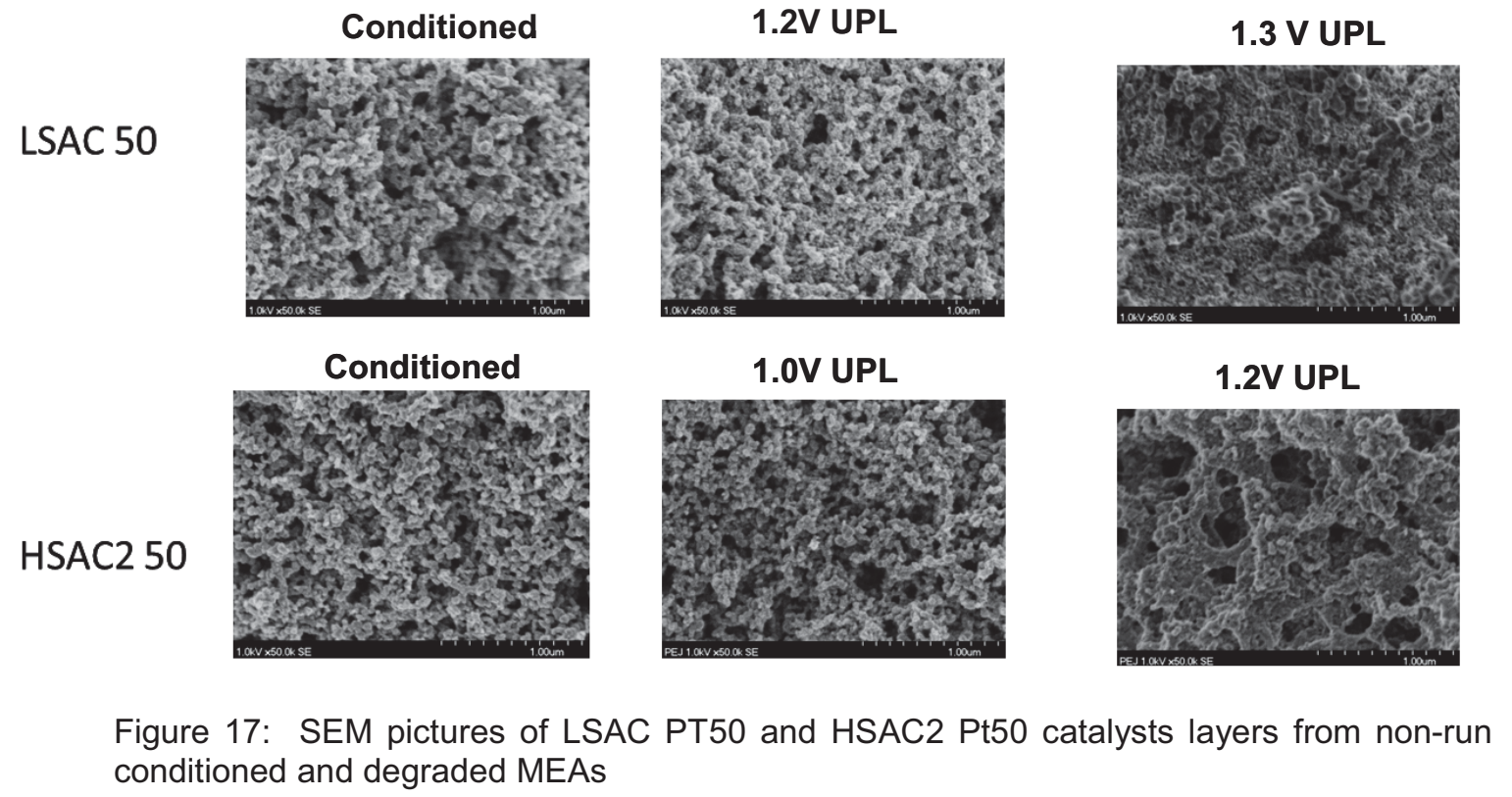

conditioned and degraded MEAs 
DE-EE0000466

Ballard Material Products Inc.

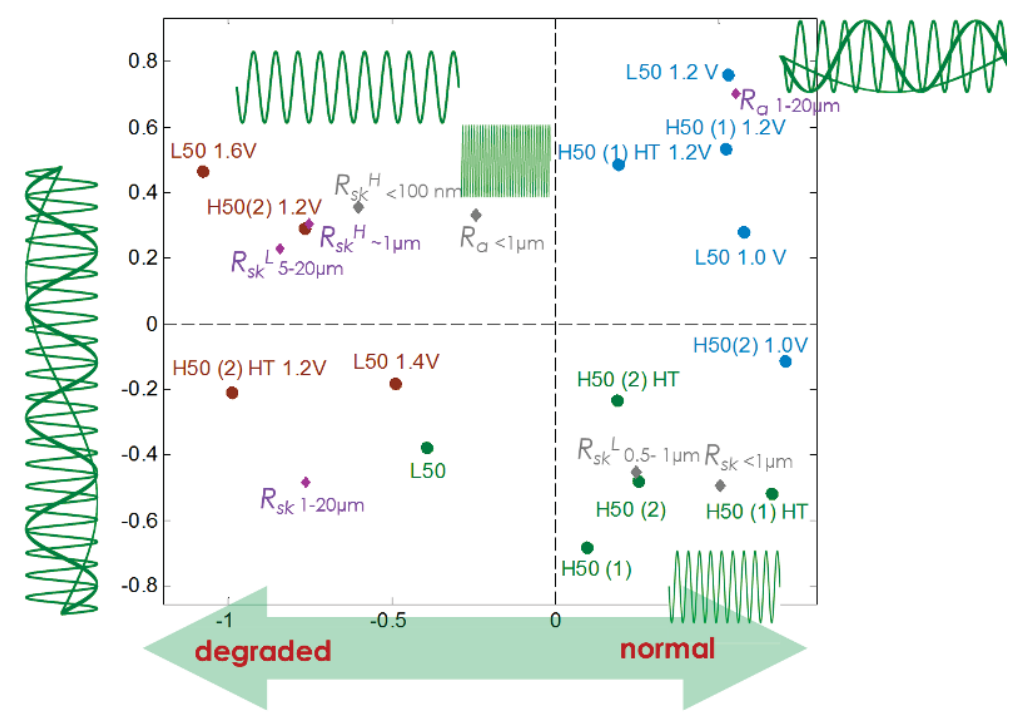

Figure 18: PCA analysis of catalyst layer morphology data for conditioned and AST tested MEA (normal $=$ Pt dissolution, degraded $=$ carbon corrosion)

Figure 18 shows PCA results of roughness and skewness parameters extracted from filtered high magnification $(50 \mathrm{~K})$ and low magnification $(2.5 \mathrm{~K})$ images. Normal AST and conditioned samples have a positive contribution to PC1 while degraded catalyst layers show a negative contribution into PC1. Conditioned samples exhibit also high micro-porosity while non-degraded samples have large overall roughness, but no changes in porosity were introduced, while degraded samples have increased nano-, micro- and meso-porosity.

In summary, conditioned MEAs show higher high frequency (0.4-1microns) roughness, while degraded MEAs show higher porosity in all dimensions; high frequency (0.4-1 microns) and low frequency (3-20 microns). Two types of porosity behavior were detected for tested MEAs; for degraded samples high frequency porosity increased as well as the low frequency porosity. For "normal" tested sample (Pt dissolution), high frequency porosity increases while low frequency porosity remains low. The degraded MEAs also revealed higher amount of smallest pores -20$100 \mathrm{~nm}$, while the conditioned and "normal" tested samples were more similar to each other at this magnification.

Macro-meso porosity at a level of 5-20 microns was not changing significantly as a function of AST UPL, however it did increase for all samples significantly in comparison with conditioned samples, while the micro ( $<1$ micro-meter) and nano $(20-100 \mathrm{~nm})$ porosities increased significantly with increasing upper potential limit. 
DE-EE0000466

Ballard Material Products Inc.

\subsubsection{Surface Chemistry - X-ray Photo-electron Spectroscopy Analysis}

Catalyst layers delaminated from as prepared, conditioned and aged MEAs for the same samples analyzed by SEM above and from the studies presented in Chapter II were also analyzed by XPS. As delamination in some cases left carbon from the GDL micro-porous layer on the catalyst layer surface, the MPL was also analyzed and used to de-convolute its impact on the XPS analysis of the catalyst layer.

Table 5 shows elemental composition of the anode and cathode layer of conditioned MEAs. The results show that overall more carbon and oxygen and less $\mathrm{Pt}$ and $\mathrm{F}$ is observed in conditioned compared to the not-run LSAC50 catalyst layer (MEA). Smaller changes are observed for the anode than cathode with conditioning and in comparison to the catalyst powders and non-run (as prepared) MEAs.

Table 5: XPS elemental composition of catalyst layers after conditioning

\begin{tabular}{|c|c|c|c|c|c|c|c|c|c|c|c|c|}
\hline & & Ano & ode & & & & & Cath & hode & & & \\
\hline & C1s \% & F1s \% & 01s \% & Pt $4 f \%$ & S 2p \% & & C1s \% & F1s \% & 01s \% & Pt $4 f \%$ & S 2p \% & \\
\hline ionomer & 25.0 & 66.4 & 7.7 & & 0.9 & ionomer & 25.0 & 66.4 & 7.7 & & 0.9 & \\
\hline MEA & 46.6 & 48.2 & 3.4 & 1.54 & 0.31 & MEA & 46.6 & 48.2 & 3.4 & 1.54 & 0.31 & \\
\hline $144 a$ & 49.6 & 44.0 & 4.7 & 1.07 & 0.60 & $144 c$ & 48.9 & 43.9 & 5.1 & 1.28 & 0.85 & LSAC50 \\
\hline $177 a$ & 48.9 & 44.4 & 5.1 & 1.01 & 0.57 & $177 c$ & 45.0 & 46.6 & 6.0 & 1.58 & 0.82 & MSAC50 \\
\hline 199a & 47.6 & 46.5 & 4.3 & 1.01 & 0.55 & $199 c$ & 49.1 & 42.7 & 6.0 & 1.41 & 0.79 & V50 \\
\hline $166 a$ & 47.6 & 45.6 & 5.2 & 0.90 & 0.69 & $166 c$ & 55.5 & 33.8 & 8.7 & 1.32 & 0.63 & HSAC(1)50 \\
\hline $200 a$ & 47.2 & 45.4 & 5.9 & 0.86 & 0.64 & $200 c$ & 57.8 & 35.1 & 5.4 & 1.18 & 0.53 & HSAC(2)50 \\
\hline $201 a$ & 49.4 & 43.6 & 5.4 & 0.90 & 0.71 & $201 c$ & 59.0 & 34.4 & 4.8 & 1.13 & 0.64 & HSAC(1)50 HT \\
\hline $204 a$ & 46.1 & 47.0 & 5.2 & 0.93 & 0.74 & $204 c$ & 60.2 & 34.1 & 4.2 & 0.87 & 0.58 & HSAC(2)50 HT \\
\hline $244 a$ & 46.2 & 46.6 & 4.9 & 1.57 & 0.76 & $244 c$ & 61.1 & 33.3 & 4.3 & 0.79 & 0.53 & LSAC30 \\
\hline $254 a$ & 46.2 & 47.3 & 4.9 & 0.92 & 0.71 & $254 c$ & 60.5 & 33.6 & 4.0 & 1.32 & 0.52 & LSAC40 \\
\hline $245 a$ & 46.2 & 47.1 & 5.1 & 0.87 & 0.72 & $245 c$ & 51.5 & 41.4 & 4.8 & 1.56 & 0.78 & LSAC60 \\
\hline $270 a$ & 47.0 & 46.3 & 5.0 & 0.93 & 0.73 & $270 c$ & 44.2 & 48.5 & 5.3 & 1.13 & 0.84 & LSAC80 \\
\hline
\end{tabular}

Analysis of $\mathrm{C} 1 \mathrm{~s}, \mathrm{~F} 1 \mathrm{~s}, \mathrm{O} 1 \mathrm{~s}$, and $\mathrm{Pt} 4 \mathrm{f}$ showed that after conditioning the $\mathrm{O}, \mathrm{F}$ and $\mathrm{Pt}$ speciations of the catalyst layer increase for conditioned MEAs compared to non-run MEAs. The fluorine spectra shows that there is a large degree of fluorination for both anode and cathode sides. The peak due to C-OF species decreased in the F1s signal, indicating that the peak at $291.3 \mathrm{eV}$ in $\mathrm{C}$ is due to $\mathrm{CF}_{2}$. The $\mathrm{O} 1 \mathrm{~s}$ peak which is hard to interpret due to large overlap between species may point towards a decrease of oxygenated CF species that are present in ionomer and fresh MEA indicating more surface oxides that are not bonded to $F$ in the catalysts layer and oxygen that is bonded to $\mathrm{CF}_{2}$ species. The peak at $292 \mathrm{eV}$ in $\mathrm{C} 1 \mathrm{~s}$, thus, may be due to O$\mathrm{CF}_{2}$ species. The $\mathrm{Pt} 4 \mathrm{f}$ peak revealed an increase in metallic $\mathrm{Pt}$ at the expense of carbides and oxides for most samples.

PCA analysis of surface species for LSAC cathode catalyst layers that were part of the AST studies discussed in Chapter II is shown in Figure 19. It was found that conditioning did not cause any changes in elemental composition, $\mathrm{F}$ and $\mathrm{O}$ speciation. Half of the $\mathrm{Pt}$ associated 
with $\mathrm{C}$ is also converted to metallic Pt. Low voltages AST $(1.0,1.1$, and $1.2 \mathrm{~V}$ all \# of cycles, 1.3 spike) causes very small similar changes, i.e. increase of $\mathrm{C}$, decrease in $\mathrm{F}$ and $\mathrm{O}$, with more graphitic $\mathrm{C}$ and more originally present surface oxides. The amount of $\mathrm{CF}_{2}$ decreases but the decrease was smaller than for the high UPL group of samples. The high UPL samples $(1.3,1.4$, $1.6 \mathrm{~V}$ ) causes decrease in $\mathrm{C}$ and increase in $\mathrm{F}$, which is hypothesized to be due to carbon loss at the higher UPLs. Increase in F-OC species is detected in O, F and C. There is also more $\mathrm{COOH}$. All types of cycling causes increase in metallic and decrease in PtO and increase in $\mathrm{O}=\mathrm{C}$. Decrease in $\mathrm{PtO}$ is larger for larger voltages. Changes to the $\mathrm{Pt}$ speciation are hypothesized to be due to the Pt dissolution and agglomeration mechanism resulting in larger Pt particles.

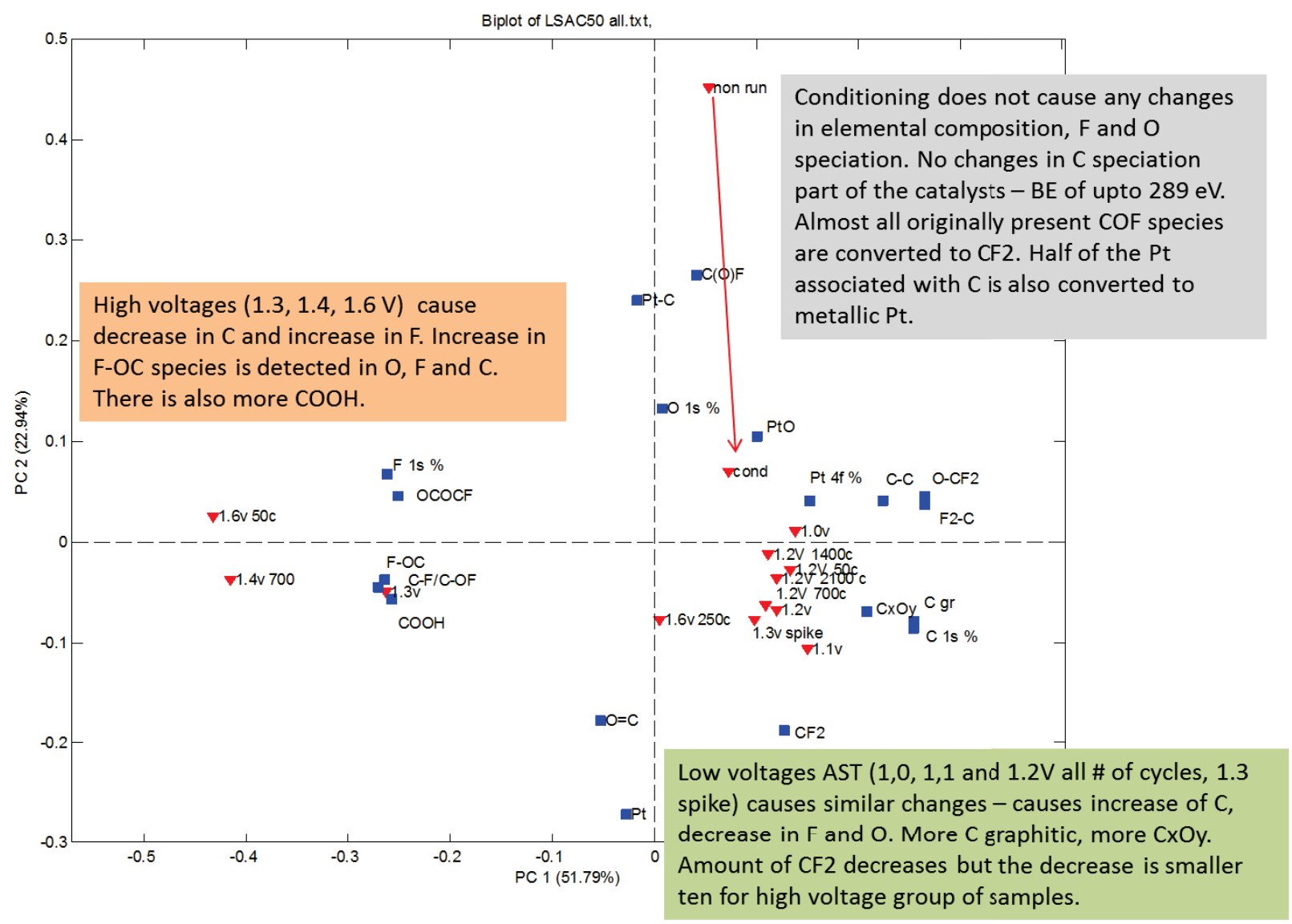

Figure 19: PCA analysis of LSAC samples subjected to AST testing at various upper potential limits 
Figure 20, showing the PCA results for samples with different Pt/C ratios, reveals that AST cycling caused an increase in $\mathrm{C}$, metallic $\mathrm{Pt}$, graphitic $\mathrm{C}$, aliphatic $\mathrm{C}, \mathrm{CxOy}$ and $\mathrm{COOH}$ contents. Figure 21 shows the PCA results for conditioned and AST tested samples. The largest changes introduced by accelerated stress testing were found to be for both the unheated HSAC samples and the HSAC 2 HT samples. The same changes in the surface chemistry (increased $\mathrm{F}, \mathrm{COOH}$ and F-OC species) were observed for the more stable LSAC50 but at substantially higher UPLs; i.e. UPLs $\geq 1$. The LSAC catalyst layers with different Pt loadings, those tested at UPL $\leq 1.1 \mathrm{~V}$, and samples from the cycle number study did not show much change after AST testing.

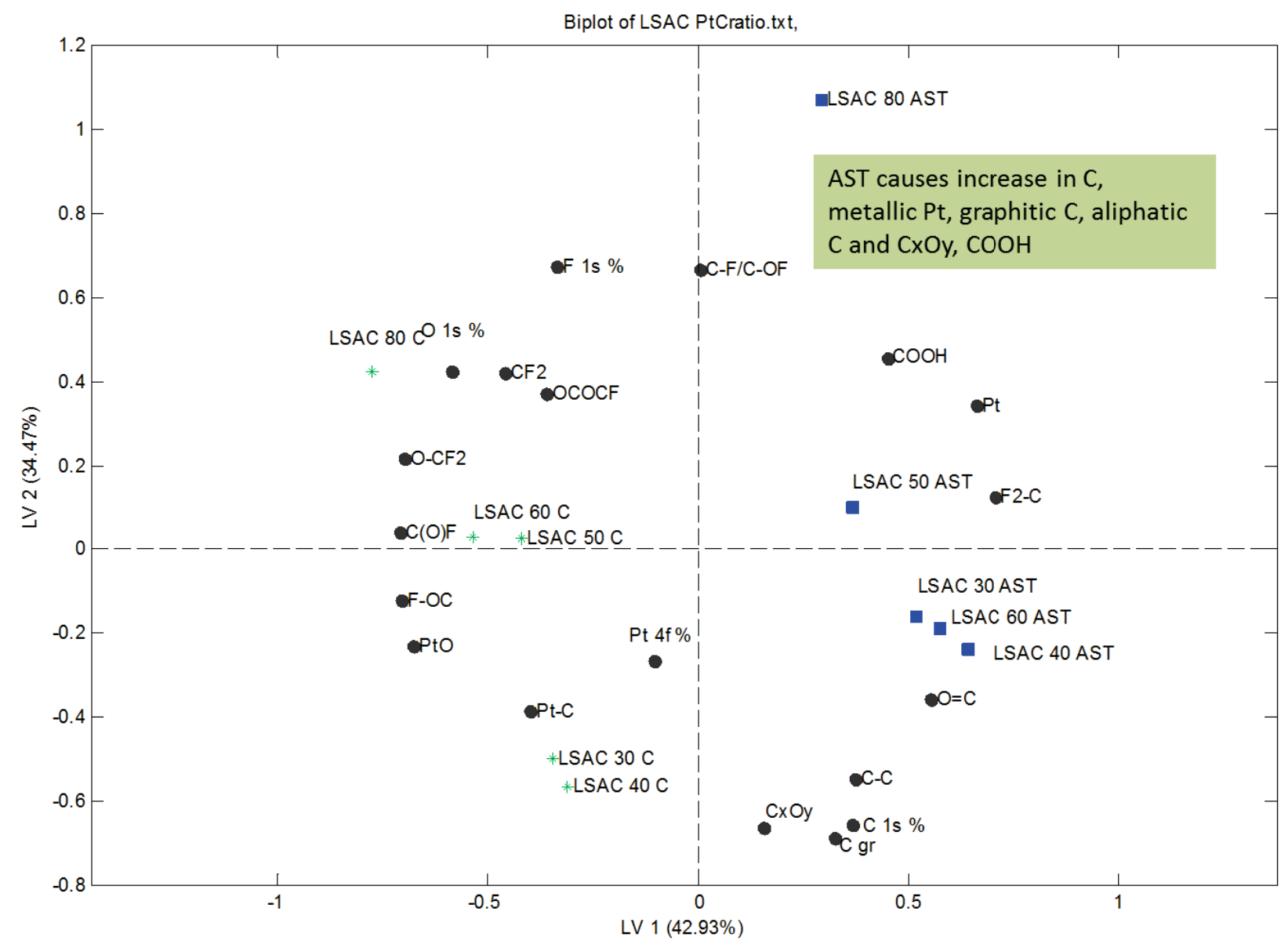

Figure 20: PCA of surface species for the Pt/LSAC ratio study (1.2V UPL) 
DE-EE0000466

Ballard Material Products Inc.

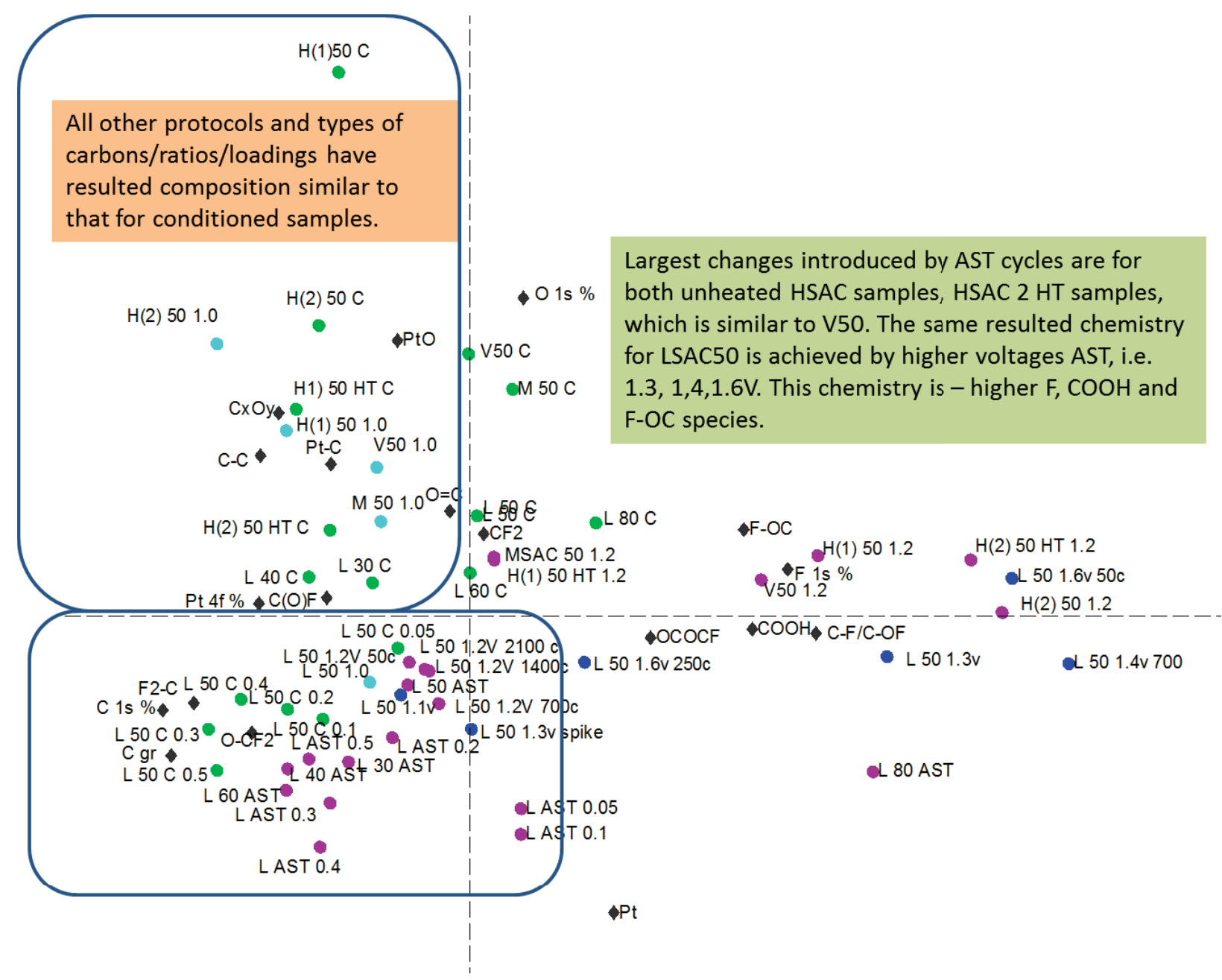

Figure 21: PCA comparing conditioned and degraded samples

In summary, tested MEAs revealed more carbon and oxygen and less Pt and $\mathrm{F}$ than in untested MEAs. Smaller changes were observed for the anode than the cathode. For both cathode and anodes an increase in graphitic $\mathrm{C}$ and in $\mathrm{CF}_{2} / \mathrm{COF}$ species at the expense of aliphatic carbons, carbides and CxOy species were found. The LSAC cathode catalyst samples (except for LSAC 80 which had different ionomer) were found to be different from the other samples in that they showed a higher amount of total $\mathrm{C}$, graphitic and aliphatic $\mathrm{C}$, total $\mathrm{Pt}, \mathrm{Pt}$ associated with $\mathrm{C}$ and

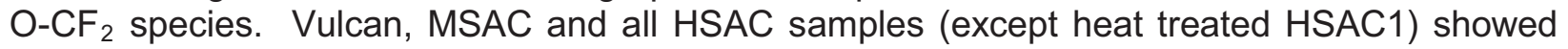
higher amounts of $\mathrm{O}$ and $\mathrm{F}$ after AST cycling. They also exhibited larger amounts of Pt metallic and associated PtO (suggesting Pt particles covered by oxide) and oxidized species such as $\mathrm{COOH}$ and $\mathrm{C}-\mathrm{OF}$.

High voltages $(1.3,1.4,1.6 \mathrm{~V})$ caused a decrease in $\mathrm{C}$ and increase in $\mathrm{F}$ species. An increase in $\mathrm{F}-\mathrm{OC}$ species was detected in $\mathrm{O}, \mathrm{F}$ and $\mathrm{C}$ chemistries. As well, cycling increased the amount of $\mathrm{COOH}$ species. These changes have been attributed to the carbon corrosion mechanism, which results in surface oxidation, followed by carbon loss. Loss of carbon results in a corresponding increase in the ionomer content in the catalyst layer, which explains the increase in F species. All cycling caused an increase in metallic Pt and decrease in PtO, as well as an increase in $\mathrm{O}=\mathrm{C}$, while the decrease in PtO species was found to be larger for catalyst layers 
DE-EE0000466

Ballard Material Products Inc.

that were degraded at higher voltages, an indication that the changes of $\mathrm{Pt}$ speciation are associated with the $\mathrm{Pt}$ dissolution and agglomeration mechanism.

The largest changes introduced by AST cycling were found to be in both unheated and heat treated HSAC samples. This agrees with durability data which showed these HSAC catalysts to be the least durable.

\subsubsection{Surface Species - Morphology-Performance Correlations}

Correlations between surface species for conditioned and AST tested catalyst layers at different upper potential limits are shown in Figure 22. The degraded samples exhibited the highest peaks due to surface oxides, specifically carbonates and carboxylates, shown in Figure 22. Interestingly, the reduction in the $\mathrm{CF}_{2}$ peak from the ionomer was also found to be largest for the degraded samples.

Figure 23 to Figure 25 show select speciations and skewness results as a function of AST UPL for LSAC50, as well as HSAC(2) and HSAC(1)HT and HSAC (2) HT. Since more catalyst degradation was observed for catalysts degraded at higher UPL, these correlations indicate how the surface species and morphology change with degradation. Figure 23 reveals that the larger the AST cycling potential the more carbonates/carboxylates and less of CF2 species are present. Pt speciation as a function of AST upper potential limit given in Figure 24 shows that with increasing upper potential limit the relative amounts of metallic Pt increase, while $\mathrm{Pt}$ bonded with carbon and PtO decrease. These changes in carbon and platinum speciations are related to carbon corrosion and Pt dissolution / agglomeration, respectively.

Figure 25 shows four values of normalized porosity extracted from both high and low magnification images as a function of AST potential. Not surprisingly, the macro-meso porosity (5-20 microns) is not changing significantly as a function of AST potential. As discussed above, it does however significantly increase for all AST samples in comparison with conditioned samples, but this does not seem to be an important factor for the degradation mechanism. Micro (<1 micron) and nano $(20-100 \mathrm{~nm})$ porosity however, increased significantly when the potential limit is increased. 
DE-EE0000466

Ballard Material Products Inc.
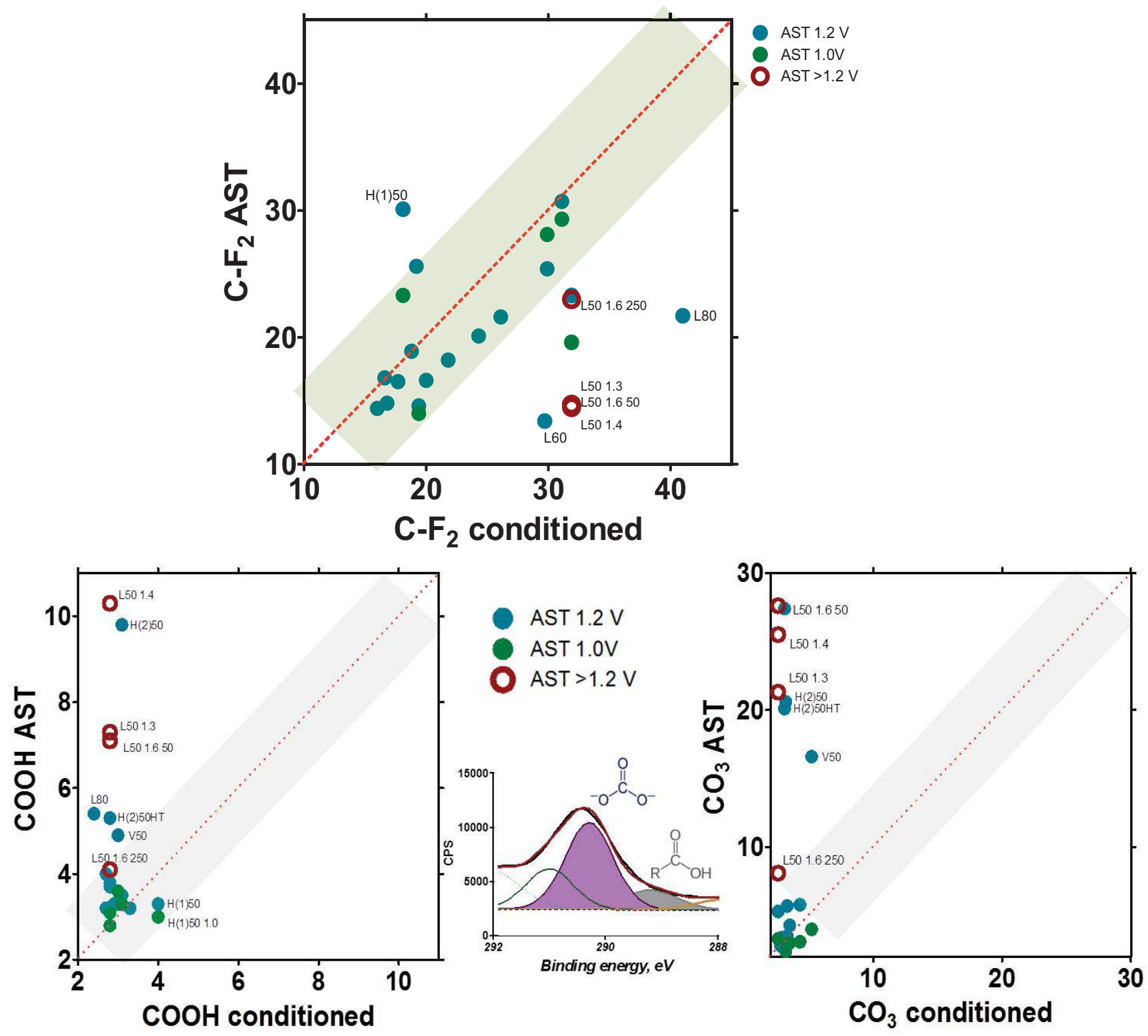

Figure 22: Change in carbonates, carboxylates and $\mathrm{CF}_{2}$ species for AST vs. conditioned samples 
DE-EE0000466

Ballard Material Products Inc.
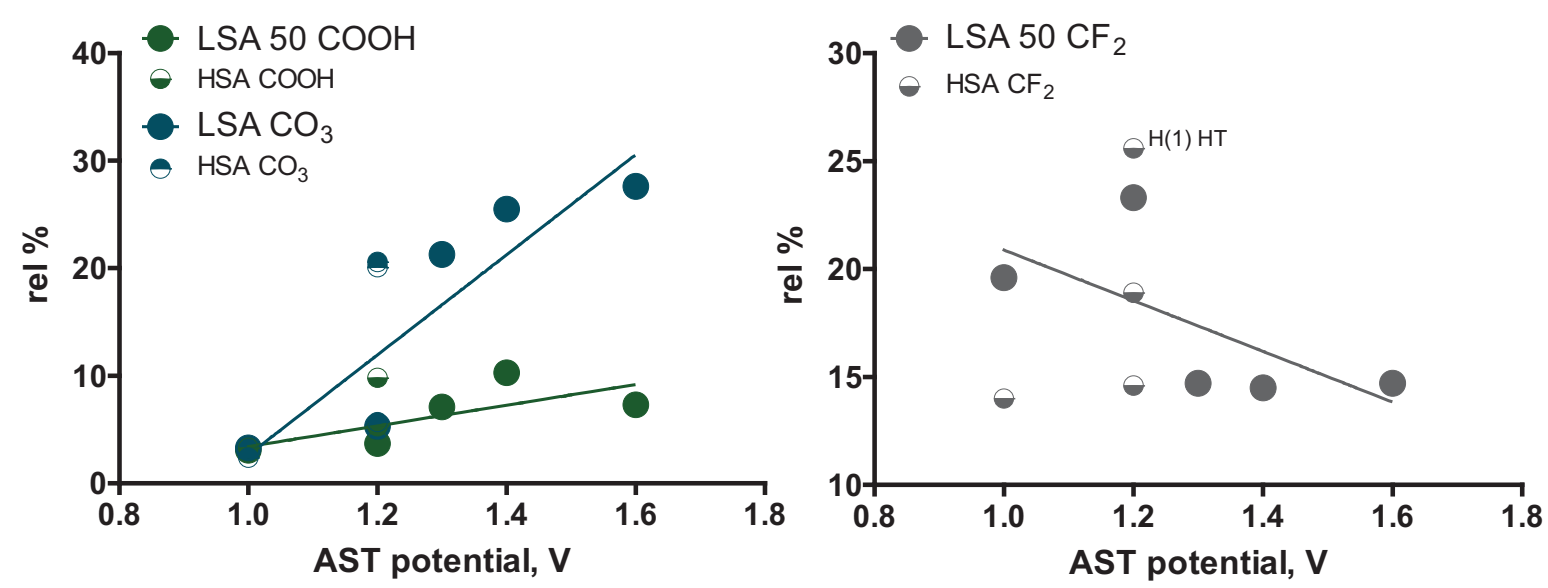

Figure 23: Change in carbonates, carboxylates and $\mathrm{CF}_{2}$ species as a function of AST upper potential limit
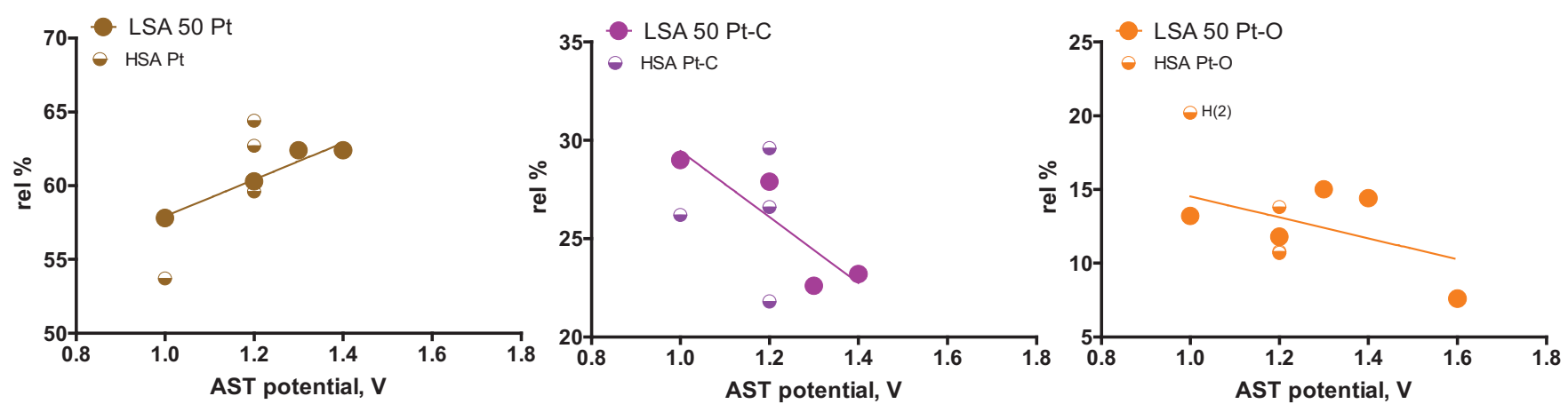

Figure 24: Change in Pt speciation as a function of AST potential 

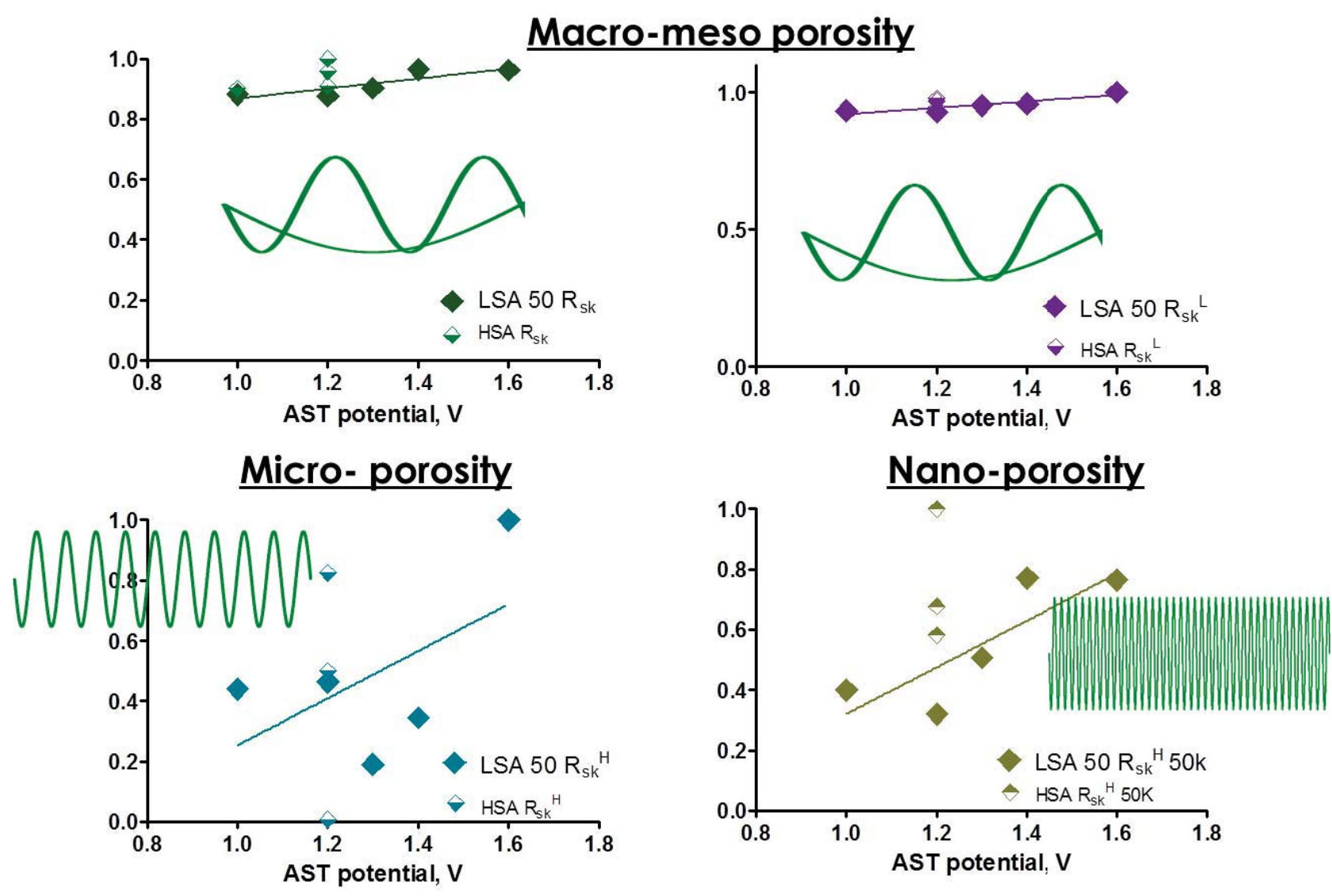

Figure 25: Skewness as a function of AST UPL for overall and different porosity scales

Figure 26 shows the structure-to-property correlations between surface chemistry and morphological parameters as obtained at UNM and other performance measures obtained by Ballard. The cathode Pt size is correlated with the growth of the relative metallic Pt content, while loss of $\mathrm{Pt}$ bound to $\mathrm{C}$ and $\mathrm{PtO}$ also correlate with loss in ESCA. These correlations confirm that the changes in $\mathrm{Pt}$ speciation are due to the $\mathrm{Pt}$ agglomeration mechanism. In addition, it was found that surface oxides correlate with MEA performance loss, while the catalyst layer thickness change correlates with the increase of porosity. These results agree with the two-step mechanism for carbon corrosion, which includes first surface oxidation of the carbon, followed by carbon loss. Moreover, the results suggest that Pt detachment and carbon corrosion introduce nano and micro-porosity. 

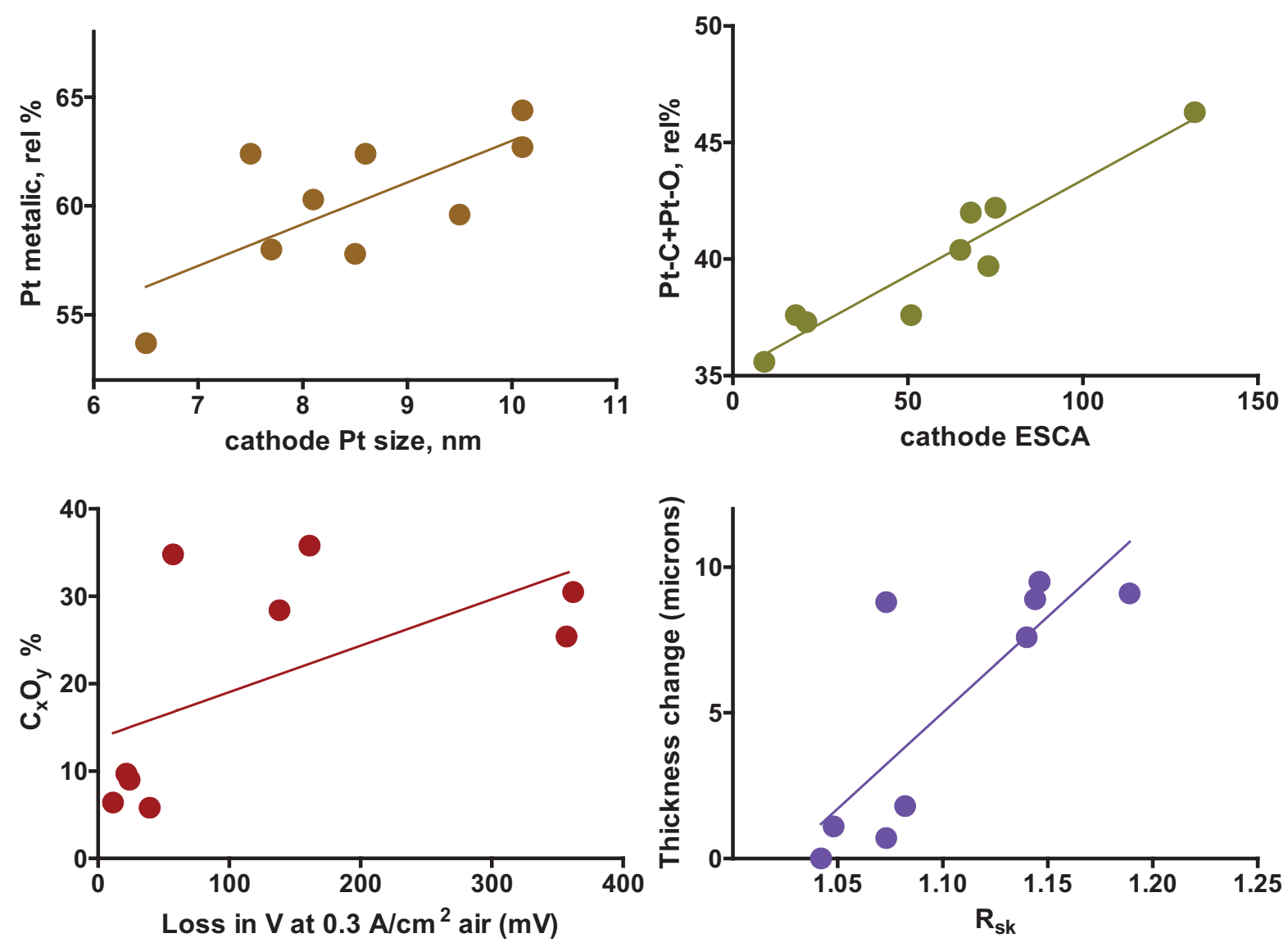

Figure 26: Structure-to-durability correlations 


\subsection{Gas adsorption measurements (LANL)}

Gas adsorption analysis (BET) was carried out on the different carbons and catalyst powders that are investigated under this project (Figure 27). The high surface area carbon powders (HSAC1 and HSAC2) showed a significant portion of micro-porous surface area compared to LSAC and MSAC with very little change observed for the corresponding catalyst powders, an indication that the Pt particles did not block the micro-pores. Catalyst powders that were heat treated freed some overall surface area from Pt blockage, which was observed to be most significant for the HSAC2 catalyst sample. Furthermore, Vulcan, HSAC1, and HSAC2 were found to have a significant fraction of micro-porous surface area (0.49-0.56), compared to LSAC $(\sim 0)$ and MSAC (0.10). Lastly, LSAC catalysts with different $\mathrm{Pt} / \mathrm{C}$ ratios showed a decrease in the cumulative surface area with increasing $\mathrm{Pt} / \mathrm{C}$ ratio.
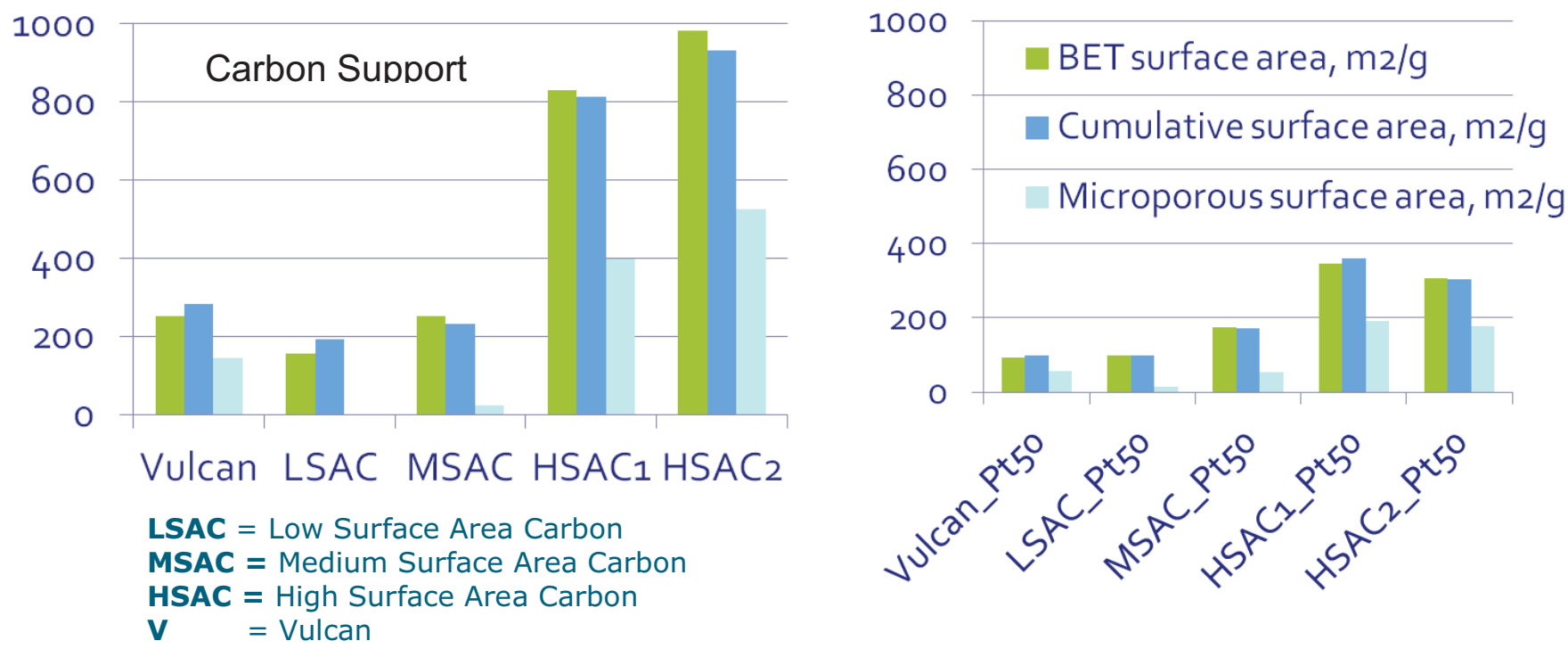

Figure 27: BET Analysis of various carbon supports and corresponding catalyst powders 
DE-EE0000466

Ballard Material Products Inc.

\subsection{Catalyst Layer Capillary Pressure (MTU)}

\subsubsection{Development of the Experimental Set-up}

The experimental set-up for the Catalyst Layer capillary pressure tests shown in Figure 28 is based on the GDL capillary pressure tool discussed below discussed below (Section 3.5). The set-up for the catalyst layers was modified and validated first with catalyst layers that were supported on a backing layer and then as a catalyst layer coated on one side of the Nafion membrane (i.e. $1 / 2$ catalyst coated membrane or $1 / 2 \mathrm{CCM}$ ).

A $4 \mathrm{~cm} \times 5.5 \mathrm{~cm}$ section of the catalyst layer was placed between two platens made of Polymethylmethacrylate (PMMA) as shown in Figure 28. In the test set-up, referred to as a pseudo-Hele-Shaw, the platens that were used for the GDL set-up were replaced by polymethylmethacrylate (PMMS) plates (Figure 29(B)) as the GDL platens that are coated by PDMS were found to degrade the catalyst layer (see Figure 30).

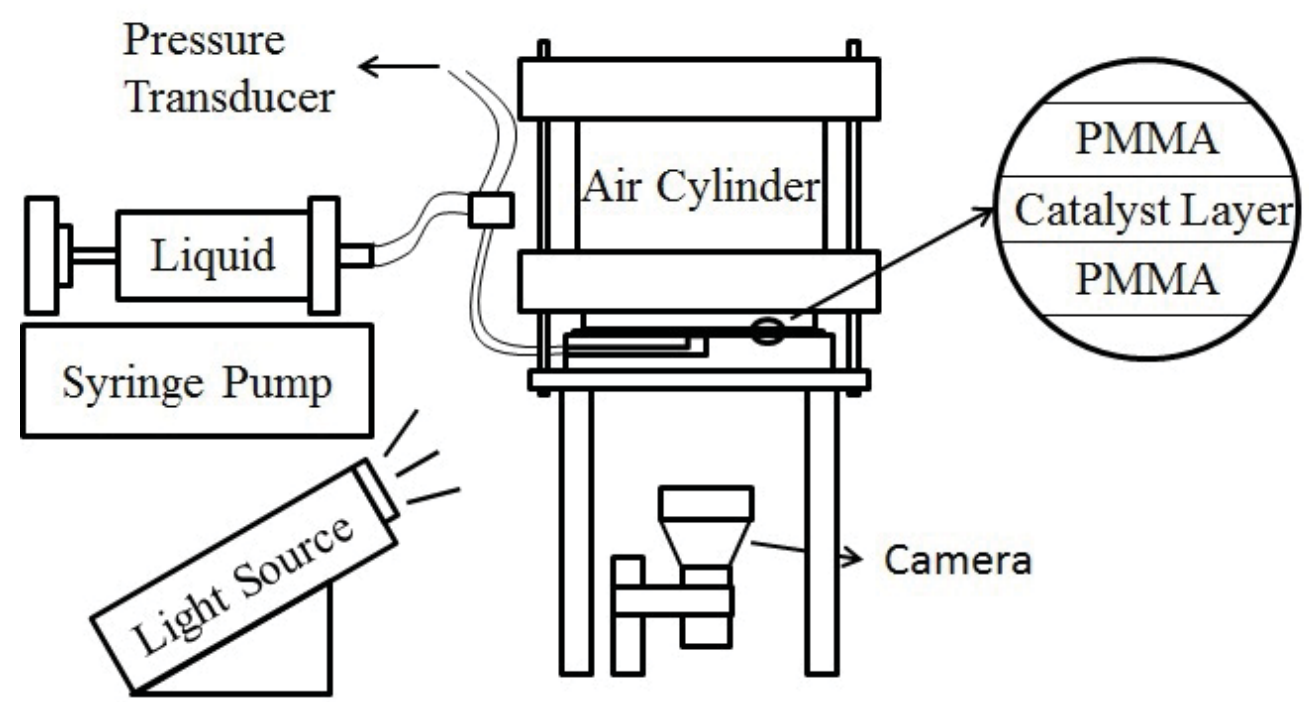

Figure 28: Schematic of final design of the Catalyst Layer Percolation Testing Experimental Set-up 
DE-EE0000466

Ballard Material Products Inc.
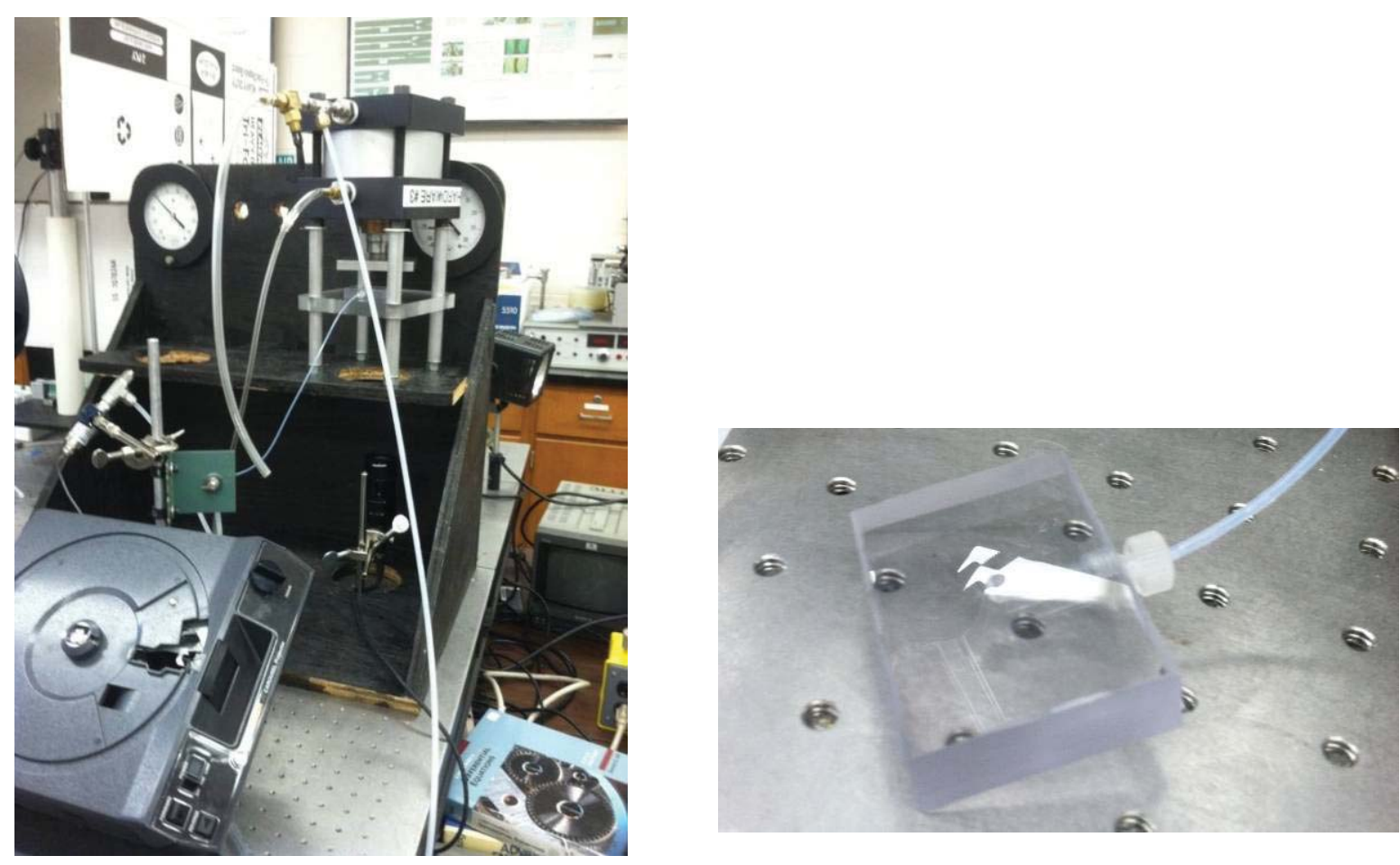

Figure 29: (A) Catalyst Layer experimental setup, (B) new Catalyst Layer Platen without PDMS coating

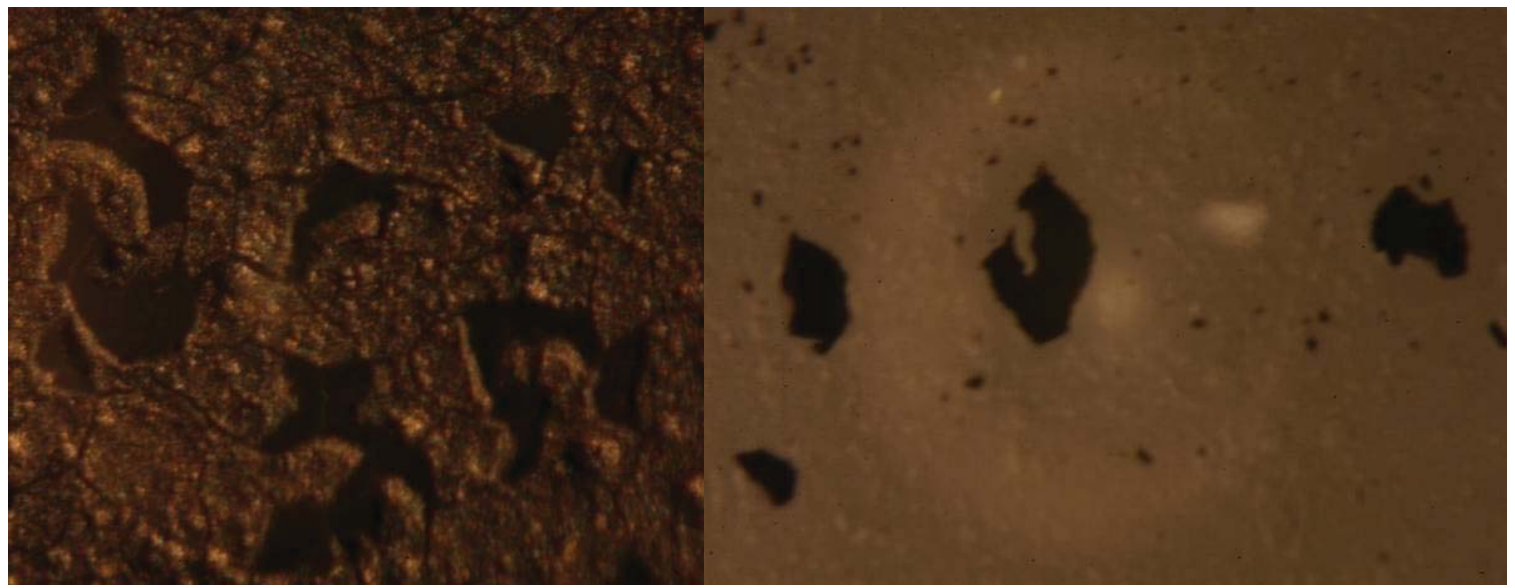

Figure 30: Degradation to the catalyst layer from the PDMS platens. On the left is the catalyst layer with pieces taken from it and on the right is the PDMS platen with the pieces of catalyst layer stuck to it 
Full CCM samples were tested at platen compression pressures of $41.37,68.95$ and $96.53 \mathrm{kPa}$. A Panasonic GP-KS125 CCD camera recorded images at 0.9, 1.8, 4.5, and 9 frames per second (fps). The test conditions are shown in Table 6. Pressure data was synchronized with video images of the percolation. Images were collected using an EPIX PIXCI frame grabber and XCAP software. The liquid was injected into the catalyst layer, through a polytetrafluoroethylene (PTFE) micro-tubing. The tubing, connected to the bottom platen has a flow passage for liquid injection. The fluid is injected into the center of the sample via a $2.5 \mathrm{ml}$ gas-tight Hamilton syringe using a pump (Harvard Apparatus model 944) at three different speeds. A pressure transducer, Omegadyne ${ }^{\circledR}$ model PX209-30V15G10V, measures the percolation pressure within the catalyst layer at intervals of $0.04,0.02$, and 0.01 seconds depending on the fluid injection rate.

Table 6: Catalyst Layer testing conditions

\begin{tabular}{|c|c|c|}
\hline $\mathbf{Q}(\mathbf{m L} / \mathbf{s})$ & Frame Rate (fps) & Data Acquisition (Hz) \\
\hline $9.483 \mathrm{e}-4$ & 0.9 & 25 \\
\hline $18.75 \mathrm{e}-4$ & 1.8 & 50 \\
\hline $37.08 \mathrm{e}-4$ & 4.5 & 100 \\
\hline $37.08 \mathrm{e}-4$ & 9 & 100 \\
\hline
\end{tabular}

The injection fluid was FC-3283, a Fluorinert ${ }^{\mathrm{TM}}$ from $3 \mathrm{M}$ which was chosen for percolation tests on catalyst layers. FC-3283 is a liquid with stable transport properties over time, is colorless, clear, thermally and chemically stable and is a fully-fluorinated fluid. FC-3283 was tested for membrane/ionomer compatibility and revealed no ionomer dissolution, nor any uptake of FC3283 that would cause swelling of the ionomer or in the Nafion membrane. FC-3283 is a wicking fluid to the catalyst layer, resulting in spontaneous imbibition. For the full CCM samples, the platens were modified into a square cross-section with dimensions of $3.8 \times 3.8 \mathrm{~cm}$ in accordance with the size of the supplied full CCMs.

\subsubsection{Capillary Pressure Results}

Table 7 and Table 8 list the full CCM samples that were tested along with their thickness and porosity.

Table 7: List of Non-Run Catalyst Layer samples. The porosity has an uncertainty of $+/-2 \%$, and the thickness $+/-0.5 \mu \mathrm{m}$.

\begin{tabular}{|c|c|c|c|}
\hline $\begin{array}{c}\text { CCL } \\
\text { Ionomer } \\
\text { Content }\end{array}$ & $\begin{array}{c}\text { Platinum } \\
\text { Loading }\end{array}$ & Porosity & Thickness \\
\hline $12 \%$ & 0.4 & $81 \%$ & $16.4 \mu \mathrm{m}$ \\
\hline $30 \%$ & 0.4 & $60 \%$ & $11.7 \mu \mathrm{m}$ \\
\hline $23 \%$ & 0.4 & $70 \%$ & $13.4 \mu \mathrm{m}$ \\
\hline $50 \%$ & 0.4 & $35 \%$ & $11.6 \mu \mathrm{m}$ \\
\hline $23 \%$ & 0.2 & $66 \%$ & $5.6 \mu \mathrm{m}$ \\
\hline $23 \%$ & 0.5 & $73 \%$ & $18.3 \mu \mathrm{m}$ \\
\hline
\end{tabular}


DE-EE0000466

Ballard Material Products Inc.

Table 8: List of Aged and Conditioned Catalyst Layer samples. The porosity has an uncertainty of $+/-2 \%$, and the thickness $+/-0.5 \mu \mathrm{m}$

\begin{tabular}{c|c|c|}
\hline $\begin{array}{c}\text { Reference MEA } \\
\text { Aged or Conditioned }\end{array}$ & Porosity & Thickness \\
\hline Conditioned & $68 \%$ & $12 \mu \mathrm{m}$ \\
\hline 1.0 V UPL & $67 \%$ & $11 \mu \mathrm{m}$ \\
\hline 1.3 V UPL & $39 \%$ & $3.3 \mu \mathrm{m}$ \\
\hline 1.4 V UPL & $36 \%$ & $3.1 \mu \mathrm{m}$ \\
\hline
\end{tabular}

Figure 31 shows the variation in percolation pressure over time for different compression pressures and flow rates for the 50\% ionomer loaded catalyst layer sample, while Figure 32 shows the same percolation pressure results plotted against the wetted volume for the sample. For the tested compression pressures, no effect on percolation pressures was observed, only the flow rates had an effect on the percolation pressure. Table 9 and

Figure 33 show test results for the same CCM sample. The BOT and EOT labels here denote the beginning and end of the tests, respectively. The slope is taken as the pressure difference over the time difference from the BOT to the EOT.

Comparison of the percolation pressures of a catalyst layer that was degraded at an upper potential limit of $1.0 \mathrm{~V}$ was found to be similar to a conditioned catalyst layer while the corroded sample that was degraded at $1.4 \mathrm{~V}$ UPL revealed a substantially lower $\Delta \mathrm{P} / \Delta \mathrm{t}$ due to a $\sim 50 \%$ porosity change and reduction in micro-pore density (Figure 34). This change is also reflected in Figure 35 which presents the percolation pressure calculated from the wetted area as a function of saturation of catalyst layer. 


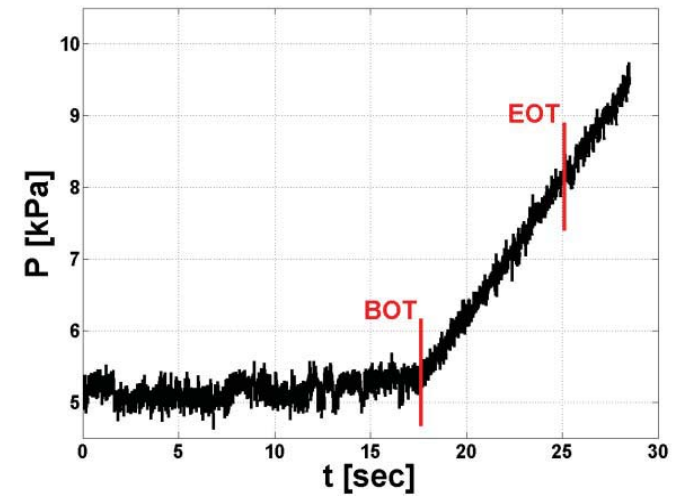

$1^{\text {st }}$ test at $41.37 \mathrm{kPa}$

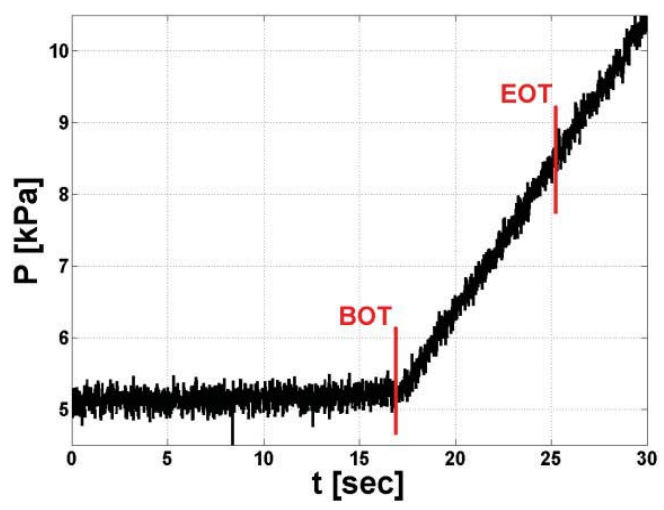

$1^{\text {st }}$ test at $68.95 \mathrm{kPa}$

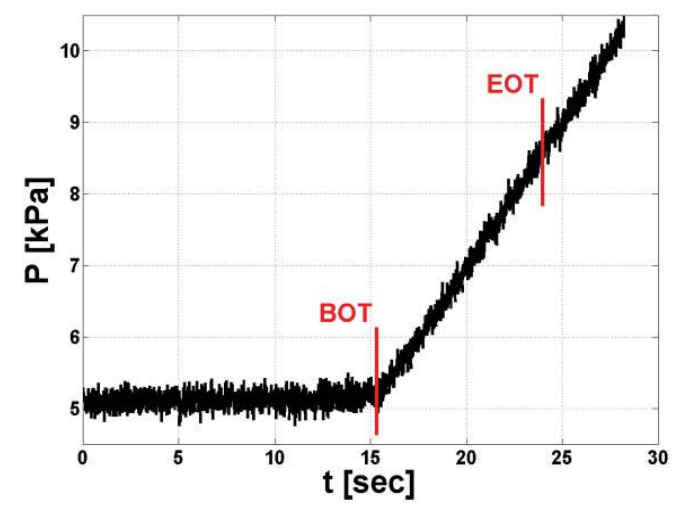

$1^{\text {st }}$ test at $96.53 \mathrm{kPa}$

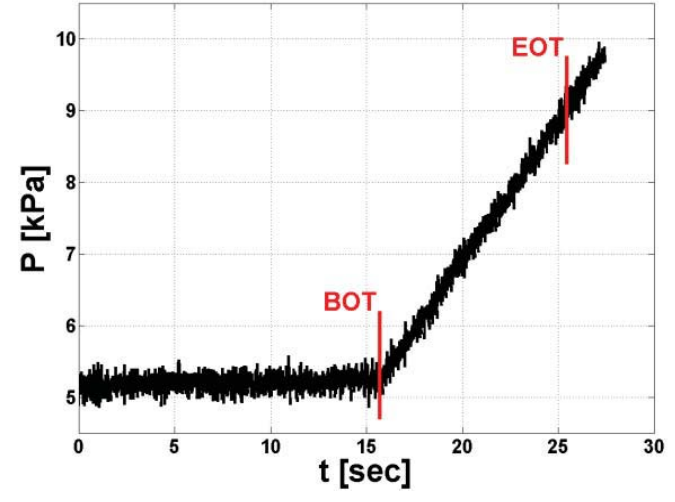

$2^{\text {nd }}$ test at $41.37 \mathrm{kPa}$

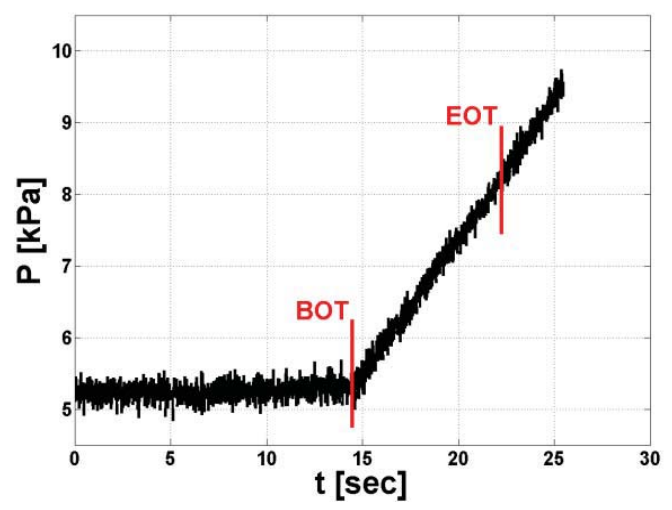

$2^{\text {nd }}$ test at $68.95 \mathrm{kPa}$

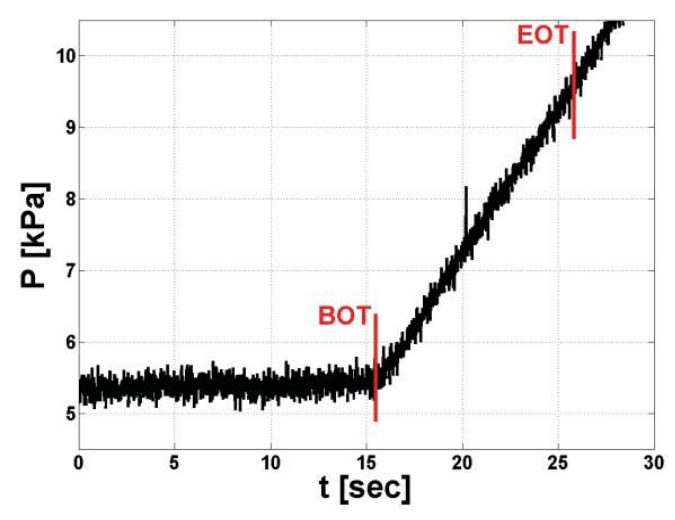

$2^{\text {nd }}$ test at $96.53 \mathrm{kPa}$

Figure 31: Percolation Pressure Plots of the $50 \%$ ionomer sample against for a flow rate of $37.08 \mathrm{e}^{-4}$ at three different compressions of $41.37,68.95$, and $96.53 \mathrm{kPa}$ 


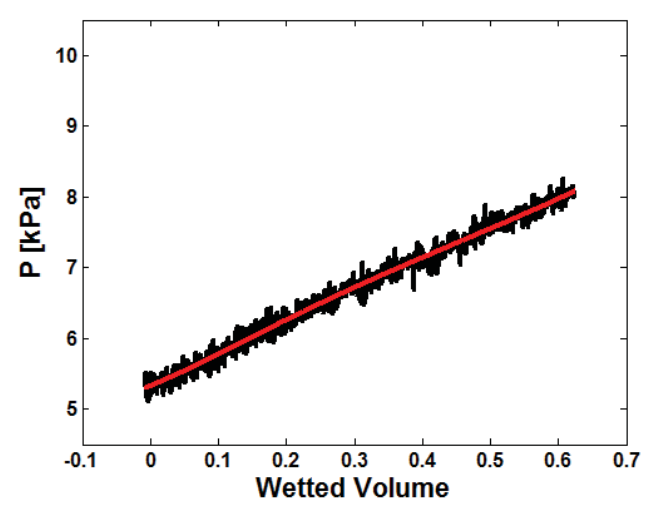

$1^{\text {st }}$ test at $41.37 \mathrm{kPa}$

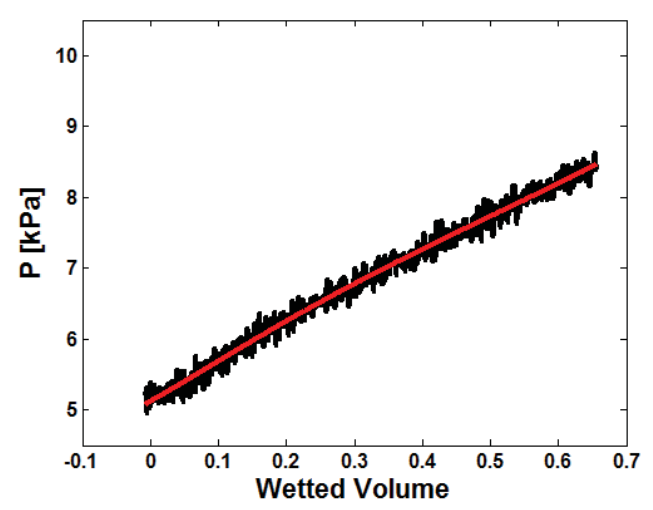

$1^{\text {st }}$ test at $68.95 \mathrm{kPa}$

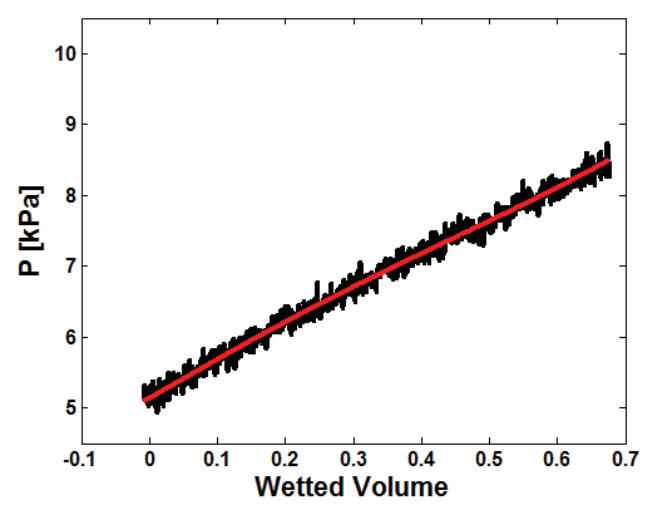

$1^{\text {st }}$ test at $96.53 \mathrm{kPa}$

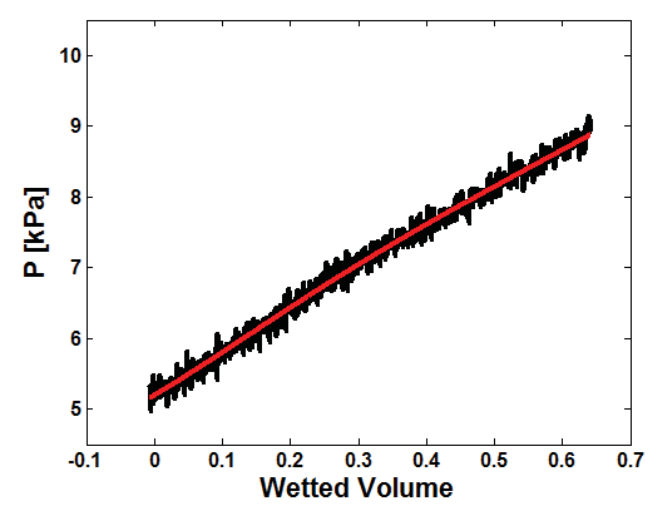

$2^{\text {nd }}$ test at $41.37 \mathrm{kPa}$

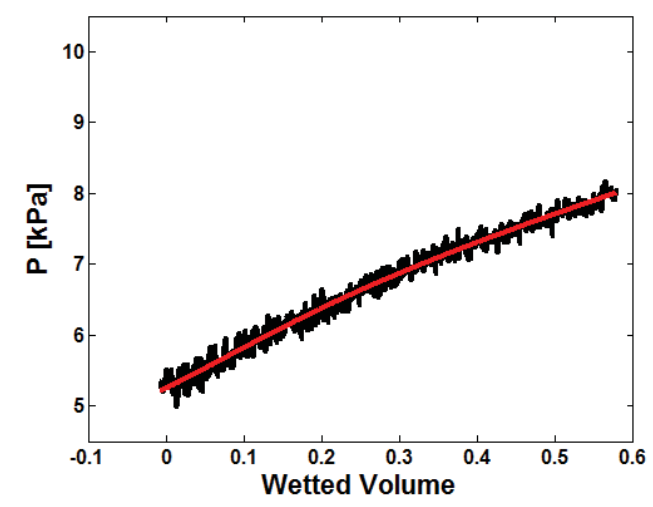

$2^{\text {nd }}$ test at $68.95 \mathrm{kPa}$

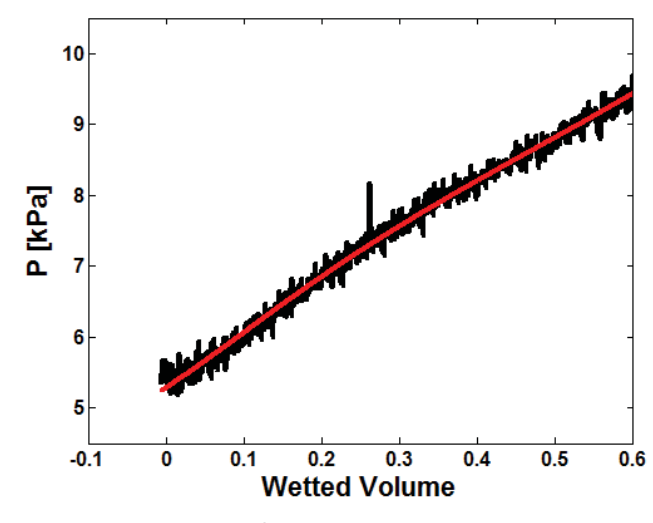

$2^{\text {nd }}$ test at $96.53 \mathrm{kPa}$

Figure 32: Percolation Pressure of the $50 \%$ ionomer sample against the Wetted Volume for a flow rate of $37.08 \mathrm{e}^{-4}$ at three different compressions of $41.37,68.95$, and $96.53 \mathrm{kPa}$ 
DE-EE0000466

Ballard Material Products Inc.

Table 9: Summary of a full CCM sample (50\% ionomer loading) Percolation Pressure testing results. The BOT and EOT labelled here are to denote the beginning of the test and end of the test. The slope is taken as the pressure difference over the time difference from BOT to EOT

\begin{tabular}{|c|c|c|c|c|c|c|c|c|}
\hline $\begin{array}{l}\text { Pump } \\
\text { Speed }\end{array}$ & $\begin{array}{l}\text { Compression } \\
\quad(\mathrm{kPa})\end{array}$ & BOT (s) & $\begin{array}{l}\text { BOT } \\
\text { (kPa) }\end{array}$ & EOT (s) & $\begin{array}{l}\text { EOT } \\
(\mathrm{kPa})\end{array}$ & $\begin{array}{c}\text { Time Diff } \\
\text { (s) }\end{array}$ & $\begin{array}{c}\text { Press } \\
\text { Diff } \\
(\mathrm{kPa})\end{array}$ & Slope \\
\hline \multirow{6}{*}{$37.08 \mathrm{e}-4$} & \multirow{2}{*}{$41.73 \mathrm{kPa}$} & 17.65 & 5.167 & 25.13 & 8.1519 & 7.48 & 2.9849 & 0.3990 \\
\hline & & 15.7 & 5.1975 & 25.46 & 9.0103 & 9.76 & 3.8128 & 0.3906 \\
\hline & \multirow{2}{*}{$68.95 \mathrm{kPa}$} & 16.91 & 5.1497 & 25.24 & 8.4824 & 8.33 & 3.3327 & 0.4000 \\
\hline & & 14.49 & 5.2495 & 22.26 & 8.2036 & 7.77 & 2.9541 & 0.3801 \\
\hline & \multirow{2}{*}{$96.53 \mathrm{kPa}$} & 15.34 & 5.1278 & 23.97 & 8.5852 & 8.63 & 3.4574 & 0.4006 \\
\hline & & 15.48 & 5.3892 & 25.82 & 9.5925 & 10.34 & 4.2033 & 0.4065 \\
\hline \multirow{6}{*}{$18.75 e-4$} & \multirow{2}{*}{$41.73 \mathrm{kPa}$} & 35.02 & 5.1906 & 46.8 & 7.4215 & 11.78 & 2.2309 & 0.189 \\
\hline & & 32.46 & 5.0553 & 44.36 & 7.0724 & 11.9 & 2.0171 & 0.1695 \\
\hline & \multirow{2}{*}{$68.95 \mathrm{kPa}$} & 34.52 & 5.0281 & 47.64 & 7.6036 & 13.12 & 2.5755 & 0.1963 \\
\hline & & 24.64 & 5.0831 & 36.52 & 7.375 & 11.88 & 2.2919 & 0.1929 \\
\hline & \multirow{2}{*}{$96.53 \mathrm{kPa}$} & 33.58 & 5.0196 & 42.08 & 6.7205 & 8.5 & 1.7009 & 0.2001 \\
\hline & & 30.06 & 5.0961 & 39.98 & 6.8096 & 9.92 & 1.7135 & 0.1727 \\
\hline \multirow{6}{*}{$9.483 e-4$} & \multirow{2}{*}{$41.73 \mathrm{kPa}$} & 73.7109 & 4.9805 & 92.1724 & 6.6612 & 18.4615 & 1.6807 & 0.0910 \\
\hline & & 70.9929 & 5.0312 & 86.87 & 6.346 & 15.8771 & 1.3148 & 0.0828 \\
\hline & \multirow{2}{*}{$68.95 \mathrm{kPa}$} & 71.7244 & 5.0425 & 88.8673 & 6.5785 & 17.1429 & 1.536 & 0.0896 \\
\hline & & 37.8614 & 5.0252 & 53.6855 & 6.3771 & 15.8241 & 1.3519 & 0.0854 \\
\hline & \multirow{2}{*}{$96.53 \mathrm{kPa}$} & 71.2595 & 4.9388 & 84.4675 & 6.0986 & 13.208 & 1.1598 & 0.0878 \\
\hline & & 71.8935 & 5.0185 & 88.9265 & 6.4962 & 17.033 & 1.4777 & 0.0867 \\
\hline
\end{tabular}

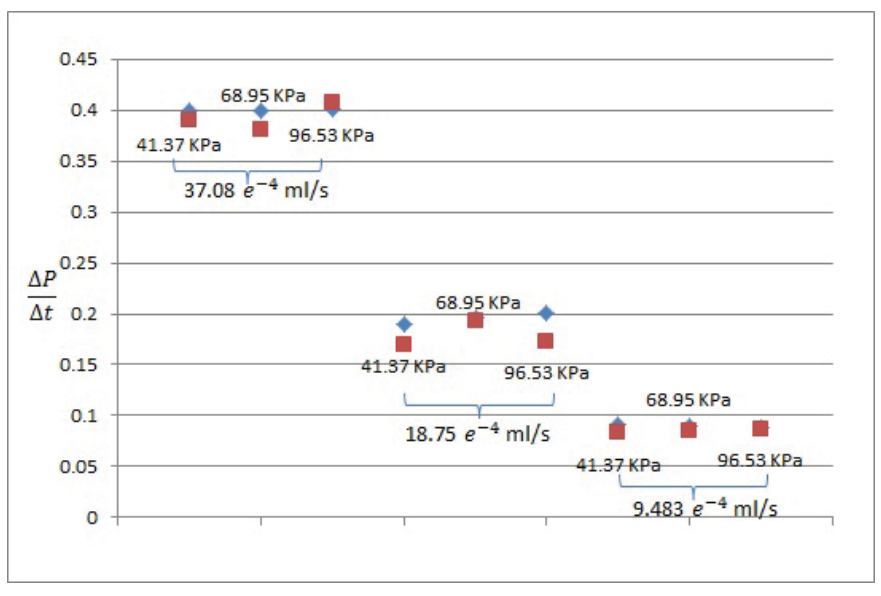

Figure 33: Summary of the Percolation Pressure testing done on the $50 \%$ ionomer catalyst layer. The plot is showing the difference in Percolation Pressure from BOT to EOT over the testing time from BOT to EOT. The points on the plot represent the test at the compression pressure and flow rate 
DE-EE0000466

Ballard Material Products Inc.
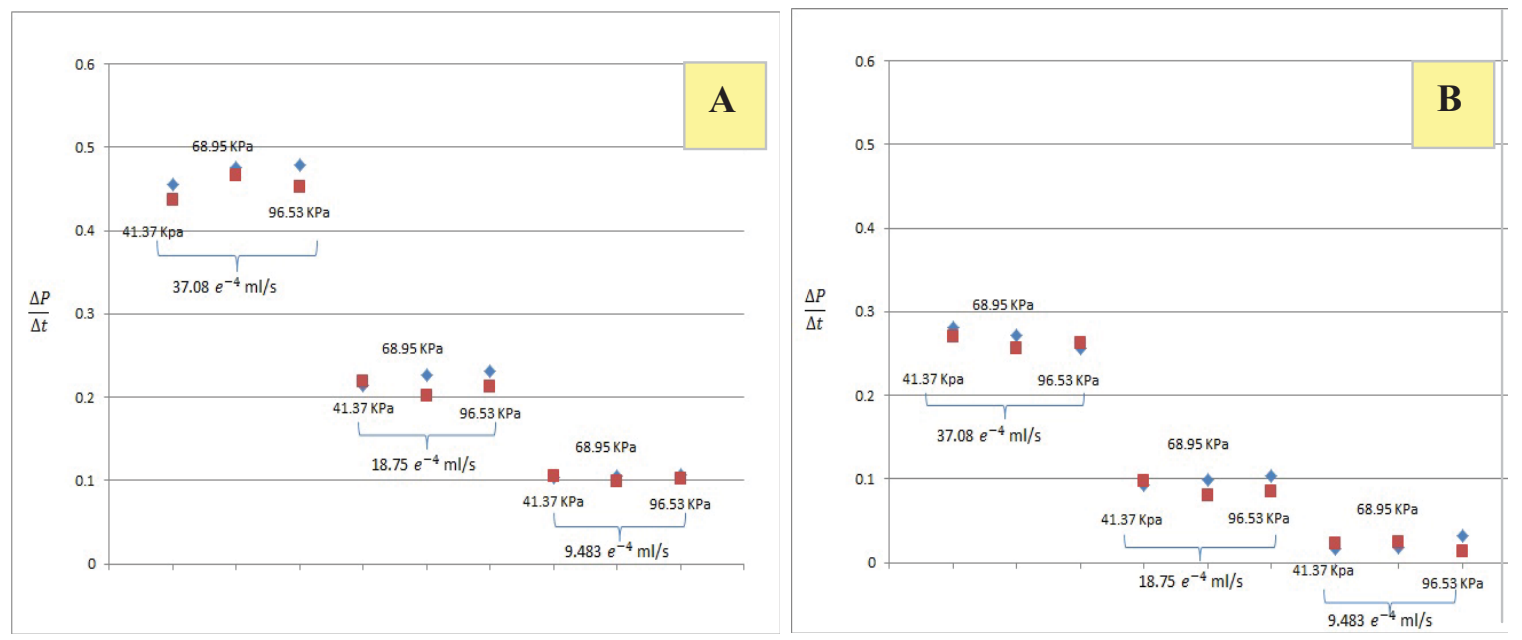

Figure 34: Percolation pressure change of MEAs that were degraded at an upper potential limit of (A) 1.0V and (B) $1.4 \mathrm{~V}$. The samples were tested under compression of $41.37 \mathrm{kPa}$ using a flow rate of $37.08 \mathrm{e}^{-4} \mathrm{~mL} / \mathrm{s}$
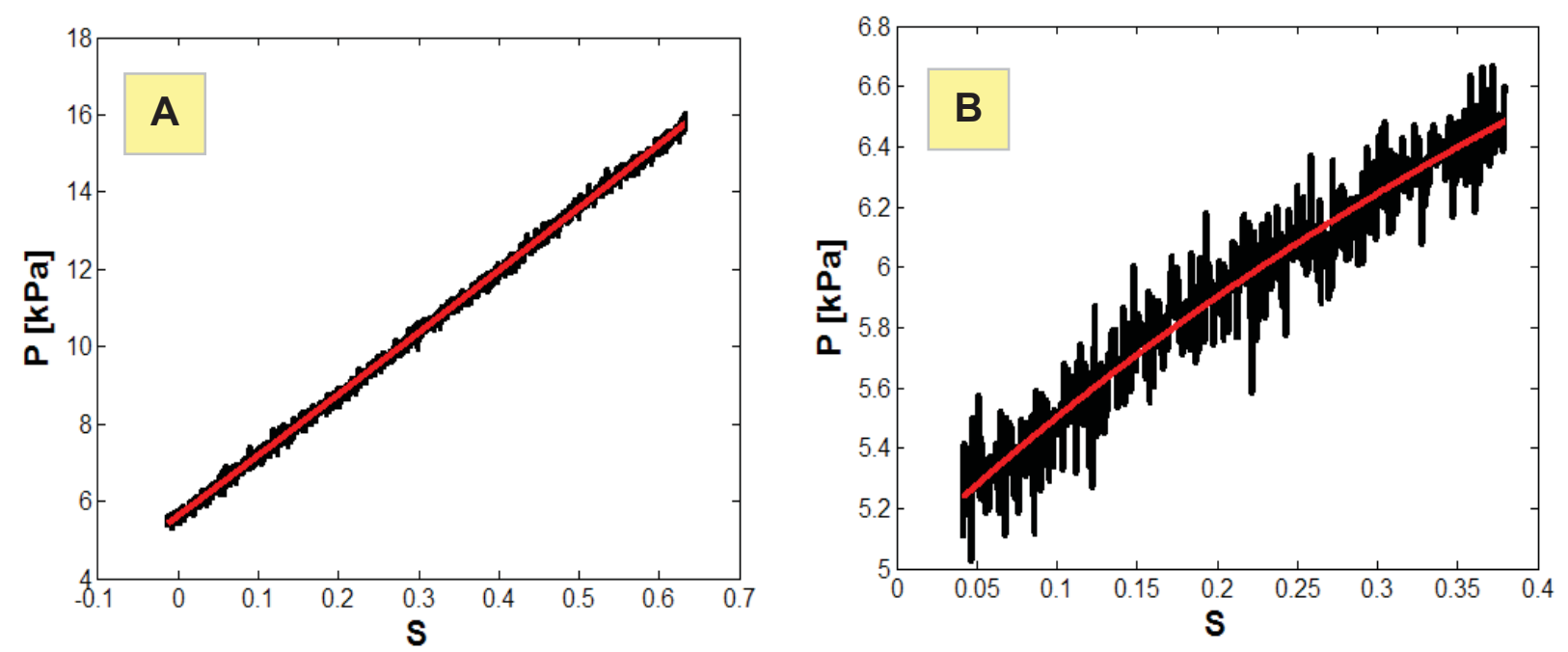

Figure 35: Pressure vs. water saturation for catalyst layers that were subjected to accelerated testing at an upper potential limit of $(A) 1.0 \mathrm{~V}$ and (B) $1.4 \mathrm{~V}$. The samples were tested under compression of $41.37 \mathrm{kPa}$ using a flow rate of $37.08 \mathrm{e}^{-4} \mathrm{~mL} / \mathrm{s}$ 
DE-EE0000466

Ballard Material Products Inc.

\subsubsection{Summary}

Chemical compatibility tests with the test fluid indicate that the FC-3283 does not react with nor absorb into the ionomer. Additionally, hydraulic permeability tests of this fluid with Nafion-211 show that Nafion is impermeable to FC-3283 at room temperature, however, some permeation at elevated temperatures are apparent.

The CCM samples have shown excellent repeatability while virgin CCMs samples delaminated from MEAs showed more variability. Furthermore, the aged sample at 1.4V UPL showed a very unique spreading pattern which was more oval like. The sample itself when investigated under a stereo microscope had parallel ridges running down the entire sample due to indentation by the flow field landings which may explain the imbibition behaviour. 
DE-EE0000466

Ballard Material Products Inc.

\subsection{GDL Capillary Pressure (MTU)}

\subsubsection{Experimental Set-up}

The experimental set-up for GDL capillary pressure characterization is shown in Figure 36. As discussed in the section above, the set-up consists of two platens, each made of Polymethylmethacrylate (PMMA) coated with Polydimethylsiloxane (PDMS). One platen has micro-tubing in the center which allows the injection of the test liquid. The platens are square. The side of the bottom platen is $10 \mathrm{~cm}$ (4 inches) in length, while the sides of the top platen are $4.7 \mathrm{~cm}$ (1.875 inches) long. Circular GDLs with a diameter of $5.4 \mathrm{~cm}$ (2.125 inches) were used in the experiments. The GDLs were made bigger than the top platen to allow the air to escape while the test fluid is injected. The platens were compressed to a pressure of $27.58 \mathrm{kPa}$ during tests. A Panasonic GP-KS125 CCD camera was used to record images at 0.033, 0.166, 1.8, and 4.5 frames per second (fps). Pressure data was synchronized with video images of the percolation. The images were collected using EPIX PIXCl frame grabber and XCAP software.

The liquid is injected into the catalyst layer, through polytetrafluoroethylene (PTFE) microtubing. The tubing is connected to the bottom platen that has a flow passage for liquid injection. The fluid is injected at the center of the sample. The fluid chosen for this study was distilled water. A syringe pump, Harvard Apparatus model 944, was used at four different speeds with a $3 \mathrm{ml}$ and $10 \mathrm{~mL}$ gas-tight Hamilton syringe. A pressure transducer, Omegadyne ${ }^{\circledR}$ model PX209030G10V, measures the percolation pressure at intervals of $1,0.1,0.02$, and 0.01 seconds depending on the fluid injection rate.

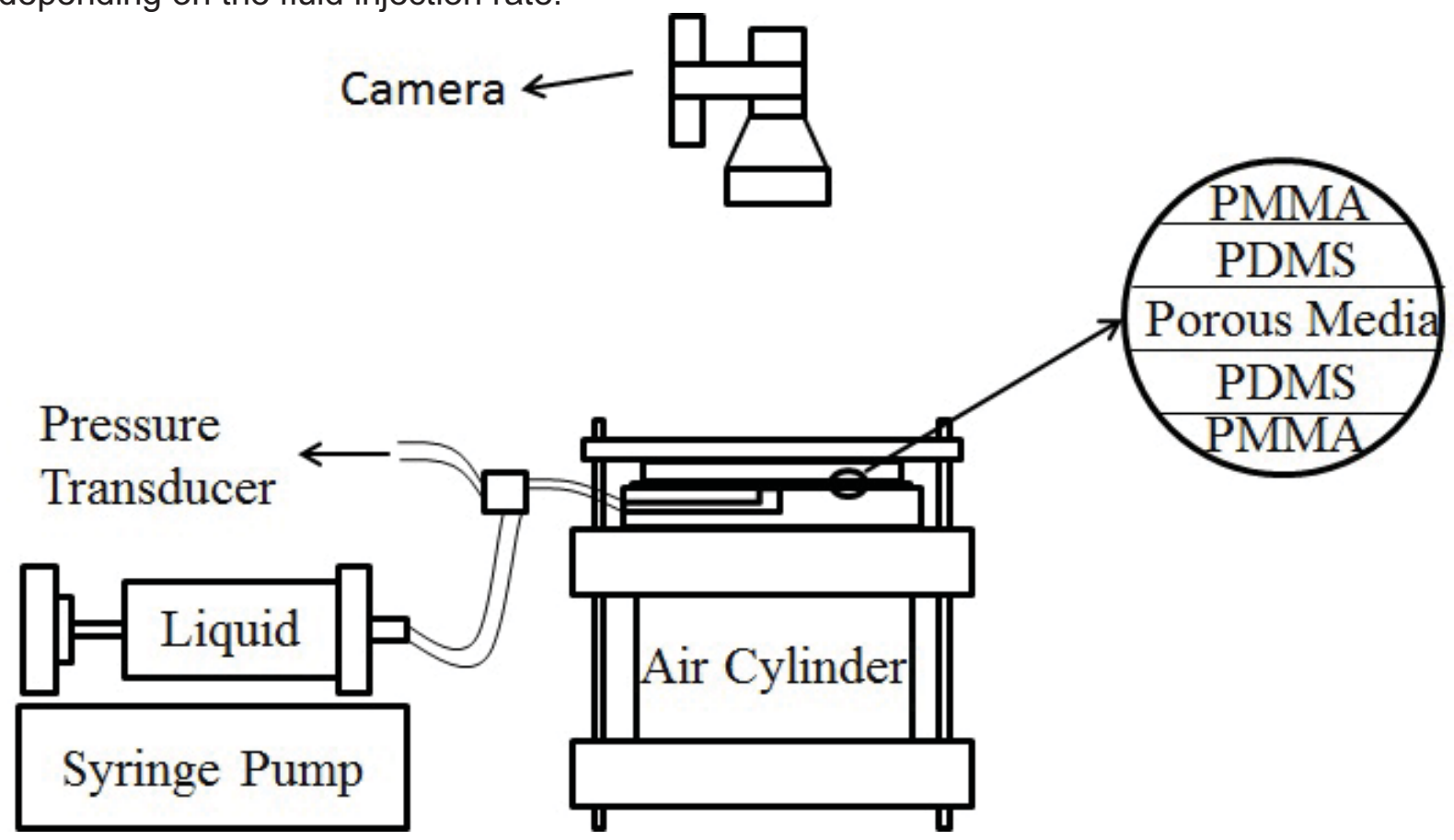

Figure 36: GDL experimental set-up diagram 
DE-EE0000466

Ballard Material Products Inc.

\subsubsection{Capillary Pressure Results}

The list of samples that were tested is shown in Table 10. Table 11 shows the flow rates and the corresponding capillary number obtained for a given setting of the syringe pump and syringe size. Syringes of two sizes were used during the testing of the PTL samples. Slightly different flow rates were obtained with the larger syringe. This change in the syringe did not affect the results as the calculated capillary numbers were of the same order.

Table 10: List of samples that have completed all testing

\begin{tabular}{|c|c|}
\hline \multicolumn{2}{|c|}{ Completed Samples } \\
\hline $\begin{array}{l}\text { - P-GDL-NR-1 }{ }^{\text {st }} \text { Batch } \\
\text { - P-Sub-NR-1 } 1^{\text {st }} \text { Batch } \\
\text { - EP-GDL-NR-1 } 1^{\text {st }} \text { Batch } \\
\text { - EP-Sub-NR-1 }{ }^{\text {st }} \text { Batch } \\
\text { - Toray-GDL-NR- } 1^{\text {st }} \text { Batch } \\
\text { - Toray-Sub-NR-1 }{ }^{\text {st }} \text { Batch } \\
\text { - P-GDL-NR-2 }{ }^{\text {nd }} \text { Batch } \\
\text { - EP-GDL-NR-2 }{ }^{\text {nd }} \text { Batch } \\
\text { - P-GDL B2-MD-NR-2 }{ }^{\text {nd }} \text { Batch } \\
\text { - P-Sub Old-A2-NR-2 }{ }^{\text {nd }} \text { Batch } \\
\text { - EP-GDL MD-AL-NR-2 }{ }^{\text {nd }} \text { Batch } \\
\text { - EP-Sub MD-B2-NR-2 }{ }^{\text {nd }} \text { Batch }\end{array}$ & $\begin{array}{l}\text { - VD360-P-NR } \\
\text { - VD360-EP-NR } \\
\text { - VD252-P-Conditioned } \\
\text { - VD252-EP-Conditioned } \\
\text { - VD302-P-Conditioned } \\
\text { - VD253-P-Aged } \\
\text { - VD253-P-Aged } \\
\text { - VD328-P-Aged } \\
\text { - VD328-EP-Aged } \\
\text { - VD275-EP-Aged }\end{array}$ \\
\hline
\end{tabular}

Table 11: Relationship between capillary numbers and flow rate

\begin{tabular}{|c|c|c|c|c|}
\hline Setting No. & Syringe Size & Pump Setting & Flow Rate & Capillary No. \\
\hline 1 & \multirow{4}{*}{$3 \mathrm{~mL}$} & Ultralow at 15 & $6.017 \times 10^{-6}$ & $3.1087 \times 10^{-8}$ \\
\hline 2 & & Ultralow at 11 & $2.167 \times 10^{-5}$ & $1.1195 \times 10^{-7}$ \\
\hline 3 & & Ultralow at 3 & $3.217 \times 10^{-4}$ & $1.662 \times 10^{-6}$ \\
\hline 4 & & Normal at 6 & $4.9 \times 10^{-3}$ & $2.5317 \times 10^{-5}$ \\
\hline 5 & \multirow{5}{*}{$10 \mathrm{~mL}$} & Ultralow at 18 & $7.296 \times 10^{-6}$ & $3.5564 \times 10^{-8}$ \\
\hline 6 & & Ultralow at 14 & $2.894 \times 10^{-5}$ & $1.3692 \times 10^{-7}$ \\
\hline 7 & & Ultralow at 6 & $1.067 \times 10^{-3}$ & $2.015 \times 10^{-6}$ \\
\hline 8 & & Normal at 7 & $5.15 \times 10^{-3}$ & $2.6609 \times 10^{-5}$ \\
\hline 9 & & Normal at 4 & $7.497 \times 10^{-3}$ & $3.8737 \times 10^{-5}$ \\
\hline
\end{tabular}


Table 12 shows the peak (or plateau) percolation pressure for each sample at a given capillary number. The values for pressure shown are in units of $\mathrm{kPa}$. Four different injection flow rates were used for each sample as shown by four different capillary numbers. The highest capillary number (far right column) resulted in a stable displacement (SD) flow regime in which the percolation pressure does not plateau. The remaining three capillary numbers are not consistent in magnitude for two samples due to a change in the test setup during the course of this project.

Table 12: Percolation pressure values in the samples listed, measured in $\mathrm{kPa}$, according to the different flow rates being tested against

\begin{tabular}{|c|c|c|c|c|}
\hline \multirow[b]{2}{*}{ Sample } & \multicolumn{4}{|c|}{ Capillary Number } \\
\hline & $3.1087 \mathrm{e}-8$ & $1.1195 \mathrm{e}-7$ & $1.662 \mathrm{e}-6$ & $\begin{array}{c}2.5317 \mathrm{e}- \\
5 \\
\end{array}$ \\
\hline P-GDL-NR-1 ${ }^{\text {st }}$ Batch & 28 & 43.85 & 73.5 & SD \\
\hline VD253-P-Aged & $18.4-40$ & $23-40$ & 65.75 & SD \\
\hline \multirow[b]{2}{*}{ Sample } & \multicolumn{3}{|c|}{ Capillary Number } & \\
\hline & $3.7696 e-8$ & $1.4951 \mathrm{e}-7$ & $5.5121 \mathrm{e}-6$ & $\begin{array}{c}2.6609- \\
5\end{array}$ \\
\hline EP-GDL-NR-1 ${ }^{\text {st }}$ Batch & 10.5 & 11 & $15.3-22.5$ & SD \\
\hline VD360-P-NR & $20-22.5$ & $23.5-25$ & $48-57$ & SD \\
\hline VD360-EP-NR & 12.75 & 13.3 & 16 & SD \\
\hline VD252-P-Conditioned & $17.5-21$ & $\begin{array}{l}20.5- \\
23.8\end{array}$ & $43.6-46$ & SD \\
\hline VD252-EP-Conditioned & 10 & 11.5 & $14.8-17.4$ & SD \\
\hline VD253-EP-Aged & $10-11.5$ & 11.5 & 14 & SD \\
\hline \multirow[b]{2}{*}{ Sample } & \multicolumn{3}{|c|}{ Capillary Number } & \\
\hline & $3.7696 \mathrm{e}-8$ & $1.4951 \mathrm{e}-7$ & $5.5121 \mathrm{e}-6$ & $\begin{array}{c}3.8737- \\
5\end{array}$ \\
\hline P-Sub-NR-1 ${ }^{\text {st }}$ Batch & $\begin{array}{c}14.4- \\
19.2\end{array}$ & $15.4-19$ & 30 & SD \\
\hline P-GDL B2-MD-NR-2 ${ }^{\text {nd }}$ Batch & 22 & 21 & $35-45$ & SD \\
\hline P-Sub Old-A2-NR-2 ${ }^{\text {nd }}$ Batch & 19 & $\begin{array}{l}21.2- \\
24.5\end{array}$ & $37.5-45$ & SD \\
\hline EP-GDL MD-AL-NR-2 ${ }^{\text {nd }}$ Batch & 11.25 & 11.5 & 15 & SD \\
\hline EP-Sub MD-B2-NR-2 ${ }^{\text {nd }}$ Batch & 11 & 12 & 16.4 & SD \\
\hline EP-Sub-NR-1 ${ }^{\text {st }}$ Batch & 10.5 & 10.5 & 11.5 & SD \\
\hline Toray-GDL-NR-1 ${ }^{\text {st }}$ Batch & 14.3 & 16.5 & 26.75 & SD \\
\hline Toray-Sub-NR-1 ${ }^{\text {st }}$ Batch & 5.3 & 5.8 & 5.7 & SD \\
\hline VD328-EP-Aged & 13.9 & 16 & 20.5 & SD \\
\hline VD275-EP-Aged & 12.2 & 14 & 21 & SD \\
\hline P-GDL-NR-2 ${ }^{\text {nd }}$ Batch & 24 & 25.5 & $53-57$ & SD \\
\hline EP-GDL-NR-2 ${ }^{\text {nd }}$ Batch & 13 & 13.3 & 16.5 & SD \\
\hline VD302-P-Conditioned & 21.5 & 24.2 & 43 & SD \\
\hline VD328-P-Aged & 22.25 & 26.5 & 46.5 & SD \\
\hline
\end{tabular}


Stable displacement occurs with flows at high capillary numbers accompanied with high pressures. For flows with low capillary numbers, a drop in pressure is observed. With this drop in pressure, there is a plateau effect, wherein the pressure starts to stabilize and attain a constant value as shown in Figure 37. From the samples, the non-run BMP-P shows a very similar trend as do the BMP-EP. Overall the trend for the GDL samples shows the cathode to have a lower overall pressure than that of the anode.

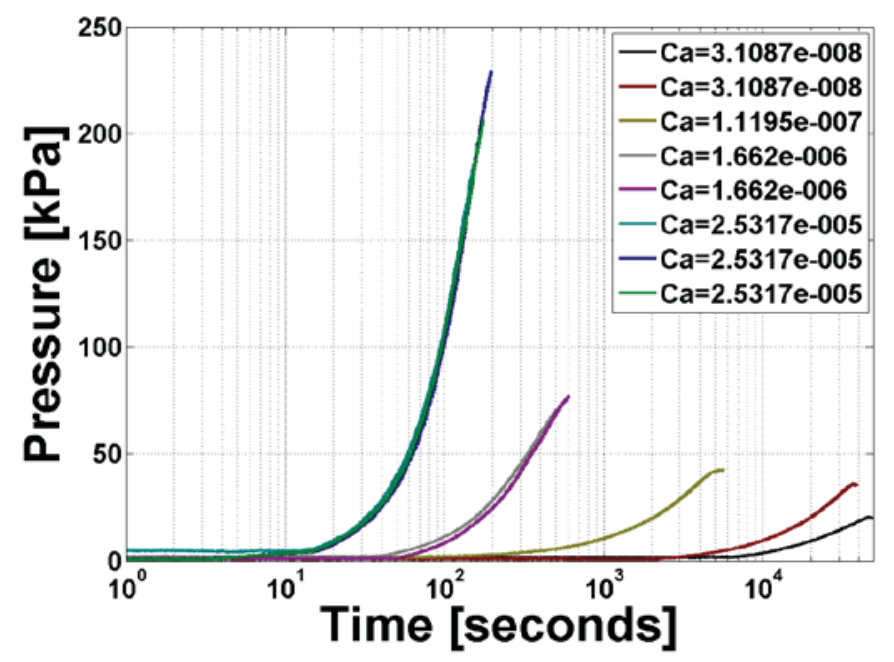

Figure 37: Showing the Percolation Pressures within the BMP-P-GDL

\subsubsection{Summary}

Percolation pressures for BMP-P GDL and BMP-P-Substrate show similar results and are higher than the BMP-EP results and Toray. The Toray and BMP EP GDL samples are very similar to one another and are noticeably different than the $\mathrm{P}$ sample. Overall, the anode samples for non-Run, conditioned, and aged GDLs show similar results to one another while also being higher than the cathode results. 


\subsection{GDL Wettability - Contact Angle Measurement Technique (MTU)}

Contact angle was measured by the method of Axisymmetrics Drop shape Analysis (ADSA). The Laplace-Young (Equation 1) defines the shapes of sessile and pendant drops depending upon the drop size represented by the radius of curvature at the apex $(b)$ and the fluid properties such as surface tension $(\sigma)$ and density $(\rho)$ and the two normal radii of curvatures $\left(R_{1}\right.$ and $R_{2}$ ). In ADSA a fit is made between the drop profile obtained from the images and the profile obtained by solving the Equation 1. Figure 38 shows a fit obtained between a drop image and the Laplacian curve.

$$
\left(\frac{1}{R_{1}}+\frac{1}{R_{2}}\right) \sigma=\frac{2 \sigma}{b}+\rho g z
$$

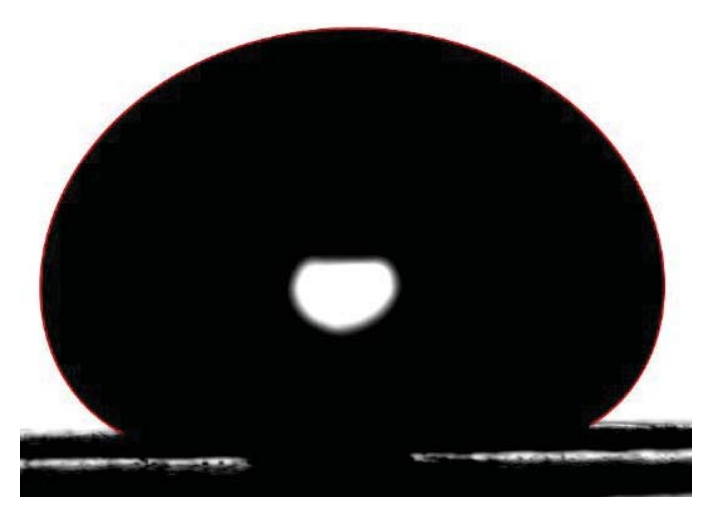

Figure 38: Fit between a sessile drop and the Laplacian curve

\subsubsection{Experimental Set-up}

Figure 39 shows the schematic of the experimental set-up for contact angle measurement. The set-up for contact angle measurement consists of Köhler illumination, acting as the source of light, a long distance microscope coupled to a CCD camera to capture the drop images and an enclosure in which the substrate is placed. Static contact angle is obtained by placing sessile drops through the opening in the enclosure using a hypodermic needle. Dynamic contact angles are obtained by injecting water on the substrate using a hypodermic needle (Figure 40) which is coupled to a syringe pump thereby enabling advancing and receding contact angle measurement. To prevent the effects of evaporation of the droplets on the contact angle, especially at higher temperatures, the heat enclosure is kept humidified. 


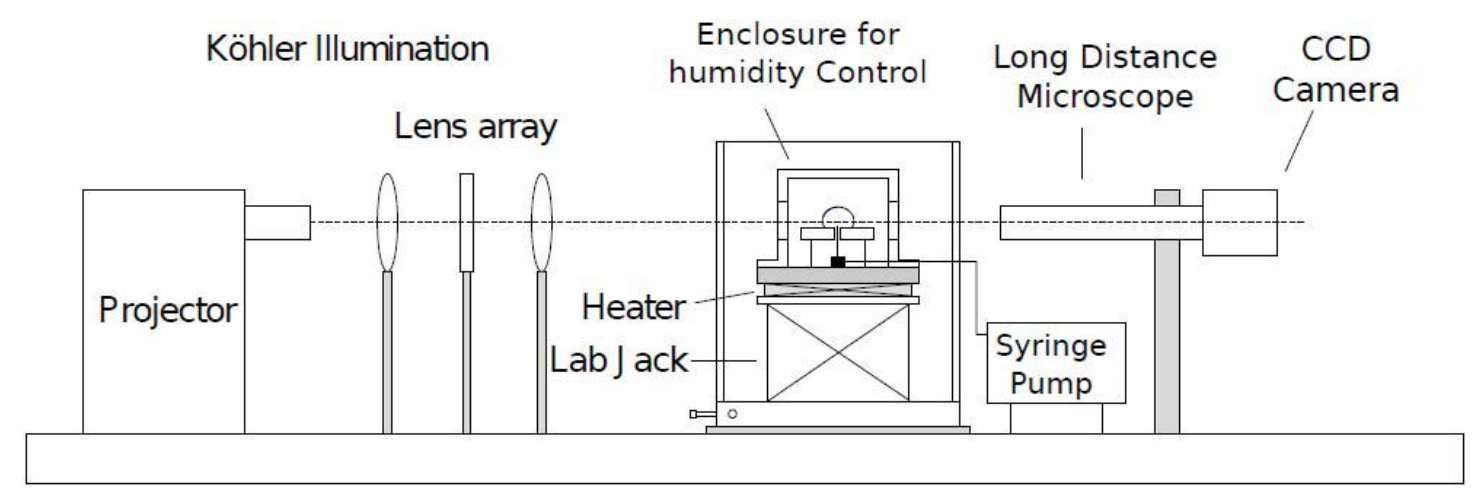

Figure 39: Schematic of the experimental set-up for contact angle measurement

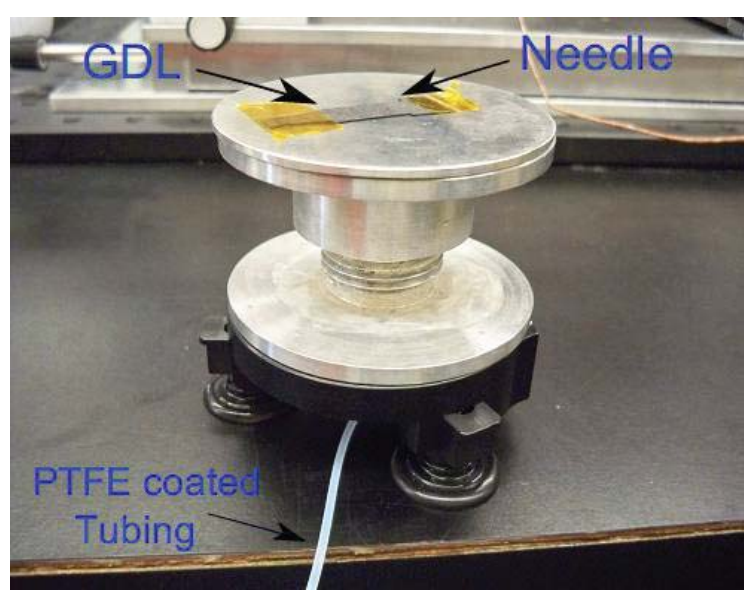

Figure 40: Droplet injection apparatus

\subsubsection{Results and Discussion}

Table 13 shows the summary of the static contact angle of the tested GDLs measured at room temperature. For the samples with an MPL, measurements were carried out on the substrate side. Measurements were also performed at $80^{\circ} \mathrm{C}$ on samples to test the effect of temperature on contact angle. Figure 41 is a graphical representation of the same data. The results reveal a minimal effect of temperature on the contact angle. The presence of a temperature effect, if any, is overshadowed by the vast hysteresis in contact angle that exists on the GDL surfaces. High hysteresis is observed on the GDL surface due to the nature of the surface. The porous structure along with the carbon fibres protruding from the surface causes the drop to pin to the surface. Very high contact angles (greater than $150^{\circ}$ ) were observed for all beginning of test samples (BOT, Figure 41a). 
DE-EE0000466

Ballard Material Products Inc.

Table 13: Summary of Static Contact Angle measurements at $20^{\circ} \mathrm{C}$

\begin{tabular}{|c|c|c|}
\hline Sr No & Sample & Contact Angle $\left({ }^{\circ}\right)$ \\
\hline 1 & P-SUB & $152 \pm 2$ \\
\hline 2 & P-GDL & $151 \pm 3$ \\
\hline 3 & EP-SUB & $156 \pm 2$ \\
\hline 4 & EP-GDL & $155 \pm 3$ \\
\hline 5 & TORAY-SUB & $151 \pm 2$ \\
\hline 6 & TORAY-GDL & $157 \pm 2$ \\
\hline 7 & End of Life - Cathode, s/n: 1444504 & $138 \pm 3$ \\
\hline 8 & End of Life - Anode, s/n: 1444504 & $139 \pm 4$ \\
\hline 9 & End of Life - Cathode, s/n: 1444917 & $153 \pm 4$ \\
\hline 10 & End of Life - Anode, s/n: 1444917 & $153 \pm 3$ \\
\hline 11 & End of Life - Cathode, s/n: 1444514 & $154 \pm 4$ \\
\hline 12 & End of Life - Anode, s/n: 1444514 & $151 \pm 7$ \\
\hline 13 & End of Life - Cathode, s/n: 1444600 & $148 \pm 5$ \\
\hline 14 & End of Life - Anode, s/n: 1444600 & $156 \pm 3$ \\
\hline 15 & Conditioned - Cathode, s/n 1444956 & $151 \pm 5$ \\
\hline 16 & Conditioned - Anode, s/n 1444956 & $148 \pm 6$ \\
\hline 17 & Non-Run - Cathode, s/n 1553371 & $156 \pm 4$ \\
\hline 18 & Non-Run - Anode, s/n 1553371 & $157 \pm 3$ \\
\hline
\end{tabular}


DE-EE0000466

Ballard Material Products Inc.

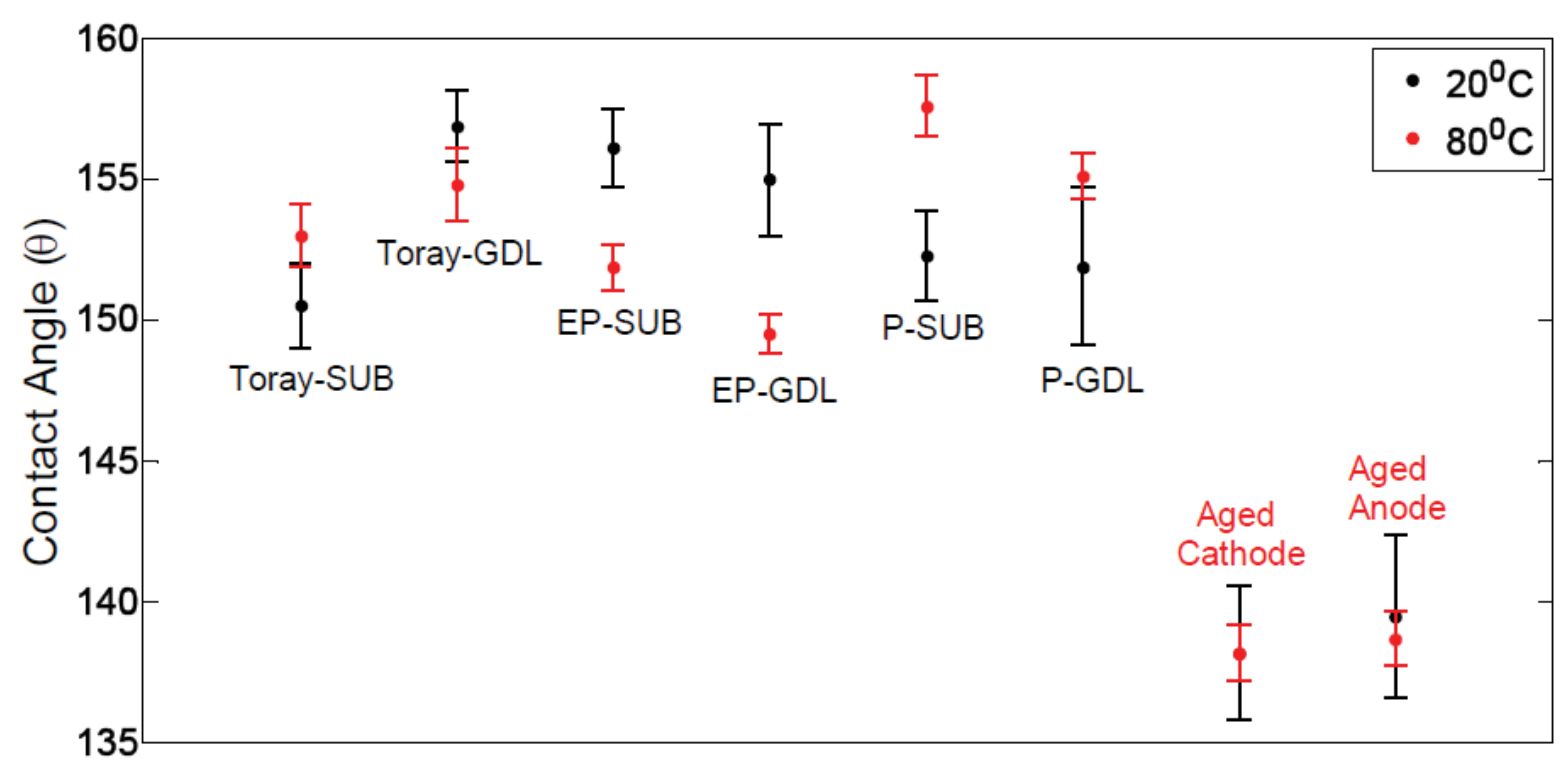

(a) Aged - s/n 1444504

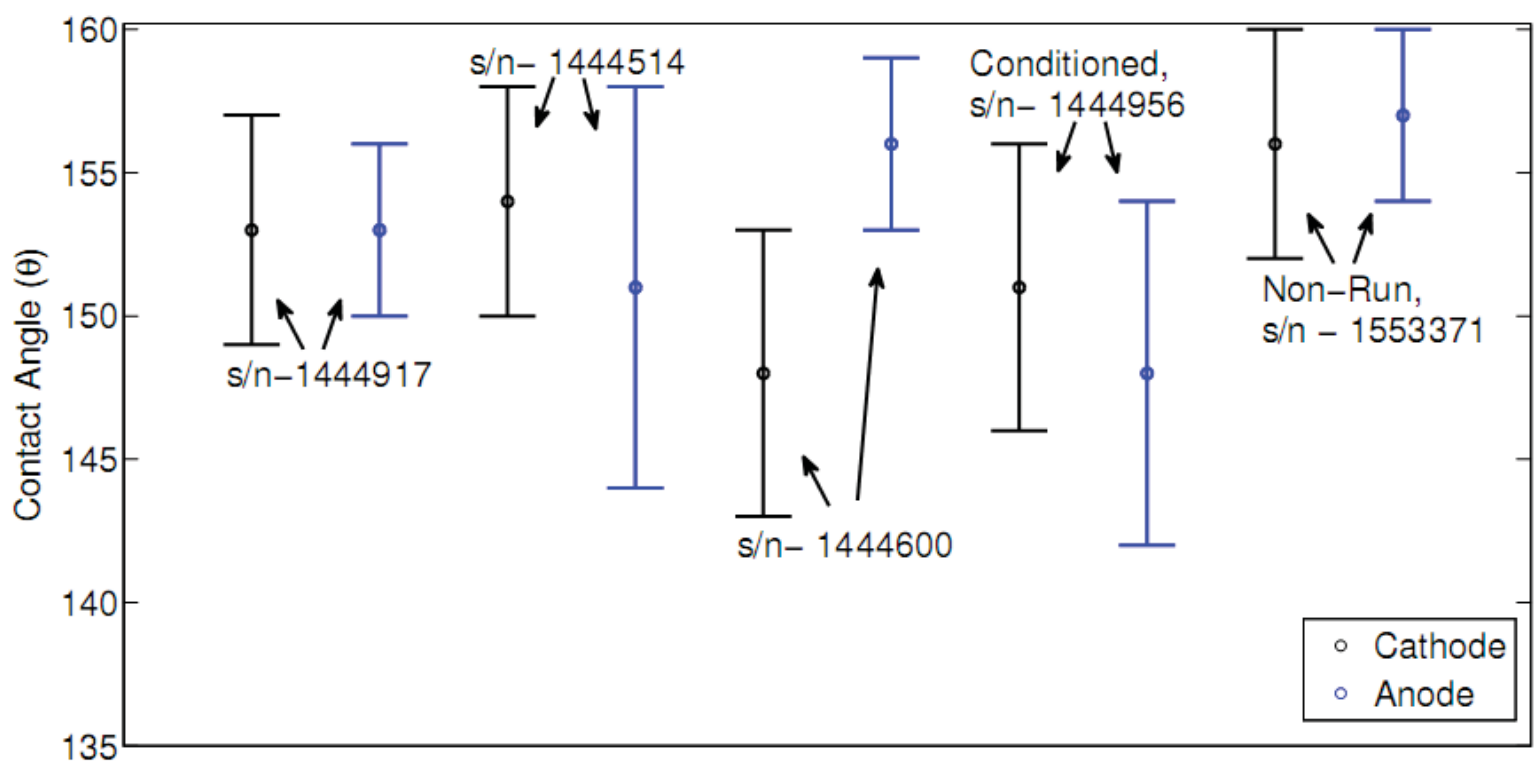

(b)

Figure 41: Static contact angle on the GDLs measured on the substrate side

Figure 42 and Figure 43 show the obtained advancing and receding contact angle data for Ptype GDL measured at different temperatures. The arrows represent the direction in which the experiment progressed. Advancing and receding experiments were conducted at three temperatures, $25^{\circ} \mathrm{C}, 55^{\circ} \mathrm{C}$, and $85^{\circ} \mathrm{C}$, while maintaining a high humidity. 
DE-EE0000466

Ballard Material Products Inc.

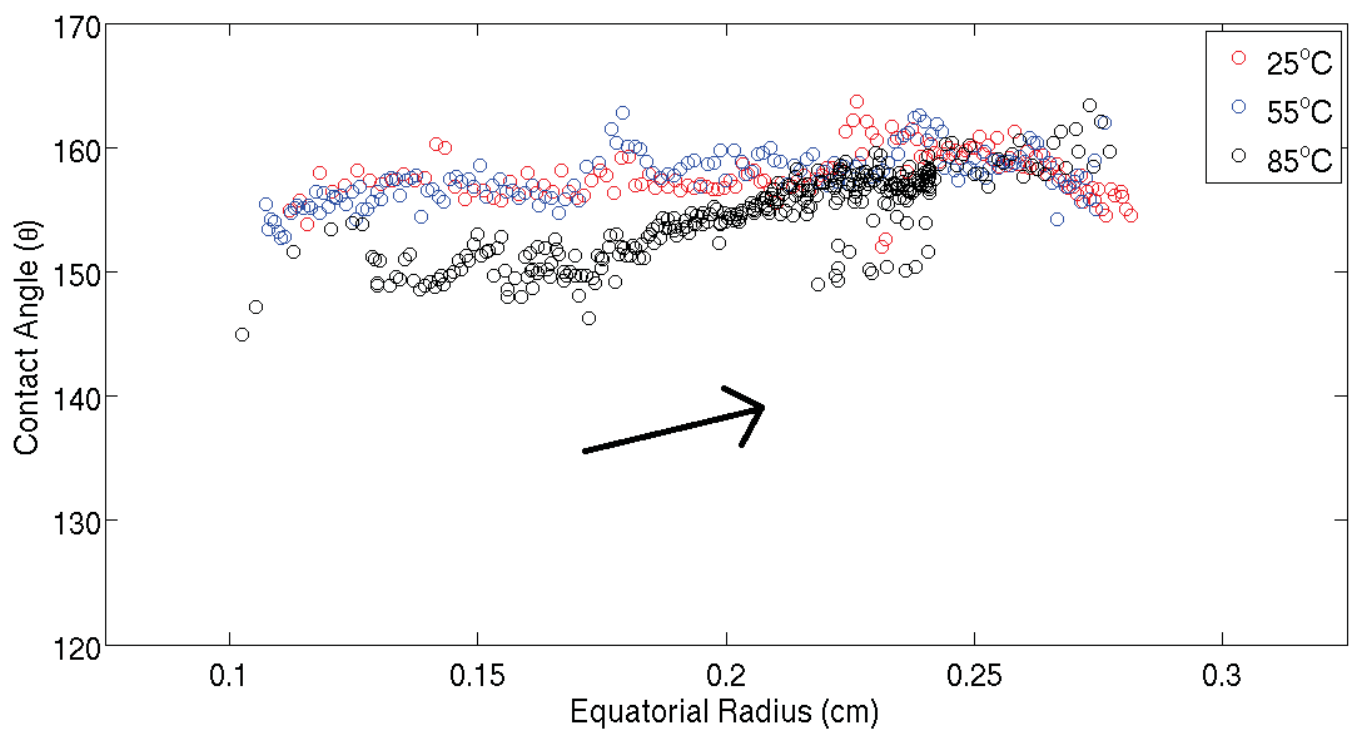

Figure 42: BMP-P GDL - Advancing

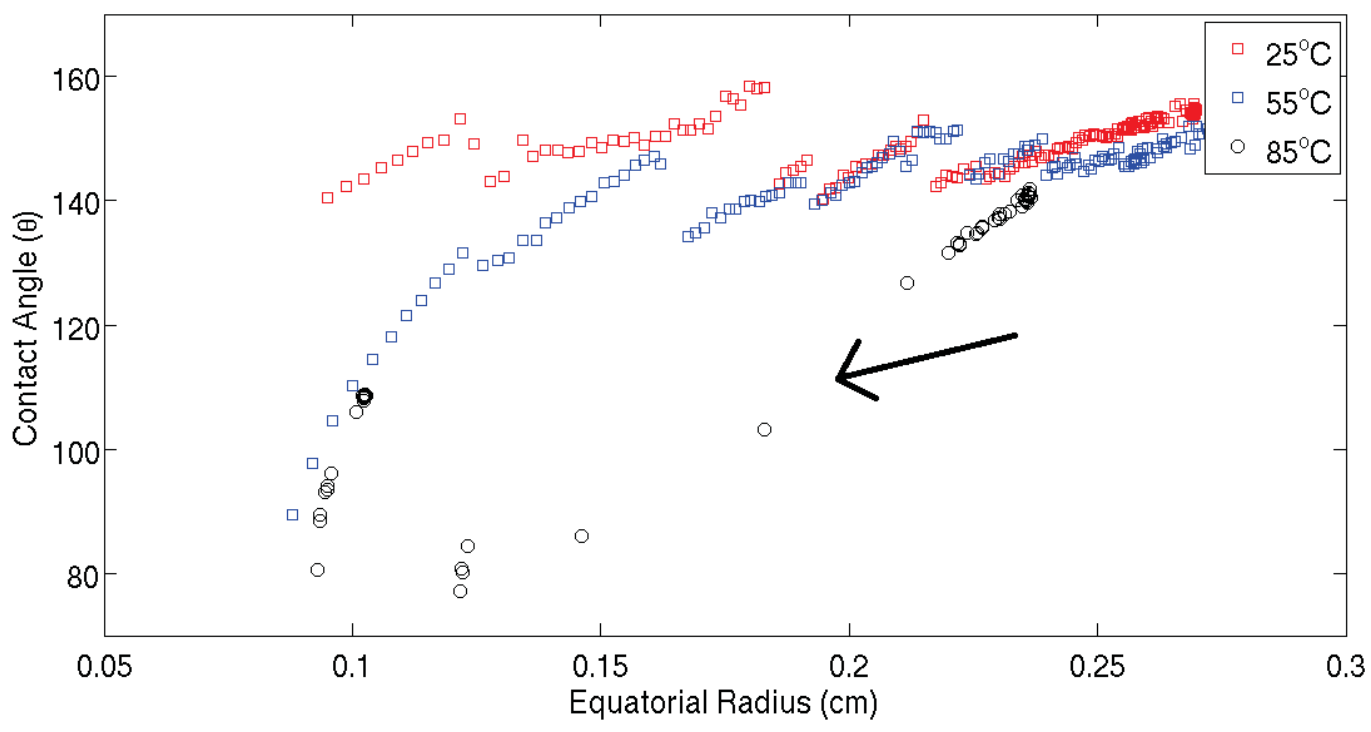

Figure 43: BMP-P GDL - Receding 
For advancing measurements, a smaller contact angle is seen during initial stages as the drop size increases. This is attributed to the increase in the drop volume with the contact line being pinned or moving slowly. A considerable difference between the contact angle at different temperatures is not seen. The rough surface of the diffusion media results in a large hysteresis and multiple runs on the same sample overlap.

For receding measurements, at higher temperatures, a drop in contact angle is seen which suggests considerable drop pinning of the diffusion media. The discontinuities that are seen in receding measurements are due to the sudden displacement of the contact line as the drop volume is reduced. Table 14 shows a summary of dynamic contact angle measurements.

Table 14: Summary of Dynamic Contact Angle measurements

\begin{tabular}{|c|l|c|c|}
\hline \multirow{2}{*}{ Sr No } & \multirow{2}{*}{ Sample } & \multicolumn{2}{c|}{ Contact Angle ( ${ }^{\circ}$} \\
\cline { 3 - 4 } & & Advancing & Receding \\
\hline 1 & P-SUB & $150-165$ & $150-80$ \\
\hline 2 & P-GDL & $160-170$ & $160-100$ \\
\hline 3 & EP-SUB & $145-160$ & $145-110$ \\
\hline 4 & EP-GDL & $155-165$ & $150-110$ \\
\hline 5 & TORAY-SUB & $155-165$ & $150-100$ \\
\hline 6 & TORAY-GDL & $150-165$ & $150-110$ \\
\hline 7 & End of Life - Anode s/n: 1444504 & $150-160$ & $140-100$ \\
\hline
\end{tabular}

\subsection{Wettability Tests after Cyclic Compression on GDL}

The compression tests were performed to assess the effect of compression on wettability of the GDLs. For these tests, the GDLs were subjected to a cyclic load. Each cycle consisted of loading the sample to $250 \mathrm{psi}(1.73 \mathrm{MPa})$ in 7 seconds, followed by keeping the sample under the rated pressure (250psi) for 30 seconds. This was followed by unloading the sample in 7 seconds to zero or minimum system load (approx. 0-3psi) and finally holding the sample under this minimum load for 15-20 seconds. Two GDL samples were used for the compression test. Sample 'A' (Non-Run, P-GDL, B2-MD) was subjected to 10 cycles while sample 'B' (Non-Run, P-GDL, B2b-MD) was subjected to 60 cycles.

Samples tested for compression:

- Sample A -10 cycles - Anode GDL (Non-Run, P-GDL, B2-MD)

- Sample B - 60 cycles - Anode GDL (Non-Run, P-GDL, B2b-MD) 


\subsubsection{Test Set-up}

Figure 44 shows the experimental setup for the compression test. The GDL sample was placed in between the two ground steel plates and placed in the load cell. A strain gauge was attached to the two steel plates to measure the compression of the GDL.

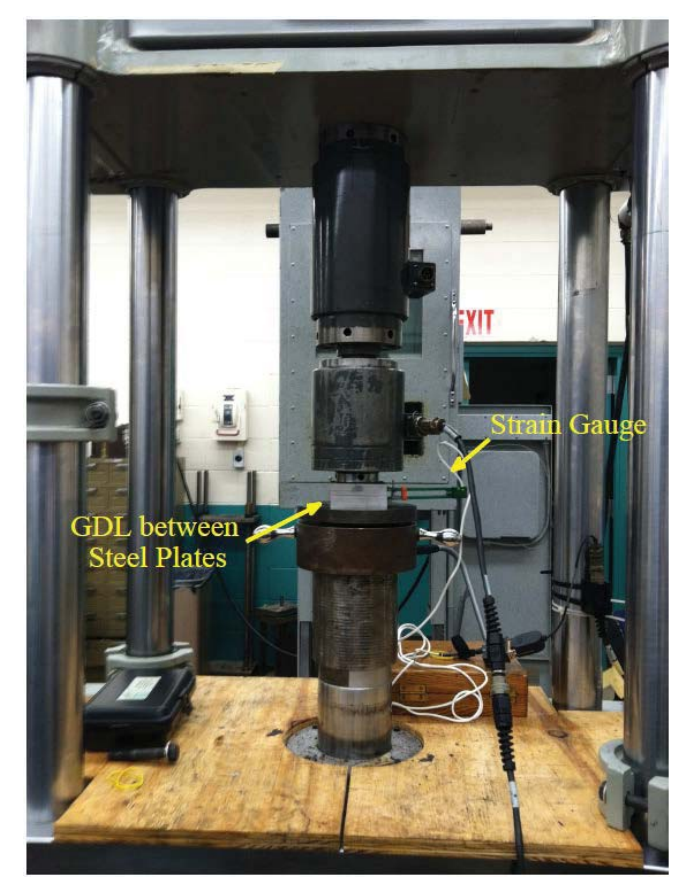

Figure 44: Experimental set-up for compression tests

\subsubsection{Results and Discussion}

Figure 45 shows the results of compression on sample 'A' and Figure 46 shows the results for sample 'B'. There appears to a small permanent compression of $10 \mu \mathrm{m}$. The maximum deformation was about $25 \mu \mathrm{m}$ which appears to have reduced to $23 \mu \mathrm{m}$ after 10 cycles. For sample ' $\mathrm{B}$ ', there is no permanent compression and it completely regained its original configuration. As the number of cycles increased, the maximum deformation in the GDL appears to have reduced. The maximum deformation in the beginning was $19 \mu \mathrm{m}$ which reduced to $15 \mu \mathrm{m}$ after 60 cycles (Figure $46 \mathrm{a}$ ). Figure 47 shows the stress-displacement curve for the two samples. The curves are obtained using an exponential fit. The GDLs "set in" after the first few cycles as seen from the stress-displacement curves. The contact angle obtained on the compressed samples do not show any change when compared to the uncompressed samples, reported previously. High contact angle of $\sim 155^{\circ}$ was observed on the tested sample, consistent with previous results. 
DE-EE0000466

Ballard Material Products Inc.

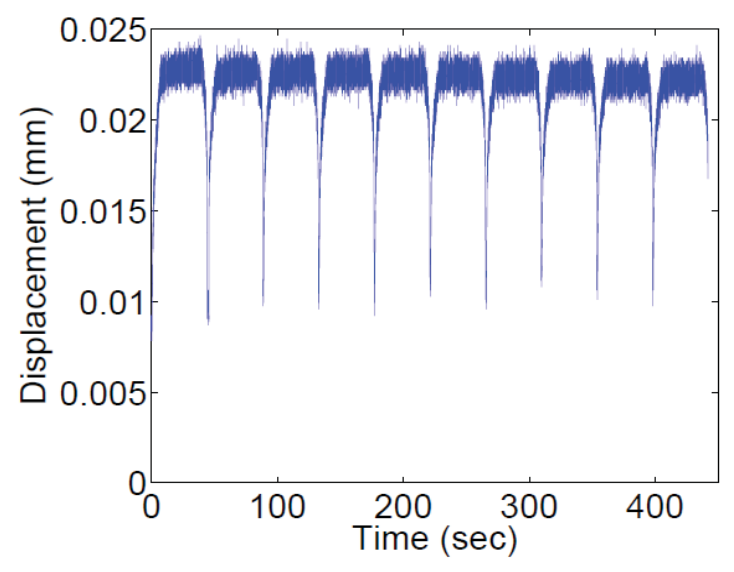

(a) GDL compression (mm) vs. Time (seconds)

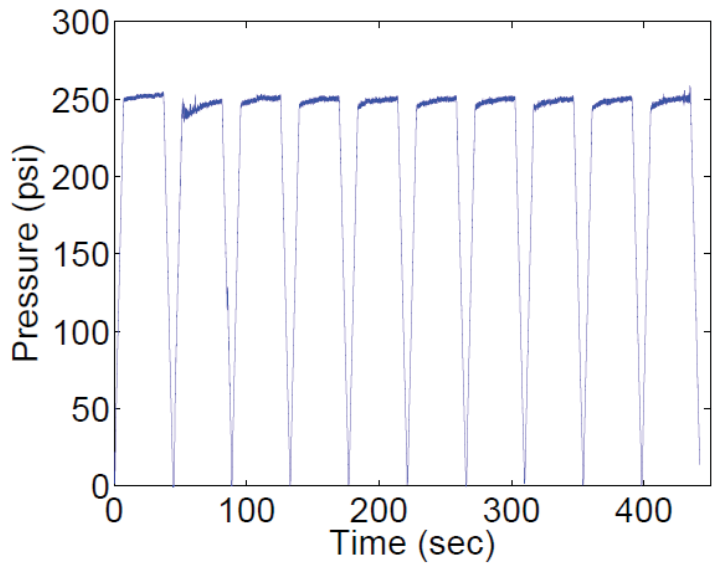

(b) Pressure (psi) vs. Time (seconds)

Figure 45: Results for Sample `A' (Non-Run, P-GDL, B2-MD)

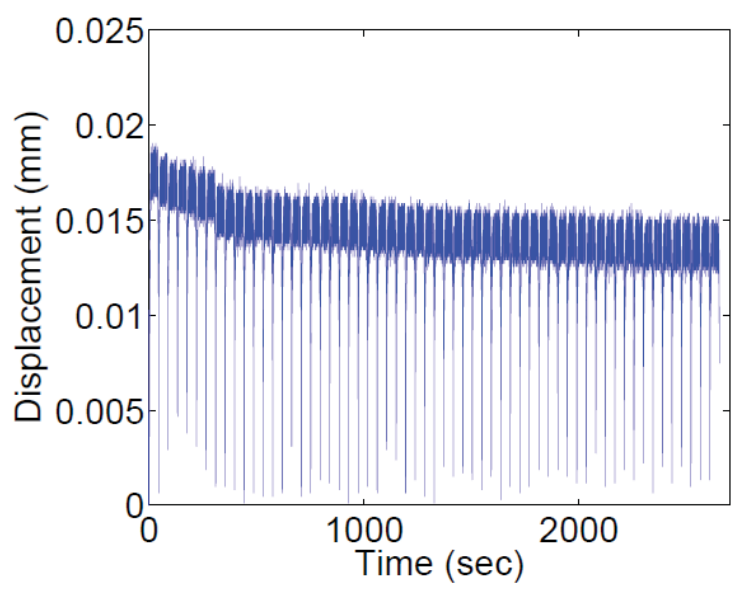

(c) GDL compression (mm) vs. Time (seconds)

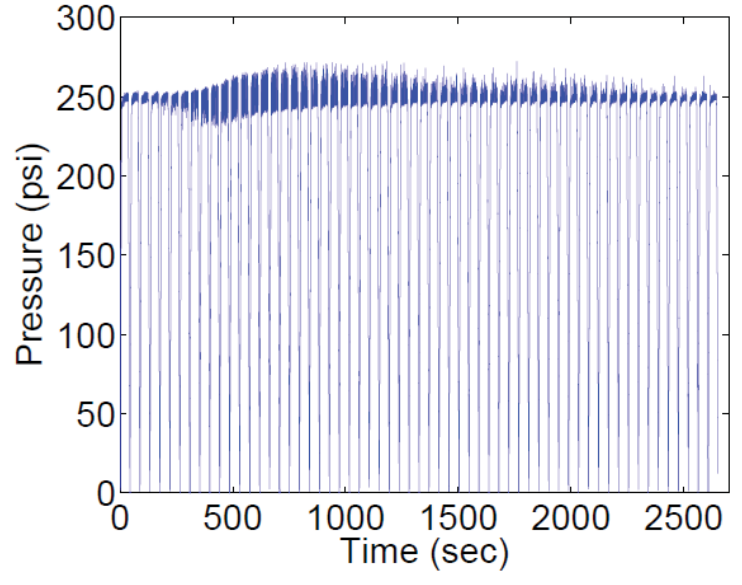

(d) Pressure (psi) vs. Time (seconds)

Figure 46: Results for Sample 'B' (Non-Run, P-GDL, B2b-MD) 


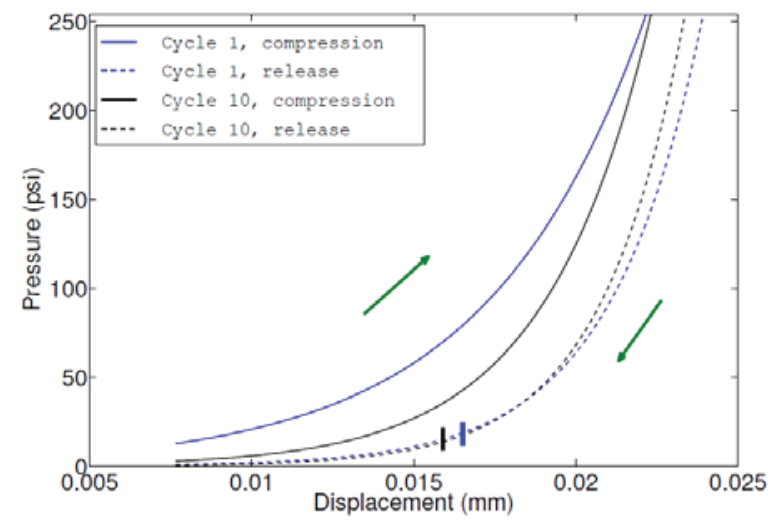

Sample A - Stress (psi) vs. Displacement (mm)

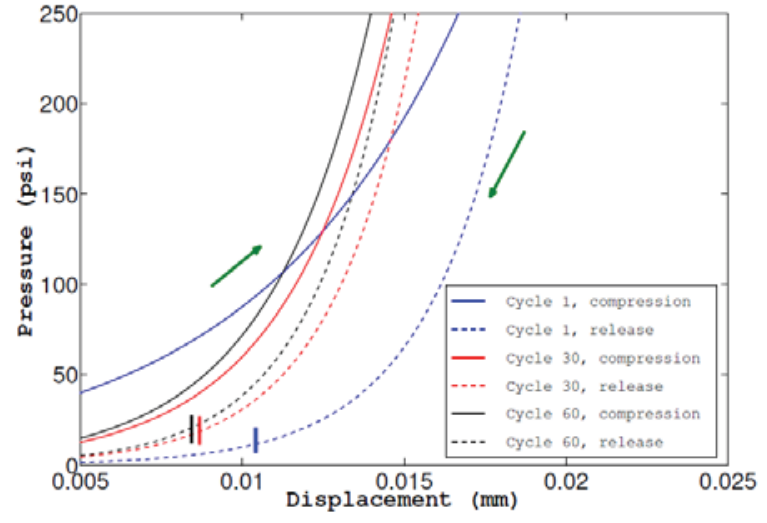

Sample B - Stress (psi) vs. Displacement (mm)

Figure 47: Stress vs. Displacement curve for the tested samples for different compression cycles. The vertical bars represent the cut-off point till which the data was collected during the release half of the cycle

Table 15: Contact Angle Measurement on the compressed samples

\begin{tabular}{|c|c|}
\hline & Contact Angle ( $^{\circ}$ ) \\
\hline Sample A, B2-MD & $155 \pm 3$ \\
\hline Sample B, B2b-MD & $156 \pm 3$ \\
\hline
\end{tabular}

\subsubsection{Summary}

The GDLs show high contact angles (greater then $150^{\circ}$ ). Drop pinning along with the porous surface resulted in large hysteresis in contact angle measurement. The advancing contact angles were found to be comparable to the measured static contact angle. The receding contact angles are much lower than the advanced/static contact angles and are of a magnitude $100-110^{\circ}$. This indicated drop pinning plays a major role on droplet dynamics on the surface of the GDL. Furthermore, an effect of temperature on the contact angle was not observed, as well no effect of compression on contact angles was observed which indicates no deterioration of the surface due to compression. 


\subsection{GDL - MPL Chemical Analysis (LANL)}

Fourier Transform Infrared (FTIR) analysis was conducted on a variety of GDL-MPLs after MEA preparation, conditioning and after the MEA was subjected to AST cycling at different upper potentials. Figure 48 shows the absorbance difference of LSAC Pt50 and MSAC Pt50 MEA GDLs that were subjected to 4700 AST cycles at an upper potential of $1.2 \mathrm{~V}$ and a virgin cathode GDL MPL. The results reveal insignificant differences between a conditioned and aged MSAC Pt50 catalyst MPL. In contrast, clear changes in the $\mathrm{C}-\mathrm{H}$ stretch wave numbers are observed for the GDL-MPL of the Pt50 LSAC MEA that was subjected to AST cycling, an indication that some GDL-MPL layer corrosion has occurred in this sample. From the carbon support study (Chapter II) it is clear that the MSAC Pt50 catalyst support exhibited more carbon corrosion, i.e. a larger change in thickness at 1.2V UPL $c$ the Pt50 LSAC MEA. Thus, the FTIR results indicate that the GDL MPL change may be dependent on the carbon support type and that some preferential carbon corrosion of the GDL MPL may occur for more corrosion resistant catalysts.

(spectrum with different absorbance scales for better view)

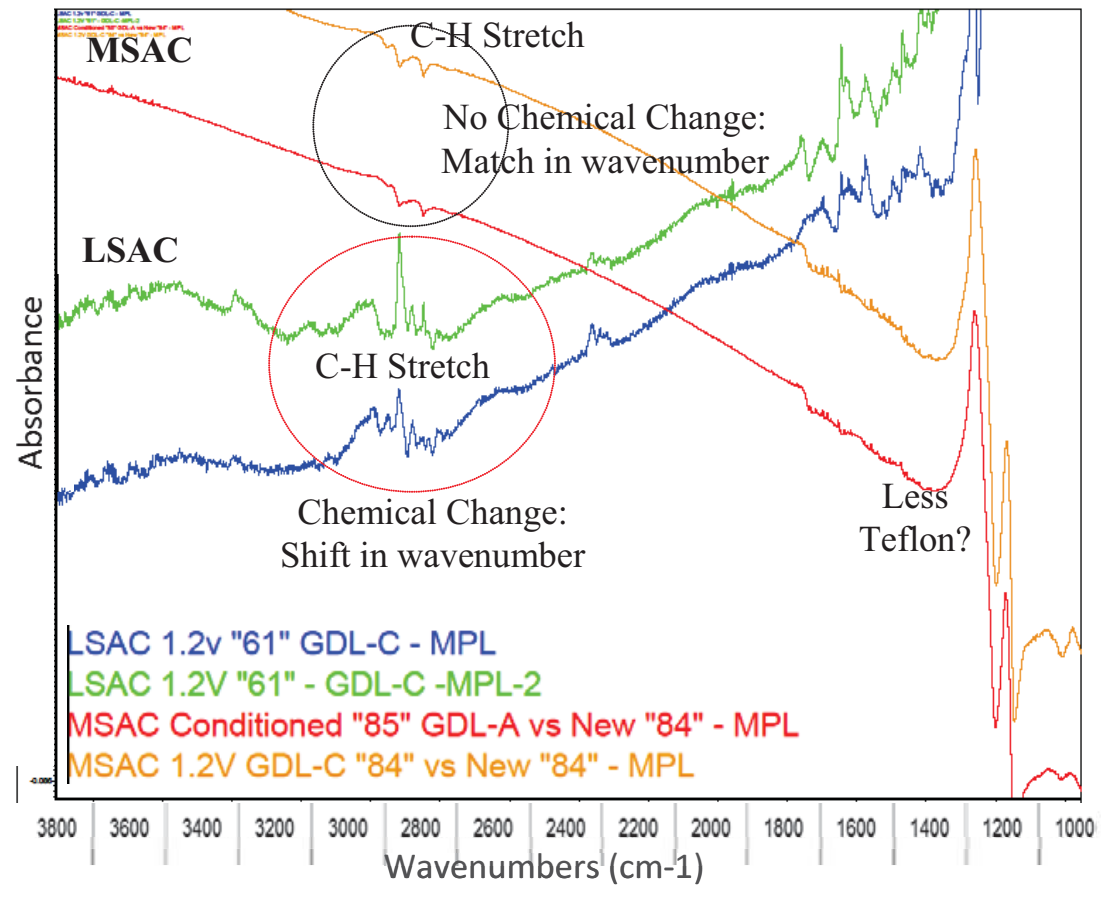

Figure 48: Differential FTIR spectrum of GDLs from MEAs with LSAC Pt50 and MSAC $\mathrm{Pt} 50$ cathode catalyst layer (compared to BOL) 
DE-EE0000466

Ballard Material Products Inc.

\subsection{Surface Roughness Measurements of GDL and Catalyst Layer (MTU)}

The surface roughness of the catalyst layer and GDL of a conditioned BOL delaminated MEA were investigated separately. The CCM and the GDLs are not bonded, thus the delamination is not causing any damage to the catalyst and GDL surface. Stereo-imaging, surface mapping microscopy, and surface profiliometry was used to evaluate the surface roughness. The same areas of the GDL and catalyst layer were investigated in order to make comparable conclusions. Figure 49 shows the imaging and profiliometry results of the two surfaces.

\subsubsection{Measurement Techniques}

\subsubsection{Stereo Imaging}

Stereo imaging is based on computing $3 D$ coordinates from the $2 \mathrm{D}$ coordinates of corresponding points from two separate images, one tilted with respect to another (by 8 deg). The scan size was $400 \times 300 \mu \mathrm{m}$. The process was carried out for other points, on both images, to find the shape of the surface. In order to get reliable results from this method, the image noise was reduced to obtain maximum volume for 3D construction. The centre point of the image was marked on the SEM screen, and the sample was then tilted until the marked point was positioned on the edge of the image. The position of the specimen was then adjusted so that the marked point was at the centre point of the image again. The process was repeated until the specimen was tilted to the desired angle of $8^{\circ}$ to the horizontal (eucentric tilting). Finally, a magnification was chosen such that the length of any diagonal line connecting two opposite corners of the image would be 70 times larger than the height of the feature to be analyzed. Three different areas of each layer were examined by SEM stereo-imaging and the surface features were investigated for validation. The visual representation of the surface is shown in pseudo-coloured depth image. For better comparison, we plotted the data of GDL and $\mathrm{CL}$ in the same scale. It was found that $\mathrm{CL}$ has smoother surface than GDL. The average roughness of $\mathrm{CL}$ and $\mathrm{GDL}$ was found to be $3.2 \mu \mathrm{m}$ and $4.1 \mu \mathrm{m}$ respectively which agrees with Hizir et al. who obtained the surface roughness of CL and GDL as $2.19 \mu \mathrm{m}$ and $5.35 \mu \mathrm{m}$ from optical profilometer. (Figure 49a) Stereo imaging in the SEM is a very complementary technique, which takes advantage of the excellent depth-of-focus of the instrument. Compared to other tactile measurement, SEM is incomparably flexible, since it enables to vary quickly and easily the magnification, in order to visualize almost immediately fields of very different size and locate accurately a particular region on the specimen. The stereo imaging is a well known technique to measure surface roughness in the research community. 
DE-EE0000466

Ballard Material Products Inc.
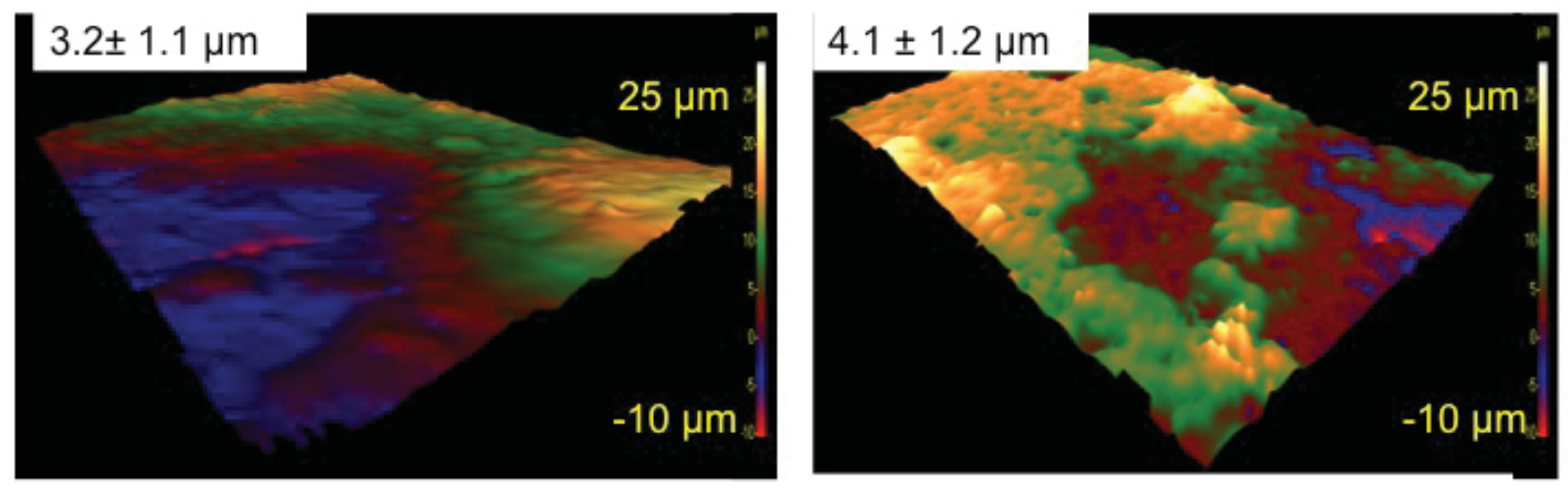

(a)
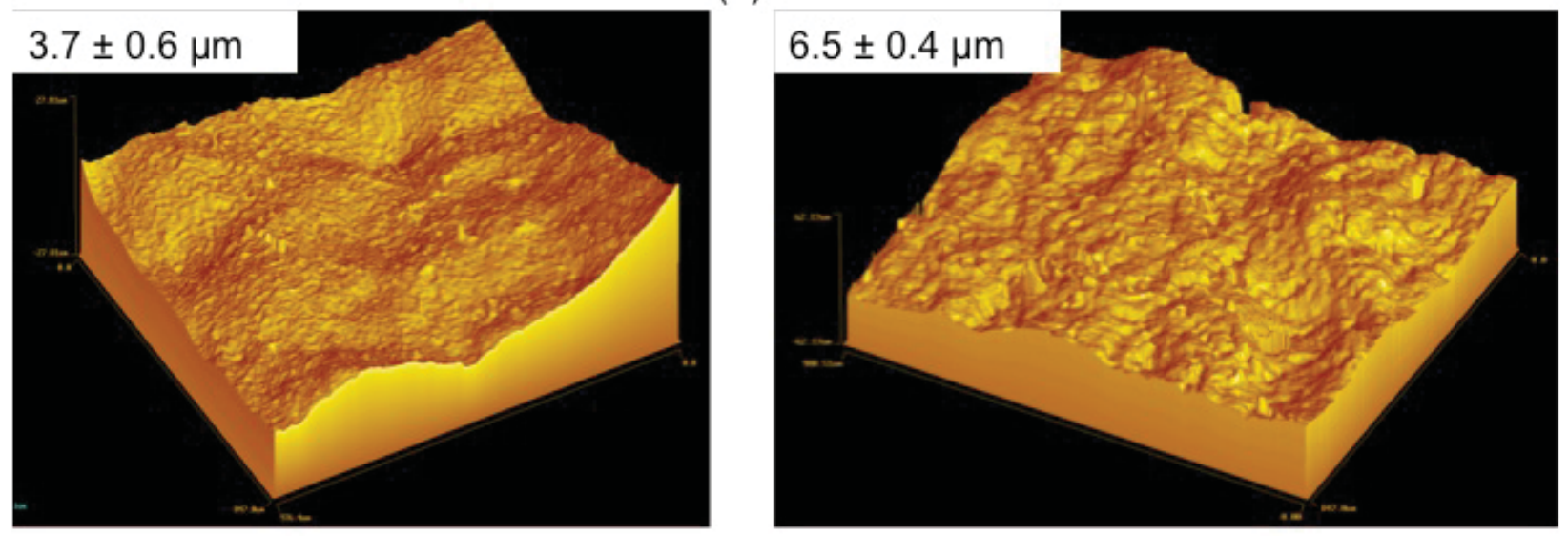

(b)

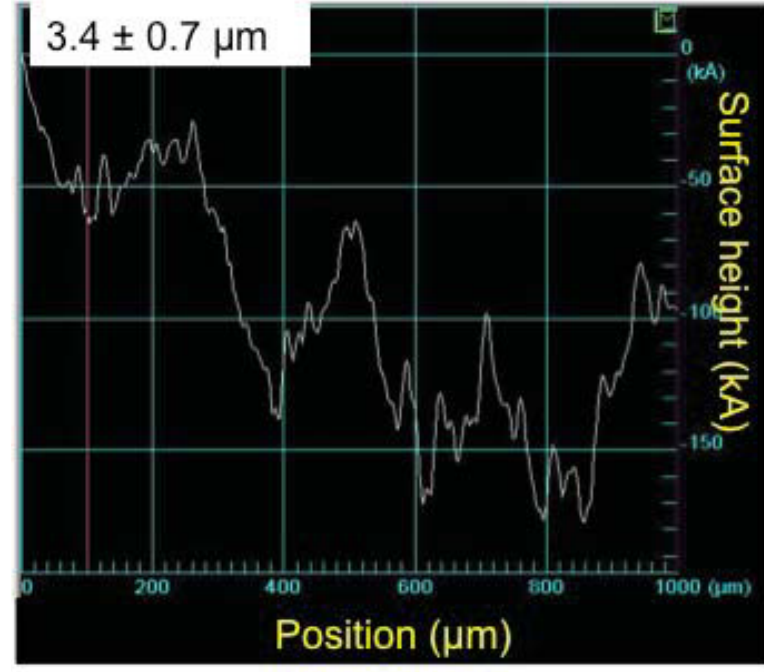

Catalyst layer

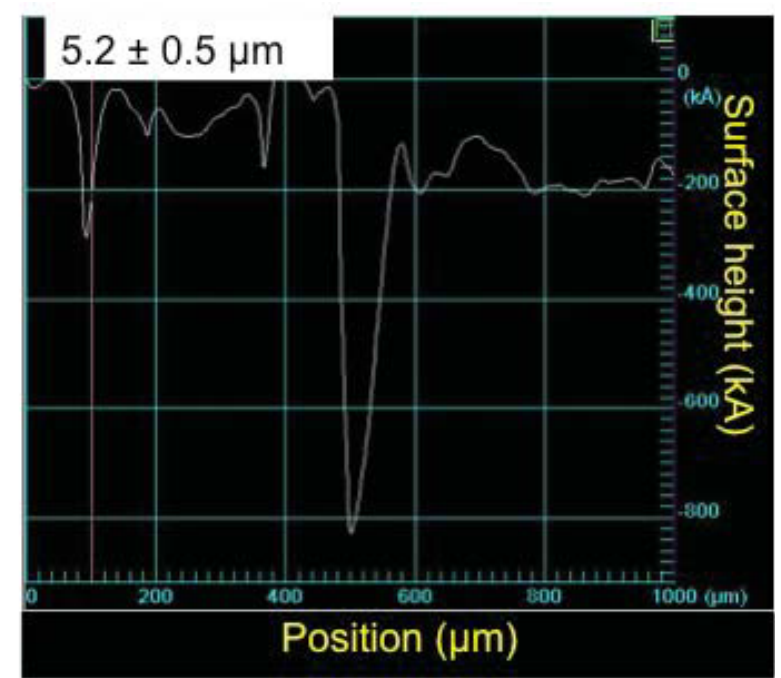

(c)

\section{GDL}

Figure 49: Surface roughness measurement of $C L$ and GDL from: (a) Stereo imaging (b) interferometer microscope (c) surface profiliometry 


\subsubsection{Surface Mapping Microscopy}

ADE Phase Shift MicroXAM surface mapping microscope was also used to measure surface roughness of $C L$ and GDL. This is a non-contact profiler having a repeatability of $0.1 \mathrm{~nm}$ and vertical resolution of $4 \mathrm{~nm}$. It was operated in quantitative mode using optical light interferometer. The scan size was $900 \times 800 \mu \mathrm{m}$. To properly capture the surface topography of the samples, surfaces should possess a high degree of reflectivity. Since the GDL and CL possess low reflectivity and have light dispersive characteristics, the surface was sputtered with a thin layer of gold. The average roughness of CL and GDL was found to be $3.7 \mu \mathrm{m}$ and $6.5 \mu \mathrm{m}$ respectively (Figure 49b). Tests were done on three different areas of each layer of GDL and $\mathrm{CL}$ for validation. This is a good technique that provides a non-contact, 3-D method of measuring surface roughness.

\subsubsection{Surface Profiliometry}

DEKTAK3ST surface profiler was also used to measure surface roughness, which is a line-scan surface measuring instrument. Measurements are made electromechanically by moving the sample beneath a $5 \mu \mathrm{m}$ radius diamond-tipped stylus with a force of $0.1 \mathrm{mN}$. The vertical resolution is $3 \mathrm{~nm}$. The average roughness of CL and GDL was found to be 3.4 and $5.2 \mu \mathrm{m}$ (Figure 49c). Here also we tested on four different areas of GDL and CL for validation. It is the fastest and precise technique for surface roughness and does not require any coating on it. The only disadvantage is that it does not give a $3 \mathrm{D}$ image.

\subsubsection{Results}

All the three methods were found to be appropriate for surface roughness measurements; however, surface mapping was preferred to measure surface roughness of the sample using surface profiliometry for validation. The surface mapping images of BOL catalyst layer was taken at different magnifications $(6.3 x, 10 x, 20.3 x$ and $63 x)$ to study its effect on roughness data. Specific crack features were examined and depth calculations were done at all magnifications (Table 16). The surface mapping images and its cross-section curves are shown in Figure 50.

Table 16: Depth of cracks

\begin{tabular}{|c|c|c|}
\hline Sr. No & $\begin{array}{c}\text { Scan Size } \\
(\boldsymbol{\mu \mathrm { m }} \times \boldsymbol{\mu \mathrm { m } )})\end{array}$ & $\begin{array}{c}\text { Depth of crack } \\
(\boldsymbol{\mu} \mathrm{m})\end{array}$ \\
\hline 1 & $1296 \times 921$ & 7.8 \\
\hline 2 & $810 \times 615$ & 8.21 \\
\hline 3 & $404 \times 326$ & 8.85 \\
\hline 4 & $130 \times 98$ & 9.27 \\
\hline
\end{tabular}

One can observe that the depth of crack increases from $7.8 \mu \mathrm{m}$ to $9.27 \mu \mathrm{m}$ as the magnification is increased. Thus, we don't see a significant effect of magnification on roughness data. 

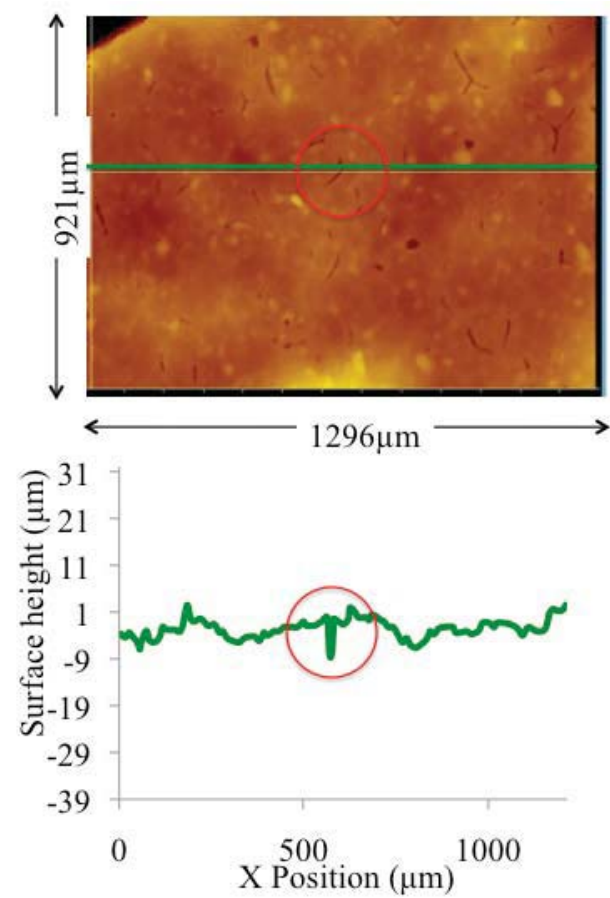

(a)

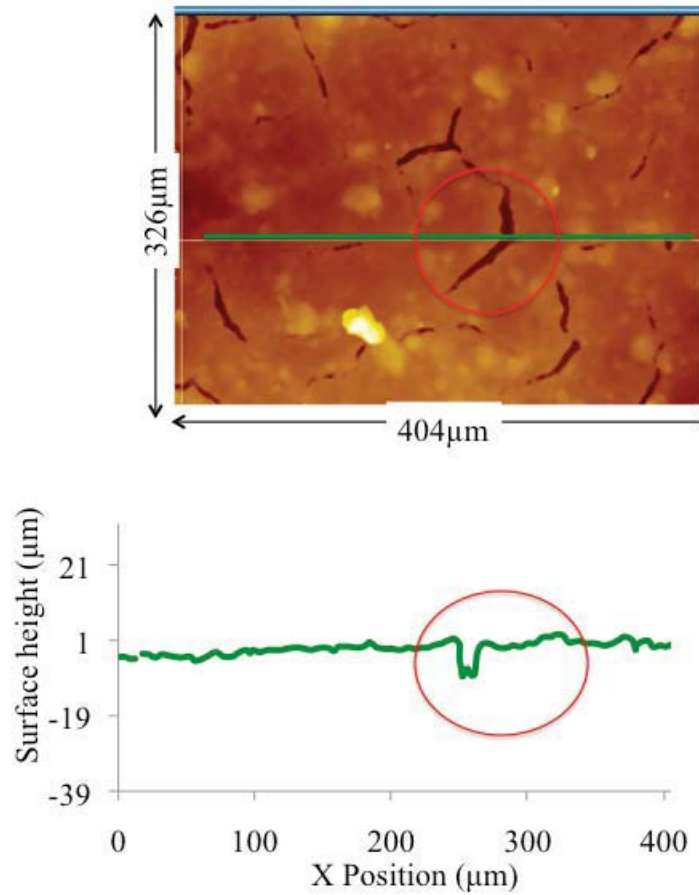

(c)
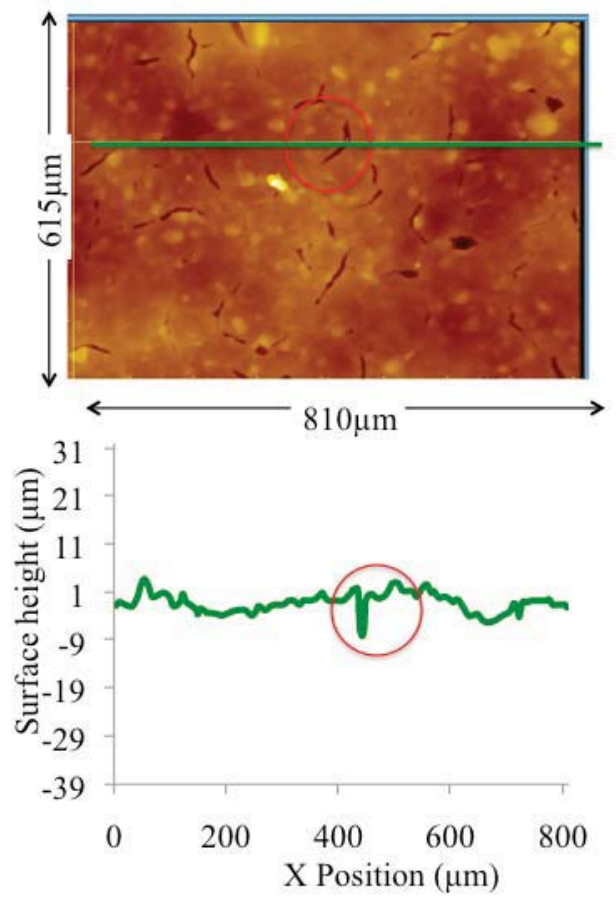

(b)
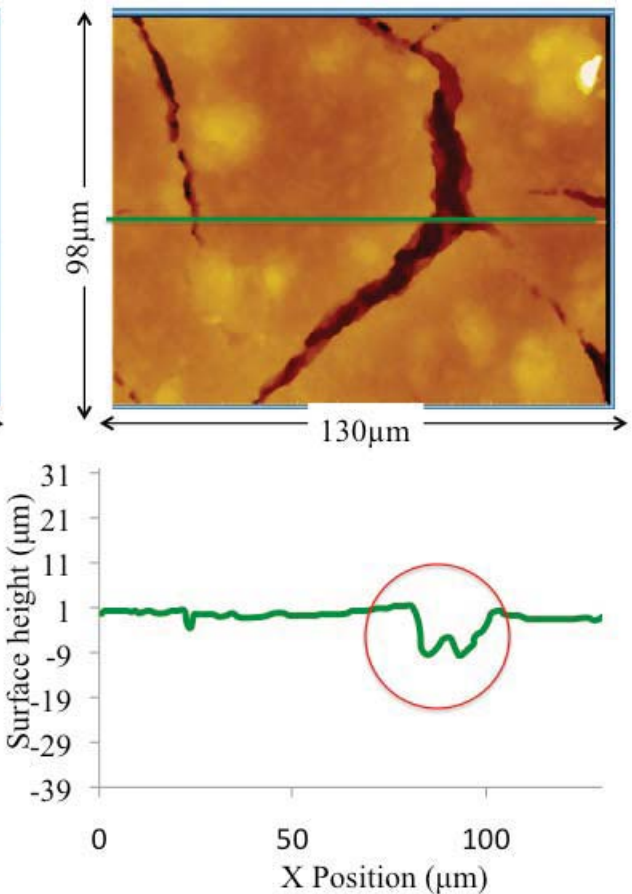

(d)

Figure 50: Surface mapping images and cross section curves at magnification of (a) $6.3 x$ (b) $10 x$ (c) $20.3 x$ (d) $63 x$. The red circle indicates the crack in the feature 


\subsubsection{Summary}

The surface roughness of catalyst layer and GDL-MPL was investigated using stereo imaging, interferometry, and surface profiliometry to characterize the surface roughness. Interferometry data has been used as an input for the contact resistance model discussed in Chapter VII. The average roughness of catalyst layer and MPL was found to be $3.7 \mu \mathrm{m}$ and $6.5 \mu \mathrm{m}$, respectively. These data were processed and exported to MATLAB. The roughness data was used to calculate the contact resistance between $\mathrm{CL}$ and GDL. 
DE-EE0000466

Ballard Material Products Inc.

\subsection{Catalyst Layer/Membrane Interface Characterizations (MTU)}

\subsubsection{SEM-EDS Analysis of Membrane-Catalyst Interface}

EDS is commonly associated with SEM or TEM analysis to obtain composition information for the selected region on the sample. Catalyst layer contains platinum which has excitation energy $(L \alpha)$ of $9.4 \mathrm{KV}$. The accelerating voltage is $2 \mathrm{x}$ excitation energy giving $18.8 \mathrm{KV}$. Thus, EDS analysis on membrane-catalyst layer interface should be done at $20 \mathrm{KV}$. It was suspected that at this voltage, the beam may damage the membrane and can affect the chemical composition. SEM images of BOL membrane-catalyst layer before and after EDS were taken to investigate the beam damage as shown in Figure 51a and Figure 51b.

Figure 51b shows the SEM images of membrane at higher magnification. A very minor change can be seen on the membrane but it is suspected that the EDS spectra are not affected. The EDS analysis at $15 \mathrm{KV}$ and $10 \mathrm{KV}$ is shown in Figure 52. Due to low accelerating voltage, it does not provide the necessary overvoltage and sufficient current from the source to excite efficiently the $\mathrm{K}$ and $\mathrm{L} \mathrm{x}$-ray lines of platinum element.

Thus, the EDS spectra for all the samples were taken at accelerating voltage of $20 \mathrm{KV}$ and dwell time of $120 \mathrm{sec}$. Figure 53 shows the EDS spectra and EDS mapping of BOL membranecatalyst layer interface. The spectra analysis indicated that the interface consist of $42 \%$ weight of carbon, $23 \%$ weight of fluorine, $22 \%$ of weight of platinum, and $10 \%$ weight of sulphur. The EDS mapping indicate a high concentration of platinum in the catalyst layer while the membrane has large concentration of fluorine and sulphur. 

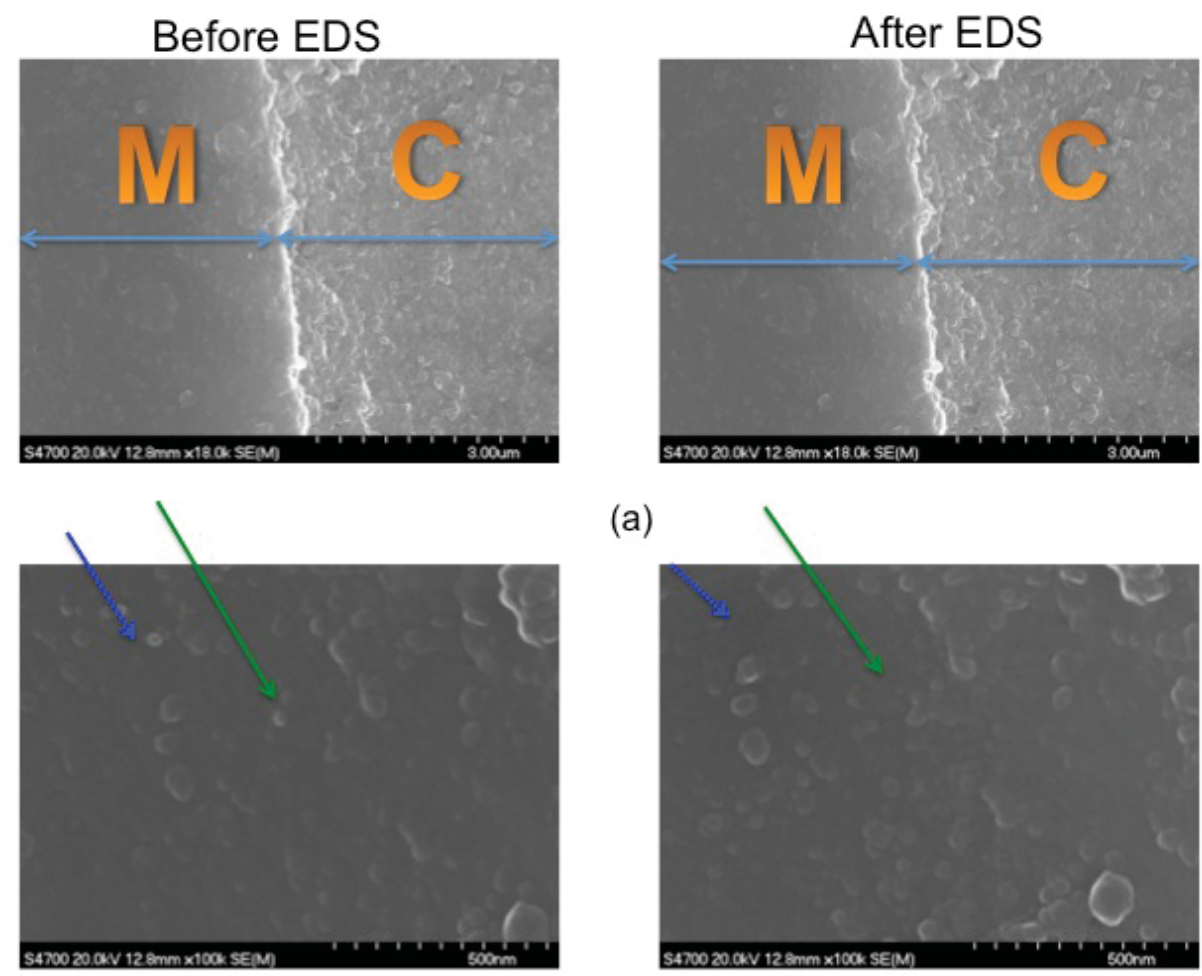

(a)

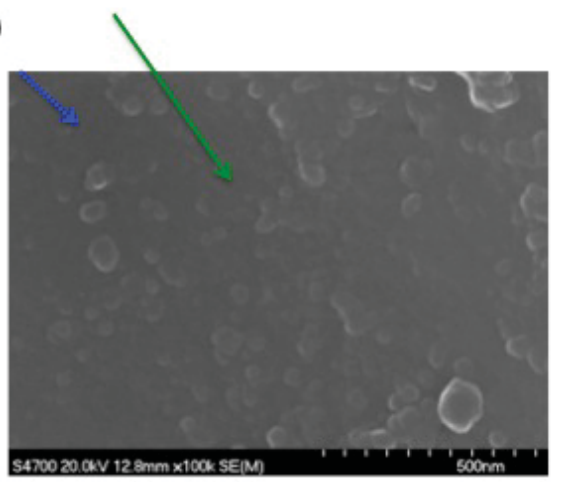

(b)

Figure 51: SEM images of: (a) BOL Membrane-catalyst interface before and after EDS. (b) BOL membrane before and after EDS. The accelerating voltage is $20 \mathrm{KeV}$. The blue and green line indicates the loss of particles in membrane after EDS. M-Membrane CCatalyst

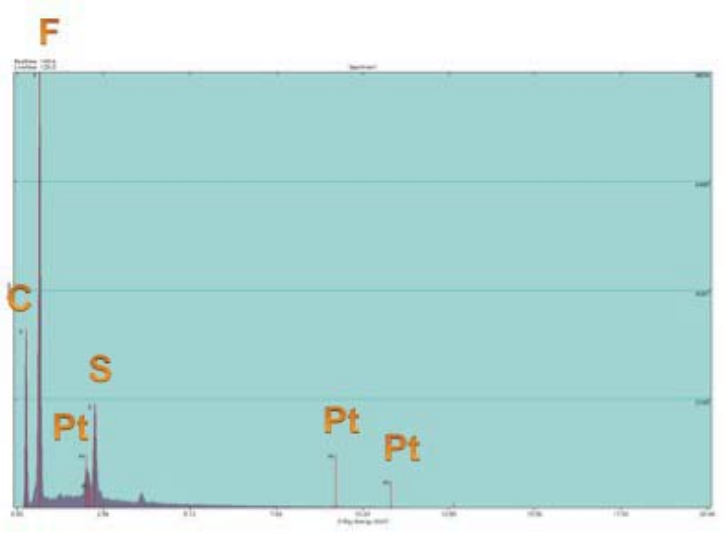

(a)

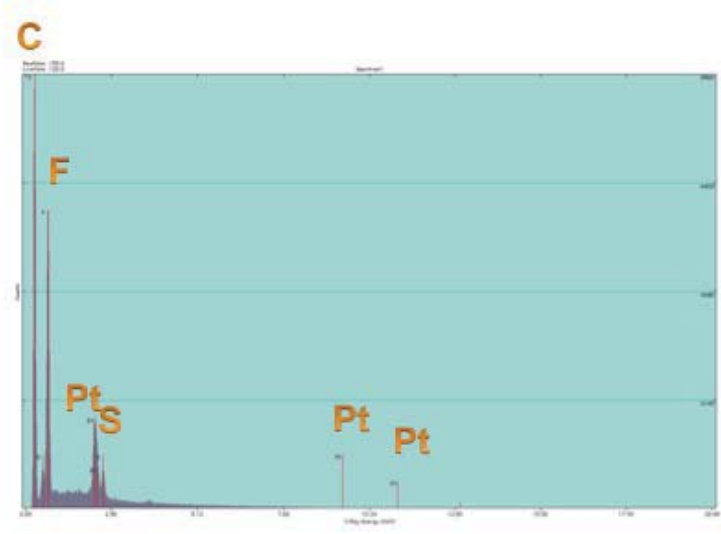

(b)

Figure 52: EDS spectra of $\mathrm{BOL}$ membrane-catalyst interface at accelerating voltage : (a) $15 \mathrm{KV}$ (b) 10KV. C-Carbon, F- Fluorine, Pt- Platinum, S-Sulphur 


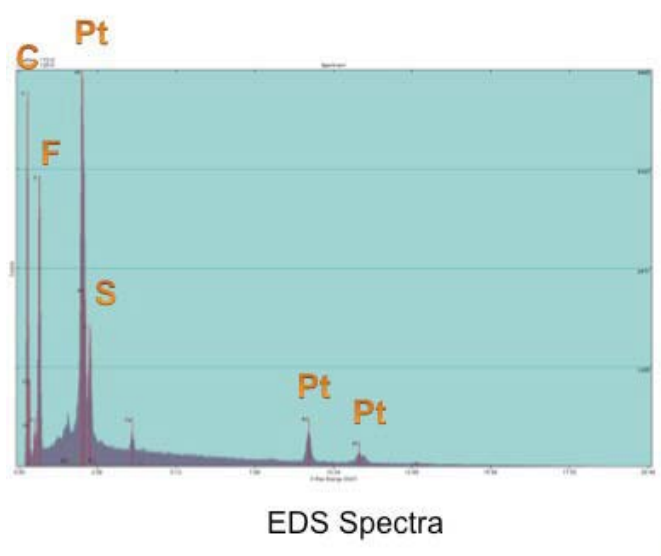

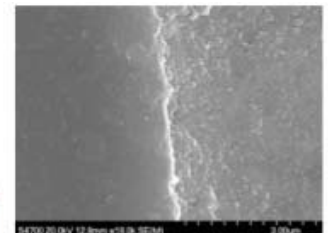

SEM

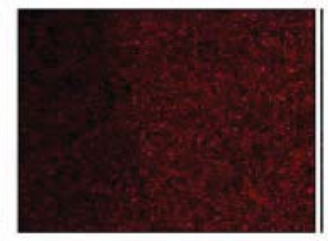

Carbon

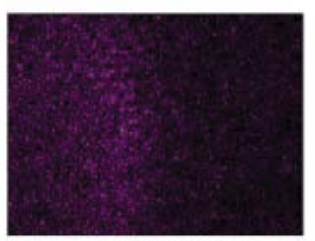

Sulphur

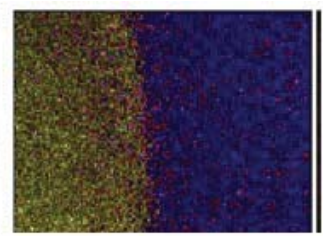

Phase

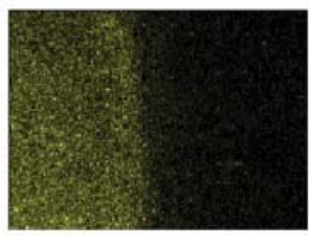

Fluorine

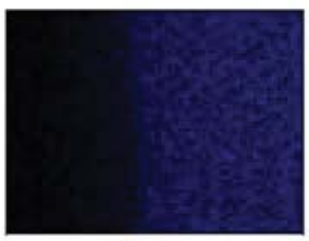

Platinum

Figure 53: EDS spectra and EDS mapping of BOL membrane-catalyst interface. The accelerating voltage and dwell time are $20 \mathrm{KV}$ and $120 \mathrm{sec}$, respectively

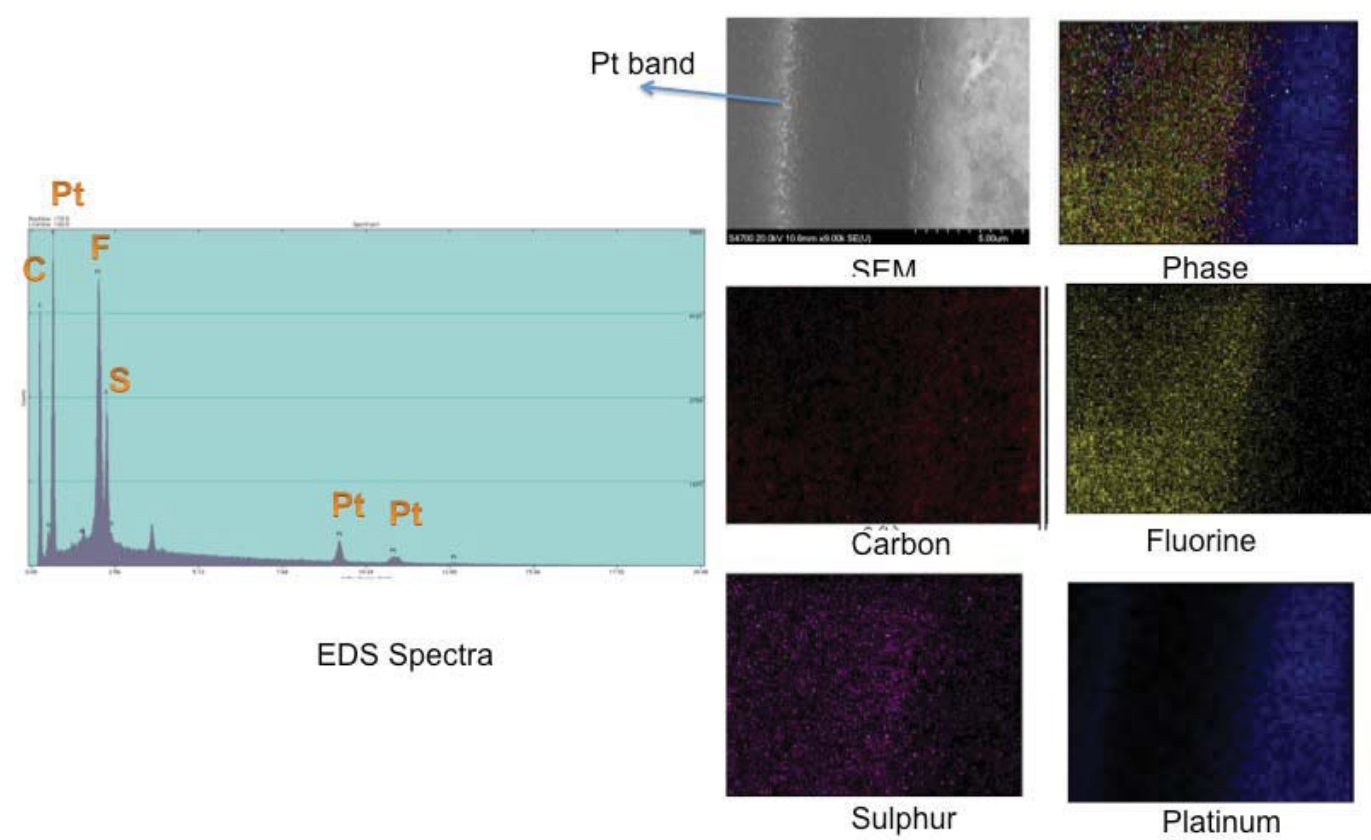

Figure 54: EDS spectra and EDS mapping of EOL-1.2V membrane-catalyst interface. The accelerating voltage and dwell time are $20 \mathrm{KV}$ and $120 \mathrm{sec}$, respectively 


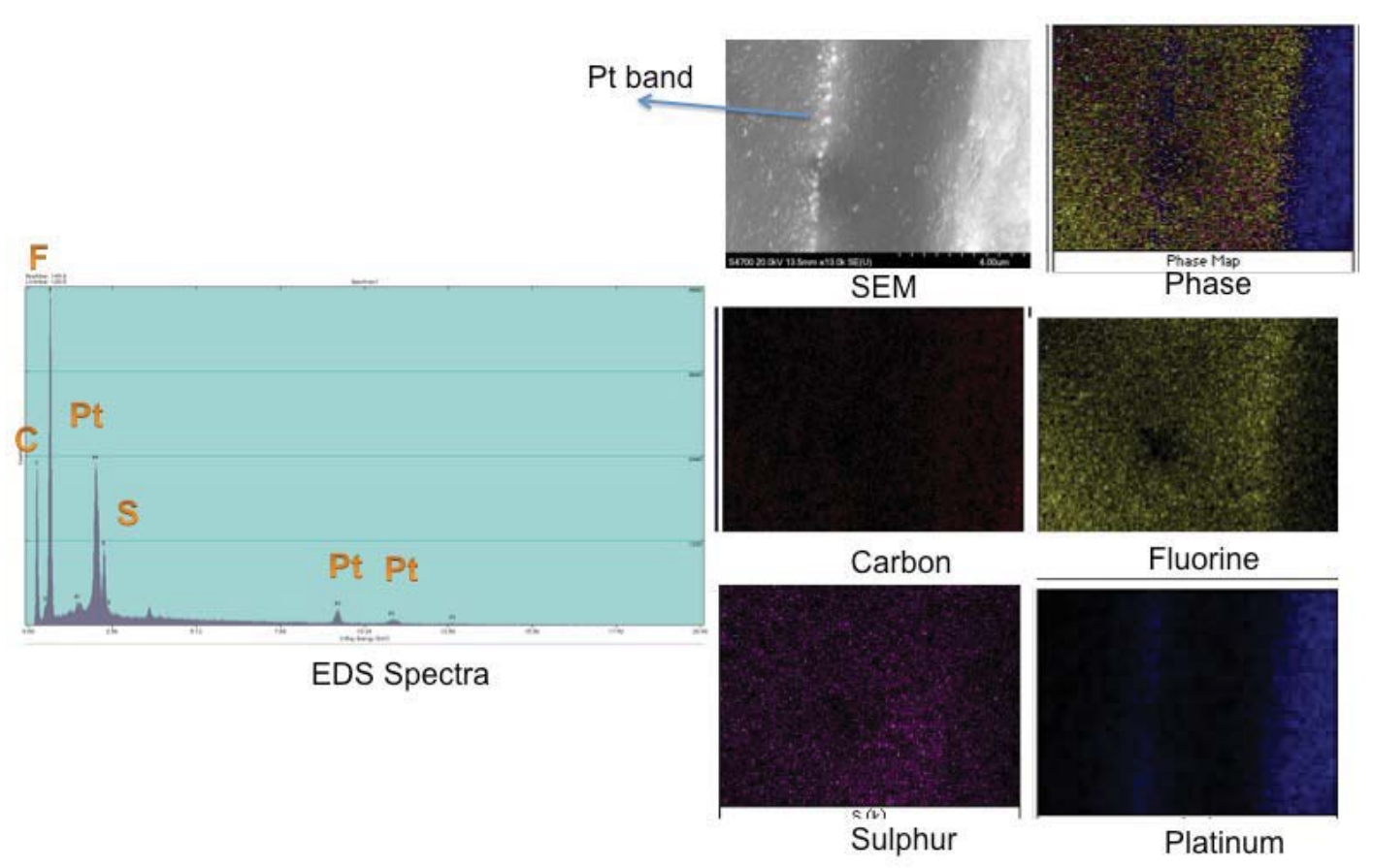

Figure 55: EDS spectra and EDS mapping of EOL-1.4V membrane-catalyst interface. The accelerating voltage and dwell time are $20 \mathrm{KV}$ and $120 \mathrm{sec}$, respectively

The EDS spectra of catalyst layer membrane interface degraded at a UPL of $1.2 \mathrm{~V}$ shown in Figure 54 consist of $32 \%$ weight of carbon, $40 \%$ weight of fluorine, $19 \%$ weight of platinum and $5 \%$ weight of sulphur. The SEM image and platinum map shows a thin platinum band formation on the membrane side. One can see a small decrease in concentration of platinum and sulphur while a minor increase in fluorine concentration.

Figure 55 shows the EDS spectra and EDS mapping of the sample degraded at a UPL of 1.4V interface. Here the platinum in the membrane side is thicker than for the $1.2 \mathrm{~V}$ UPL sample. The weight concentration of carbon, fluorine, platinum and sulphur are $51 \%, 35 \&, 4 \%$ and $5 \%$, respectively. Here, the platinum band is better defined as compare to EOL-1.2V. One can see a significant decrease in concentration of platinum and no major change is observed in other elemental composition.

\subsubsection{Mechanical Characterization of Membrane-Catalyst Interface}

Peak-force tapping AFM was used to detect the aging process of catalyst in a PEM fuel cell subjected to accelerated stress tests. In peak-force tapping mode, force-distance curve is reconstructed at every image point. With this mode, besides topography and phase image, local data on adhesion force, deformation, dissipation, and elastic modulus can be obtained. This information is crucial to better understand the degradation mechanisms of the catalyst layer.

Peak-force tapping mode is a new operating mode of AFM that can generate nanomechanical mapping of surfaces. The conventional tapping mode in AFM is vibrated near the resonant 
frequency of the cantilever while it is scanned across the sample. The AFM tip only contacts the sample surface for a small percentage of the time, keeping the tapping force low and the lateral forces negligible. A drawback to this technique is that the resonant behaviour of the tip also acts as a filter, making it impractical to reconstruct force curves with sufficient precision to extract quantitative mechanical information. The peak-force mode has the ability to acquire and analyze the individual force curves from each tap that occurs during the imaging process. To quantitatively measure different material properties such as modulus, adhesion, dissipation, and deformation, it is necessary to measure the instantaneous force on the AFM tip rather than timeaverage of the force over time, as is done in tapping mode. In peak-force tapping, the modulation frequency is intentionally chosen to be significant lower than the cantilever resonant frequency. The novelty of this mode lies in the control of the maximum normal force ("peak force") that is applied at each location of the surface. Unlike in tapping mode where the feedback is based on the amplitude value, the imaging feedback is based on the peak-force of the force-distance curves. Figure 56a shows how the tip is subject to attractive (negative) and repulsive (positive) forces during an approach-retract cycle. Figure 56b represents how the different mechanical properties can be extracted from each individual force curve.
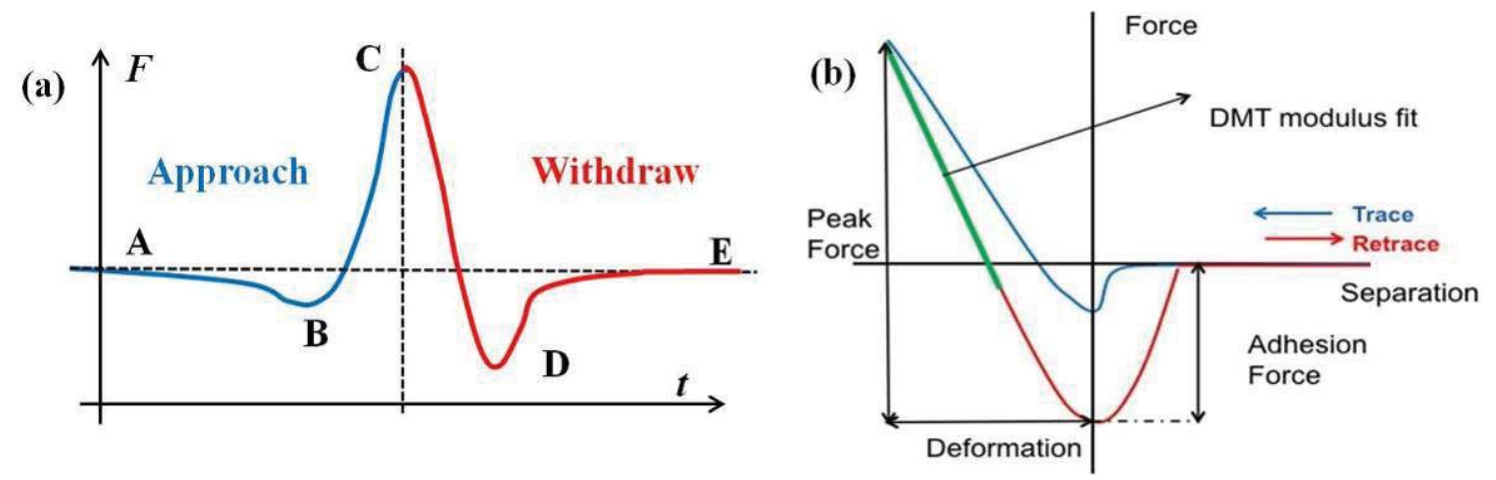

Figure 56: Peak-force tapping mode principles (a) plot of the AFM tip force interactions during a single approach-retract cycle. (A) The AFM tip is close to the surface and experiences an attractive field first until it reaches the contact point (B). At this junction it experiences a repulsive field as it starts to indent into the sample. (C) Represents the maximum peak-force that is used for the feedback loop. In the second half of the cycle, the AFM tip pulls off until it reaches its maximum adhesion (D) and then returns to its starting position (E). (b) Plot of the force vs. displacement showing how the different material properties are extracted from the force curve. In the peak-force tapping AFM the maximum force ('peak force') is set to be constant for each measurement position

To obtain elastic modulus, the retract curve is fitted using contact mechanic models. The adhesion force is the minimum force between sample surface and the AFM tip while the dissipation can be calculated by integrating the area between the two curves. Deformation is defined as the difference of separation at zero force and peak force.

Prior to the experiment and in order to obtain quantitative data directly, the AFM probe was calibrated as follows: first, the tip was engaged on a stiff sample (sapphire), a representative force curve was captured, and the deflection sensitivity was calculated from its slope. The spring constant was calculated by using a thermal tune (a sweep in frequency allows finding the resonance peak). Finally, the tip radius was automatically calculated by scanning a calibration sample, which enables the calculation of the tip shape by using the blind reconstruction technique provided in the Nanoscope Analysis software. This last step is of particular 
DE-EE0000466

Ballard Material Products Inc.

importance since the sample's mechanical properties, especially the Young's modulus, are strongly dependent on the tip shape. All AFM images consisted of $512 \times 256$ pixels with the scanning rate of $0.2 \mathrm{~Hz}$.

The most common and widely used fit for modulus measurements is the Hertz fit. JohnsonKendall-Robert (JKR) and Derjaguin-Muller-Toporov (DMT) models are well known extensions from the classical Hertz model. The three models are mathematically represented as:

$$
\text { Hertz: } \begin{aligned}
& F & =\frac{\mathbf{4}}{\mathbf{3}} E^{*} \sqrt{R} \delta^{\frac{\mathbf{3}}{2}} \\
\mathrm{DMT}: & F & =\frac{\mathbf{4}}{\mathbf{3}} E^{*} \sqrt{R} \delta^{\frac{\mathbf{3}}{2}}-2 \pi R \gamma \\
\mathrm{JKR}: & F & =\frac{\mathbf{9}}{\mathbf{4}} E^{*} \sqrt{R} \delta^{\frac{\mathbf{3}}{2}} \Delta \gamma \pi \\
& \frac{\mathbf{1}}{E^{*}} & =\frac{1-v_{s}{ }^{2}}{E_{s}}+\frac{1-v_{t}{ }^{2}}{E_{t}}
\end{aligned}
$$

where $\mathrm{F}$ is force, $\delta$ is indentation, $\mathrm{R}$ tip radius, $E^{*}$ the reduced elastic modulus and $\gamma$ the work of adhesion defined as in Equation (2). The reduced elastic modulus comprises the modulus of elasticity and Poisson ratio of the sample, $E_{s}$ and $v_{s}$, and the modulus of elasticity and Poisson ratio of the AFM tip, $E_{t}$ and $v_{t}$. Hertz model does not consider adhesion between the two surfaces. In our work, adhesion force was 43 and $24 \mathrm{nN}$ for membrane and catalyst, respectively. This was a significant factor and thus more sophisticated approaches, such as the JKR and the DMT models were used. The JKR and DMT models assume the presence of short-range adhesion forces within the contact area and long-range adhesion forces outside the contact area, respectively. In general, DMT model is more appropriate for systems with hard materials having low surface energy and small radii of probe curvature. The JKR model applied better to softer materials with higher surface energy and larger probes. The choice between the JKR and DMT models can be made on the basis of the use of Maugis parameter $\alpha$, which is a dimensionless parameter ranging from 0 to 1 ( $\alpha=1$ corresponds to the JKR case and $\alpha=0$ corresponds to the DMT case).

$$
\begin{aligned}
& \alpha=-\frac{50}{51\left(e^{\frac{250 \lambda}{231}}-1\right)} \\
& \lambda=1.157\left(\frac{w_{a d h}^{2} R}{E^{* 2} D_{0}^{3}}\right)^{\frac{1}{3}},
\end{aligned}
$$

where $w_{a d h}$ is the adhesion energy per unit area and $D_{o}$ is the inter-atomic distance between the atom of the probe and sample at contact. To evaluate the Maugis factor, The $E_{t}$ was assumed to be $160 \mathrm{GPa}$. The poison ratio for the AFM tip and sample is 0.3 . The tip radius was calculated to be $14.3 \mathrm{~nm}$. $\mathrm{D}_{0}$ was assumed to be $0.16 \mathrm{~nm}$ for both membrane and catalyst. Using these values, we estimate the Maugis parameter to be 0.27 and 0.08 for membrane and catalyst, respectively, thus justifying the use of DMT model. 
Figure 57 shows the three-dimensional maps of topography, peak force error, elastic modulus, adhesion, and deformation of a fresh catalyst-membrane interface sample as acquired by peakforce tapping mode of AFM, respectively. Figure 57a compares the surface topography of catalyst and the Nafion membrane. It is evident from the scale bar in Figure 57a, the catalyst layer has rougher topography with average roughness of $64 \mathrm{~nm}$ in comparison to Nafion membrane with average roughness of $21 \mathrm{~nm}$. This is expected considering that the catalyst consists of carbon particles, while the membrane is a continual film of polymer. The peak force error map, shown in Figure 57b is essentially the peak force set point plus the error. It provides a sensitive edge detection technique and is very helpful in visualizing fine details in topography that are difficult to see in the regular height image data of Figure 57a.
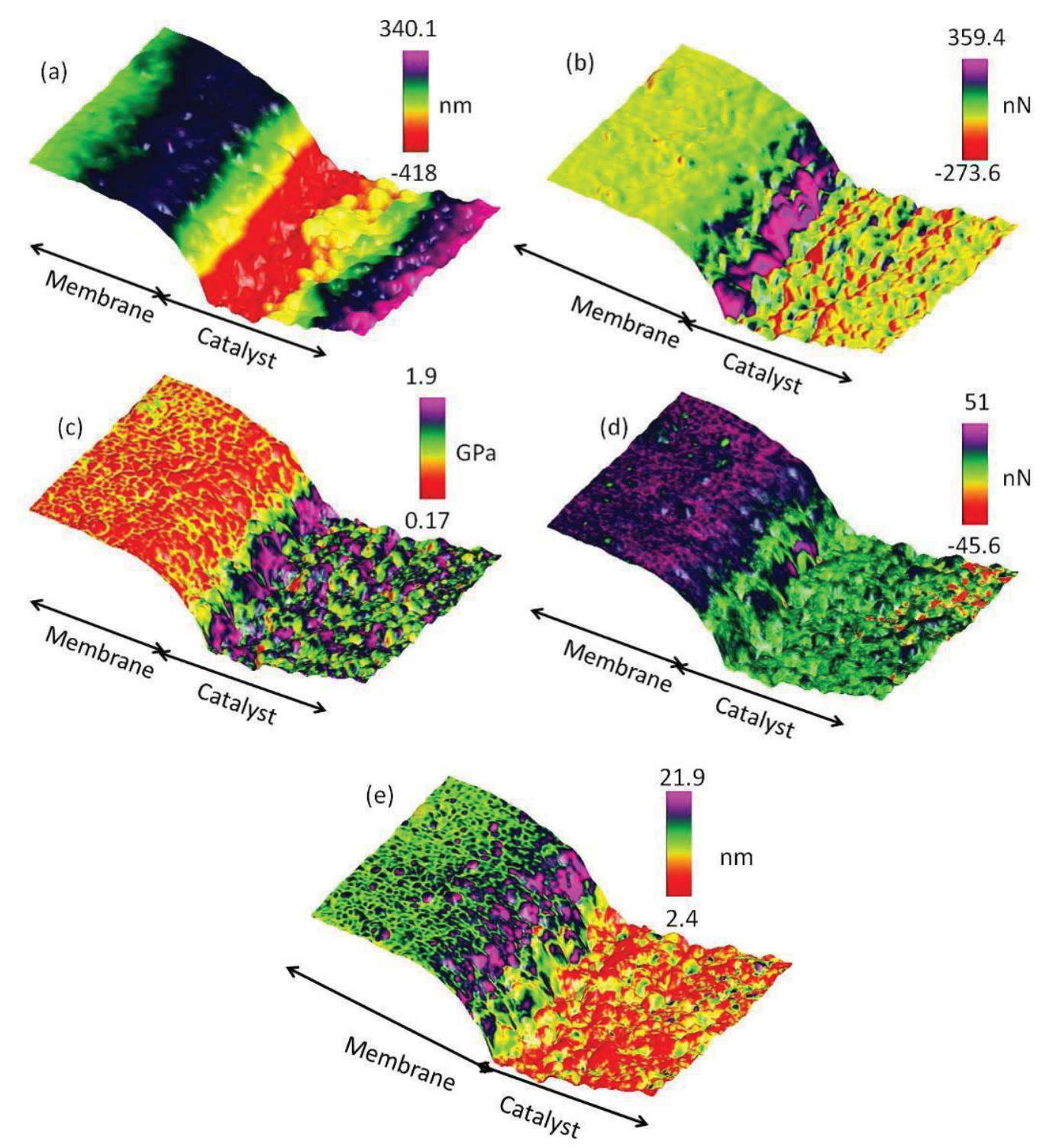

Figure 57: Three-dimensional AFM images of Fresh membrane-catalyst interface: (a) Height (b) Peak force error (c) Modulus (d) Adhesion (e) Deformation. The scan size and scan rate are $10 \times 5 \mu \mathrm{m}$ and $0.5 \mathrm{~Hz}$, respectively 
Since the AFM tip is calibrated, we can obtain quantitative maps of Young's modulus as shown in Figure 57c. The average modulus of membrane and catalyst layer is 0.54 and $1.2 \mathrm{GPa}$ respectively. The adhesion map shown in Figure $57 \mathrm{~d}$ reflects the atomic interaction between the AFM tip and CCM sample. The adhesion force between Nafion ionomer particles and the AFM tip $(37 n N)$ was found to be significantly higher as compare to carbon black particles (9nN). The deformation map in Figure 57e shows the maximum penetration of AFM tip into the surface at the peak force. The deformation is high for Nafion ionomer $(14 \mathrm{~nm})$ and relatively low for the rigid carbon particles $(3.7 \mathrm{~nm})$.

The dry Nafion membrane behaves as an elastomer, which has a lower strength but much greater elongation. With its perfluorinated carbon backbone it might be expected that the large fluorine atoms in Nafion would cause conformational rigidity due to restricted bond rotations. The fluoropolymer chains are rather flexible in the sense of torsion. Thus, one can see Nafion membrane has low stiffness but high adhesion and deformation value as compare to catalyst layer.

Figure 58a shows 2-D AFM height image of fresh membrane-catalyst interface. To calculate the elastic modulus, one has to precisely measure the interaction forces between the AFM tip and sample surface. The force-distance curves for membrane (Figure 58b) and catalyst layer (Figure 58c) was obtained at a maximum applied force of $30 \mu \mathrm{N}$ using Nanoindentation method in AFM. The penetration depth for both components is $\sim 300 \mathrm{~nm}$. The stiffness of the sample can be extracted from the part of the curve where the AFM tip is in contact with the sample.

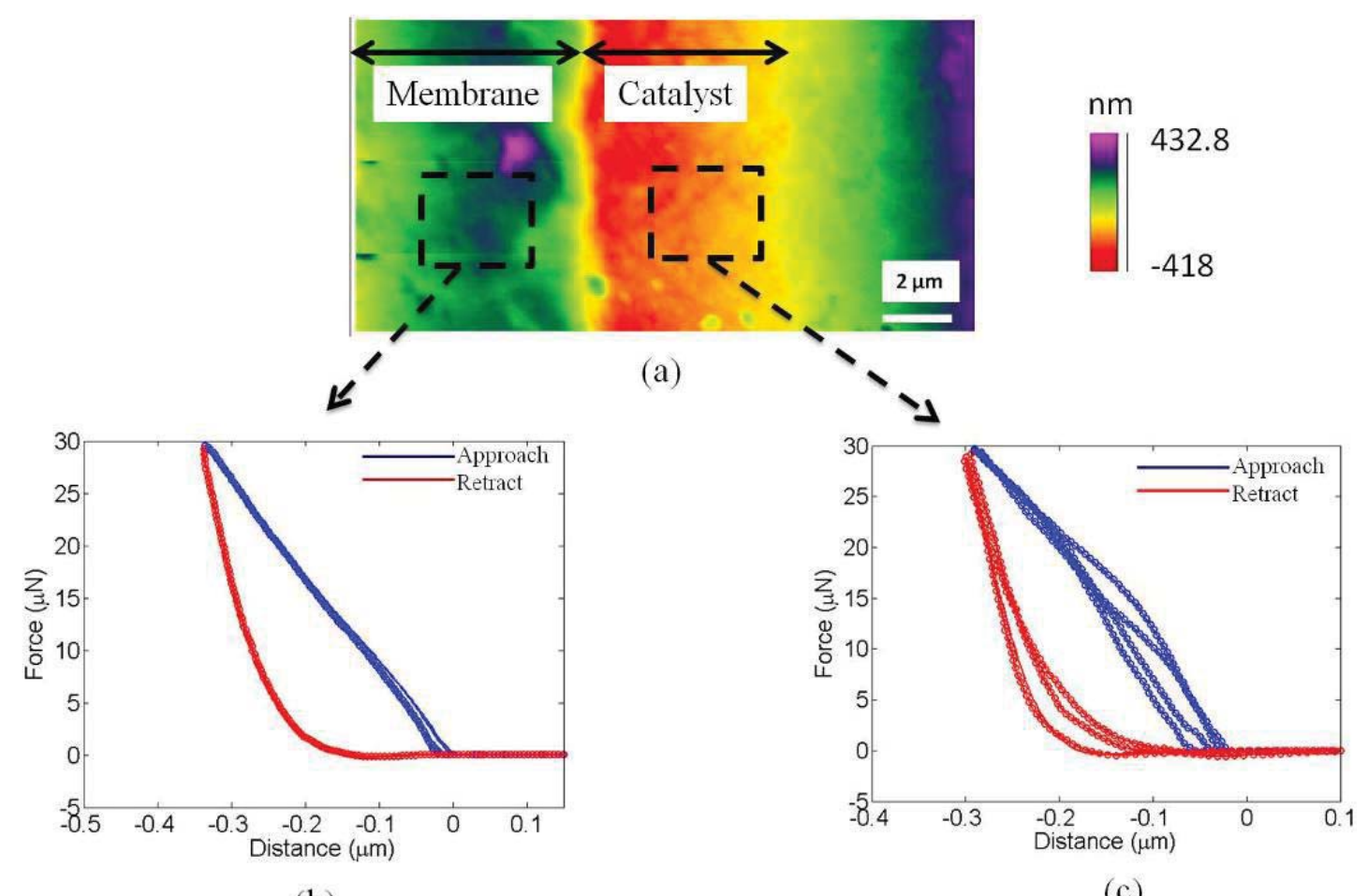

(b)

(c)

Figure 58: AFM height images of membrane-catalyst interface. Force indentation curves of (b) Membrane (c) Catalyst layer. The indentations were done with $30 \mu \mathrm{N}$ load forces. 5 curves were obtained at different locations on the surface 


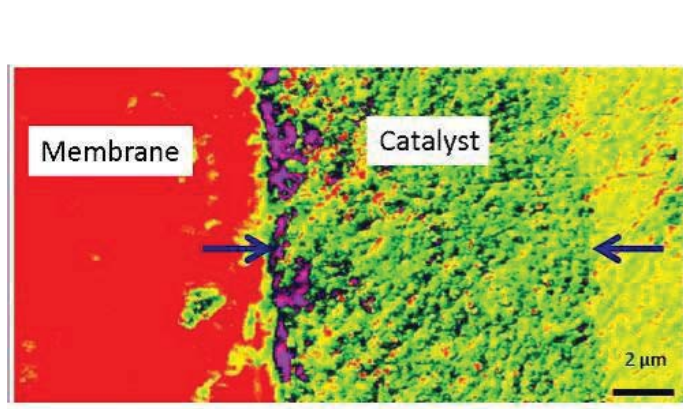

(a)

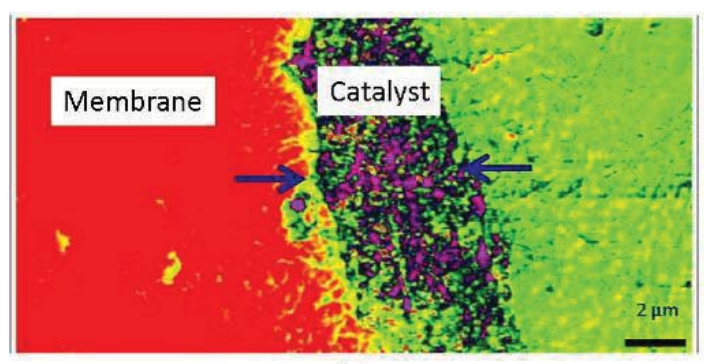

(c)

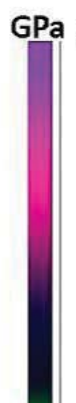

2

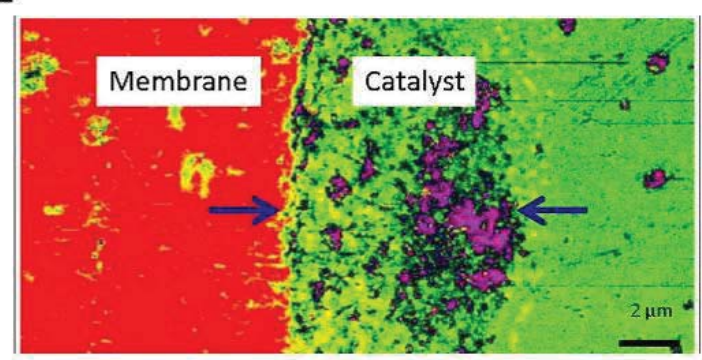

(b)

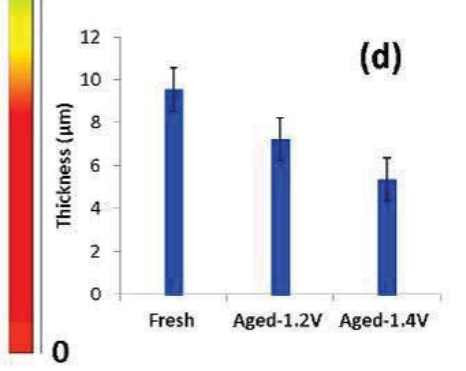

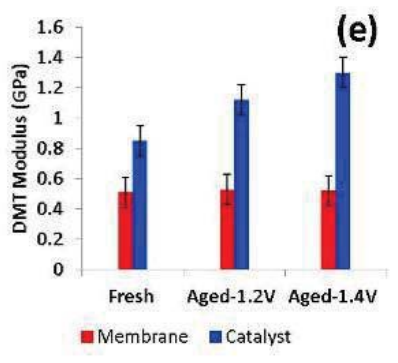

Figure 59: DMT modulus maps of membrane-cathode catalyst interface: (a) Fresh, (b)
Aged-1.2V, and (c) Aged-1.4V. (d) Thickness variation in catalyst layer (e) Average elastic modulus of membrane and catalyst layer. The scan size is $20 \mu \mathrm{m}$. The arrows indicate the region of catalyst layer. The red areas in modulus map represent the modulus range between $0.4-0.6 \mathrm{GPa}$, while yellow-green areas have fairly high modulus in the order of $1 \mathrm{GPa}$. The pink areas represent cluster of particles having high modulus in range of $1.5-2 \mathrm{GPa}$

Figure 59 shows DMT modulus image of membrane-catalyst interface of fresh, aged-1.2V and aged-1.4V sample. The thickness of catalyst layer in Fresh CCM sample was reported to be $9.45 \mu \mathrm{m}$ (Figure $59 \mathrm{a}$ ). At $1.4 \mathrm{~V}$, its thickness decreased by more than $40 \%$ (Figure 59c). The average elastic modulus of the membrane and catalyst layer is 0.83 and $1.34 \mathrm{GPa}$, respectively. The high modulus peaks (represented by pink regions) in Fresh CCM are largely concentrated at the interface of membrane and cathode catalyst layer, while in Aged-1.2V, it appears to be at the end of catalyst layer. It is interesting to see the modulus value increase by $\sim 50 \%$ in Aged$1.4 \mathrm{~V}$, while the thickness of the layer reduced by $\sim 40 \%$, indicating a strong correlation between modulus and thickness. The gain in the modulus of Aged-1.4V can be due to Nafion ionomer degradation (loss of soft material) in catalyst. Some of the Nafion ionomer may dissolve and wash out during the fuel cell operation. The key to the strength of any material is dislocation. According to Halls-Petch equation, as the resistance to dislocation motion increases, the strength of the material also increases. Catalyst layer may undergo strain age hardening effect during the fuel cell operation, which can be caused by change in temperature (freeze/thaw cycle). This process produces fine particles of an impurity phase, which impede the movement of dislocations. Since dislocation is often the dominant carrier of plasticity, this serves to harden the catalyst layer. The change in modulus of membrane is less significant, possibly due to retained crystallinity after aging. While a significant amount of work has focused on the degradation of Nafion membrane, little has been done regarding the degradation of ionomer in the catalyst. This information can be retrieved from adhesion image as shown in Figure 60. 


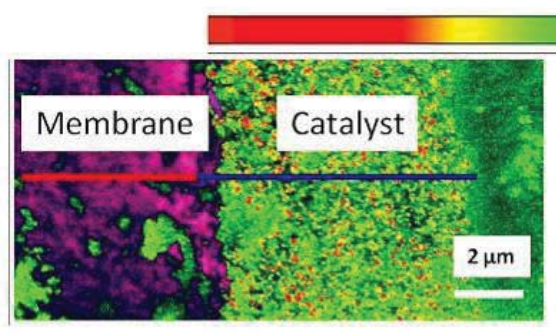

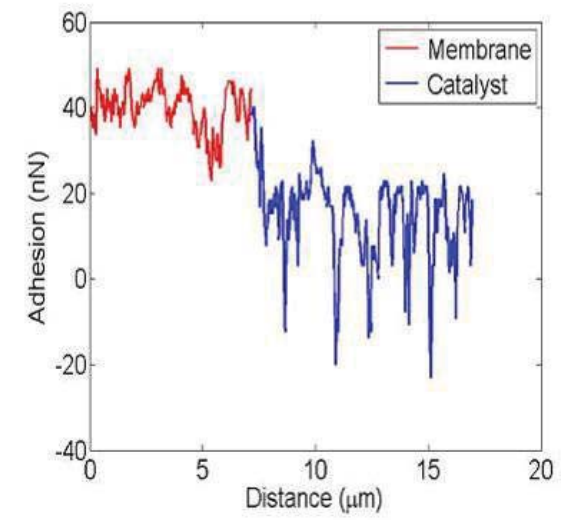

(a)
$\mathrm{nN}$
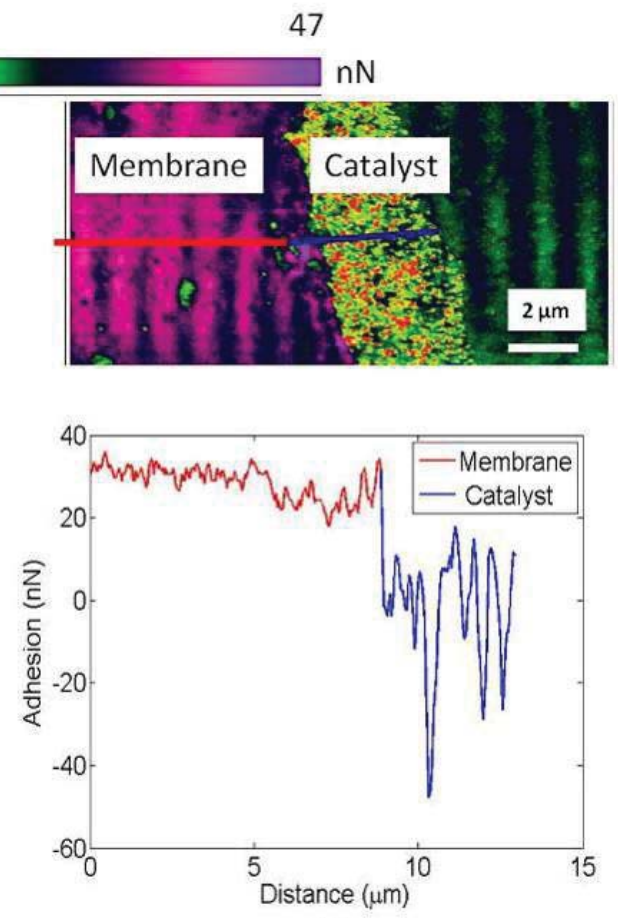

(b)

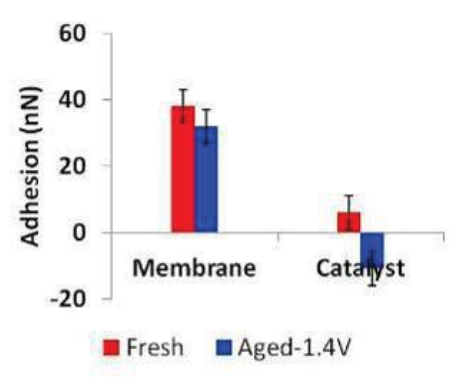

(c)

Figure 60: Adhesion map and section line of membrane-cathode catalyst interface: (a) Fresh (b) Aged-1.4V c) Average Adhesion value of membrane and catalyst. The scan size is 20um. (The pink region represents high adhesion areas while green and red areas dominantly in catalyst have low adhesion

A high value of the adhesion force is an indication of high molecular forces between the AFM tip and sample surface. From Figure $60 a$, the adhesion force of membrane is significantly high as compared to catalyst layer. The average adhesion for membrane and catalyst layer in fresh and aged condition is summarized in Figure 60c. The comparison revealed an overall decrease of adhesion for both the components after aging. The Nafion ionomer in the catalyst is susceptible to many of the degradation mechanisms that are described for the fuel cell membrane. As a result of chemical ionomer degradation in the catalyst, the interface between carbon particle and ionomer is lost, consequently reducing the electrochemically active surface of the catalyst. As the ionomer acts as a binder in the catalyst, it has a strong impact on the ionic conductivity of the electrode. The structural instability due to ionomer dissolution during long-time exposure in high-humidified conditions can change the adhesion properties, leading to degradation of the fuel cell.

Nafion has many characteristics that makes it suitable for use as a membrane in fuel cells, most importantly, when hydrated it exhibits high intrinsic proton conductivity and it is chemically and electrochemically stable in an acidic and oxidizing environment. The structure of Nafion has phase-separation morphology of distinct hydrophilic and hydrophobic regions. The hydrophilic phase provides an ionically conductive water-filled network for proton conduction. Although the study of Nafion ionomer structure is an on-going process, it is well known that the microstructure of Nafion strongly depends upon the temperature, the hydration state, and the current flow. It is 


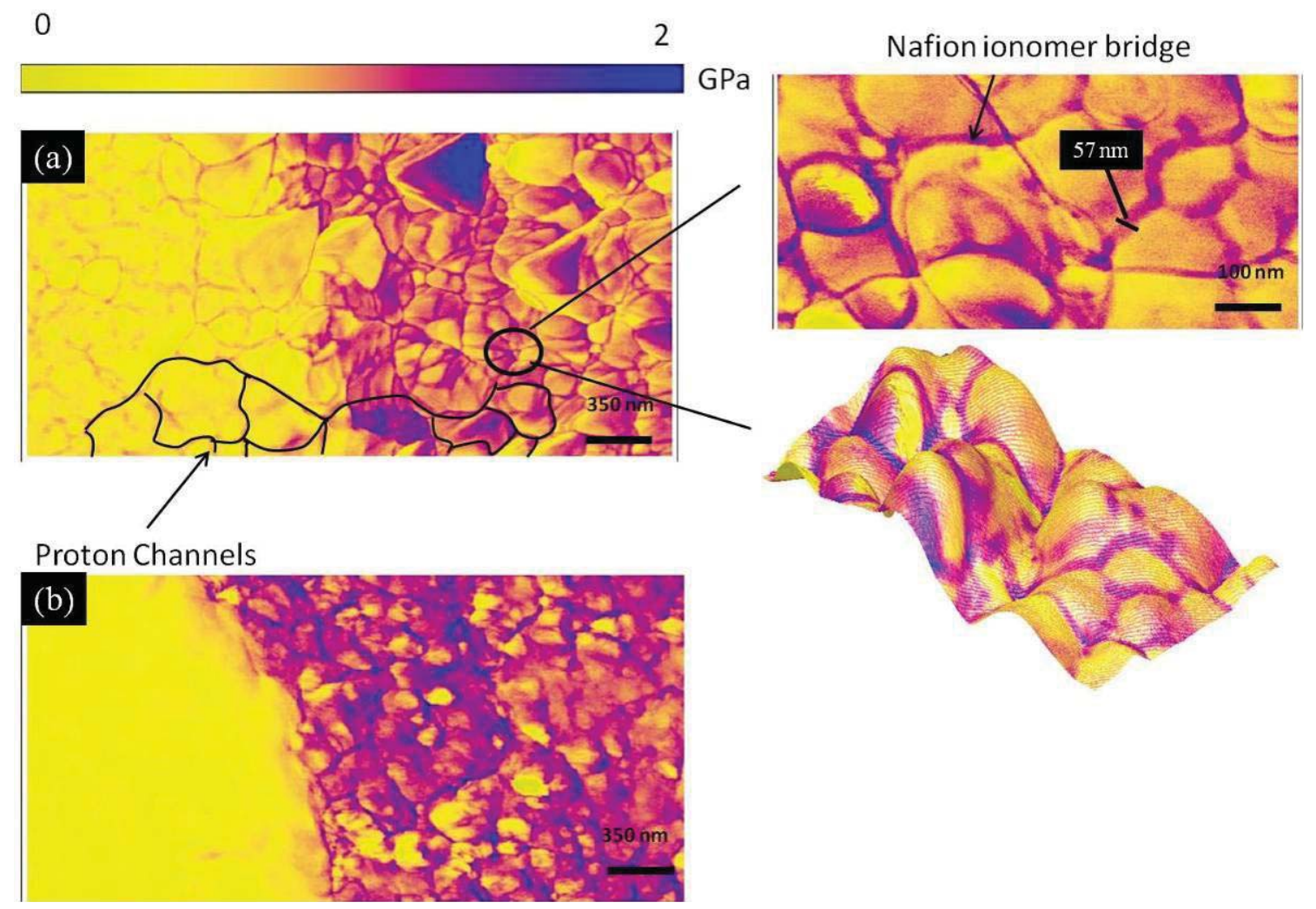

Figure 61: DMT modulus image of BOL membrane-catalyst interface: (a) BOL (b) EOL 1.4V

evident that proton channels can only be seen in membrane and catalyst layer when hydrated but we observed formation of Nafion ionomer connectivity in DMT modulus AFM image at high magnification. It is important to note that these channels were not observed in height and peak force AFM image.

Figure 61 shows the high magnification DMT modulus image of fresh and aged membranecatalyst interface. The dense network of polymer strands can be seen in Fresh CCM (Figure 61a), while no channels was observed in aged membrane. These channels have slightly higher modulus that the Nafion and carbon particles. The diameter of these channels is around 50$60 \mathrm{~nm}$. This result does not support or confirm the proton channels and further investigation will be required to test the hypothesis but it shows how peak-force AFM can be applied to study an interesting phenomenon at nanoscale. 


\subsubsection{Mechanical Properties of Membrane-CL Interface for Samples with Different Ionomer Content}

The variation in mechanical properties at interface with MEA of different ionomer was studied using peak-force tapping mode in AFM. Figure 62 compares the membrane-catalyst interfaces of $12,23,30$, and $50 \%$ ionomer.

There is no significant change in modulus of membrane. Catalyst layer of $12 \%$ ionomer has average modulus of $2.01 \mathrm{GPa}$, while it is $1.12 \mathrm{GPa}$ for $50 \%$ ionomer. The modulus of catalyst layer decreases with increase in ionomer content. The thickness of catalyst layer for all the samples is between $8-12 \mu \mathrm{m}$. Three different areas were analyzed and the slope of elastic modulus at the interface was calculated for each sample. Figure 63 summarize all the results

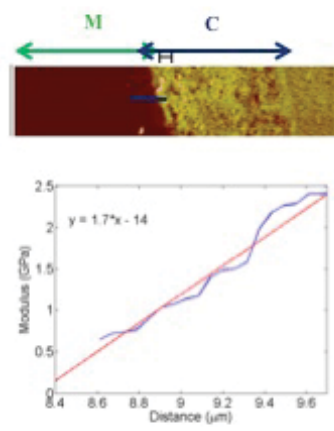

(a)

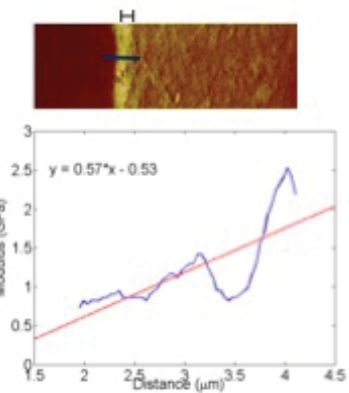

(b)

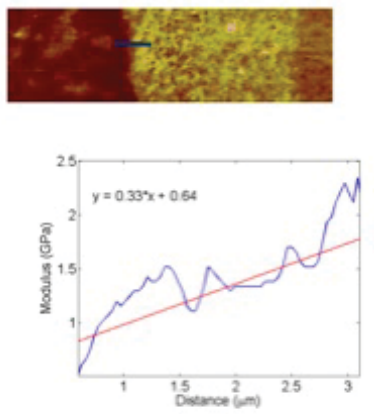

(c)

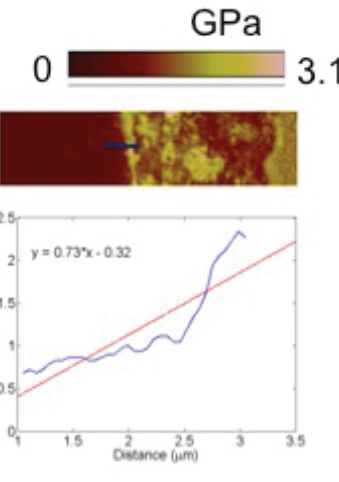

(d)

Figure 62: DMT modulus image of membrane-catalyst interface of MEA with different ionomer: (a) $12 \%$ (VD420) (b) $23 \%$ (VD421) (c) $30 \%$ (VD422) (d) 50\% (VD423)

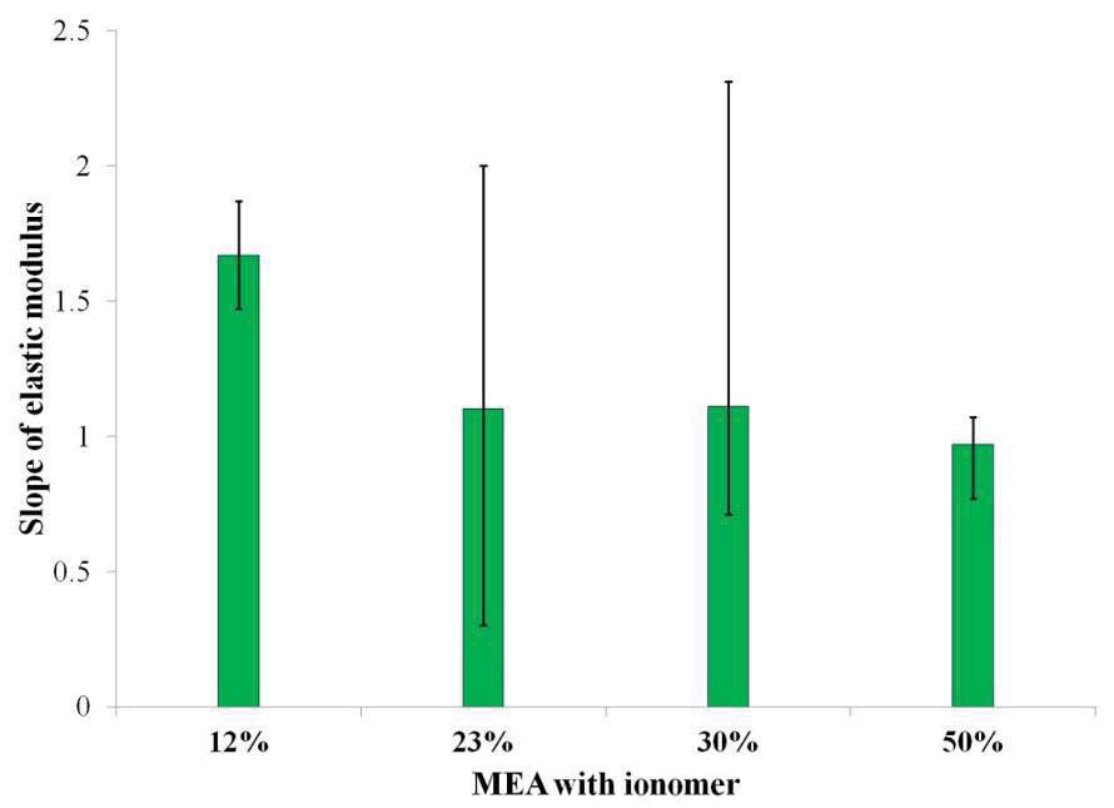

Figure 63: Plot of slope of elastic modulus vs. MEA with ionomer 
and shows the slope of elastic modulus for MEAs with different ionomer contents. One can see 12 and $50 \%$ ionomer have a consistent slope while a big variation is observed for 23 and $50 \%$ ionomer. Due to the large error in the measurement it is difficult to derive conclusions; however, a weak correlation between slope of elastic modulus and Nafion content seems to exist as the average value of slope decreases with increasing ionomer content.

\subsubsection{Electrical Characterization of Membrane-Catalyst Interface}

A measuring technique based on CAFM may give insight into the catalyst structure and to provide details of the structure-electrical conductivity correlation at the nanoscale. This information is crucial to better understand the degradation mechanisms of catalyst. The experiment was performed using an extended tunnelling (TUNA) module. The working principle of cAFM is shown in Figure 64. All the measurements were recorded using a Pt/lr coated Multi$75 \mathrm{G}$ (BudgetSensors ${ }^{\mathrm{TM}}$ ) AFM tip with a resonance frequency of $75 \mathrm{KHz}$ and spring constant of $40 \mathrm{~N} / \mathrm{m}$. Silver paint was used to coat the bottom of the CCM sample to make a reliable electrical contact with the AFM stage.

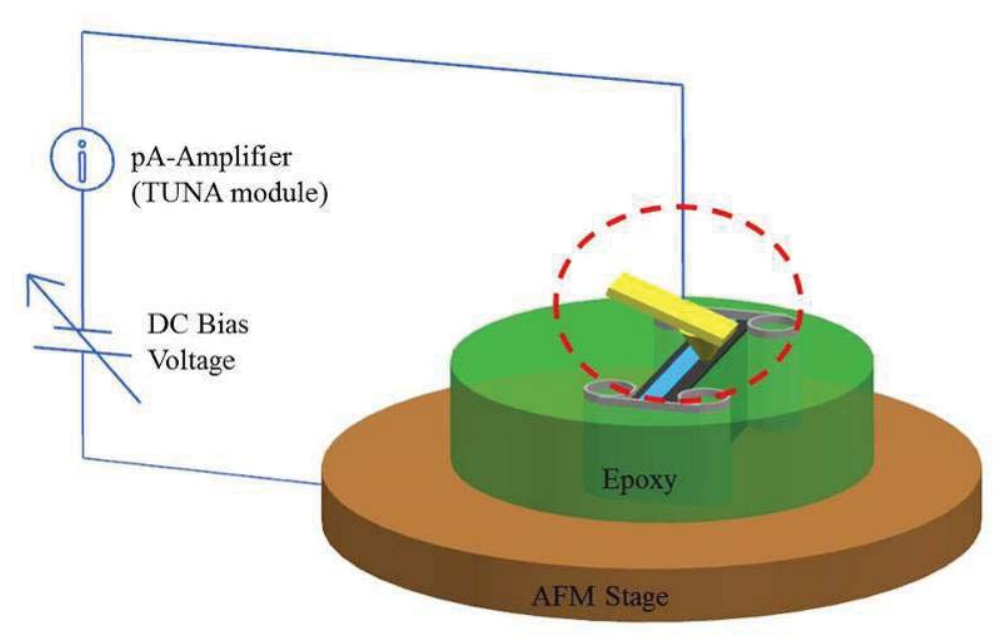

(a)

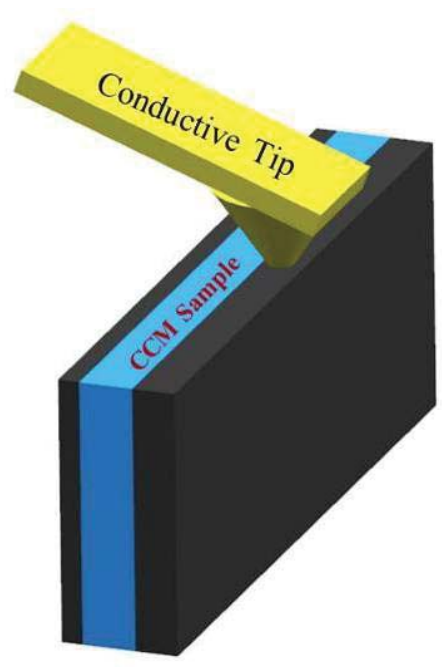

(b)

Figure 64: (a) Experimental set-up for cAFM. (b) Detail diagram showing the conductive AFM tip and CCM sample. A bias voltage is applied between a conductive tip and CCM sample embedded in epoxy. Silver paint is applied on the AFM stage-CCM cross section interface to make electrical contact between the CCM sample and the stage

The geometry of tip determines the lateral resolution, which is roughly equal to the end radius of the tip. With the tip close to the CCM, a selectable bias voltage is applied between the conductive tip and the sample. While scanning in contact mode, a low-noise linear current amplifier with a range of 80 to $120 \mathrm{pA}$ and extra gain filter senses the resulting current passing through the sample. By maintaining a constant force between the AFM probe and the sample, simultaneous topographic and current images are generated, enabling the direct correlation of local topography with electric properties. To determine the appropriate voltage bias, a cAFM 
test was done on catalyst layer with known Nafion content of $30 \mathrm{wt} \%$. Figure 65 shows a series of electrical current images taken at bias voltages ranging from 100 to $1000 \mathrm{mV}$. Since the catalyst is made of carbon particles, high electrical conductivity is expected and the current sensitivity was set to $100 \mathrm{nA} / \mathrm{V}$ to detect the current signal. The blue-coloured area shows high current ranges larger than $1 \mu \mathrm{A}$, representing carbon/Pt structure. The areas coloured in yellow indicate regions with no or very little current signal corresponding to non-conductive Nafion material. The non-conductive area was then plotted vs. the bias voltage as shown in Figure 65b. As expected, the percentage of non-conductive area decreases with increase in bias voltage. In other words, bias voltage is directly proportional to electrical conductivity. At $500 \mathrm{mV}$, the percentage of non-conductive area is $\sim 33 \%$, which is nearly equal to the Nafion content in catalyst $(30 \%)$. Thus $500 \mathrm{mV}$ bias voltage was selected to generate the electrical current map for CCM samples.

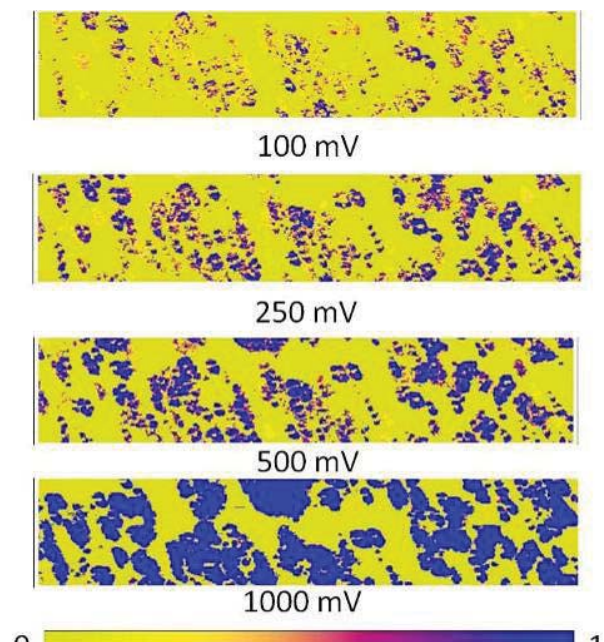

0

(a)

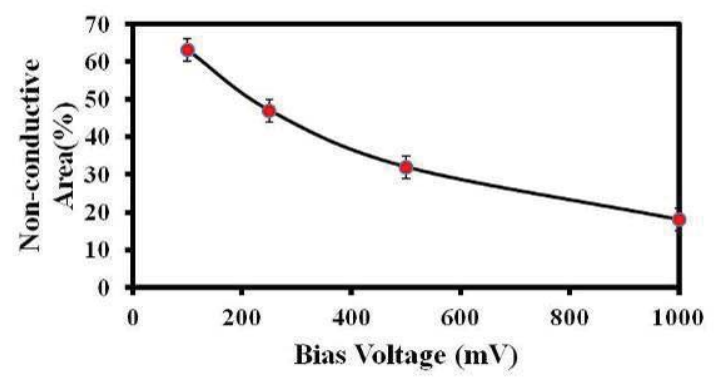

(b)

Figure 65: (a) Electrical current images of catalyst at different bias voltage. (b) Percentage of non-conductive area for the catalyst with $30 \%$ Nafion content. Scan size is $5 \times 1 \mu \mathrm{m}$. Error bar in the plot represent $95 \%$ confidence intervals 


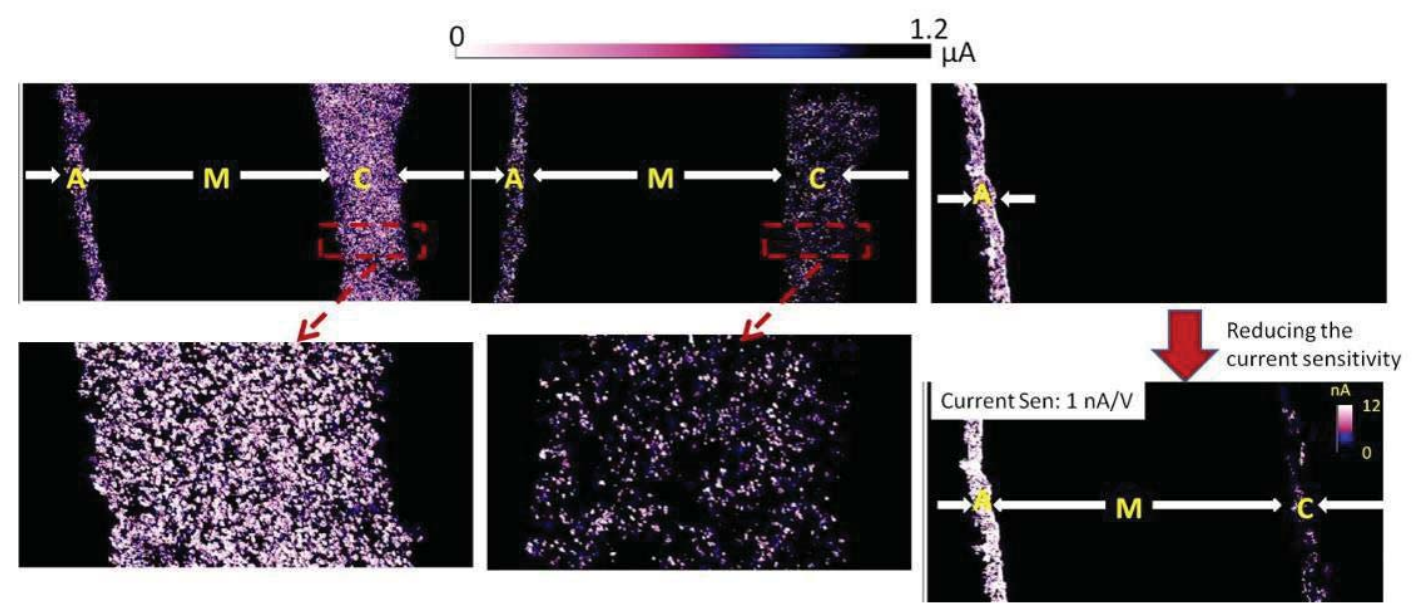

(a)

(b)

(c)

Figure 66: Electrical current images of $\mathrm{CCM}$ and $\mathrm{CL}$ at $100 \mathrm{nA} / \mathrm{V}$ current sensitivity: (a) Fresh (b) Aged-1.2V. Current images of Aged-1.4V CCM at current sensitivity of 100nA/V $\& 1 \mathrm{nA} / \mathrm{V}$. The bias voltage is $500 \mathrm{mV}$. A, M and $\mathrm{C}$ represent the anode catalyst layer, membrane and the cathode catalyst layer respectively. The white areas represent cluster of $\mathrm{Pt} / \mathrm{C}$ particles having high current regions above $1 \mu \mathrm{A}$, pink areas represent the current in the range of $0.5-1 \mu \mathrm{A}$. The black areas have negligible or no current signal

CAFM was performed to study the electrical conductivity of catalyst layer from fresh to aged condition. CAFM is a variant of contact-mode AFM in which a potential is applied to the sample, causing current to flow between the sample and a conductive tip. The current measurement module of cAFM has fixed sensitivities ranging from 10pA/V to 100nA/V. Selection of 100nA/V can detect maximum current signal of $1.2 \mu \mathrm{A}$. The noise level of this module (typ. 50fA) allows one to take extremely sensitive current images of the sample. Figure 66 shows the current images of Fresh, Aged1-2.V and Aged-1.4V CCM samples.

The Nafion membrane is non-conductive at dry condition, thus no electrical current signal was detected from the membrane. On the other hand, the electrical current signal can be detected from anode and cathode catalyst layers. The intensity of electrical current signal is much less on cathode catalyst of Aged-1.2V than fresh sample. Interestingly, no current electrical signal is detected on Aged-1.4V. As one reduces the current sensitivity to $1 \mathrm{nA} / \mathrm{V}$, some current patches of cathode catalyst can be observed. The electrical current signal in fresh and Aged-1.2V are in order of micro-ampere while in Aged-1.4V, the maximum current signal is just $12 \mathrm{nA}$. 


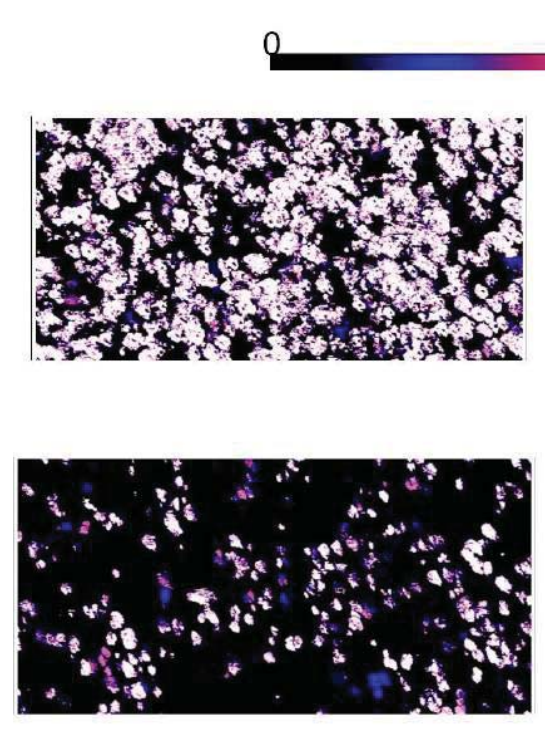

(a)
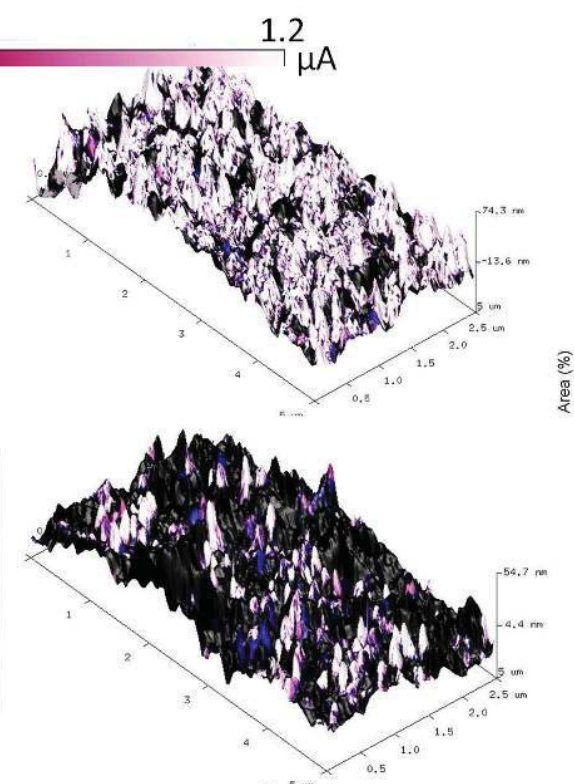

(b)

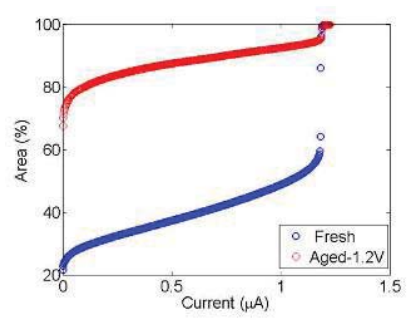

(c)

Figure 67: The2D electrical current and 3D overlay of electrical current on topography: (a) Fresh CL (b) Aged-1.2V CL. (c) Current distribution curves

Scan size of $5 \times 2.5 \mu \mathrm{m}$ of cathode catalyst of was selected to measure the current variation in $\mathrm{BOL}$ and EOL-1.2V samples (Figure 67). One can see, non-conductive areas increase from 25 to $70 \%$ in EOL-1.2V surface. From the current distribution curves, $51 \%$ of the area in BOL catalyst have high current peaks of $1.2 \mu \mathrm{A}$ while in EOL-1.2V catalyst surface, it is barely $3 \%$. Thus, the conductivity drops by $94 \%$ in EOL-1.2V catalyst layer.

Platinum nanoparticles are dispersed onto the carbon support to increase the active surface area. For this, the BOL catalyst has a good electronic network, providing pathway for electrons to reach platinum nanoparticles. One can see very high conductive regions in fresh catalyst (pink areas in Figure 67a) in the range of $1-1.2 \mu \mathrm{A}$. The second pathway being the Nafion, it must be present at the active site to provide pathway of protons. This is shown by enclosed black area in catalyst. The oxidation of carbon support affects the catalyst structure, which leads to loss of electrical conductivity. Carbon corrosion destroys the active sites and leads to the separation of carbon electrical network. Thus one can see a significant drop of conductivity in aged catalyst layer surface (Figure 67b). In BOL catalyst surface, the carbon black particles have dense and uniform conductive network giving high electrical conductivity. The conductivity of carbon black depends on number of parameters like volume fraction (percolation threshold), its structure and the particle size, etc. Due to carbon corrosion, the conductive network of carbon black in catalyst layer breaks down resulting in dramatic loss of conductivity and collapse of the structure. 
DE-EE0000466

Ballard Material Products Inc.

\subsubsection{Summary}

The surface of catalyst layer and GDL-MPL were investigated using stereo imaging, interferometry, and surface profilometer to characterize the surface roughness. Interferometry data has been used as an input for the contact resistance model (described in Chapter VII). The average roughness of catalyst layer and MPL was found to be $3.7 \mu \mathrm{m}$ and $6.5 \mu \mathrm{m}$, respectively. These data were processed and exported to MATLAB. The roughness data can help to calculate the contact resistance between $\mathrm{CL}$ and GDL.

The mechanical properties of membrane-catalyst interface at the nanoscale were characterized by peak-force tapping mode atomic force microscopy (AFM). The technique demonstrated the capability to provide information on the local distribution of modulus and adhesion of membrane and catalyst from pristine to aged condition. The Modulus value increase by $\sim 50 \%$ in Aged$1.4 \mathrm{~V}$ samples, while the thickness of the layer reduced by $\sim 40 \%$, indicating a strong correlation between modulus and thickness. The modulus of the catalyst layer decreased with increasing catalyst layer ionomer content.

The CL showed substantial conductivity loss after aging at $1.4 \mathrm{~V}$ UPL as compared to BOT samples. The conductive area of aged cathode catalyst layer was reduced by $\sim 65 \%$ while no significant changes were observed in anode CL. Carbon corrosion was found to be the key factor for the loss of conductivity in the aged catalyst. While the cAFM technique shows promise and provided some insight into electrical conductivity changes within the catalyst, the contact resistance between the stage and sample was found to be a significant contributor to the overall results. 


\title{
DE-EE0000466
}

\section{Final Project Report}

Development of Micro-Structural Mitigation Strategies for PEM Fuel Cells: Morphological Simulations and Experimental Approaches

\author{
CHAPTER IV
}

\section{CORRELATIONS OF CATALYST LAYER STRUCTURE AND PERFORMANCE/DEGRADATION}

\author{
DESIGN WINDOWS
}




\section{TABLE OF CONTENTS}

4.1 Correlations of Catalyst Layer Structure and Performance /Degradation .................. 149

4.2 Impact of Composition, Component Characteristics and Processing on Catalyst

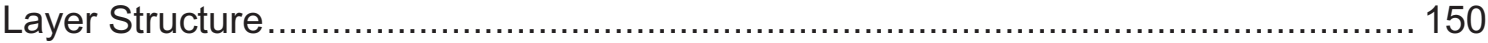

4.2.1 Catalyst and Ionomer Dispersion .................................................. 150

4.2.2 Impact of Catalyst Layer Property Parameters on Performance .................. 159

4.2.3 Structure Changes with Degradation .................................................. 165

4.2.4 Correlations Overall Summary......................................................... 167

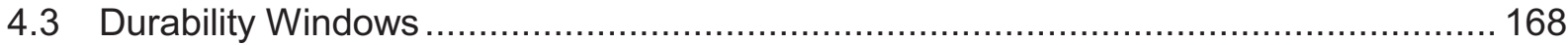

4.3.1 Structural Parameter Durability Windows …........................................ 168

4.3.2 Operational Durability Windows ..................................................... 170

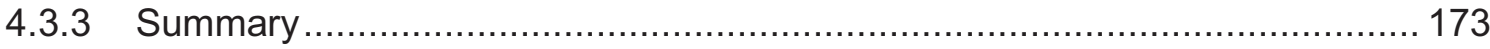


DE-EE0000466

Ballard Material Products Inc

\section{LIST OF FIGURES}

Figure 1: Interactions of component characteristics and catalyst layer composition, structure, properties and performance

Figure 2: Cathode Catalyst Layer Thickness Relationship with Catalyst Powder Bulk Density

Figure 3: Catalyst Powder Bulk Density Relationship to Catalyst Powder Pt Content

Figure 4: $\quad$ Catalyst Layer Thickness Relationship to Pt Loading

152

Figure 5: $\quad$ Catalyst Layer Thickness Relationship to catalyst layer lonomer Content

153

Figure 6: Catalyst Layer Mesopore Size Distribution Relationship to Catalyst Layer Ionomer Content

153

Figure 7: $\quad$ ECSA Relationship to Pt Loading

Figure 8: $\quad$ ECSA Relationship to Pt Size

154

155

Figure 9: $\quad$ ECSA Relationship to Pt Loading and Pt Size 155

Figure 10: Ionomer Volume Fraction and Porosity Relationship to lonomer Content 155

Figure 11: Ionomer Volume Fraction Relationship to Catalyst Powder Pore Volume 155

Figure 12: Ionomer Tortuosity Relationship to Ionomer Volume Fraction ${ }^{2} 156$

Figure 13: Catalyst Layer Ionic Conductivity Relationship to lonomer Content 157

Figure 14: Catalyst Layer lonic Conductivity Relationship to lonomer Tortuosity 157

Figure 15: Pore Size Distribution Relationship to lonomer Content 158

Figure 16: Catalyst Layer Porosity Relationship to Catalyst Powder Pore Volume 158

Figure 17: Effective Diffusivity Relationship to Porosity 158

Figure 18: Types of Polarization Losses Observed ${ }^{1} \quad 160$

Figure 19: Kinetic Loss Relationship to ECSA 162

Figure 20: Mass Transport Loss Relationship to Effective Diffusivity (Deff) 162

Figure 21: Mass Transport Loss Relationship to Catalyst Layer Thickness 162

Figure 22: Catalyst Layer Ionic Loss Relationship to Catalyst Layer lonic Resistivity 162

Figure 23: Catalyst Layer lonic Relationship to ECSA when Poor ECSA, Resistivity and

Diffusivity Occur Concurrently

163

Figure 24: Kinetic Loss and Mass Transport losses increase with increasing Catalyst Layer Ionic Resistivity

Figure 25: Kinetic Loss increases with decreasing Effective Diffusivity

Figure 26: Catalyst Layer lonic Loss increases with decreasing Effective Diffusivity

Figure 27: Catalyst Layer lonic Loss Increases with Increasing Catalyst Layer Thickness and Pt Depletion at the Membrane / Catalyst Interface.

164

164

164

165

Figure 28: STXM Volume Fraction Profiles across BOT and Degraded MEA Crosssections

167

$\begin{array}{lll}\text { Figure 29: } & \text { Comparison of Ballard square wave AST and DOE triangular sweep AST } & 168 \\ \text { Figure 30: } & \text { Cathode Pt Loading Durability Windows for 15\% performance loss } & 169\end{array}$

Figure 31: Durability window for ionomer loading for 15\% performance loss 169

Figure 32: Durability window for graphitic content of the catalyst carbon support for $15 \%$ performance loss

Figure 33: Time at UPL to cause 15\% performance loss and 40\% ECSA loss

Figure 34: Comparison of time at UPL to reach (a) $10 \%$ and $15 \%$ performance loss and (b) $40 \%, 50 \%$, and $60 \%$ ECSA loss

Figure 35: Effect of temperature on the time at UPL to reach $15 \%$ performance loss and $40 \%$ ECSA loss

Figure 36: Effect of temperature on the time at UPL to reach $15 \%$ performance loss and $40 \%$ 
DE-EE0000466

Ballard Material Products Inc

\section{LIST OF TABLES}

Table 1: Summary Table of Design Levers for BOT Catalyst Layer Structure and

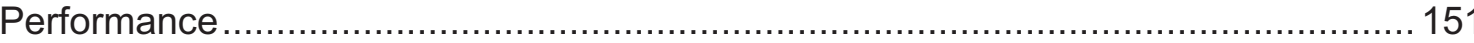

Table 2: Impact of catalyst layer properties on Polarization losses ................................ 161

Table 3: Effect of Degradation on Catalyst Layer Structure, Properties and Polarization

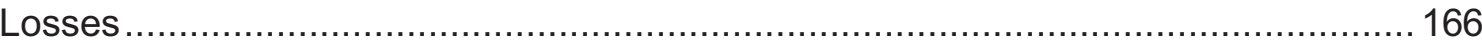

Table 4: Durability Windows for Structural Design Levers ............................................ 173

Table 5: Durability Windows for Operational Parameter .............................................. 173 


\subsection{Correlations of Catalyst Layer Structure and Performance /Degradation}

Correlations between composition, structure, properties and performance have been identified for the cathode catalyst layer $(\mathrm{CL})$. The purpose of this work is to:

- Correlate catalyst performance/catalyst structural change as a function of unit cell operational conditions and catalyst layer morphology/composition,

- Demonstrate the theoretical flow path of the model and allow for verification and validation of relationships between model predictions and experimental observations,

- Identify parameters that are key levers for the MEA designer, and

- Identify and propose parameters for optimization by the catalyst manufacturers.

The interactions of the measurables for the component characteristics and catalyst layer composition, structure, properties and performance are demonstrated through a flow chart, as shown in Figure 1. Flowing from left to right, the flow chart displays how the component characteristics and composition link to the catalyst layer structure, which in turn impact the catalyst layer effective properties and affect the reaction distribution, polarization losses and performance. Although most of the measurables have been quantified, the yellow and pale blue boxes highlight the gaps in quantification methods for important parameters which are included in and calculated by the model.

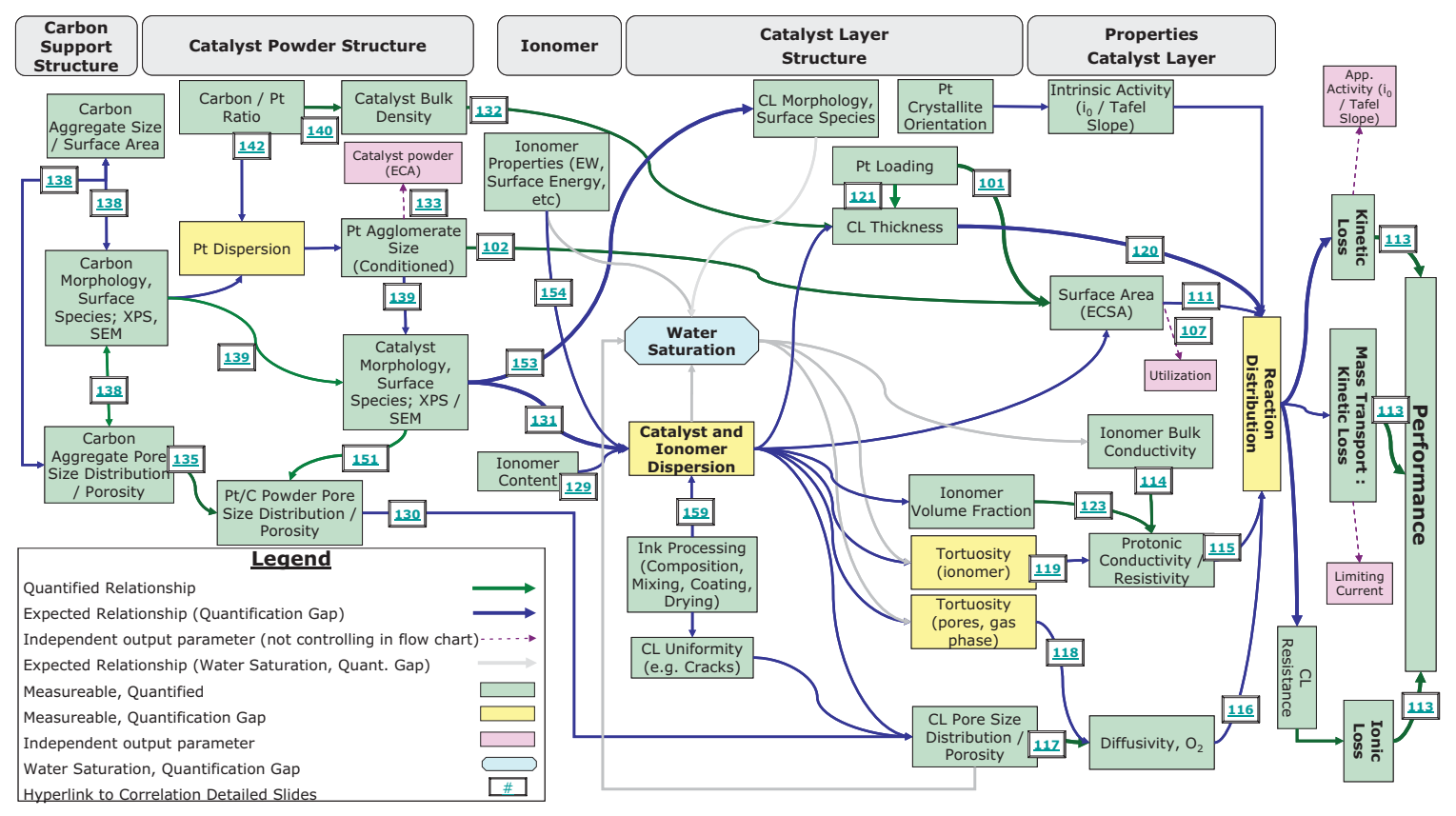

Figure 1: Interactions of component characteristics and catalyst layer composition, structure, properties and performance

Details of the main parameters and interactions shown on the flow chart are given in this section and have been subdivided into three main sections: i) impact of composition, component characteristics and processing on catalyst layer structure; ii) impact of catalyst layer structure on performance; and iii) structure changes with degradation. 
DE-EE0000466

Ballard Material Products Inc

\subsection{Impact of Composition, Component Characteristics and Processing on Catalyst Layer Structure}

\subsubsection{Catalyst and Ionomer Dispersion}

The catalyst layer structure is dependent on the catalyst and ionomer surface interactions and how these two components aggregate and disperse within the layer. Catalyst and lonomer Dispersion is used as a collective term to describe the overall catalyst layer structure and is a central component in the flow chart.

The Catalyst and lonomer Dispersion at present cannot be measured directly. It is therefore described by six parameters that describe the overall nature of the structure:

1) Thickness (measured)

2) Ionomer Volume Fraction (calculated)

3) Ionomer Tortuosity (calculated)

4) Pore Size Distribution / Porosity (measured)

5) Pore Tortuosity (calculated)

6) Catalyst Surface Area, ECSA (measured)

The Catalyst and lonomer Dispersion is controlled by the composition of the layer, the properties of the components, and the processing steps used to create the layer.

- The composition directly affects the structure due to the component volume fractions. In the flow chart, only the ionomer content $(w t \%)$ is shown, as the catalyst amount becomes redundant.

- Component properties affect the structure through interactions of their surface groups (catalyst-catalyst interactions, ionomer-ionomer interactions and catalyst - ionomer interactions). Therefore the properties of both the ionomer and catalyst are important and included.

- Processing of the components, ink and fabrication parameters all affect the resulting structure. These can include mixing, coating and drying processes, for example. Processing is outside of the scope of this project and therefore is not discussed in detail, but is included in the flow chart for completion.

Table 1 shows a summary of the main design levers which affect the Catalyst and lonomer Dispersion and their effects on performance. The individual design levers will be discussed in detail below. 
Table 1: Summary Table of Design Levers for BOT Catalyst Layer Structure and Performance

\begin{tabular}{|c|c|c|c|c|c|c|c|c|c|c|c|c|c|}
\hline \multicolumn{10}{|c|}{$\begin{array}{c}\text { Impact Matrix } \\
\text { Effect of Composition, Component Properties and Processing on BOT CL Structure }\end{array}$} & \multicolumn{4}{|c|}{$\begin{array}{c}\text { Impact Matrix } \\
\text { Effect on BOT Performance }\left(0.5 \mathrm{~A} / \mathrm{cm}^{2}\right)\end{array}$} \\
\hline & Design Lever & Range Studied & ECSA & Thickness & $\begin{array}{c}\text { Porosity, } \\
\text { CL }\end{array}$ & $\begin{array}{l}\text { Mesopore } \\
\text { Diameter, }\end{array}$ & \begin{tabular}{l|l} 
lonomer \\
Volume
\end{tabular} & $\begin{array}{l}\text { Diffusivity, } \\
\text { Effective }\end{array}$ & $\begin{array}{c}\text { Resistivity, } \\
\text { lonic }\end{array}$ & Kinetic Loss & MT Loss & $\begin{array}{l}\text { CL lonic } \\
\text { Loss }\end{array}$ & $\begin{array}{l}\text { Air } \\
\text { Performance }\end{array}$ \\
\hline \multirow{2}{*}{ 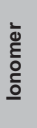 } & Ionomer Content & $\stackrel{\uparrow}{\uparrow}$ & & L & $\downarrow$ & $\downarrow$ & $\uparrow$ & $\downarrow$ & L & $\cup$ & $\cup$ & $\cup$ & $\cap$ \\
\hline & $\begin{array}{l}\text { lonomer } \\
\text { Property: EW }\end{array}$ & $\begin{array}{c}\uparrow \\
850 \mathrm{EW}-1050 \mathrm{EW}\end{array}$ & & $\cup$ & $\cup$ & N/A & $\cap$ & & $\uparrow$ & $\Gamma$ & $\uparrow$ & & $\downarrow$ \\
\hline \multirow{2}{*}{$\frac{\frac{E}{3}}{\frac{\mathrm{I}}{\bar{T}}}$} & Pt loading & $\begin{array}{c}\uparrow \\
0.05 \mathrm{mg} / \mathrm{cm}^{2}- \\
0.5 \mathrm{mg} / \mathrm{cm}^{2}\end{array}$ & $\uparrow$ & $\uparrow$ & $\cup$ & & $\cap$ & $\Gamma$ & & $\downarrow$ & & $\cup$ & $\uparrow$ \\
\hline & Pt Size & $\stackrel{\uparrow}{\uparrow} \underset{4.2 \mathrm{~nm}-7.0 \mathrm{~nm}}{ }$ & $\downarrow$ & & & $\uparrow$ & & & & $\uparrow$ & & $\downarrow$ & $\uparrow$ \\
\hline \multirow{4}{*}{ 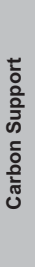 } & $\begin{array}{l}\text { Bulk Density, } \\
\text { Catalyst (MIP) }\end{array}$ & $\begin{array}{c}\uparrow \\
0.23 \mathrm{~g} / \mathrm{mL}-0.43 \mathrm{~g} / \mathrm{mL}\end{array}$ & & $\downarrow$ & $\downarrow$ & & & & & & & $\downarrow$ & $\uparrow$ \\
\hline & \begin{tabular}{|c|} 
Total Pore Area, \\
Catalyst (MIP)
\end{tabular} & $\begin{array}{c}\uparrow \\
28 \mathrm{~m}^{2} / \mathrm{g}-176 \mathrm{~m}^{2} / \mathrm{g}\end{array}$ & & & & & & & & & & & \\
\hline & $\begin{array}{c}\text { Mesopore } \\
\text { Diameter, } \\
\text { Catalyst }\end{array}$ & $\begin{array}{c}\uparrow \\
0.032 \mu \mathrm{m}-0.047 \mu \mathrm{m}\end{array}$ & & $\downarrow$ & $\downarrow$ & & $\uparrow$ & & $\uparrow$ & $\cap$ & & & $\uparrow$ \\
\hline & $\begin{array}{l}\text { O1s content, } \\
\text { Catalyst (XPS) }\end{array}$ & 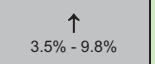 & & & & $\downarrow$ & & & & & & & $\uparrow$ \\
\hline \multicolumn{14}{|c|}{${ }^{a} 60 \%$ RH Only } \\
\hline \multicolumn{6}{|c|}{$\cap$ Data goes through a maximum } & \multicolumn{3}{|c|}{ Legend } & & & & & \\
\hline \multicolumn{6}{|c|}{$\cup$ Data goes through a minimum } & \multicolumn{3}{|c|}{ Negligible Effect } & & & & & \\
\hline \multicolumn{6}{|c|}{$\mathrm{L}$ One high point at lower range } & \multicolumn{3}{|c|}{$\begin{array}{c}\text { Small Effect } \\
<20 \mathrm{mV} \text { Air Performance Variation }\end{array}$} & & & & & \\
\hline & N/A Not Available or Not & Applicable & & & & \multicolumn{3}{|c|}{$\begin{array}{l}\text { Large Effect } \\
>20 m V \text { Air Performance Variation }\end{array}$} & & & & & \\
\hline
\end{tabular}

\section{(a) Water Saturation}

Under fuel cell operation, another element of the structure is the water content. The Catalyst and lonomer Dispersion and Catalyst Layer structure will affect and also be affected by the water saturation. The water saturation is also a stressor for degradation mechanisms such as Pt dissolution and carbon corrosion and therefore is an important parameter for consideration.

Water saturation is defined as the volume fraction of liquid water in the pores of the catalyst layer and is calculated in the model. Measurement methods of water saturation do exist, but have low resolution and accuracy. Therefore, this parameter is identified as a quantification gap.

Water saturation is dependent on hydrophobicity effects related to the ionomer and catalyst surface characteristics, capillary condensation effects related to the pore size distribution and the fuel cell operating conditions. Water is introduced to the cell through humidification of the oxidant and/or the fuel and is also produced on the cathode with operation. Additionally, water can cross the membrane in both directions due to diffusion gradients and/or electro-osmostic drag and can exist as a liquid or as water vapour.

Water saturation can affect the catalyst layer structure and properties. For example, increased water saturation leads to ionomer swelling and may decrease the ionomer tortuosity. Moreover, pore tortuosity can be reduced by ionomer swelling into pores, or the narrowing of pores due to liquid water. Furthermore, increased water saturation will increase the ionomer water content which in turn increases the ionomer bulk conductivity. Overall, these structural changes will result in changes to the catalyst layer diffusivity and CL ionic resistivity. 


\section{(b) Design Levers Impacting CL Thickness}

The catalyst layer thickness is impacted by catalyst powder bulk density, Pt loading and in some cases, the ionomer content. The catalyst layer thickness is a function of the catalyst layer density and the loading as given in Equation 1:

$$
\mathrm{CL} \text { Thickness }=(\text { Total Mass } / \text { Area }) / C L \text { Density }
$$

The catalyst layer density will be a function of the density of its components (i.e. ionomer and catalyst). Since the catalyst is over $75 \%$ of the catalyst layer by mass, the density of the catalyst powder will be dominating. As shown in Figure 2, the thickness was found to be inversely dependant on catalyst powder bulk density. In this example, the catalyst powder density was varied by the $\mathrm{Pt} / \mathrm{C}$ ratio of the catalyst powder, while other catalyst design parameters, such as Pt loading and ionomer content were held constant. Figure 3 shows how the catalyst powder density increased with increasing Pt content on the carbon powder. In addition to varying $\mathrm{Pt}$ content of the catalyst powder, the catalyst bulk density can also be varied by the carbon support type. For example, Pt supported on low surface area carbon supports are denser and lead to thinner catalyst layers than Pt catalysts using high surface area carbon supports.

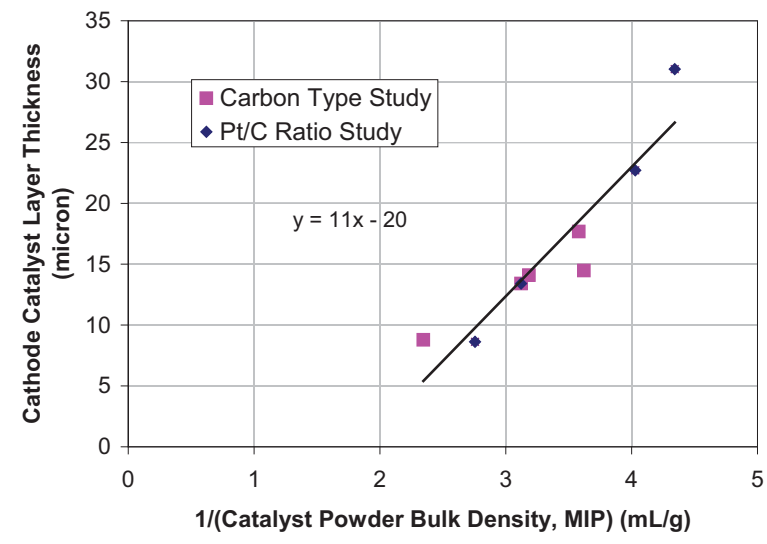

Figure 2: Cathode Catalyst Layer Thickness Relationship with Catalyst Powder Bulk Density

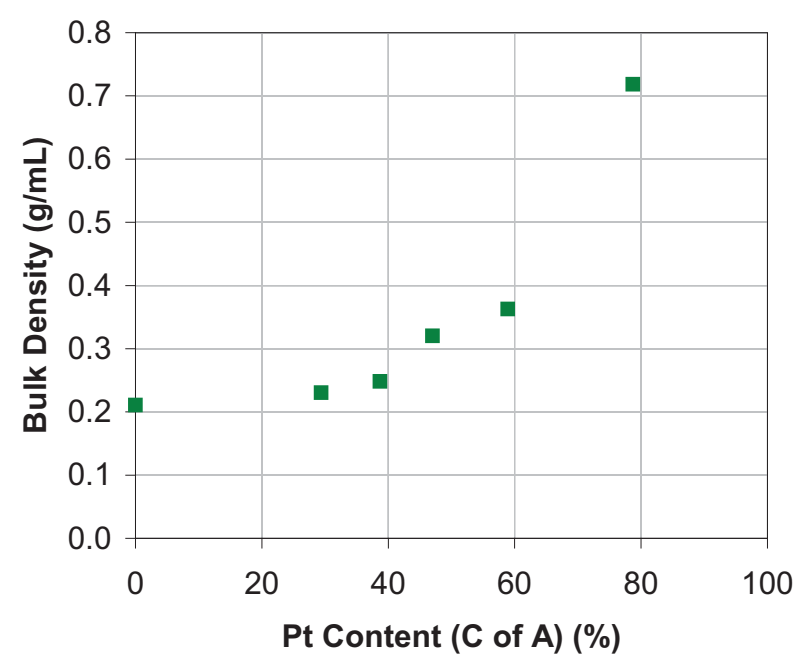

Figure 3: Catalyst Powder Bulk Density Relationship to Catalyst Powder Pt Content

The dependency of the catalyst layer thickness on the Total Mass/Area is observed by varying the loading of the catalyst ink. This can be seen in Figure 4, which shows a linear relationship of catalyst layer thickness and Pt loading. Here, the same catalyst ink formulation was used so the total mass/area would be proportional to the Pt loading/area. 


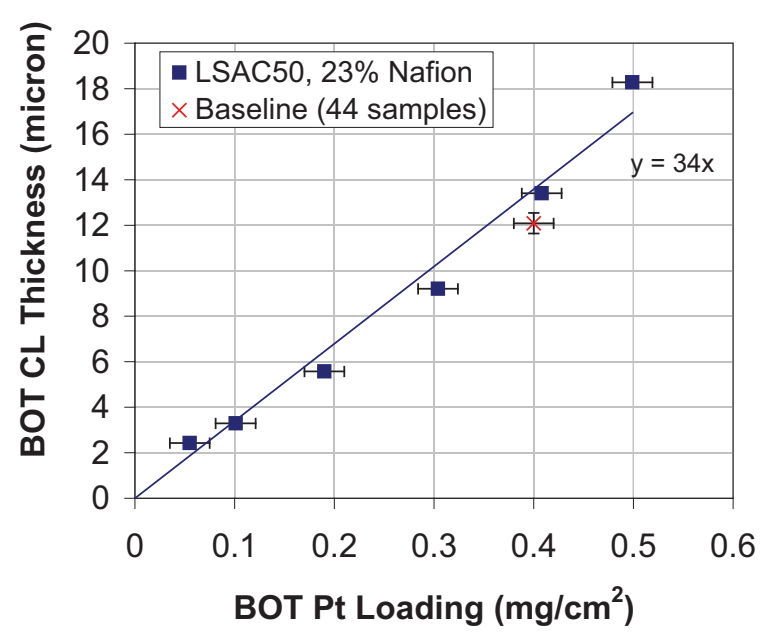

Figure 4: Catalyst Layer Thickness Relationship to Pt Loading

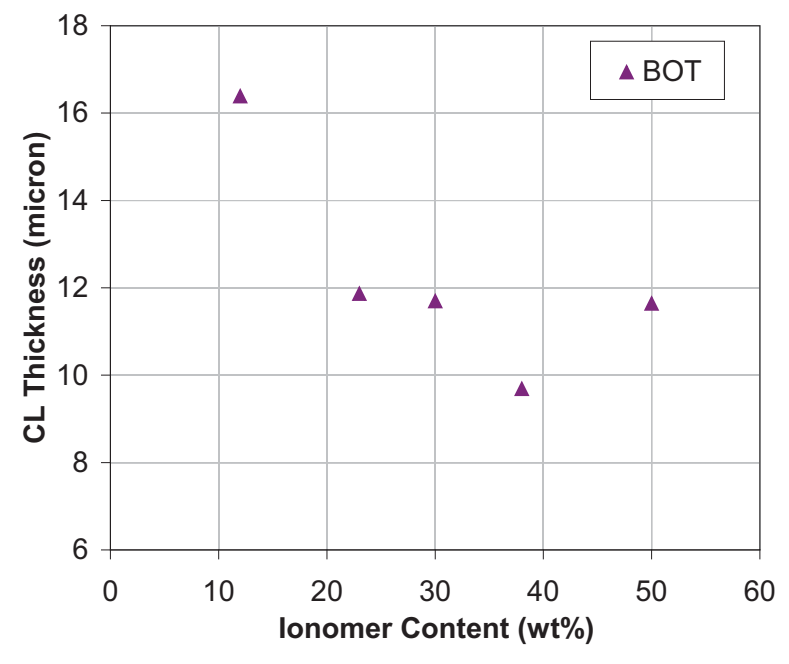

Figure 5: Catalyst Layer Thickness Relationship to catalyst layer lonomer Content

The total catalyst layer mass/area can also be affected by the catalyst ink composition, i.e. changing the ionomer content, while keeping the catalyst loading constant. According to Equation 1, an initial hypothesis would suggest that the catalyst layer increases in thickness as the ionomer content increases. However, this was not observed, as shown in Figure 5. Interaction effects of the catalyst and ionomer such as filling of pores with ionomer affected the catalyst layer density such that the catalyst layer thickness did not show a significant change from $23 \%$ to $50 \%$ ionomer content. Interestingly, at an ionomer content of $12 \%$, a thicker catalyst layer was observed. The pore size data shown in Figure 6 sheds some light on this observation. The $12 \%$ ionomer content catalyst layer had a larger pore size distribution (i.e. peak width) than the other structures, due to a bimodal distribution. This bimodal distribution suggested the presence of filled and unfilled pores due to insufficient ionomer. This resulted in a more porous structure than the higher ionomer content catalyst layers which had sufficient ionomer to form a more compact layer with a homogeneous pore size distribution. 


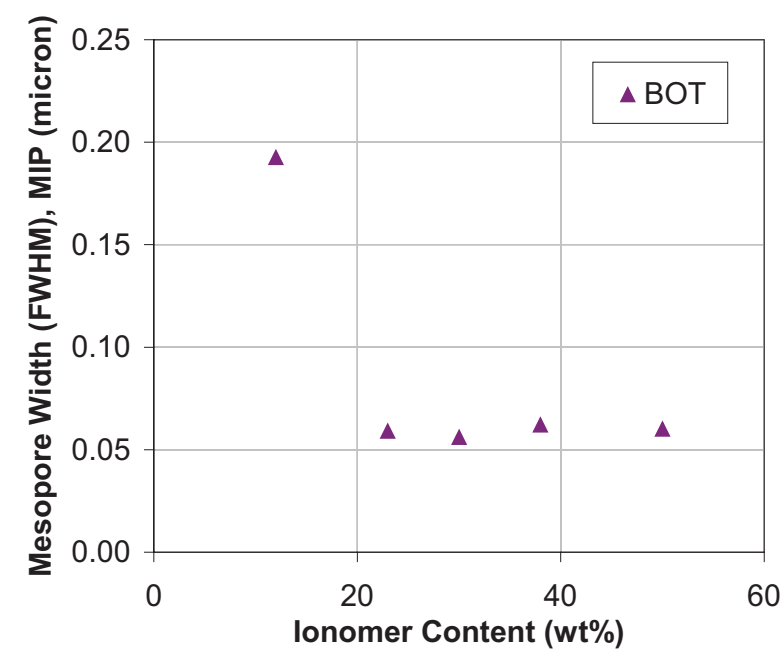

Figure 6: Catalyst Layer Mesopore Size Distribution Relationship to Catalyst Layer Ionomer Content

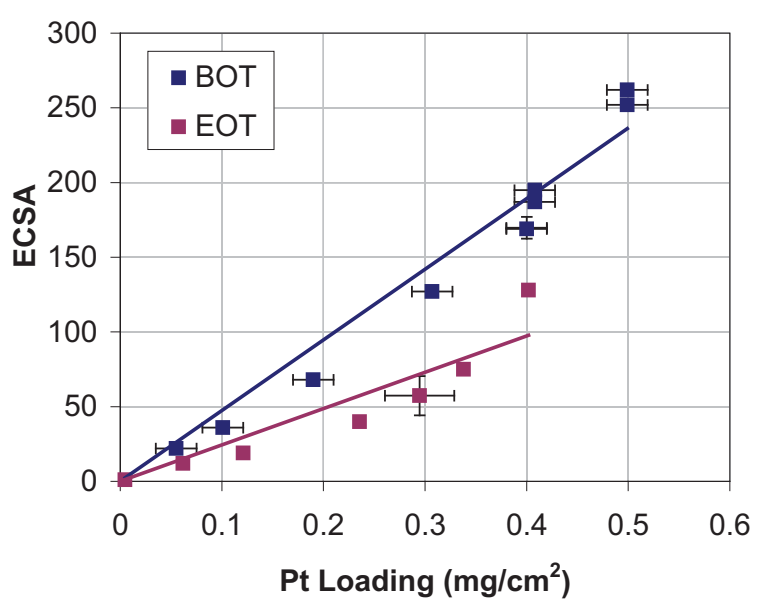

Figure 7: ECSA Relationship to Pt Loading

(c) Design Levers Impacting ECSA

Effective catalyst surface area or ECSA is a function of the Pt loading $\left(L_{P t}\right)$, Pt size $\left(r_{P t}\right), P t$ density $\left(\rho_{\mathrm{Pt}}\right)$ and utilization or three-phase contact region, as shown by Equation 2.

$$
E C S A=3 * L_{P t} /\left(r_{P t} * \rho_{P t}\right) * \text { utilization factor }
$$

This relationship assumes that the Pt particles are perfect spheres of uniform diameter, equal to the average crystallite diameter as measured by XRD and that $100 \%$ of the Pt surface is accessible. Assuming that the utilization factor is constant, ECSA has a linear relationship to Pt loading and inverse relationship to Pt size. Figure 7 and Figure 8 show these relationships that hold for both BOT and EOT data with EOT results shifting further towards zero due to degradation, i.e. agglomeration and Pt loss. When both $\mathrm{Pt}$ loading and $\mathrm{Pt}$ size are considered together, as shown in Figure 9, BOT and EOT data fall on the same line with a slope that indicates a Pt utilization of $82 \%$. 


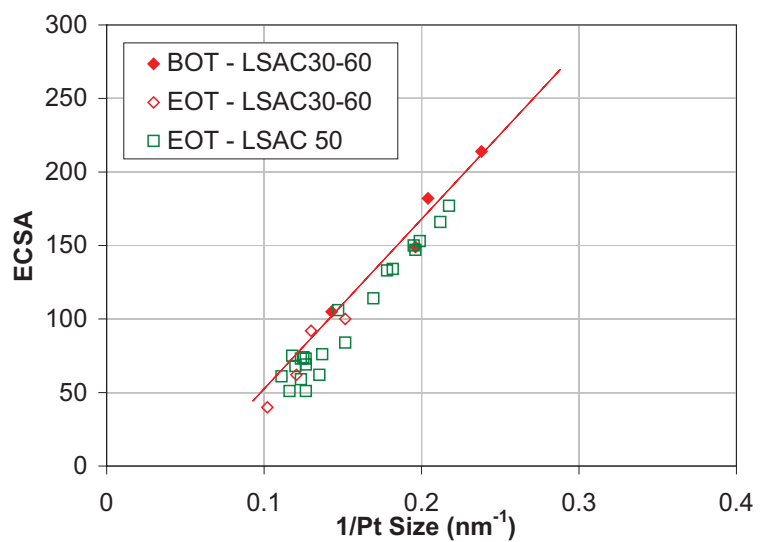

Figure 8: ECSA Relationship to Pt Size

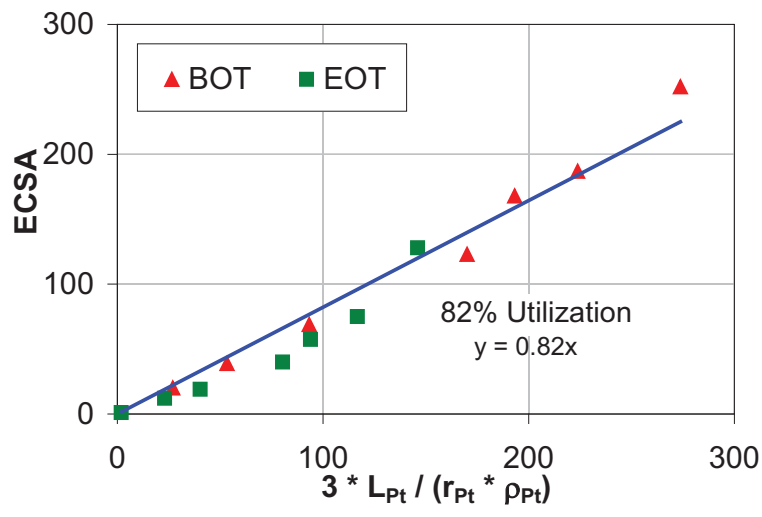

Figure 9: ECSA Relationship to Pt Loading and Pt Size

(d) Design Levers Impacting lonomer Volume Fraction

lonomer volume fraction is impacted by the ionomer content and catalyst powder porosity. The ionomer content has a linear relationship to ionomer volume fraction as shown in Figure 10. As ionomer content is increased, the ionomer volume fraction increases linearly. It is also observed that the porosity decreases linearly, suggesting that ionomer fills pores within the catalyst agglomerates.

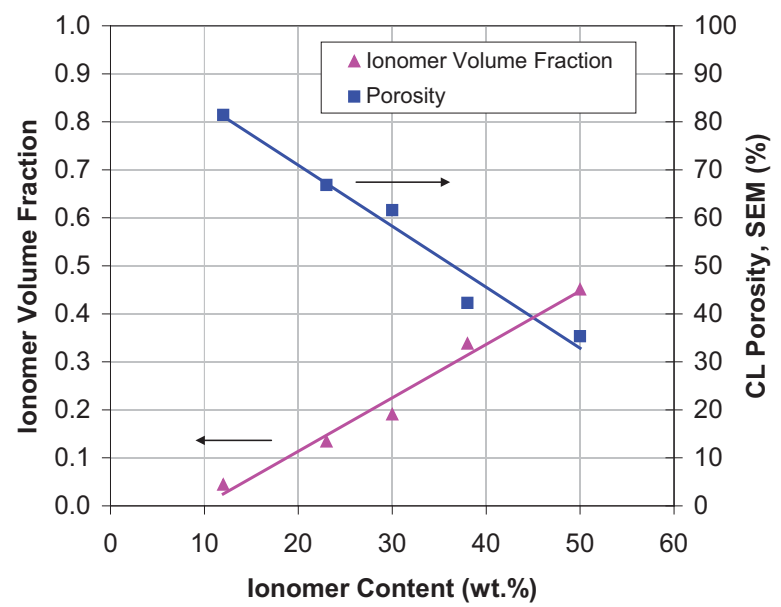

Figure 10: lonomer Volume Fraction and Porosity Relationship to Ionomer Content

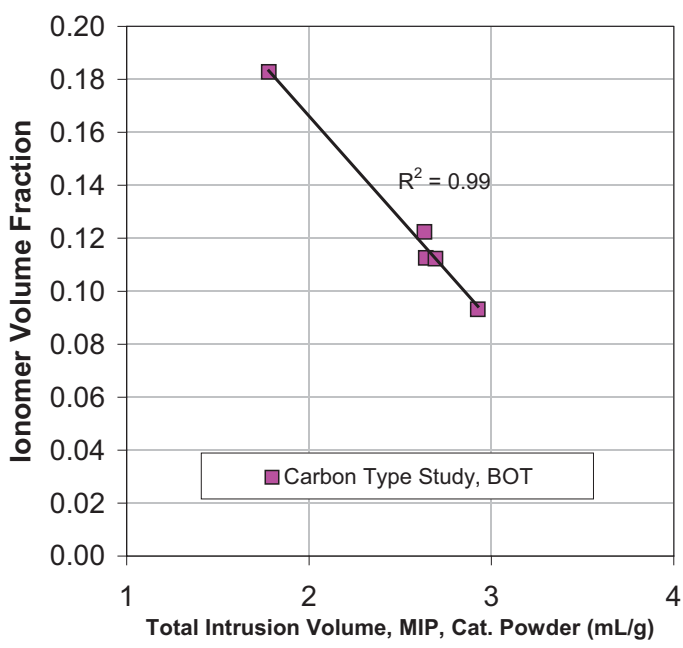

Figure 11: Ionomer Volume Fraction Relationship to Catalyst Powder Pore Volume

In addition to ionomer content, the catalyst powder porosity also impacts ionomer volume fraction. The catalyst powders with higher pore volume (intrusion volume) result in catalyst layers with greater pore volumes and lower ionomer volume fractions, as shown in Figure 11. 
DE-EE0000466

Ballard Material Products Inc

(e) Design Levers Impacting lonomer Tortuosity

The ionomer tortuosity is not measured directly, but calculated according to Bruggeman ${ }^{1}$ as shown in Equation 3.

$$
\sigma_{\mathrm{CL} \text { ionomer }}=\sigma_{\text {bulk ionomer }} \varepsilon^{\tau}
$$

where $\sigma_{C L}$ ionomer is the proton conductivity of catalyst layer ionomer, $\sigma_{\text {bulk ionomer }}$ is the proton conductivity of bulk ionomer in membrane, $\varepsilon$ is the ionomer volume fraction, and $\tau$ is the ionomer tortuosity factor which typically varies from 1 to 2 .

Liu et al (Figure 12) have shown a relationship of ionomer tortuosity to ionomer volume fraction $^{2}$. As shown below for an ionomer volume fraction of 0.15 or lonomer/Carbon ratio of 0.8 the tortuosity increases exponentially due to disruption in the ionomer percolation. Increasing the ionomer volume fractions from 0.15 to 0.25 , results only in a small decrease in ionomer tortuosity.

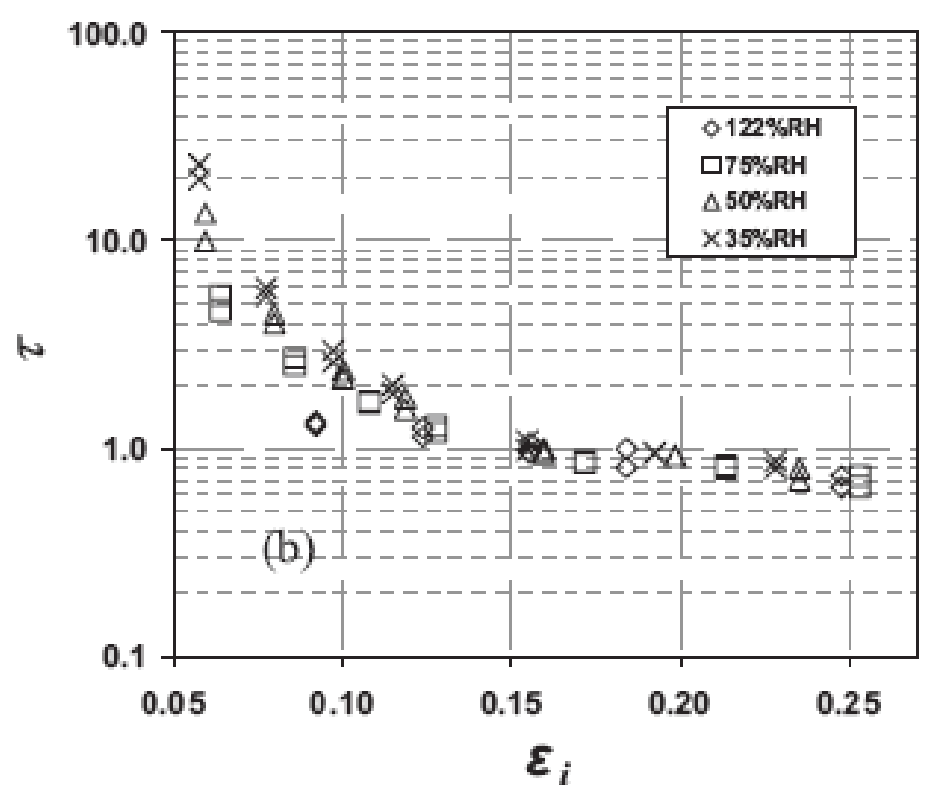

Figure 12: Ionomer Tortuosity Relationship to lonomer Volume Fraction

\footnotetext{
${ }^{1}$ Young, A.P.; Stumper, J.; Knights, S.; Gyenge, E., Journal of The Electrochemical Society, 1573 B425-B436 2010

${ }^{2}$ Liu,Y.; Murphy, M.; Baker, D.; Gu, W, Ji, C.; Jorne, J.; Gasteiger, H., Journal of The Electrochemical Society, 1568 B970-B980 2009.
} 
DE-EE0000466

Ballard Material Products Inc

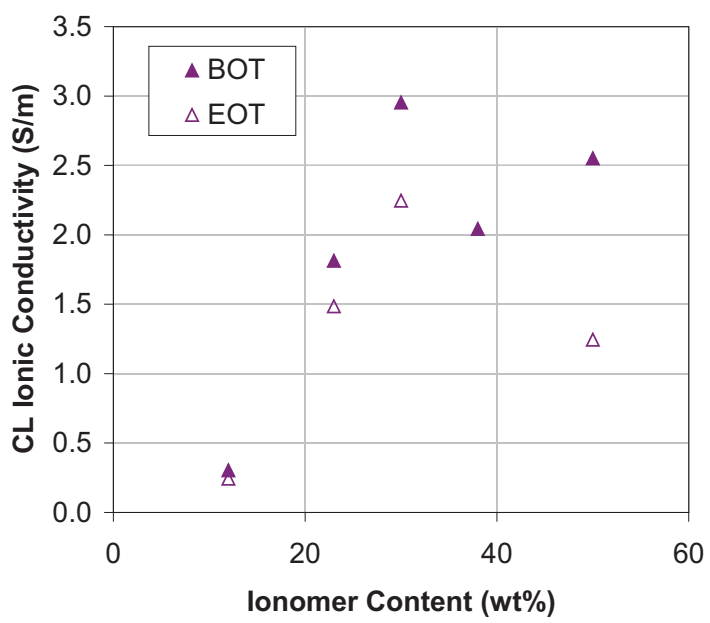

Figure 13: Catalyst Layer lonic Conductivity Relationship to lonomer Content

\section{(f) Design Levers Impacting Catalyst Layer lonic Resistivity}

The catalyst layer ionic resistivity, (1/catalyst layer ionic conductivity) is dependent on the bulk ionomer conductivity, the ionomer tortuosity and ionomer volume fraction, as described above by the Bruggeman relationship $(\sigma C L$ ionomer $=\sigma \beta \nu \lambda \kappa$ ionomer $\varepsilon \tau$

Figure 13 and Figure 14, show that the relationship holds for both BOT and EOT samples, where ionic conductivity is shown to increase with ionomer content (i.e. ionomer volume fraction) and decrease with increasing ionomer tortuosity. The ionic conductivity vs. ionomer content results showed some outliers, which can be explained by noise factors such as processing conditions and carbon surface oxidation effects with AST cycling. Increased carbon surface oxidation with AST cycling increases wettability and improves the bulk proton conductivity. Therefore, EOT conductivities were found to be lower than at BOT.
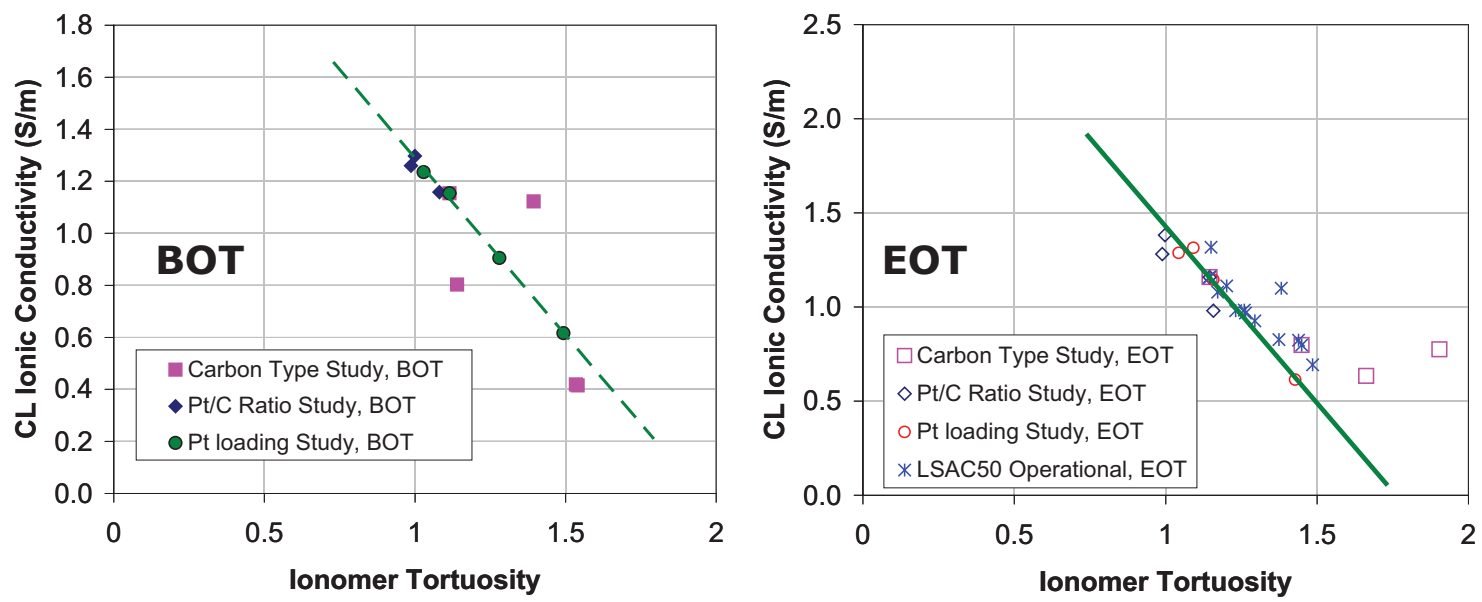

Figure 14: Catalyst Layer Ionic Conductivity Relationship to lonomer Tortuosity 


\section{(g) Design Levers Impacting Porosity / Pore Size Distribution}

Since the porosity of the catalyst layer has an inverse relationship to the ionomer volume fraction, it is expected that that both parameters show correlations with the same design levers Consequently, similar to the ionomer volume fraction, ionomer content and catalyst powder porosity were also found to affect the catalyst layer porosity. As shown in Figure 15, increased ionomer content fills the catalyst pores and shifts the mesopore diameter to smaller pore size. The mesopore diameter was found to have a linear relationship to the ionomer content and the filling of pores with ionomer causes a drop in the porosity as shown in Figure 10.
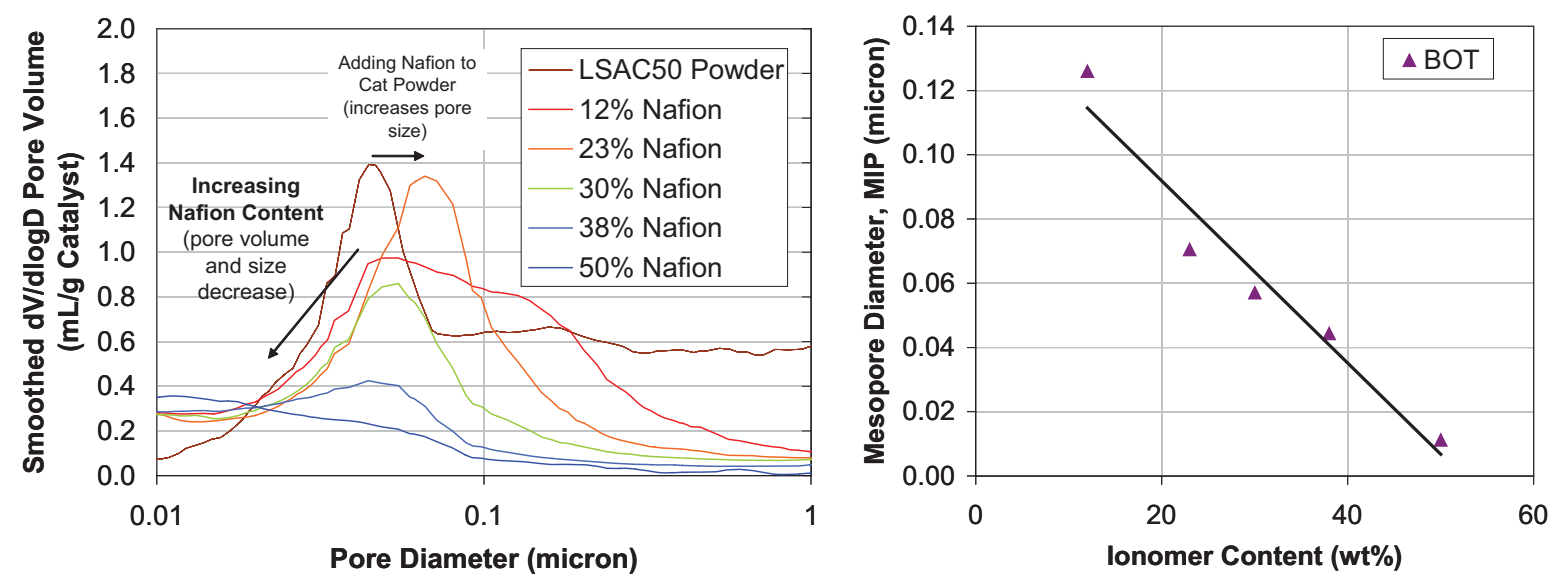

Figure 15: Pore Size Distribution Relationship to lonomer Content

In addition to the ionomer content, the catalyst powder porosity also affects the catalyst layer porosity. As the catalyst powder porosity is increased, a corresponding increase in catalyst layer porosity is observed. Figure 16 displays this linear relationship.

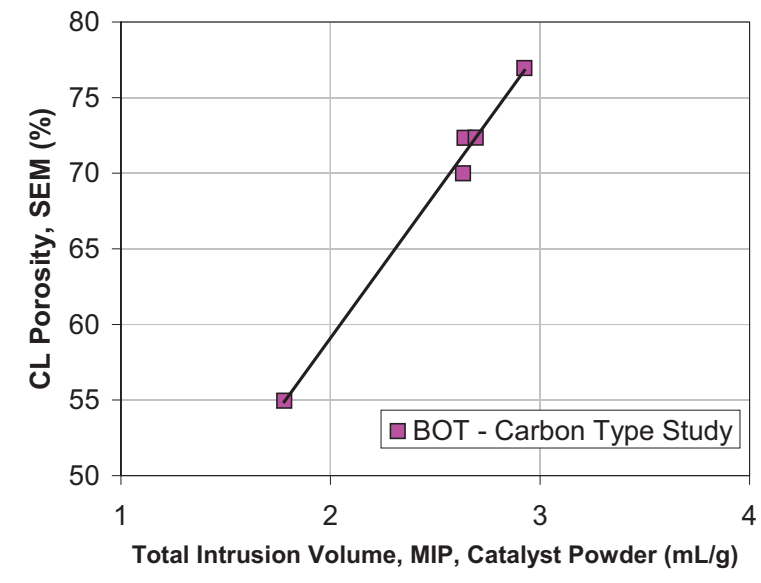

Figure 16: Catalyst Layer Porosity Relationship to Catalyst Powder Pore Volume

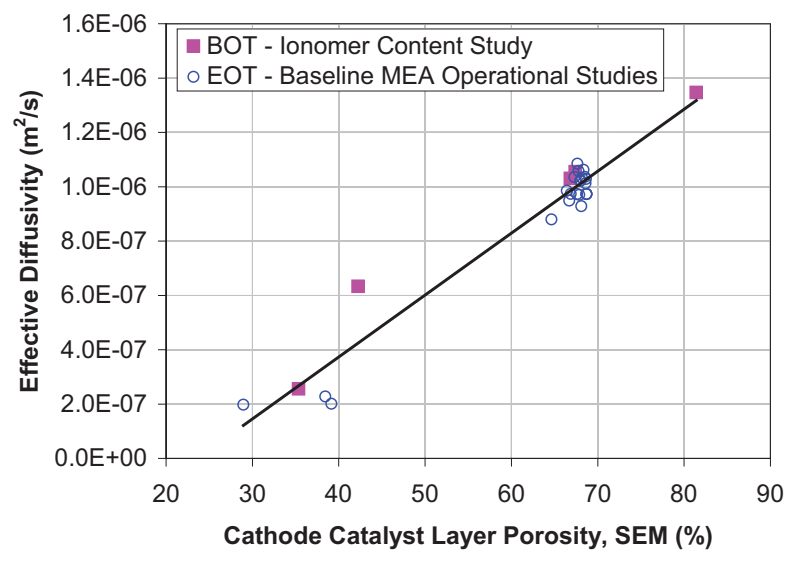

Figure 17: Effective Diffusivity Relationship to Porosity 


\section{(h) Design Levers Impacting Pore Tortuosity}

Although, pore tortuosity cannot be quantified directly, however, it can be calculated from the measured diffusivity, as shown in Equation 4 below, Model simulations by Suzuki et al., for two different catalyst layers show that while the porosity was varied significantly $(40 \%$ to $50 \%$ porosity), there was only a small change in pore tortuosity (1.13 to 1.09$)^{3}$. Therefore, these results suggest that minimal effects on pore tortuosity are expected for medium range changes to porosity.

\section{(i) Design Levers Impacting Catalyst Layer Diffusivity}

Effective diffusivity is a function of the porosity, the gas tortuosity factor and diffusion coefficients, as shown in Equation 4.

$$
D_{\text {eff, } 02}=\varepsilon / \gamma *\left(1 /\left(1 / D_{O 2}+1 / D_{k n}\right)\right)
$$

where $D_{\text {eff }}$ o2 is the effective oxygen gas diffusion in the catalyst layer, $\varepsilon$ is the porosity, $\gamma$ is the gas tortuosity factor and $D_{\mathrm{O}_{2}}$ and $D_{\mathrm{kn}}$ are the Fickian and Knudsen diffusion coefficients which represent the bulk diffusivity, $\mathrm{D}_{\text {Bulk }}$ as defined by the Bosanquet Relation (Equation 5).

$$
D_{\text {Bulk }}=\left(1 /\left(1 / D_{O 2}+1 / D_{K n}\right)\right)
$$

The diffusivity shows a linear correlation to porosity as expected, for both BOT and degraded samples, (Figure 17).

\section{(j) Summary}

The main design levers affecting the catalyst and ionomer dispersion of the catalyst layer were found to be $\mathrm{Pt}$ loading, $\mathrm{Pt}$ size, $\mathrm{Pt} / \mathrm{C}$ ratio, ionomer content, catalyst powder porosity and bulk density.

\subsubsection{Impact of Catalyst Layer Property Parameters on Performance}

The fuel cell performance is dependent on the individual polarization losses: Kinetic, Anode, Cell Resistance (membrane plus electrical), CL lonic (i.e. ionic resistance) and Mass Transport induced kinetic losses (Figure 18). These polarization losses are impacted by the properties of the catalyst layer, such as ECSA, diffusivity, catalyst layer thickness and ionic resistivity.

${ }^{3}$ Suzuki, A.; Hattori, T.; Miura, R.; Tsuboi, H.; Hatakeyama, N.; Takaba, H.; Williams, M. and Miyamoto, A., Int. J. Electrochem. Sci., 5, 1948 - 1961, 2010. 
DE-EE0000466

Ballard Material Products Inc

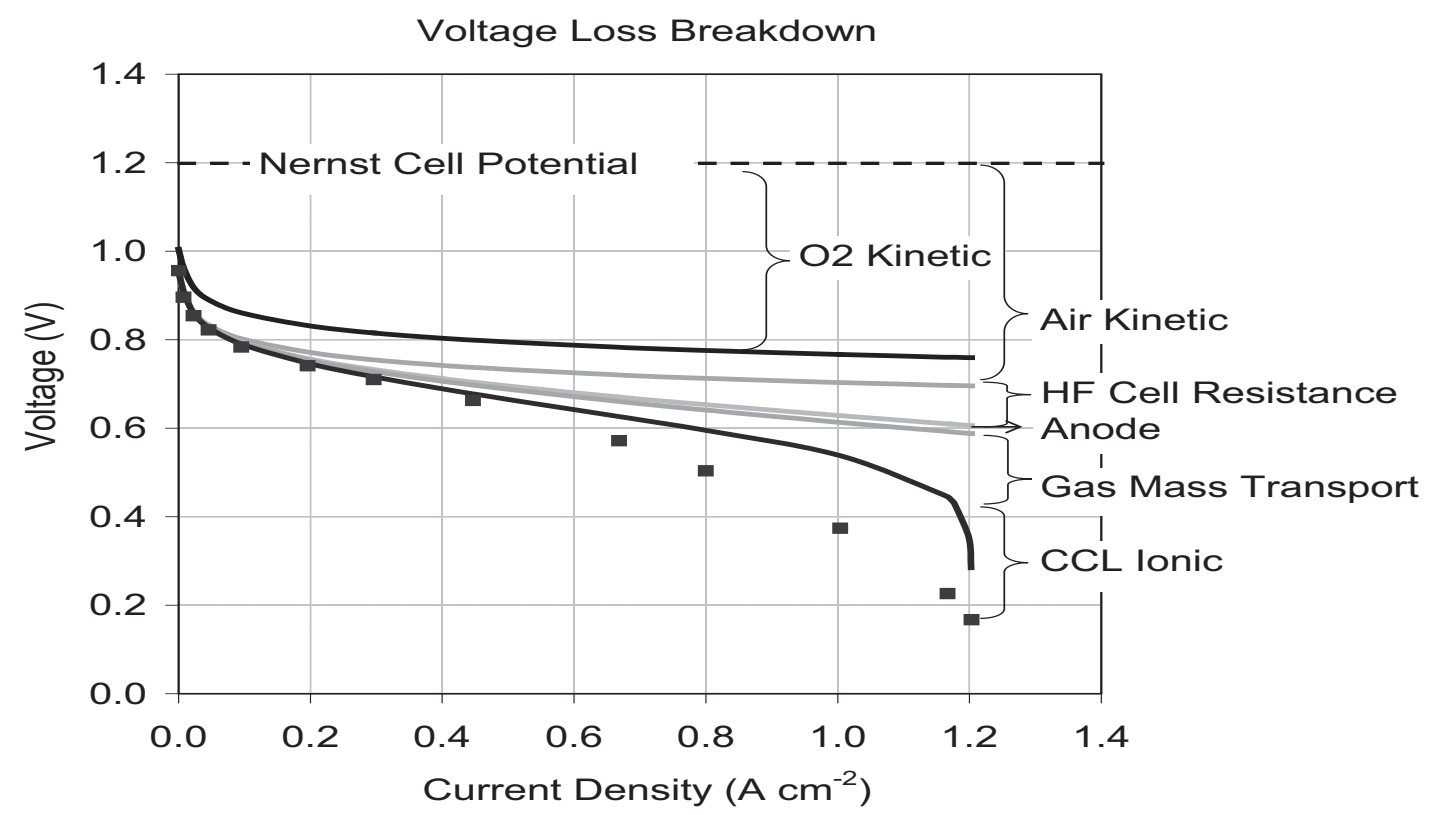

Figure 18: Types of Polarization Losses Observed

The reaction distribution in the catalyst layer is optimized by a balancing of the different overpotentials in order to minimize the total polarization losses. Therefore, changes to one catalyst layer property can have an effect on each of the different overpotentials that are inherent in the polarization characteristics. For example, mass transport limitations, caused by reduced diffusivity or increased catalyst layer thickness, will reduce the local oxygen concentration at the catalyst surface increasing kinetic losses. This results in a shift of the reaction distribution into the catalyst layer to access more oxygen. This will lower the mass transport losses, but also causes an associated increase to the catalyst layer ionic loss. Similarly, the proton conductivity, intrinsic kinetic activity and catalyst surface area can also affect the reaction distribution and polarization losses. Table 2 shows a summary of the impact of catalyst layer properties on the polarization losses and performance. 
Table 2: Impact of catalyst layer properties on Polarization losses

\begin{tabular}{|c|c|c|c|c|c|c|c|c|c|}
\hline \multicolumn{10}{|c|}{$\begin{array}{c}\text { Impact Matrix } \\
\text { Effect of CL Properties on Polarization Losses }\end{array}$} \\
\hline \multirow[b]{2}{*}{ Design Lever } & \multirow[b]{2}{*}{ Data Set } & \multicolumn{4}{|c|}{ Control Factor Levels } & \multirow[b]{2}{*}{ Kinetic } & \multirow{2}{*}{$\begin{array}{l}\text { Mass } \\
\text { Transport }\end{array}$} & \multirow[b]{2}{*}{ CL lonic } & \multirow[b]{2}{*}{ Performace } \\
\hline & & ECSA & Diffusivity & $\begin{array}{l}\text { Resistivity, } \\
\text { lonic }\end{array}$ & Thickness & & & & \\
\hline \multirow{3}{*}{ ECSA } & вот & $\begin{array}{c}\downarrow \\
\text { High to Low }\end{array}$ & High & Low to Medium & High to Low & $\uparrow$ & & & $\downarrow$ \\
\hline & Pt Dissolution & \begin{tabular}{|c|}
$\downarrow$ \\
High (No Pt Depletion), \\
Medium (Pt Depletion: $4 \mu \mathrm{m}$ ) \\
\end{tabular} & High & Low & Medium & $\uparrow$ & & $\uparrow$ & $\downarrow$ \\
\hline & $\begin{array}{l}\text { Pt Dissolution } \\
\text { Corrosion }\end{array}$ & $\stackrel{\downarrow}{\downarrow}$ Low to Very Low & Low & Medium to High & Low & & & $\uparrow$ & $\downarrow$ \\
\hline \multirow{3}{*}{ Diffusivity } & Вот & Medium to High & $\begin{array}{c}\downarrow \\
\text { High to Low }\end{array}$ & Low & Medium & $\uparrow$ & $\uparrow$ & $\uparrow$ & $\downarrow$ \\
\hline & Pt Dissolution & Low to Medium & $\begin{array}{c}\downarrow \\
\text { High to Low }\end{array}$ & Low & Medium & $\uparrow$ & $\uparrow$ & & $\downarrow$ \\
\hline & $\begin{array}{l}\text { Pt Dissolution } \\
\text { Corrosion }\end{array}$ & Low to Medium & $\stackrel{\downarrow}{\text { Medium to Low }}$ & High & Low & $\uparrow$ & $\uparrow$ & $\uparrow$ & $\downarrow$ \\
\hline \multirow[b]{2}{*}{$\begin{array}{l}\text { Resistivity, } \\
\text { Ionic }\end{array}$} & вот & High & High & $\begin{array}{c}\uparrow \\
\text { Low to Medium }\end{array}$ & Medium & $\uparrow$ & $\uparrow$ & & $\downarrow$ \\
\hline & \begin{tabular}{|c} 
BOT \\
Pt Dissolution \\
Corrosion
\end{tabular} & Medium & Low to Medium & $\begin{array}{c}\uparrow \\
\text { Low to High }\end{array}$ & Low to Medium & $\uparrow$ & $\uparrow$ & $\uparrow$ & $\downarrow$ \\
\hline \multirow{2}{*}{ Thickness } & вот & Medium to High & High & Low & $\begin{array}{c}\uparrow \\
\text { Medium to High }\end{array}$ & & $\uparrow$ & $\uparrow$ & $\downarrow$ \\
\hline & Pt Dissolution & Low to Medium & High & Low & $\begin{array}{c}\uparrow \\
\text { Medium to High }\end{array}$ & $\uparrow$ & $\uparrow$ & $\uparrow$ & $\downarrow$ \\
\hline
\end{tabular}

\begin{tabular}{|c|}
\hline Legend \\
\hline Negligible Effect \\
\hline Small Effect \\
\hline$<20 m V$ Air Performance Variation or Impact on Air Performance \\
\hline Large Effect \\
\hline
\end{tabular}

Data Set Definitions

BOT: Beginning of Test

EOT: End of Test

Pt Dissolution: EOT samples showing ECSA Loss

Corrosion: EOT samples showing cathode thinning

Although, traditionally it is expected that the kinetic loss is dependent on catalyst activity and catalyst surface area (Figure 19), the mass transport loss is dependent on diffusivity and thickness (Figure 20 and Figure 21), while the catalyst layer ionic loss is dependent on catalyst layer ionic resistivity (Figure 22). Table 2 shows that the catalyst layer properties can also affect other polarization losses. These unforeseen dependencies occur due to shifts in reaction distribution, especially when the catalyst layer properties are non-ideal or degraded. 
DE-EE0000466

Ballard Material Products Inc
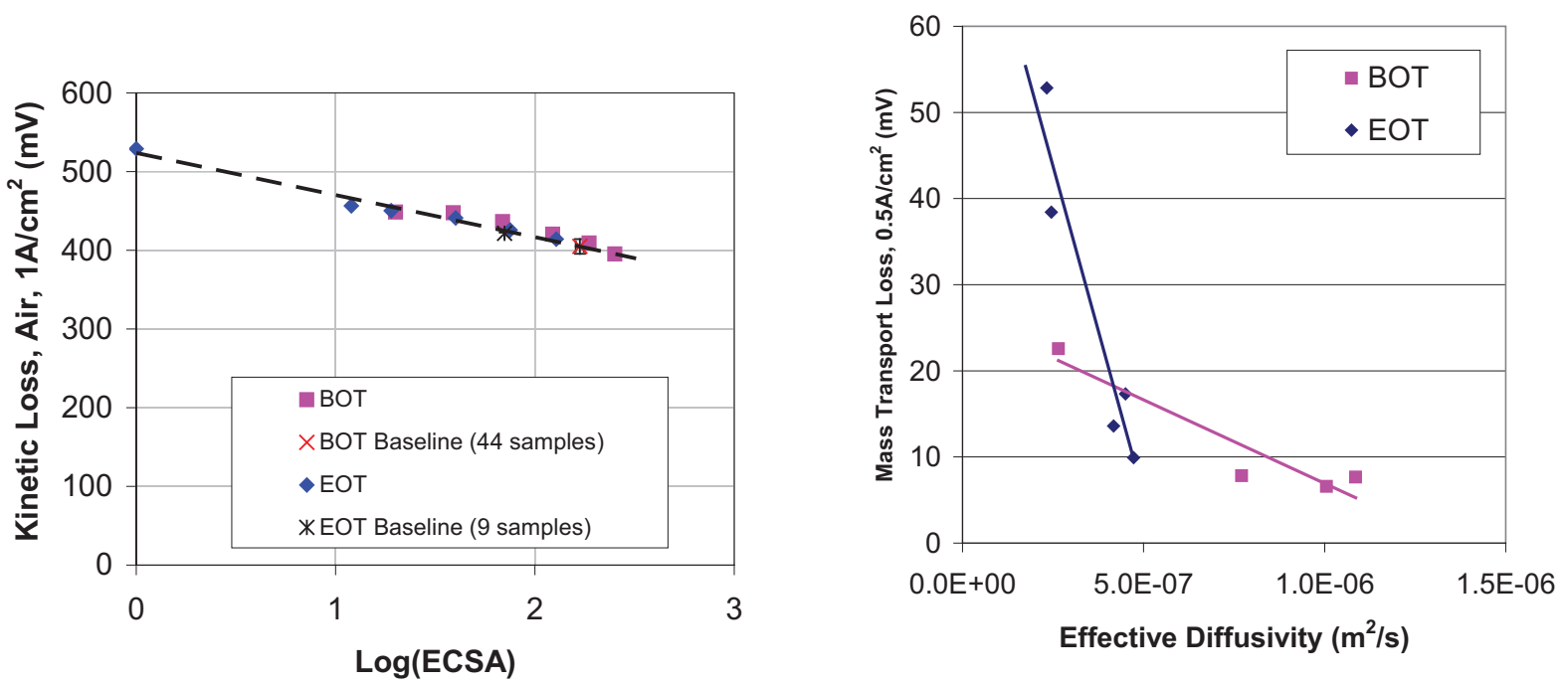

Figure 19: Kinetic Loss Relationship to ECSA

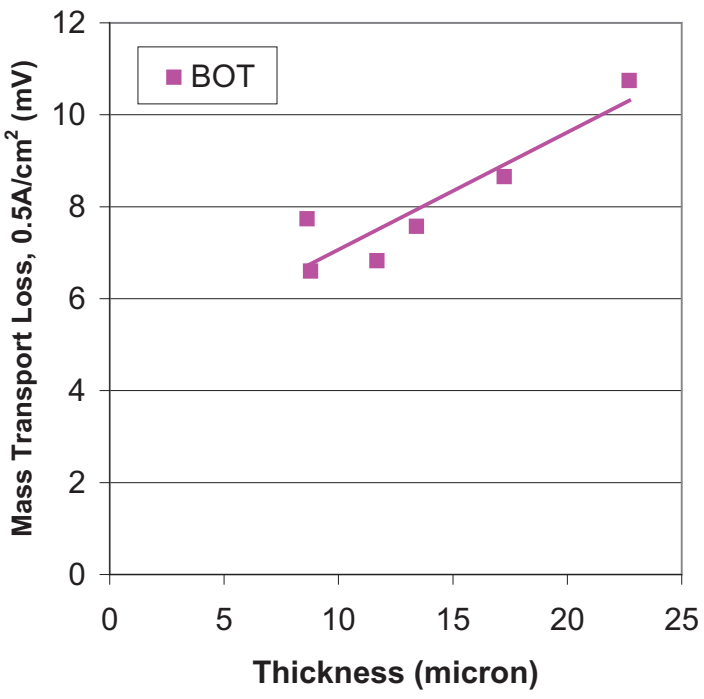

Figure 21: Mass Transport Loss Relationship to Catalyst Layer Thickness

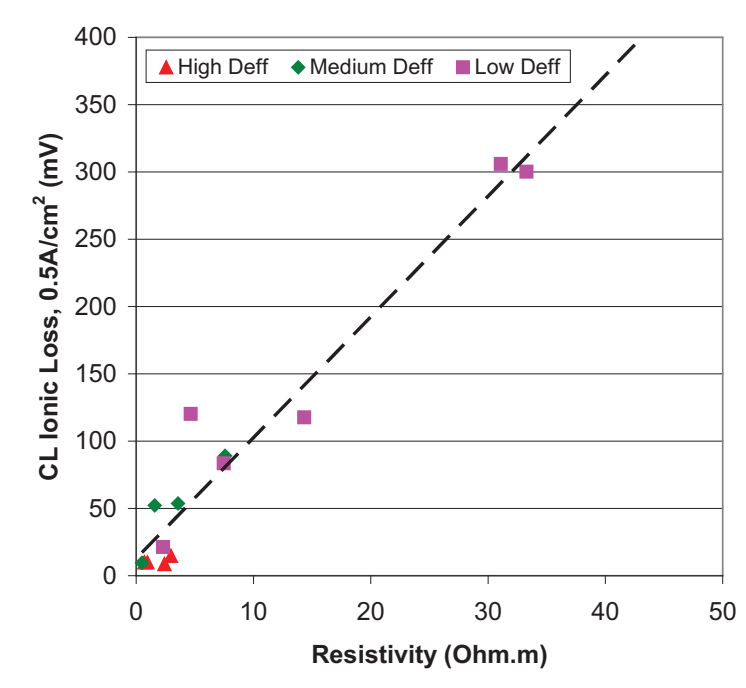

Figure 22: Catalyst Layer lonic Loss Relationship to Catalyst Layer lonic Resistivity

Figure 20: Mass Transport Loss Relationship to Effective Diffusivity (Deff) 
In cases where the catalyst layer design gives non-optimal properties, such as high proton resistivity or low oxygen diffusivity, the reaction distribution varies to compensate for the high catalyst layer ionic and mass transport losses. In these cases, the apparent ECSA may be lower than the total ECSA so a kinetic loss to ECSA correlation is not observed.

In some extreme cases of poor ECSA, proton resistivity and diffusivity, it was observed that the ECSA had an effect on the catalyst layer ionic loss rather than kinetic loss, as shown in Figure 23. In such cases, the reaction zone is very limited due to low oxygen content at membrane and reaction penetration is hindered by the high resistivity, whereas the low ECSA is limiting the reaction. Therefore greater penetration is required to access more catalyst sites at the cost of higher catalyst layer ionic losses. In this case, even small increases in ECSA allow for less penetration and an improvement in catalyst layer ionic losses results.

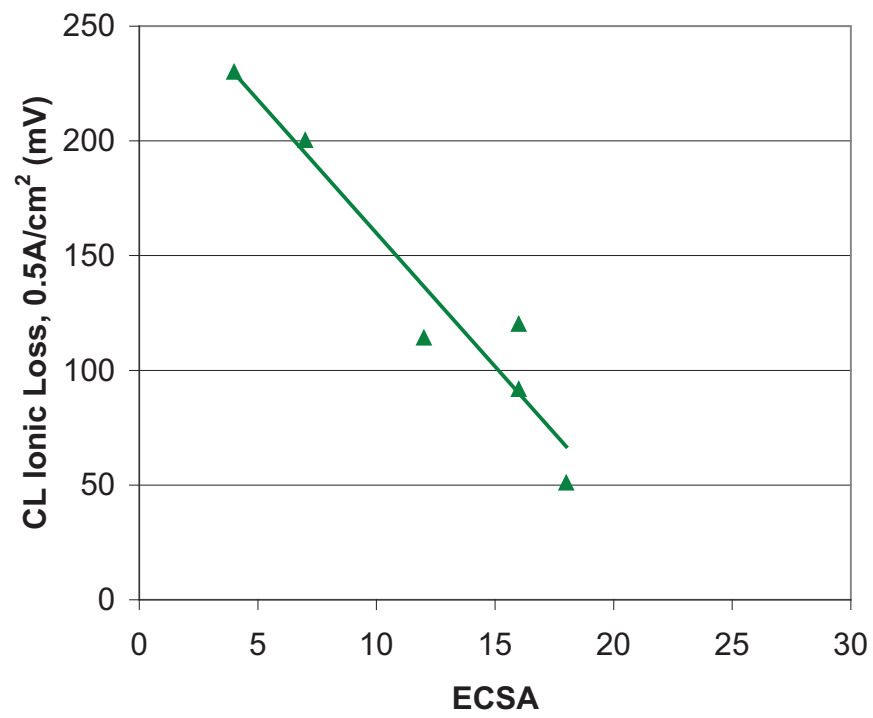

Figure 23: Catalyst Layer lonic Relationship to ECSA when Poor ECSA, Resistivity and Diffusivity Occur Concurrently

Catalyst layer ionic resistivity has been shown to have effects on both kinetic and mass transport losses, as shown in Figure 24. Increasing catalyst layer resistivity restricts the reaction penetration in the catalyst layer due to ohmic losses. Restricting the reaction to a limited region near the membrane results in greater local oxygen depletion in the reaction zone and leads to greater mass transport losses. The limited reaction zone also limits the apparent ECSA and subsequently, increases kinetic losses. 

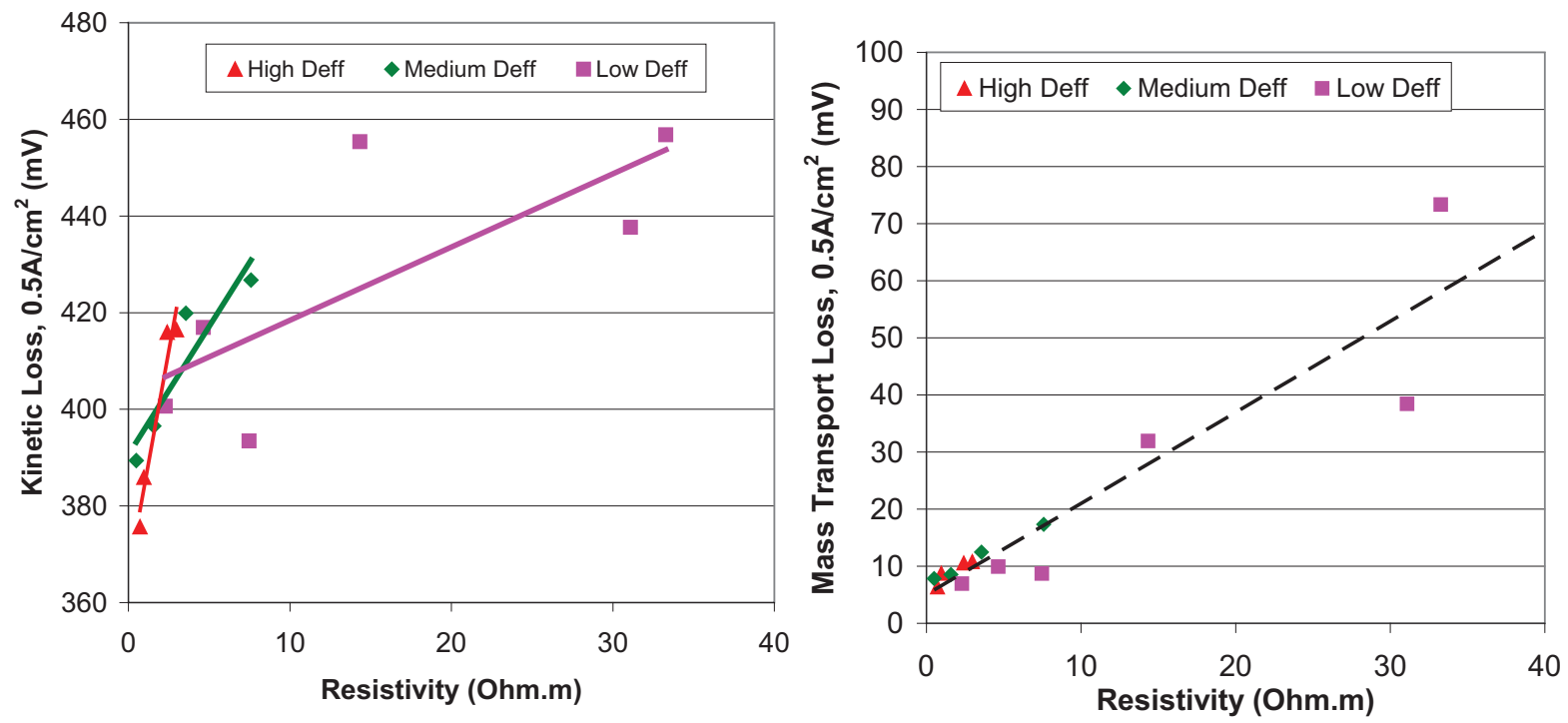

Figure 24: Kinetic Loss and Mass Transport losses increase with increasing Catalyst Layer lonic Resistivity

Diffusivity changes can affect kinetic losses and catalyst layer ionic losses when other properties, such as catalyst layer resistivity and ECSA are satisfactory (Figure 25 and Figure 26). As diffusivity is decreased, an oxygen depleted zone occurs near the membrane leaving that region of the catalyst layer inoperable. This causes the apparent ECSA to drop and results in increased kinetic losses. In addition, the shift further into the catalyst layer causes additional catalyst layer ionic losses. As shown in Figure 25 and Figure 26, with degradation, the kinetic loss values are shifted up due to the lower ECSA caused by the Pt dissolution mechanism and the catalyst layer ionic dependence on diffusivity becomes greater due to increased losses caused by lower resistivity as a result of carbon corrosion.

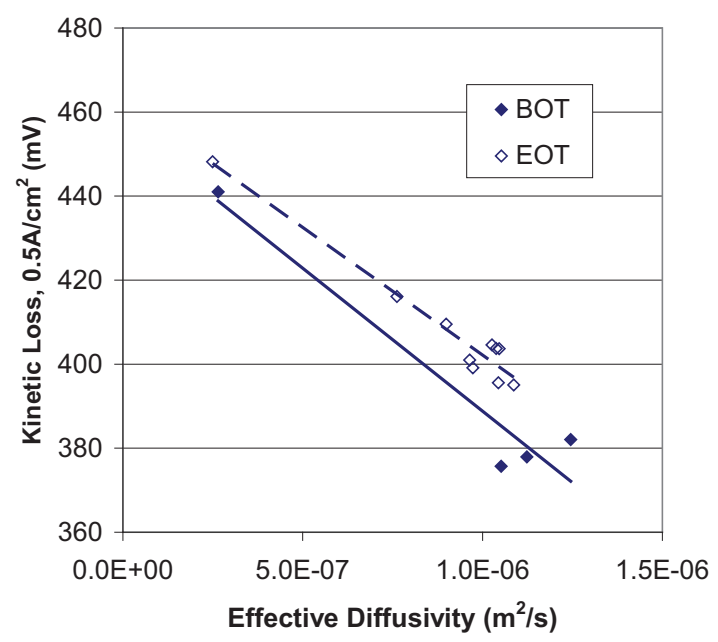

Figure 25: Kinetic Loss increases with decreasing Effective Diffusivity

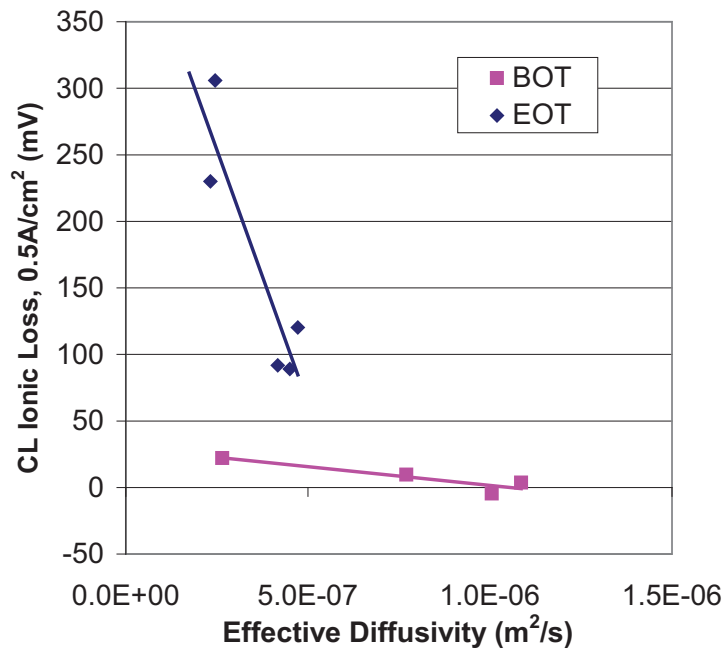

Figure 26: Catalyst Layer lonic Loss increases with decreasing Effective Diffusivity 
An increase in catalyst layer thickness will affect Mass Transport losses, due to oxygen depletion effects; however, the effect on catalyst layer ionic losses due to resistive effects is more notable as shown in Figure 27. In this example, the Pt loading is held constant, while the thickness is increased due to a lower $\mathrm{Pt} / \mathrm{C}$ ratio within the catalyst layer. The current distribution is forced further into the catalyst layer for the thicker catalyst layer to access the same amount of ECSA as the thinner catalyst layer. As a result, the thicker catalyst layer gives a higher catalyst layer ionic loss. With degradation, the catalyst layer ionic loss increase from BOT to EOT due to Pt depletion which also shifts the reaction penetration. An Effective Thickness was calculated from the CL lonic Loss and the resistivity and shows that the increase in Effective Thickness (penetration) agrees with the thickness of the Pt depleted region $(\sim 3 \mu \mathrm{m}$ Pt depletion for the $9 \mu \mathrm{m}$ layer and 6-8 $\mu \mathrm{m}$ Pt depletion for the $31 \mu \mathrm{m}$ layer).
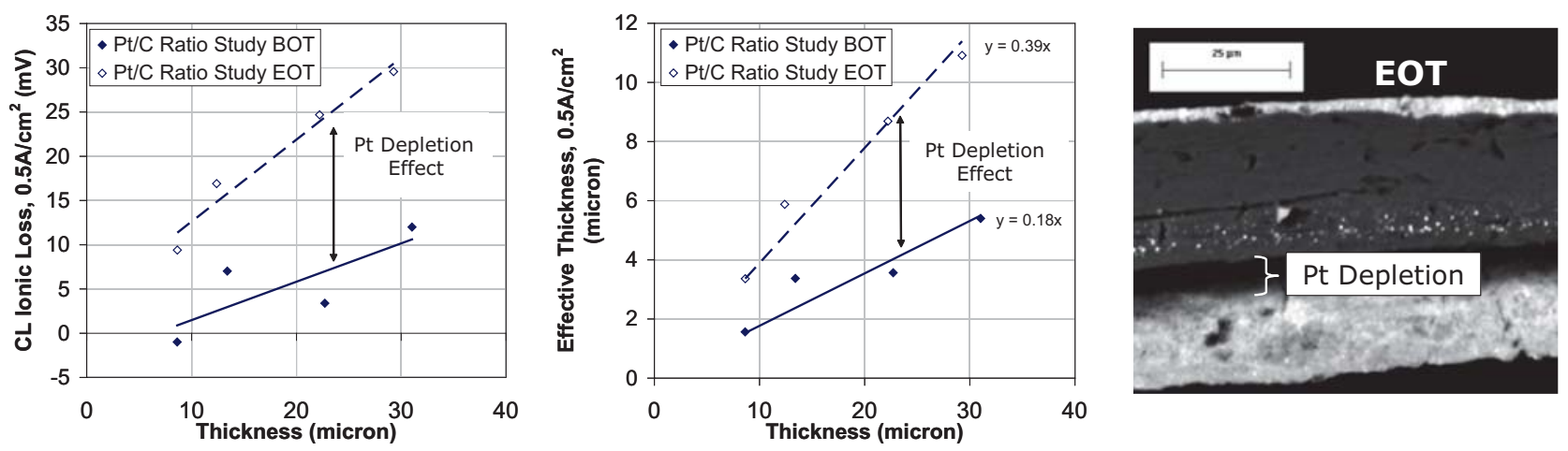

Figure 27: Catalyst Layer lonic Loss Increases with Increasing Catalyst Layer Thickness and Pt Depletion at the Membrane / Catalyst Interface.

\subsubsection{Structure Changes with Degradation}

The two main catalyst layer degradation mechanisms, Pt dissolution and carbon corrosion were investigated by AST testing followed by detailed structural analysis. Table 3 summarizes the effects of Pt Dissolution and Carbon Corrosion on the catalyst layer structure and resulting performance changes.

\section{(a) Pt Dissolution}

$\mathrm{Pt}$ dissolution results in $\mathrm{Pt}$ loss, which reduces the $\mathrm{Pt}$ loading in the catalyst layer, and $\mathrm{Pt}$ agglomeration, which increases the Pt particle size. The Pt dissolution and migration rate is greater at the membrane/catalyst interface, possibly due to potential gradients through the thickness of the catalyst layer and/or proximity to the bulk membrane due to diffusion gradients. This results in a Pt depleted zone adjacent to the membrane. Scanning transmission X-ray microscopy (STXM), results in Figure 28 confirm that the Pt content is not uniform across the catalyst layer thickness and is depleted near the membrane. This can also be observed visually by the changes in brightness as shown in the SEM image of Figure 27. The overall affect for $\mathrm{Pt}$ dissolution is a drop in the ECSA and increases to both kinetic and catalyst layer ionic losses. 


\section{(b) Carbon Oxidation/corrosion}

Carbon degradation is a two-step process. In the first step, the surface of the carbon particles becomes oxidized with oxygen containing surface groups. These surface groups are more hydrophilic than the base carbon material and aid in increasing the water content at the catalyst surface which results in a decrease in the catalyst layer ionic resistivity and associated improvements in kinetic and catalyst layer ionic losses.

In the second step of carbon degradation, the carbon is fully oxidized to $\mathrm{CO}_{2}$ which is lost to the exhaust. The carbon content of the catalyst layer decreases and significant changes to the catalyst layer structure occur. For example, the catalyst thickness and associated porosity decreases, while the ionomer volume fraction increases. Moreover, it is hypothesized that the electronic percolation drops, due to loss of carbon to carbon contact and an increased ionomer content, while the pore tortuosity increases due to loss of pore structure and increased ionomer content. The STXM results in Figure 28 show an increase to the ionomer volume fraction when the carbon content decreases with corrosion. Another point of note is that the carbon and ionomer contents are relatively uniform across the thickness for the degraded structures, suggesting that the carbon corrosion mechanism occurs more uniformly across the catalyst layer thickness, in contrast to the Pt dissolution mechanism.

Table 3: Effect of Degradation on Catalyst Layer Structure, Properties and Polarization Losses

\begin{tabular}{|c|c|c|c|c|c|}
\hline \multicolumn{6}{|c|}{$\begin{array}{l}\text { Impact Matrix } \\
\text { Effect of Degradation on CL Structure / Properties and Polarization Losses }\end{array}$} \\
\hline \multicolumn{2}{|c|}{ Degradation Mechanism } & $\begin{array}{c}\text { Composition / } \\
\text { Component Property } \\
\text { Affected }\end{array}$ & CL Structure Change & CL Property Change & $\begin{array}{l}\text { Polarization Loss } \\
\text { Change }\end{array}$ \\
\hline \multirow{2}{*}{ Pt Dissolution } & $\begin{array}{l}\text { Pt Loss: } \\
\text { PITM } \\
\text { Washout }\end{array}$ & Pt Content $\downarrow$ & \multirow{2}{*}{$\begin{array}{l}\text { Pt depletion at membrane / } \\
\text { catalyst interface } \uparrow\end{array}$} & \multirow{2}{*}{ ECSA $\downarrow$} & \multirow{2}{*}{$\begin{array}{l}\text { Kinetic Loss } \uparrow \\
\text { CL lonic Loss } \uparrow\end{array}$} \\
\hline & Pt Agglomeration & Pt Size $\uparrow$ & & & \\
\hline \multirow{3}{*}{ Carbon Degradation } & Carbon Oxidation & $\begin{array}{l}\text { Oxygen Species on } \\
\text { Carbon Surface } \uparrow\end{array}$ & & lonomer Resistivity $\downarrow$ & $\begin{array}{l}\text { Kinetic Loss } \downarrow \\
\text { CL lonic Loss } \downarrow\end{array}$ \\
\hline & \multirow[t]{2}{*}{$\begin{array}{l}\text { Carbon Corrosion I } \\
\text { Loss }\end{array}$} & Carbon Content $\downarrow$ & $\begin{array}{c}\text { Thickness } \downarrow \\
\text { Porosity } \downarrow \\
\text { Electronic Percolation* } \downarrow \\
\text { Ionomer Vol. Frac. } \uparrow \\
\text { Tortuosity, Pore* } \uparrow\end{array}$ & $\begin{array}{c}\text { Diffusivity } \downarrow \\
\text { Electronic Resistivity* } \uparrow\end{array}$ & $\begin{array}{l}\text { Kinetic Loss } \uparrow \\
\text { CL lonic Loss } \uparrow\end{array}$ \\
\hline & & Pt Content $\downarrow$ & & ECSA $\downarrow$ & $\begin{array}{l}\text { Kinetic Loss } \uparrow \\
\text { CL lonic Loss } \uparrow\end{array}$ \\
\hline
\end{tabular}


DE-EE0000466

Ballard Material Products Inc.
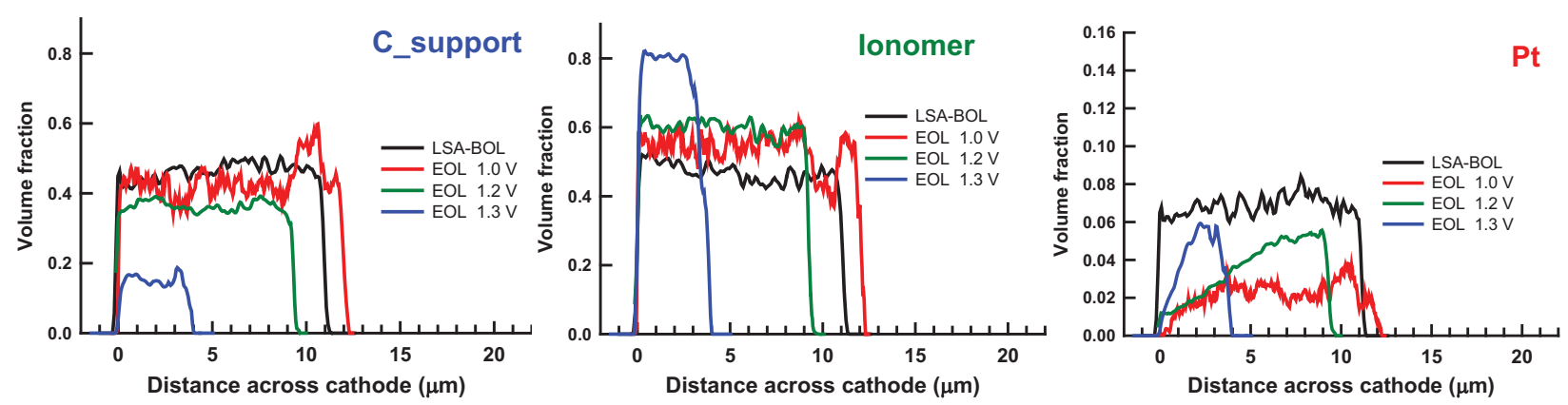

Figure 28: STXM Volume Fraction Profiles across BOT and Degraded MEA Cross-sections

\subsubsection{Correlations Overall Summary}

Correlations have been developed between composition, structure and properties of cathode components and catalyst layer. The BOT and EOT show similar curves for structure to property relationships so the same model predictions can be applied. The correlations serve additionally as validation for the FCPEM Performance and Durability Model. 
DE-EE0000466

Ballard Material Products Inc.

\subsection{Durability Windows}

A selected number of durability windows were developed for both the catalyst layer structural design levers and operational conditions. The criteria for acceptable durability, was based on Ballard Bus durability requirements of $\leq 15 \%$ performance degradation at $1.0 \mathrm{~A} / \mathrm{cm}^{2}$ after 12,000 operational hours and the DOE target for ECSA loss of $\leq 40 \%$.

While the DOE recommended AST for electro-catalyst durability evaluation uses a triangular sweep, this work used square wave ASTs as these are better suited for model validation, i.e. water production at the lower potential can be accurately modeled. Figure 29 shows a comparison of both ASTs at EOT. The results show that at low current density the performance losses are very similar and consistent with predominately kinetic changes for both ASTs which is also evident in the similar ECSA losses and mass activity losses vs. cycle time for both ASTs. At higher current density of $0.8 \mathrm{~A} / \mathrm{cm}^{2}$, the Ballard AST had a larger performance loss of $29 \mathrm{mV}$ versus the $14 \mathrm{mV}$ observed for the DOE AST.
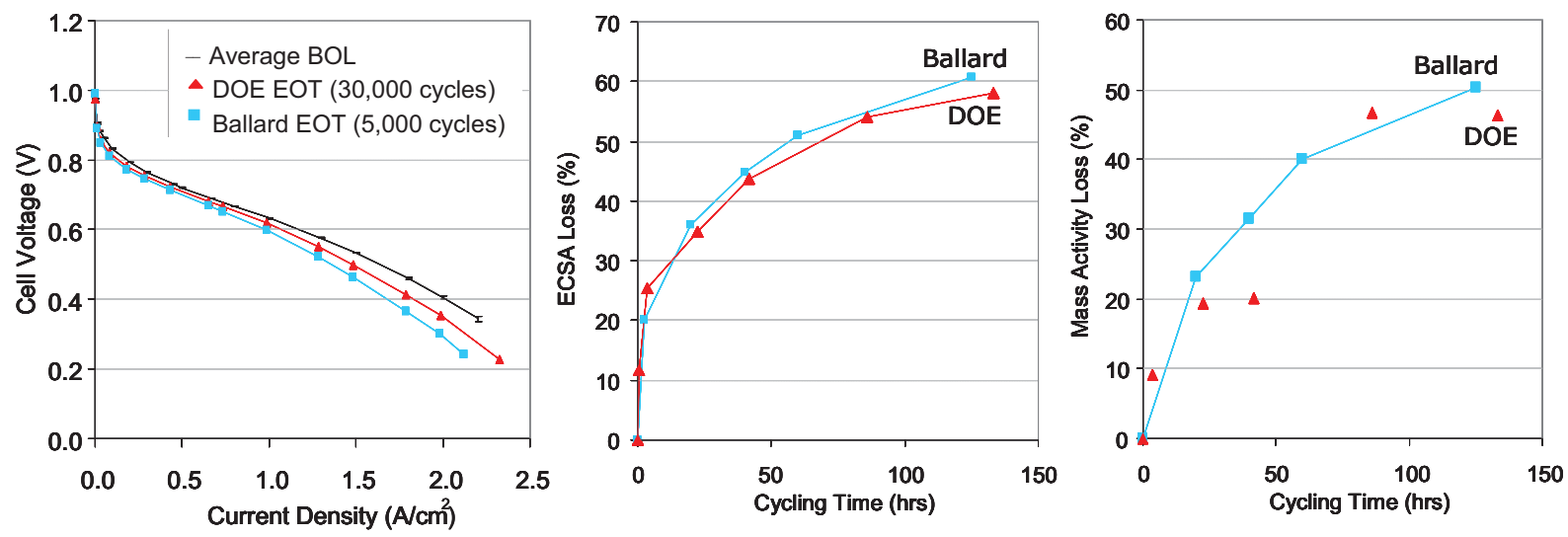

Figure 29: Comparison of Ballard square wave AST and DOE triangular sweep AST

\subsubsection{Structural Parameter Durability Windows}

\section{(a) Pt Loading Durability Window}

Platinum loading is an important design lever that impacts performance, durability and cost. Figure 30 shows the BOT performance and EOT performance loss as a function of cathode $\mathrm{Pt}$ loading. As the low-loaded MEAs could not be cycled to the standard 4700 cycles, results at 2100 cycles are also presented. It was found that a cathode Pt loading $\geq 0.13 \mathrm{mg} / \mathrm{cm}^{2}$ and $\geq 0.21 \mathrm{mg} / \mathrm{cm}^{2}$ is needed to ensure a $\leq 15 \%$ performance loss after 2100 and $\overline{4} 700$ AST cycles, respectively. Although the performance losses were below $15 \%$ for the durability window, ECSA losses over $40 \%$ were observed, even at 2100 cycles for the full loading range. This may suggest that the $40 \%$ ECSA loss criteria may be too conservative, compared to the performance loss criteria of $15 \%$ loss at $1 \mathrm{~A} / \mathrm{cm}^{2}$. 
DE-EE0000466

Ballard Material Products Inc.
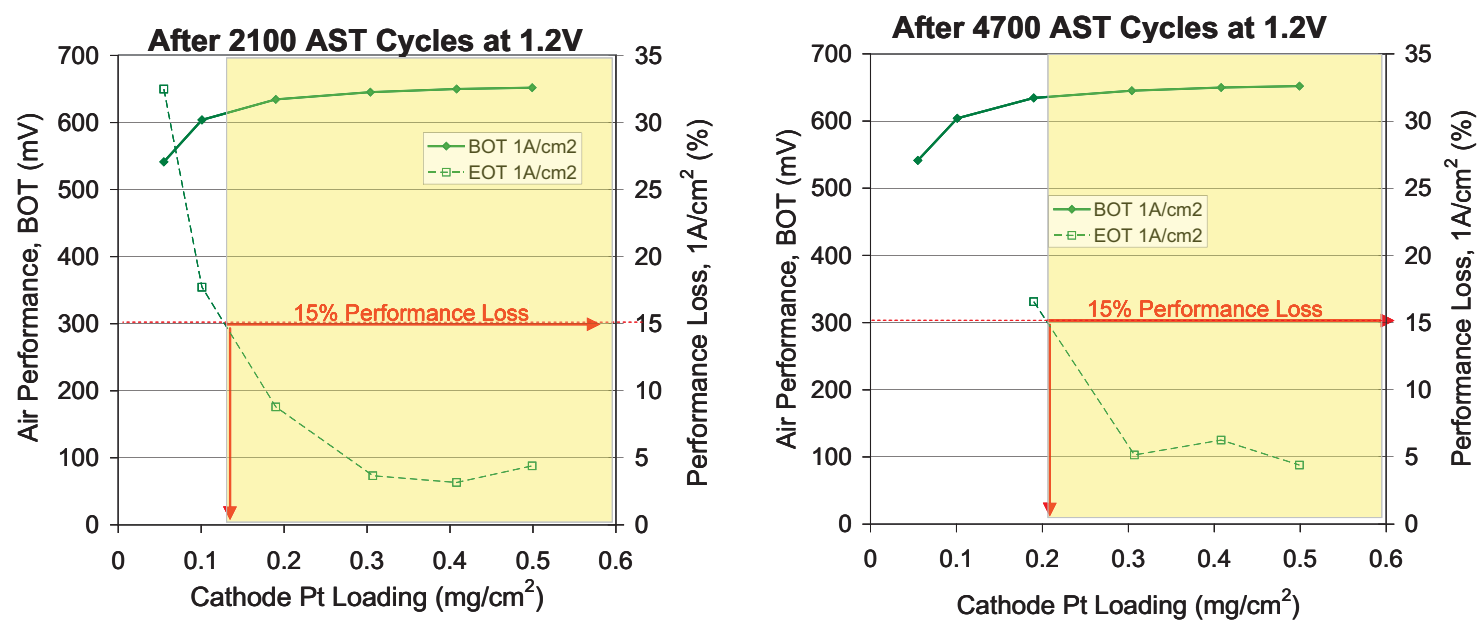

Figure 30: Cathode Pt Loading Durability Windows for 15\% performance loss

\section{(b) Ionomer Content Durability Window}

The cathode catalyst layer ionomer content has a strong effect on both the BOT performance and durability, as shown in Figure 31. A catalyst layer ionomer content of 23 to $40 \%$ would meet a durability target of $15 \%$ performance loss after 4700 AST cycles, while optimizing the BOT performance. Similar to the Pt loading study, ECSA losses of $>40 \%$ were observed for this durability window.

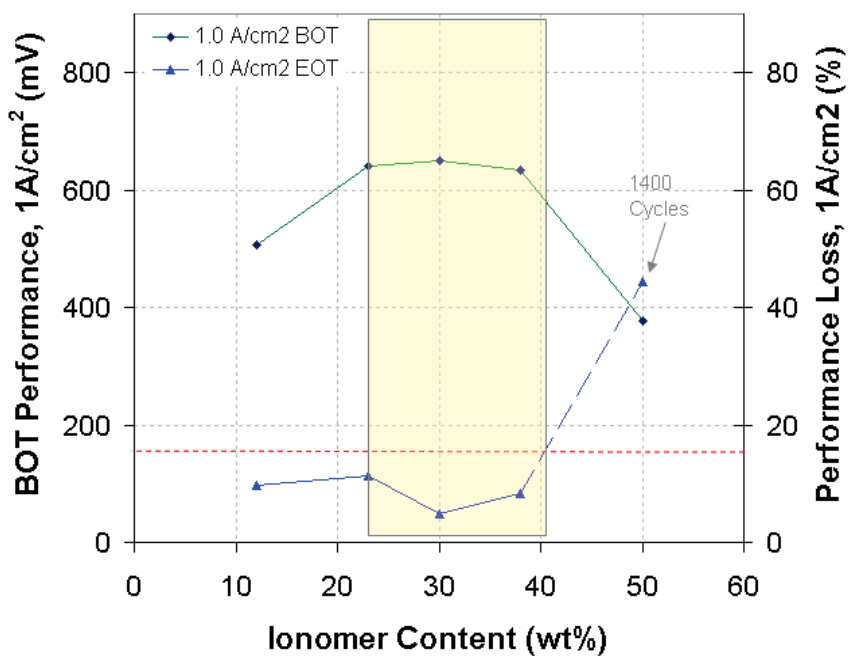

Figure 31: Durability window for ionomer loading for $15 \%$ performance loss 
DE-EE0000466

Ballard Material Products Inc.

\section{(c) Carbon Support Graphitic Content Durability Window}

The carbon support type, evaluated by surface graphitic content, showed a linear relationship to performance loss, as shown in Figure 32. This example used $\mathrm{Pt} / \mathrm{C}$ ratio of $50 \mathrm{wt} \%$ supported on low, medium, and high surface area carbon powders. The results show a surface graphite content of $\geq 55 \%$ will meet a durability target of $15 \%$ performance loss at $1 \mathrm{~A} / \mathrm{cm}^{2}$.

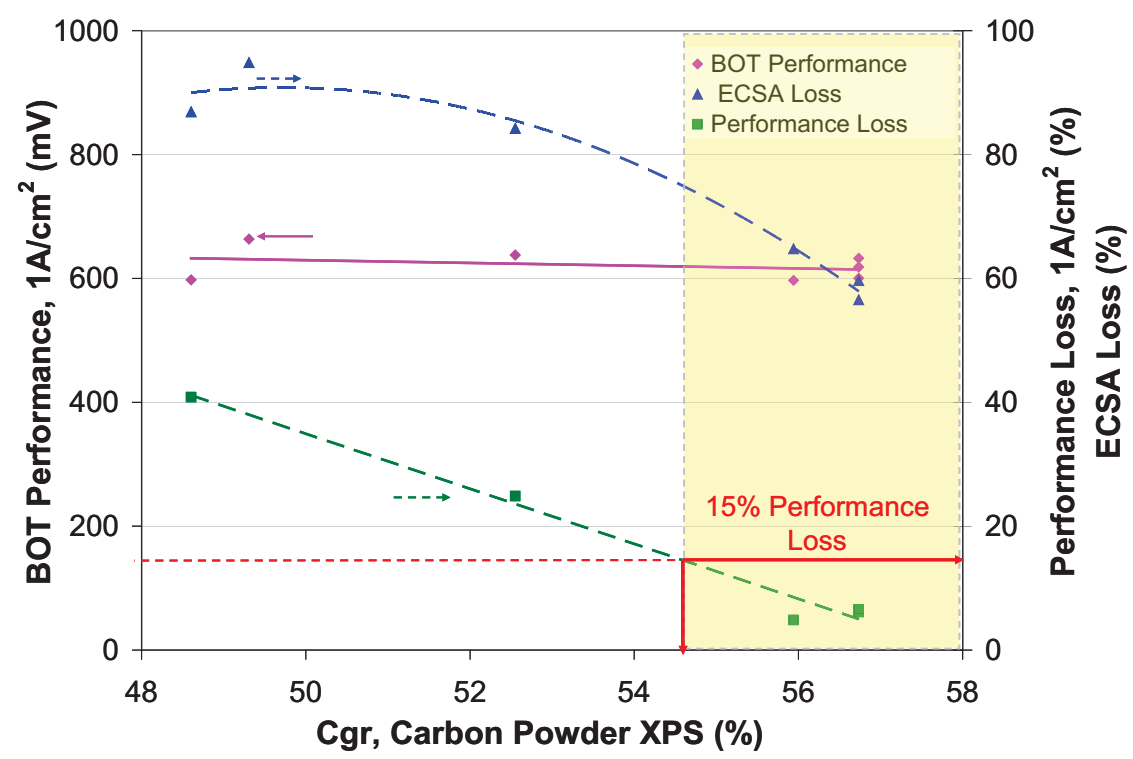

Figure 32: Durability window for graphitic content of the catalyst carbon support for $15 \%$ performance loss

\subsubsection{Operational Durability Windows}

In order to evaluate and demonstrate durability windows for operational conditions, the time held at UPL to reach $15 \%$ performance loss or $40 \%$ ECSA loss was determined for each operational condition. In some cases these values needed to be extrapolated beyond the tested data ( 5000 minutes at UPL). It is anticipated that this evaluation method will be more sensitive to the corrosion mechanism due to its sensitivity to UPL hold time, rather than Pt dissolution which is more sensitive to the number of cycles.

\section{Upper Potential Limit Durability Window}

Figure 33 shows the time at UPL to reach 15\% air performance loss and $40 \%$ ECSA loss. The time at UPL to $15 \%$ air performance loss increases exponentially with decreasing upper potential limit due carbon corrosion, while the time at UPL to $40 \%$ ECSA loss is linearly dependent on the UPL. A 20x increase in lifetime can be achieved by reducing UPL of $1.4 \mathrm{~V}$ to $1.2 \mathrm{~V}$, based on the $15 \%$ air performance criteria, while a $10 \mathrm{x}$ increase occurs for the $40 \%$ ESCA loss criteria. 
Figure 34 displays the sensitivity of the Time at UPL to a range in evaluation criteria of performance and ECSA loss. Very little difference between the $10 \%$ and $15 \%$ air performance loss criteria from $1.2 \mathrm{~V}$ to $1.4 \mathrm{~V}$ is evident due to the rapid degradation rates of the corrosion mechanism. However, at the lower UPLs, differences become bigger as the Pt dissolution mechanism is the dominant degradation process. This is also reflected in the ECSA losses, i.e. small differences in time are observed at a UPL of $1.4 \mathrm{~V}$ while, the operational times are substantially longer by moving the target from $40 \%$ to $60 \%$ ECSA loss.
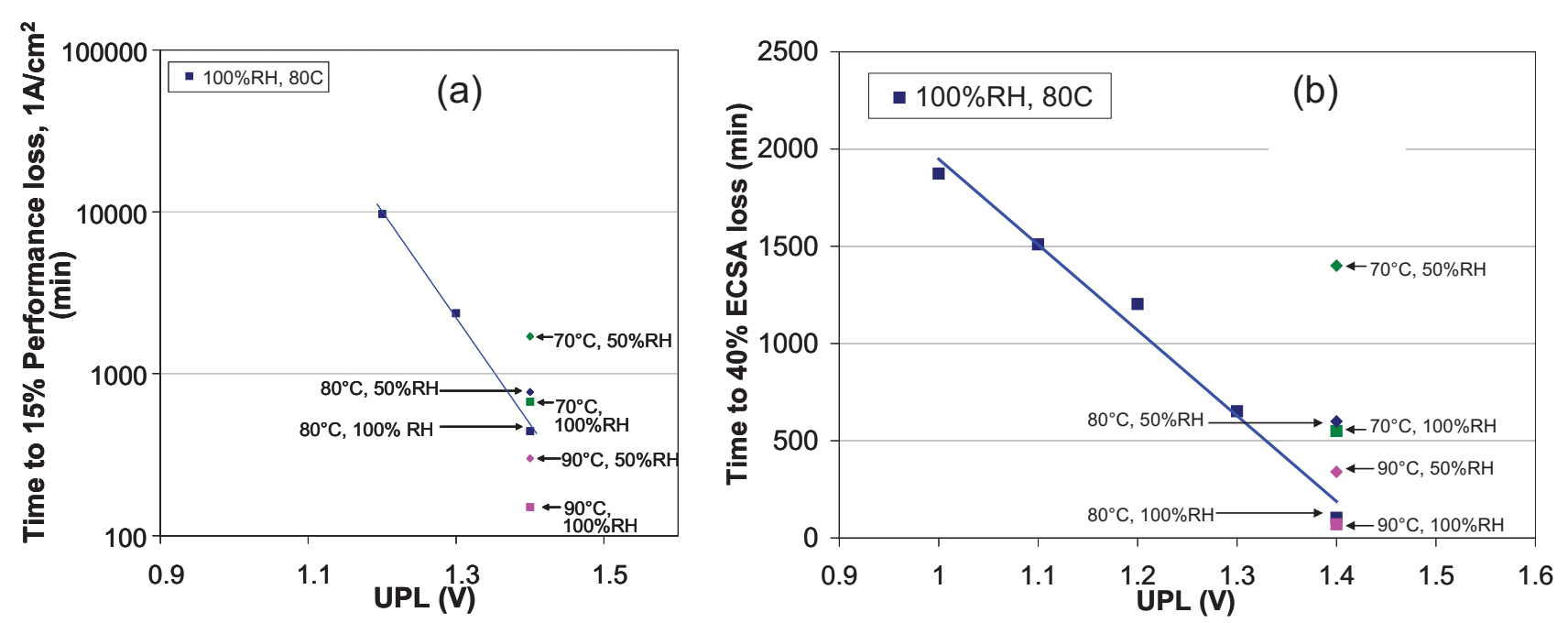

Figure 33: Time at UPL to cause 15\% performance loss and 40\% ECSA loss
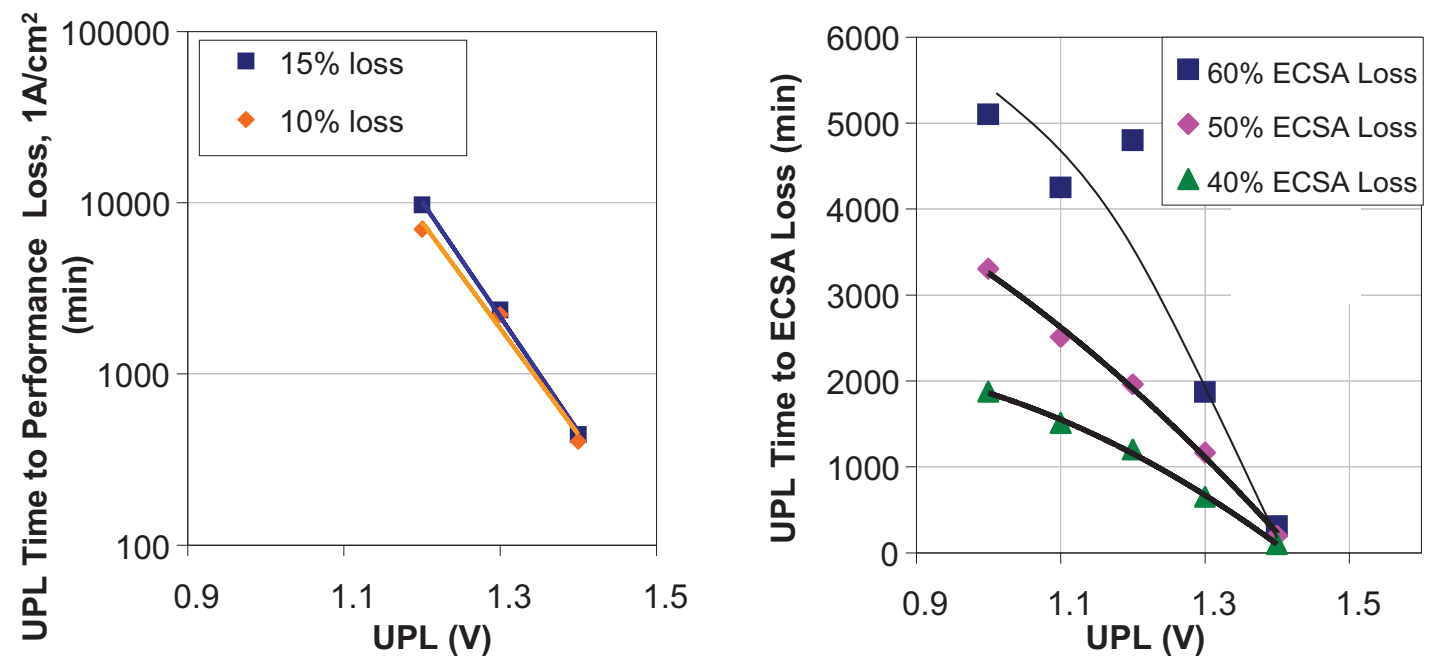

Figure 34: Comparison of time at UPL to reach (a) $10 \%$ and $15 \%$ performance loss and (b) $40 \%, 50 \%$, and $60 \%$ ECSA loss 


\section{(a) Temperature Durability Window}

The dependence on temperature follows an Arrhenius type behaviour under both wet $(100 \%$ $\mathrm{RH})$ and dry $(50 \% \mathrm{RH})$ conditions. This behaviour is observed for both air performance and ECSA losses. A $\sim 15$ times increase in lifetime at $1.4 \mathrm{~V}$ UPL can be achieved by reducing the temperature from $90^{\circ} \mathrm{C}$ to $60^{\circ} \mathrm{C}$.
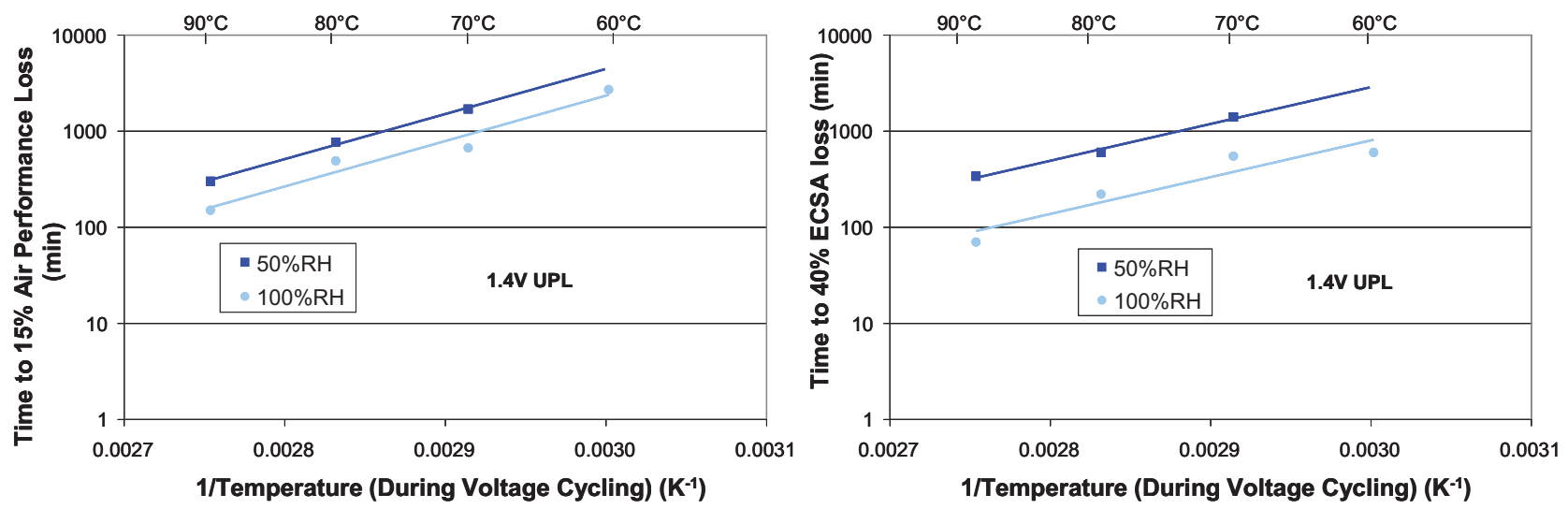

Figure 35: Effect of temperature on the time at UPL to reach $15 \%$ performance loss and $40 \%$ ECSA loss

\section{(b) Relative Humidity Durability Window}

The durability windows for relative humidity $(\mathrm{RH})$ on the cathode are developed for an upper potential limit at $1.2 \mathrm{~V}$ and $1.4 \mathrm{~V}$. The data show that $\mathrm{Pt}$ dissolution and corrosion degradation rates increase with increasing $\mathrm{RH}$ in the range of $50 \% \mathrm{RH}$ to $100 \% \mathrm{RH}$. The Time at UPL values decrease exponentially with increasing $\mathrm{RH}$. Once the oxidant gas is saturated with water, adding more liquid water has a minimal effect to the durability. By reducing the cathode $\mathrm{RH}$ from $100 \% \mathrm{RH}$ to $60 \% \mathrm{RH}$ an approximately 10 -fold increase in lifetime can be achieved. 
DE-EE0000466

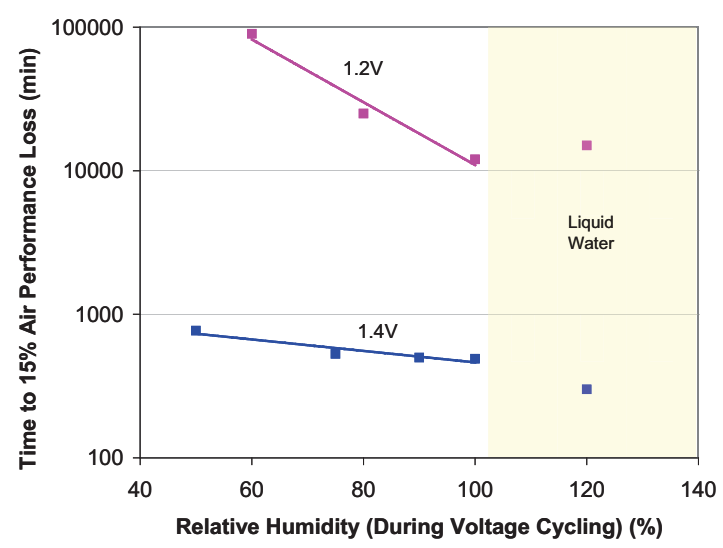

Ballard Material Products Inc.

Figure 36: Effect of temperature on the time at UPL to reach $15 \%$ performance loss and $40 \%$ ECSA loss at 1.4V UPL (Figure 35)

\subsubsection{Summary}

Durability windows for structural design levers including cathode Pt loading, ionomer content and carbon support graphitic content are summarized in Table 4. The operational durability windows are summarized in Table 5.

Table 4: Durability Windows for Structural Design Levers

\begin{tabular}{|l|l|}
\hline Design Lever & Durability Window \\
\hline Pt loading & $>0.21 \mathrm{mg} / \mathrm{cm}^{2}$ \\
\hline lonomer Content & 23 to $40 \mathrm{wt} . \%$ \\
\hline Carbon Support Surface Graphitic Content & $\geq 55 \%$ \\
\hline
\end{tabular}

Table 5: Durability Windows for Operational Parameter

\begin{tabular}{|c|c|c|c|c|}
\hline $\begin{array}{c}\text { Operational } \\
\text { Parameter }\end{array}$ & Lower Level & $\begin{array}{c}\text { Lower Level Time to } \\
15 \% \text { Performance } \\
\text { Loss }\end{array}$ & Upper Level & $\begin{array}{c}\text { Upper Level Time to } \\
15 \% \text { Performance } \\
\text { Loss }\end{array}$ \\
\hline $\begin{array}{c}\text { UPL } \\
(100 \% \text { RH, } \\
\left.80^{\circ} \mathrm{C}\right)\end{array}$ & $1.0 \mathrm{~V}$ & 243 hours & $1.4 \mathrm{~V}$ & 7 hours \\
\hline $\begin{array}{c}\text { Temperature } \\
(100 \% \mathrm{RH}, 1.4 \mathrm{~V} \\
\text { UPL) }\end{array}$ & $60^{\circ} \mathrm{C}$ & 46 hours & $90^{\circ} \mathrm{C}$ & 2.5 hours \\
\hline $\begin{array}{c}\text { Temperature } \\
(50 \% \mathrm{RH}, 1.4 \mathrm{~V} \\
\text { UPL })\end{array}$ & $60^{\circ} \mathrm{C}$ & 70 hours & $90^{\circ} \mathrm{C}$ & 5 hours \\
\hline $\begin{array}{c}\mathrm{RH}, \text { cathode } \\
\left(1.4 \mathrm{~V} \text { UPL, } 80^{\circ} \mathrm{C}\right)\end{array}$ & $60 \% \mathrm{RH}$ & 11 hours & $100 \%$ & 8 hours \\
\hline $\begin{array}{c}\mathrm{RH}, \text { cathode } \\
\left(1.2 \mathrm{~V} \text { UPL, } 80^{\circ} \mathrm{C}\right)\end{array}$ & $60 \% \mathrm{RH}$ & 1500 hours & $100 \%$ & 200 hours \\
\hline
\end{tabular}


DE-EE0000466

Ballard Material Products Inc. 


\title{
DE-EE0000466
}

Final Project Report

\section{Development of Micro-Structural Mitigation Strategies for PEM Fuel Cells: Morphological Simulations and Experimental Approaches}

\section{CHAPTER V}

\section{MOLECULAR MODEL}

\author{
PI: Seung Soon Jang \\ Georgia Institute of Technology \\ Giuseppe F. Brunello, PhD Candidate \\ Ji II Choi, Post Doctoral Fellow
}


DE-EE0000466

Ballard Material Products Inc.

\section{TABLE OF CONTENTS}

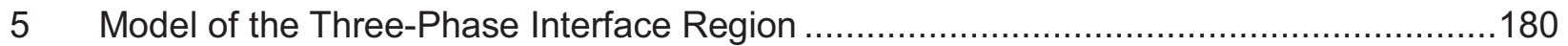

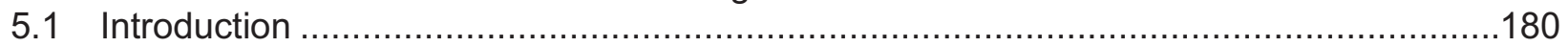

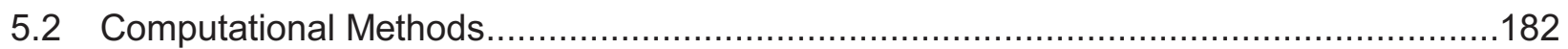

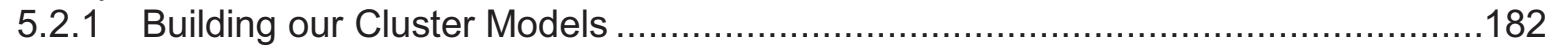

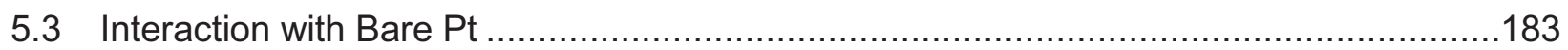

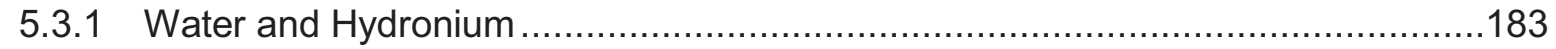

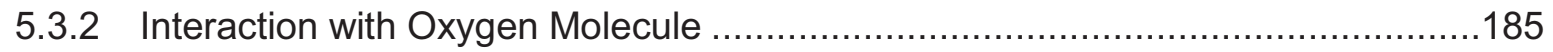

5.3.3 Interaction with a Polymer Electrolyte (Nafion) ……...................................188

5.3.4 Three-Phase Molecular Dynamics Model....................................................190

5.4 Interaction with Oxide Covered Pt ..................................................................191

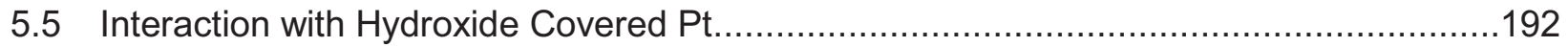

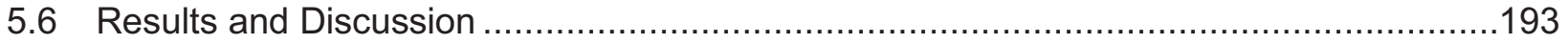

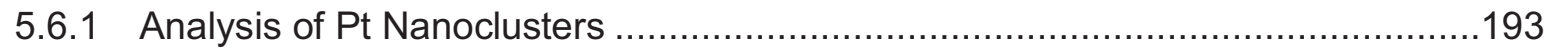

5.6.2 Coordination Number (CN) Model for Pt Nanoparticles ...................................197

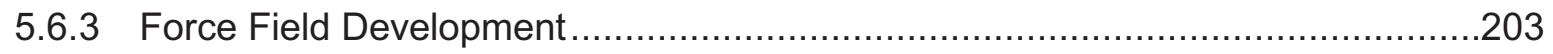

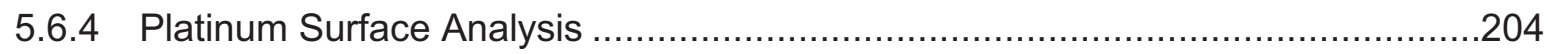

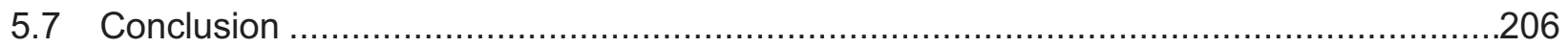

5.8 Pt Dissolution Study by the Nudged Elastic Band Method ......................................207

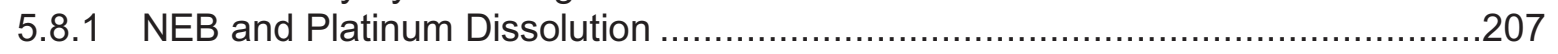

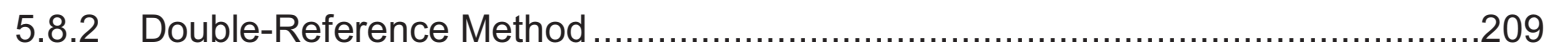

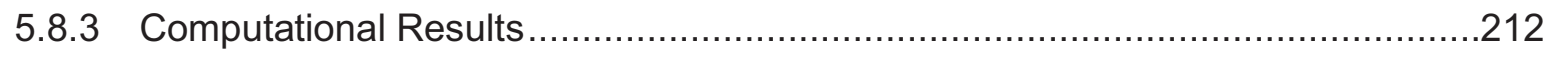

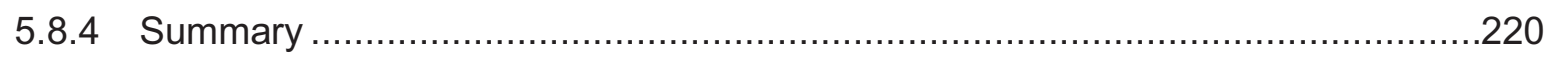

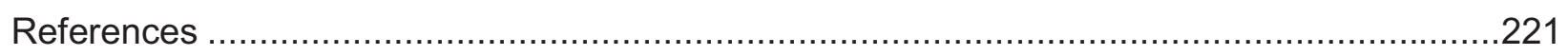


DE-EE0000466

Ballard Material Products Inc.

\section{LIST OF FIGURES}

Figure 1: Adsorptive binding energy of $\mathrm{H}_{2} \mathrm{O}$ (water) and $\mathrm{H}_{3} \mathrm{O}^{+}$(hydronium) molecule on $\mathrm{Pt}$ (111) surface: (a) optimized geometry of $\mathrm{H}_{2} \mathrm{O}-\mathrm{Pt}_{14-13-8}$; (b) optimized geometry of $\mathrm{H}_{3} \mathrm{O}^{+}-\mathrm{Pt}_{12-7}$; (c) change of binding energy of $\mathrm{H}_{2} \mathrm{O}$ and $\mathrm{H}_{3} \mathrm{O}^{+}$on $\mathrm{Pt}$ (111) surface as a function of distance from the surface along the surface normal. For DFT calculations, M06 functional is used with LACVP** basis set. For force field calculations, Morse potential function is used with newly optimized offdiagonal van der Waals parameters.

Figure 2: Side and top views of the six initial configurations before geometrically optimizing molecular oxygen on the Pt surface.

Figure 3: Adsorptive binding energy of $\mathrm{O}_{2}$ (oxygen) molecule on $\mathrm{Pt}$ (111) surface: (a) tile view of optimized geometry of $\mathrm{O}_{2}-\mathrm{Pt}_{14-13-8}$; (b) top view of optimized geometry; (c) change of binding energy of $\mathrm{O}_{2}$ on $\mathrm{Pt}$ (111) surface as a function of distance from the surface along the surface normal. For DFT calculations, M06 functional is used with LACVP ${ }^{* *}$ basis set. For force field calculations, Morse potential function is used with newly optimized off-diagonal van der Waals parameters

Figure 4: Adsorptive binding energy of fragments of polymer electrolyte (Nafion) on $\mathrm{Pt}$ (111) surface: (a) fragments of Nafion; (b) optimized geometry of $\mathrm{CF}_{3} \mathrm{CF}_{3}-\mathrm{Pt}_{12-}$ 7 ; (c) optimized geometry of $\mathrm{CF}_{3} \mathrm{OCF}_{3}-\mathrm{Pt}_{12-7} ;$ (d) optimized geometry of $\mathrm{CF}_{3} \mathrm{SO}_{3}{ }^{-}-\mathrm{Pt}_{12-7} ;(\mathrm{e})$ change of binding energy for $\mathrm{CF}_{3} \mathrm{CF}_{3}-\mathrm{Pt}_{12-7}$ and $\mathrm{CF}_{3} \mathrm{OCF}_{3-}$ $\mathrm{Pt}_{12-7}$ Vs. distance from the surface along the surface normal; (f) change of binding energy for $\mathrm{CF}_{3} \mathrm{SO}_{3}{ }^{-}-\mathrm{Pt}_{12-7} \mathrm{Vs}$. distance from the surface along the surface normal. For DFT calculations, M06 functional is used with LACVP** basis set. For force field calculations, Morse potential function is used with newly optimized off-diagonal van der Waals parameters.....

Figure 5: Water in on an oxide covered platinum surface.....

Figure 6: The DFT and force field fits for CF3CF3, CF3OCF3 and CF3SO3The DFT and force field fits for water, oxygen, and hydronium.

Figure 7: Various $\mathrm{Pt}$ nanoparticles $\left(\mathrm{Pt}_{\mathrm{X}-\mathrm{Y}-\mathrm{Z}}\right)$ calculated in this study. $\mathrm{X}, \mathrm{Y}$ and $\mathrm{Z}$ denote the number of $\mathrm{Pt}$ atoms in the first, the second and the third layer, respectively, of the nanoparticles. If the number of $\mathrm{Pt}$ in the layer is zero, it is not shown

Figure 8: Change in relative energy of Pt nanoparticle as a function of spin multiplicity. The relative energy is the energy difference from the minimum energy with a optimal spin multiplicity. Other cases are not presented due to their similarity

Figure 9: Change of atomic cohesive energy (ACE) with various layers as a function of the number of $\mathrm{Pt}$ atoms in a cluster. The ACE values of Pt clusters are higher than that of Pt in bulk Phase....

Figure 10: A Pt nanoparticle has atoms of various coordination number (CN): (a) For example, $\mathrm{Pt}_{6-3-1}$ has $4 \mathrm{Pt}$ atoms with $\mathrm{CN}=3$ and $6 \mathrm{Pt}$ atoms with $\mathrm{CN}=6$; (b) Change in atomic cohesive energy of $\mathrm{Pt}$ as a function of coordination number $(\mathrm{CN})$ reported in Table 3.

Figure 11: Pt nanoparticles used to validate the $\mathrm{CN}$ model in Eq. (2). The number of Pt atoms are 21 for all cases, and the positions of the two red colored Pt atoms are determined to generate different $\mathrm{CN}$ 
Figure 12: (a) Energy and (b) radius of gyration of a Pt cluster vs count from a MC simulation of a 95-atom cluster. 200

Figure 13: Pt surfaces with various facets: (a) (111) direction; (b) (100) direction. The colors are introduced to clarify the structural repetition of the atomic layers

Figure 14: Per cent occupation on the Pt170 surface 202

Figure 15: Schematic representation of the Nudged Elastic Band method. Starting from an initial reaction path (initial images), the chain settles to the Minimum Energy Path (MEP) images after convergences 207

Figure 16: Platinum dissolution (a) on bare Pt (111) surface; (b) and (c) show optimized initial and final models surrounded by water molecules, respectively 208

Figure 17: Activation of water on the bare Pt (111) surface in the applied electric field of $0.06 \mathrm{~V}$. It shows hydroxyl species and hydronium in the solution.

Figure 18: Results of double-reference method to compute applied electrostatic potentials for corresponding charges. Plane averaged electrostatic potential plots are illustrated: (a) (b) as an example, illustrates models with vacuum and water phase under the corresponding electrostatic potential plots; in the middle of the vacuum, first reference point is established, and second reference point is in the middle of water phase of the second plot (without vacuum phase); (d) potential plots of system of oxygen covered surfaces; and (e) hydroxide covered surfaces.

Figure 19: Energy minimized structures at potential, (a) $-2.12 \mathrm{~V},-0.67 \mathrm{~V}$, and $0.50 \mathrm{~V}$ on bare Pt (111) surface; (b) $-0.74 \mathrm{~V}, 1.98 \mathrm{~V}$, and $2.44 \mathrm{~V}$ on Oxygen covered surface; and (c) $0.98 \mathrm{~V}, 1.12 \mathrm{~V}$, and $2.08 \mathrm{~V}$ on $\mathrm{OH}$ covered surface. Water molecules surround the dissolute Pt atom and prevent re-clustering to the surface. Dotted red circle on $\mathrm{Pt}(111)$ surface on figure (a) indicates the initial position of the dissociated Pt atom.

Figure 20: Free energy changes are plotted as a function of reaction coordinates, (a) bare $\mathrm{Pt}(111)$ surface, (b) oxygen covered Pt(111) electrode, (c) hydroxide covered $\mathrm{Pt}(111)$ surface. The reaction coordinates are measured from the initial position of a Pt atom on the surface to the coordinates of dissociated Pt atom. Energy barriers of Oxygen- or Hydroxide-covered surfaces do not show clear dependency to applied potentials.

Figure 21: First energy barrier in $\mathrm{eV}$ as a function of applied potential. In the range of voltage $(0 \mathrm{~V} \sim 1.2 \mathrm{~V})$, the Pt dissolution hardly happens on the bare surface of platinum. Oxygen covered surface shows lowest energy barrier in the range.

Figure 22: Fitted harmonic oscillator model along the energy curve. It is supposed that the dissociated Pt atom vibrates about fixed average positions until it crosses over the transition point.

Figure 23: Transmission coefficients of the Pt dissolution on the bare platinum surface using the harmonic oscillator model. The plot shows that the Pt dissolution depends on the applied potentials 
DE-EE0000466

Ballard Material Products Inc.

\section{LIST OF TABLES}

Table 1: Interaction parameters for oxygen on top of $\mathrm{Pt} . \mathrm{O} \_\mathrm{H} 3 \mathrm{O}, \mathrm{H} \_\mathrm{H} 3 \mathrm{O}$ refer respectively to oxygen and hydrogen of hydronium, $\mathrm{C} 33$ to the carbon in the polymer backbone, O_2 to the oxygen molecule, O_CF3 the oxygen in Nafion's ether group, S_3 and O_2a to the sulfur and oxygen of the sulfonate respectively, $F_{-}$is the fluorine, the O_F3C and H_F3C are the oxygen and hydrogen of the water molecules

Table 2: Force field parameters for CF3CF3, CF3OCF3 and CF3SO3

Table 3: The atomic cohesive energy with respect to the coordination number. As expected, more coordinated atoms contribute more to form more stable $\mathrm{Pt}$ nanoparticles by lowering its cohesive energy

Table 4: Performance of $\mathrm{CN}$ model to predict surface energies and cluster CE 201

Table 5: Energy of losing an atom and the relative order

Table 6: Force field parameters for the ionomer moieties interacting with platinum using the Morse Potential function. The parameter $\alpha$ is unitless

Table 7: (a) The occupancy for various moieties on the platinum surface; (b) The occupancy for various moieties on the oxygen covered platinum surface; (c) The occupancy for various moieties on the hydroxide covered platinum surface.

Table 8: Used potential values. Applied charges and corresponding electrode potentials of with respect to the NHE potential of the bare Pt(111), Oxygen covered, and $\mathrm{OH}$ covered surface in water phase. $Q$ values are total net charges applied to each system, for instance, -1e means that one electron was added into the system.

Table 9: Energy barriers along forward (from initial state to transition state), and reverse (from final state to the transition state) directions

Table 10: Energy barriers for oxygen-, hydroxide- covered surfaces. It shows two barriers, first, it appears on the surfaces. There exists energy barrier to escape the surface. Second, the barrier exists when the dissolved Pt-complex moves into the water phase

Table 11: Calculated transmission coefficients. Transition rate was calculated following the definition given above 
DE-EE0000466

Ballard Material Products Inc.

\section{Model of the Three-Phase Interface Region}

In the three-phase boundary region of Polymer Electrolyte Membrane Fuel Cells (PEMFC), the electrolyte, catalyst and electrode work in concert to ensure operation. Technical challenges in developing a simulation study of this region are numerous; the first step in developing a model is a quantum mechanical study of the materials and their interaction. To this end, we preformed various Density Functional Theory simulations of platinum clusters and their interaction with various moieties. We developed a DFT simulation protocol, a model for the cohesive energy of Pt clusters and found the binding energy curves for various moieties on $\mathrm{Pt}$, which will allow us to develop an MD model of the three-phase region. To validate our simulations we compared our results with others and their methods. Having developed the protocol, we developed a cohesive energy model that uses the coordination model of the $\mathrm{Pt}$ atoms in the cluster. From this we determined which Pt atoms are least tightly bound to the cluster and the energy and shape distribution. Finally our adsorption simulations gave us the binding energy curves for the $\mathrm{Pt}$ clusters with adsorbed moieties.

\subsection{Introduction}

There has been a significant amount of interest in metal particles with small sizes down to the nanometer scale. This is due to their engineering importance[1-4] since the property of materials with size below a certain length scale is distinctly different from that from the bulk phase.[5, 6] This is true for transition metals, as quantum effects are more prominent as the surface-volume ratio increases. [7, 8] In particular, platinum nanoparticles, despite their high price, are considered as the catalyst of choice for fuel cell technology due to their excellent catalytic abilities. Therefore, theoretical and computational[9-15] investigations as well as experimental approaches[16-18] have been performed extensively to understand this material. The aim of this research is the maximizing of the benefit from the use of such precious metal and also understand the underpinning fundamentals of the observed properties.

There have been many experimental studies on Pt nanoparticles as well as theoretical work. The experimental research has investigated crystallographic changes of structures through spectroscopic study. At the same time, theoretical studies were trying to explain the shape of clusters while overcoming artifacts of DFT in describing heavy metal atoms by considering various electronic correlations.

In experiments it was found that the metallic character depends on the size of the cluster; Eberhart et al. [16] were able to create metal clusters of 1-6 atoms and found using photoemission spectroscopy that such metal clusters did not have metallic character. Also, the Pt dimer was found to have a different bond length than the bulk. Airola[18] and Morse studied the Pt dimer using fluorescence spectroscopy and obtained the bond length of $2.33 \AA$ for the ground state, compared to the bulk length of $2.774 \AA$. Given how different these clusters are, the smallest Pt clusters might not be representative of a catalytic material. Wang and his coworkers[17] reported that Pt clusters of tetrahedral symmetry maintain their shape up to 350 $450^{\circ} \mathrm{C}$ beyond which Pt clusters tend to become spherical. They also found that the melting temperature of the clusters is significantly lower than the bulk melting temperature. 
DE-EE0000466

Ballard Material Products Inc.

Compared to experimental characterization, the theoretical and computational approaches may have distinct advantages in tackling small systems with nanoscale dimensions by directly investigating the atomic structures and the corresponding properties. To improve DFT calculations, various theoretical aspects were introduced. To obtain better energy and geometrical results, dynamical and non-dynamic electron correlation was considered by Lee et al, [15] to obtain reliable spectroscopic data relativistic effects for binding energy for heavy metals were introduced by Varga et al, [9] and Grönbeck and Andreoni[10] studied neutral and anion various shapes to $\mathrm{Pt}_{5}$ using SLDA and BLYP. Their calculations found that 3-D geometries are not favored up to $\mathrm{Pt}_{5}$ clusters. Through a DFT study on large size of $\mathrm{Pt}$ clusters shapes using PW91 and plane wave basis set, however, Xiao and Wang[13] found that the planar shape is preferred for small clusters up to 9 atoms, because the layered Pt clusters are very stable compared to closed-packed spherical shapes. This is in agreement with other research groups.[14, 19, 20] However, a hybrid functional DFT study using B3LYP by Sebetci [21] showed contradictory results reporting that 3-D geometries are energetically preferred on $\mathrm{Pt}_{\mathrm{n}}(\mathrm{n}=4-6)$. It is thought that small size clusters show their peculiar energy state due to their size and energy state. The quantum mechanical properties of confined electrons on small clusters is not fully understood, but it is known that change in geometry also leads to specific stabilized electronic energy states even for an equal number of electrons. To avoid problems with shape and spin dependency when studying small-size clusters, it is required to research on various size clusters, DFT functionals and spin multiplicity of the cluster. Apra and Fortunelli [12] reported that $\mathrm{Pt}_{n}(\mathrm{n}=13,38$ and 55) start developing metallic characteristics already, especially $\mathrm{Pt}_{55}$ favors the icosahedral geometry $\left(\mathrm{I}_{h}\right)$ whereas $\mathrm{Pt}_{13}$ favors the $\mathrm{D}_{4 h}$ geometry. In addition, Chepulskii and Curtarolo[22] calculated the energy of various common Pt nanoparticle shapes as a function of size up to 400 atoms using various GGA functionals and plane wave basis set. From such large cluster calculations, they found that the surface energy depends on the lattice parameter and the bulk surface energies are only valid for nanoparticles with the diameter greater than 1.46 1.57 nm.

Our work on platinum nanocluster uses DFT to model the energy of a large set of clusters. From this we build an energy model of the platinum nanocluster similar to cluster expansion models.[23-27] From this we obtain the cluster size distribution for a given number of atoms.

Fuel cell molecular species are also present on the catalyst surfaces. Investigating the interaction of $\mathrm{Pt}$ with molecular species is difficult. Work on Pt-water interaction includes work by Langenbach et al.[28] who used via IR-reflection and UV-photoemission and found that water molecules are adsorbed on the Pt (111) surface via its oxygen atom. Michaelides et al.[29] confirmed the water adsorption on the on-top site of Pt (111) surface using DFT. DFT studies agree that water's oxygen atom is positioned on the on-top position of Pt nearly parallel to the surface. There is also agreement on the binding energy of water on a plane (111) surface, but there is no such consensus for the binding energy of water on a Pt cluster. [29-44]

Oxygen adsorption on $\mathrm{Pt} 111$ has also been studied, both experimentally[45-48] and computationally[49-52], and it is found that oxygen can be in many different states. Chemisorbed molecular oxygen can be in both paramagnetic (top-bridge-top) and nonmagnetic state (tilted bridge on the fcc and hcp sites). Experiment and computation agree on the preferred configuration for the molecular physisorbed state, the top-bridge-top configuration, however most computation find an adsorption of around $0.7 \mathrm{eV}$, whereas the experimental results are $0.38 \mathrm{eV}[47]$ and $0.5 \mathrm{eV}$. However, Ohma et al. obtained a binding energy of $0.39 \mathrm{eV}$ for the oxygen molecule adsorbed in the bridge site using DFT.[53] 
DE-EE0000466

Ballard Material Products Inc.

Little research has been done on Nafion adsorbed on Pt with DFT. Of note, Kendrick et al. used DFT to confirm his experimental work suggesting that the sulfonate group is strongly bound to the Pt surface. He also found that the CF3CF3 groups are also strongly bound.[54] In this study we studied various adsorbed species on $\mathrm{Pt}$, including water, oxygen, hydronium, and various Nafion fragments. From these simulations, we built a computational system to study the three-phase interface of a PEMFC. To this end we have probed the phase space of these moieties on the $\mathrm{Pt}$ surface to obtain the strength of their interaction, the position and conformation of the adsorbed species. From this we developed a force field for use in a molecular dynamics study of the three phase interface of the PEMFC system.

\subsection{Computational Methods}

In order to investigate electronic structure and energies from various $\mathrm{Pt}$ nanoparticles, we used spin-unrestricted Density Functional Theory (DFT) through Jaguar.[55] By employing three widely used functionals M06,[56, 57] PBE[58, 59] and B3LYP[60, 61] with LACVP** basis set containing $6-31 \mathrm{G}^{* *}$ and LANL2DZ effective core basis set,[62] we attempted to compare the electronic structures and interactions of Pt nanoparticles of various shapes and sizes (Figure 7) which have up to three layers.

For our larger systems, we also used spin-unrestricted periodic DFT through CASTEP[63] implemented in Materials Studio[64] with PBE functional and a plane wave basis set, to compare to our non-periodic DFT calculations with the local basis set. By design, some functionals are more suited for certain materials than others and users must select the appropriate one suited for their study. In particular, PBE is widely used for metallic studies, and B3LYP is widely used for organic systems.

The electronic structure of platinum particles depends both on the size and shape of the particle. Consequently, different clusters have different minimum energy multiplicities. To perform our jaguar calculations we started with a guess of the initial spin states, using the interstitial electron model described by McAdon and Goddard and as used by Kua and Goddard.[65, 66] This method identifies the location, and therefore number, of the interstitial electrons. From this, the number of unpaired electrons is obtained. By having a good guess of the initial spin state, the amount of work required to get the correct spin state is reduced. Then we simulated different multiplicities around this initial guess until we arrived at the lowest energy one. We verified that the various functionals agree on the lowest energy multiplicity.

\subsubsection{Building our Cluster Models}

We built clusters of different shapes and sizes. The smallest clusters (up to 5 atoms), were carved from the crystal structure by trying to exhaustively obtain all of the combinations. Due to their small size, these structures carved from the crystal structure are not expected to be the lowest possible energy. This is because the crystal lattice is for an infinite bulk having, therefore, insignificant surface energy.

In addition to small clusters, we also investigated medium-sized clusters (up to $n=35$ ). Our method for making medium-sized clusters is different from the method to build small clusters. Whereas the small clusters were built trying to exhaust all possible shapes within our constraint of being on lattice, this is no longer possible, for large $n$, because the number of possible shapes increases greatly as the number of atoms is increased. Instead, our clusters were built by carving out the crystal structure of platinum into various shapes. Shapes chosen include highly symmetric shapes, as well as random shapes (see below). As the number of atoms is increased clusters become more bulk-like. 
The set of clusters built by carving out the crystal structure introduces some bias because they were built by the user carving them out from the lattice. These clusters were usually very symmetric. To eliminate this bias, we sought to add clusters without this systematic bias. These clusters are of random shape and were built by a computer program.

This program was a Monte Carlo program that used Boltzmann sampling to generate different shapes constrained to the Pt crystal lattice and also constrained to be a connected graph (a move is allowed only if the cluster remains whole). After inserting $n$ atoms during the initialization, the program randomly picks an atom and attempts to move it according to the move's resulting change in energy and the simulated temperature. The energy model used for these MC calculations was from the preliminary results from the coordination-model developed in the course of the study. Since we wanted to sample a large portion of phase space, we annealed the clusters by bringing the temperature up to $5 \mathrm{eV}$. We then added these clusters to our energy model and generated new ones to validate it.

\subsection{Interaction with Bare Pt}

\subsubsection{Water and Hydronium}

To describe the interaction of water $\left(\mathrm{H}_{2} \mathrm{O}\right)$ and hydronium $\left(\mathrm{H}_{3} \mathrm{O}^{+}\right)$molecules with $\mathrm{Pt}$ (111) surface, we obtained the adsorptive binding energy using M06 functional with LACVP** basis set. For this purpose, the structure of $\mathrm{H}_{2} \mathrm{O}-\mathrm{Pt}_{14-13-8}$ and $\mathrm{H}_{3} \mathrm{O}^{+}-\mathrm{Pt}_{12-7}$ nanoparticle was optimized (Figure 1a and b) and then, the binding energy was calculated as a function of phase space. This is done by moving the molecules in many directions, especially along the direction normal to the surface. Usually the direction normal to the surface had the strongest change in energy, however, in the case of the water molecule we found that in order to obtain a good force field we had to change its orientation extensively along two angles as well. Figure 1c shows that the binding energies are $15.01 \mathrm{kcal} / \mathrm{mol}$ at the distance of $2.411 \AA$ and $\sim-40.43 \mathrm{kcal} / \mathrm{mol}$ at the distance of $2.899 \AA$ for water and hydronium, respectively. Due to the magnitude of the binding energy, and no spin change, it is clear that the water molecule is physisorbed on the $\mathrm{Pt}$ (111) surface. On the other hand, we observed that the binding of hydronium with Pt surface is much stronger than that of water due to the charge. This type of strong adsorption of the charged molecular species onto the metal surface is due to the polarization of the metal surface induced by the charges in the molecule; that is, the positive $\mathrm{H}_{3} \mathrm{O}^{+}$molecules change the available energy states on the $\mathrm{Pt}$ surface near the hydronium. As a consequence, there are more electrons occupying states on the platinum atoms near the $\mathrm{H}_{3} \mathrm{O}^{+}$than there otherwise would be. This can also be explained succinctly with the classical electromagnetism results of a charge near a conductor surface: an opposite "mirror" charge is induced. The charge-charge interaction between the positive hydronium and the excess electrons cause the strong observed binding energy. Thus, these results indicate that the hydronium molecule is preferable on the $\mathrm{Pt}$ nanoparticle. 


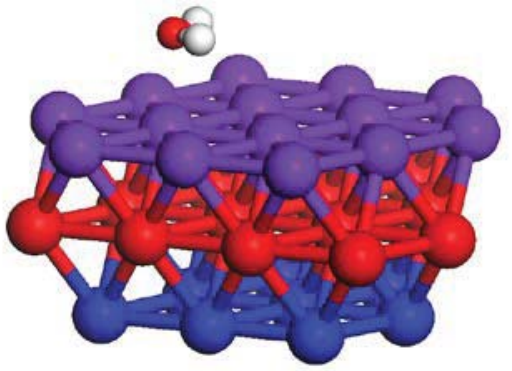

(a)

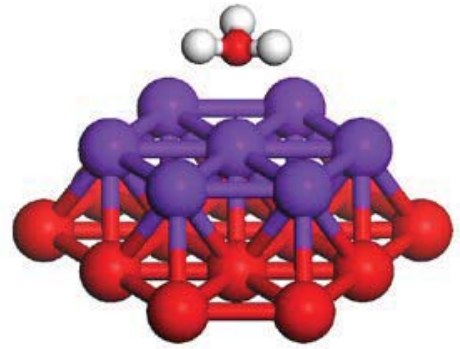

(b)

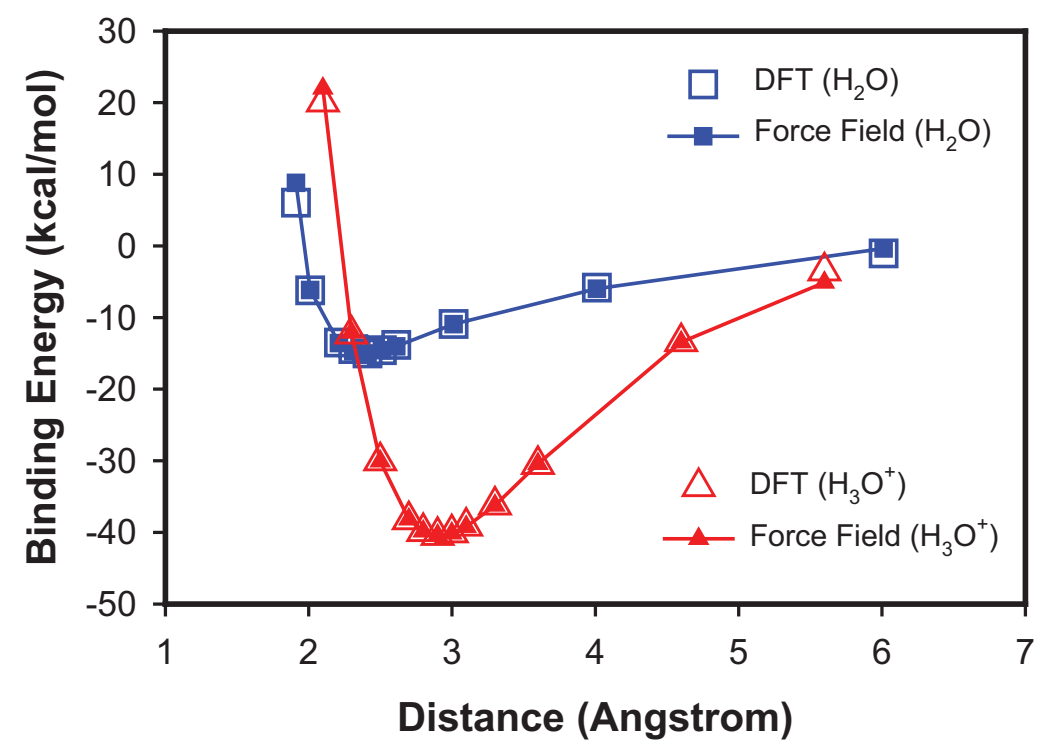

(c)

Figure 1: Adsorptive binding energy of $\mathrm{H}_{2} \mathrm{O}$ (water) and $\mathrm{H}_{3} \mathrm{O}^{+}$(hydronium) molecule on Pt (111) surface: (a) optimized geometry of $\mathrm{H}_{2} \mathrm{O}-\mathrm{Pt}_{14-13-8}$; (b) optimized geometry of $\mathrm{H}_{3} \mathrm{O}^{+}-\mathrm{Pt}_{12-7}$; (c) change of binding energy of $\mathrm{H}_{2} \mathrm{O}$ and $\mathrm{H}_{3} \mathrm{O}^{+}$on $\mathrm{Pt}$ (111) surface as a function of distance from the surface along the surface normal. For DFT calculations, M06 functional is used with LACVP $^{* *}$ basis set. For force field calculations, Morse potential function is used with newly optimized off-diagonal van der Waals parameters 
DE-EE0000466

Ballard Material Products Inc.

\subsubsection{Interaction with Oxygen Molecule}

Currently experimental [45-48] and computational [49-52] studies agree that the lowest physisorbed state for molecular oxygen is along the Pt-Pt bond. However most computation find an adsorption of around $0.7 \mathrm{eV}$, whereas the experimental results are $0.38 \mathrm{eV}[47]$ and $0.5 \mathrm{eV}$, although Ohma et al. in 2010 found a binding energy of $0.39 \mathrm{eV}$ using DFT.[53]

In our study we started by geometrically optimizing various configuration of the oxygen $\left(\mathrm{O}_{2}\right)$ molecule adsorbed on the Pt (111) with DFT. Many different starting configurations are needed because there are many local minima in the Pt-molecular oxygen energy phase space. The initial states used are reproduced in Figure 2. We found that an oxygen $\left(\mathrm{O}_{2}\right)$ molecule lies along the Pt-Pt bond on Pt (111) surface (Figure 3a), in agreement with previous studies. Furthermore, many oxygen molecules in the various initial configurations ended up along the Pt bond. For the geometric optimization we tried six different initial positions because the $\mathrm{O}_{2}-\mathrm{Pt}$ interaction is very complex. For thoroughness, we also tried many different spin states. By analyzing the change of the binding energy as a function of the distance from the Pt surface, the $\mathrm{O}_{2}$ adsorption on $\mathrm{Pt}$ (111) surface takes place through two mechanisms, chemisorption or physisorption. As shown in Figure $3 \mathrm{~b}$, through chemisorption, $\mathrm{O}_{2}$ molecule is adsorbed at $2.12 \AA$ of the distance from the Pt surface with $-7.71 \mathrm{kcal} / \mathrm{mol}$ of the binding energy $(\mathrm{S}=11$ where $S$ is spin multiplicity). On the other hand, the physisorption occurs at $2.72 \AA$ of the distance with a smaller binding energy $(-7.07 \mathrm{kcal} / \mathrm{mol}$ or $0.31 \mathrm{eV})(\mathrm{S}=13)$. Our physisorbed result is closer than most other DFT results. Also, it is observed that the physisorption has a longer distance from the Pt surface compared to the chemisorption. Figure $3 \mathrm{c}$ shows that, after $\mathrm{O}_{2}$ physisorbed Pt surface at $2.72 \AA$, can form a chemisorbed state by overcoming the energy barrier at $\sim 2.5 \hat{A}$. This is a first step towards the oxygen reduction reaction which is not within the scope of this study since the oxygen reduction reaction has been studied very intensively. 


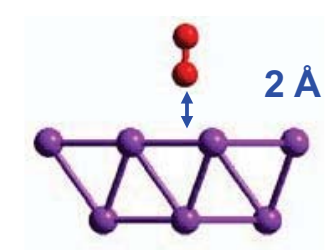

HCP
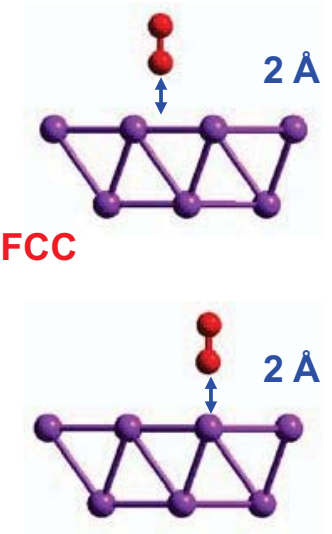

On top Pt
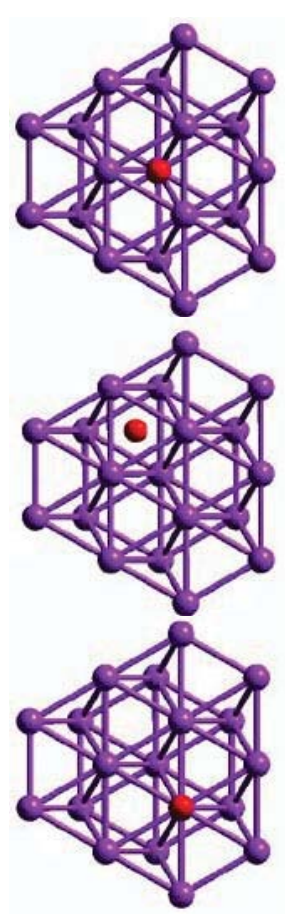

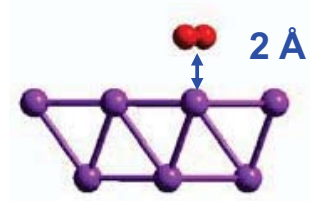

Lie diagonal

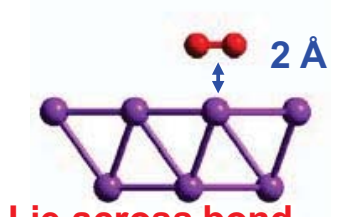

Lie across bond

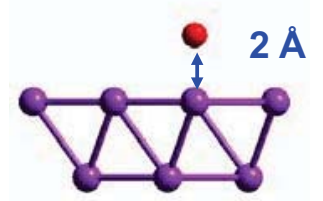

Lie Along Pt
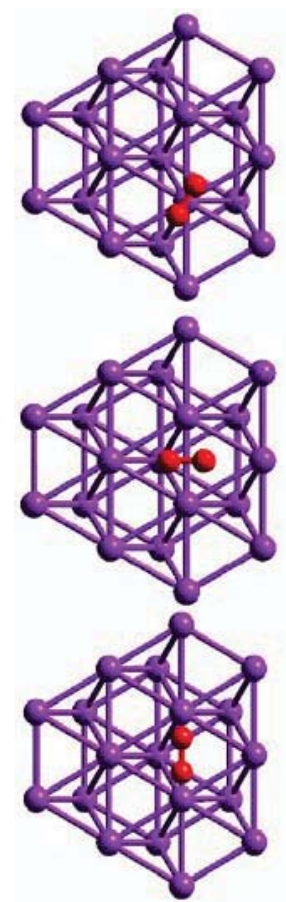

Figure 2: Side and top views of the six initial configurations before geometrically optimizing molecular oxygen on the Pt surface 


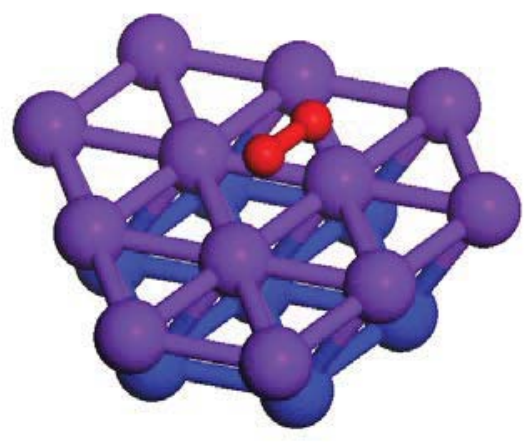

(a)

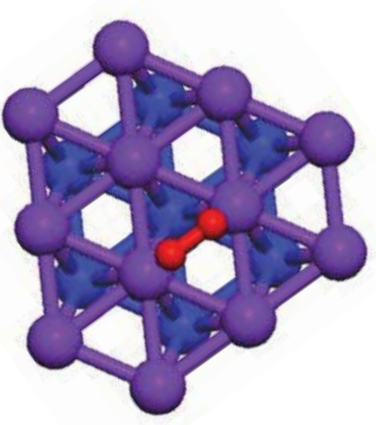

(b)

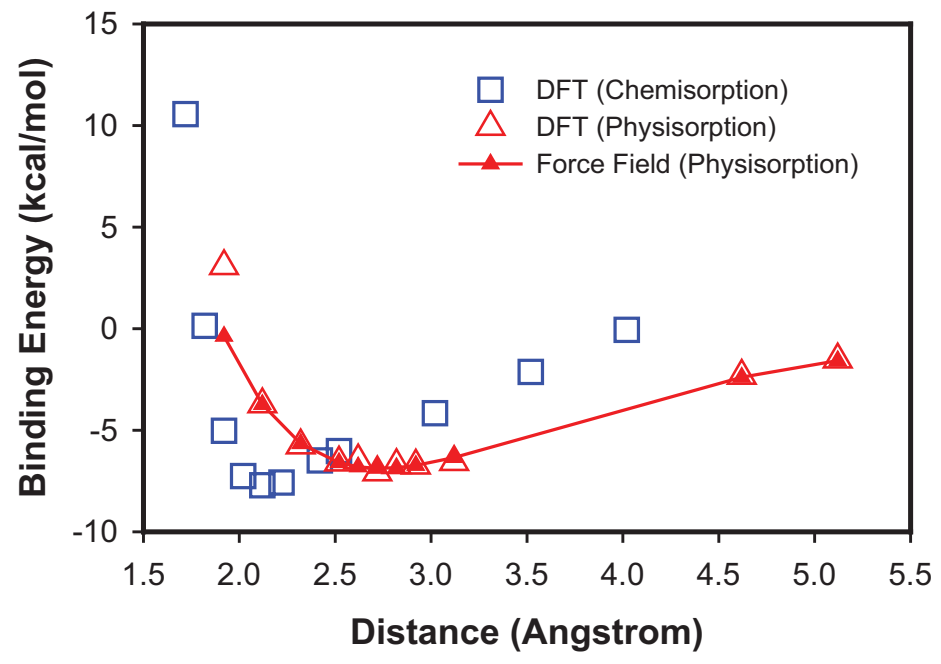

(c)

Figure 3: Adsorptive binding energy of $\mathrm{O}_{2}$ (oxygen) molecule on $\mathrm{Pt}$ (111) surface: (a) tile view of optimized geometry of $\mathrm{O}_{2}-\mathrm{Pt}_{14-13-8}$; (b) top view of optimized geometry; (c) change of binding energy of $\mathrm{O}_{2}$ on $\mathrm{Pt}$ (111) surface as a function of distance from the surface along the surface normal. For DFT calculations, M06 functional is used with LACVP ${ }^{* *}$ basis set. For force field calculations, Morse potential function is used with newly optimized off-diagonal van der Waals parameters 
DE-EE0000466

Ballard Material Products Inc.

\subsubsection{Interaction with a Polymer Electrolyte (Nafion)}

Although the surface of the electrode is coated by an ionomer, its interaction with the Pt catalyst is not thoroughly understood. In order to investigate the Nafion-Pt interaction, first, we prepared three small model molecules by segmenting the Nafion unit structure (Figure 4a) such as perfluorinate $\left(\mathrm{CF}_{3} \mathrm{CF}_{3}\right)$, perfluorodimethyl ether $\left(\mathrm{CF}_{3} \mathrm{OCF}_{3}\right)$, and perfluoromethyl sulfonate $\left(\mathrm{CF}_{3} \mathrm{SO}_{3}{ }^{-}\right) . \mathrm{CF}_{3} \mathrm{CF}_{3}, \mathrm{CF}_{3} \mathrm{OCF}_{3}$ and $\mathrm{CF}_{3} \mathrm{SO}_{3}{ }^{-}$represent the backbone chain, the side chain ether and the sulfonate group of side chain in Nafion, respectively. This division of the polymer chain is inspired, and justified, by the common abstraction in polymer science that treats a polymer chain like a chain of beads. In this abstraction model, the polymer chain is treated like linked beads. Likewise we divide our Nafion chain into smaller segments and assume that each atom type's interaction with the platinum does not change if they are in a representative segment or the polymer.

We performed a DFT geometry-optimization of these model molecules on the Pt (111) surface as presented in Figure 4b, Figure 4c and Figure 4d. For each system, we tried several initial positions since the geometric optimizer cannot guarantee global minimization. Our computation resulted in the following binding energies: $-11.88 \mathrm{kcal} / \mathrm{mol}$ at $3.70 \AA \hat{,},-13.25 \mathrm{kcal} / \mathrm{mol}$ at $3.25 \hat{\AA}$, and $-51.33 \mathrm{kcal} / \mathrm{mol}$ at $2.71 \AA$ for $\mathrm{CF}_{3} \mathrm{CF}_{3}-\mathrm{Pt}_{12-7}$ (b), $\mathrm{CF}_{3} \mathrm{OCF}_{3}-\mathrm{Pt}_{12-7}$ (c) and $\mathrm{CF}_{3} \mathrm{SO}_{3}^{-}-\mathrm{Pt}_{12-7}$ (d), respectively. All the distance are from the surface along the surface normal. The size of the binding energy indicates that $\mathrm{CF}_{3} \mathrm{CF}_{3}$ and $\mathrm{CF}_{3} \mathrm{OCF}_{3}$ are physisorbed, and no change in optimal spin state was found. Like the $\mathrm{H}_{3} \mathrm{O}^{+}$, the $\mathrm{CF}_{3} \mathrm{SO}^{+}$charged nature causes significant changes in the metals' electronic state resulting in a large binding energy. From the binding energies of these model molecules, it is inferred that the surface of Pt nanoparticle is occupied by the sulfonate groups of Nafion, hydronium, and water molecules while $\mathrm{CF}_{3} \mathrm{CF}_{3}$ and $\mathrm{CF}_{3} \mathrm{OCF}_{3}$ may not be able to occupy the surface of Pt nanoparticle. However, these segments must be near the catalyst since the sulfonate group is attached to the backbone through these segments. 
DE-EE0000466

Ballard Material Products Inc.

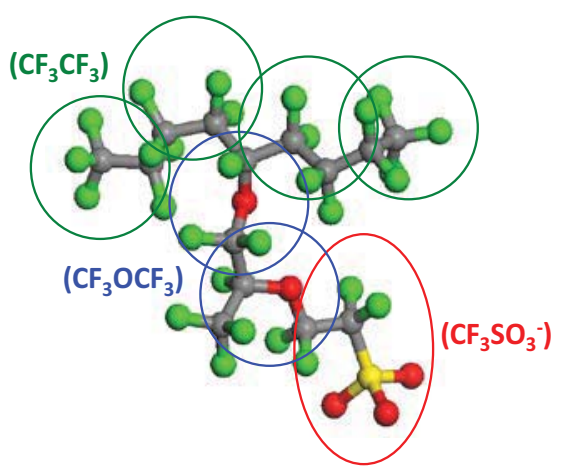

(a)

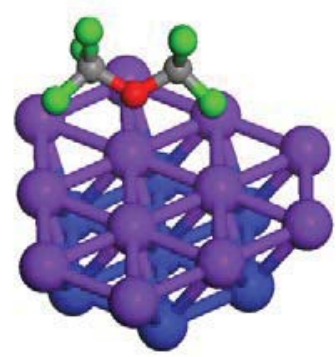

(c)

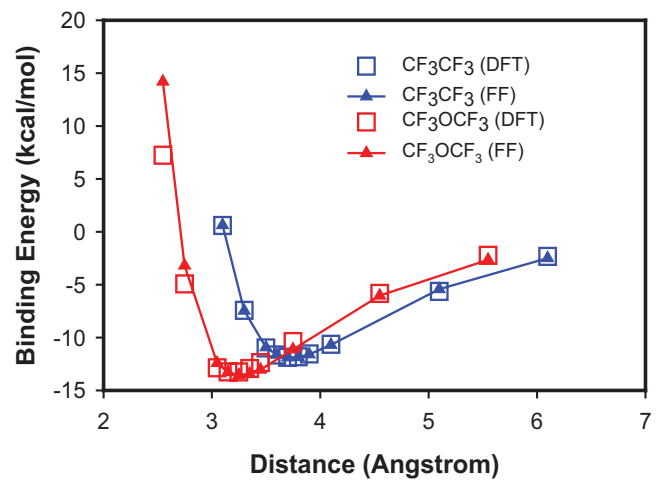

(e)

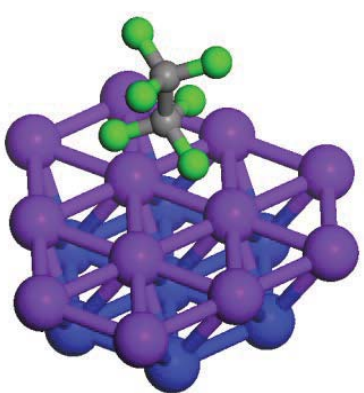

(b)

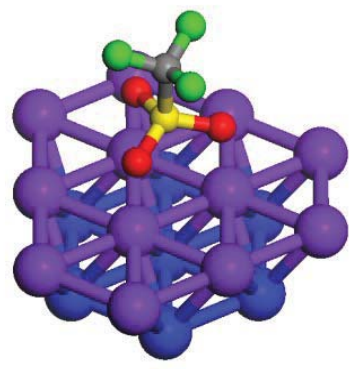

(d)

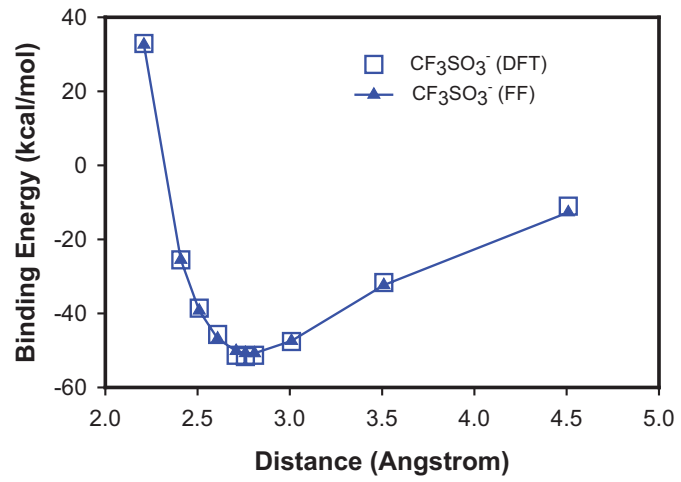

(f)

Figure 4: Adsorptive binding energy of fragments of polymer electrolyte (Nafion) on Pt (111) surface: (a) fragments of Nafion; (b) optimized geometry of $\mathrm{CF}_{3} \mathrm{CF}_{3}-\mathrm{Pt}_{12-7}$; (c) optimized geometry of $\mathrm{CF}_{3} \mathrm{OCF}_{3}-\mathrm{Pt}_{12-7}$; (d) optimized geometry of $\mathrm{CF}_{3} \mathrm{SO}_{3}-\mathrm{Pt}_{12-7}$; (e) change of binding energy for $\mathrm{CF}_{3} \mathrm{CF}_{3}-\mathrm{Pt}_{12-7}$ and $\mathrm{CF}_{3} \mathrm{OCF}_{3}-\mathrm{Pt}_{12-7}$ vs. distance from the surface along the surface normal; (f) change of binding energy for $\mathrm{CF}_{3} \mathrm{SO}_{3}{ }^{-}-\mathrm{Pt}_{12-7} \mathrm{Vs}$. distance from the surface along the surface normal. For DFT calculations, M06 functional is used with LACVP ${ }^{* *}$ basis set. For force field calculations, Morse potential function is used with newly optimized off-diagonal van der Waals parameters 
DE-EE0000466

Ballard Material Products Inc.

\subsubsection{Three-Phase Molecular Dynamics Model}

Having developed a force field for Nafion-Pt interaction we built a MD model of the three-phase region of the fuel cell. This model contains the electrolyte, the electrode, and the catalyst at the cathode region. Furthermore, a vacuum layer was added. This vacuum layer is the channel by which gaseous molecules are introduced or removed from the electrolyte. During the simulation, therefore, we find that water and oxygen molecules leave the electrolyte and establish a gas phase.

Like in previous chapters, we utilized the DREIDING force field[67] and the F3C water model.[68] These force field parameters are described in the original papers[67-69]. Our force field has the form:

$$
E_{\text {total }}=E_{v d W}+E_{Q}+E_{\text {bond }}+E_{\text {angle }}+E_{\text {torsion }}+E_{\text {inversion }}
$$

where $E_{\text {total }}, E_{v d W}, E_{Q}, E_{\text {bond }}, E_{\text {angle }}, E_{\text {torsion }}$ and $E_{\text {inversion }}$ are total energies, van der Waals, electrostatic, bond stretching, angle bending, torsion and inversion components, respectively.

The charge equilibration (QEq) method[70] optimized to reproduce the Mulliken charges of small molecules. The atomic charges of water molecule were from the F3C water model.[68]

For this study, the annealing and MD simulations were performed using the MD code LAMMPS (Large-scale Atomic/Molecular Massively Parallel Simulator) from Plimpton at Sandia[71, 72]. The equations of motion were integrated using the velocity Verlet algorithm[73] with a time step of 1.0fs. The Nose-Hoover temperature thermostat[74-76] for the NVT and NPT MD simulations used a damping relaxation time of $0.1 \mathrm{ps}$ and the dimensionless cell mass factor of 1.0 . We annealed the system and allowed $5 \mathrm{~ns}$ of equilibration time. Then we collected the data from another 5 ns run.

Due to the large size and simulation time of system, our simulations were performed at Keeneland at the Oak Ridge National Labs, a supercomputer cluster of GPU machines. Therefore, we used the CUDA modules of LAMMPS to exploit this architecture.

Our simulation box size was $51.3 \times 51.91 \times 509$ Angstroms. The simulation box size was chosen so that a graphite sheet can fit unstrained in the $x$ and $y$ direction. For the $z$ direction, our Nafion ionomer was around 100 angstroms thick, and the graphite layer was around $9 \AA$ $(0.335 \mathrm{~nm} \times 3)$. The remaining $400 \AA$ were free volume. The electrode was four layers of graphite, the bottom most layer being fixed and the topmost layer had a vacancy defect on it. The graphite had no charge on it. Then we placed a platinum cluster of 170 atoms, all with zero charge, on top of the defect on the top most layer of graphite. We found that a defect is necessary to anchor the Pt nanoparticle onto the graphite surface otherwise the particle drifts away from the electrode.

The amorphous hydrated Nafion was built in a separate simulation box and equilibrated for $5 \mathrm{~ns}$. Then we copied it to our new simulation box. This was done to speed up the equilibration of the amorphous Nafion. The hydrated ionomer we introduced had a $\lambda$ of 14 . There were 16 Nafion chains, with a molecular mass of 11468Da and ten sulfonate groups per chain. Our Nafion chains, therefore, have a similar equivalent weight to the commonly used Nafion 117 material (equivalent weight $1100 \mathrm{~g}$ ). 


\subsection{Interaction with Oxide Covered Pt}

The development of a force field for oxygen covered platinum necessitated a new set of simulations similar to the ones preformed for bare platinum. A periodic system with oxygen atoms on the FCC position of the platinum surface was used. The initial positions of the moieties were determined by geometric optimizing the various species on the platinum surface. Because geometric optimization does not guarantee that the absolute minimum will be found, this was done for many initial positions guided by physical and geometrical insight.

The development of a force field for oxygen covered platinum largely followed the scheme illustrated in the bare platinum model. One major complication is that our assumption that the cluster particle is polarizable is no longer valid. The oxygen atoms on top of the platinum clusters impose a strict charge on the platinum atoms below it. Therefore, we must treat the electrostatic interaction between the various moieties and the cluster explicitly. This was done with the Ewald summation.

Table 1: Interaction parameters for oxygen on top of $\mathrm{Pt}$. $\mathrm{O} \_\mathrm{H} 3 \mathrm{O}, \mathrm{H} \_\mathrm{H} 3 \mathrm{O}$ refer respectively to oxygen and hydrogen of hydronium, C_3 to the carbon in the polymer backbone, O_2 to the oxygen molecule, O_CF3 the oxygen in Nafion's ether group, S_3 and O_2a to the sulfur and oxygen of the sulfonate respectively, $\mathrm{F}_{\text {_ }}$ is the fluorine, the $\mathrm{O}_{-} \mathrm{F} 3 \mathrm{C}$ and $\mathrm{H}_{-} \mathrm{F} 3 \mathrm{C}$ are the oxygen and hydrogen of the water molecules

\begin{tabular}{|c|c|c|c|}
\hline & De (kcal) & Re (A) & Gamma \\
\hline OH30-O_PtO & 0.395 & 5.34 & 6.96 \\
\hline HH30-O_PtO & 0.668 & 3.89 & 2.17 \\
\hline C_3-O_PtO & 0.233 & 4.034 & 15.47 \\
\hline O_2-O_PtO & 0.033 & 4.675 & 8.81 \\
\hline O_CF3-O_PtO & $4.59 e-5$ & 6.89 & 27.1 \\
\hline S_3-O_PtO & 1.258 & 5.91 & 6.09 \\
\hline O_2a-O_PtO & 3.100 & 2.70 & 18.9 \\
\hline F_-O_PtO & 0.00139 & 8.373 & 5.362 \\
\hline OF3C-O_PtO & 0.577 & 3.307 & 6.637 \\
\hline HF3C-O_PtO & 0.372 & 3.67 & \\
\hline
\end{tabular}

The PtO force field must include parameters for both $\mathrm{Pt}$ and $\mathrm{O}$. There are two possibilities for the Pt parameters. One is to refit the Pt parameters. Another is to use the Pt parameters from the bare $\mathrm{Pt}$ case above. We choose the later because it will allow us to perform simulations of partially covered Pt clusters. In Table 1 we report the fitting parameters used. 
DE-EE0000466

Ballard Material Products Inc.

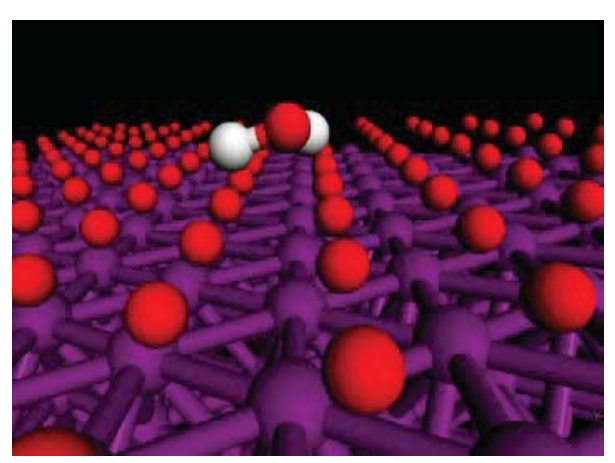

Figure 5: Water in on an oxide covered platinum surface

\subsection{Interaction with Hydroxide Covered Pt}

Similar to the work on the oxide covered Pt, we calculated the interaction energy of hydroxide covered Pt, as shown in Figure 6. The resulting fits are shown in Table 2.

Table 2: Force field parameters for CF3CF3, CF3OCF3 and CF3SO3

\begin{tabular}{|c|c|c|c|c|c|c|c|}
\hline Element & D & re & $\mathbf{a}$ & Element & D & re & $\mathbf{a}$ \\
\hline Pt_C_3 & 0.3880 & $\begin{array}{l}4.4 \\
760\end{array}$ & 1.5400 & H_OH-O_CF3 & $\begin{array}{c}1.0 \\
019\end{array}$ & 4.2528 & 2.1963 \\
\hline O_OH-C_3 & 0.2000 & $\begin{array}{l}4.0 \\
917 \\
\end{array}$ & 1.7570 & Pt_S_3 & $\begin{array}{r}3.0 \\
411 \\
\end{array}$ & 3.4127 & 2.1497 \\
\hline H_OH-C_3 & 0.0334 & $\begin{array}{l}2.7 \\
798\end{array}$ & 0.2746 & O_OH-S_3 & $\begin{array}{c}1.1 \\
332\end{array}$ & 10.9432 & 0.1192 \\
\hline Pt_F & 0.2050 & $\begin{array}{c}3.6 \\
740 \\
\end{array}$ & 0.8160 & H_OH-S_3 & $\begin{array}{l}4.0 \\
103 \\
\end{array}$ & 2.9784 & 6.2539 \\
\hline O_OH-F & 0.0047 & $\begin{array}{l}8.3 \\
556 \\
\end{array}$ & 0.4848 & Pt_0_2a & $\begin{array}{c}2.3 \\
511 \\
\end{array}$ & 2.5113 & 1.0243 \\
\hline H_OH-F_ & 5.5543 & $\begin{array}{l}0.4 \\
989\end{array}$ & 1.8613 & O_OH-O_2a & $\begin{array}{c}0.0 \\
230\end{array}$ & 4.6326 & 1.6089 \\
\hline Pt_O_CF3 & 3.4998 & $\begin{array}{l}2.7 \\
867 \\
\end{array}$ & 2.5512 & H_OH-O_2a & $\begin{array}{c}0.0 \\
207 \\
\end{array}$ & 9.1361 & 0.2760 \\
\hline O_OH-O_CF3 & 0.0124 & $\begin{array}{c}11 . \\
444 \\
4\end{array}$ & 0.3069 & & & & \\
\hline
\end{tabular}


DE-EE0000466

Ballard Material Products Inc.

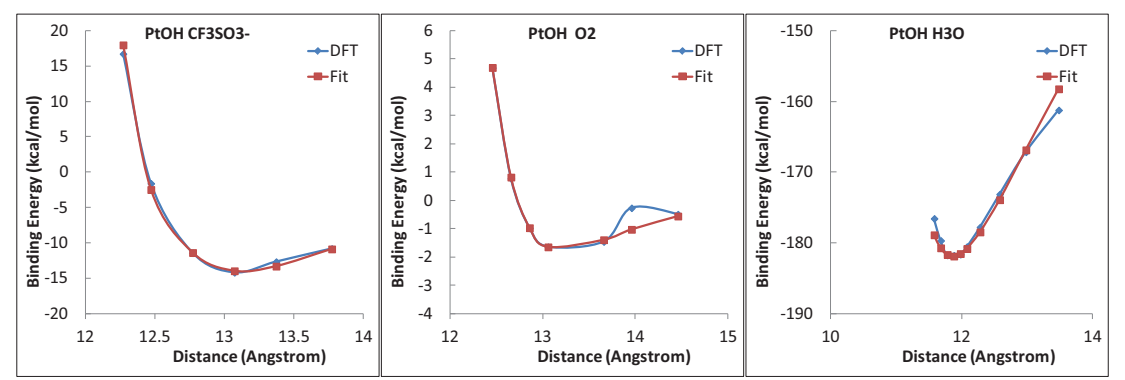

Figure 6: The DFT and force field fits for CF3CF3, CF3OCF3 and CF3SO3The DFT and force field fits for water, oxygen, and hydronium

\subsection{Results and Discussion}

\subsubsection{Analysis of Pt Nanoclusters}

We evaluated the energy of the Pt nanoparticles shown in Figure 7. In order to obtain their most probable energy state, we performed the spin-unrestricted calculations with various spin multiplicities. Some of the calculations are presented in Figure 8 in which the relative energies are calculated as the energy difference from the minimum energy and the optimal spin state. The optimal spin state is defined as a physically possible spin state which gives the lowest energy. After specifying the minimum energy value of Pt nanoparticle with the optimal spin state, we calculated the normalized cohesive energy (NCE) defined as:

$$
N C E=\left[E\left(P t_{X-Y-Z}[m]\right)-n \times E(P t)\right] / n
$$

where $\mathrm{m}$ and $\mathrm{n}$ denote the spin multiplicity and the total number of $\mathrm{Pt}$ atoms in the nanoparticle, and $X, Y$ and $Z$ denote the number of $P t$ atoms in the first, the second, and the third layer of the nanoparticle, respectively. The sum of $\mathrm{X}, \mathrm{Y}$ and $\mathrm{Z}$ is equal to $\mathrm{n}$. $E\left(P t_{X-Y-Z}[m]\right)$ and $E(P t)$ are the energies of the $\mathrm{Pt}$ nanoparticle and the single $\mathrm{Pt}$ atom, respectively.

In Figure 9, we summarize the NCEs calculated from the Pt nanoparticles shown in Figure 7. First, it is observed that the NCE decreases as a function of the number of Pt atoms in a nanoparticle, approaching the experimental value of NCE for bulk Pt $(-5.8 \mathrm{eV})$.[77] As expected, the stability of the Pt nanoparticle increases with increasing particle size and the three different functionals converge to the asymptote in a similar manner. 
DE-EE0000466

Ballard Material Products Inc.

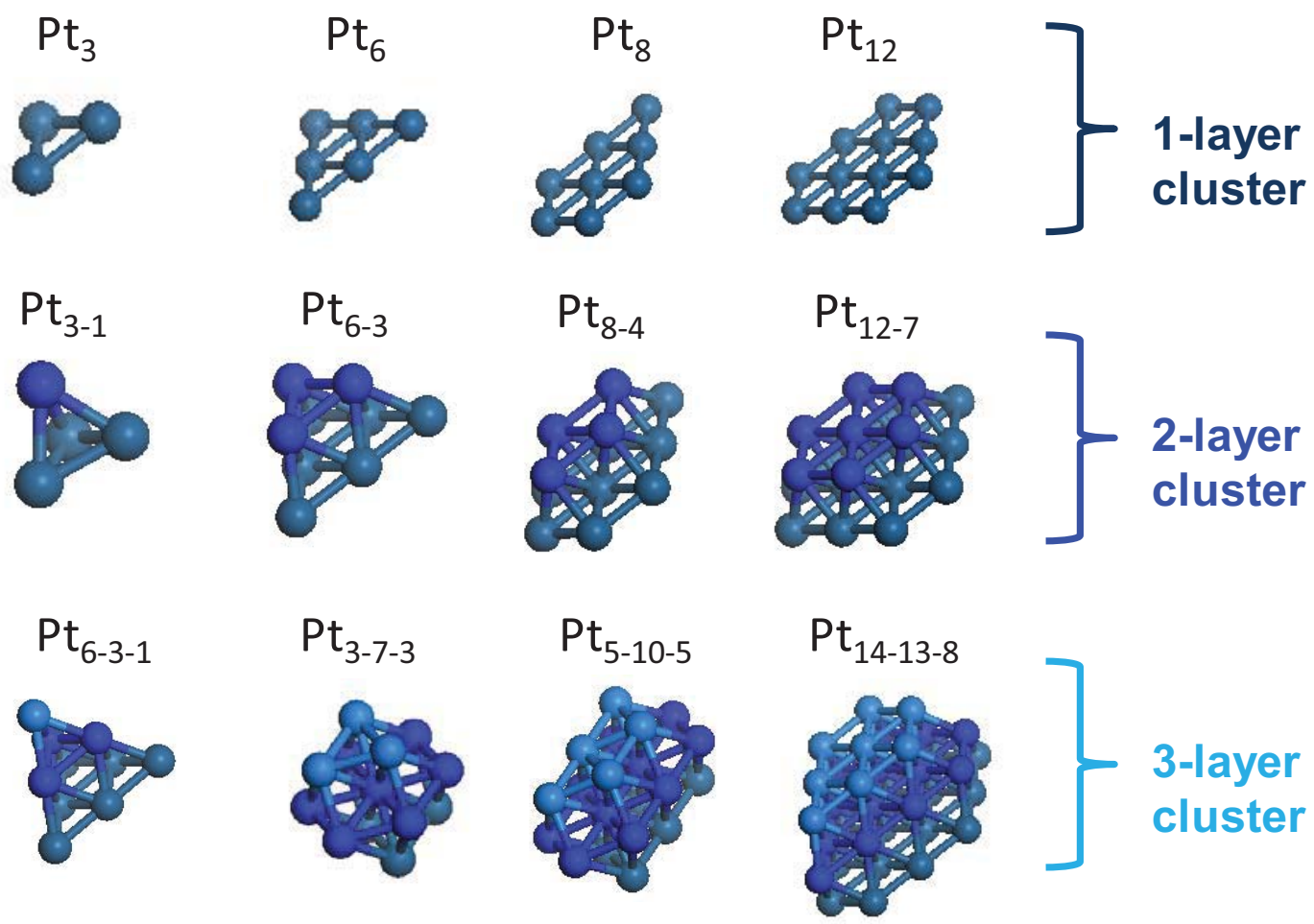

Figure 7: Various $\mathrm{Pt}$ nanoparticles $\left(\mathrm{Pt}_{\mathrm{X}-\mathrm{Y}-\mathrm{Z}}\right)$ calculated in this study. $\mathrm{X}, \mathrm{Y}$ and $\mathrm{Z}$ denote the number of $\mathrm{Pt}$ atoms in the first, the second and the third layer, respectively, of the nanoparticles. If the number of $\mathrm{Pt}$ in the layer is zero, it is not shown 
DE-EE0000466

Ballard Material Products Inc.

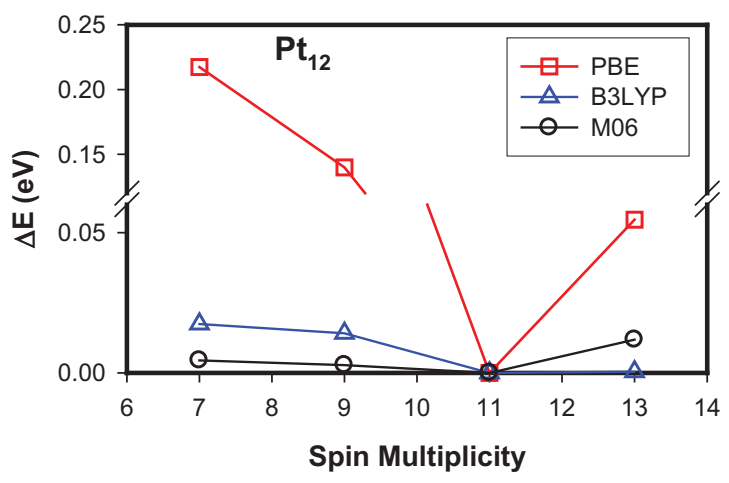

(a)

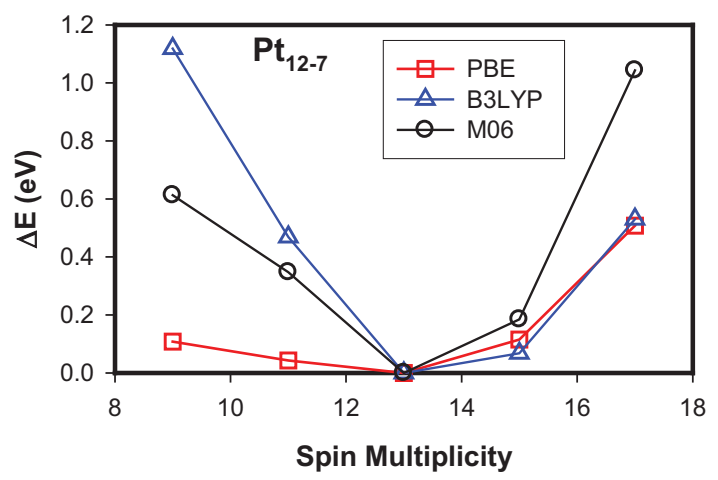

(b)

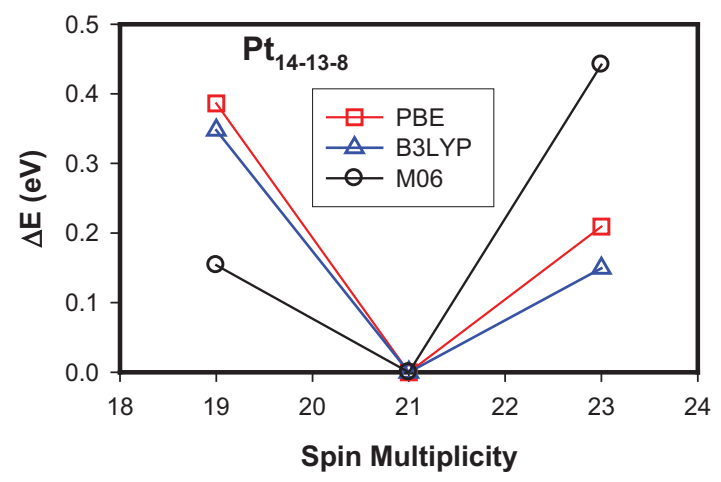

(c)

Figure 8: Change in relative energy of $\mathrm{Pt}$ nanoparticle as a function of spin multiplicity. The relative energy is the energy difference from the minimum energy with an optimal spin multiplicity. Other cases are not presented due to their similarity 
Although the general trend of the NCE is the same for all functionals we used in this study, it should be noted that the values of NCE are different depending on the functionals: NCE $(\mathrm{PBE})<\mathrm{NCE}(\mathrm{M} 06)$ < NCE $(\mathrm{B} 3 \mathrm{LYP})$. This indicates that PBE predicts the largest stabilization energy for the $\mathrm{Pt}$ nanoparticle, whereas B3LYP gives the lowest stability for the same Pt nanoparticle.

Binding energy differences amongst the functionals is expected since, so far, no functional fully describes the energy for a quantum system. Instead, functionals are built to describe certain systems well. PBE is widely used for metallic studies, and B3LYP is widely used for organic systems. M06, on the other hand, was designed specifically to describe the interaction between organic and metallic systems, including the dispersion energy. For purely metallic systems, therefore, we trust the PBE functional most, but we use M06 to describe the adsorption. Another point in Figure 9 is that the 2-layer and 3-layer Pt nanoparticles have nearly the same stability and the data points from these nanoparticles seem to be on an identical line for each functional, while the 1-layer nanoparticles are less stable with higher NCE since the Pt atoms in the 1-layer model lack the higher coordination with neighboring atoms. Our results agree with Sebetci's finding. [21]

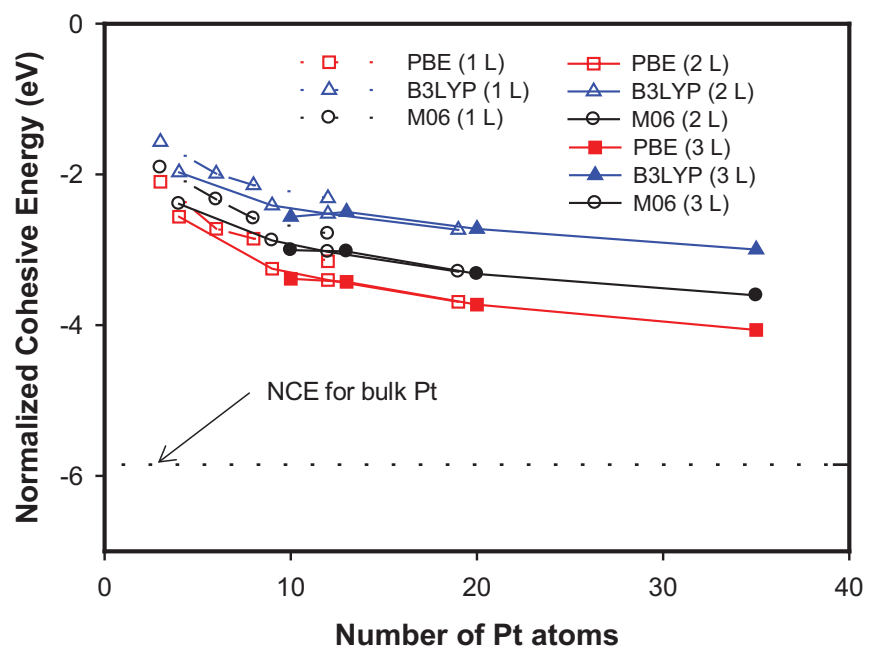

Figure 9: Change of atomic cohesive energy (ACE) with various layers as a function of the number of $\mathrm{Pt}$ atoms in a cluster. The ACE values of Pt clusters are higher than that of $\mathrm{Pt}$ in bulk Phase 
DE-EE0000466

Ballard Material Products Inc.

\subsubsection{Coordination Number (CN) Model for Pt Nanoparticles}

Based on our DFT calculations for various Pt nanoparticles, we developed a model to evaluate the energy of $\mathrm{Pt}$ nanoparticle from its size and shape. The $\mathrm{CN}$ model is to predict the energetically stable state based on shape and size. It is an on-lattice model with constant bond lengths. It can be used to predict the formation energy of these types of clusters.

In general, however, the smallest clusters (few atoms) have the strongest and shortest bonds than the larger clusters and geometrically optimized larger structures provide more reasonable energy states for the clusters; however, considering off lattice geometries considerably increases the search space. Furthermore, since Pt clusters asymptotically approach the crystal lattice an on lattice model should describe most features.

Our model of the cluster energy classifies each atom according to the number of nearest neighbors. For example, a platinum atom inside a crystalline bulk has a $\mathrm{CN}$ of 12 , while an atom on the (111) surface has a CN of 9. In our model, the energy of a cluster is the sum of the contribution of each atom. Each atom's contribution is determined uniquely by its $\mathrm{CN}$.

For this purpose, we counted the $\mathrm{CN}$ of each atom for all the Pt nanoparticles (Figure 7 shows some of the 52 different clusters). An example in Figure 10a shows that $\mathrm{Pt}_{6-3-1}$ nanoparticle has four $\mathrm{Pt}$ atoms with $\mathrm{CN}=3$, three $\mathrm{Pt}$ atoms with $\mathrm{CN}=6$, and three $\mathrm{Pt}$ atoms with $\mathrm{CN}=4$. The atomic cohesive energy, $(A C E)_{C N}$, is determined by least squares fit of Eq (3) to the DFT CE.

$$
C E=\sum_{C N=1}^{12}(A C E)_{C N} N_{C N}
$$

where $\mathrm{CE}$ and $\mathrm{N}_{\mathrm{CN}}$ denote the cohesive energy and the number of $\mathrm{Pt}$ atoms for each $\mathrm{CN}$, respectively. A similar, but not identical, model for metallic clusters is known as the cluster expansion model, derived from the Ising spin model, and widely used especially for alloy systems. [23-27] The governing equation is shown in (3):

$$
E_{C E}(\sigma)=N_{s i t e s}+J_{p} \sum_{i} \sigma_{i}+\sum_{i, j} J_{i j} \sigma_{i} \sigma_{j}+\sum_{i, j, k} J_{i j k} \sigma_{i} \sigma_{j} \sigma_{k}+\ldots
$$

where $E_{C E}$ is the cluster energy, $N_{\text {sites }}$ is the number of available sites, the $\mathrm{J}$ terms are energy contribution terms and the $\sigma$, with $a+1$ or -1 value, represents the Ising spin state. In equation (4) we see that the cluster expansion model is a sum over all pairs in a cluster. By contrast our coordination number only considers the energy of the nearest neighbor, but considers the number of nearest neighbors.

In Figure 10b, we observed that the determined $(\mathrm{ACE})_{\mathrm{CN}}$ decreases with increasing $\mathrm{CN}$, along the line connecting two reference points (zero for $\mathrm{CN}=0$ and $5.8 \mathrm{eV}$ for $\mathrm{CN}=12$ which is the experimentally reported formation energy [77]), which means that the stability of Pt atom in the 
nanoparticle increases with increasing the number of bonds (or the number of bonded neighboring atoms).

However, it should also be noted in Figure $10 \mathrm{~b}$ that $(\mathrm{ACE})_{\mathrm{CN}}$ has deviation from the line to have more negative numbers, which seems more distinct for lower CN. Considering that the dashed line between $\mathrm{CN}=0$ and $\mathrm{CN}=12$ predicts the stability of $\mathrm{Pt}$ atom increases linearly as a function of $\mathrm{CN}$ by assuming that each bond has the constant strength regardless of the number of bonds, such negative deviation indicates that the bond strength is not constant, but decreases as a function of $\mathrm{CN}$. In other words, the bond characteristics of a Pt with neighboring Pt atoms depends on the number of the neighboring $\mathrm{Pt}$ atoms. This interpretation could be clearly confirmed by displaying $\mathrm{ACE} / \mathrm{CN}$ as a function of $\mathrm{CN}$ as also shown in Figure 10b: the contribution of individual Pt-Pt bond to ACE is the most significant for $\mathrm{CN}=1$ and becomes weaker with increasing $\mathrm{CN}$.

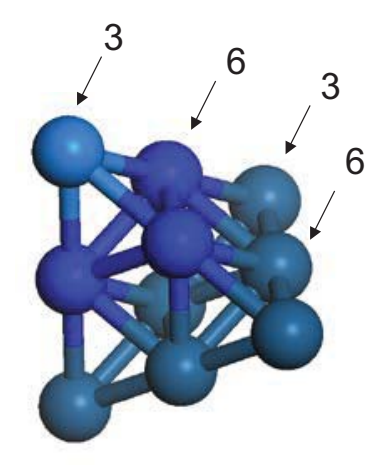

(a)

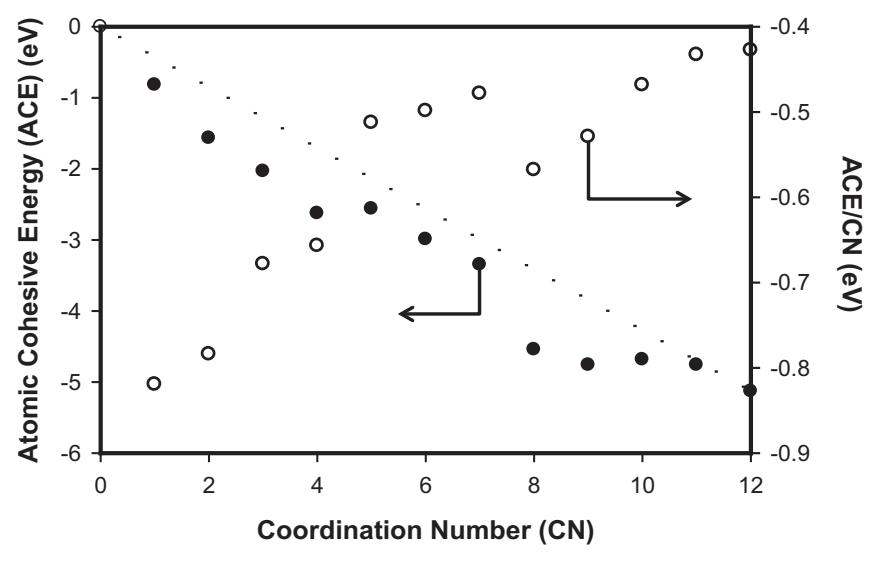

(b)

Figure 10: A Pt nanoparticle has atoms of various coordination number (CN): (a) For example, $\mathrm{Pt}_{6-3-1}$ has $4 \mathrm{Pt}$ atoms with $\mathrm{CN}=3$ and $6 \mathrm{Pt}$ atoms with $\mathrm{CN}=6$; (b) Change in atomic cohesive energy of $\mathrm{Pt}$ as a function of coordination number $(\mathrm{CN})$ reported in Table 3 
In order to validate our $\mathrm{CN}$ model in Eq. (2) with the atomic cohesive energy contributions of each $\mathrm{CN}$ in Table 3, we calculated the CE of new Pt nanoparticles that were not used in developing the $\mathrm{CN}$ model presented in Figure 11. As shown in Figure 11, the CN model predicts to at most $12 \%$ the correct cohesive energy. Note that the NCE, or CE divided by the number of atoms, is less than Pt's formation energy, as predicted by the simulation and the clusters system. This is reasonable since the particle is very small; only the first two $\mathrm{Pt}_{21}$ clusters have any $\mathrm{CN}=12$ atoms. The remaining atoms are under coordinated surface and vertex atoms. As the clusters become larger, the proportion of bulk atoms increases and the NCE will approach Pt's formation energy.
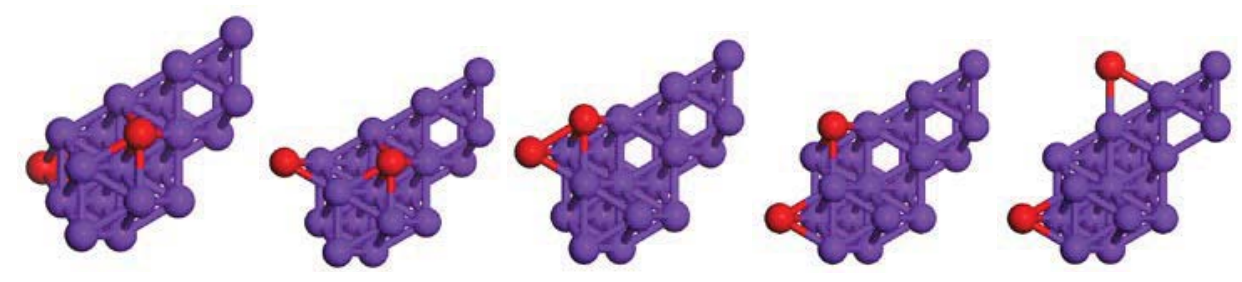

(a)

(b)

(c)

d)

(e)

Figure 11: Pt nanoparticles used to validate the $\mathrm{CN}$ model in Eq. (2). The number of Pt atoms are 21 for all cases, and the positions of the two red colored Pt atoms are determined to generate different $\mathrm{CN}$

In addition, we compared the surface energies calculated using the $\mathrm{CN}$ model with the value reported from literature. [22, 78-82] For this, we investigated several different facets such as (111), and (100) as shown in Figure 13. 
DE-EE0000466

Ballard Material Products Inc.

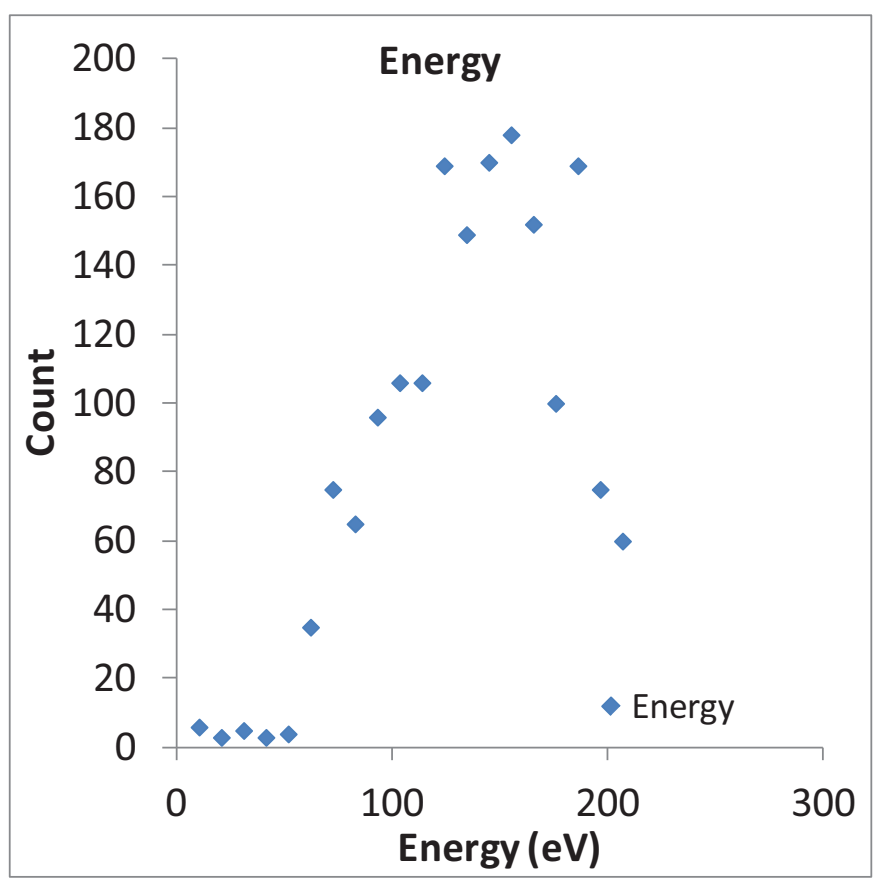

(a)

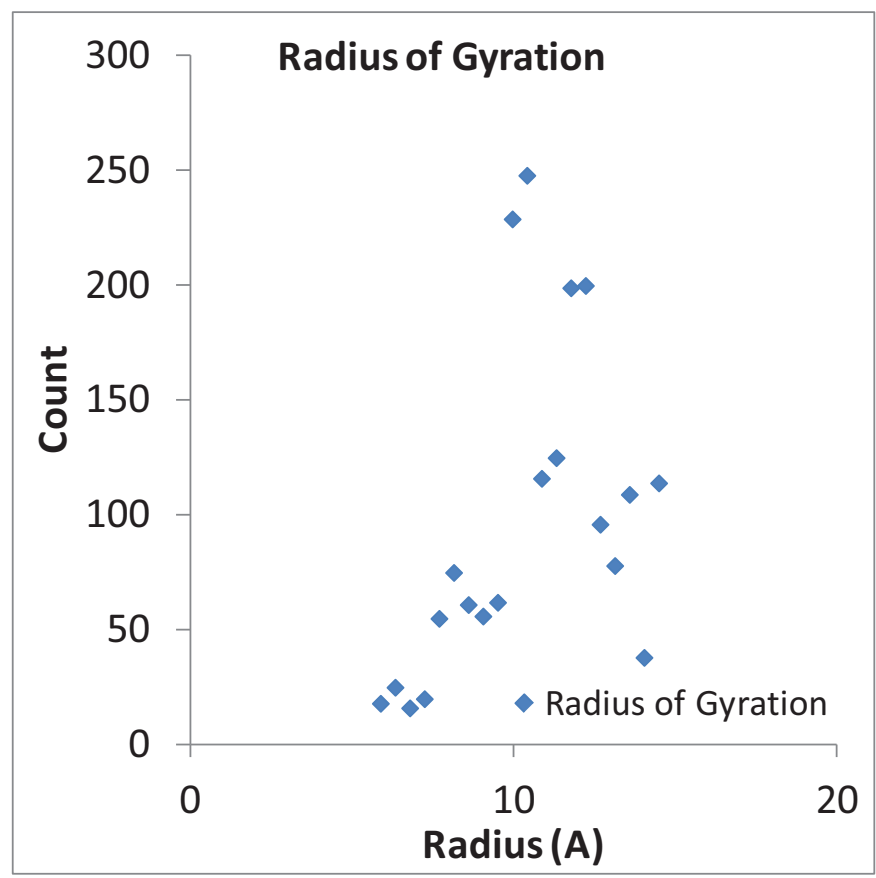

(b)

Figure 12: (a) Energy and (b) radius of gyration of a Pt cluster vs count from a MC simulation of a 95-atom cluster 
DE-EE0000466

Ballard Material Products Inc.

Table 3: The atomic cohesive energy with respect to the coordination number. As expected, more coordinated atoms contribute more to form more stable Pt nanoparticles by lowering its cohesive energy

\begin{tabular}{lllllll}
\hline CN & 1 & 2 & 3 & 4 & 5 & 6 \\
\hline Energy $(\mathrm{eV})$ & -0.82 & -1.57 & -2.03 & -2.63 & -2.56 & -2.99 \\
& & & & & & \\
\hline $\mathrm{CN}$ & 7 & 8 & 9 & 10 & 11 & 12 \\
\hline Energy $(\mathrm{eV})$ & -3.35 & -4.54 & -4.76 & -4.69 & -4.76 & -5.13 \\
\hline
\end{tabular}

Table 4: Performance of CN model to predict surface energies and cluster CE

\begin{tabular}{|c|c|c|}
\hline Surfaces & $\begin{array}{l}\text { CN Bond Model } \\
\left(m e V / \AA^{2}\right)\end{array}$ & References (meVIA $)$ \\
\hline$(111)$ & 95.9 & $\begin{array}{c}93,[22] 95,[78] 96,[79] 124,[80] 104,[81] \\
97[82]\end{array}$ \\
\hline$(100)$ & 129.2 & 116,[22] 114,[78]116,[79] 147[80] \\
\hline Cluster & $\begin{array}{l}\text { CN bond model } \\
\text { (eV/atom) }\end{array}$ & DFT (eV/atom) \\
\hline $\mathrm{Pt} 21_{1}$ & -3.30 & -3.04 \\
\hline $\mathrm{Pt} 21_{2}$ & -3.25 & -2.96 \\
\hline $\mathrm{Pt} 21_{3}$ & -3.25 & -2.95 \\
\hline $\mathrm{Pt} 21_{4}$ & -3.25 & -2.91 \\
\hline $\mathrm{Pt} 21_{5}$ & -3.19 & -2.85 \\
\hline
\end{tabular}

These surface energies were obtained by characterizing the (111) and the (100) surfaces using our CN model. Therefore, a (111) surface is a surface of $\mathrm{CN}=9$ atoms, the (100) surface is a surface of $\mathrm{CN}=8$ atoms. Therefore, for the (111) surface we calculate the energy required to create a surface of $\mathrm{CN}=9$ from a bulk of $\mathrm{CN}=12$ atoms. Although the $\mathrm{CN}$ model results are a little off from the reported DFT surface energies of the (100) surfaces, respectively, it seems that the $\mathrm{CN}$ model captures the surface energy reasonably well, especially for the (111) surface (Table 4), further validating our model. 
DE-EE0000466

Ballard Material Products Inc.

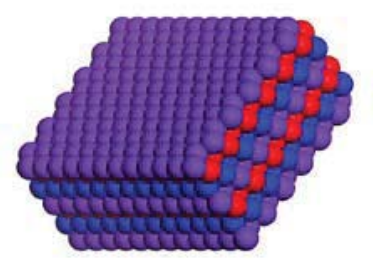

(a)

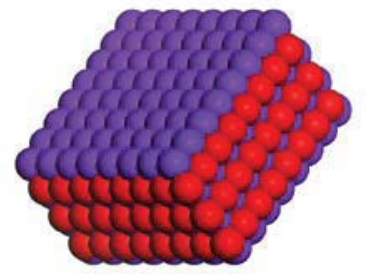

(b)

Figure 13: Pt surfaces with various facets: (a) (111) direction; (b) (100) direction. The colors are introduced to clarify the structural repetition of the atomic layers

In Figure 12, we show the results of using our model to generate the energy distribution and radius of gyration of a platinum cluster with 95 atoms. These are the results from a $10^{7}$ step monte carlo simulation at $\mathrm{T}=0.025 \mathrm{eV}$ after several annealing steps. Only 1900 moves were accepted. From this data we calculated the mean radius of gyration of particle of 95 atoms as $6.2 \AA$. This calculation, needing 10 million steps to obtain a mere 1900 accepted states would be difficult using DFT with 95 atoms. Conversely, surface approaches are not accurate enough to properly describe the energy of such small cluster.

Finally, we used our model for purpose it was not intended for - to identify the most loosely held. The DFT results for this calculation and the methodology to train our CN model were very different. In this calculation, we allowed the clusters to relax (geometric optimization), and we had graphite with a defect. All these features are absent in the $\mathrm{CN}$ model. However, as can be seen, the model still identifies the most loosely held atom, and the subsequent order of most tightly held atom. The model is even able to distinguish between two corner atoms that are formed by the intersection of the same planes. This is possible because the two atoms nearest neighbors are different. Note that the energy predicted is not accurate, as expected since the cluster in our model is not allowed to relax.

Table 5: Energy of losing an atom and the relative order

\begin{tabular}{|l|c|c|c|c|}
\hline & DFT (eV) & Order & CN model (eV) & Order \\
\hline Lower corner & -2.46 & 1 & -4.38 & 1 \\
\hline Lower edge & -3.02 & 2 & -4.49 & 3 \\
\hline Top corner & -3.08 & 3 & -5.16 & 4 \\
\hline Along edge & -3.61 & 4 & -5.91 & 6 \\
\hline Top & -4.09 & 5 & -7.97 & 5 \\
\hline Side face & -4.27 & 6 & -7.55 & \\
\hline
\end{tabular}


DE-EE0000466

Ballard Material Products Inc.

\subsubsection{Force Field Development}

From the results above, we used the Morse potential to obtain force fields of the interaction of the above molecular species with platinum. The form of the Morse potential is:

$$
V(r)=D_{e}\left(1-e^{-\alpha\left(r-r_{e}\right)}\right)^{2}
$$

where $D_{e}$ is the depth of the potential, $\alpha$ is the width and $r_{e}$ is the equilibrium distance. The resulting force field fits are shown in the figures above. The parameters obtained are shown in

Table 6. These parameters were developed with a pragmatic approach in mind, namely that the force field would include all the DFT interaction energy. To avoid the problems of mirror charges on the platinum surface moving as the charged atoms move, we set the charges of $\mathrm{Pt}$ to be fixed and equal to zero. The strong binding energy between platinum and the sulfonate and hydronium ions was absorbed into Morse Potential.

The resulting fits for all the species are well within a kT of the original DFT points on the graphs. For $\mathrm{O}_{2}$ we did not fit the chemisorbed oxygen, just the physisorbed one. However, for completeness, in we report the physisorbed, the chemisorbed and the resulting fit to the physisorbed state.

Table 6: Force field parameters for the ionomer moieties interacting with platinum using the Morse Potential function. The parameter $\alpha$ is unitless

\begin{tabular}{|c|c|c|c|}
\hline Pair & Depth (kcal/mol) & $\operatorname{Re}(\AA)$ & $\alpha$ \\
\hline Pt-C & 0.34 & 4.48 & 1.54 \\
\hline Pt-F & 0.204 & 3.67 & 5.99 \\
\hline Pt-S & 3.04 & 3.41 & 2.15 \\
\hline $\mathrm{Pt}-\mathrm{O}_{\mathrm{SO}}$ & 2.35 & 2.51 & 1.02 \\
\hline Pt-O ${ }_{\text {ether }}$ & 3.45 & 2.79 & 2.55 \\
\hline $\mathrm{Pt}-\mathrm{O}_{\mathrm{O} 2}$ & 0.430 & 3.47 & 1.01 \\
\hline $\mathrm{Pt}-\mathrm{O}_{\mathrm{H} 2 \mathrm{O}}$ & 2.50 & 2.416 & 2.40 \\
\hline $\mathrm{Pt}-\mathrm{H}_{\mathrm{H} 2 \mathrm{O}}$ & 1.36 & 3.05 & 1.60 \\
\hline Pt-O hydronium & 6.43 & 3.47 & 1.12 \\
\hline Pt-H $\mathrm{H}_{\text {hydronium }}$ & 0.24 & 1.61 & 3.73 \\
\hline
\end{tabular}


DE-EE0000466

Ballard Material Products Inc.

\subsubsection{Platinum Surface Analysis}

The surface of the platinum nanocluster in our molecular dynamics simulation was characterized to measure what was on its surface. Specifically, we measured the percentage that each moiety in our MD simulation occupies on the Pt surface.

The measurement was done using the Connolly surface method.[83] This method uses a probe to measure the surface area of a molecule that is accessible to a sphere of radius $r$. For our purposes we used a $1.4 \AA$ probe radius since this is the radius of a water molecule. Water is the smallest molecule in our system that is electrochemically relevant, therefore, any space the water can access is electrochemically important. Using the Connolly surface method, we measure the contact area of a molecule on $\mathrm{Pt}$. This area was defined as the area on the $\mathrm{Pt}$ surface that is no longer accessible by the probe due to another molecule on top of it. The results are shown in Figure 14 as percentages of the exposed $\mathrm{Pt}$ area. That is, we do not consider the area that is in contact with the electrode.

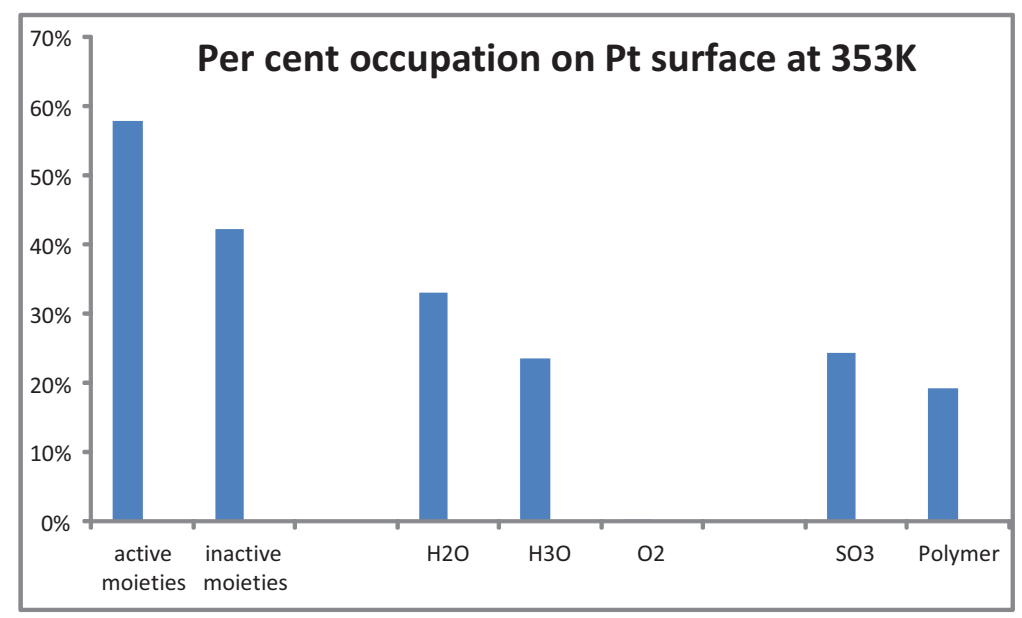

Figure 14: Per cent occupation on the Pt170 surface

In the above graph, area occupied by water, hydronium, and oxygen are considered electrochemically active, while the polymer segments are not. The measured electrochemically active area is $58 \%$ of the exposed Pt surface area. Experimentally reported values are very variable as it depends on many factors, and our value fits within the range of the reported values.[84-87] It must be kept in mind that the experimental value and our own value are very dependent on how the system is made. These differences can cause very large changes in the amount of surface area that a moiety occupies. Also, there is an error of around $5 \%$ in our measurement due to how the surface area is assigned. Specifically, at the seams between two different moiety types, we measure an overlap in the surface area occupied by each moiety on the Pt surface. This is purely a measurement error, and the overlapped area is being double counted. To correct for this, we assign the overlapping area proportionally. The overlap area is around $5 \%$ of any measurement. Finally, there is another source of area from the sulfonate ion. 
It is known that the sulfonate ion affects an area on the Pt surface larger than its Van der Waals radius. However, this is not captured in a classical MD simulation so it is not reflected here.

Our method can also measure the individual contributions from each moiety. Here we see that water occupies the largest share of the surface area. This is due to several factors including the strong dipole interaction with the surface, the water molecule's small size enabling it to occupy areas inaccessible to other moieties, and most significantly the larger number of water molecules in the system. Water's high occupancy is followed by the sulfonate ion and the hydronium ion. They are on the surface mostly due to their strong interaction with the $\mathrm{Pt}$ surface. The percentage they occupy is similar, probably due to the fact that they are counterions. The ionomer backbone occupies some area, despite its weak interactions, probably due to sulfonate ions attached to it. Finally, there was no measured $\mathrm{O}_{2}$ on the surface. Since oxygen molecules are present in small concentration, it is very expensive to add a significant amount of oxygen molecules to the simulation, therefore this last results is due to simulation error on account of the few oxygen molecules in the simulation system and subsequent high error of measurement.

Table 7: (a) The occupancy for various moieties on the platinum surface; (b) The occupancy for various moieties on the oxygen covered platinum surface; (c) The occupancy for various moieties on the hydroxide covered platinum surface

\begin{tabular}{cccccccc}
\hline & Active & Inactive & $\mathbf{H}_{2} \mathbf{O}$ & $\mathbf{H}_{3} \mathbf{O}^{+}$ & $\mathbf{O}_{2}$ & $\mathbf{S O}_{3}$ & Polymer \\
\hline $353 \mathrm{~K}$ & 0.58 & 0.42 & 0.33 & 0.24 & $<0.01$ & 0.24 & 0.19 \\
$363 \mathrm{~K}$ & 0.59 & 0.42 & 0.35 & 0.23 & $<0.01$ & 0.22 & 0.20 \\
$373 \mathrm{~K}$ & 0.58 & 0.43 & 0.33 & 0.25 & $<0.01$ & 0.23 & 0.19 \\
$383 \mathrm{~K}$ & 0.58 & 0.43 & 0.38 & 0.23 & $<0.01$ & 0.23 & 0.17 \\
\hline
\end{tabular}

(a)

\begin{tabular}{cccccccc}
\hline & Active & Inactive & $\mathbf{H}_{\mathbf{2}} \mathbf{O}$ & $\mathbf{H}_{3} \mathbf{O}^{+}$ & $\mathbf{O}_{2}$ & $\mathbf{S O}_{3}$ & Polymer \\
\hline $353 \mathrm{~K}$ & 0.49 & 0.51 & 0.30 & 0.20 & $<0.01$ & 0.25 & 0.25 \\
$363 \mathrm{~K}$ & 0.47 & 0.53 & 0.28 & 0.19 & $<0.01$ & 0.25 & 0.27 \\
$373 \mathrm{~K}$ & 0.47 & 0.53 & 0.30 & 0.18 & $<0.01$ & 0.25 & 0.27 \\
$383 \mathrm{~K}$ & 0.43 & 0.57 & 0.29 & 0.15 & 0.01 & 0.22 & 0.33 \\
\hline
\end{tabular}

(b)

\begin{tabular}{cccccccc}
\hline & Active & Inactive & $\mathrm{H}_{2} \mathbf{O}$ & $\mathrm{H}_{3} \mathbf{O}^{+}$ & $\mathbf{O}_{2}$ & $\mathbf{S O}_{3}$ & Polymer \\
\hline $353 \mathrm{~K}$ & 0.56 & 0.44 & 0.38 & 0.20 & $<0.01$ & 0.23 & 0.20 \\
$363 \mathrm{~K}$ & 0.63 & 0.39 & 0.40 & 0.21 & $<0.01$ & 0.22 & 0.18 \\
\hline
\end{tabular}

(c) 


\subsection{Conclusion}

Our overall goal is to study the role of $\mathrm{Pt}$ in the three-phase region. Given the relative size of the three-phase region, multiscale tools are required. That is, depending on the phenomenon and the size of the system, different tools should be employed. Therefore, DFT is suited for cluster stability, force field development and chemical dissolution studies. Molecular Dynamics, on the other hand, is more suited to investigate how all three phases of the catalyst layer interact with each other. In this study we presented our work on Pt cluster stability, developed a model to predict the energy of a cluster and predict which atoms in a cluster are more loosely held, and finally developed the force field for ionomer-Pt interaction. From this force field we created an MD simulation. Our coordination number model of cluster stability is used in our work to predict likely candidates for Pt dissolution, successfully. Our model is a consistent way to predict the atomic cohesive energy of an arbitrary Pt cluster of $n$ atoms, constrained to the crystal structure. This model does not take lattice relaxation into account explicitly. It successfully predicts energies and likely atoms for dissolution.

The force field we developed was used to create a MD simulation to study the three-phase region. In particular, we found the species that occupy the surface of a platinum cluster. Namely water occupies a plurality of the surface followed by the hydronium and sulfonate ions. These two occupy roughly the same amount of surface area since they are together. 
DE-EE0000466

Ballard Material Products Inc.

\subsection{Pt Dissolution Study by the Nudged Elastic Band Method}

\subsubsection{NEB and Platinum Dissolution}

Identification of Minimum Energy Pathway (MEP) of a chemical reaction from one stable state to another is essential to understand the reaction energetics of atomic or molecular reaction such as platinum dissolution. Complex reaction surfaces consist of complex energy landscapes where a very large number of relevant MEPs exist. Nudged Elastic Band (NEB) method has been applied successfully to a wide range of problems to find the reaction path and saddle point energy or transition state, which gives activation energy barrier. The Nudged Elastic Band Method is a chain-of-states method, in which a string of images or 'states' is generated between the end point configurations, and intermediate images are optimized simultaneously.

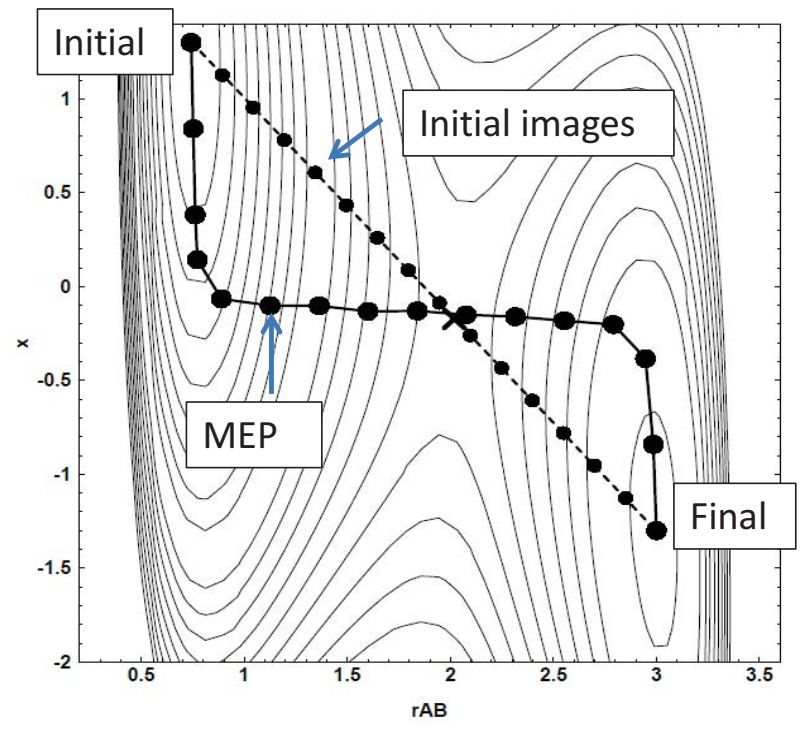

Figure 15: Schematic representation of the Nudged Elastic Band method. Starting from an initial reaction path (initial images), the chain settles to the Minimum Energy Path (MEP) images after convergences

To derive all images to same MEP, the images are connected by the theoretical spring. In NEB method, N-1 images are optimized with respect to all degrees of freedom except that of the reaction pathway. A spring constant is added to ensure the continuity of the path. The initial guess has to be good enough to converge to a realistic MEP. The highest energy image gives a good estimate of the transition state. We use transition state search algorithms implemented in VASP.

Transition state for the platinum dissolution depends on external potential and environmental molecular fields. It has been observed that the oxygen coverage on Pt surface varies with respect to applied external potential to electrodes. To investigate the free energy change and transition states during the dissolution effectively, we prepared computational models required to use the NEB method. Figure 16 illustrates a model of no atomic or molecular coverage of the platinum surface, where a surface platinum is dissolving into water phase. Dissolution on bare Pt (111) surface is referenced for comparison with a model surrounded by water molecules. 
DE-EE0000466

Ballard Material Products Inc.

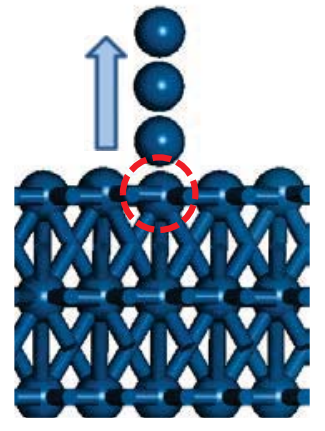

(a)

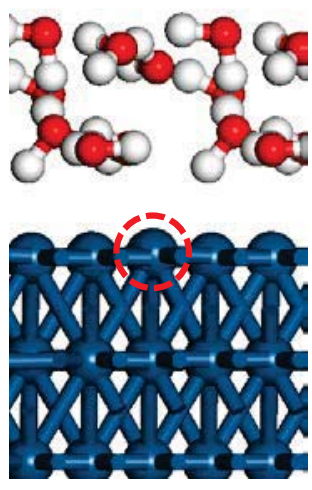

(b)

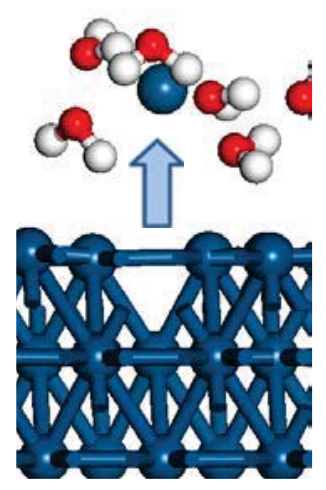

(c)

Figure 16: Platinum dissolution (a) on bare Pt (111) surface; (b) and (c) show optimized initial and final models surrounded by water molecules, respectively

NEB method requires well optimized initial final structures, shown on Figure 16 (b) and (c) for example. Intermediate images are prepared by linear interpolation between two models, initial images on Figure 15. We prepared three models with different surface coverage; bare, oxygen covered, and hydroxide covered surfaces. External fields are applied on each model. Potential dependent activation barriers are calculated using the NEB method for the three models.

Reaction features at the water-metal interface can alter bonding arrangement and/or ionic transfer. The reactions are affected and controlled by the applied electrode potential. The true treatment of a constant potential system would require a full quantum mechanical simulation of the anode, cathode, ion transport across the membrane as well as electron conduction through the circuit. Using quantum mechanics, however, the simulation of fuel cell operation in steady state conditions is seriously restricted by cost and computation time. We used instead predeveloped a half-cell approach [C.D. Tayler, et al., Molecular Simulation, Vol. 33, 429-436, 2007], where the electrode is treated as periodic metal slabs together with condensed water and an explicit charge to the metal. By charging the metal we can induce an electric field across the water-metal interface which polarizes the water molecules and results in a potential change. The water molecules may become activated to form a series of products:

$$
\begin{aligned}
& \mathrm{H}_{2} \mathrm{O}^{*}+e^{-} \rightarrow \mathrm{H}^{*}+\mathrm{OH}^{-} \\
& \mathrm{H}_{2} \mathrm{O}^{*} \rightarrow \mathrm{OH}^{*}+\mathrm{H}^{+}+e^{-} \\
& \mathrm{H}_{2} \mathrm{O}^{*} \rightarrow \mathrm{O}^{*}+2 \mathrm{H}^{+}+2 e^{-}
\end{aligned}
$$

The dissociation of platinum at the metal/water phase also depends on the complex bonding arrangement and the degree of surface polarization, resulting in the formation of platinum complexes such as $\mathrm{Pt}-\mathrm{OH}, \mathrm{Pt}-\mathrm{H} 2 \mathrm{O}$ and $\mathrm{Pt}-\mathrm{O}$. The products might vary according to the surface coverage and intensity of applied potentials.

The applied potential can be simulated by adding or subtracting electrons to the models. J.S. Filhol and M. Neurock introduced Double Reference method [J.S. Filhol and M. Neurock, Angew. Chem. 45, 2006] to compute the magnitude of applied potential with respect to the number of electrons added or subtracted. 
DE-EE0000466

Ballard Material Products Inc.

\subsubsection{Double-Reference Method}

It is possible to apply an arbitrary charge to the metal slab. The applied net charge introduces uniformly distributed countercharge density across a supercell to maintain the cell neutrality, mimicking the presence of a diffuse ionic distribution close to the interface. The resulting electric field that develops at the interface leads to the potential drop and quantified by comparing the Fermi level of the system with a reference potential established at a distance from the metal/water interface. The electrode potential by the homogeneous countercharge density can be tuned by Double-reference method. The first reference point is referenced to the potential at vacuum phase, and the water phase reference represents the second reference point. Finally, calculated system potential is referenced to the normal hydrogen electrode (NHE) scale by subtracting $4.8 \mathrm{~V}$, which is the approximate work function of the NHE.

Double-reference method in this $\mathrm{Pt}$ dissolution study, replicate the applied electric field at metal/water system. The method is applied to three different Pt (111) surfaces, bare, oxygencovered, and hydroxide-covered surface, and is estimated to have an absolute error of approximately $\pm 0.5 \mathrm{~V}$ [C.D. Taylor, S.A. Wasileski, J.S. Filhol, M. Neurock, Phys. Rev. B 73 (2006) 165402]. However, the actual error in the potential dependence of reaction energies is known to be much less due to the different errors when comparing similar systems [M.J. Janik, M. Neurock, Electrochimica Acta 52 (2007) 5517]. Details of the preparation of model and calculation of the potential are followed by.

\subsubsection{Electrode Surface: Half-Cell Approach}

\subsubsection{Water Molecules}

Water molecules are explicitly introduced on the top of the electrode surface to form the meal/water interface. To obtain the well known density of bulk water, $\sim 0.999 \mathrm{~g} / \mathrm{cm}^{3}$, and networked hydrogen-bond structure, molecular dynamics simulation was performed for 500ps $\sim 1 \mathrm{~ns}$, where we developed force field to describe the Pt and water interactions, and the force field was used for molecular dynamics simulations. Figure 16 illustrates the water structure on different surface states.

The water molecules rotate or experience flip-flop mechanism according to the applied potential, which shows different degree of polarization. Various numbers of structures are possible at the metal/water interface. Those structures may lead to the change of degree of electro-oxidation reduction, or polarizability of the surface, even if the quantity is small. However, we use the most thermodynamically stable structures as mentioned in other papers.

In presence of applied external field, $>0.06 \mathrm{~V}$ in this study, DFT computation shows that the reaction of water molecules with platinum surface forms surface hydroxyl species and hydronium $\left(\mathrm{H}_{3} \mathrm{O}\right)$ by releasing a proton into the solution. Figure 17 illustrates the formation of hydroxyl species and hydronium. 
DE-EE0000466

Ballard Material Products Inc.

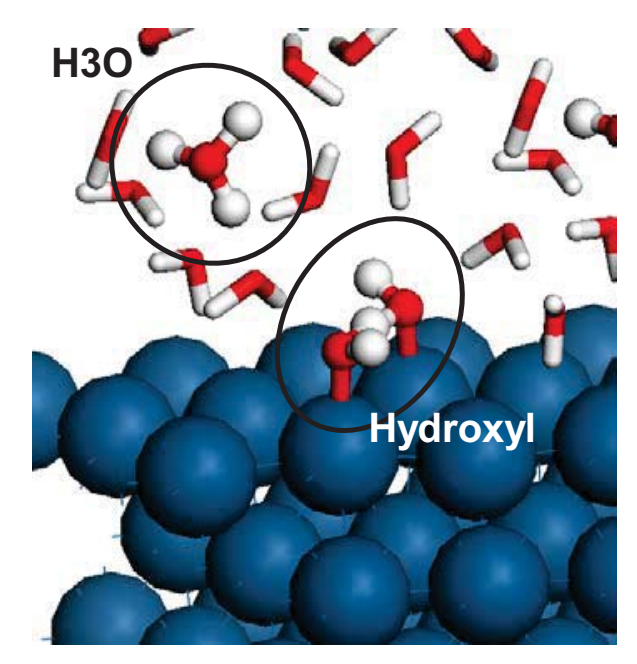

Figure 17: Activation of water on the bare $\mathrm{Pt}$ (111) surface in the applied electric field of $0.06 \mathrm{~V}$. It shows hydroxyl species and hydronium in the solution.

\subsubsection{Determination of Surface Potential: Computation of Applied Potential}

The surface potential is determined by the reference Fermi level. First, water-Pt (111) interface system is initially optimized at neutral charge. A vacuum layer of $\sim 20 \AA$ (Figure 18 (a)) is then introduced and a single point calculation is carried out in order to determine the vacuum potential. The surface Fermi level is then referenced to this vacuum potential in the closed (no vacuum layer) neutral system. This vacuum-reference represents the first reference potential in the double-reference method.

The system potential is then tuned by adding or subtracting electrons. Compensating background charge (counter-charge) is subsequently distributed homogeneously to maintain neutrality within the periodic unit cell. The atomic positions are optimized at each charge. The added charge polarizes the metal surface and alters the potential at the interface. The magnitude of potential difference at each charge is determined by shifting the potentials in the unit cell, including the Fermi level of platinum surface, such that the potential at the center of the aqueous layer remains constant. This reference represents the second reference in the doublereference method. The internal vacuum-referenced system potential is referenced to the normal hydrogen electrode (NHE) scale by subtracting $4.85 \mathrm{~V}$, the approximate work function of the normal hydrogen electrode. Figure 18 shows all plots of the electrostatic potentials before scaling to the NHE.

All calculations were performed using the VASP with generalized gradient approximation (GGA) using PBE functional. Electronic structure calculations are based on the projector augmented wave (PAW) method. The Brillouin Zone is sampled by $3 \times 3 \times 1$ Monkhorst-Pack grid with energy cutoff of $400 \mathrm{eV}$. Electronic self-consistent field cycles are converged to $1.0 \times 10^{-5} \mathrm{eV}$ and geometry optimization using conjugate-gradient method and residual minimization method direct inversion in the iterative subspace (RMM-DIIS) was converged to $1.0 \times 10^{-5} \mathrm{eV}$. 
DE-EE0000466

Ballard Material Products Inc.

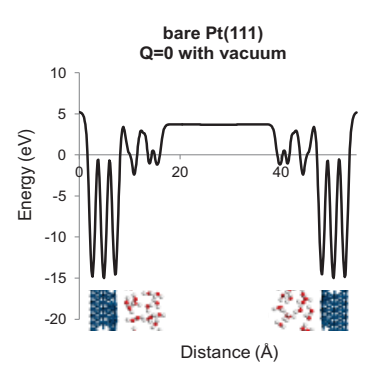

(a)

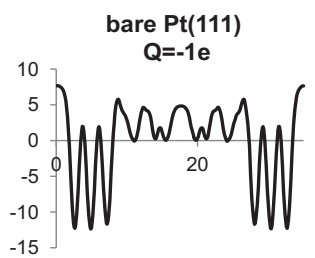

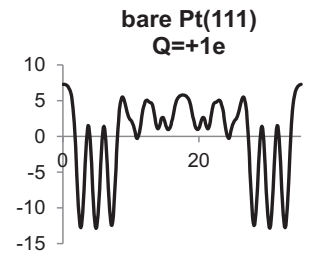

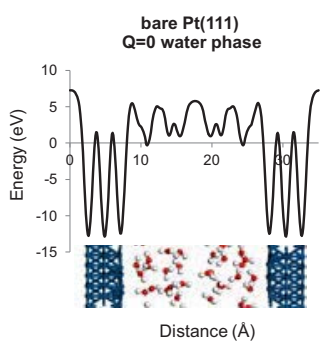

(b)

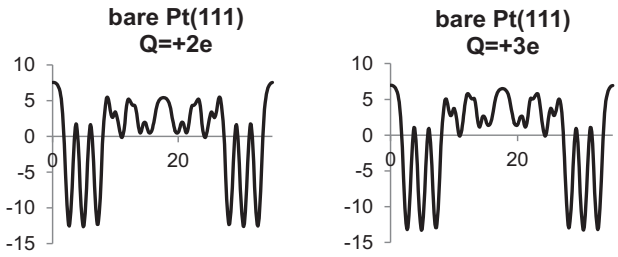

(c)
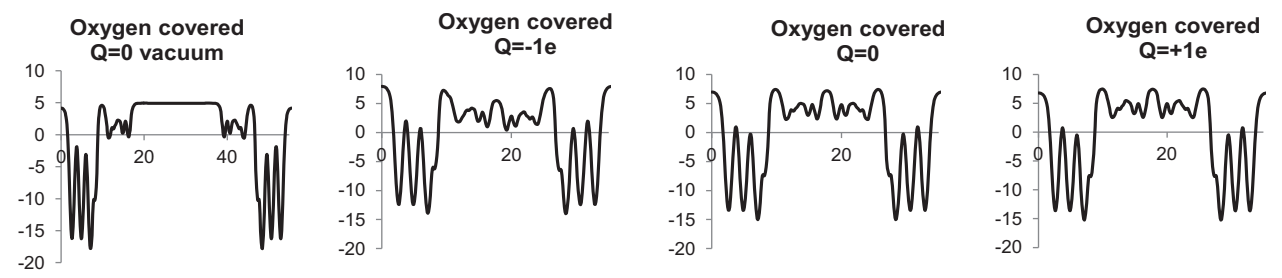

(d)
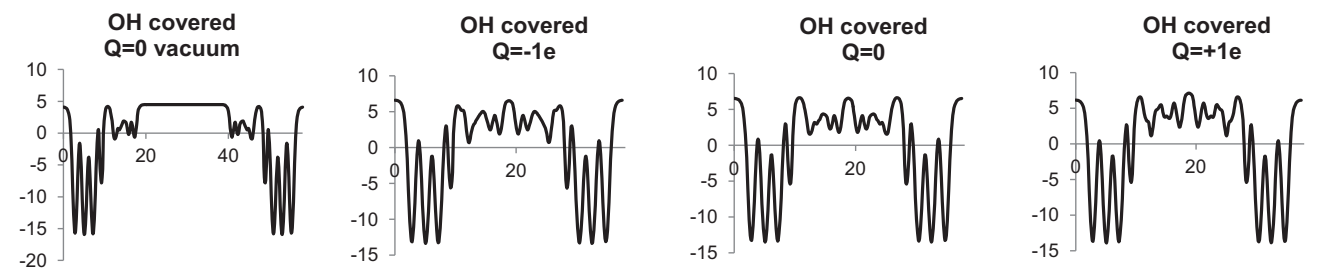

(e)

Figure 18: Results of double-reference method to compute applied electrostatic potentials for corresponding charges. Plane averaged electrostatic potential plots are illustrated: (a) (b) as an example, illustrates models with vacuum and water phase under the corresponding electrostatic potential plots; in the middle of the vacuum, first reference point is established, and second reference point is in the middle of water phase of the second plot (without vacuum phase); (d) potential plots of system of oxygen covered surfaces; and (e) hydroxide covered surfaces 


\subsubsection{Correction of Energy}

DFT energy $\left(E_{D F T}\right)$ must be corrected for the interaction with the background charge. The DFT energy can be described as

$$
E_{D F T}\left(n_{e}, n_{g}\right)=E_{s l a b}\left(n_{e}\right)+E_{s l a b-b g}\left(n_{e}, n_{g}\right)+E_{b g}\left(n_{g}\right)
$$

where, $E_{\text {slab }}$ is the energy of the slab without background, $E_{b g}$ is the energy of the background and $E_{\text {slab-bg }}$ is the interaction energy between the slab and background. $n_{\mathrm{e}}$ is the added number of electrons to the system and $n_{g}$ is the number of compensating background charge. The last two terms correspond to the integration of volume averaged electrostatic potential,

$$
-\int_{0}^{q}<\phi>d Q
$$

The correction energy is obtained by subtracting the energy related to the background charge. Total free energy includes contributions for the excess electrons $(q)$ at the referenced Fermi potential, $\phi_{\text {vac }}$. Thus the corrected free energy is given by

$$
\Delta E_{\text {Free }}=E_{D F T}+\int_{0}^{q}\langle\phi\rangle d Q-q \phi_{\text {vac }}
$$

The free energy change during platinum dissolution will be investigated in the applied electrochemical potential.

\subsubsection{Computational Results}

\subsubsection{Determination of Reference Potential by Double-Reference Method}

First reference potential is determined from the plane-averaged electrostatic potential in the middle of inserted vacuum region in Figure 18(a) of the uncharged system. The reaction coordinates are function of position of the dissociated $\mathrm{Pt}$ atom. Assuming uncharged electrochemical potential of electrode when the vacuum region is omitted, shown in Figure 18(b), the second reference potential for a charged electrode can be determined at the center of the water phase of the uncharged system in Figure 18(b).

Using both reference potentials, reference Fermi potential of a charged system, $\phi_{q}(f)$, is given by

$$
\varphi_{q}(f)=\varphi_{q}{ }^{\prime}(f)-\varphi_{q}^{\prime}(w)+\varphi_{0}(w)
$$

where, primed potentials indicate calculated potentials of the electrode interacting with water molecules at a given charge, $q$. 
Table 8: Used potential values. Applied charges and corresponding electrode potentials of with respect to the NHE potential of the bare $\mathrm{Pt}(111)$, Oxygen covered, and $\mathrm{OH}$ covered surface in water phase. $Q$ values are total net charges applied to each system, for instance, -1 e means that one electron was added into the system.

\begin{tabular}{|c|c|c|c|c|}
\hline Bare Surface & $Q=-1 e$ & $Q=0$ & $\mathrm{Q}=+2 \mathrm{e}$ & $\mathrm{Q}=+3 \mathrm{e}$ \\
\hline$\Phi_{\mathrm{NHE}}$ & $-2.12 \mathrm{~V}$ & $-0.67 \mathrm{~V}$ & $0.50 \mathrm{~V}$ & $2.27 \mathrm{~V}$ \\
\hline \multicolumn{2}{|c|}{ Oxygen/OH covered surface } & $Q=-1 e$ & $Q=0$ & $Q=+1 e$ \\
\hline \multicolumn{2}{|c|}{$\begin{array}{l}\Phi_{\mathrm{NHE}} \\
\text { (Oxygen } 1 \mathrm{ML} \text { covered surface) }\end{array}$} & $-0.74 \mathrm{~V}$ & $1.98 \mathrm{~V}$ & $2.44 \mathrm{~V}$ \\
\hline \multicolumn{2}{|c|}{$\Phi_{\mathrm{NHE}}$} & $0.98 \mathrm{~V}$ & $1.12 \mathrm{~V}$ & $2.08 \mathrm{~V}$ \\
\hline
\end{tabular}

Potential at an electrode with respect to Normal Hydrogen Electrode (NHE) in solvent is calculated from following relation:

$$
\varphi_{\text {NHE }}=-4.85-\varphi_{a}(f)
$$

Where, $\Phi_{\text {NHE }}$ is the potential with respect to NHE, and $-4.85 \mathrm{~V}$ is the absolute potential of $\mathrm{Pt}$ electrode in standard condition, and $\Phi_{q}(f)$ indicates the referenced Fermi potential of a charged system. Using the relation above, electrode potentials with respect to applied charges are given at Table 8. At $Q=0$, it shows non-zero potential, which tells that the Pt surface potential was changed by water-Pt surface interaction at even uncharged state.

\subsubsection{Pt Dissociation by Nudged Elastic Band Theory}

The nudged elastic band (NEB) is a method for finding saddle points and minimum energy paths between known reactants and products by optimizing intermediate images along the reaction path. The optimization is done by adding spring forces along the band between images and by projecting out the component of the force due to the potential perpendicular to the band. For the Pt dissolution study, energy minimized initial and final states are prepared for applied three different electrode potentials. Optimized final images are illustrated at Figure 19, which contain a Platinum atom dissociated from the surface. The dissociated $\mathrm{Pt}$ atoms are surrounded by water molecules and interact at very close distance. The bond distance was

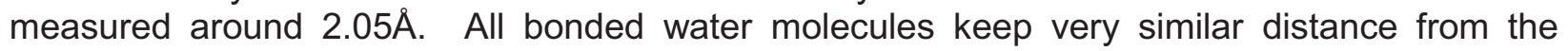
dissociated Pt atom and inhibit the atom from re-clustering to the surface. Applied electrode potentials are also closely related to the reactivity of the dissociated Pt atom. Water molecules and/or hydroxides are favorably bonded to the dissociated Pt atom as the potential increases, which may lead to the more stabilized dissolution state. 
DE-EE0000466

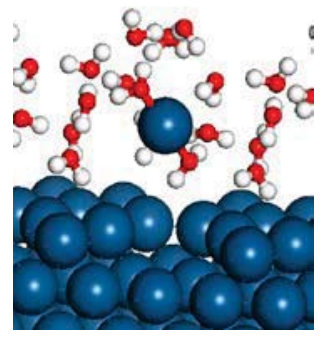

$-2.12 \mathrm{~V}$

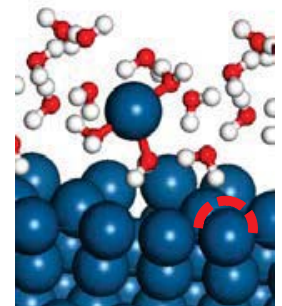

$-0.67 \mathrm{~V}$

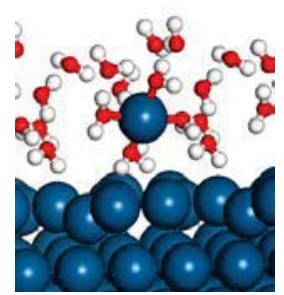

$0.50 \mathrm{~V}$

(a)

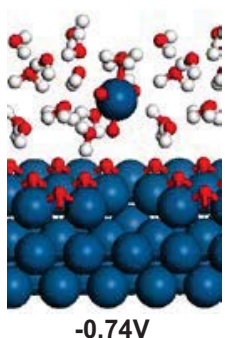

$-0.74 \mathrm{~V}$

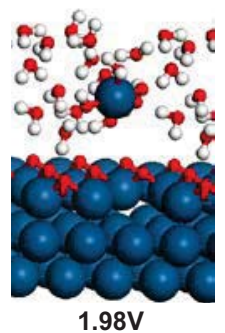

(b)

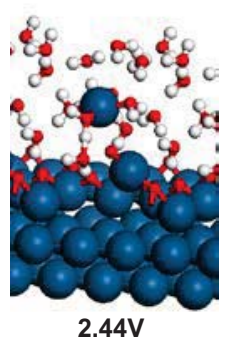

$2.44 \mathrm{~V}$
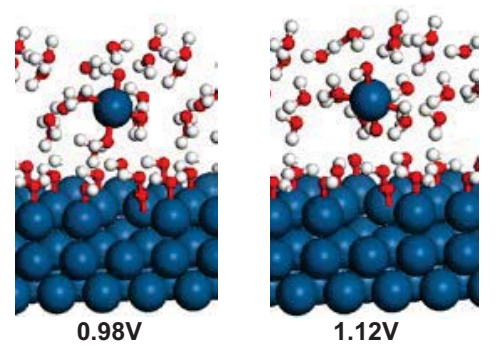

(c)
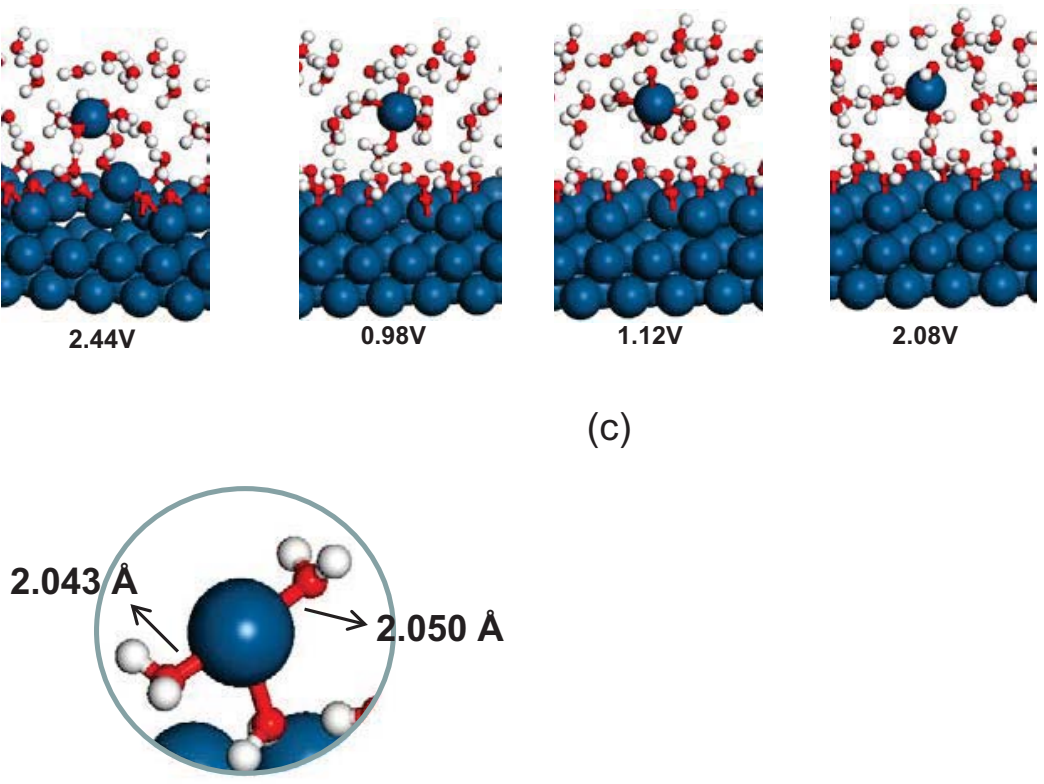

(d) Bond Length

Figure 19: Energy minimized structures at potential, (a) $-2.12 \mathrm{~V},-0.67 \mathrm{~V}$, and $0.50 \mathrm{~V}$ on bare $\mathrm{Pt}$ (111) surface; (b) $-0.74 \mathrm{~V}, 1.98 \mathrm{~V}$, and $2.44 \mathrm{~V}$ on Oxygen covered surface; and (c) $0.98 \mathrm{~V}, 1.12 \mathrm{~V}$, and $2.08 \mathrm{~V}$ on $\mathrm{OH}$ covered surface. Water molecules surround the dissolute $\mathrm{Pt}$ atom and prevent re-clustering to the surface. Dotted red circle on $\mathrm{Pt}(111)$ surface on figure (a) indicates the initial position of the dissociated Pt atom

Calculated energy differences between final and initial states for given potentials are $+6.867 \mathrm{eV}$ (at $-2.12 \mathrm{~V}$ ), $+2.310 \mathrm{eV}$ (at $-0.67 \mathrm{~V}$ ), $-6.435 \mathrm{eV}$ (at $0.50 \mathrm{~V}$ ), and $-12.758 \mathrm{eV}$ (at $2.08 \mathrm{~V}$ ) for models reacting on bare $\mathrm{Pt}(111)$ surface. Positive sign implies that the energy of final state is higher than that of initial state. The increase of potential significantly stabilizes the final structures, which will hardly derive the dissolved $\mathrm{Pt}$ atom return to the surface again. Table 9 shows the energy barriers measured in forward direction and reverse direction with the gap between initial and final state. 
DE-EE0000466

Ballard Material Products Inc.

Table 9: Energy barriers along forward (from initial state to transition state), and reverse (from final state to the transition state) directions

\begin{tabular}{|l|c|c|c|c|}
\hline & $-2.12 \mathrm{~V}$ & $-0.67 \mathrm{~V}$ & $0.50 \mathrm{~V}$ & $2.08 \mathrm{~V}$ \\
\hline $\begin{array}{l}\text { Forward } \\
\text { Direction }\end{array}$ & $7.07 \mathrm{eV}$ & $4.41 \mathrm{eV}$ & $3.74 \mathrm{eV}$ & $2.20 \mathrm{eV}$ \\
\hline $\begin{array}{l}\text { Reverse } \\
\text { Direction }\end{array}$ & $0.20 \mathrm{eV}$ & $1.40 \mathrm{eV}$ & $10.17 \mathrm{eV}$ & $14.87 \mathrm{eV}$ \\
\hline $\begin{array}{l}\text { Gap } \\
\text { (Final-Initial) }\end{array}$ & $+6.867 \mathrm{eV}$ & $+2.310 \mathrm{eV}$ & $-6.435 \mathrm{eV}$ & $-12.758 \mathrm{eV}$ \\
\hline
\end{tabular}

From Table $9(-2.12 \mathrm{~V})$ and $(-0.67 \mathrm{~V})$, it shows a relatively lower barrier in reverse direction compared to forward direction. The energy $0.20 \mathrm{eV}$ (at $-2.12 \mathrm{~V}$ ) can be easily overcome by external environment such as thermal fluctuation. Thus, it is thought that the Pt dissolution hardly happens at lower voltages. Thus the platinum dissolution occurs more favorably as the electrode potential increases. The highest peak at the plots of Figure 20 indicates the transition state during the Pt dissolution and heights are summarized at Table 10.

For the oxygen- or hydrogen-covered surfaces, the energy barriers become more complicated. As shown on Figure 19, platinum surfaces at initial stage have already well-formed PtO or PtOH species. Thus, the Pt dissolutions are accompanied by oxygen or hydroxide at the beginning of dissolution process. The activation energy barriers, shown at Figure 20 (b) and (c), show two transition states. Considering the position of $\mathrm{Pt}$ atom along the reaction coordinates, the first barrier at around $1.2 \AA$ indicates the energy barrier to escape from the surface with attached oxygen or hydroxide, and the subsequent energy barrier at around $3 \sim 5 \AA$ indicates a position in water phase. Higher second barrier shows that the molecules, $\mathrm{PtO}$ or $\mathrm{PtOH}$, cannot easily move into the water phase but requires additional reaction to move into water phase forming various complexes, such as $\mathrm{Pt}(\mathrm{O}) x, \mathrm{Pt}(\mathrm{OH}) x$ or PtO- $(\mathrm{OH}) x$, which models are illustrated in Figure 19 (a), (b), and (c). The first and second activation energy barriers are tabulated at Table 10. 


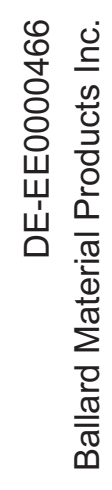

a
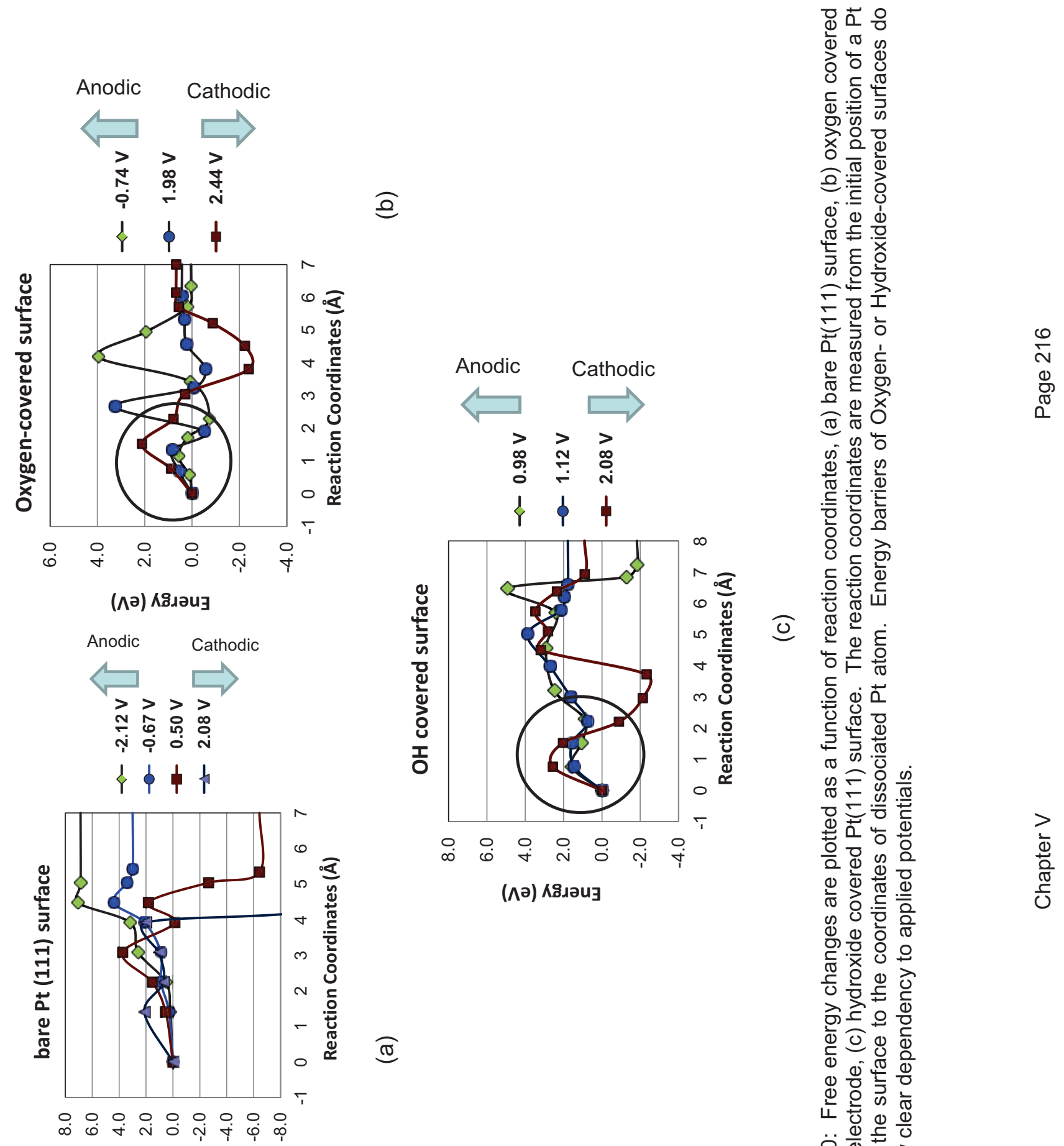

( 
DE-EE0000466

Ballard Material Products Inc

Table 10: Energy barriers for oxygen-, hydroxide- covered surfaces. It shows two barriers, first, it appears on the surfaces. There exists energy barrier to escape the surface. Second, the barrier exists when the dissolved Pt-complex moves into the water phase

\begin{tabular}{|l|c|c|c|}
\hline $\begin{array}{l}\text { Oxygen-covered } \\
\text { Surface }\end{array}$ & $-0.74 \mathrm{~V}$ & $1.98 \mathrm{~V}$ & $2.44 \mathrm{~V}$ \\
\hline $\begin{array}{l}\text { First } \\
\text { (leaving surface) }\end{array}$ & $0.57 \mathrm{eV}$ & $0.55 \mathrm{eV}$ & $2.12 \mathrm{eV}$ \\
\hline $\begin{array}{l}\text { Second } \\
\text { (into water phase) }\end{array}$ & $4.68 \mathrm{eV}$ & $3.77 \mathrm{eV}$ & $3.06 \mathrm{eV}$ \\
\hline $\begin{array}{l}\text { Hydroxide-covered } \\
\text { Surface }\end{array}$ & $0.98 \mathrm{~V}$ & $1.12 \mathrm{~V}$ & $2.08 \mathrm{~V}$ \\
\hline $\begin{array}{l}\text { First } \\
\text { (leaving surface) }\end{array}$ & $1.57 \mathrm{eV}$ & $1.55 \mathrm{eV}$ & $2.56 \mathrm{eV}$ \\
\hline $\begin{array}{l}\text { Second } \\
\text { (into water phase) }\end{array}$ & $4.92 \mathrm{eV}$ & $3.12 \mathrm{eV}$ & $5.52 \mathrm{eV}$ \\
\hline
\end{tabular}

The activation energy barriers on the bare platinum surfaces are much higher than the oxygen or hydroxide covered surface in the range of $0 \mathrm{~V} \sim 1.2 \mathrm{~V}$. Figure 21 plots the energy barriers as a function of applied potentials.

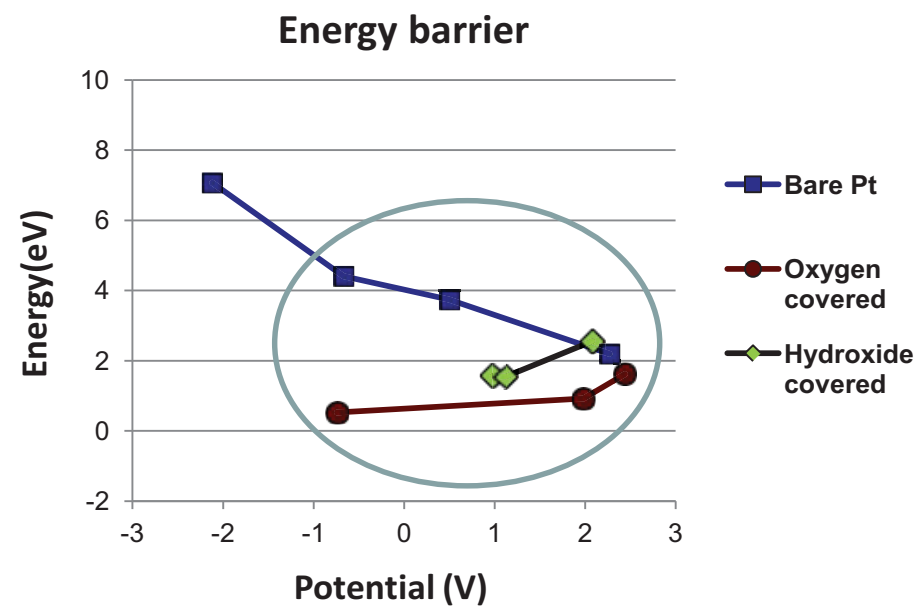

Figure 21: First energy barrier in $\mathrm{eV}$ as a function of applied potential. In the range of voltage $(0 \mathrm{~V} \sim 1.2 \mathrm{~V})$, the $\mathrm{Pt}$ dissolution hardly happens on the bare surface of platinum. Oxygen covered surface shows lowest energy barrier in the range 
Pt atom can move $\sim 3 \AA$ away from the surface experiencing the first barrier. Figure 21 plots the first energy barriers during the Pt dissolution. While the applied potential on the bare platinum surface increases, the barrier decreases, oxygen or hydroxide covered surfaces behave in opposite way. The height of energy barrier, as shown in Figure 20, decreases as the electrode potential increases. Comparing the first energy barriers, the height of energy barrier does not change much for the applied potentials.

Determining transition state and activation energy barrier by the NEB method, reaction rate can be quantified by calculating transmission coefficients using Transition State Theory (TST). TST is a widely used tool for analyzing rate constant of chemical reactions. In general, dissociation rate constant can be written as the product of Transition State Theory (TST) rate constant $\left(k^{T S T}\right)$, and transmission coefficient $(k)$ as follows:

$$
\begin{aligned}
k_{T S T} & =\kappa \frac{k_{b} T}{h} e^{-\Delta G^{0} / R T} \\
\kappa & =1-e^{-\hbar \omega_{0} / k_{B} T}
\end{aligned}
$$

Where, $k^{T S T}$ is the transition state theory rate constant, $k$ is the transmission coefficient, and $\omega_{0}$ indicates associated frequency. Free energy variation is given by $\Delta G^{\circ}$. Supposing dissociated $\mathrm{Pt}$ atom as a reacting atom on the $\mathrm{Pt}(111)$ surface, it can be approximated as vibrating atom about fixed average positions until it moves over the transition point as shown in Figure 22. From this assumption, the region of potential surface can be represented by a harmonic approximation.

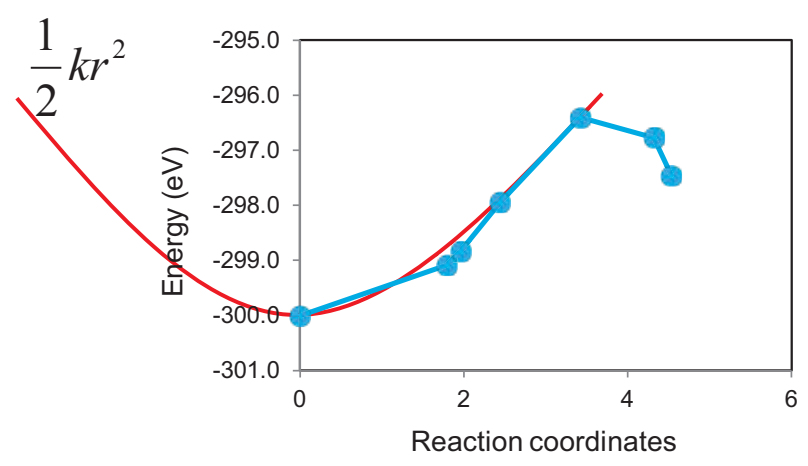

Figure 22: Fitted harmonic oscillator model along the energy curve. It is supposed that the dissociated Pt atom vibrates about fixed average positions until it crosses over the transition point 
The construction of the normal modes of vibration can be carried out by fitting the potential energy surface as

$$
\frac{1}{2} \mu \omega_{0}^{2}\left(r-r_{0}\right)^{2}
$$

where $\mu$ is the reduced mass of dissociated Pt atom and bulk Pt surface atoms. The coefficient of harmonic oscillator $(k)$, was set to have the dimension of [eV/(atomic mass $\left.\times \AA^{2}\right)$ ], and the transition rate constant was calculated accordingly. Transmission coefficients and transition rate by fitting the parabolic curves are listed at Table 11.

Table 11: Calculated transmission coefficients. Transition rate was calculated following the definition given above

\begin{tabular}{|l|c|c|c|c|}
\hline & $-2.12 \mathrm{~V}$ & $-0.67 \mathrm{~V}$ & $0.50 \mathrm{~V}$ & $2.08 \mathrm{~V}$ \\
\hline $\begin{array}{l}\text { Transmission } \\
\text { coefficient(K) }\end{array}$ & 0.0573 & 0.0736 & 0.0962 & 0.1723 \\
\hline$\left(\mathrm{k}^{\mathrm{TST}}\right)$ & $2.20 \times 10^{-26}$ & $2.56 \times 10^{-52}$ & $1.56 \times 10^{-63}$ & $2.88 \times 10^{-108}$ \\
\hline
\end{tabular}

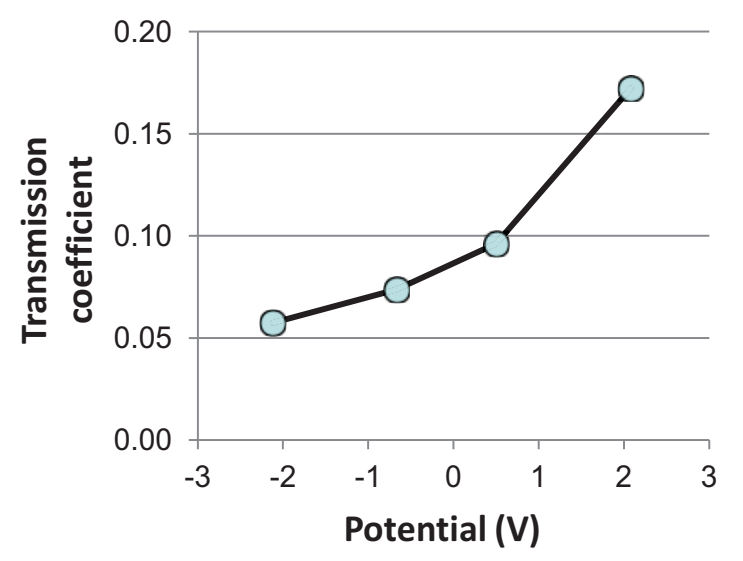

Figure 23: Transmission coefficients of the $\mathrm{Pt}$ dissolution on the bare platinum surface using the harmonic oscillator model. The plot shows that the Pt dissolution depends on the applied potentials

The transmission coefficient gradually increases as the applied potential value increases. The transition rate constant shows extremely small values implying harder transition, which is agreed with higher energy barrier in the transition. 


\subsubsection{Summary}

The nudged elastic band (NEB) method enables us to research about the transition state and calculate the activation energy barrier for the platinum dissolution. Considering the existence of electrode potential and variable surface adatoms during fuel cell operation, we adopted pre-established double-reference method to apply the electrode potential for various surface adatom states, bare surface, oxygen-covered, and hydroxide-covered surfaces. We successfully determined potential values with respect to the NHE for the various surface states.

Quantum mechanical computations predicted dissolved platinum particle in the solvent, $\mathrm{H}_{2} \mathrm{O}$, with various energy barriers according to the applied potential and surface adatoms. Dissolved platinum atoms were captured in water phase by forming various Ptcomplexes, such as $\mathrm{Pt}(\mathrm{O}) \mathrm{x}, \mathrm{Pt}(\mathrm{OH}) \mathrm{x}, \mathrm{Pt}(\mathrm{H} 2 \mathrm{O}) \mathrm{x}$. The $\mathrm{Pt}$ complexes are making quite strong bonds with the oxygen, hydroxide, or water molecules. We also found that the surface reconstruction is contributing to the platinum dissolution by removing the site where Pt could recombine.

Bare Pt surface showed highest activation energy barrier compared to oxygen or hydroxide covered surfaces. It is attributed to the higher reactivity of the oxygen atom and hydroxide to the $\mathrm{Pt}$ atom. In addition, the surface adatoms are bonded to the dissolving platinum atoms forming Pt-complexes to stay in water phase. The surface adatoms seem to screen the effects from applied potentials. The activation energy barriers show quite similar values even though the applied potentials are different. Oxygen covered surface showed lowest activation energy barrier among three kinds of surfaces. The transition state analysis performed for the bare platinum surface showed same tendency in comparison with transition coefficients and activation energy barriers. 
DE-EE0000466

Ballard Material Products Inc

\section{References}

[1] K. Koga, T. Ikeshoji, and K. Sugawara, "Size- and Temperature-Dependent Structural Transitions in Gold Nanoparticles," Physical Review Letters, vol. 92, p. 115507, 2004.

[2] Y. Sun and Y. Xia, "Shape-Controlled Synthesis of Gold and Silver Nanoparticles," Science, vol. 298, pp. 2176-2179, December 13, 20022002.

[3] R. Sardar, A. M. Funston, P. Mulvaney, and R. W. Murray, "Gold Nanoparticles: Past, Present, and Future," Langmuir, vol. 25, pp. 13840-13851, 2012/05/16 2009.

[4] S. Link, C. Burda, B. Nikoobakht, and M. A. El-Sayed, "Laser-Induced Shape Changes of Colloidal Gold Nanorods Using Femtosecond and Nanosecond Laser Pulses," The Journal of Physical Chemistry B, vol. 104, pp. 6152-6163, 2012/05/16 2000.

[5] G. Hodes, "When Small Is Different: Some Recent Advances in Concepts and Applications of Nanoscale Phenomena," Advanced Materials, vol. 19, pp. 639-655, 2007.

[6] M. J. Yacaman, J. A. Ascencio, H. B. Liu, and J. Gardea-Torresdey, "Structure shape and stability of nanometric sized particles," Journal of Vacuum Science \& Technology B: Microelectronics and Nanometer Structures, vol. 19, pp. 1091-1103, 2001.

[7] J. P. Wilcoxon and B. L. Abrams, "Synthesis, structure and properties of metal nanoclusters," Chemical Society Reviews, vol. 35, pp. 1162-1194, 2006.

[8] H. Häkkinen and M. Manninen, "How "Magic" is a Magic Metal Cluster?," Physical Review Letters, vol. 76, pp. 1599-1602, 03/04/ 1996.

[9] S. Varga, B. Fricke, H. Nakamatsu, T. Mukoyama, J. Anton, D. Geschke, et al., "Fourcomponent relativistic density functional calculations of heavy diatomic molecules," The Journal of Chemical Physics, vol. 112, pp. 3499-3506, 2000.

[10] H. Grönbeck and W. Andreoni, "Gold and platinum microclusters and their anions: comparison of structural and electronic properties," Chemical Physics, vol. 262, pp. 1-14, 2000 .

[11] J. Wang, G. Wang, and J. Zhao, "Density-functional study of Aun ( $n=2-20)$ clusters: Lowest-energy structures and electronic properties," Physical Review B, vol. 66, p. 035418, 2002.

[12] E. Aprà and A. Fortunelli, "Density-Functional Calculations on Platinum Nanoclusters: Pt13, Pt38, and Pt55," The Journal of Physical Chemistry A, vol. 107, pp. 2934-2942, 2003/04/01 2003.

[13] L. Xiao and L. Wang, "Structures of Platinum Clusters: Planar or Spherical?," The Journal of Physical Chemistry A, vol. 108, pp. 8605-8614, 2004/10/01 2004.

[14] W. Q. Tian, M. Ge, B. R. Sahu, D. Wang, T. Yamada, and S. Mashiko, "Geometrical and Electronic Structure of the Pt7 Cluster: A Density Functional Study," The Journal of Physical Chemistry A, vol. 108, pp. 3806-3812, 2004/04/01 2004. 
[15] D.-K. Lee, G.-H. Jeung, and Y. S. Lee, "Two component calculations of Pt2 with relativistic effective core potential including spin-orbit operator," International Journal of Quantum Chemistry, vol. 109, pp. 1975-1983, 2009.

[16] W. Eberhardt, P. Fayet, D. M. Cox, Z. Fu, A. Kaldor, R. Sherwood, et al., "Photoemission from mass-selected monodispersed Pt clusters," Physical Review Letters, vol. 64, pp. 780783, 1990.

[17] Z. L. Wang, J. M. Petroski, T. C. Green, and M. A. El-Sayed, "Shape Transformation and Surface Melting of Cubic and Tetrahedral Platinum Nanocrystals," The Journal of Physical Chemistry B, vol. 102, pp. 6145-6151, 1998/08/01 1998.

[18] M. B. Airola and M. D. Morse, "Rotationally resolved spectroscopy of Pt2," The Journal of Chemical Physics, vol. 116, pp. 1313-1317, 2002.

[19] M. N. Huda, M. K. Niranjan, B. R. Sahu, and L. Kleinman, "Effect of spin-orbit coupling on small platinum nanoclusters," Physical Review A, vol. 73, p. 053201, 2006.

[20] K. Bhattacharyya and C. Majumder, "Growth pattern and bonding trends in Ptn $(n=2-13)$ clusters: Theoretical investigation based on first principle calculations," Chemical Physics Letters, vol. 446, pp. 374-379, 2007.

[21] A. Sebetci, "Does spin-orbit coupling effect favor planar structures for small platinum clusters?," Physical Chemistry Chemical Physics, vol. 11, pp. 921-925, 2009.

[22] R. V. Chepulskii and S. Curtarolo, "Ab Initio Insights on the Shapes of Platinum Nanocatalysts," Acs Nano, vol. 5, pp. 247-254, Jan 2011.

[23] R. Kikuchi, "A THEORY OF COOPERATIVE PHENOMENA," Physical Review, vol. 81, pp. 988-1003, 1951.

[24] J. M. Sanchez, F. Ducastelle, and D. Gratias, "GENERALIZED CLUSTER DESCRIPTION OF MULTICOMPONENT SYSTEMS," Physica A, vol. 128, pp. 334-350, 1984.

[25] M. H. F. Sluiter and Y. Kawazoe, "Invariance of truncated cluster expansions for firstprinciples alloy thermodynamics," Physical Review B, vol. 71, p. 212201, 06/09/ 2005.

[26] R. Drautz and A. Díaz-Ortiz, "Obtaining cluster expansion coefficients in ab initio thermodynamics of multicomponent lattice-gas systems," Physical Review B, vol. 73, p. 224207, 06/29/ 2006.

[27] D. J. Schmidt, "Cluster Expansion Studies of Oxygen Adsorption on Transition Metal Surfaces," Doctorate of Philosophy, Chemical and Biomolecular Engineering Notre Dame, 2012.

[28] E. Langenbach, A. Spitzer, and H. Lüth, "The adsorption of water on Pt(111) studied by irreflection and UV-photoemission spectroscopy," Surface Science, vol. 147, pp. 179-190, 1984.

[29] A. Michaelides, V. A. Ranea, P. L. de Andres, and D. A. King, "General Model for Water Monomer Adsorption on Close-Packed Transition and Noble Metal Surfaces," Physical Review Letters, vol. 90, p. 216102, 2003.

[30] A. B. Anderson, "REACTIONS AND STRUCTURES OF WATER ON CLEAN AND OXYGEN COVERED PT(111) AND FE(100)," Surface Science, vol. 105, pp. 159-176, 1981. 
DE-EE0000466

Ballard Material Products Inc

[31] H. P. Bonzel, G. Pirug, and J. E. Muller, "REVERSIBLE H2O ADSORPTION ON PT(111) $+\mathrm{K}$ - WORK-FUNCTION CHANGES AND MOLECULAR-ORIENTATION," Physical Review Letters, vol. 58, pp. 2138-2141, May 1987.

[32] T. Jacob and W. A. Goddard, "Agostic interactions and dissociation in the first layer of water on Pt(111)," Journal of the American Chemical Society, vol. 126, pp. 9360-9368, Aug 2004.

[33] S. Kandoi, A. A. Gokhale, L. C. Grabow, J. A. Dumesic, and M. Mavrikakis, "Why Au and $\mathrm{Cu}$ are more selective than $\mathrm{Pt}$ for preferential oxidation of $\mathrm{CO}$ at low temperature," Catalysis Letters, vol. 93, pp. 93-100, Mar 2004.

[34] J. Kua and W. A. Goddard, "Oxidation of methanol on 2nd and 3rd row Group VIII transition metals (Pt, Ir, Os, Pd, Rh, and Ru): Application to direct methanol fuel cells," Journal of the American Chemical Society, vol. 121, pp. 10928-10941, Dec 1999.

[35] S. Meng, E. G. Wang, and S. W. Gao, "Water adsorption on metal surfaces: A general picture from density functional theory studies," Physical Review B, vol. 69, May 2004.

[36] T. Ohwaki, K. Kamegai, and K. Yamashita, "Electric field effects on the adsorption, charge transfer and vibrational state at metal electrodes: A DFT study on H2O/Pt(111), $(\mathrm{H} 2 \mathrm{O}) / \mathrm{Pt}(100)$ and $(\mathrm{H} 2 \mathrm{O})(2) / \mathrm{Pt}(111), "$ Bulletin of the Chemical Society of Japan, vol. 74, pp. 1021-1029, Jun 2001.

[37] P. Vassilev, R. A. van Santen, and M. T. M. Koper, "Ab initio studies of a water layer at transition metal surfaces," Journal of Chemical Physics, vol. 122, Feb 2005.

[38] P. A. Thiel and T. E. Madey, "THE INTERACTION OF WATER WITH SOLID-SURFACES FUNDAMENTAL-ASPECTS," Surface Science Reports, vol. 7, pp. 211-385, 1987.

[39] A. Michaelides and K. Morgenstern, "Ice nanoclusters at hydrophobic metal surfaces," Nature Materials, vol. 6, pp. 597-601, Aug 2007.

[40] A. Michaelides, A. Alavi, and D. A. King, "Insight into H2O-ice adsorption and dissociation on metal surfaces from first-principles simulations," Physical Review B, vol. 69, Mar 2004.

[41] A. Hodgson and S. Haq, "Water adsorption and the wetting of metal surfaces," Surface Science Reports, vol. 64, pp. 381-451, Sep 2009.

[42] G. B. Fisher and J. L. Gland, "THE INTERACTION OF WATER WITH THE PT(111) SURFACE," Surface Science, vol. 94, pp. 446-455, 1980.

[43] S. Haq, J. Harnett, and A. Hodgson, "Growth of thin crystalline ice films on Pt(111)," Surface Science, vol. 505, pp. 171-182, May 2002.

[44] J. L. Daschbach, B. M. Peden, R. S. Smith, and B. D. Kay, "Adsorption, desorption, and clustering of $\mathrm{H} 2 \mathrm{O}$ on Pt(111)," Journal of Chemical Physics, vol. 120, pp. 1516-1523, Jan 2004.

[45] C. Puglia, A. Nilsson, B. Hernnas, O. Karis, P. Bennich, and N. Martensson, "PHYSISORBED, CHEMISORBED AND DISSOCIATED O-2 ON PT(111) STUDIED BY DIFFERENT CORE-LEVEL SPECTROSCOPY METHODS," Surface Science, vol. 342, pp. 119-133, Nov 1995. 
DE-EE0000466

Ballard Material Products Inc

[46] B. C. Stipe, M. A. Rezaei, W. Ho, S. Gao, M. Persson, and B. I. Lundqvist, "Singlemolecule dissociation by tunneling electrons," Physical Review Letters, vol. 78, pp. 44104413, Jun 1997.

[47] J. L. Gland, B. A. Sexton, and G. B. Fisher, "OXYGEN INTERACTIONS WITH THE PT(111) SURFACE," Surface Science, vol. 95, pp. 587-602, 1980.

[48] H. Steininger, S. Lehwald, and H. Ibach, "ADSORPTION OF OXYGEN ON PT(111)," Surface Science, vol. 123, pp. 1-17, 1982.

[49] A. Eichler and J. Hafner, "Molecular precursors in the dissociative adsorption of O-2 on Pt(111)," Physical Review Letters, vol. 79, pp. 4481-4484, Dec 1997.

[50] A. Eichler, F. Mittendorfer, and J. Hafner, "Precursor-mediated adsorption of oxygen on the (111) surfaces of platinum-group metals," Physical Review B, vol. 62, pp. 4744-4755, Aug 2000.

[51] M. L. Bocquet, J. Cerda, and P. Sautet, "Transformation of molecular oxygen on a platinum surface: A theoretical calculation of STM images," Physical Review B, vol. 59, pp. 1543715445, Jun 1999.

[52] J. Roques and A. B. Anderson, "Electrode potential-dependent stages in OHads formation on the Pt3Cr alloy (111) surface," Journal of the Electrochemical Society, vol. 151, pp. E340-E347, 2004.

[53] A. Ohma, T. Ichiya, K. Fushinobu, and K. Okazaki, "Theoretical analysis of oxygen reduction reaction and $\mathrm{H} 2 \mathrm{O} 2$ formation and the impact of $\mathrm{CF} 3 \mathrm{SO} 3 \mathrm{H}$ coverage on $\mathrm{Pt}$ (111)," Surface Science, vol. 604, pp. 965-973, 6/15/ 2010.

[54] I. Kendrick, D. Kumari, A. Yakaboski, N. Dimakis, and E. S. Smotkin, "Elucidating the Ionomer-Electrified Metal Interface," Journal of the American Chemical Society, vol. 132, pp. 17611-17616, 2010/12/15 2010.

[55] "Jaguar," 7.5 ed. New York: Schrödinger, LLC, 2008.

[56] Y. Zhao and D. G. Truhlar, "The M06 suite of density functionals for main group thermochemistry, thermochemical kinetics, noncovalent interactions, excited states, and transition elements: two new functionals and systematic testing of four M06-class functionals and 12 other functionals," Theoretical Chemistry Accounts, vol. 120, pp. 215241, May 2008.

[57] Y. Zhao and D. G. Truhlar, "Exploring the Limit of Accuracy of the Global Hybrid Meta Density Functional for Main-Group Thermochemistry, Kinetics, and Noncovalent Interactions," Journal of Chemical Theory and Computation, vol. 4, pp. 1849-1868, Nov 2008.

[58] J. P. Perdew, K. Burke, and Y. Wang, "Generalized gradient approximation for the exchange-correlation hole of a many-electron system," Physical Review B, vol. 54, p. 16533, 1996.

[59] J. P. Perdew, K. Burke, and M. Ernzerhof, "Generalized gradient approximation made simple," Physical Review Letters, vol. 77, pp. 3865-3868, Oct 281996.

[60] A. D. Becke, "DENSITY-FUNCTIONAL THERMOCHEMISTRY .3. THE ROLE OF EXACT EXCHANGE," Journal of Chemical Physics, vol. 98, pp. 5648-5652, Apr 1993. 
DE-EE0000466

Ballard Material Products Inc

[61] P. J. Stephens, F. J. Devlin, C. F. Chabalowski, and M. J. Frisch, "AB-INITIO CALCULATION OF VIBRATIONAL ABSORPTION AND CIRCULAR-DICHROISM SPECTRA USING DENSITY-FUNCTIONAL FORCE-FIELDS," Journal of Physical Chemistry, vol. 98, pp. 11623-11627, Nov 1994.

[62] P. J. Hay and W. R. Wadt, "Ab initio effective core potentials for molecular calculations. Potentials for $\mathrm{K}$ to $\mathrm{Au}$ including the outermost core orbitals," The Journal of Chemical Physics, vol. 82, pp. 299-310, 1985.

[63] M. C. Payne, M. P. Teter, D. C. Allan, T. A. Arias, and J. D. Joannopoulos, "ITERATIVE MINIMIZATION TECHNIQUES FOR ABINITIO TOTAL-ENERGY CALCULATIONS MOLECULAR-DYNAMICS AND CONJUGATE GRADIENTS," Reviews of Modern Physics, vol. 64, pp. 1045-1097, Oct 1992.

[64] "Materials Studio," 5.0 ed. San Diego: Accelrys Software Inc., 2009.

[65] J. Kua and W. A. Goddard, "Chemisorption of Organics on Platinum. 2. Chemisorption of C2Hx and CHx on Pt(111)," The Journal of Physical Chemistry B, vol. 102, pp. 9492-9500, 1998/11/01 1998.

[66] J. Kua and W. A. Goddard, "Chemisorption of Organics on Platinum. 1. The Interstitial Electron Model," The Journal of Physical Chemistry B, vol. 102, pp. 9481-9491, 1998/11/01 1998.

[67] S. L. Mayo, B. D. Olafson, and W. A. Goddard, III, "DREIDING - A GENERIC FORCEFIELD FOR MOLECULAR SIMULATIONS," J. Phys. Chem., vol. 94, pp. 8897-8909, 1990.

[68] M. Levitt, M. Hirshberg, R. Sharon, K. E. Laidig, and V. Daggett, "Calibration and Testing of a Water Model for Simulation of the Molecular Dynamics of Proteins and Nucleic Acids in Solution," Journal of Physical Chemistry B, vol. 101, pp. 5051-5061, 1997.

[69] S. S. Jang, M. Blanco, W. A. Goddard, III, G. Caldwell, and R. B. Ross, "THE SOURCE OF HELICITY IN PERFLUORINATED N-ALKANES," Macromolecules, vol. 36, pp. 5331-5341, 2003.

[70] A. K. Rappe and W. A. Goddard, III, "Charge Equilibration for Molecular-Dynamics Simulations," Journal of Physical Chemistry, vol. 95, pp. 3358-3363, Apr 181991.

[71] S. J. Plimpton, "Fast Parallel Algorithms for Short-Range Molecular Dynamics," J. Comp. Phys., vol. 117, pp. 1-19, 1995.

[72] S. J. Plimpton, R. Pollock, and M. Stevens, "Particle-Mesh Ewald and rRESPA for Parallel Molecular Dynamics Simulations," in the Eighth SIAM Conference on Parallel Processing for Scientific Computing, Minneapolis, 1997.

[73] W. C. Swope, H. C. Andersen, P. H. Berens, and K. R. Wilson, "A COMPUTERSIMULATION METHOD FOR THE CALCULATION OF EQUILIBRIUM-CONSTANTS FOR THE FORMATION OF PHYSICAL CLUSTERS OF MOLECULES - APPLICATION TO SMALL WATER CLUSTERS," Journal of Chemical Physics, vol. 76, pp. 637-649, 1982 1982.

[74] S. Nose, "A Unified Formulation of the Constant Temperature Molecular- Dynamics Methods," Journal of Chemical Physics, vol. 81, pp. 511-519, 1984.

[75] S. Nose, "A MOLECULAR-DYNAMICS METHOD FOR SIMULATIONS IN THE CANONICAL ENSEMBLE," Molecular Physics, vol. 52, pp. 255-268, 19841984. 
DE-EE0000466

Ballard Material Products Inc

[76] S. Nose, "AN EXTENSION OF THE CANONICAL ENSEMBLE MOLECULAR-DYNAMICS METHOD," Molecular Physics, vol. 57, pp. 187-191, Jan 1986.

[77] J. A. Dean, Lange's Handbook of Chemistry

New York, NY: McGraw-Hill Book Company, 1979.

[78] N. E. Singh-Miller and N. Marzari, "Surface energies, work functions, and surface relaxations of low-index metallic surfaces from first principles," PHYSICAL REVIEW B, vol. 80, p. 235407, 2009.

[79] H. Iddir, V. Komanicky, S. Ogut, H. You, and P. Zapol, "Shape of platinum nanoparticles supported on SrTiO3: Experiment and theory," JOURNAL OF PHYSICAL CHEMISTRY C, vol. 111, pp. 14782-14789, 2007.

[80] S. Baud, C. Ramseyer, G. Bihlmayer, S. Blugel, C. Barreteau, M. C. Desjonqueres, et al., "Comparative study of ab initio and tight-binding electronic structure calculations applied to platinum surfaces," PHYSICAL REVIEW B, vol. 70, p. 235423, 2004.

[81] J. L. F. Da Silva, C. Stampfl, and M. Scheffler, "Converged properties of clean metal surfaces by all-electron first-principles calculations," SURFACE SCIENCE vol. 600, pp. 703-715, 2006.

[82] G. Boisvert, L. J. Lewis, and M. Scheffler, "Island morphology and adatom self-diffusion on Pt(111)," PHYSICAL REVIEW B, vol. 57, pp. 1881-1889, 1998.

[83] M. Connolly, "Analytical molecular surface calculation," Journal of Applied Crystallography, vol. 16, pp. 548-558, 1983.

[84] B. Krishnamurthy and S. Deepalochani, "Performance of Platinum Black and Supported Platinum Catalysts in a Direct Methanol Fuel Cell," International Journal of Electrochemical Science, vol. 4, pp. 386-395, Mar 2009.

[85] I. Esparbé, E. Brillas, F. Centellas, J. A. Garrido, R. M. Rodríguez, C. Arias, et al., "Structure and electrocatalytic performance of carbon-supported platinum nanoparticles," Journal of Power Sources, vol. 190, pp. 201-209, 5/15/ 2009.

[86] A. Ignaszak, S. Ye, and E. d. Gyenge, "A Study of the Catalytic Interface for O2 Electroreduction on Pt: The Interaction between Carbon Support Meso/Microstructure and Ionomer (Nafion) Distribution," The Journal of Physical Chemistry C, vol. 113, pp. 298-307, 2009/01/08 2008.

[87] G. Sasikumar, J. W. Ihm, and H. Ryu, "Dependence of optimum Nafion content in catalyst layer on platinum loading," Journal of Power Sources, vol. 132, pp. 11-17, 5/20/ 2004. 


\section{DE-EE0000466}

\section{Final Project Report}

\section{Development of Micro-Structural Mitigation Strategies for PEM Fuel Cells: Morphological Simulations and Experimental Approaches}

\section{CHAPTER VI}

\section{MICRO-STRUCTURAL MODEL}

Simon Fraser University:

Zahra Yoosefabadi, PhD Candidate

PI: Dr. J. Pharoah

Queen's University:

Mobin Khakbazbaboli, PhD Candidate

Hae-Won Choi, Post-Doctoral Fellow

Chih-Che Chueh, Post-Doctoral Fellow 
DE-EE0000466

Ballard Material Products Inc

\section{TABLE OF CONTENTS}

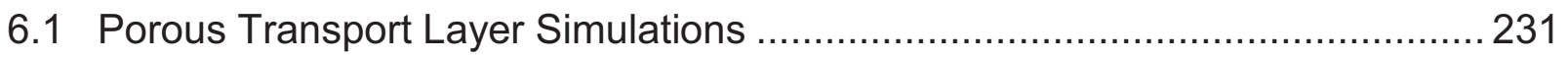

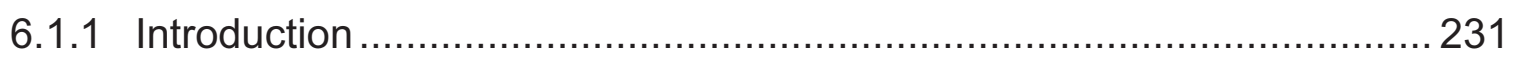

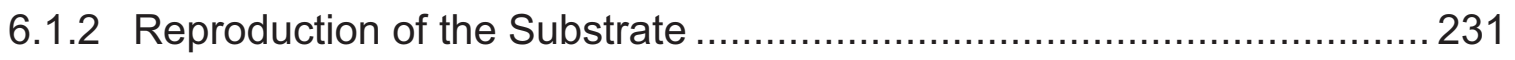

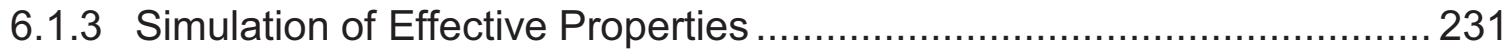

6.2 Micro-structural Catalyst Layer Model ...................................................... 245

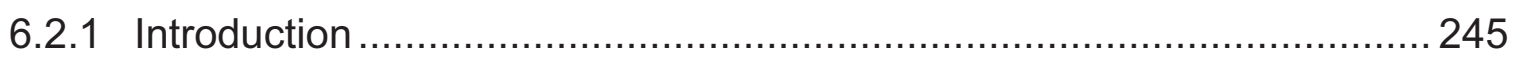

6.2.2 Pore Distribution and Knudsen Number.......................................... 247

6.2.3 Oxygen Dissolution in the lonomeric Phase ..................................... 250

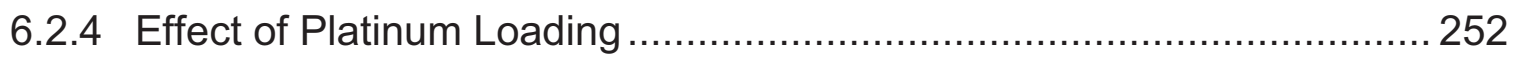

6.2.5 Capillary Pressure in Micro-structural Catalyst Layers........................ 257

6.2.6 Transient Cycling of a Catalyst Micro-structure ….............................. 262 


\section{LIST OF FIGURES}

Figure 1: Numerical Reconstruction process to simulate a PTL, (left) Generation of Fibers, (middle)

Placement of Binder, and (right) Impregnation with Teflon

Figure 2: Comparison of the model predicted porosity, after the three-step generation process, with

experimental data. Note that this result is for a Toray based paper ................................... 232

Figure 3: Comparison of the model cross-section images with those taken using SEM imaging.......... 232

Figure 4: Effective diffusivity as a function of porosity of the substrate and MPL ................................2 233

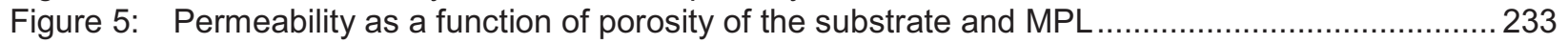

Figure 6: Thermal conductivity as a function of the porosity of the substrate and MPL ....................2234

Figure 7: Comparison of simulated effective diffusivity with experimental data and empirical correlations for different PTFE loadings (0-30\% wt. PTFE) ............................................. 235

Figure 8: Comparison of simulated in-plane (IP) and through-plane (TP) permeability with

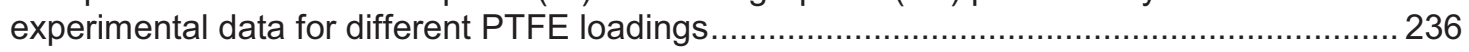

Figure 9: Comparison of simulated in-plane (IP) and through-plane (TP) effective thermal conductivity with experimental data and analytical model for different PTFE loadings .......... 237

Figure 10: Comparison of simulated in-plane (IP) and through-plane (TP) effective electrical conductivity with experimental data for different PTFE loadings

Figure 11: Simulated capillary pressure curves as a function of liquid water saturation for........................ PTFE loadings and (b) contact angles.

Figure 12: Comparison of simulated relative in-plane (IP) and through-plane (TP) gas diffusivity ( $0 \%$ wt. PTFE) with empirical correlations as a function of liquid water saturation ........................ 240

Figure 13: Simulated relative through-plane (TP) gas diffusivity as a function of liquid water saturation

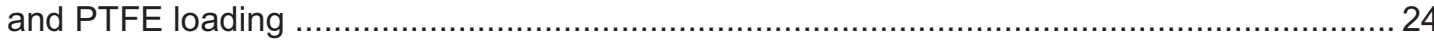

Figure 14: Simulated in-plane (IP) and through-plane (TP) relative permeability ( $0 \%$ wt. PTFE) as a function of liquid water saturation for both wetting (WP) and non-wetting (NWP) phases ..... 242

Figure 15: Simulated through-plane (TP) relative permeability as a function of liquid water saturation and PTFE loading for both wetting (WP) and non-wetting (NWP) phases ....

Figure 16: Comparison of simulated through-plane (TP) relative permeability with empirical correlations.......

Figure 17: Simulated through-plane (TP) effective thermal conductivity as a function of liquid water

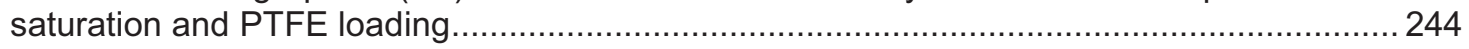

Figure 18: Schematic showing the representative regions and interfaces within a PEMFC CL............ 246

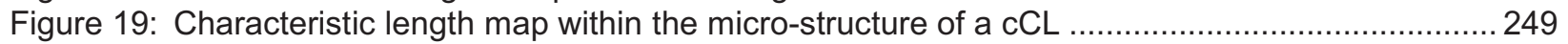

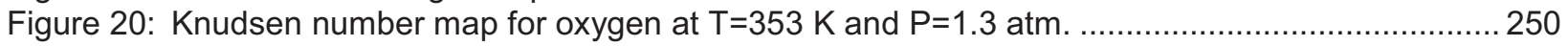

Figure 21: Local Knudsen number map for water vapour at $\mathrm{T}=353 \mathrm{~K}$ and $\mathrm{P}=1.3 \mathrm{~atm}$........................2 250

Figure 22: Effect of the description/inclusion of oxygen dissolution in the ionomer phase..................... 252

Figure 23: Polarization curves for the 4 different micro-structures with the current density on a

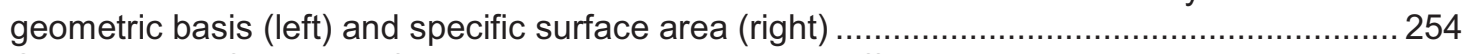

Figure 24: Comparison of the role of protonic transport, reactant diffusion, and base kinetics as function of the path length of the domain (a function of loading) ........................................ 255

Figure 25: Decrease in the local Oxygen as a function of the micro-structure depth based on different

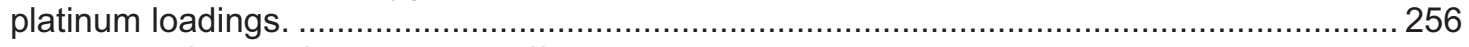

Figure 26: The shape factor of the oxygen diffusive transport around the ionomer particles as a function of platinum loading.

Figure 27: Non-wetting pore algorithm for determination of the capillary pressure, (a) the erosion, (b) the component of the erosion that is connecting to the NWP reservoir, (c) NWP-filled portion .....

Figure 28: Sample slices at left: original geometry, centre: $67 \%$ pore space remaining, right: $15 \%$ pore space remaining....

Figure 29: Capillary pressure curves generated from the Young-Laplace equation at two different length scales. Left: assuming a voxel size of $1 \mathrm{~nm}$, Right: assuming a voxel size of 20 Angstroms 
Figure 30: Capillary pressure saturation curves predicted with a Euclidean and a Manhattan distance maps.

Figure 31: Capillary pressure saturation curves predicted using the old and new algorithms applied to cubic geometries (left) and elongated geometries (right)

Figure 32: Capillary pressure saturation curves predicted using the new algorithms applied to various catalyst geometries

Figure 33: Capillary pressure generated from the micro-structural model.......................................... 262

Figure 34: Catalyst layer micro-structures at various saturation levels for AST cycling. ...................... 263

Figure 35: The current response to a 30 s square wave voltage cycle at two different temporal resolutions.

Figure 36: A time series of the oxygen concentrations at one point in the ionomer and at one point in the pore during voltage cycling.

\section{LIST OF TABLES}

Table 1: Summary of all of the sub models in which the mathematical model is subdivided................247

Table 2: Tabulated Current Density and Overpotential for the 3 Dissolution Models........................... 252

Table 3: Tabulated current produced for the four micro-structures 


\section{Micro-structural Models}

\subsection{Porous Transport Layer Simulations}

\subsubsection{Introduction}

In this section of the overall project, the Porous Transport Layer (PTL) that was used in the experimental testing was reproduced using a micro-structural based simulation. The purpose of these simulations was to extract the effective properties of the layer and enable those relationships to be applied within the unit cell models.

\subsubsection{Reproduction of the Substrate}

The substrate paper was recreated based on the information pertaining to the basis weight, Teflon content, manufacturing process, thickness, and measured porosity. Figure 1 shows the steps of reproducing the micro-structure: (1) Generation of Fibers, (2) Placement of Binder, and (3) Impregnation with Teflon.
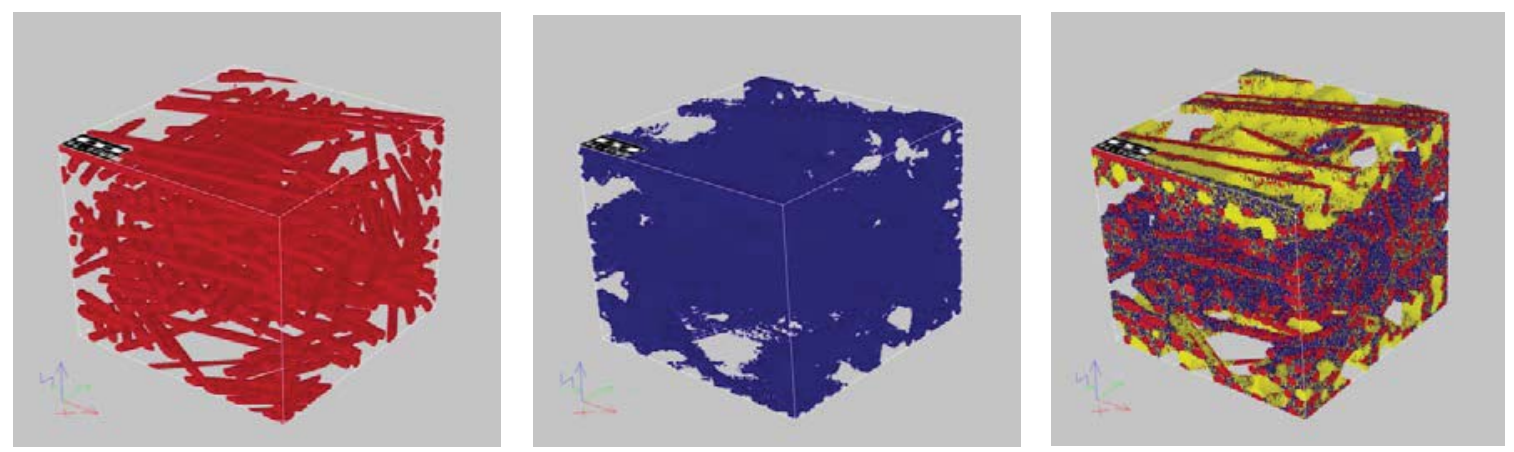

Figure 1: Numerical Reconstruction process to simulate a PTL, (left) Generation of Fibers, (middle) Placement of Binder, and (right) Impregnation with Teflon

Based on this reconstruction technique, the comparison of the resulting model porosity compared with experiment is shown in Figure 2. Further to this, the comparison of the model cross-sections with SEM cross-section imaging also show good qualitative agreement - Figure 3.

The model was also moved through a process in which a Micro-Porous Layer was added in order to fully assemble the final PTL part that is used in the unit cell.

\subsubsection{Simulation of Effective Properties}

\section{Effect of the MPL:}

The micro-structural model was run in order to extract effective properties on a dry and saturated basis. The measured list of properties included permeability, thermal conductivity, electronic conductivity, capillary pressure, and diffusivity. 
DE-EE0000466

Ballard Material Products Inc

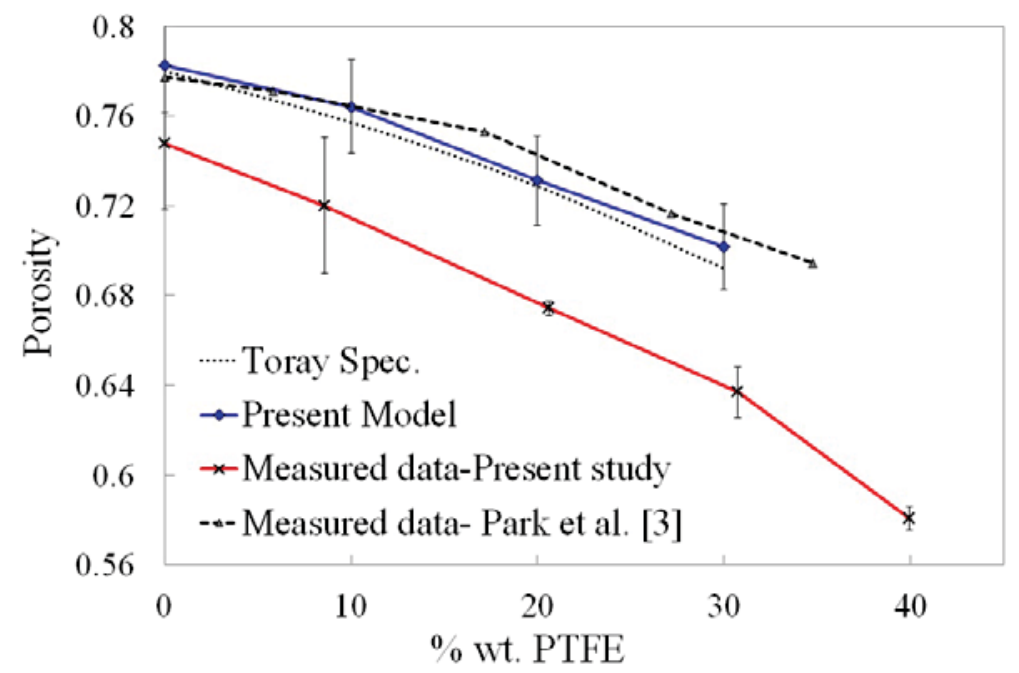

Figure 2: Comparison of the model predicted porosity, after the three-step generation process, with experimental data. Note that this result is for a Toray based paper

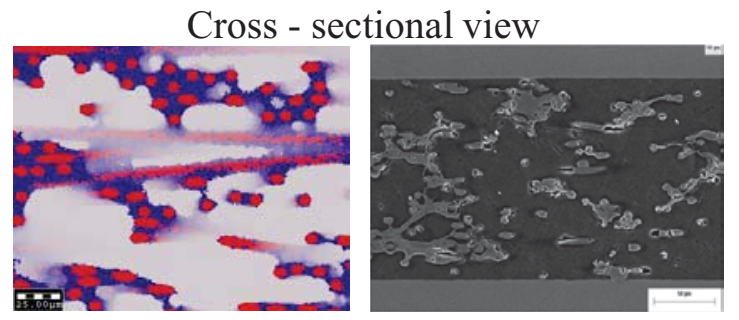

Top - down view

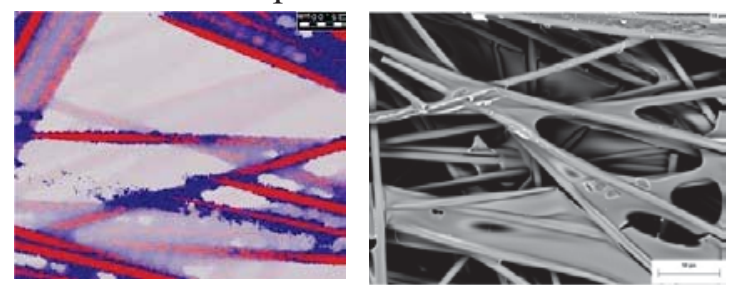

Figure 3: Comparison of the model cross-section images with those taken using SEM imaging

The diffusivity was studied as a function of saturation but also as a function of the combination of the MPL and substrate layers. Figure 4 shows the initial values of the substrate as predicted by the model and the effect of the addition of the MPL on the diffusivity of the final PTL part. From this figure, we see a larger reduction in the in-plane diffusivity as compared to the throughplane. This would likely affect the ability of the cell to re-distribute gases across the landchannel regions of the cell. 
DE-EE0000466

Ballard Material Products Inc

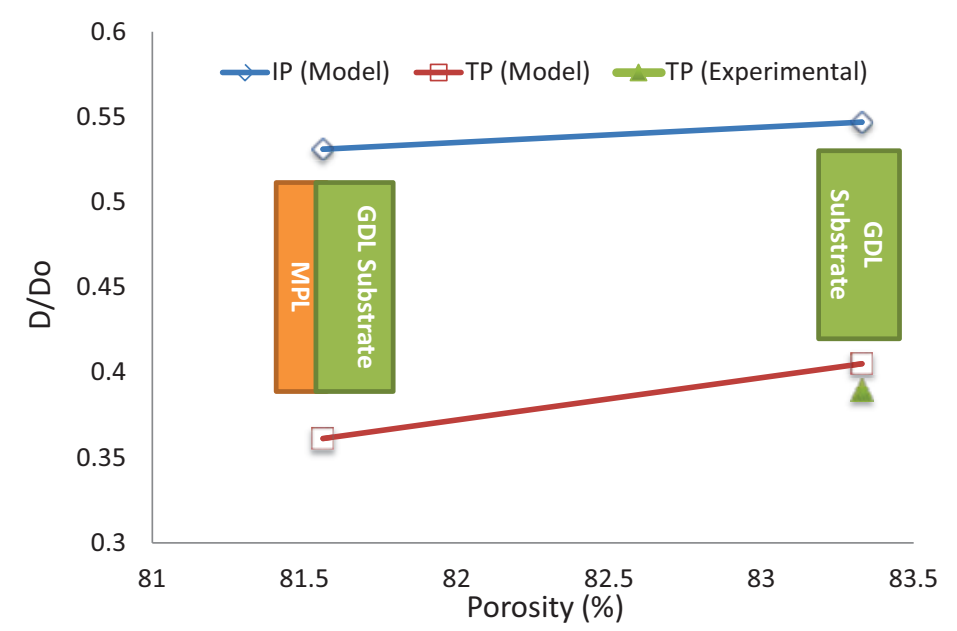

Figure 4: Effective diffusivity as a function of porosity of the substrate and MPL

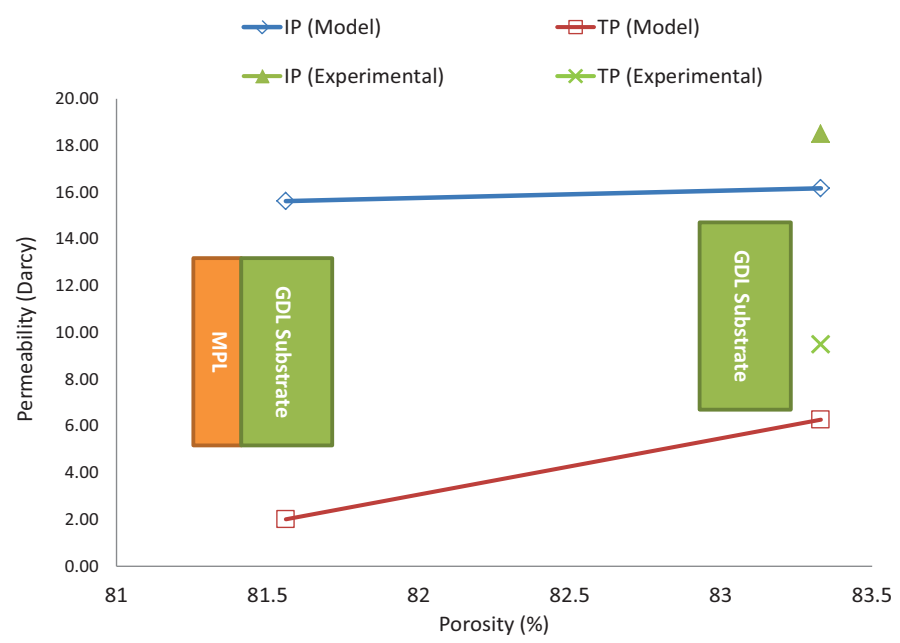

Figure 5: Permeability as a function of porosity of the substrate and MPL

From Figure 5, the permeability is seen to demonstrate a similar behaviour to the diffusivity experiencing a larger reduction in the in-plane direction as compared to the through-plane. The values reported here estimate the permeability to be around 16 Darcy in the through-plane direction.

The effective thermal conductivity was also mapped as a function of the addition of the MPL to the substrate. Figure 6 , shows a relatively flat trend due to the addition of the MPL. 
DE-EE0000466

Ballard Material Products Inc

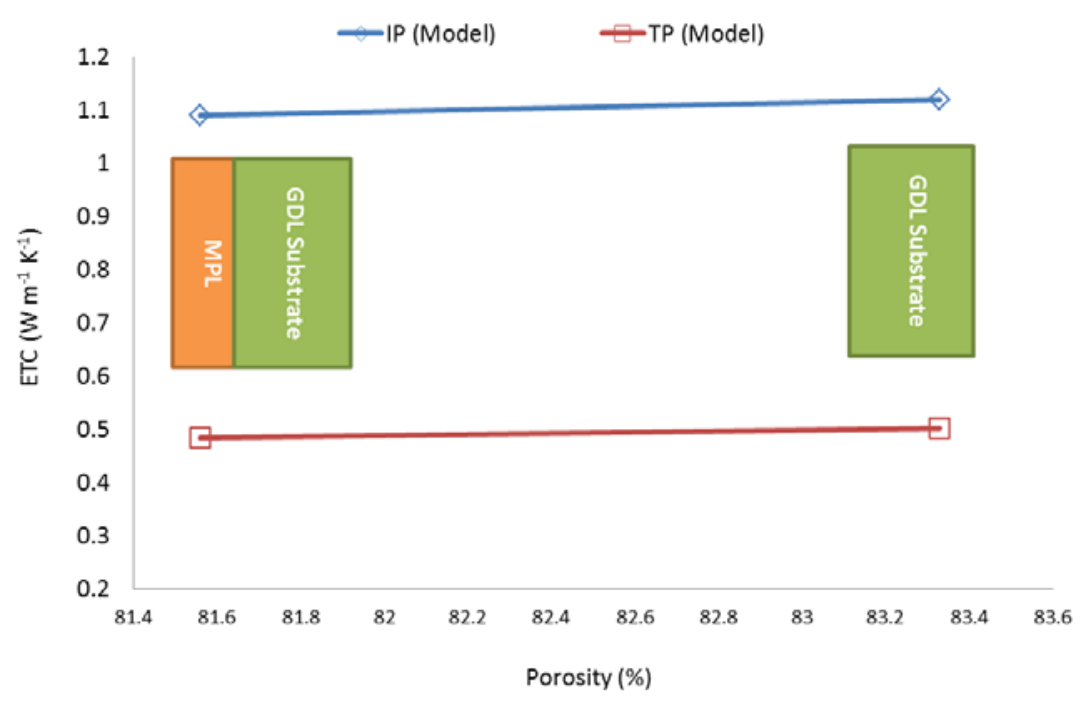

Figure 6: Thermal conductivity as a function of the porosity of the substrate and MPL

\section{DRY CONDITIONS}

\section{Effective Diffusivity:}

The variation of effective diffusivity in the present model versus the porosity as a result of adding PTFE to the bare GDL is illustrated in Figure 7 and compared with empirical correlations available in the literature and in-house measured data. Both through-plane (TP; $z$ ) and in-plane (IP; $x$ and $y$ ) properties are considered in the three-principal directions of diffusion. As anticipated, increasing the PTFE content decreases the average porosity of the GDL by covering areas that were previously available for gas diffusion, and the effective gas diffusivity is consequently reduced in all three principal directions. The trends exhibited by the simulated data are in good agreement with the corresponding ex-situ measured TP data, although the measured GDL batch had lower porosity than the Toray specification of $78 \%$ used for the model. The simulated results reveal that the preferential IP orientation of the fibers results in IPoriented, connected pores with enhanced diffusive capabilities. 
DE-EE0000466

Ballard Material Products Inc

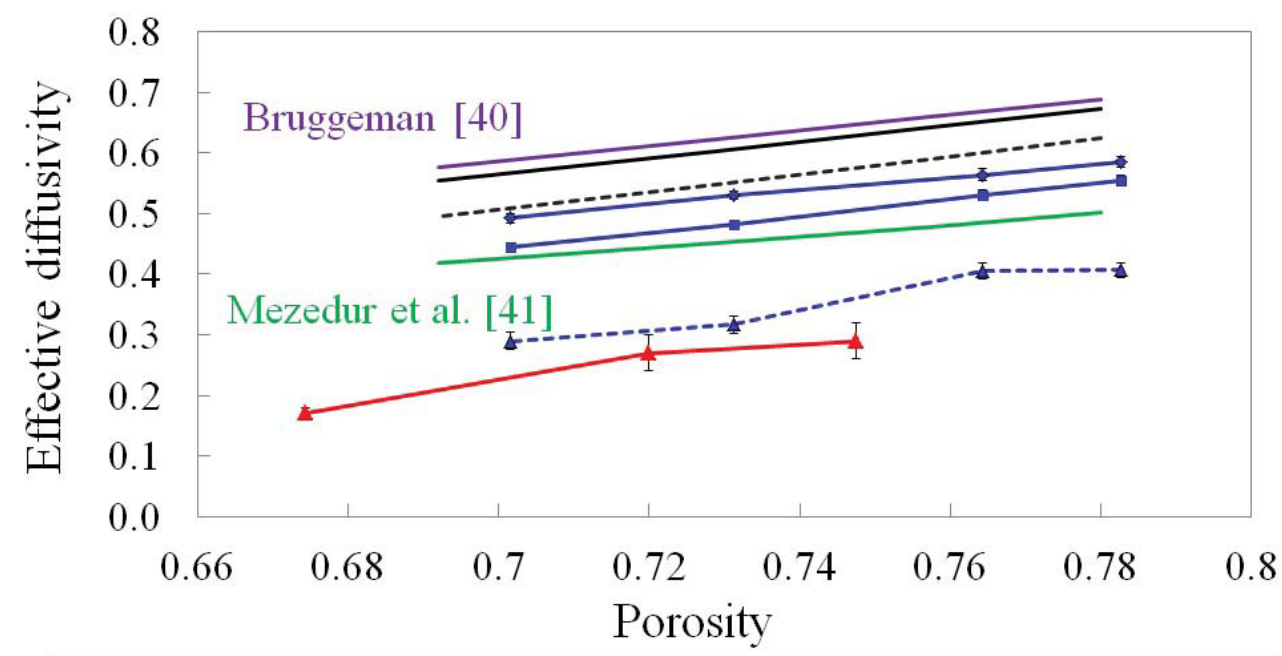

\begin{tabular}{|ll|}
\hline-- TS [43] - TP & $\rightarrow-$ Present model- IP (x) \\
-TS [43]- IP & $\rightarrow-$ Present model- IP (y) \\
- - - Present model- TP (z) & $\rightarrow$-Experimental data- TP (z) \\
\hline
\end{tabular}

Figure 7: Comparison of simulated effective diffusivity with experimental data and empirical correlations for different PTFE loadings (0-30\% wt. PTFE)

On average, the IP diffusivities are $\sim 50 \%$ larger than the TP diffusivities due to the dominant IP fiber orientation. Further, the reduction of TP diffusivity as a result of adding PTFE is higher compared to the IP direction. This is a direct result of increased tortuosity, which is less significant in the IP direction. Thus, PTFE mainly reduces the TP interconnectivity of the pores and magnifies the diffusional anisotropy of the material. Overall, although PTFE treatment is essential for water management it may also interfere with the diffusion and increase gas transport losses in GDLs. Accurate information on diffusive properties is therefore important when selecting PTFE loading for GDL prototyping assignments and optimization studies. Established empirical correlations are compared with the numerical data in Figure 7 in order to put the simulated and measured results into context; however, none of the empirical correlations account for binder or PTFE influences. It can be seen in Figure 7 that the spherical agglomerate model by Bruggeman predicts the highest diffusivity while the more realistic fiber model of Tomadakis and Sotirchos results in smaller, anisotropic values. Mezedur et al. fitted their lattice network to experimental data, and the results are comparable to the present IP diffusivity results. However, it is noteworthy that the present micro-structural modeling framework results, when compared to the commonly employed empirical correlations, are considerably more accurate with the detailed results (TP, IP, binder, PTFE impact) more closely resembling the physical materials and measured data.

\section{Intrinsic Permeability:}

The effect of PTFE loading on the simulated intrinsic, single-phase IP and TP permeability and comparison with the corresponding in-house measured ex-situ data are shown in Figure 8. As expected, the permeability decreases slightly with increasing PTFE loading due to the filling of pores originally available to participate in convective transport. This implies that the flow resistance of the gas phase increases in the GDL as the porosity decreases. Similarly to the diffusivity results, the permeability is higher in the IP direction than the TP direction due to the 
tendency of fiber alignment in the $x-y$ plane and larger connected pathways for the flow. Both simulated and measured data are approximately a factor of three higher in the IP direction than TP. Although the measured TP data are in some cases restricted by the maximum flow rate of the measurement tool, the observed values and trends are consistent with the simulated results. The small deviations are likely a result of the material variability and lower overall porosity of the physical samples used for the measurements. Overall, the simulated results are in good agreement with the measured data, and the modeling framework again demonstrates higher accuracy and versatility in relation to existing empirical correlations. For instance, the permeability obtained using the Kozeny-Carmanand Tomadakis-Robertson correlations was 7.47 and 12.41 Darcy, respectively, which does not capture the large differences observed between the IP and TP directions.

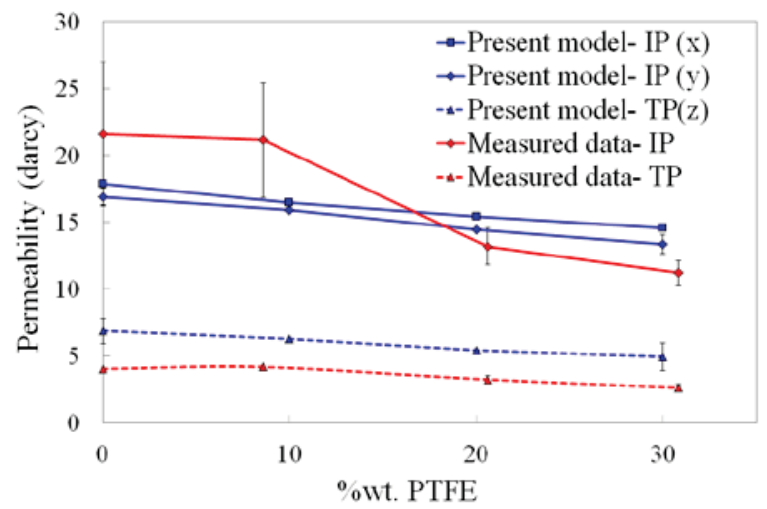

Figure 8: Comparison of simulated in-plane (IP) and through-plane (TP) permeability with experimental data for different PTFE loadings

\section{Effective Thermal Conductivity:}

Heat transfer through porous materials is a complex process comprising conduction, convection, and radiation. However, heat transfer in GDLs is presumed to be dominated by conduction, which occurs in both the solid matrix and the pores, and is therefore the focus of the present study. As shown in Figure 9, the simulated effective thermal conductivity $\left(k_{e f f}\right)$ of the non-teflonated GDL model is about 5.3 and $18 \mathrm{~W} \mathrm{~m}^{-1} \mathrm{~K}^{-1}$ in the TP and IP directions, respectively, and increases marginally with PTFE loading. 
DE-EE0000466

Ballard Material Products Inc

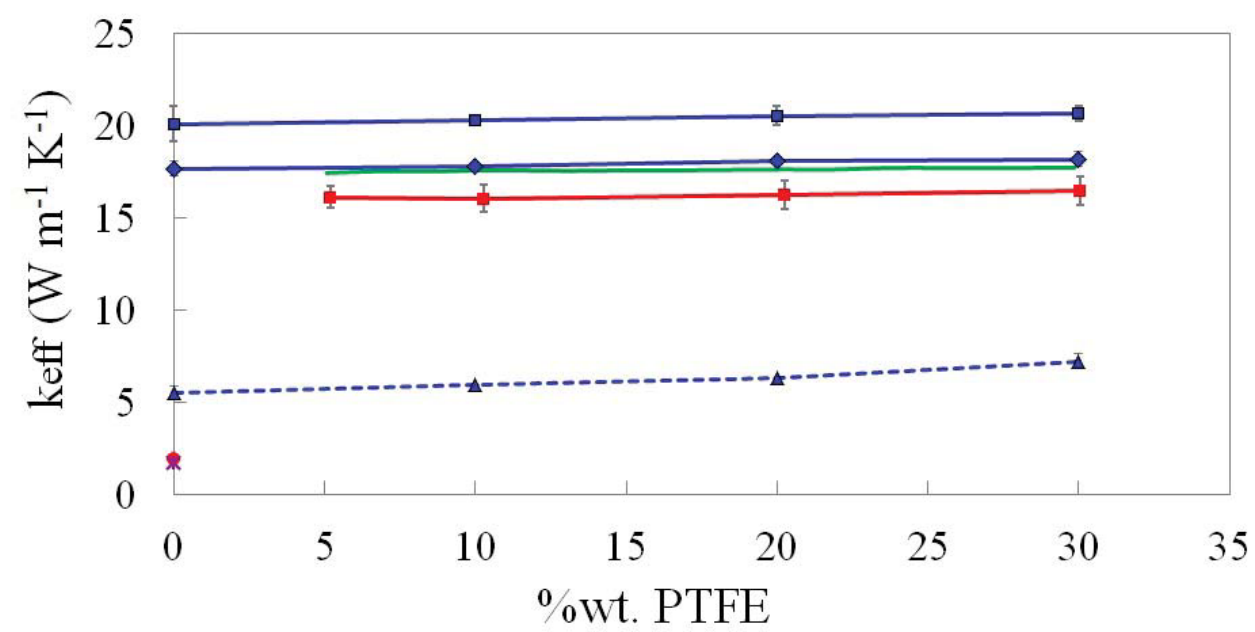

-Analytical model [20]- IP $\rightarrow$-Present model- IP (x)
--Experimental [16]- IP $\rightarrow$ - Present model- IP (y)
- Experimental [16]- TP $\quad$-₫-Present model- TP (z)
* Toray Spec.- TP

Figure 9: Comparison of simulated in-plane (IP) and through-plane (TP) effective thermal conductivity with experimental data and analytical model for different PTFE loadings

The specific thermal conductivity of PTFE $\left(0.25 \mathrm{~W} \mathrm{~m}^{-1} \mathrm{~K}^{-1}\right)$ is higher than air but low relative to carbon and graphite materials (10.5 and $24 \mathrm{~W} \mathrm{~m}^{-1} \mathrm{~K}^{-1}$, respectively), and the small increase can therefore be attributed to replacing air with PTFE in the model. Again, $k_{e f f}$ is highly dependent on the fiber distribution, and the IP value is approximately 3-4x higher than the TP value, which is an important consideration for both GDL and MEA design. The present results are in good agreement with the measurements and analytical model of Sadeghi et al. in the IP direction; however, the results are $2-3 x$ higher than the Toray specification and measured data of Khandelwal et al. in the TP direction. The difference between simulated and measured TP results is attributed to a thermal contact resistance (TCR), which can contribute up to $65-90 \%$ of the measured thermal resistance of the GDL but is not captured by the present model. TCR studies involving porous materials can be relatively complex and are beyond the scope of this work. The simulated IP $k_{e f f}$ results are comparable to the maximum and minimum $k_{e f f}$ of the empirical correlations developed by Hashin and Shtrikman and Kaviany et al. which are 15.8 and $18.3 \mathrm{~W} \mathrm{~m}^{-1} \mathrm{~K}^{-1}$. However, in contrast to the present modeling approach, the empirical correlations are not capable of capturing the strong anisotropy of the GDL.

\section{Effective Electrical Conductivity:}

The effective electrical conductivity $\left(\sigma_{e f f}\right)$ of the Toray TGP GDL is estimated by simulation of steady state electrical conduction in the solid phase of the micro-structural models. Figure 10 illustrates the obtained $\sigma_{\text {eff }}$ as a function of PTFE. The simulated TP electrical conductivity is approximately $600 \mathrm{~S} \mathrm{~m}^{-1}$ and the IP property is $4 \mathrm{x}$ higher due to the anisotropic fiber alignment. As a result of the low electrical conductivity of PTFE, the simulated results are essentially independent of PTFE loading. The in-house measured TP data was significantly lower as a consequence of contact resistance between the tool and the sample. Although there is limited 
experimental verification of anisotropic $\sigma_{e f f}$ in the literature, it has been demonstrated that the IP electrical conductivity is about an order of magnitude higher than the TP conductivity. High IP conductivity is essential to achieve a uniform current density distribution in the catalyst layers relative to the land/channel and along-the-channel directions.

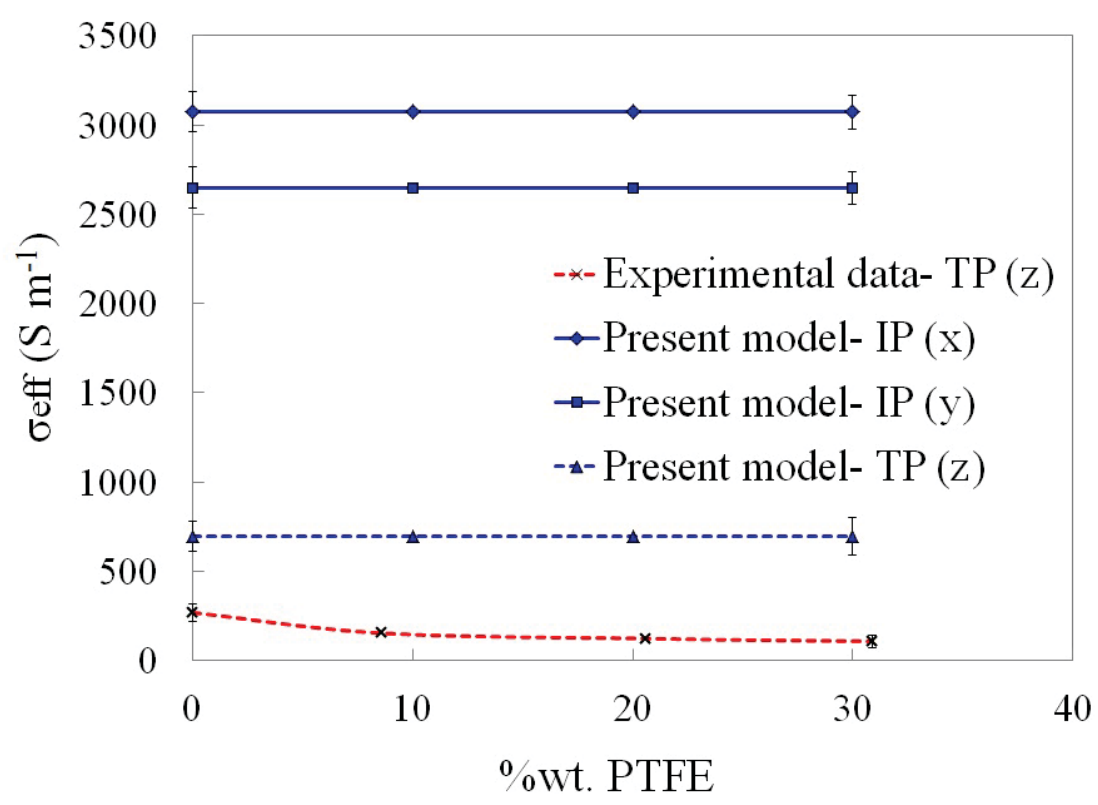

Figure 10: Comparison of simulated in-plane (IP) and through-plane (TP) effective electrical conductivity with experimental data for different PTFE loadings

\section{WET CONDITIONS}

The simulated GDL transport properties presented in the previous section are relevant for fuel cell operation at dry conditions. However, provided that PEMFCs produce water during operation and typically run at low temperature and fully humidified conditions, the presence of liquid water in the GDL must also be considered. This section therefore presents a complete set of simulated GDL transport properties as a function of PTFE content and liquid water saturation that can be used more generally in the context of GDL and MEA design for fuel cell operation under wet conditions.

\section{Capillary Pressure:}

Figure 11 presents the simulated capillary pressure variation in the GDL versus the liquid water saturation in the case of imbibition. A contact angle of $150^{\circ}$ and a surface tension of $0.064 \mathrm{~N} \mathrm{~m}^{-1}$ for water (NWP) in air (WP) at $70^{\circ} \mathrm{C}$ are assumed. Under the assumption of a fixed contact angle, the simulated saturation characteristics are almost independent of PTFE loading. For larger contact angles, as illustrated in Figure 11, higher capillary pressures are required to reach the same saturation level. The simulated percolation path diameter for the non-teflonated GDL model is approximately $20 \mu \mathrm{m}$ and equivalent to a TP tortuosity of $\sim 2$. 
DE-EE0000466

Ballard Material Products Inc
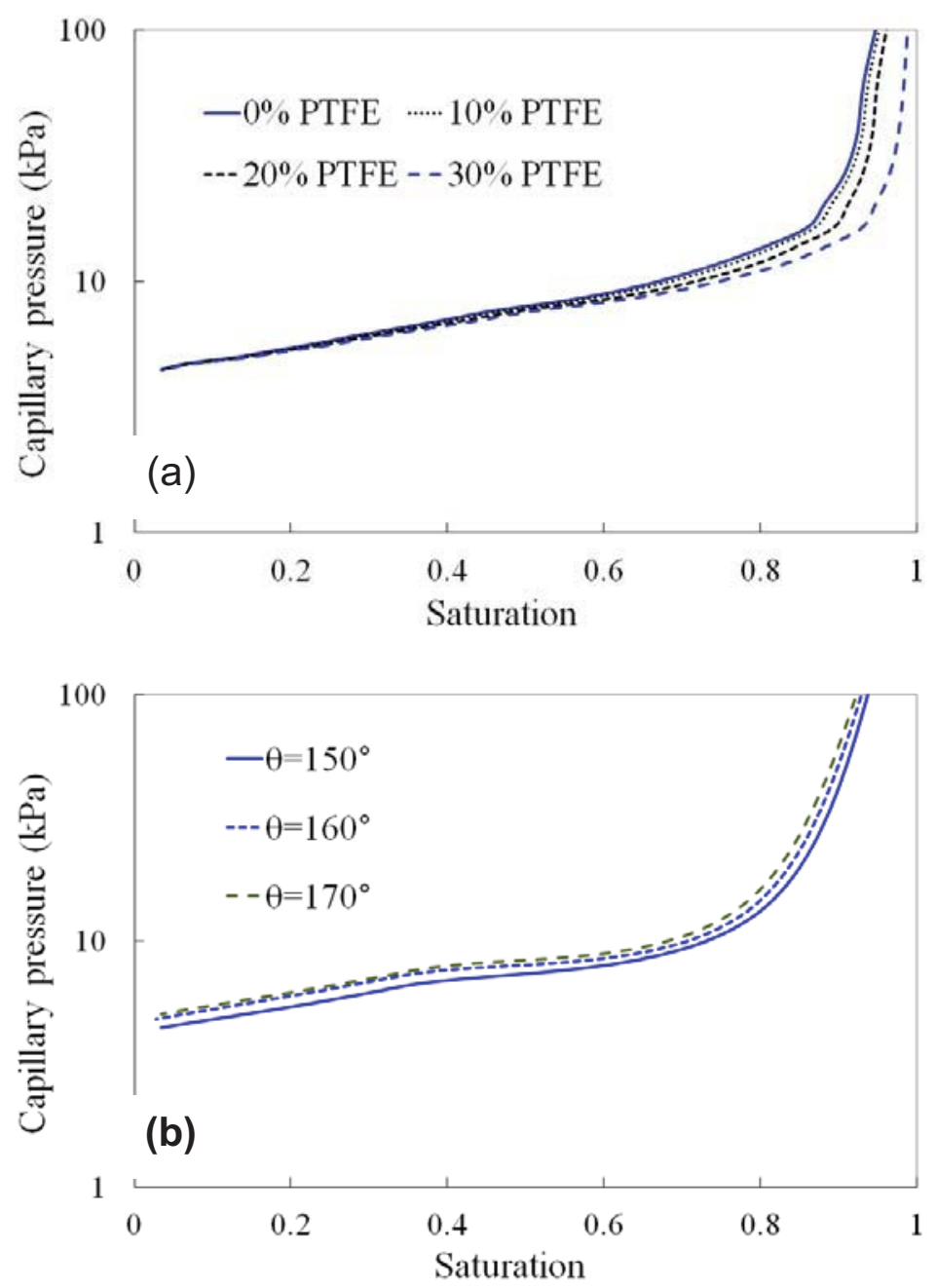

Figure 11: Simulated capillary pressure curves as a function of liquid water saturation for different (a) PTFE loadings and (b) contact angles

\section{Relative Diffusivity:}

The relative gas diffusivity is an important transport property that demonstrates the capability of the GDL to allow reactant gas transport to the active sites in the presence of liquid water. The water situated in (or moving through) the GDL limits the rate of gas diffusion compared to dry conditions. Therefore, the amount of water should be controlled for optimum reactant diffusion through the GDL. The simulated relative diffusivity of the non-teflonated GDL is illustrated in Figure 12 as a function of liquid water saturation. As can be expected, the relative diffusivity decreases with increasing saturation as the pores available for gas diffusion are gradually filled with liquid water. The simulated results in this regard are consistent with previously established empirical correlations for diffusion as a function of porosity in the in-plane direction; however, a notable difference is found in the through-plane direction. 
DE-EE0000466

Ballard Material Products Inc

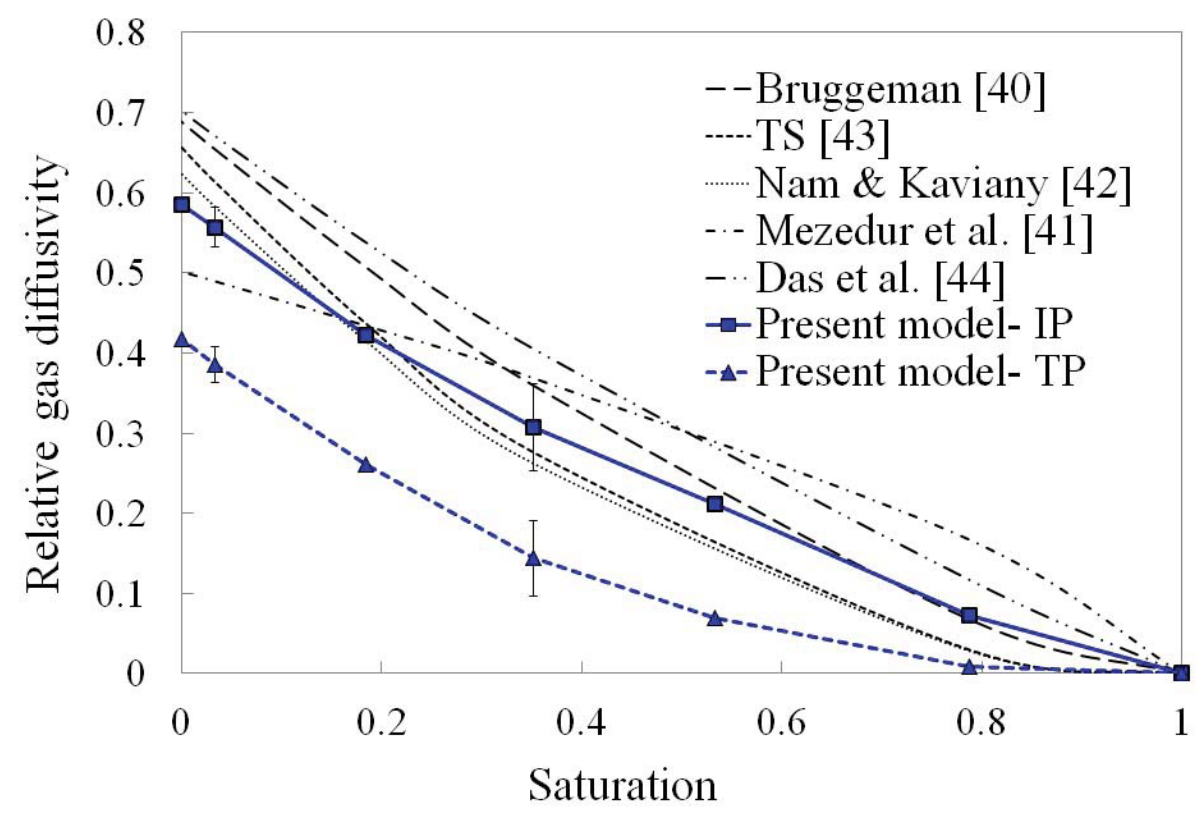

Figure 12: Comparison of simulated relative in-plane (IP) and through-plane (TP) gas diffusivity $(0 \%$ wt. PTFE) with empirical correlations as a function of liquid water saturation

As briefly discussed for diffusion under dry conditions, while the empirical correlations are reasonably accurate for IP properties, the present model is expected to provide considerably more realistic results in the TP direction. In addition, the relative diffusivity is more significantly reduced in the TP direction compared with the IP direction due to the higher tortuosity in the presence of water. A saturation of 0.5 , for instance, is found to reduce the diffusivity by more than $80 \%$ from approximately 0.4 in the dry state to 0.08 , which is of great significance in the context of fuel cell design and operation. Unfortunately, relative diffusivity is challenging to measure and no experimental data are available for comparison. At the same time, the lack of data further justifies the usefulness and application of the present modeling approach and results.

The hydrophobic treatment that is necessary to improve the water management can also impede gas diffusion by reducing the porosity and increasing the tortuosity of the GDL. Figure 13 determines the combined effect of PTFE loading and liquid water saturation on relative TP gas diffusivity. Decreasing the porosity by adding PTFE results in even lower gas diffusivity than for the non-teflonated material under wet conditions and must also be taken into account for accurate predictions of effective diffusivity during fuel cell operation. Furthermore, the obtained curves are found to be non-linear functions of saturation; i.e., the diffusivity drops more rapidly than the gas phase porosity at low saturation levels. Overall, the simulated results are expected to provide a reasonable estimation of the true gas diffusivities in wet conditions during fuel cell operation and can be used as a guide for GDL and MEA design. 
DE-EE0000466

Ballard Material Products Inc

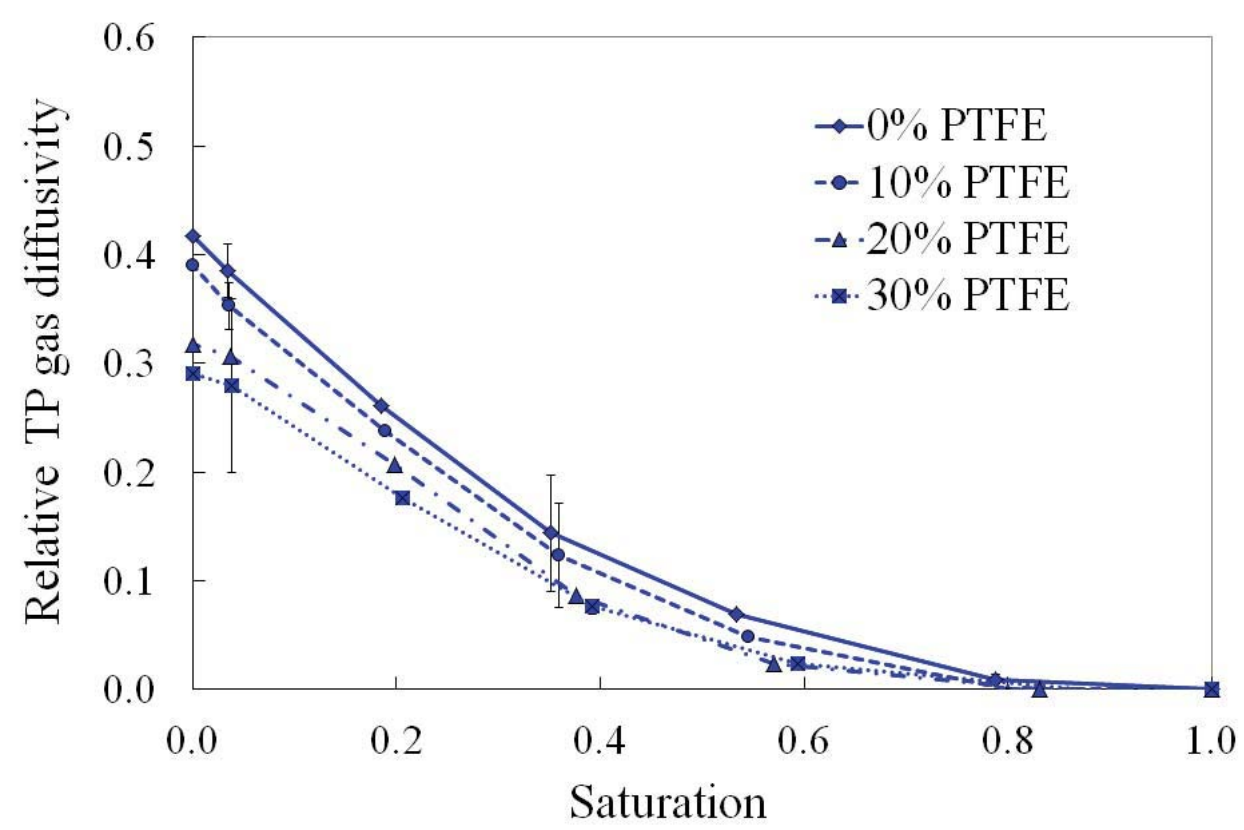

Figure 13: Simulated relative through-plane (TP) gas diffusivity as a function of liquid water saturation and PTFE loading

\section{Relative Permeability:}

In the presence of liquid water in the GDL, the need for estimation of phase pressures of gas and liquid leads to calculation of the relative permeability of both phases. The simulated relative permeability of the non-wetting phase (water), denoted NWP and the wetting phase (gas), denoted WP, as a function of saturation is given in Figure 14 for the non-teflonated GDL. Decreasing the permeability of WP and increasing the NWP permeability with increasing saturation is demonstrated, and order-of-magnitude changes in permeability are readily seen due to the competition for pore space available for the flow. The relative permeability is found to be zero under certain conditions, indicating that no continuous percolation path exists for the given phase due to the prevalence of the opposite phase. This occurs in the gas phase when the saturation level reaches 0.8 and in the liquid phase for saturations less than 0.3. 
DE-EE0000466

Ballard Material Products Inc

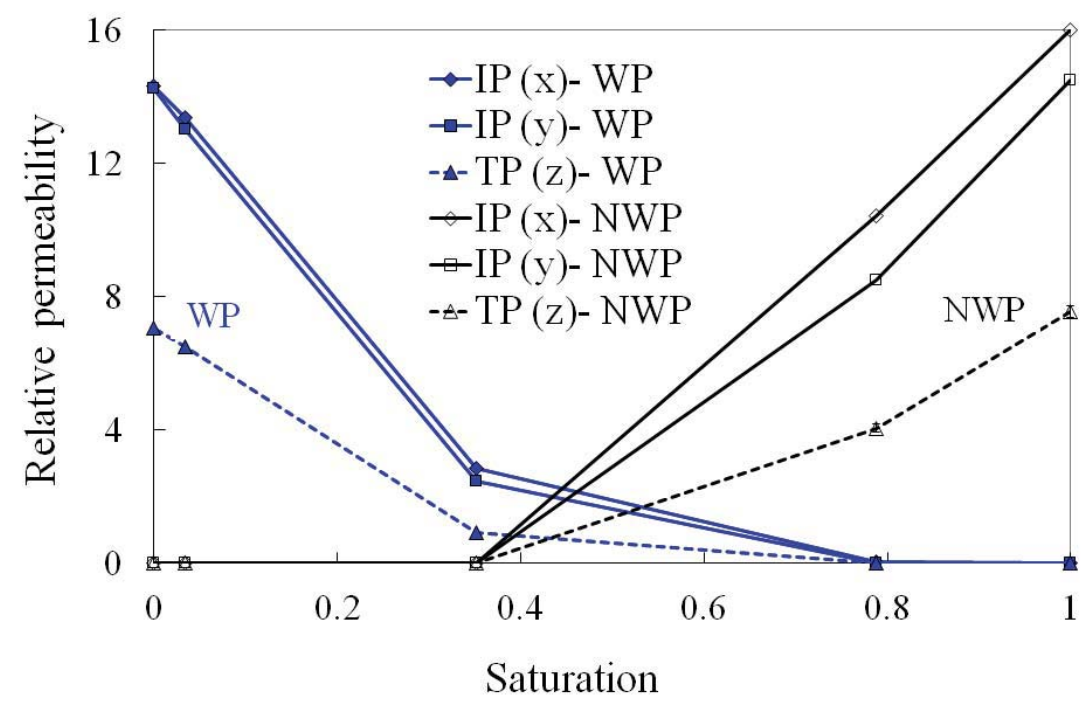

Figure 14: Simulated in-plane (IP) and through-plane (TP) relative permeability $(0 \% \mathrm{wt}$. PTFE) as a function of liquid water saturation for both wetting (WP) and non-wetting (NWP) phases

The effect of PTFE loading on the TP relative permeability of liquid and gas phases is illustrated in Figure 15. The relative permeability decreases overall when PTFE is added and the sensitivity to saturation is moderately reduced. As shown in Figure 16, the simulated relative TP permeability is in relatively good agreement with the empirical correlations of the Power Law, Brooks Corey (BC), and Van Genuchten (VG) models.

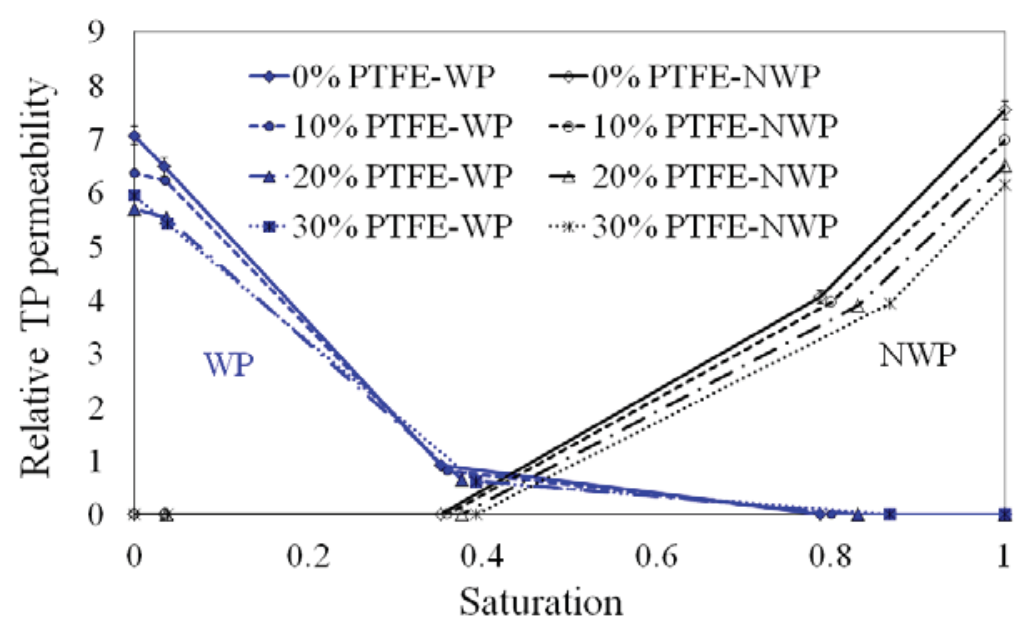

Figure 15: Simulated through-plane (TP) relative permeability as a function of liquid water saturation and PTFE loading for both wetting (WP) and non-wetting (NWP) phases 
DE-EE0000466

Ballard Material Products Inc

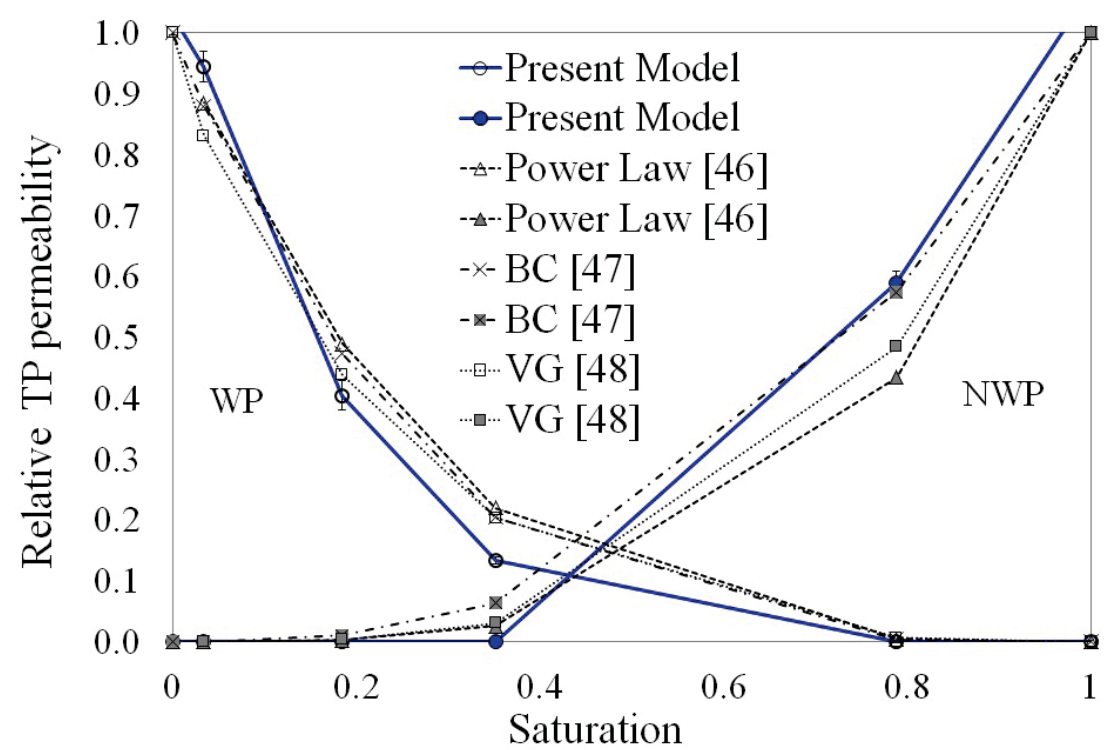

Figure 16: Comparison of simulated through-plane (TP) relative permeability with empirical correlations

\section{Thermal Conductivity:}

While the electrical conductivity of the GDL is independent of liquid water saturation, it is interesting to evaluate the effective thermal conductivity under wet conditions due to the relatively high thermal conductivity of liquid water. Figure 17 presents the numerical results for the TP thermal conductivity of the GDL as a joint function of liquid water saturation and PTFE loading. The rise in $k_{e f f}$ obtained by increasing the water saturation is significant. In the fully saturated state, the TP property is nearly three times higher than in dry conditions. The liquid water is found to decrease the thermal resistance between the fibers and hence increase the rate of heat conduction. Scarce literature is available for the effect of liquid water on the $k_{e f f}$ of GDLs. Burheim et al. measured the TP $k_{\text {eff }}$ of a similar carbon paper with $80 \%$ porosity under various degrees of compression and a liquid water saturation of 0.26 to be $0.57 \pm 0.06 \mathrm{~W} \mathrm{~m}^{-1} \mathrm{~K}^{-}$ 1. The results of the present study are in good agreement with this measurement as the simulated $k_{\text {eff }}$ at 0.26 saturation is approximately $0.6 \mathrm{~W} \mathrm{~m}^{-1} \mathrm{~K}^{-1}$, which is within the measured uncertainty range. The coupled effect of adding PTFE and liquid water is also noteworthy; at low saturation levels, the PTFE loading has a positive effect on $k_{\text {eff }}$, while at high saturation levels, the effect is opposite. Overall, the effect of liquid water is much more significant due to its higher thermal conductivity, and the simulated $k_{\text {eff }}$ trend with saturation is an important contribution of the present work that may have significant impact on the thermal engineering of fuel cells. 
DE-EE0000466

Ballard Material Products Inc

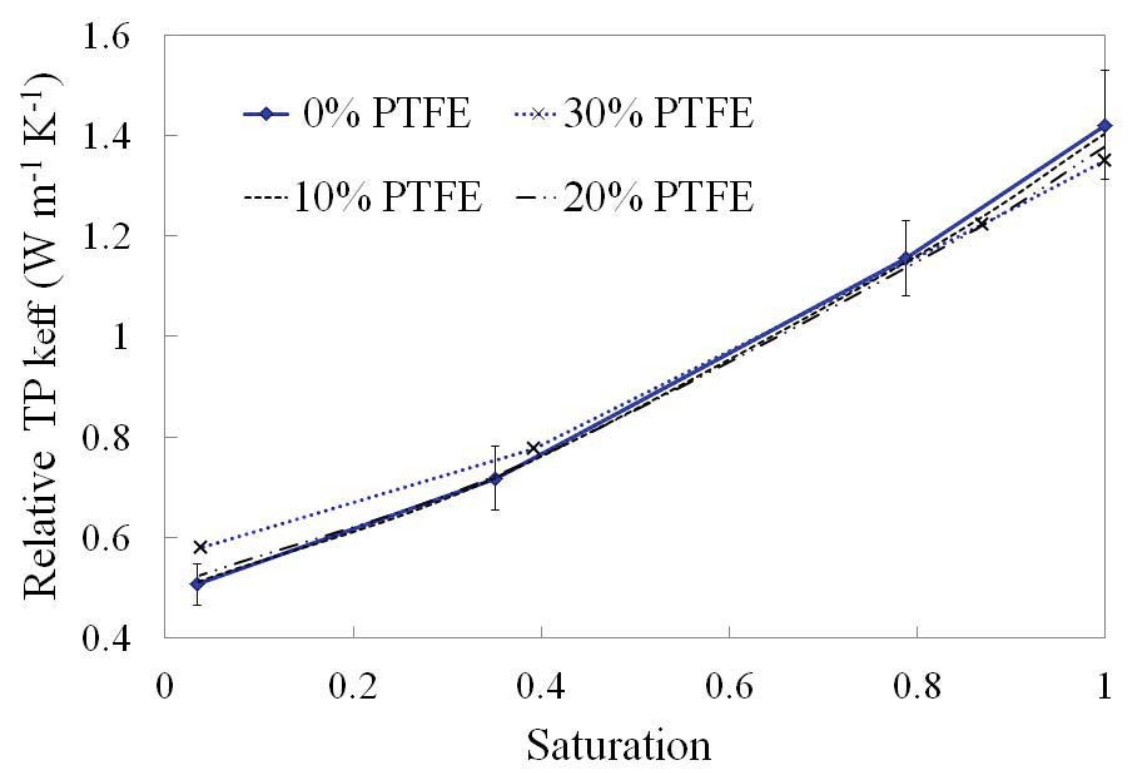

Figure 17: Simulated through-plane (TP) effective thermal conductivity as a function of liquid water saturation and PTFE loading 
DE-EE0000466

Ballard Material Products Inc

\subsection{Micro-structural Catalyst Layer Model}

\subsubsection{Introduction}

In this work, a micro-model of the catalyst layer of polymer electrolyte membrane fuel cell (PEMFC) was developed. The micro-model includes the transport phenomena and the reaction kinetics within a three-dimensional micro-structure representing a sample of a PEMFC catalyst layer (CL). Proper physical boundary conditions have been described on the surfaces of the sample as well as on the interfaces between the regions through which all constituents are solved in a coupled manner. A four-phase micro-structure of CL was reconstructed, the platinum particles were resolved in the computational grid generation and the governing equations were solved within platinum region. A body-fitted computational mesh was generated for the reconstructed micro-structure of the CL. The number of computational cells was optimized based on how close the magnitude of the surface area of a discretized sphere was compared to the analytic surface area for a sphere of the same size. The interfaces relevant to important physical phenomena were refined compared to the rest of the interfaces, specifically the electrochemically active reaction surface and the overall computational mesh was refined to deliver a grid independent numerical solution. The Knudsen effects were included by calculating the characteristic length in the pore region. Four different approaches to including Knudsen effects were studied. Also, a comparison was made between solutions with and without Knudsen effects. A physical model describing the process of oxygen dissolution into the ionomer and liquid phase was developed, with the oxygen dissolution at the interface between pore and ionomer was treated as a superficial phenomenon. Performance curves were produced and provided for the reconstructed micro-structure along with the distribution of field variables, such as local, dissolved oxygen concentration. A catalyst thickness study of the reconstructed micro-structure was conducted such that the results from the micro-modeling were recast allowing for the interpretation of the trend in variable distributions observed in the macro-modeling compared to those observed in experiments. A platinum loading study was performed and the anomalous phenomena of dramatic increases in oxygen transport resistance observed in some experimental works was explained by isolating the ionomer region of the CL micro-structure and numerically calculating the shape factor for diffusive transport. It was found that the increase in oxygen transport resistance is due to the increase in diffusion pathway and decrease in the overall transport surface area. 


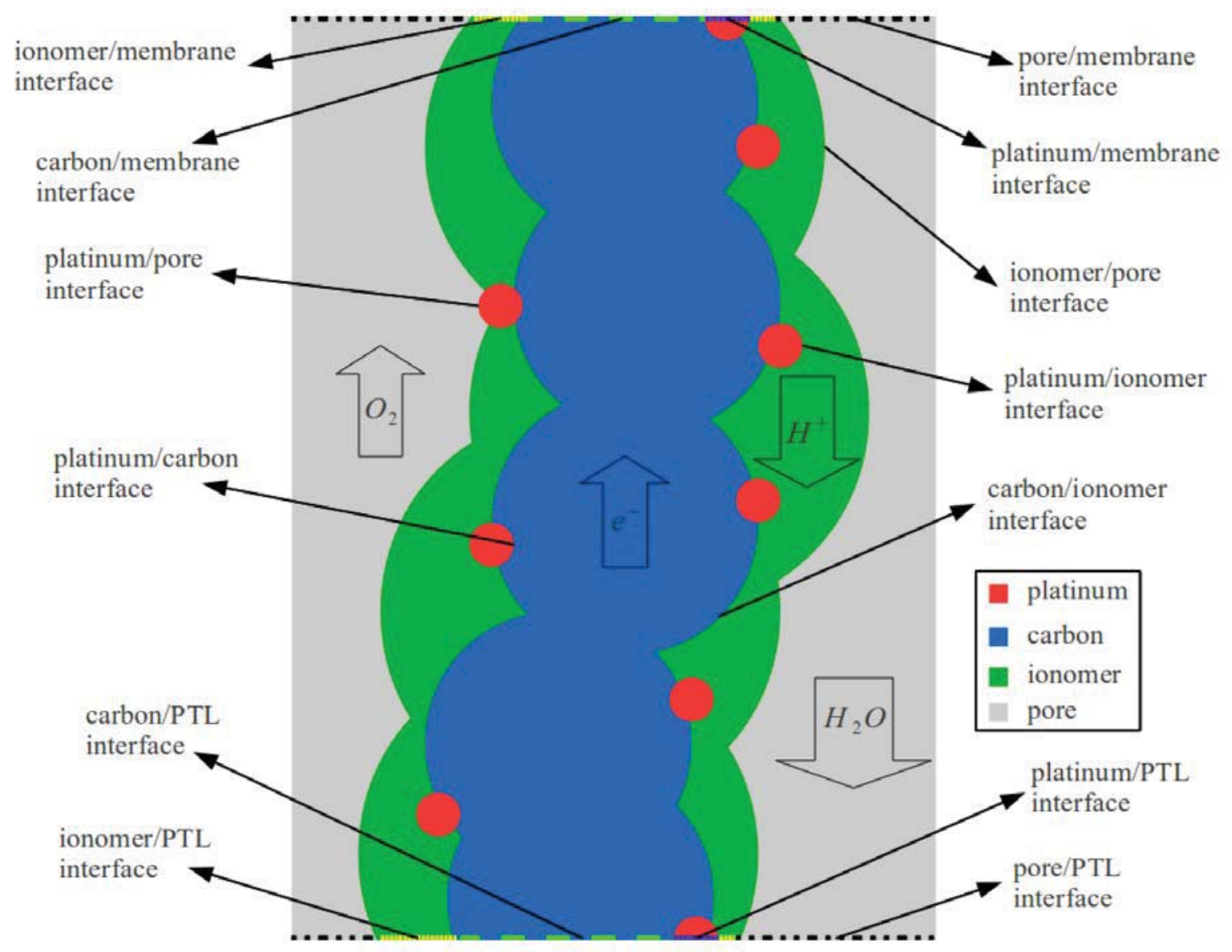

Figure 18: Schematic showing the representative regions and interfaces within a PEMFC CL

The micro-scale operating principle of PEMFC CL involves a complex network of coupled physical phenomena, all of which take place simultaneously within the numerous phases present within the CL. Depending on the CL constituents; the governing equations that describe the physical phenomena may be different. This is due to the different nature and properties of the CL constituents (e.g. carbon, platinum, ionomer and pore). For instance, the formulation of the species conservation equation in the ionomer phase differs from the one describing the same phenomenon in pore phase, where Knudsen diffusion has to be considered alongside bulk diffusion transport. To model each phenomenon in a PEMFC CL with the appropriate equation, the whole mathematical model has been categorized into a set of sub-models. The resulting mathematical sub-models for the CL constituents are: (1) pore model, (2) ionomer model, (3) platinum model, and (4) carbon model. There are a total of four sub-models in total. In addition, the mathematical model includes two other sub-models that account for the electrochemistry and interfacial phenomena. These two sub-models are not part of any of the former region sub-models; both phenomena are relevant only at the interfaces between regions as it is assumed that electrochemical reactions solely occurs at the interface between platinum and ionomer regions (superficial reaction) and the interface between pore and platinum regions is not electrochemically active.

In addition to platinum and ionomer interface, there are other interfaces to be treated according to the associated physical model such as pore and ionomer interface. Figure 18 schematically 
schematically shows all the interfaces between regions in a micro-structure. The five mathematical sub-models are summarized in Table 1, where the physical phenomena and governing equations that are accounted for in each sub-model are presented.

Table 1: Summary of all of the sub-models in which the mathematical model is sub-divided

\begin{tabular}{|clc|}
\hline Region & Phenomena & Governing equations \\
\hline pore & Species transport & Multi-component Fick's law \\
& Energy transport & Fourier-Multicomponent \\
\hline ionomer & Species transport & Fick's law \\
& Energy transport & Fourier \\
& Charge transport & Ohm \\
\hline \multirow{2}{*}{ carbon } & Energy transport & Fourier \\
& Charge transport & Ohm \\
\hline platinum & Energy transport & Fourier \\
& Charge transport & Ohm \\
\hline
\end{tabular}

Among all the physics included in sub-models, the species conservation equation and energy equation are used in most of the sub-regions. It is helpful to first discuss the general form of these two frequently used equations: (1) species conservation equation, and (2) energy conservation equation, then, a detailed description of the mathematical model is presented in the following sequence for micro-model (3) the pore model, (4) the ionomer model, (5) the carbon and platinum model, (6) the electrochemically reactive interface model, and (7) the nonreactive interface model.

\subsubsection{Pore Distribution and Knudsen Number}

The micro-structure dimension is of order magnitude $1 \times 10^{-6} \mathrm{~m}$. While considering the local transport within the pore region of the CL micro-structure, the order of magnitude gets even smaller around $1 \times 10^{-9} \mathrm{~m}$. The mean free path of oxygen in operating condition, $T=353.05 \mathrm{~K}$ and $p=1.3 \mathrm{~atm}$, is around $5 \times 10^{-9} \mathrm{~m}$. This means the molecules not only interact with each other (bulk diffusion), they may even interact with the solid walls of the micro-structure (Knudsen diffusion). The interaction with solid wall of the micro-structure will hinder the diffusive transport of the reactant and product gases. In this section, the necessary information for modeling the diffusive transport of the gaseous reactant and product through the pore region of the microstructure will be provided for the micro-model.

The Knudsen number is defined as the ratio between the mean free path and the characteristic length of the geometry through which the diffusive transport occurs. In the case of a tube, its diameter is the characteristic length while in more complicated geometries this characteristic length is not as straightforward. When the Knudsen number becomes large $(\mathrm{Kn}>10)$, transport is dominated by Knudsen diffusion. For the $\mathrm{Kn}<0.01$, the transport is dominated by bulk diffusion. There is one more regime $(0.01<\mathrm{Kn}<10)$, called the transition regime, in which both the bulk and Knudsen diffusion are important. The diffusive transport of reactant and product gas mostly falls into this regime. The Bosanquet equation is generally used in this range allowing for the consideration of both bulk diffusion and Knudsen diffusion to the overall observed value for the diffusion coefficient. 
Proper calculation of the Knudsen number requires a proper evaluation of the characteristic length, d. For simple geometries, such as diffusive transport through a tube, it is straightforward to evaluate the characteristic length as the diameter of a tube does not change with the respect to the other coordinates such as axial direction. Whereas, the pore region of the micro-structure is by comparison rather complicated - some locations within the pore region can be very confined in different angles and directions by the solid regions of the micro-structure (carbon, platinum and ionomer) resulting in highly irregular pore shapes wherein the characteristic length is changing dramatically. Due to the highly varying characteristic length, the Knudsen effect is also found to vary spatially depending on the location within the pore region. As such, the Knudsen effect must be defined for each location within the pore region. In some works, a single characteristic length is defined as an equivalent tube with constant diameter.

There are different, both experimental and mathematical, methods to calculate pore size distribution, characteristic length distribution, within the pore region of a porous media. A few of these methods are described as follows:

\section{Mercury Intrusion Porosimetry}

Mercury Intrusion Porosimetry (MIP) is an experimental technique often used to determine mean pore size and other pore related properties within a porous structure. In this method, mercury which is a liquid that does not wet most solids and does not spontaneously penetrate pores, is forced to in infiltrate pores by an external pressure. From pressure versus intrusion data, pore volume and size distributions are determined. Mathematically, the pore size distribution can be calculated morphologically. In this method, there are many ways to calculate the pore size distribution such as the area size distribution of intersected pores by Murphy et al., the chord size distribution by Jongerius et al. and the distribution of the diameter of a sphere with the same surface area of the pores by Bouma et al. These measures are difficult to interpret in terms of the three-dimensional reality and do not admit any physical interpretation.

\section{Sphere Growth Methods}

There are two different sphere growth methods presented here. In both methods, a point is picked within the pore space and a sphere is grown until a set of conditions are met. In the first method, the sphere is grown until its surface makes contact with the solid phase of the porous structure. This will be repeated for new points. After a reasonable number of grown spheres, their diameters determine the pore size distribution for the domain. This method generally under-estimates the pore size distribution since the spheres rarely fill the rest of the space they grow in. The second method is similar to the previous method but spheres are grown from a picked point; however the coordinate of the picked point is not fixed in order to let the sphere grow as much as possible in order to fill the whole space of the pore. The diameters of the spheres are analyzed for the characteristic length of the pore region.

\section{Chord Length Methods}

There are a few similar pore size measurement techniques that involve the collection of chord lines to represent pore diameters. In this method, a random point is chosen within the pore domain and a chord is drawn in a random direction from that point until it contacts the surface of the solid phase. The chord is then extended in the opposite direction to contact with the solid phase. This is repeated for new points. After repeating this for a large number of points, the length of the chords are used to calculate the characteristic length of the pore. 
Taking a look at the complexity of the pore region of the micro-structure might bring a question as to why a single Knudsen number is used to model diffusive transport of species, more specifically - is the Knudsen effect the same at any location inside the pore region. The point is at some locations; the pore region is so confined and surrounded by solid regions such that after producing the computational cells, there might be a narrow tube-shape path of single computational cell thick for the gaseous species to transport through. It is possible to calculate a rough Knudsen number at for those computational cells. It is clear that the Knudsen effect must be more intense at some locations. The idea is to find a local characteristic length for each computational cell rather than using a single characteristic length for all of the computational cells in pore region. A new method was used by Blore et al. to calculate the local characteristic and eventually local Knudsen number in order to have a better estimation of the Knudsen effect in diffusive transport. In this way, the diffusion coefficient can be locally evaluated in terms of Knudsen number. Random walk is not a suitable method for the purpose of calculating local characteristic length as, in the current work, the characteristic length at cell centre coordinate of each computational cell was found while in random walk the initial point is randomly chosen. In this approach, a large number of chords are extended in random directions from each of cell centre coordinate of the pore region until both ends of the chord come into contact with a pore-solid interface. The total length of all chords at each starting point is at least 500 times the size of the micro-structure sample. This technique does not allow for the key aspects to be determined because it is not a true random walk algorithm.

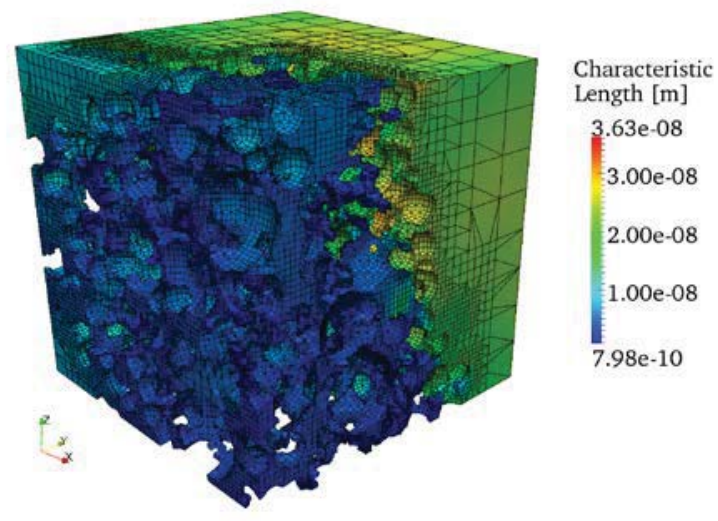

Figure 19: Characteristic length map within the micro-structure of a $\mathrm{CCL}$

Figure 19 illustrates the characteristic length distribution within pore region of CL microstructure. This characteristic length is used to find the local Knudsen number for oxygen and water diffusive transport. As a comparison between the global characteristic length calculated in previous section and the local characteristic length calculated in this section, the average value of the characteristic length in the pore region is $6.44 \%$ larger than the global characteristic length. Almost the same deviation, 5\%, comparing the global and local characteristic lengths was reported by Blore et al. Figure 20 shows the local Knudsen number for oxygen diffusive transport. Figure 21 shows the local Knudsen number for water diffusive transport. 


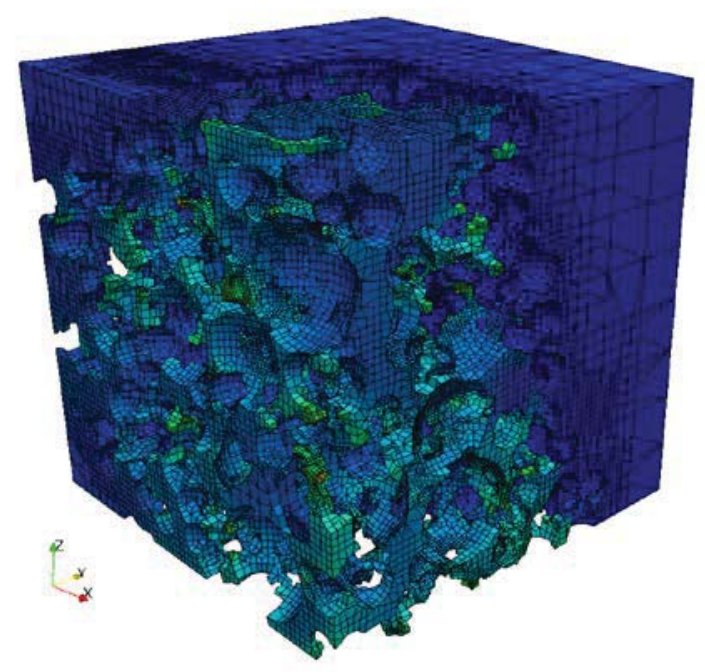

Knudsen Number for Oxygen Diffusive Transport

10.86

10.00

8.000

6.000

4.000

2.000

0.2386

Figure 20: Knudsen number map for oxygen at $\mathrm{T}=353 \mathrm{~K}$ and $\mathrm{P}=1.3 \mathrm{~atm}$

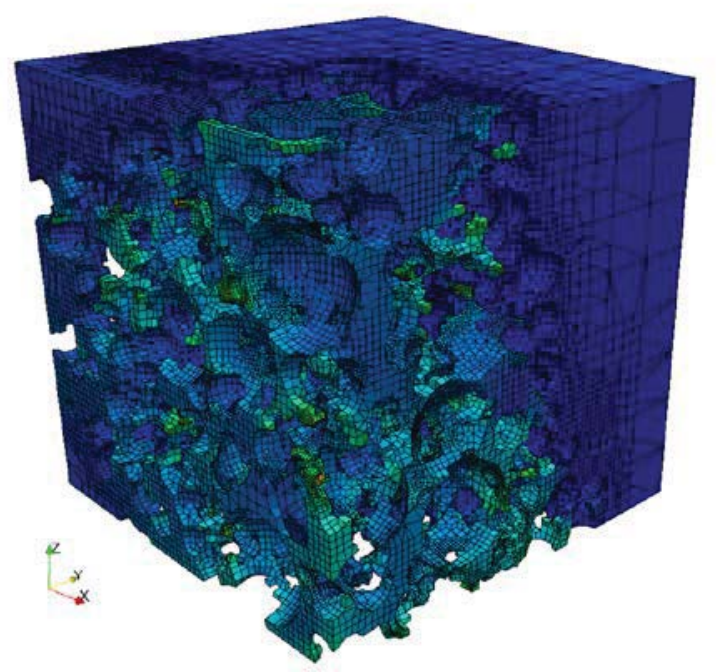

Knudsen Number for Water

Diffusive Transport

35.89

30.00

20.00

10.00

0.7887

Figure 21: Local Knudsen number map for water vapour at $T=353 \mathrm{~K}$ and $\mathrm{P}=1.3 \mathrm{~atm}$

\subsubsection{Oxygen Dissolution in the lonomeric Phase}

Oxygen concentration is different in the pore and ionomer regions adjacent to the interface between these two regions. There is a jump in oxygen concentration at this interface. The oxygen dissolution at the interface between pore and ionomer was mostly ignored in literature. In those works, the diffusion coefficient of oxygen inside ionomer region is set to a smaller value in comparison to the oxygen diffusion coefficient in pore region in order to differentiate between ionomer and pore regions. Since the electrochemical reaction occurs at the interface between ionomer and platinum, the reference oxygen concentration, $\mathrm{C}_{\text {ref, } \mathrm{O} 2}$, in Butler-Volmer equation, must consistently be described regarding the oxygen transport within ionomer region in which electrochemical reaction occurs. The reference oxygen concentration is measured 
experimentally. Since the electrochemical reaction occurs within the ionomer region, the reference oxygen concentration must be adjusted for the micro-models with no oxygen dissolution model but it is kept the same for micro-models with the oxygen dissolution model. In a work by Hattori et al., it is mentioned that the oxygen dissolution in the ionomer region was included in their micro-model, no further information is provided in how this phenomenon was included in their numerical model and the jump in oxygen concentration was included by others in the open literature. Generally, a discontinuity introduced by the jump in oxygen concentration is described and has been attributed to observed computational instabilities. In this model, this was overcome by using an equivalent oxygen concentration that was solved in ionomer region. The oxygen diffusion coefficient within ionomer region was modified by Henry's constant instead of the oxygen concentration so that a continuous oxygen concentration profile across the interface is maintained. After convergence of the numerical solution, the real oxygen concentration within ionomer was back calculated using Henry's Law. So, oxygen dissolution was treated in the volume of the ionomer region. After back calculation, the oxygen gradient within the ionomer region will change, but using the real oxygen diffusion coefficient within ionomer region, the fluxes on the boundaries do not change. If the equivalent oxygen concentration within the ionomer region was used in Butler-Volmer equation to calculate the local current density, the reference oxygen concentration must be adjusted otherwise the local current density will be over predicted in the same way as a micro-model with no oxygen dissolution model. This was not reported in the literature and here it is assumed that they adjusted the reference oxygen concentration. In the current study, Henry's Law was used to model oxygen dissolution at the interface between ionomer and pore regions and it is assumed that oxygen within the gas phase is in equilibrium with the oxygen in ionomer region. The oxygen dissolution was treated as a surface phenomenon and it is assured that the oxygen flow rates at both sides of the interface are the same while a jump in oxygen concentration was maintained at the interface; in other words a physical boundary condition was built and used at the interface between pore and ionomer regions according to equilibrium transport at this interface.

The performance curves of three models, oxygen dissolution model in current study, oxygen dissolution model by Siddique et al., and no oxygen dissolution model are in shown in Figure 22. The micro-model with no oxygen dissolution model over-predicts the performance of the micro-structure. The oxygen dissolution model used by Siddique et al. matches with the performance curve predicted in the current work.

Table 2 shows the total current densities at different overpotentials for the three different models. The reference oxygen concentration was modified according to Henry's constant, $\mathrm{H}=4.9 \mathrm{~cm}^{2} \mathrm{~mol} / \mathrm{atm}$, for the micro-model with no oxygen dissolution and the approach used by Siddique et al. and was left untouched for the micro-model developed in the current work. The micro-model developed by Siddique et al. is in good agreement with the micro-model developed in the current work. 
DE-EE0000466

Ballard Material Products Inc

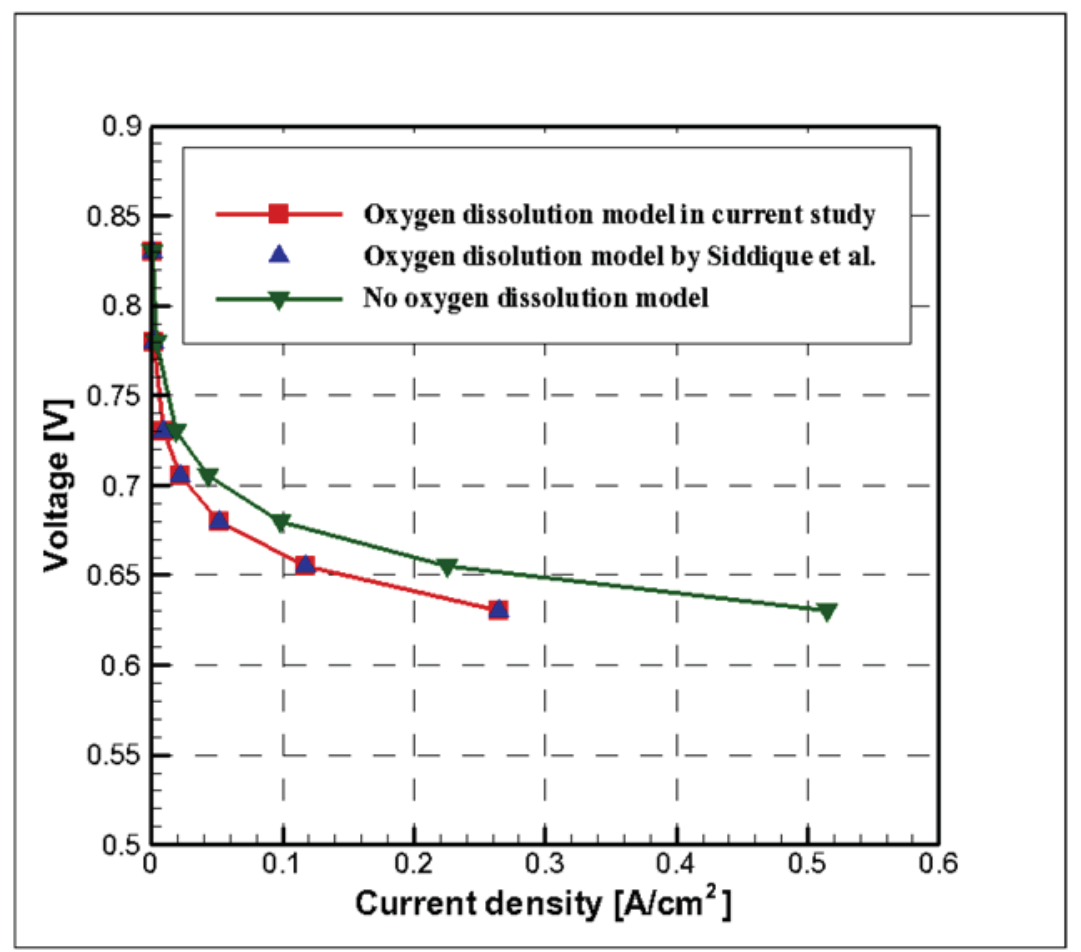

Figure 22: Effect of the description/inclusion of oxygen dissolution in the ionomer phase

Table 2: Tabulated Current Density and Overpotential for the three Dissolution Models

\begin{tabular}{|c|c|c|c|}
\hline $\begin{array}{c}\text { Overpotential } \\
{[\mathrm{V}]}\end{array}$ & $\begin{array}{c}\text { With oxygen dissolution } \\
\text { model in } \\
\text { current study }\left[\mathrm{A} / \mathrm{cm}^{2}\right]\end{array}$ & $\begin{array}{c}\text { With oxygen dissolution } \\
\text { model used by } \\
\text { Seddique et al. }\left[\mathrm{A} / \mathrm{cm}^{2}\right]\end{array}$ & $\begin{array}{c}\text { With no oxygen dissolution } \\
\text { model }\left[\mathrm{A} / \mathrm{cm}^{2}\right]\end{array}$ \\
\hline-0.4 & $3.5878 \times 10^{-4}$ & $3.5875 \times 10^{-4}$ & $6.8250 \times 10^{-4}$ \\
\hline-0.45 & $1.8813 \times 10^{-3}$ & $1.8809 \times 10^{-3}$ & $3.5792 \times 10^{-3}$ \\
\hline-0.50 & $9.8596 \times 10^{-3}$ & $9.8591 \times 10^{-3}$ & $1.8768 \times 10^{-2}$ \\
\hline-0.525 & $2.2554 \times 10^{-2}$ & $2.2549 \times 10^{-2}$ & $4.2973 \times 10^{-2}$ \\
\hline-0.55 & $5.1522 \times 10^{-2}$ & $5.1517 \times 10^{-2}$ & $9.8371 \times 10^{-2}$ \\
\hline-0.575 & $1.1732 \times 10^{-1}$ & $1.1726 \times 10^{-1}$ & $2.2506 \times 10^{-1}$ \\
\hline-0.6 & $2.6524 \times 10^{-1}$ & $2.6518 \times 10^{-1}$ & $5.1432 \times 10^{-1}$ \\
\hline
\end{tabular}

\subsubsection{Effect of Platinum Loading}

In this section, a set of CL micro-structures with different platinum loadings are generated and the micro-model was used to predict the performance curves and compare the results. Four different cases with $0.05,0.2,0.4$, and $0.6 \mathrm{mg} / \mathrm{cm}^{2}$ platinum loadings are considered here; also, a micro-structure of depth $1000 \mathrm{~nm}$ is considered for each of the cases. The objective is to keep all other parameters the same so that the only difference will be the platinum loading between all the cases, even the configuration of all carbon, platinum, and ionomer particles are kept the same. The CL micro-structure with the dimensions of $100 \mathrm{~nm} \times 100 \mathrm{~nm} \times 100 \mathrm{~nm}$ was reconstructed with platinum loading of $0.6 \mathrm{mg} / \mathrm{cm}^{2}$ for a $12 \mu \mathrm{m}$ thick catalyst layer sample. The same micro-structure was mirrored and attached in order to make thicker micro-structures. The 
number of platinum particles can be changed within this micro-structure in order to produce micro-structures with lower platinum loadings while having the same configuration of all particles. To do so, any number of the platinum particles can be treated as carbon particles so that the electronic percolation is maintained and the configuration and all other parameters are kept the same. The platinum particles to be added to the carbon region are randomly chosen within the micro-structure of dimensions $100 \mathrm{~nm} \times 100 \mathrm{~nm} \times 100 \mathrm{~nm}$. Then, the CL microstructure of depth $100 \mathrm{~nm}$, with new platinum loading, was mirrored and attached to make a 1000nm deep micro-structure.

Figure 23 shows the performance curves for the four cases with $0.05,0.2,0.4$, and $0.6 \mathrm{mg} / \mathrm{cm}^{2}$ platinum loadings. Higher platinum loadings result in larger surface area of the electrochemically active surfaces, the interface between platinum and ionomer regions, and consequently larger electrochemical reaction rates, currents $[A]$. So, the case with the highest platinum loading is predicted to have the highest current density $\left[\mathrm{A} / \mathrm{cm}^{2}\right]$ based on the nominal area of the micro-structure. Figure 23 shows the performance curves based on the total surface area of the electrochemically active surfaces, the interface between platinum and ionomer regions. As it is seen, the case with lowest platinum loading is predicted to have the highest current per unit area of the electrochemically active surfaces, $\left[A / \mathrm{cm}^{2} \mathrm{pt}\right]$. In other words, the $\mathrm{CL}$ with lower platinum loading is predicted to have higher current per platinum particles. This shows that the CL with a lower platinum loading is more effectively used. Table 3 shows the total current, $I_{m p t}$, as a function of the platinum loading, $m_{p t}$, for the four cases with different platinum loadings at overpotential, $n=0.6 \mathrm{~V}$.

The total surface area of the electrochemically active surfaces for each platinum loading, $S_{\mathrm{mpt}}$, are also included in Table 3. The ratio between $I_{m p t}$ and the ratio between $S_{\text {mpt }}$ for different platinum loadings are also provided. Comparing the last two columns shows that $I_{\mathrm{mpt}}$ did not increase as much as the $S_{\text {mpt }}$ with the platinum loading. This is the result of larger transport losses as larger fluxes are required. So, larger transport losses are expected in CLs with higher platinum loadings due to the effect of increasing thickness.

Table 3: Tabulated current produced for the four micro-structures

\begin{tabular}{|c|c|c|c|c|}
\hline $\begin{array}{c}\text { Platinum loading } \\
m_{p t}\left[\mathrm{mg} / \mathrm{cm}^{2}\right]\end{array}$ & $\begin{array}{c}\text { Total current } \\
i[A] \times 10^{-11}\end{array}$ & $\begin{array}{c}\text { Total area of electrochemically } \\
\text { active surfaces } S_{m_{p t}}\left[\mathrm{~cm}^{2}\right] \times 10^{-13}\end{array}$ & $\begin{array}{c}\text { Ratio of } \\
I_{m_{p t}} / I_{m_{p t}=0.05}\end{array}$ & $\begin{array}{c}\text { Ratio of } \\
S_{m_{p t}} / S_{m_{p t}=0.05}\end{array}$ \\
\hline 0.05 & 0.3993 & 0.0878 & 1.00 & 1.00 \\
\hline 0.2 & 1.8665 & 0.4507 & 4.67 & 5.13 \\
\hline 0.4 & 3.3685 & 0.8927 & 8.43 & 10.16 \\
\hline 0.6 & 4.7250 & 1.3565 & 11.83 & 15.44 \\
\hline
\end{tabular}



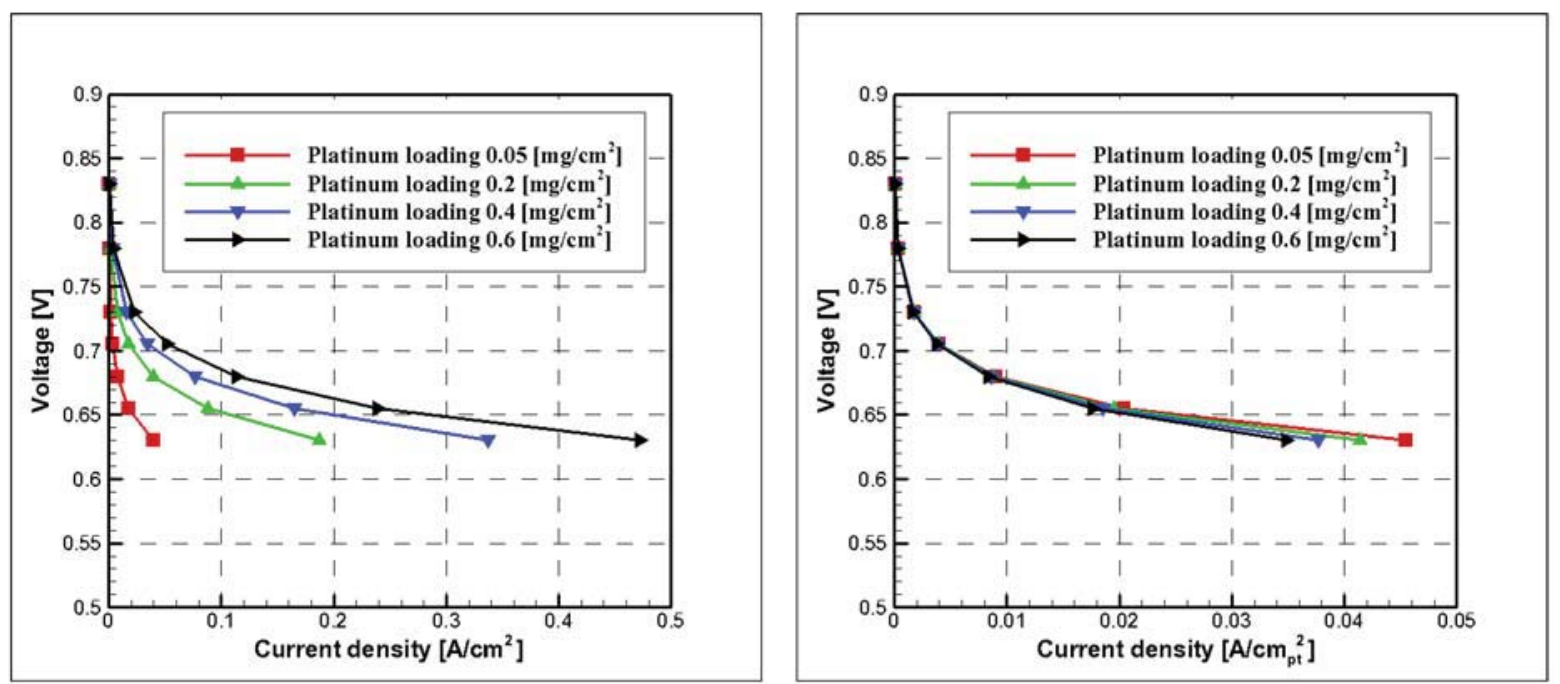

Figure 23: Polarization curves for the 4 different micro-structures with the current density on a geometric basis (left) and specific surface area (right)

The transport losses are related to both species and charge transport. The electronic transport loss is negligible in comparison to either of the protonic and species transport losses; this is due to high electronic conductivity. The electronic transport was neglected by some investigators and it was assumed that electrons are abundantly available to complete the electrochemical reaction; the same approach was followed in this work. The two significant transport losses are the oxygen and protonic transport losses. In order to compare the two losses, it is planned to assume one of the reactants, say oxygen, is abundantly available and use the micro-model without considering oxygen transport to predict the performance of the CL micro-structure by just solving for protonic transport. In this case, the oxygen concentration is uniform within the ionomer region. The same approach can be repeated but the other reactant, proton, is assumed to be abundantly available and use the micro-model to predict the performance curve by just solving for oxygen transport. In this case, the protonic potential will be uniform. Two performance curves are produced from the two cases of uniform oxygen and uniform protonic potential; two other performance curves can be produced from the fully coupled, Figure 24 and pure kinetics cases which solely considers Butler-Volmer equation.

Four performance curves will be produced for each micro-structure with different platinum loading:

1. The performance curve from fully coupled model of species and charge transport.

2. The performance curve from uniform protonic potential model, protons abundantly available.

3. The performance curve from uniform oxygen concentration model, oxygen is abundantly available.

4. The performance curve from pure kinetics model.

Figure 24 illustrates the four above-mentioned performance curves for the four micro-structures of different platinum loadings, $0.05,0.2,0.4$, and $0.6 \mathrm{mg} / \mathrm{cm}^{2} \mathrm{pt}$. As it is seen for all the platinum loadings, the performance curve from the uniform protonic potential model is far from the fully coupled model and closer to the pure kinetics while the performance curve from uniform oxygen is close to the fully coupled model and farther from the pure kinetics. These show the changes 
in protonic potential have a large influence on the performance curve. This is due to the functionality between current density, oxygen concentration, and protonic potential in ButlerVolmer equation. The protonic potential is exponentially related to the current density while oxygen concentration is linearly related to the current density. So, an infinitesimal fluctuation in protonic potential will change the current density more than the same fluctuation in oxygen concentration. The effects of the changes in protonic potential on the performance curve is not always the same as the platinum loading is decreased. The differences between the four performance curves for the case with platinum loading of $0.05 \mathrm{mg} / \mathrm{cm}^{2}$ are small in comparison to the case of platinum loading $0.6 \mathrm{mg} / \mathrm{cm}^{2}$.
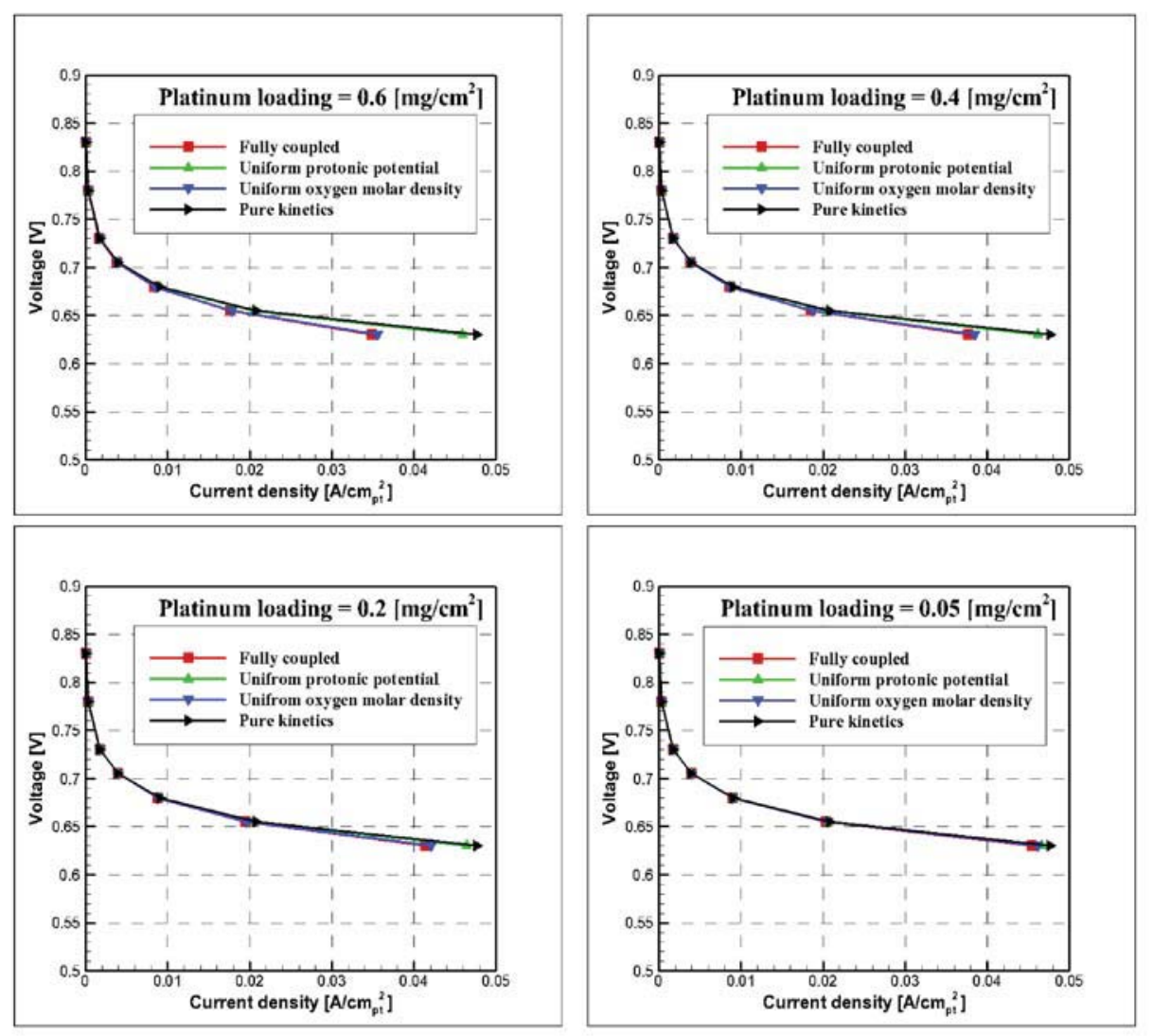

Figure 24: Comparison of the role of protonic transport, reactant diffusion, and base kinetics as function of the path length of the domain (a function of loading)

The oxygen transport through different parts of such as gas channel, porous transport layer and CL was studied by several investigators. Nonoyama et al. investigated oxygen diffusive transport experimentally. They broke down the total oxygen transport resistance into three components:

1. The oxygen diffusive resistance within diffusion media including porous transport layer and micro-porous layer, RDM.

2. The oxygen diffusive resistance within $C L$ pore region, $R_{C L ; g a s}$.

3. The oxygen diffusive resistance within $\mathrm{CL}$ ionomer region, $\mathrm{R}_{\mathrm{CL} ; i o n}$. 
The performance is only dependent on oxygen transport, so they investigated the effect of platinum loading on each of the above-mentioned resistances by running at limiting current density in order to isolate oxygen transport for their measurements. It can be seen that the oxygen diffusive transport within $\mathrm{CL}$ varies inversely with platinum loading while the oxygen diffusive resistance within diffusion media stays constant with platinum loading. Furthermore, they showed in their experiments that the major part of the total diffusive resistance is due to

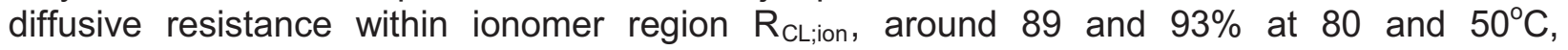
respectively. They explained the rapid increase in oxygen diffusive transport resistance within ionomer region at low platinum loadings to be related to the decrease of effective ionomer surface area for oxygen permeation to reach platinum surfaces. This effective ionomer surface is thought to be related to the electrochemically active surface area of the platinum particles and decreases with platinum surface area.

In another experimental investigation by Greszler et al., the oxygen transport resistance was investigated at different platinum loadings. They also used the limiting current density technique. They showed that the oxygen partial pressure drop from the electrode to the platinum surface varies inversely with roughness factor which is the ratio between the area of electrochemically active reaction surfaces and the nominal surface area of the CL. The measurements were done at the same current density (i.e. $1.5 \mathrm{~A} / \mathrm{cm}^{2}$ ), geometric surface area of the cell, and varied the electrode roughness factor which is related to platinum loading. The increase in resistance requires larger gradients at the same current density. With the same oxygen partial pressure, oxygen concentration, within the electrode, larger gradients result in smaller oxygen partial pressure, oxygen concentration, at platinum surfaces. In fact, Figure 25 tells the same story about rapid increase in oxygen transport resistance at low platinum loadings.

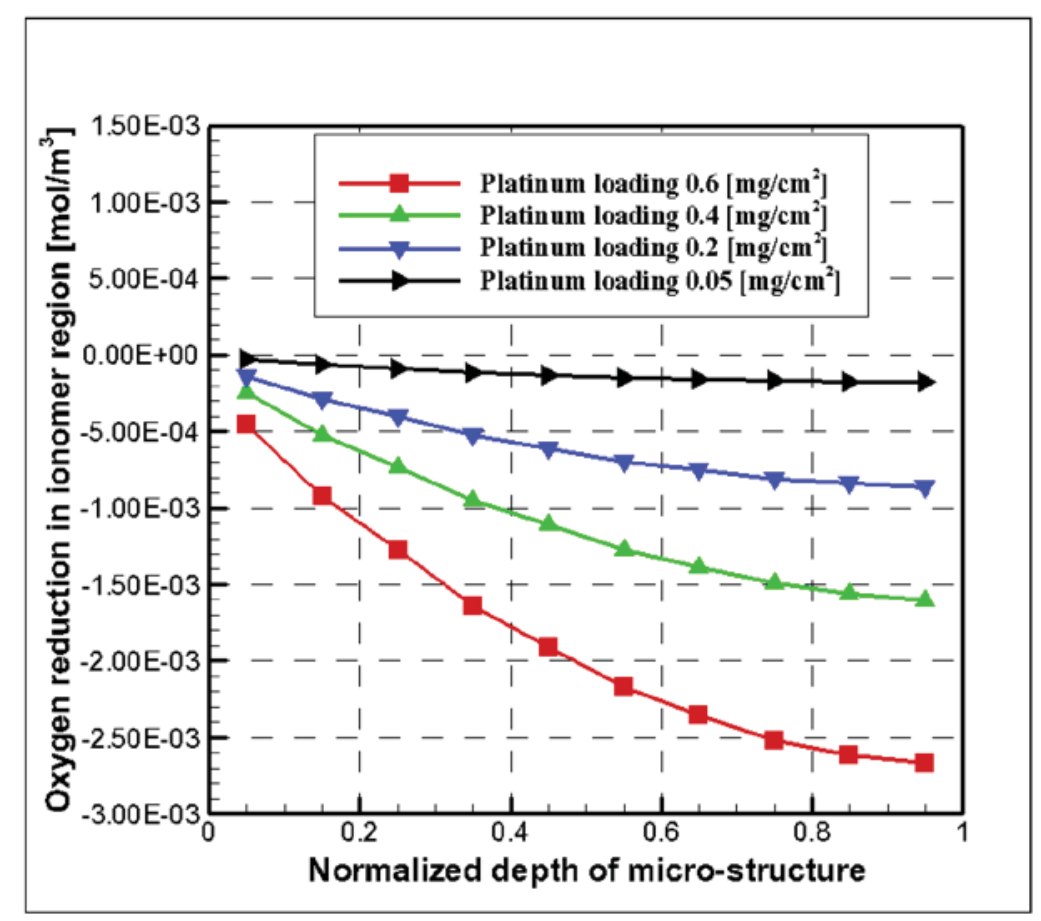

Figure 25: Decrease in the local oxygen as a function of the micro-structure depth based on different platinum loadings 
These investigations in literature also calculated an equivalent resistance of $35 \mathrm{~nm}$ for the ionomer film on the surface of platinum particles based on the resistance they observed at low platinum loading in their experiments, while the possible thickness of ionomer film in their CL is estimated to actually be between 2 and $10 \mathrm{~nm}$. They mentioned the source of dramatic increase in oxygen diffusive transport resistance at low platinum loadings being of unknown origin and mathematically is similar to a thin, resistive film covering the platinum surfaces.

Figure 26 shows that the oxygen transport resistance within the ionomer region dramatically increases at low platinum loadings as the inverse of shape factor for the case with platinum loading of $0.05 \mathrm{mg} / \mathrm{cm}^{2}$ is almost 16 times larger than the one for the case with platinum loading of $0.6 \mathrm{mg} / \mathrm{cm}^{2}$ while the inverse of shape factor does not change much from platinum loadings $0.6 \mathrm{mg} / \mathrm{cm}^{2}$ to $0.4 \mathrm{mg} / \mathrm{cm}^{2}$. This is due to the configuration of the ionomer and platinum particles and also the areas of the transport interfaces, pore/ionomer and platinum/ionomer interfaces. The shape factor study reveals that the rapid increase in the oxygen diffusive transport resistance is due to the increased length of species pathways and the decreased surface area of the interface between the platinum and the ionomer regions as the platinum loading is reduced within the complicated ionomer geometry.

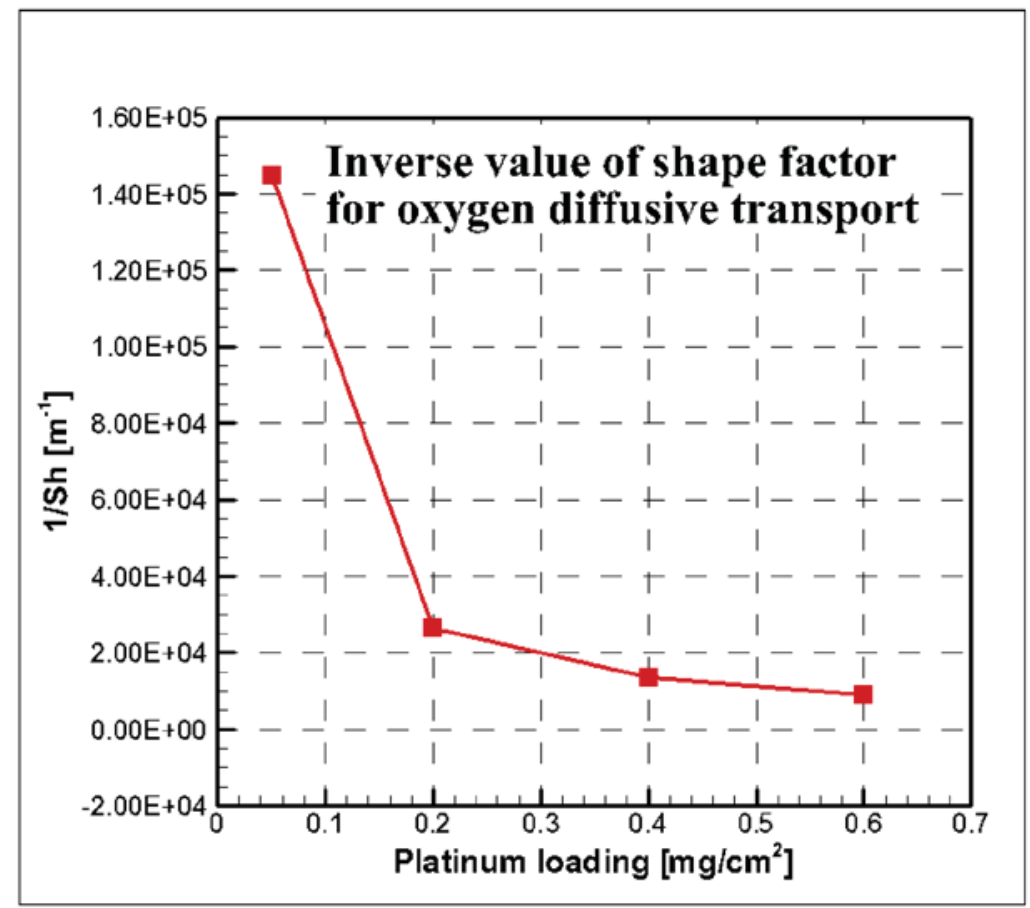

Figure 26: The shape factor of the oxygen diffusive transport around the ionomer particles as a function of platinum loading

\subsubsection{Capillary Pressure in Micro-structural Catalyst Layers}

Numerical analysis of the capillary pressure of the catalyst layers was completed using a NWP (non-wetting pore) analysis algorithm. This method returns the typical pressure vs. saturation relationship that is used by macro-level cell models. Figure 27 shows the general algorithm for this technique. 
DE-EE0000466

Ballard Material Products Inc
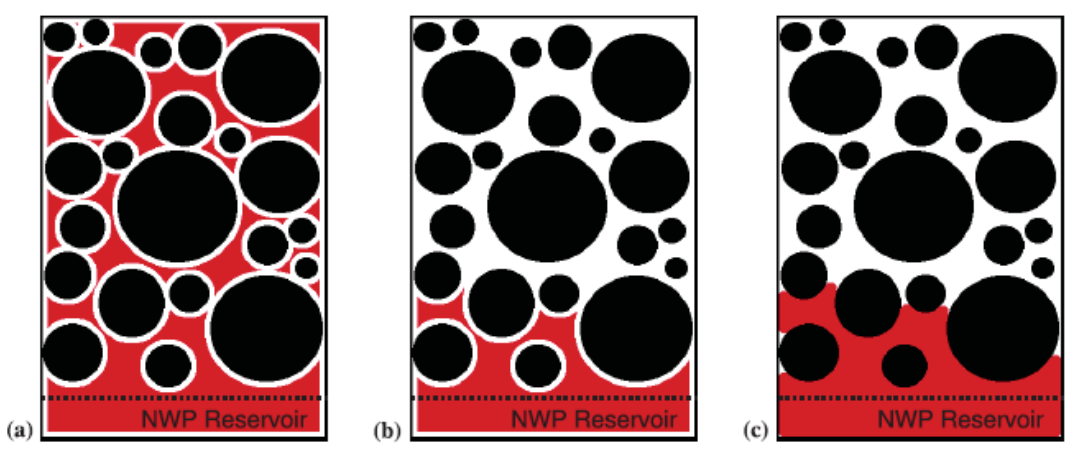

Figure 27: Non-wetting pore algorithm for determination of the capillary pressure, (a) the erosion, (b) the component of the erosion that is connecting to the NWP reservoir, (c) NWP-filled portion

The morphological approach to generating capillary pressure curves has been implemented within the three-dimensional catalyst morphology. On a high-level, the method 'erodes' the pore space of a porous morphology by a given amount which is equivalent to dilating the solid portion of the domain by that length scale. At each level of erosion (up to full saturation), the remaining percolating pore space is used to determine the saturation level at that length scale, and the Young Laplace equation is then applied to determine the capillary pressure for the chosen parameters. At the moment, the implementation assumes that the ionomer is already fully saturated with liquid water such that there is no uptake, and that there is a single contact angle in the domain. The erosion is carried out using a distance transform (in 3D) via the application of a Manhattan distance map.
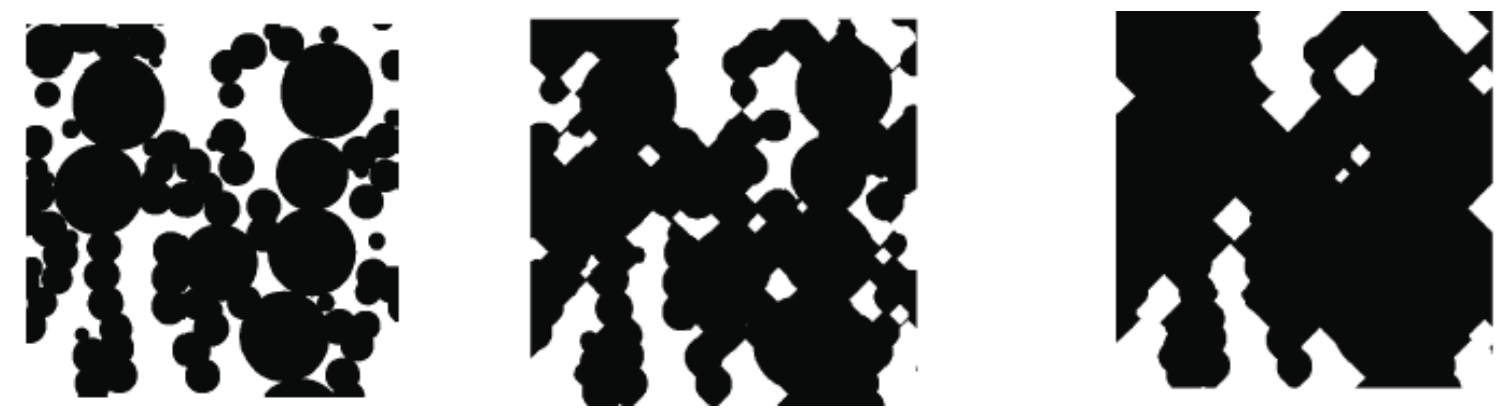

Figure 28: Sample slices at left: original geometry; centre: $67 \%$ pore space remaining; right: $15 \%$ pore space remaining

In Figure 28, a depiction of a slice from a 3D catalyst micro-structure and two levels of erosion of the pore space are shown; while Figure 29 shows the predicted capillary pressure assuming two different length scales for the catalyst. As expected, a very large dependency on the length scale is observed and the predicted capillary pressures are extremely high - probably quite a bit higher than experimental determinations. As mentioned previously, these results are achieved by assuming a constant contact angle (which is physically not the case in the real morphology) and in future the model will be extended to include the effect of varying contact angle. 

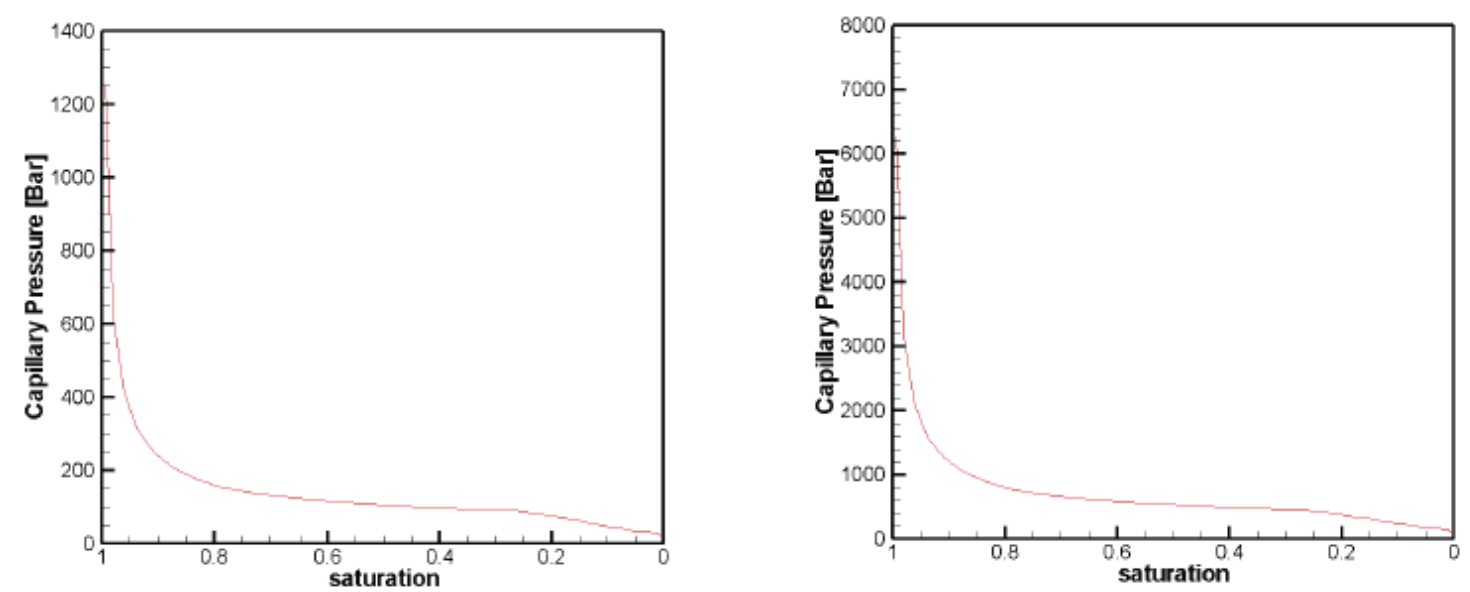

Figure 29: Capillary pressure curves generated from the Young-Laplace equation at two different length scales. Left: assuming a voxel size of $1 \mathrm{~nm}$; Right: assuming a voxel size of 20 Angstroms

The water placement algorithms using morphology have been significantly improved, first to include a Euclidean distance option (previous versions exclusively used Manhattan distance) that is believed to provide a placement closer to the desired physical situation.

Figure 30 compares two distance maps for the case of a voxel map assuming a $0.2 \mathrm{~nm}$ voxel size, a surface tension of $6.2 \times 10-2 \mathrm{~N} / \mathrm{m} 2$ and a contact angle of 180 degrees. While the differences appear small on this scale, they are not insignificant, and are largest at the higher saturations. The Euclidean distance is a more physical measure in this context as it is the normal straight line distance from the solid surface, whereas the Manhattan distance is akin to taking a piecewise linear route through city blocks.

In addition, a refinement was made to the algorithm for computing saturation distributions. In the previous results, the order of operations was: i) erosion, ii) dilation, iii) percolation identification; while in the new algorithm, steps ii) and iii) have been swapped such that it is now (New Algorithm) i) erosion, ii) percolation identification, iii) dilation. This change makes physical sense, as the percolation identification should occur when the pores of the desired length scale are blocked by the erosion algorithm. Figure 31 compares results on both a cubic and an elongated geometry using these two algorithms. Not surprisingly, the new algorithm predicts slightly higher capillary pressures for the same saturation. More interestingly however, is the large difference between a cubic and an elongated geometry, the latter of which requires substantially higher capillary pressures to achieve an initial saturation. 
DE-EE0000466

Ballard Material Products Inc

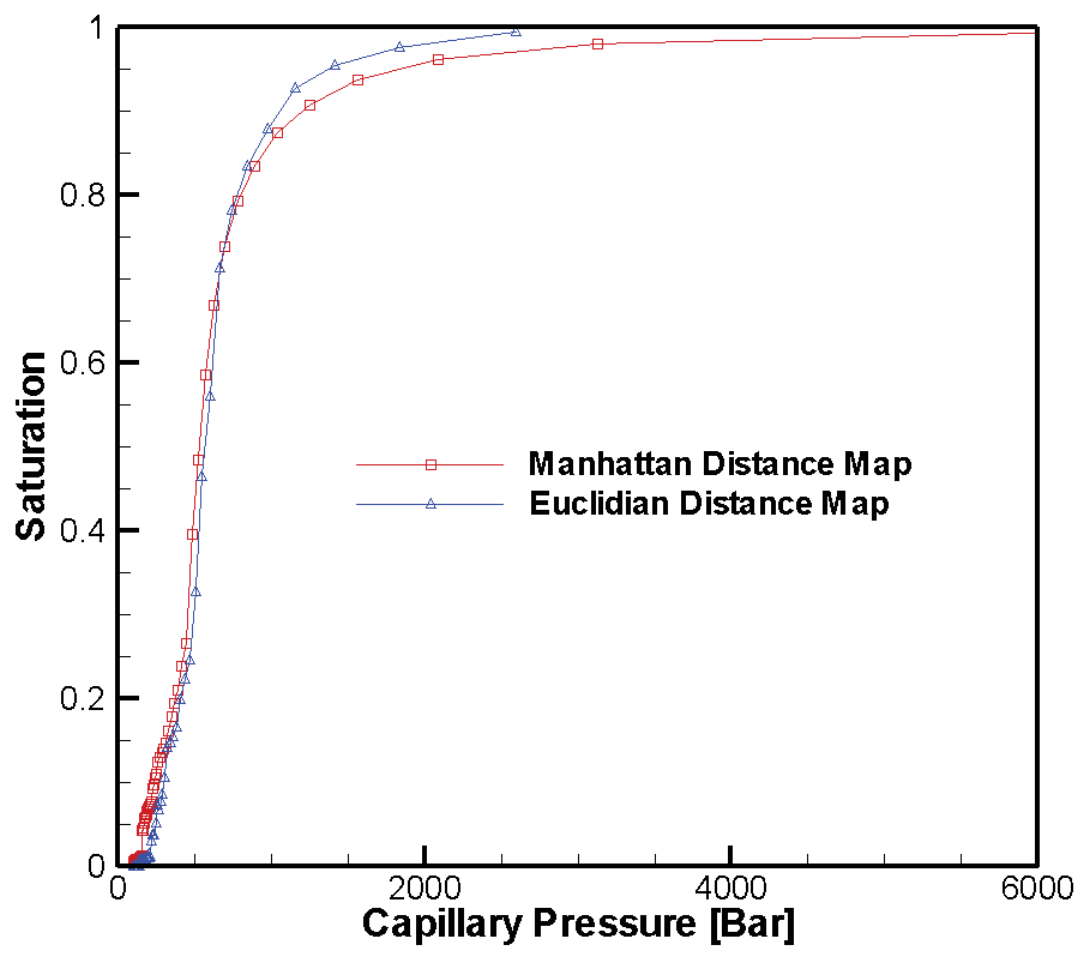

Figure 30: Capillary pressure saturation curves predicted with a Euclidean and a Manhattan distance maps
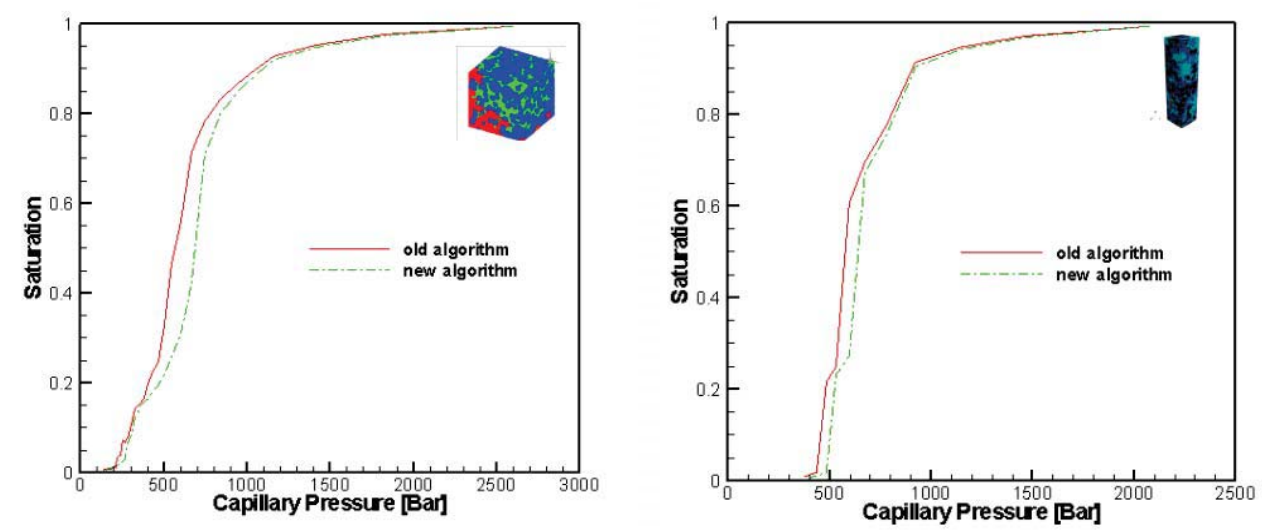

Figure 31: Capillary pressure saturation curves predicted using the old and new algorithms applied to cubic geometries (left) and elongated geometries (right) 

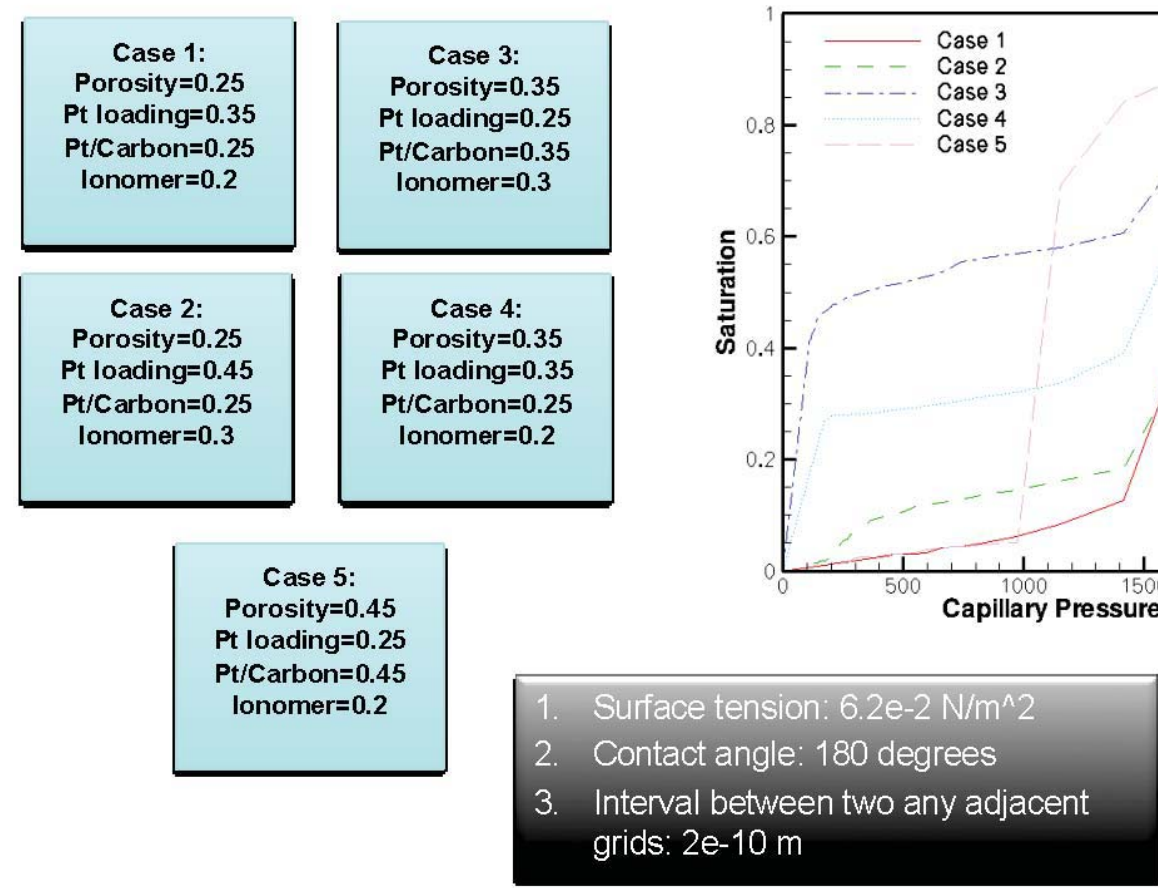

Figure 32: Capillary pressure saturation curves predicted using the new algorithms applied to various catalyst geometries

Figure 32 presents part of a parametric study of capillary pressure-saturation behaviour as a function of compositional catalyst parameters. It is clear that there is a very large variation of the capillary pressure dependent on the catalyst parameters.

For the broader parametric study the capillary pressure was investigated as a function of the catalyst compositional parameters of loading, ionomer content, and platinum/carbon ratio. It should be noted that the input parameters were moved parametrically rather than in response to a specific combination that mimicked physical catalyst combination. The parameters were varied within the following ranges:

- $\quad$ platinum loading, $0.25-0.45 \mathrm{mg} / \mathrm{cm}^{2}$

- ionomer mass ratio, $20-40 \%$

- $\quad$ latinum/carbon ratio, $25-50 \%$

A comparison along the test case showed the model prediction for capillary pressure change as a function of loading results in a shift of the capillary pressure to the left as the loading increased. 

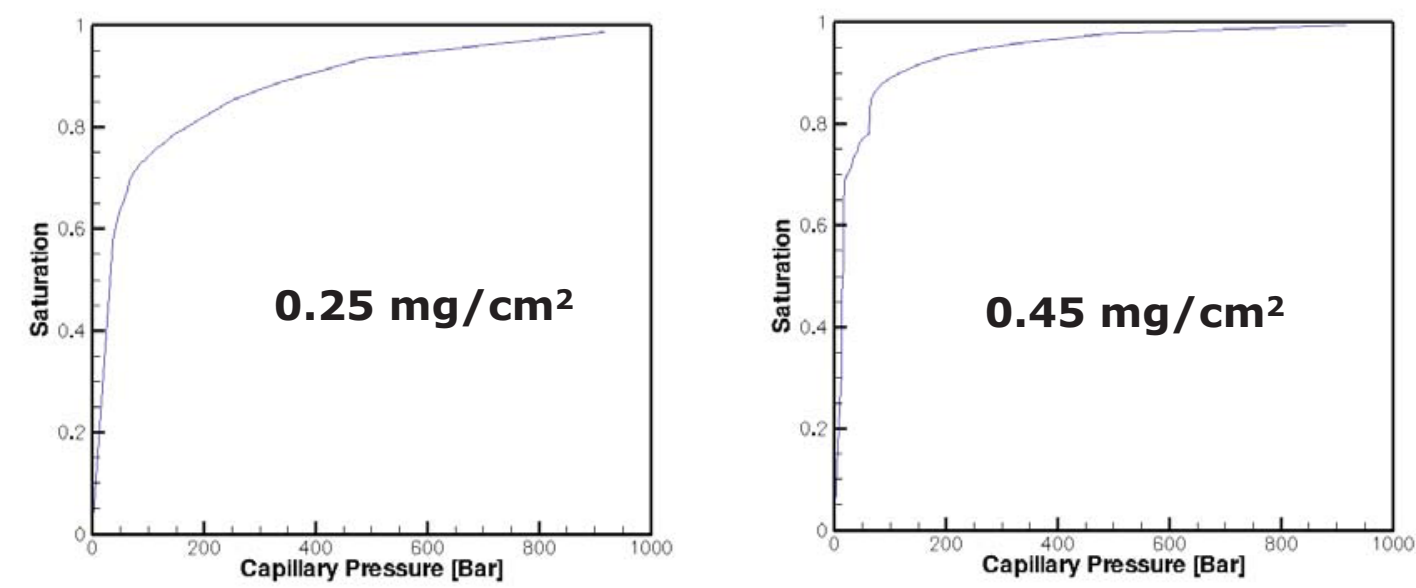

Figure 33: Capillary pressure generated from the micro-structural model

\subsubsection{Transient Cycling of a Catalyst Micro-structure}

A series of micro-structure representations with liquid water were created and sent through simulated AST cycling. Representations of these structures are seen in Figure 34.

Figure 35 shows the current predicted from the unsteady performance code at two different temporal resolutions. It is clear from the figure that a two-second time step is too large, and also that for the chosen parameters the bulk of the system responds to the transient on a time scale much quicker than the applied voltage cycle such that the bulk of the domain has reached the steady solution at each applied voltage. This will be the case when no liquid water is present; however it is likely to be very different in the case of saturated samples. This behaviour has been explored in this project but it must be noted that presently no models exist that predict the water transport in a catalyst layer reliably and as such developing a meaningful approximation and appropriate transport schemes has remained a challenge. The structures created with liquid water are then simulation cycled via the square wave voltage profile. 

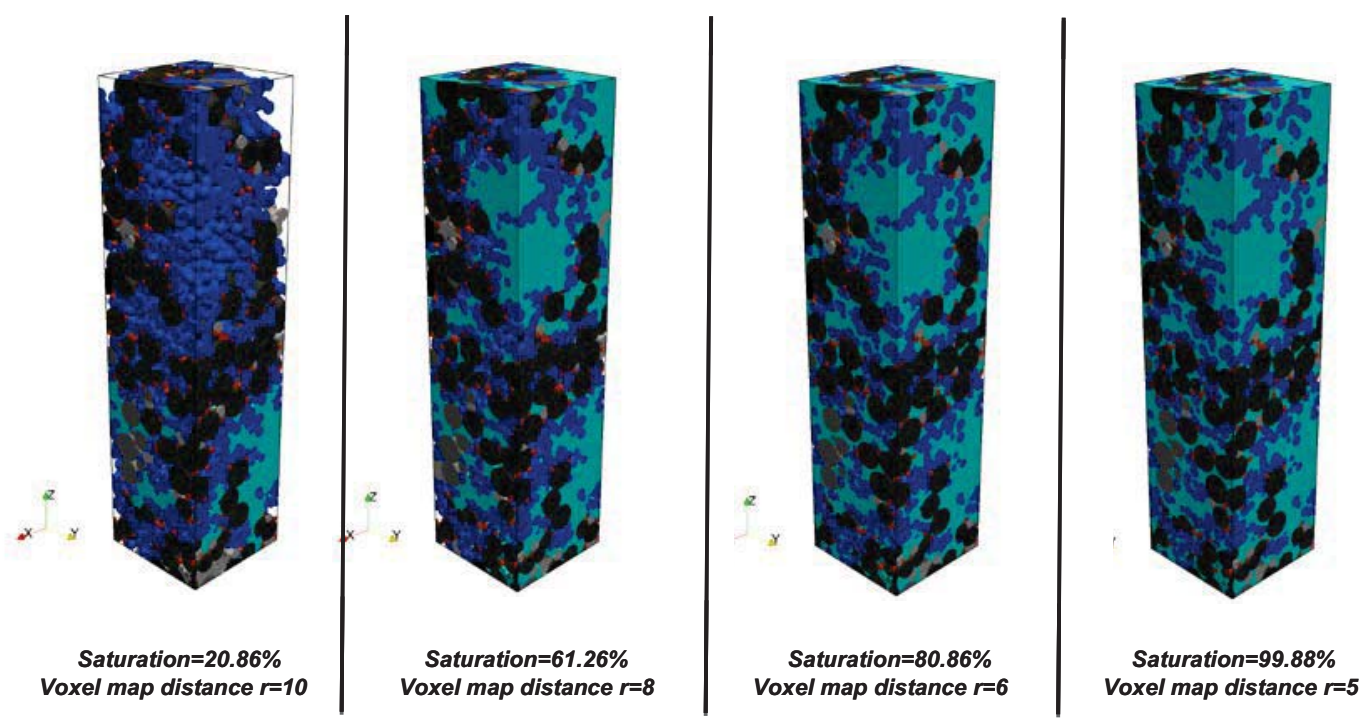

Figure 34: Catalyst layer micro-structures at various saturation levels for AST cycling

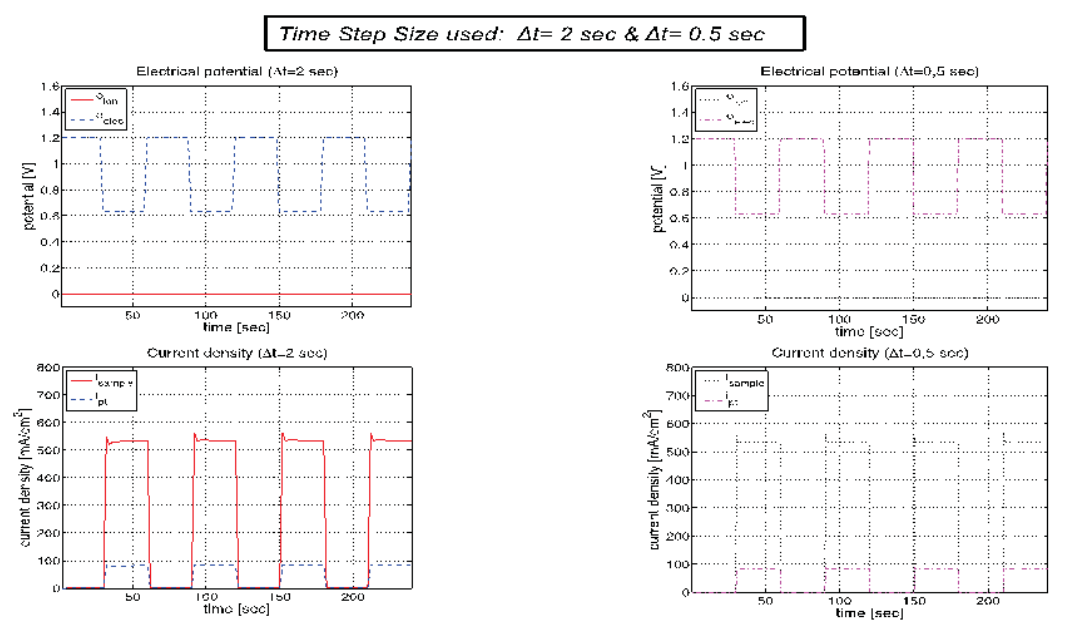

Figure 35: The current response to a 30 s square wave voltage cycle at two different temporal resolutions

Figure 36 depicts the time variation of the oxygen concentration at one location in the ionomer region and at one location in the pore region during the simulation. Evident in the simulation is the initial transient as the simulation adjusts to the initial condition, and then a periodic region where the concentrations are varying in response to the voltage cycle. Again, it is clear that a $2 s$ time step is too large and that the properly resolved system responds much more quickly than the frequency of the voltage cycling. It is also clear that for these conditions the variations are very small. This once again points to the importance of treating liquid water in these 
DE-EE0000466

Ballard Material Products Inc

domains which is important for the degradation mechanisms and which also can significantly impact the predicted concentrations and hence performance.

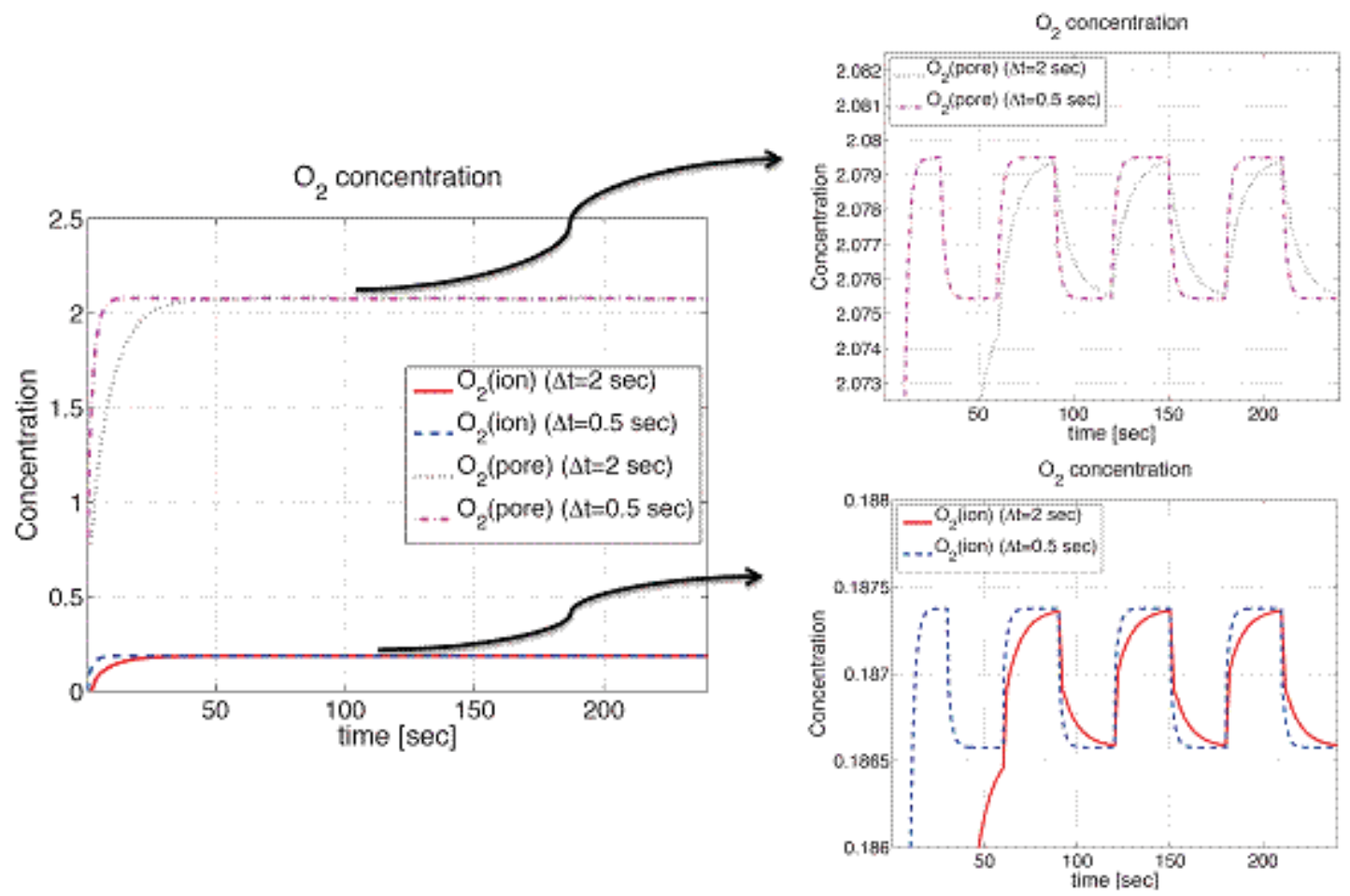

Figure 36: A time series of the oxygen concentrations at one point in the ionomer and at one point in the pore during voltage cycling. 


\section{DE-EE0000466}

Final Project Report

Development of Micro-Structural Mitigation Strategies for PEM Fuel Cells: Morphological Simulations and Experimental Approaches

\section{CHAPTER VII}

\section{FCPEM PERFORMANCE AND DURABILITY MODEL}

Multi-Path ORR Model

PI: Kunal Karan

Queen's University

Barath Jayasankar, PhD Candidate 


\section{TABLE OF CONTENTS}

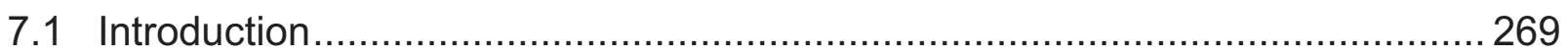

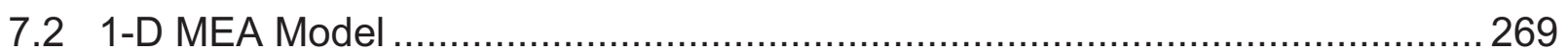

7.2.1 Non-statistical BOT Performance Model .......................................... 269

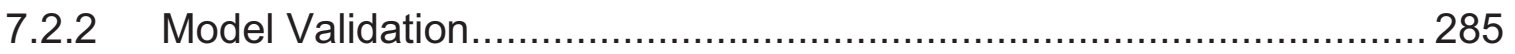

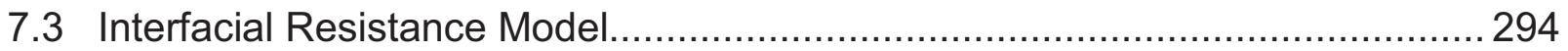

7.4 Statistical BOT Performance Model............................................................ 296

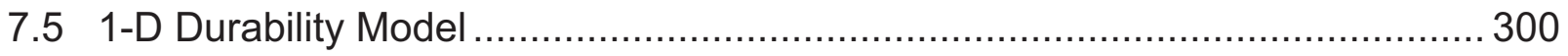

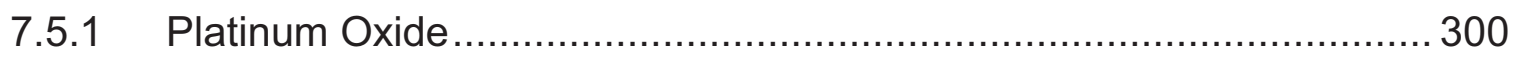

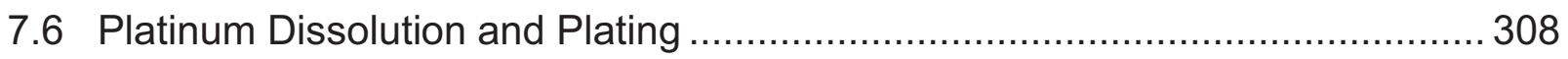

7.7 Carbon Surface Oxidation and Carbon Corrosion ......................................... 310 


\section{LIST OF FIGURES}

Figure 1: Representation of the 1-D Domain and Agglomerate Description .......................270

Figure 2: Comparison of the effect of Agglomerate vs. Discrete Morphology Formulations...272

Figure 3: Potential Nomenclature and Activation Overpotential ........................................276

Figure 4: Permeability of the PTL predicted by microstructural simulations........................280

Figure 5: Thermal conductivity predicted by the microstructural models for the PTL .............282

Figure 6: Parametric Study on the Effect of Permeability on the Liquid Water Model ............285

Figure 7: Schematic of the coupling between the catalyst layer composition and the appropriate terms in the governing equations ..............................................28

Figure 8: Comparison of the model predictions without the liquid water model and using the base parameter set.

Figure 9: Comparison of the model predictions without liquid water and using adjusted agglomerate parameters.

Figure 10: Validation of the two-phase model with ionomer composition (a) $23 \%$ (b) $30 \%$ (c) $38 \%$ by weight. .288

Figure 11: Measured Proton Conductivity of the Catalyst Layer vs. $\mathrm{RH}$ as a function of Catalyst Layer lonomer Content .... .289

Figure 12: Predicted Catalyst Layer Protonic Conductivity based on the Springer Relationship 290

Figure 13: Predicted effect of the $\mathrm{RH}$ change and comparison to the cell polarization data for the same conditions.

Figure 14: Model Validation for a platinum loading of $0.408\left[\mathrm{mg} / \mathrm{cm}^{2}\right]$

Figure 15: Model Validation for a platinum loading of $0.19\left[\mathrm{mg} / \mathrm{cm}^{2}\right]$...........

Figure 16: Model Validation for a platinum loading of $0.05\left[\mathrm{mg} / \mathrm{cm}^{2}\right]$

Figure 17: Relative saturation levels between a high loaded and low loaded catalyst layer. ...293

Figure 18: Schematic Methodology for the Contact Resistance Model ................................294

Figure 19: Surface roughness maps measured on the Catalyst Layer and PTL...................294

Figure 20: Predicted and Experimental Contact Resistance vs. Compression .......................295

Figure 21: Methodology for the modification of the model into a statistically based code......296

Figure 22: Statistical test variation for variable sensitivity. .........................................297

Figure 23: Statistical variation of platinum loading for 75 repeats.................................297

Figure 24: Statistical variation in ECSA as a result of platinum loading variation over 75 repreats.

Figure 25: Statistical model validation using the single phase module . ............................298

Figure 26: Statistical model validation using the two-phase module.

Figure 27: ORR "Dual Pathway" Model as published by Wang et al. 300

Figure 28: Initial attempt to use the double pathway ORR model to simulate the Cyclic Voltammogram for an MEA.

Figure 29: Comparison of the Dual Pathway model to experimental EQCM data for oxide

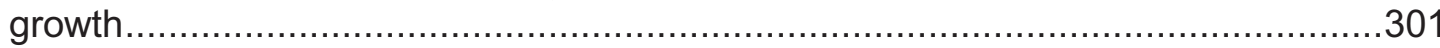

Figure 30: Oxide Coverage predictions using the Dual Pathway model for the ORR. ............302

Figure 31: Modified Dual Pathway model fit to RDE data.

Figure 32: CV predicted from the Modified Dual Pathway model using RDE fit parameters at low oxygen concentration

Figure 33: Coverage response to step changes in potential from $0.43 \mathrm{~V}$ to a UPL, similar to

Figure 34: Parametric analysis for the oxide formation peak using the Modified Dual Pathway model ..... 
Figure 35: Parametric analysis for the oxide reduction peak using the Modified Dual

Pathway model .305

Figure 36: Effect of the active site correction factor based on the surface species.................306

Figure 37: Comparison of the EQCM predictions based on the model reformulation to include a description of the available active sites.

Figure 38: Comparison of Modified Dual Pathway Model with experimental CV data.............307

Figure 39: Effect of proton concentration variation on the predicted location and magnitude of the lower CV peak.

Figure 40: Multi-step ORR pathway with the addition of platinum dissolution.........................308

Figure 41: Initial results for the platinum dissolution AST simulations, based on a wave profile from $0.6-1.1 \mathrm{~V}$ and $\sim 500$ cycles.

Figure 42: Model validation based on the prediction of the per cent loss of carbon surface area versus the per cent loss of carbon as measured in the gas exhaust

\section{LIST OF TABLES}

Table 1: RDE analysis for the exchange current density based on a $60 \mathrm{mV} / \mathrm{dec}$ tafel slope ...273 
DE-EE0000466

Ballard Material Products Inc

\subsection{Introduction}

\subsection{1-D MEA Model}

The purpose of the model development was to construct a numerical representation of the MEAs described and tested in CHAPTER II. This model needed to capture the relevant phenomena at beginning of test and then accommodate the addition of degradation mechanisms such that the performance change over the course of an accelerated stress test (AST) could be described. The model was developed to represent a standard test cell hardware developed at Ballard Power Systems. This hardware was designed to result in uniform test conditions across the active area of the MEA, though there would exist distributions of reactants and products through the thickness. As such the following assumptions are made and remain consistent across each of the following sections, which describe the model development:

\section{Assumption:}

- Negligible gradients exist along the length of the cell in:

○ Potential (Electronic, Protonic)

- Liquid water saturation

- Reactant/Product Concentrations

- Temperature

- Pressure

- Channel Flows are sufficiently fast such that liquid water does not accumulate within the channels implying that the:

- Water removal rate is infinite at the PTL/Channel interface

- Polymer Membrane is impermeable, such that:

- Gas-crossover is negligible

- Convective flow is negligible

\subsubsection{Non-statistical BOT Performance Model}

The initial derivation of the model was done in a deterministic manner, such that for each set of inputs there would result a unique but repeatable performance result. The developed model included both the polymeric membrane and anode/cathode components, with the onedimensional approximation lying directly under the channel, as seen in Figure 1. 
DE-EE0000466

Ballard Material Products Inc

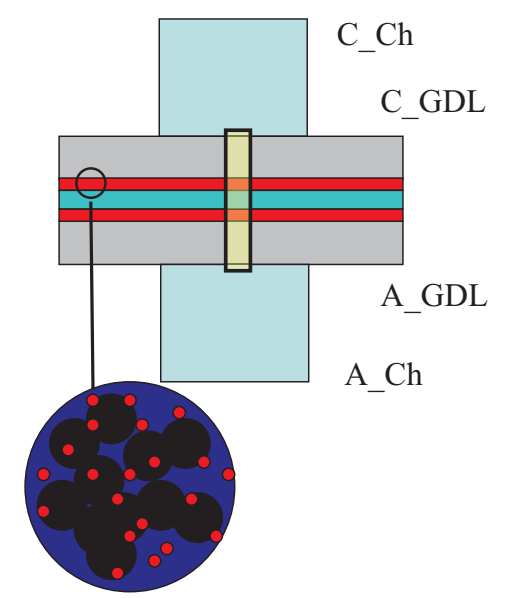

Figure 1: Representation of the 1-D Domain and Agglomerate Description

This configuration prompted an additional assumption for the model development:

\section{Assumption:}

- Negligible gradients between landing and channel regions in

- Potential (Electric, Protonic)

- Liquid water fraction

- Reactant/Product Concentrations

- Temperature

- Pressure

On the one-dimensional level, the model included a description for the following transport processes:

1. Electrons

2. Protons

3. Mass (re-cast for Pressure drop)

4. Species (Hydrogen, Oxygen, Nitrogen, and Water Vapour)

5. Energy

6. Liquid water (saturation)

The transport equations that relate to (1) - (6) will be described on a component by component basis.

\section{Electron Transport}

Anode Porous Transport Layer (aPTL):

The electron transport in the anode GDL was modelled to follow Ohm's law such that the transport equation took the following form:

$$
C_{D}\left(\frac{\partial \phi_{e}}{\partial t}\right)+\nabla \cdot\left(\sigma_{e}^{e f f} \nabla \phi_{e}\right)=0
$$

Where $C_{d}$ is the double layer capacitance and was set to zero for the anode PTL. 
$\sigma_{e}{ }^{\text {eff }}$ is the effective electrical conductivity of the porous layer and is generally described through the use of an effective medium theory relationship that typically takes the following form:

$$
\sigma_{e}^{e f f}=\sigma_{e}\left(v_{f}^{e}\right) \tau^{e}
$$

Where, $\sigma_{e}$ is the conductivity of the base material the comprises the porous layer, $v_{f}^{e}$ is the volume fraction, and $\mathrm{T}^{\mathrm{e}}$ is the tortuosity of the electron conducting material, respectively.

\section{Anode Catalyst Layer (aCL):}

The electron transport within the anode catalyst was governed by the same transport equation used for the aPTL. However, in the case of the anode catalyst layer the double layer capacitance, $\mathrm{C}_{\mathrm{d}}$, was ascribed a value derived from the measurement of double layer capacitance in the experimental set-up. The application of effective medium theory within the $\mathrm{aCL}$ to describe the effective electrical conductivity was modified to reflect the transport of electrons through the metallic and carbon phases and their resulting tortuosity. It should be noted that the determination of the appropriate volume fraction was based on a catalyst material formulation/volume packing method that is discussed later. Within the anode catalyst layer a source of electrons exists derived from the appropriate electrochemical reaction process. This process is governed by the Tafel-Herovsky-Volmer Multi-step mechanism to describe Hydrogen Oxidation Reduction Kinetics. As such the governing equation within the aCL takes the following form:

$$
C_{D}\left(\frac{\partial \phi_{e}}{\partial t}\right)+\nabla \cdot\left(\sigma_{e}^{e f f} \nabla \phi_{e}\right)=S_{c u r r e n t}
$$

Where $S_{\text {current }}$ represents the source of electrons generated from the electrochemical reactions and the appropriate descriptions for these kinetics can be found further in the report.

\section{Cathode Catalyst Layer (cCL):}

The electron transport within the anode catalyst was governed by the same transport equation used for the $\mathrm{aCL}$. The double layer capacitance, conducting volume fraction, and phase tortuosity were determined in a similar manner to that carried out within the aCL. With respect to the electrochemical reaction, two different catalyst layer descriptions were investigated in describing the layer morphology, while again two different descriptions were investigated in describing the electrochemical kinetic process. For the morphological description, there are typically two standard approaches for describing the layer: the discrete model and the agglomerate model. Figure 1 shows a typical representation of an agglomerate model less the exterior film thickness (the existence of the lonomer within the agglomerate and the film and its associated thickness tend to be points of differing opinion within literature). The agglomerate model includes the effect of additional resistances due to simultaneous reaction/diffusion in the core of the agglomerate, resulting in the existence of a utilization factor in most models which generally points to an underutilized core. The discrete model is one in which the layer has a finite thickness (as does the agglomerate model) such that bulk transport resistances and distributions through the thickness of the electrode are accounted for. Based on analysis completed in the experimental section of the report the agglomerate sizes were considered to be smaller than that usually identified arbitrarily through SEM style images of the catalyst layer (in part this may also reflect improvements made in electrode preparation since the original analysis was published). As such the overall transport resistance at higher currents seen in 
most agglomerate models does not exist in this case, that notwithstanding Figure 2 clearly shows an influence of moving between the discrete and agglomerate models such that, at least in part, the mass transport losses seen at high current density is partially explained from a morphology standpoint. It should be noted that one of the previous (and potentially ongoing) failings of the existing agglomerate models is the result of sufficient free parameters such that the concentration loss related effects at higher loads can be completely described by adjusting the morphological parameters. In the case of this model, however, the parameters were fixed based on what was observed experimentally for the agglomerate size and the ionomeric film thickness was removed as a free parameter by linking it to the layer formulation.

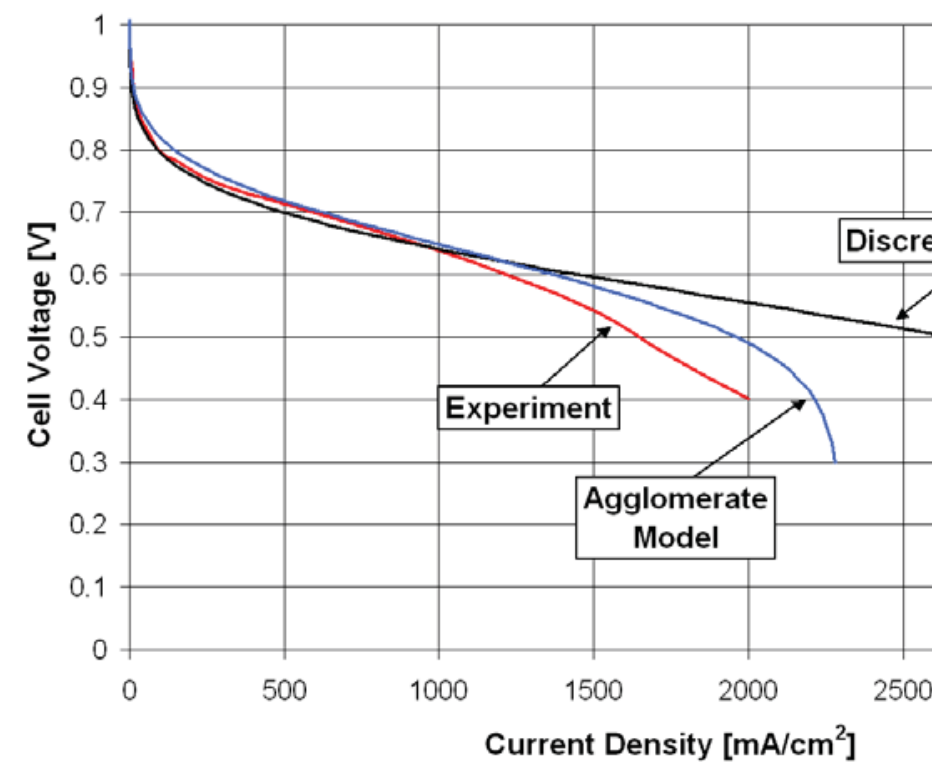

Figure 2: Comparison of the effect of Agglomerate vs. Discrete Morphology Formulations

With respect to the kinetic description, three approaches were considered:

1. Butler-Volmer, two-electron rate determining step

2. Multi-step pathway, double trap oxide formation kinetics

3. Revised multi-step pathway, modified reverse reaction kinetics

At this point the description of the Butler-Volmer will be discussed and the Dual pathway implementations will be discussed in the section related to the formation of platinum oxide.

For the purpose of both the agglomerate and discrete catalyst layer morphology formulations, the same Butler-Volmer formulation was used.

$$
i=i_{o, \text { geom }}^{(T, C, S)}\left(\exp \left(\frac{n(1-\beta) F}{R T} \eta_{a c t}\right)-\exp \left(\frac{-n \beta F}{R T} \eta_{a c t}\right)\right)
$$

Where, $\mathrm{n}$ is the number of electrons in the rate determining step, $\beta$ is the symmetry factor, $\mathrm{F}$ is Faraday's constant, $\mathrm{R}$ is the universal gas constant, and $\mathrm{T}$ is the local temperature within the layer. 
$\eta_{\text {act }}$ and $\mathrm{i}_{\mathrm{o}, \mathrm{geom}}{ }^{(\mathrm{T}, \mathrm{C}, \mathrm{S})}$ are the activation overpotential and reference exchange current density on a geometric basis (i.e. not on a platinum surface basis but volume of electrode) which are determined by:

$$
\eta_{a c t}=\phi_{e}-\phi_{p}-\phi_{0}
$$

Where, $\varphi_{\mathrm{e}}, \varphi_{\mathrm{p}}$, and $\varphi_{\mathrm{o}}$ are the electric, protonic, and reference potentials, respectively.

And, the reference exchange current density on a geometric basis is determined according to:

$$
i_{o, g e o m}^{(T, C, S)}=i_{o, p t}^{r e f(T, C)} S_{p t}\left(\frac{C_{O 2}}{C_{O 2}^{r e f}}\right)^{\gamma_{O 2}} \exp \left[\frac{-E_{a}}{R}\left(\frac{1}{T}-\frac{1}{T_{r e f}}\right)\right]
$$

Where, $\mathrm{i}_{\mathrm{o}, \mathrm{pt}}{ }^{\mathrm{ref}(\mathrm{T}, \mathrm{C})}$ is the specific exchange current density at a reference temperature and oxygen concentration on a platinum basis, $S_{p t}$ is the specific available surface area of platinum per unit volume of the electrode, $\mathrm{C}_{\mathrm{O} 2}$ and $\mathrm{C}_{\mathrm{O} 2}{ }^{\text {ref }}$ are the oxygen concentration and reference oxygen concentration respectively, $\mathrm{E}_{a}$ is the energy of activation for platinum with respect to the ORR, and $\mathrm{Y}_{\mathrm{O} 2}$ is the reaction order with respect to oxygen.

With respect to the earlier mention of the number electrons in the rate determining step, $\mathrm{n}$,(in the case of this model) a value of 2 was assigned. Strictly speaking this is not in keeping with the Butler-Volmer model as it is meant to represent a single electron transfer. However, the analysed Tafel slope approximations of the experimental data (both cell and RDE) yielded approximations of $\sim 60 \mathrm{mV} / \mathrm{dec}$ at $25^{\circ} \mathrm{C}$ such that in order to reconcile the transfer coefficients and symmetry factors a value of $\sim 0.49-0.55$ was ascribed to $\beta$ in keeping with a value of $n=2$. It is possible that there are other combinations that are valid here; however the symmetry factor was required to be between 0 and 1 and from this constraint the values arrived at appear reasonable within the limitations of the kinetic description. Based on this analysis, the exchange current density from the RDE based experiments was determined to have a mean of approximately $\mathrm{i}_{\mathrm{o}} \sim 4.5 \mathrm{e}^{-4} \mathrm{~A} / \mathrm{m}^{2}$ pt with a standard deviation of approximately $16 \%$ of the mean, $a$ subset of the those results are shown in Table 1.

\begin{tabular}{|c|c|c|c|c|c|c|c|c|}
\hline$\#$ & Catalyst & IPA:H2O & $\begin{array}{c}\text { Pt Area } \\
{\left[\mathrm{m}^{\wedge} 2 / \mathrm{gm}\right]}\end{array}$ & $\begin{array}{c}\mathrm{i}^{\mathrm{k}}(0.9 \mathrm{~V}) \\
{\left[\mathrm{uA} / \mathrm{cm}_{\mathrm{pt}}{ }^{\wedge} 2\right]}\end{array}$ & $\begin{array}{c}\text { Tafe } \\
\text { Slope } \\
{[\mathrm{mV} / \mathrm{dec}]}\end{array}$ & $\begin{array}{c}-\log \left(\mathrm{i}_{\mathrm{o}}\right) \\
{\left[\mathrm{A} / \mathrm{cm}_{\mathrm{pt}}{ }^{2}\right]}\end{array}$ & $\begin{array}{c}\mathrm{i}_{\mathrm{o}} \\
{\left[\mathrm{A} / \mathrm{m}_{\mathrm{pt}}{ }^{2}\right]}\end{array}$ & $\begin{array}{l}\text { correction for } \\
02 \text { Solubility to } \\
1 \text { bar at } 20 \mathrm{C}\end{array}$ \\
\hline 7 & $50 \% \mathrm{Pt} / \mathrm{LSAC}$ & $1: 4$ & 39 & 267 & 60 & 8.92 & $1.2 \mathrm{E}-05$ & $4.5 \mathrm{E}-04$ \\
\hline 8 & $50 \% \mathrm{Pt} / \mathrm{LSAC}$ & $1: 4$ & 43.9 & 349 & 60 & 8.89 & $1.3 \mathrm{E}-05$ & 4.9E-04 \\
\hline 9 & $50 \% \mathrm{Pt} / \mathrm{LSAC}$ & $1: 1$ & 37.6 & 250 & 60 & 8.98 & $1.0 \mathrm{E}-05$ & $4.0 \mathrm{E}-04$ \\
\hline 10 & $50 \% \mathrm{Pt} / \mathrm{LSAC}$ & $4: 1$ & 40.9 & 352 & 60 & 8.81 & $1.5 \mathrm{E}-05$ & $5.9 \mathrm{E}-04$ \\
\hline
\end{tabular}

Table 1: RDE analysis for the exchange current density based on a $60 \mathrm{mV} / \mathrm{dec}$ tafel slope

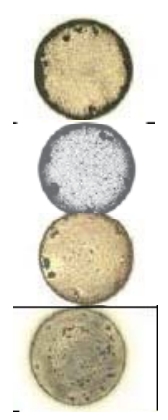


DE-EE0000466

Ballard Material Products Inc

\section{Cathode Porous Transport Layer (cPTL):}

The electron transport within the cathode porous transport layer was governed by the same transport equation used for the aPTL.

\section{Proton Transport}

\section{Anode Catalyst Layer (aCL):}

The proton transport within the anode catalyst is governed by Ohm's Law, similar to that used to describe the electron transport.

$$
C_{D}\left(\frac{\partial \phi_{p}}{\partial t}\right)-\nabla \cdot\left(\sigma_{p}^{e f f} \nabla \phi_{p}\right)=S_{c u r r e n t}
$$

Note that the transient term is included with the appropriate preceding capacitance variable, however the capacitance for the anode catalyst layer is accounted for in the electron transport equation and as such the capacitance for the proton transport is assumed to be zero here. Also the source term for proton generation, $S_{\text {current }}$, is equivalent to that discussed previously in the electron transport section.

The effective protonic conductivity, $\sigma_{p}{ }^{\text {eff }}$, is determined using the same effective medium theory relation as was used for the effective electronic conductivity:

$$
\sigma_{p}^{e f f}=\sigma_{p}\left(v_{f}^{\mathrm{p}}\right)^{\tau^{p}}
$$

Where, $v_{f}^{p}$ and $T^{p}$ are the volume fraction and tortuosity of the proton conducting phase, respectively.

$\sigma_{p}$ is the base conductivity of the proton conducting material in the anode catalyst layer which is determined by the equilibrium correlation determined by Springer et al.

$$
\sigma_{p}=\left(5.139 e^{-3} \lambda-3.26 e^{-3}\right) \cdot e^{\left[1268 \cdot\left(\frac{1}{303}-\frac{1}{T_{\text {local }}}\right)\right]}
$$

(Of note, for the purposes of this model the water phase within the catalyst layer is assumed to not participate in the bulk transport of protons.)

Where, $\lambda$ is the number of water molecules per sulfonic acid site and is generally determined via an empirical relationship for a given lonomer. The following equation represents the $\lambda$ versus $\mathrm{RH}$ correlation based on the measurements done by Springer for Nafion 117.

$$
\lambda=0.043+17.81 \cdot a_{H 2 O}-39.85 \cdot\left(a_{H 2 O}\right)^{2}+36 \cdot\left(a_{H 2 O}\right)^{3}
$$

Where, $\mathrm{a}_{\mathrm{H} 2 \mathrm{O}}$ is the activity of water vapour. 
It should be noted that the empirical relationship for $\lambda$ in this context is only valid for activities between 0 and 1 .

Within this performance model however, the movement of water dissolved in the ionomeric phase is solved for such that $\lambda$ is determined from the solution of that transport equation.

\section{Polymer Membrane (pMEM):}

The same governing equation for proton transport that was applied in the anode catalyst is applied in the polymer membrane, less the source term representing the production of protons from the electrochemical reaction:

$$
C_{D}\left(\frac{\partial \phi_{p}}{\partial t}\right)-\nabla \cdot\left(\sigma_{p}^{e f f} \nabla \phi_{p}\right)=0
$$

Again, the value of the double layer capacitance is set to zero as we are not in the electrode regions.

Within the membrane, for consistency, the effective medium theory approach is still applied, however the volume fraction of the proton conducting material is set to 1.0 such that the value of the effective protonic conductivity equates to:

$$
\sigma_{p}^{e f f}=\sigma_{p}
$$

With the determination of $\sigma_{p}$ done similarly with the relationship used in the anode catalyst layer and the local dissolved water content used to establish the local value of $\lambda$.

\section{Cathode Catalyst Layer (CCL):}

The transport of protons within the cathode catalyst layer was similar to that described for the anode catalyst layer.

\section{Formulation of the Potential Nomenclature:}

In deriving the equations for proton and electron transport across the membrane electrode assembly (MEA) a consistent description was required in order to formulate the coupling to other equations and the nature of the electrochemical reactions.

As such the proton transport was formulated so that protons flow from a high potential to a low potential, while the electron transport was formulated to allow electrons to flow from low potential to high potential.

This formulation has several implications: the first is that coupled with the definition for the activation over-potential and setting the reference electrode as the reference hydrogen electrode - the determined activation over-potential follows the following convention:

- A positive overpotential drives an anodic current

- A negative overpotential drives a cathodic current 
A plot of the results of this convention can be seen in Figure 3, here we see that based on the activities of each of the catalysts for their respective reactions and the respective derivations for the activation overpotential for the HOR and ORR reactions respectively, the anode has a small but positive overpotential (hence a anodic reaction) while the cathode has a large but negative overpotential (hence a cathodic reaction).

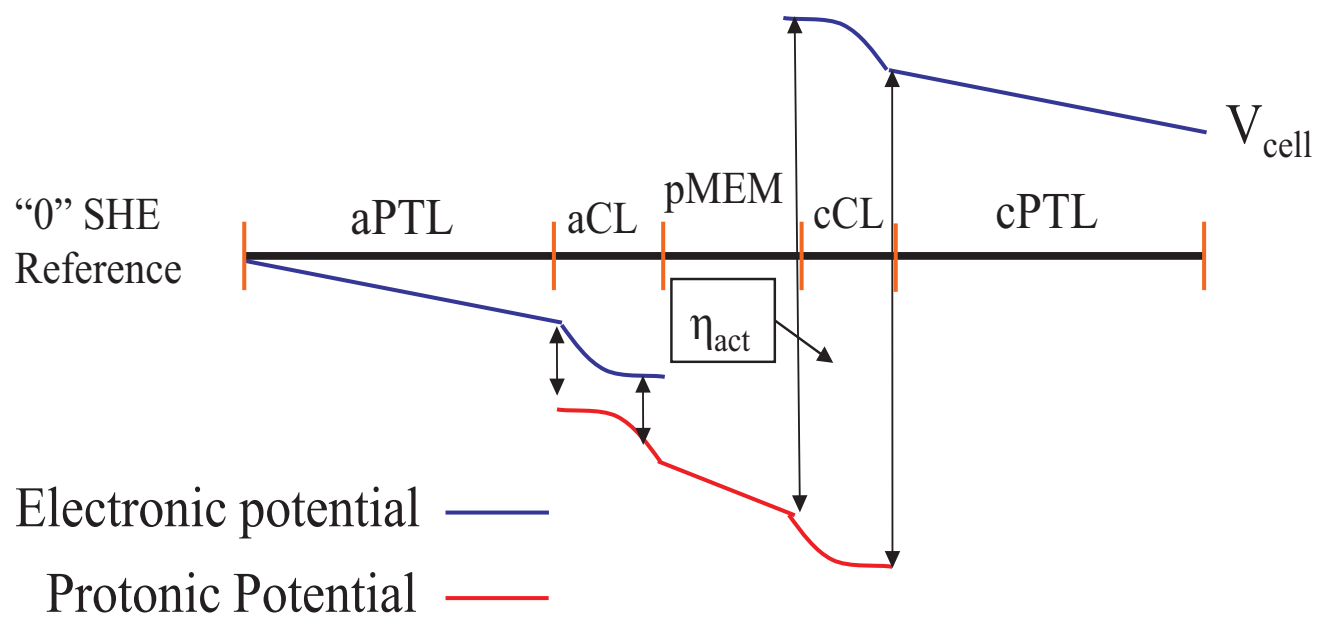

Figure 3: Potential Nomenclature and Activation Overpotential

\section{Species Transport}

The species transport equations are solved in each of the PTLs and CLs, but the assumption at this stage is that the membrane is impermeable to the gases and as such any dissolved reactants are not considered. Within the anode, the species of interest are hydrogen $\left(\mathrm{H}_{2}\right)$, nitrogen $\left(\mathrm{N}_{2}\right)$, and water in the vapour phase $\left(\mathrm{H}_{2} \mathrm{O}^{v}\right)$, whereas on the cathode side the species are the same except that hydrogen is omitted and oxygen considered instead $\left(\mathrm{O}_{2}\right)$.

There are several different approaches to describing the movement of species due to various driving forces, in particular the use of formulations such as Fick's Law or the Stefan-Maxwell equations for Multi-component diffusion are common. With respect to the two approaches just mentioned, Fick's Law tends to be computationally of low overhead and as such is routinely used as an easy implementation, while Stefan-Maxwell is recognized as more accurate and a better model for multiple species that are not near a dilute limit. This model has been developed in an attempt to find a balancing point between these two models though the use of a Fickian-approach but a mixture dependent effective diffusivity.

\section{Anode Porous Transport Layer (aPTL):}

The species transport equation considers driving forces from both, diffusion and convection, but neglecting thermal. As such the local species mass fraction, $y_{i}$, is determined via:

$$
\rho_{\text {mix }}\left(\frac{\partial y_{i}}{\partial t}\right)-\nabla \cdot\left(\rho_{\text {mix }} D_{i, m i x}^{e f f} \nabla y_{i}-\rho_{\text {mix }} y_{i} \overrightarrow{\mathrm{v}}_{\text {mix }}\right)=S_{i}
$$


This equation is solved for $\mathrm{i} \equiv\left\{\mathrm{H}_{2}, \mathrm{~N}_{2}, \mathrm{H}_{2} \mathrm{O}^{\mathrm{v}}\right\}$, noting that $\mathrm{N}_{2}$ exists only for closure as the gas composition on the anode side was simulated as humidified, pure hydrogen.

The source term, $S_{i}$, reflects that need for a source/sink term when $i=\left\{\mathrm{H}_{2} \mathrm{O}^{v}\right\}$, such that the exchange of mass between the vapour and liquid phases are accounted for and appropriately coupled.

In the species transport equation $\rho_{\text {mix }}$ is the local mixture density, $V_{\text {mix }}$ is the mixture velocity, $D_{i, m i x}{ }^{\text {eff }}$ is the effective diffusivity of species i into the background mixture, which is determined using effective medium theory:

$$
D_{i, \text { mix }}^{\text {eff }}=D_{i, \text { mix }}(1-s)^{\tau_{\text {liq }}^{P T L}}\left(v_{f, G D L}^{\text {pore }}\right)^{\tau_{\text {pore }}^{P T L}}
$$

With, $v_{f}$ pore representing the gas transporting volume (i.e. unsaturated pore space), $s$ is the local liquid water saturation, $\mathrm{T}_{\text {liq }} \mathrm{PTL}_{\mathrm{T}}$ is the correction order of the liquid water saturation on the gas transporting volume, and $\mathrm{T}_{\text {pore }}{ }^{\mathrm{PTL}}$ is the tortuosity of the pore space for gas transport on a drybasis.

$D_{i, m i x}$ is the diffusivity of species $i$ in the background mixture and it is determined using a molar weighting method to account for the change in the background composition such that:

$$
D_{i, m i x}=\frac{1-x_{i}}{\sum_{j, j \neq i}^{N}\left(\frac{x_{j}}{D_{i j}}\right)}
$$

In which, $x_{i}$ is the mole fraction and $D_{i, j}$ is the binary diffusivity conventionally used in the StefanMaxwell approach.

There are several methods available to estimate the binary diffusivity, the method employed here is the empirical correlation developed by Fuller, Schettler, and Giddlings (which is generally attributed to being within a few per cent of the estimates determined from kinetic theory). The Fuller, Schettler, Giddings correlation is written as:

$$
D_{i j}=1 e^{-7} \times \frac{T_{\text {mix }}^{1.75}\left(\frac{1}{M_{w, i}}+\frac{1}{M_{w, j}}\right)}{p_{\text {mix }}\left(\left(\sum_{i} V_{i}\right)^{(1 / 3)}+\left(\sum_{j} V_{j}\right)^{(1 / 3)}\right)^{2}}
$$

Applying this correlation requires tabulation of the molecular weights, $M_{w}$, and the atomic diffusion volumes, $\Sigma V_{i}$ for each of the species of interest. 
DE-EE0000466

Ballard Material Products Inc

\section{Anode Catalyst Layer (aCL):}

The species transport equation in the catalyst layer is the same as that which is applied within the porous transport layers, with the exception of an added source term:

$$
\rho_{\text {mix }}\left(\frac{\partial y_{i}}{\partial t}\right)-\nabla \cdot\left(\rho_{\text {mix }} D_{i, m i x}^{e f f} \nabla y_{i}-\rho_{\text {mix }} y_{i} \overrightarrow{\mathrm{v}}_{\text {mix }}\right)=S_{i}
$$

Where the source term, $S_{i}$, is defined the sink of either hydrogen due to consumption via the electrochemical reaction or a source/sink term for water vapour based on evaporation/condensation and/or adsorption/desorption from the ionomer phase dependent on the local conditions and which is the species of interest.

\section{Cathode Catalyst Layer (cCL):}

The species transport equation for the cathode catalyst layer is the same as that described for the anode catalyst layer with the exception that the species subset becomes $\mathrm{i} \equiv\left\{\mathrm{O}_{2}, \mathrm{~N}_{2}, \mathrm{H}_{2} \mathrm{O}^{\mathrm{v}}\right\}$ and the associated sink term becomes one for Oxygen and there is an added source term for the production of water in the vapour phase.

Cathode Porous Transport Layer (cPTL):

The species transport equation for the cathode porous transport layer is the same as that described for the anode porous transport layer with the exception that the species subset becomes $\mathrm{i} \equiv\left\{\mathrm{O}_{2}, \mathrm{~N}_{2}, \mathrm{H}_{2} \mathrm{O}^{\mathrm{v}}\right\}$.

\section{Continuity (Mass Transport)}

The continuity equation was recast in the model in order to solve for the pressure variation based on the mass consumption and Darcy's Law. As such, the equation is used to determine the local velocity field and appropriately couple this to the convective terms in the species and liquid water transport equations.

Anode Porous Transport Layer (aPTL):

Within the aPTL the continuity equation took the following form:

$$
\left(\frac{\partial \rho_{\text {mix }}}{\partial t}\right)+\nabla \cdot\left(\rho_{\text {mix }} \overrightarrow{\mathrm{v}}_{\text {mix }}\right)=S_{\text {mass }}
$$

Where the term $S_{\text {mass }}$ is the source term for the exchange of mass between the liquid and vapour phases.

At this stage, the continuity equation was then recast using a relationship relating pressure and velocity (Darcy's Law) such that the equation could be recast to solve for the local pressure variation: 
DE-EE0000466

Ballard Material Products Inc

$$
\overrightarrow{\mathrm{v}}_{\text {mix }}=-\left(\frac{K_{p}^{G D L}}{\mu}\right) \nabla P_{\text {mix }}
$$

As such the continuity equation takes the final form,

$$
\frac{1}{\bar{R} T_{\text {mix }}}\left(\frac{\partial P_{\text {mix }}}{\partial t}\right)+\nabla \cdot\left(-\rho_{\text {mix }}\left(\frac{K_{p}^{G D L}}{\mu}\right) \nabla P_{\text {mix }}\right)=S_{\text {mass }}
$$

Where $\rho_{\text {mix }}$ is determined via,

$$
\rho_{m i x}=\frac{P_{m i x}}{\bar{R} T_{m i x}}
$$

\section{Anode Catalyst Layer (aCL):}

Within the anode catalyst layer, the continuity equation is the same as that within the aPTL, with the exception of a modification to the source term such that it accounts for any water generated due to electro-osmotic drag, adsorption/desorption related to vapour exchange with the ionomer, and condensation/evaporation resulting from local phase change.

\section{Cathode Catalyst Layer (cCL):}

Within the cathode catalyst layer, as in the $\mathrm{aCL}$, the source term represents the only modification from the equation listed for the aPTL. However, an additional term is added within the cathode catalyst layer to represent the production of water from the electrochemical reaction.

\section{Cathode Porous Transport Layer (cPTL):}

The cathode porous transport layer employs the same equation set for continuity as is applied within the aPTL.

Within each of the layers of porous media (the porous transport layers and catalysts) the permeability was determined through the use of microstructural model simulations. More information about these simulations can be found in the section describing the microstructural models. For the PTL (both anode and cathode), the permeability was extracted from flow simulations and determined to be roughly on the order of $1 \mathrm{e}^{-13} \mathrm{~m}^{2}$ - Figure 4 shows a comparison of the permeability between the initial substrate paper and the finished PTL. 
DE-EE0000466

Ballard Material Products Inc

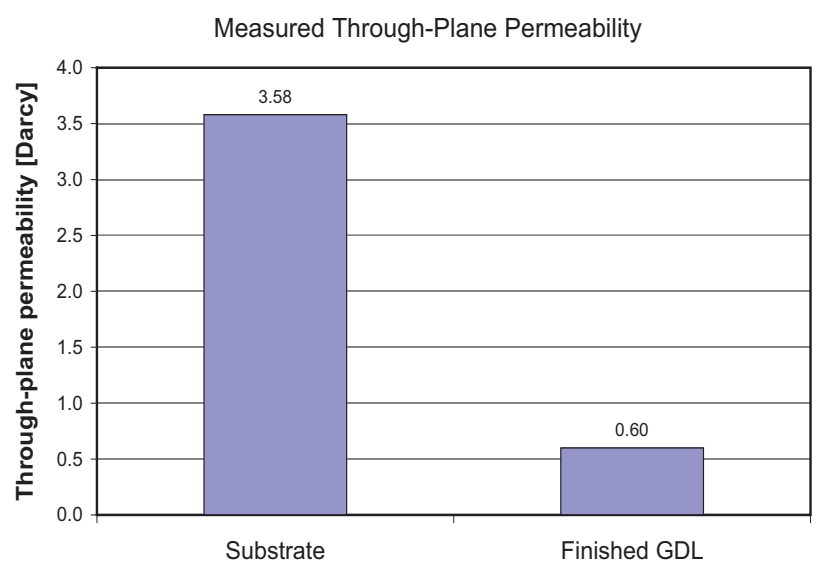

Figure 4: Permeability of the PTL predicted by microstructural simulations

Overall, the use of different values for the permeability is directly related to two primary coupling points in the model, both related to convective transport terms. The first of these coupling points is in the gas phase equations and the second is in the liquid water transport equation. The effect of the value of the permeability will be discussed in section related to the latter.

\section{Energy Transport}

The solved model is non-isothermal, with the primary mode of heat transfer assumed to occur through conduction. In the absence of solving for the momentum equations in the channel, it is assumed the all of the heat flux occurs in the direction of the coolant channel in the plate (or vice versa, depending on the conditions of the cell). Given the large thermal conductivity of the plate material and the fact that the plate geometry is not expressly included in the onedimensional code, a temperature boundary condition is applied at the MEA surface and it is assumed that this "collection" surface is essentially isothermal.

\section{Anode Porous Transport Layer (aPTL):}

Given the assumption that conduction is the primary driving force for the flow of energy within the domain, the governing equation takes the following form:

$$
\nabla \cdot\left(-\mathrm{K}_{\mathrm{PTL}}^{\text {eff }} \nabla \mathrm{T}\right)=\mathrm{S}_{\text {energy }}
$$

Where, $\mathrm{K}_{\mathrm{PTL}}{ }^{\text {eff }}$ represents the effective thermal conductivity of the porous media and $\mathrm{S}_{\text {energy }}$ is the source term representing the release or consumption of energy due to other processes such as ohmic heating, evaporation/condensation, and contact resistance (an ohmic term as well). As such the source term takes the following form:

$$
S_{\text {energy }}=\mathrm{S}_{\text {ohmic }}+\mathrm{S}_{\text {phase change }}
$$


DE-EE0000466

Ballard Material Products Inc

The ohmic heating term is determined by:

$$
S_{\text {ohmic }}=\sigma_{e}^{e f f}\left(\nabla \phi_{e}\right)^{2}
$$

The phase change term is related to the mass of water condensed or vaporized multiplied by the latent heat of vaporization/condensation.

Anode Catalyst Layer (aCL):

The governing equation for energy within the anode catalyst follows the same form as that in the aPTL, however the source term definitions follow a form related to the electrochemical kinetics:

$$
\nabla \cdot\left(-\mathrm{K}_{\mathrm{PTL}}^{\text {eff }} \nabla \mathrm{T}\right)=\mathrm{S}_{\text {energy }}
$$

Where $S_{\text {energy }}$ takes a form that includes a term related to the entropy change in the reactants and the overall heat released due to irreversibility in the electrochemical reaction:

$$
S_{\text {energy }}=\mathrm{S}_{\text {ohmic }}+\mathrm{S}_{\text {phase change }}+S_{\text {reaction }}
$$

$S_{\text {reaction }}$ has two terms, one related to the entropy change between the reactants and products and the other related to the energy released due to the irreversibility of the reaction.

$$
S_{\text {reaction }}=\left|\frac{\mathrm{S}_{\text {current }}}{\mathrm{zF}}\right|(\mathrm{T} \delta \mathrm{s})-\mathrm{S}_{\text {current }} \eta_{\text {act }}
$$

Where $\delta s$ is the entropic change for the reaction and in the case of the anode is assumed to be zero. The value of $z$ in this equation is the number of moles of electrons transferred per mole of reactant.

\section{Cathode Catalyst Layer (cCL):}

The governing equation for the cathode catalyst layer follows the same form shown previously for the aCL. Within the cathode catalyst, however, the value of the entropy change between reactants and products is assigned as the difference between the reactants and products of the global reaction:

$$
\mathrm{O}_{2}+2 \mathrm{H}_{2} \longleftrightarrow 2 \mathrm{H}_{2} \mathrm{O}+\text { heat }
$$

Essentially this assumption implies that the bond breakage in the half-reaction for the hydrogen reaction is negligible, while the bond breakage of the oxygen is dominating such that all of the entropic release essentially occurs on the cathode side. 
DE-EE0000466

Ballard Material Products Inc

\section{Cathode Porous Transport Layer (cPTL):}

The energy transport within the cathode porous transport layer is consistent with the governing equation presented in the anode porous transport layer section.

As was done with the permeability of the porous media, the thermal conductivity was also determined as a function of composition, using the microstructural models. Figure 5 shows the two pictures of the representative microstructures for the PTLs and the resulting estimates of the thermal conductivity for those components. Generally, the thermal conductivity was seen to be on the order of $0.1-1.3[\mathrm{~W} / \mathrm{m} \mathrm{K}]$ consistent with sources in the open literature.

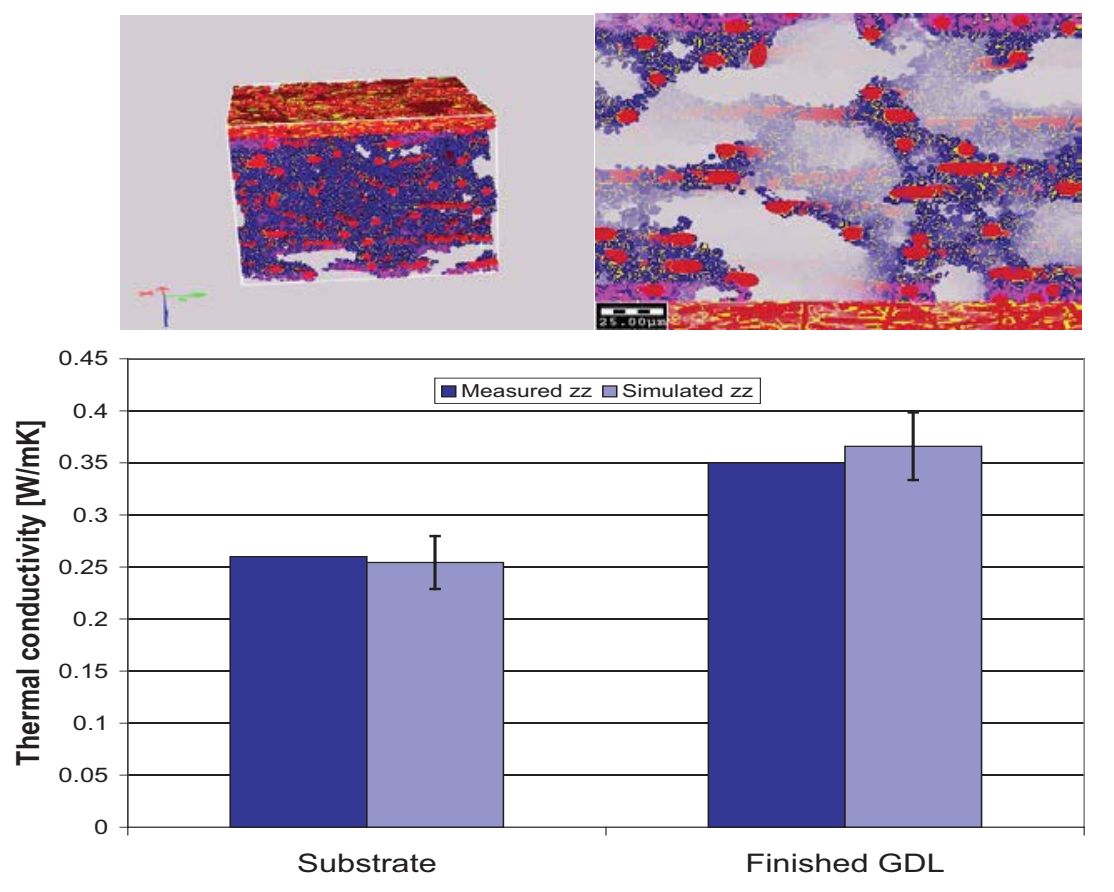

Figure 5: Thermal conductivity predicted by the microstructural models for the PTL

\section{Liquid Water Transport}

In review of the available liquid water models, there were three primary approaches identified that describe the movement of the liquid phase: (1) Pressure driven capillary models, (2) Saturation driven capillary models, (3) Volume of Fluid based approaches. Given the implementation approach for the model and considerations on the computational expense for the liquid water module addition, the Volume of Fluid approach was rejected. Most PEMFC models to date use the second approach, a saturation-based driving force, however the first option is generally considered more thermo-dynamically correct in that pressure is a more appropriate driving force. However, both options (1) and (2) are still "fitted" models in that different key aspects are fitted in order to obtain the respective behaviour/shape of the polarization curve due to the mass transport limiting effects of water accumulation. As such, given the ease of implementation, the second option was selected as the initial liquid water model. 
DE-EE0000466

Ballard Material Products Inc

\section{Anode Porous Transport Layer (aPTL):}

The water transport equation within the porous transport layer is governed by a derivation that arises from the description for capillary pressure:

$$
\nabla \cdot\left(\rho_{l i q} \vec{v}_{l i q}\right)=S_{\text {mass }}^{\text {liq }}
$$

Where $\mathrm{v}_{\text {liq }}$ is defined according to:

$$
\vec{v}=-\frac{\tau_{l i q}^{r l} K}{\mu_{l i q}} \nabla p_{l i q}
$$

$\mathrm{T}_{\text {liq }}{ }^{\mathrm{rl}}$ is the correction factor for the permeability based on the local value of the saturation, $\mathrm{K}$ is the permeability of the medium based on zero saturation, $\mu_{\text {liq }}$ is the viscosity of liquid water. The value of the gradient in the liquid pressure is determined by re-arranging the capillary pressure relation:

$$
p_{\text {liq }}=p_{\text {gas }}-p_{c}
$$

Inserting the relation for the liquid pressure into the governing equation results in a modification to the following form:

$$
\nabla \cdot\left(\frac{\rho_{l} \mathrm{~K}_{\mathrm{rl}}}{\mu_{l}} \frac{d P_{c}}{d s} \frac{d s}{d x}\right)-\nabla \cdot\left(\frac{\rho_{l} \mathrm{~K}_{\mathrm{rl}}}{\mu_{l}} \frac{d P}{d x}\right)=S_{\text {phase-change }}
$$

The solved variable now becomes the saturation which is denoted by the variable, s. Also of note is that the variable $\mathrm{K}$, which represents the permeability, now has a subscript, $\mathrm{rl}$, which represents that it has been corrected, based on the local saturation.

The value of the partial derivative of the capillary pressure with local saturation is determined through the use of the capillary pressure equation:

$$
P_{c}=\sigma_{l i q} \cdot \cos \left(\theta_{c}^{P T L}\right)\left(\sqrt{\frac{v_{f}^{\text {pore }}}{K}}\right) J(s)
$$

The term $\sigma_{\text {liq }}$ represents the surface tension of liquid water, $\theta_{c}$ represents the contact angle, and $\mathrm{J}(\mathrm{s})$ represents the Leverett-J function.

The Leverett-J function contains the dependence of the capillary pressure on saturation and as such its partial derivative multiplied by the preceding terms in the previous equation result in the partial derivate necessary in the governing equation. 
Returning to the terms in the governing equation, the value of the source term, $S_{\text {phase-change }}$ is determined by using a switching term that corresponds with the local conditions in determining whether condensation or evaporation is occurring.

$$
S_{\text {phase-change }}=M_{w}^{H 2 O} h_{p c}\left(P_{\mathrm{H} 2 \mathrm{O}}^{\text {vap }}-P_{\mathrm{H} 2 \mathrm{O}}^{\text {sat }}\right)
$$

Where, the term $h_{p c}$ is defined as:

$$
h_{p c}=\frac{k_{\text {cond }} V_{f}^{\text {Pore }}(1-\mathrm{s}) x_{\mathrm{H} 2 \mathrm{O}}^{\text {vap }}}{2 R T}\left[1+\frac{\left|P_{\mathrm{H} 2 \mathrm{O}}^{\text {vap }}-P_{\mathrm{H} 2 \mathrm{O}}^{\text {sat }}\right|}{p_{\mathrm{H} 2 \mathrm{O}}^{\text {vap }}-P_{\mathrm{H} 2 \mathrm{O}}^{\text {sat }}}\right]+\frac{k_{\text {evap }} V_{f}^{\text {Pore }} s \rho_{l}}{2 \mathrm{M}_{\mathrm{w}}^{\mathrm{H} 2 \mathrm{O}}}\left[1-\frac{\left|P_{\mathrm{H} 2 \mathrm{O}}^{\text {vap }}-P_{\mathrm{H} 2 \mathrm{O}}^{\text {sat }}\right|}{p_{\mathrm{H} 2 \mathrm{O}}^{\text {vap }}-P_{\mathrm{H} 2 \mathrm{O}}^{\text {sat }}}\right]
$$

In this equation, $\mathrm{k}_{\text {cond }}$ and $\mathrm{k}_{\text {evap }}$ represent the rate of condensation and evaporation respectively.

\section{Anode Catalyst Layer (aCL):}

Within the catalyst layer, the formation of the Leverett function can take two forms, principally there is a hydrophobic relation and a hydrophilic one, based on the composition of the layer there is assumed a volume weighted average such that the layer may or may not be hydrophobic according to this composition. For the purpose of this model, this weighting process was used and the form of the leveret/contact angle was modified accordingly. The hydrophobic Leverett-J function is determined via:

$$
J(s)=1.417 s-2.120 s^{2}+1.263 s^{3}
$$

It should be noted however that based on the desire for numerical stability the final model assumes that the layers are each hydrophobic and as such a strict derivation of the meaning of the contact angle and specific Leverett-J function are used.

\section{Cathode Catalyst Layer (cCL):}

The form of the liquid water transport in the cathode catalyst layer follows that implemented in the anode catalyst layer, including the concept of a blended estimation on the contact angle and the use of the appropriate Leverett-J function.

\section{Cathode Porous Transport Layer (cPTL):}

The cathode porous transport layer follows the same form as that shown for the APTL. Both the cathode and anode PTLs are assumed to demonstrate hydrophobic behaviour though it has been suggested in literature that layers such as these may have hydrophilic and hydrophobic pores. However, this concept is largely a geometric one, unless the process of forming the layer has been done in such a manner that the behaviour of the constituent material is itself hydrophilic.

\section{The Effect of Permeability on the Liquid Water Model:}

As was mentioned in the section discussing the governing equation for mass continuity, the permeability affects the convective driving force in several different equations and also impacts the ease of movement in the liquid water model. Frequently, both the contact angle, relative permeability correction, and the base material permeability are used as the fitting parameters for the liquid water model. In order to understand the effect of the permeability as a fitting 
parameter a parametric study was run observing the effect of different orders of permeability on the resulting cell polarization. Figure 6 shows the resulting behaviour; of note is that the permeability determined from the microstructural models approximated the permeability of the PTL to be on the order of $1 \mathrm{e}^{-13}$ to $1 \mathrm{e}^{-14} \mathrm{~m}^{2}$.

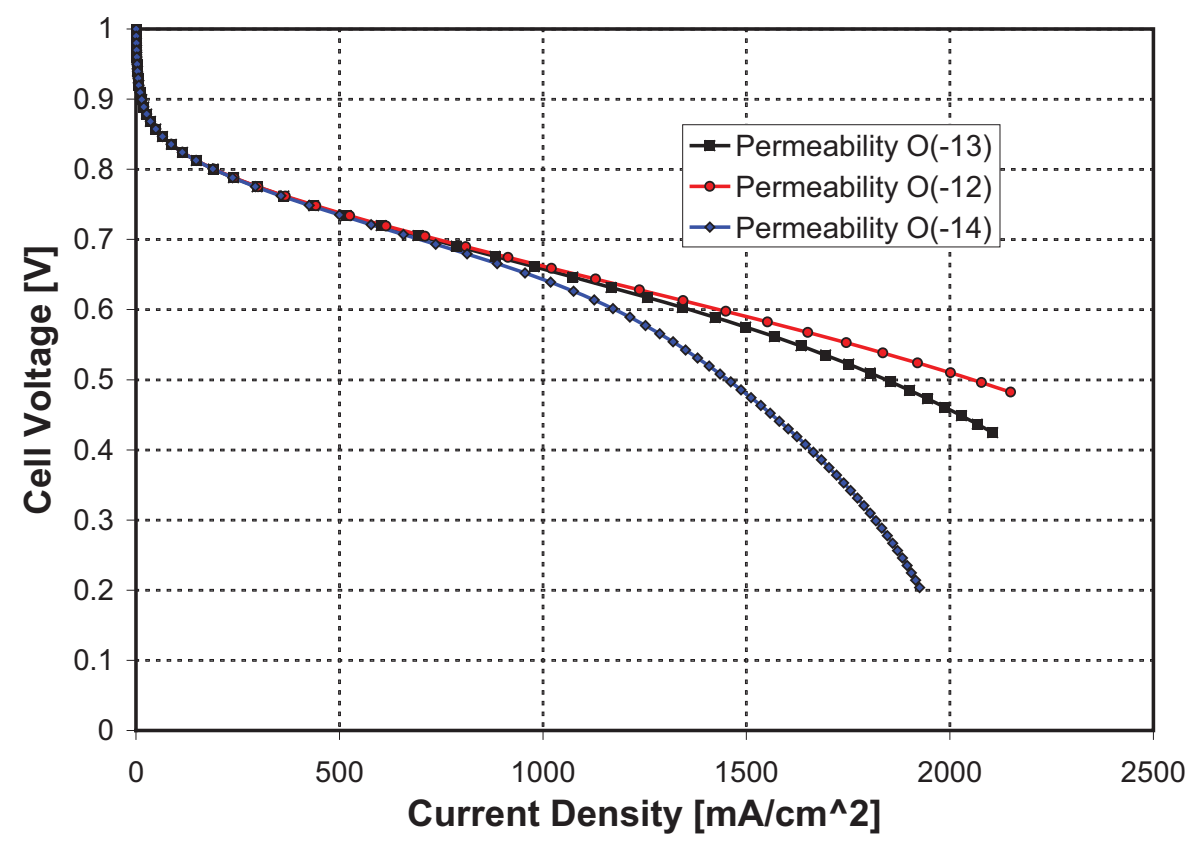

Figure 6: Parametric Study on the Effect of Permeability on the Liquid Water Model

\subsubsection{Model Validation}

In order to validate the model, there were a number of uncoupled parameters that are generally used in describing parametric studies on layer compositions (in particular the catalyst layers). In order to appropriately capture the effect of these inputs, the model inputs for the catalyst composition were derived such that the mass related composition inputs were used and then recast based on material densities to the appropriate terms used in calculating terms required for the governing equations. Figure 7 shows a schematic of the process. 
DE-EE0000466

Ballard Material Products Inc

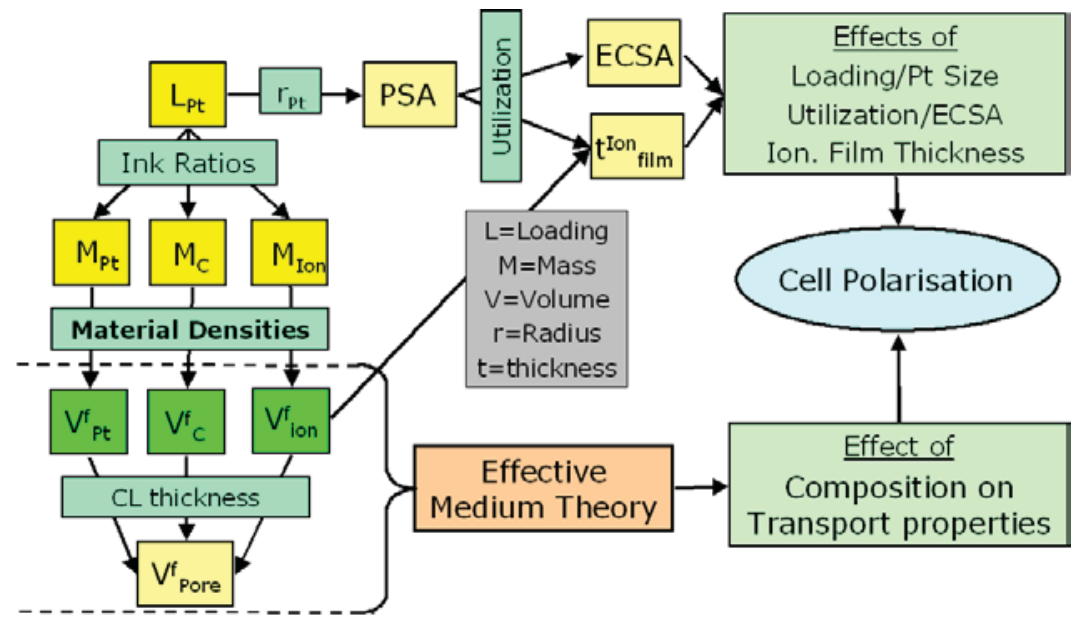

Figure 7: Schematic of the coupling between the catalyst layer composition and the appropriate terms in the governing equations.

The model validation was focused largely on compositional variation of the cathode catalyst as the purpose of the project was to capture performance and durability across a range of catalyst formulations.

Figure 8 and Figure 9 show the initial results of the model in comparing compositions of catalyst ionomer for $12 \%$ and $28 \%$ by weight. These initial runs were done without the liquid water module added on and as such we observe in the $12 \%$ ionomer case a lack of accuracy from the model at values of the current density greater than $1000\left[\mathrm{~mA} / \mathrm{cm}^{2}\right]$, however in the second case attempts to fit the model using the free parameters within the agglomerate model demonstrate that there exist parameter sets that can entirely attribute the observed transport resistances to the morphology of the layer - even in the absence of liquid water (illustrating the aforementioned issue of an excessive number of free parameters).

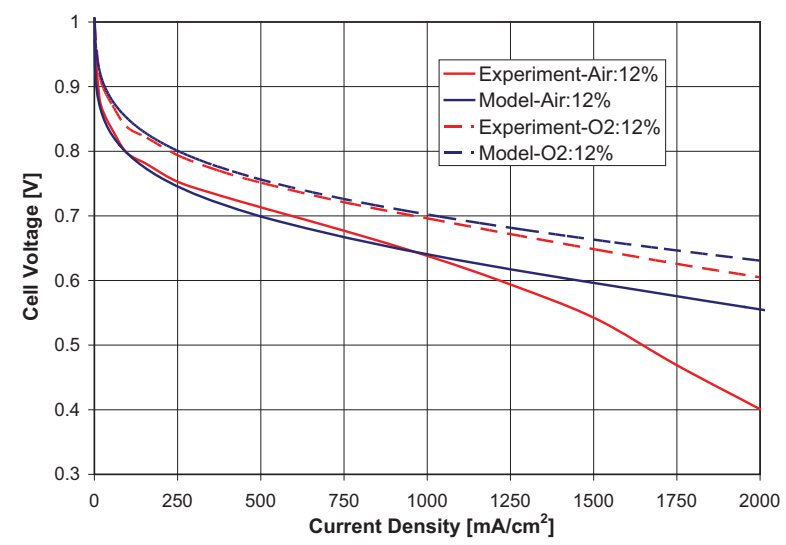

Figure 8: Comparison of the model predictions without the liquid water model and using the base parameter set 
DE-EE0000466

Ballard Material Products Inc

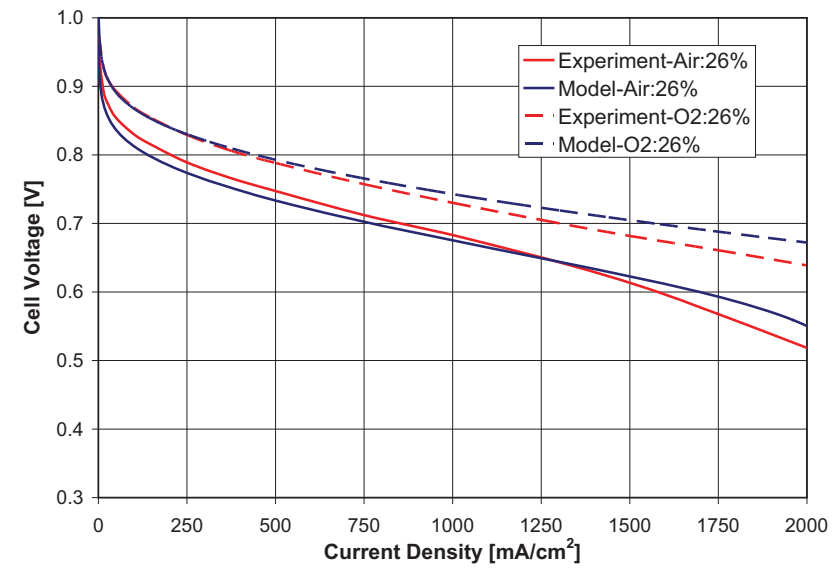

Figure 9: Comparison of the model predictions without liquid water and using adjusted agglomerate parameters 
DE-EE0000466

Ballard Material Products Inc

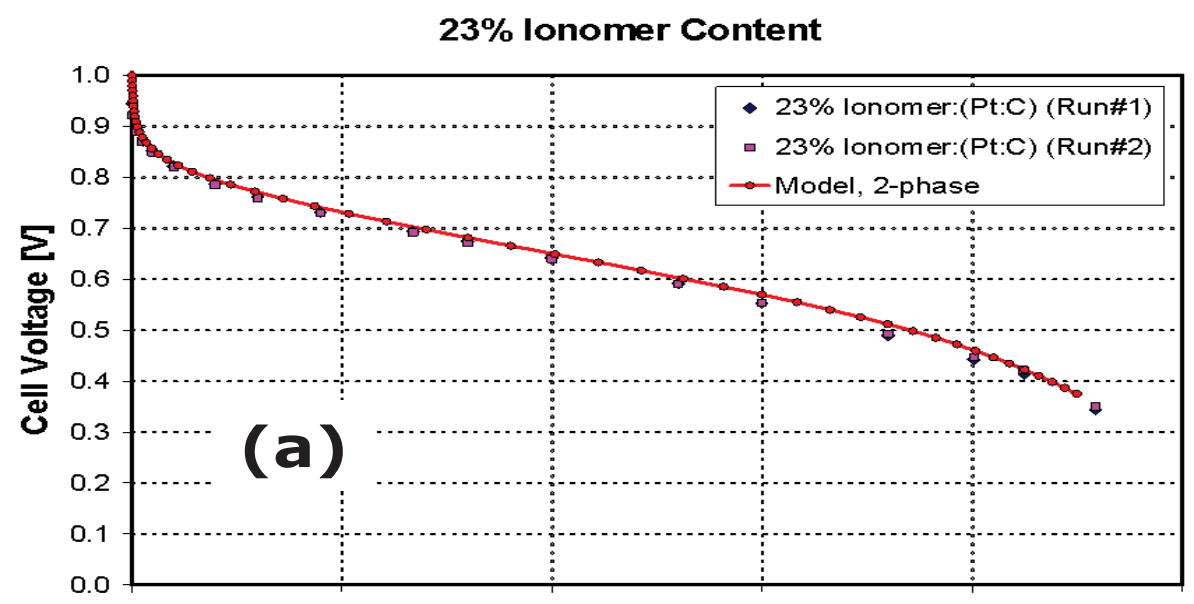

$30 \%$ lonomer Content

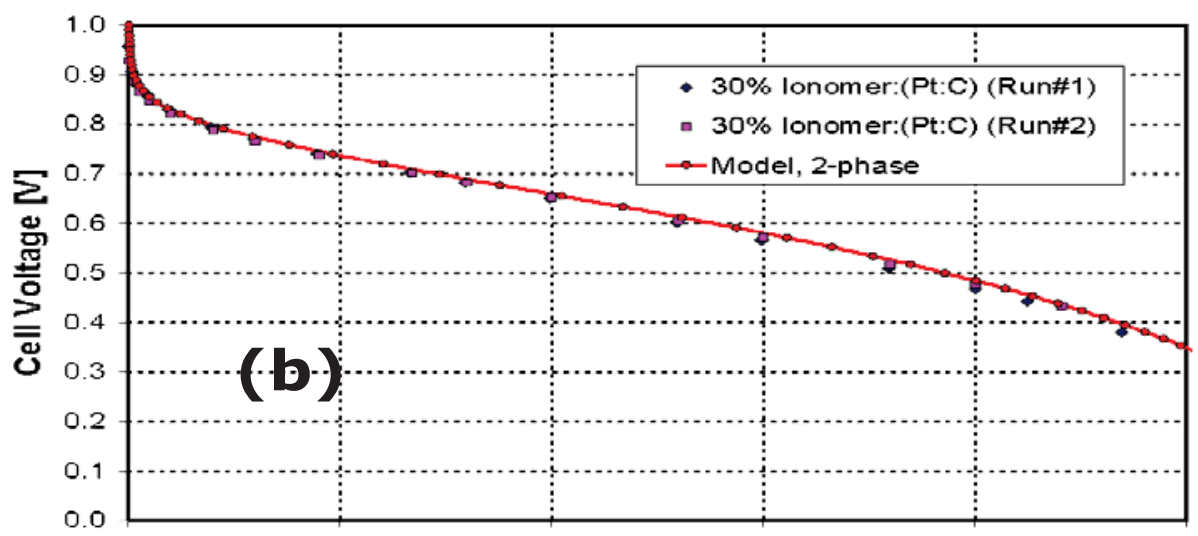

$38 \%$ lonomer Content

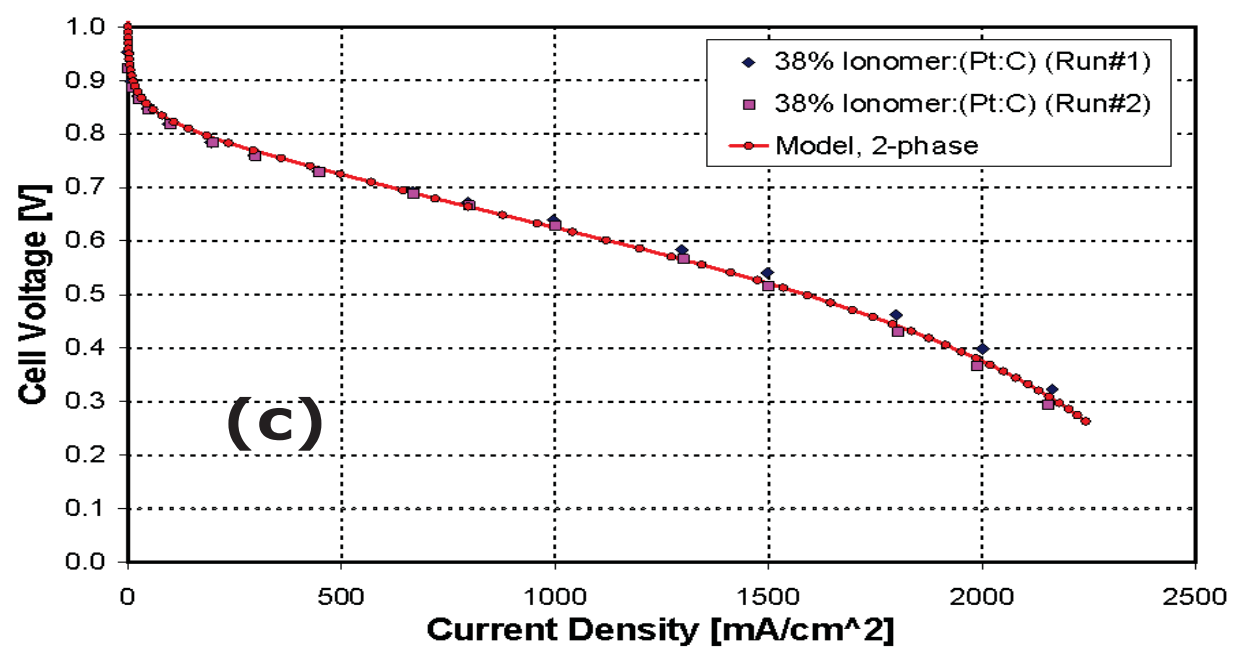

Figure 10: Validation of the two-phase model with ionomer composition (a) $23 \%$ (b) $30 \%$ (c) $38 \%$ by weight

However, the parameters that would be required by the agglomerate model in order to account for the observed transport resistances are generally not physically observed (i.e. substantially 
large agglomerate particle sizes and extremely thick ionomeric films). As such, the parameters used in the coupled model are based on any available measurements for the actual agglomerate size and an estimation on the film thickness based on the assumption that any active platinum surface area is active due to contact with the ionomeric films. This process results in rather small agglomerates and thin ionomeric films such that a liquid water model is necessary to capture the polarization behaviour - further, the permeability of the porous media was then constrained to the values predicted by the microstructural models.

Figure 10 shows the validation of the two-phase model with changing ionomer content and using a consistent set of baseline parameters. In part, the effect of changing ionomer composition affects the local porosity and gas composition but also directly impacts the water content via the capillary pressure relation and the local protonic conductivity via an increased participating volume fraction.

The effect of the local proton conductivity is also known to vary with local $\mathrm{RH}$ and as such the overall cell conditions. Frequently, cell polarizations are seen to respond strongly to changes in the inlet RH. However this response is a complicated scenario in that the local RH change can affect the local liquid water content/transport across the membrane and hence the local mass transport, the local reaction distribution within the catalyst layer, and also the local transport rate of protons via changes in the conductivity.

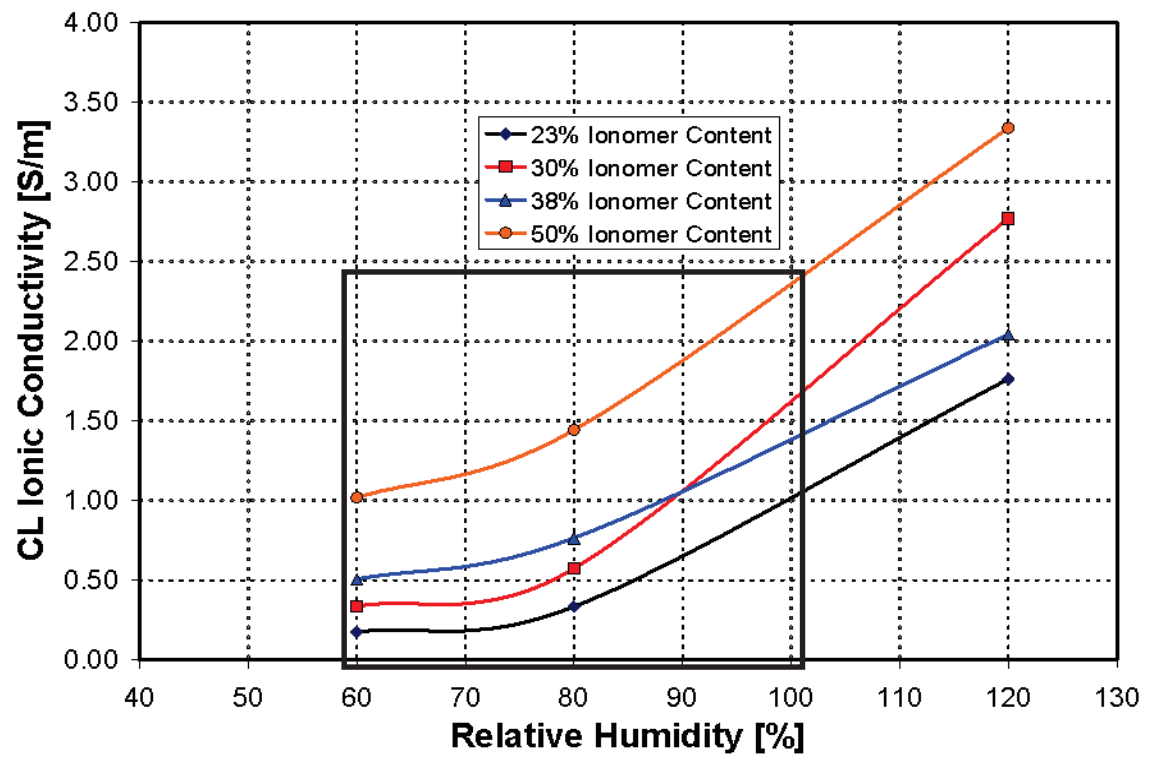

Figure 11: Measured Proton Conductivity of the Catalyst Layer vs. RH as a function of Catalyst Layer lonomer Content 
DE-EE0000466

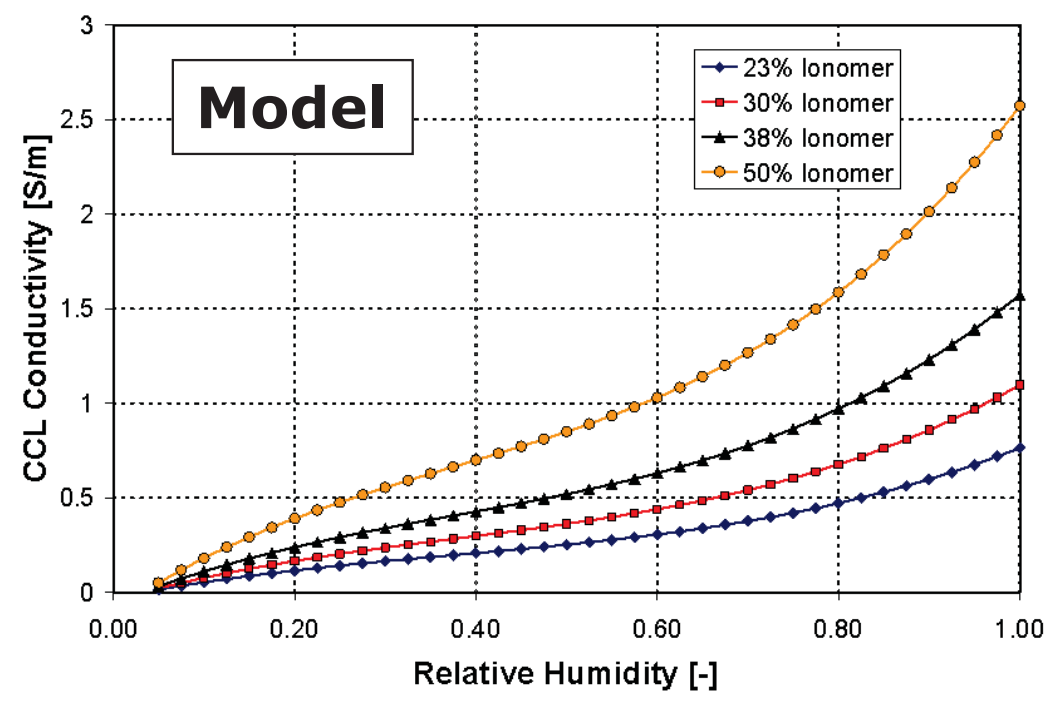

Figure 12: Predicted Catalyst Layer Protonic Conductivity based on the Springer Relationship

The ability of the model to capture the effects related to changes in the local proton conductivity is governed in part to the dissolved water transport, but also to the local dissolved water content and conductivity relationship. Using the published Springer model for proton conductivity and comparing the results of that model with analysis of catalyst layer proton conductivity determined by EIS it is observed, in Figure 11, that the measured proton conductivity may suffer from accuracy issues based on the data for the $30 \%$ and $38 \%$ ionomer contents by weight however the overall trend along the path of increasing $\mathrm{RH}$ is consistent. Comparing the predicted conductivities using the Springer model, Figure 12, shows that in general the conductivities are the correct order of magnitude but the values differ by a margin of up to $50 \%$ of the measured value along the $\mathrm{RH}$ range. This variation will ultimately control in part the response of the model to changes in the cell $\mathrm{RH}$. As the accuracy of the water content vs. conductivity relationship is improved the model predictions will increase in accuracy accordingly. Based on the existing relationship, the model predictions between a relative humidity of $60 \%$ (low end on the EIS measurement) and 100\% (upper end on the Springer relationship) show the ability to capture the resistivity decreases with respect to the proton transport (noting that the bulk of this resistance is accounted for by the same shift in conductivity which also occurs within the membrane). 
DE-EE0000466

Ballard Material Products Inc

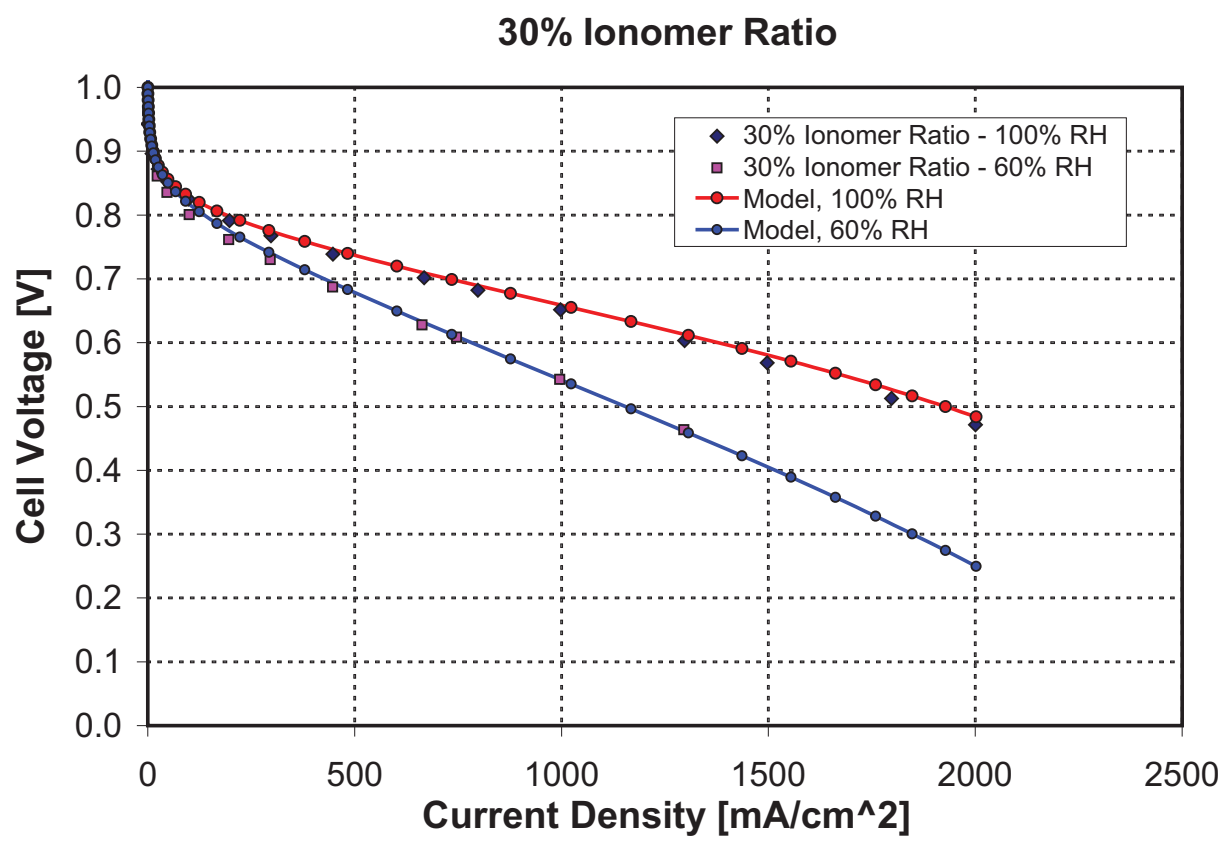

Figure 13: Predicted effect of the $\mathrm{RH}$ change and comparison to the cell polarization data for the same conditions

Further validation of the model from a composition and operational standpoint was done with consideration for the platinum loading. Variations in the platinum loading were tested from 0.05 to $0.408 \mathrm{mg} / \mathrm{cm}^{2}$ under a constant relational composition with ionomer. The operating conditions for the set were consistent between the samples with the exception that the sensitivity to oxygen was also probed.

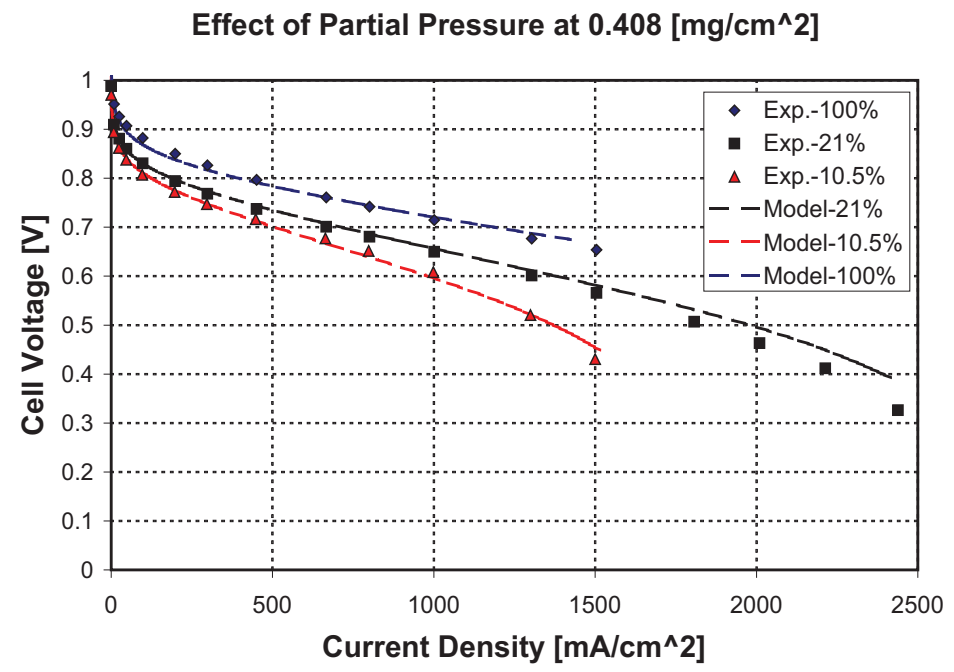

Figure 14: Model Validation for a platinum loading of $0.408\left[\mathrm{mg} / \mathrm{cm}^{2}\right]$ 
DE-EE0000466

Ballard Material Products Inc

Effect of Partial Pressure at $0.19\left[\mathrm{mg} / \mathrm{cm}^{\wedge} 2\right]$

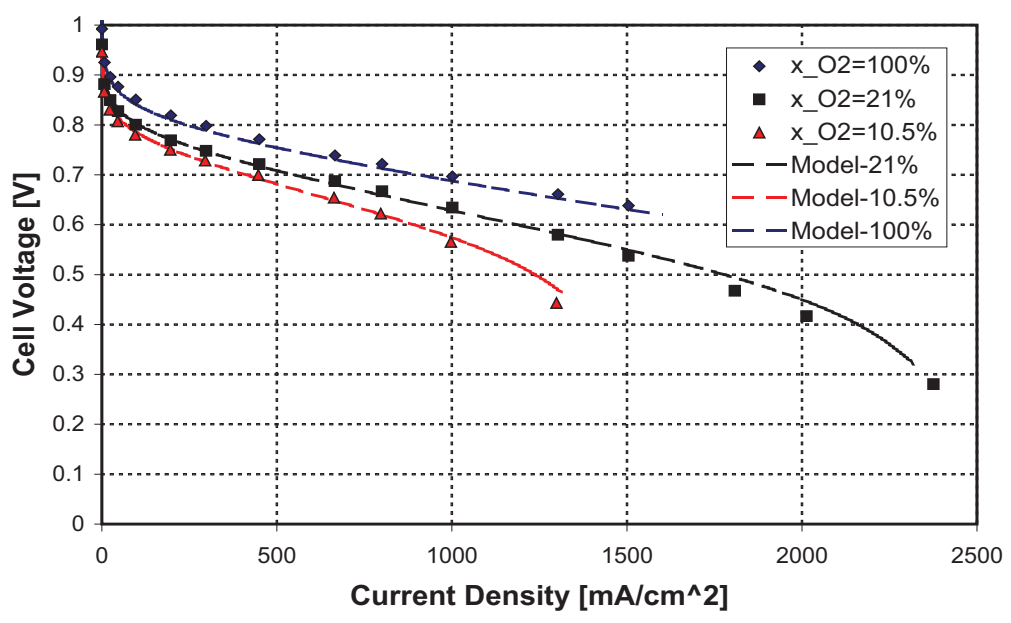

Figure 15: Model Validation for a platinum loading of $0.19\left[\mathrm{mg} / \mathrm{cm}^{2}\right]$

Effect of Partial Pressure at $0.05\left[\mathrm{mg} / \mathrm{cm}^{\wedge} 2\right]$

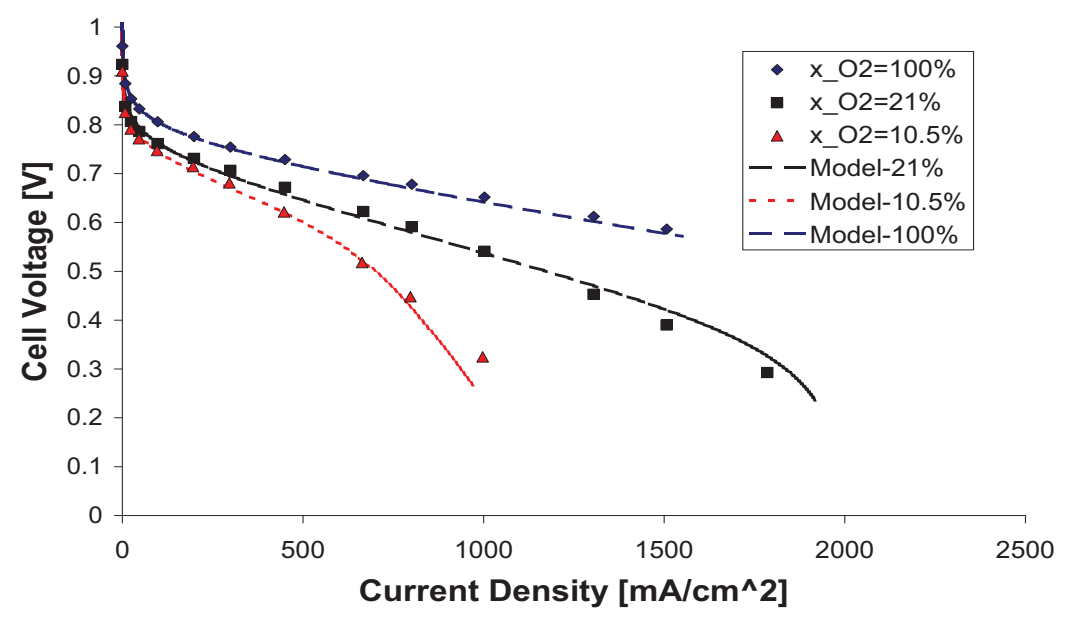

Figure 16: Model Validation for a platinum loading of $0.05\left[\mathrm{mg} / \mathrm{cm}^{2}\right]$

Figure 14, Figure 15, and Figure 16 demonstrate that the model captures the relevant changes in performance due to reductions in platinum loading, but also the increased sensitivity in oxidant mass fraction. This latter effect is due in part to two aspects: the first is that the thickness of the electrodes was allowed to decrease in the model as the loading dropped (consistent with the physically measured layers) and as it turns out this is a significant aspect in capturing the shifts in behaviour for different layers; the second aspect is that the liquid water saturation level was also observed to increase in the thinner catalyst layers at points of similar current density, Figure 17. This increase in water content plays a significant role in the reduction of the performance as the same current density requires higher cell overpotential to drive cell, hence resulting in lower operating cell voltages. 
DE-EE0000466

Ballard Material Products Inc
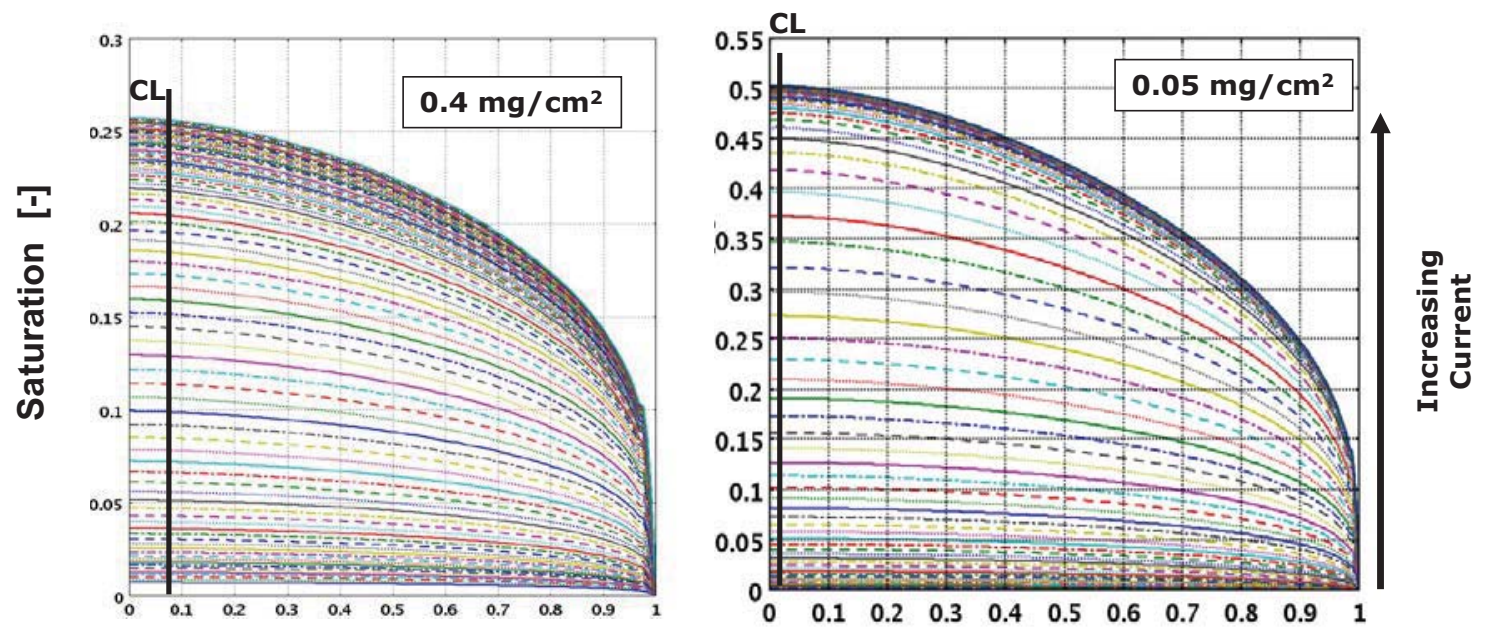

Figure 17: Relative saturation levels between a high-loaded and low-loaded catalyst layer

Additional validation was completed for carbon:platinum ratios and temperature, but these are not shown as there was no significant shift in the performance for the ranges tested. 


\subsection{Interfacial Resistance Model}

An electrical contact resistance model was developed following a procedure and method published in the available literature (Swamy et al.). This method employed a methodology, based on tribology studies, in which spectral and statistical analysis of the surface and associated roughness is carried out in order to estimate the surface contact area between components. Figure 18 shows the general process in which the imaged surfaces are analysed: (1) raw profilometry data is collected via an imaging process, (2) data is analyzed for the spectral moments and characteristic parameters, (3) Contact conditions are calculated and the average surface pressure and contact conductance are determined.

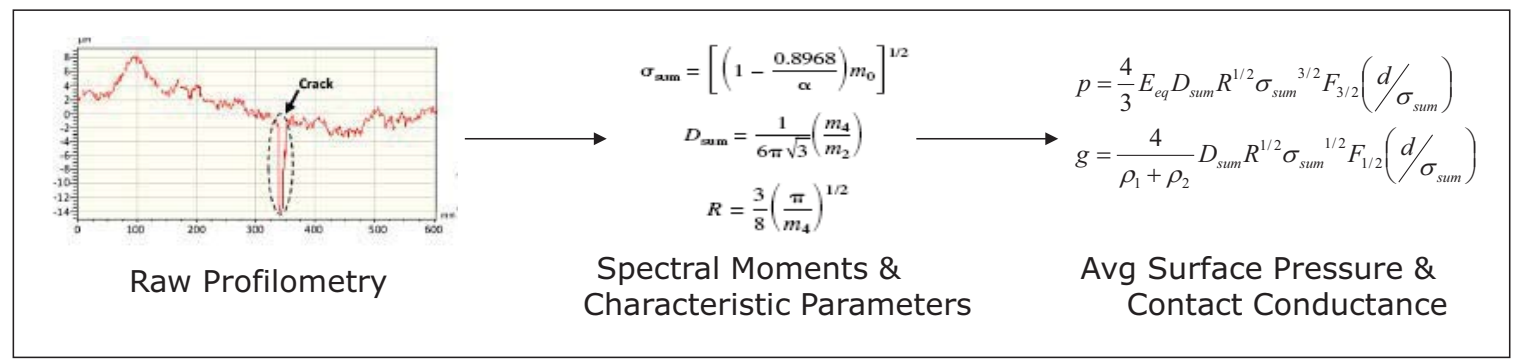

Figure 18: Schematic Methodology for the Contact Resistance Model

The raw profilometry data could be collected from a number of sources, but principally data collected in two-dimensions was preferable as was a method that had the resolution to capture the overall peak to valley roughness of the associated surface. Figure 19 shows a representative surface map of the PTL and Catalyst Layer on their respective mating sides.
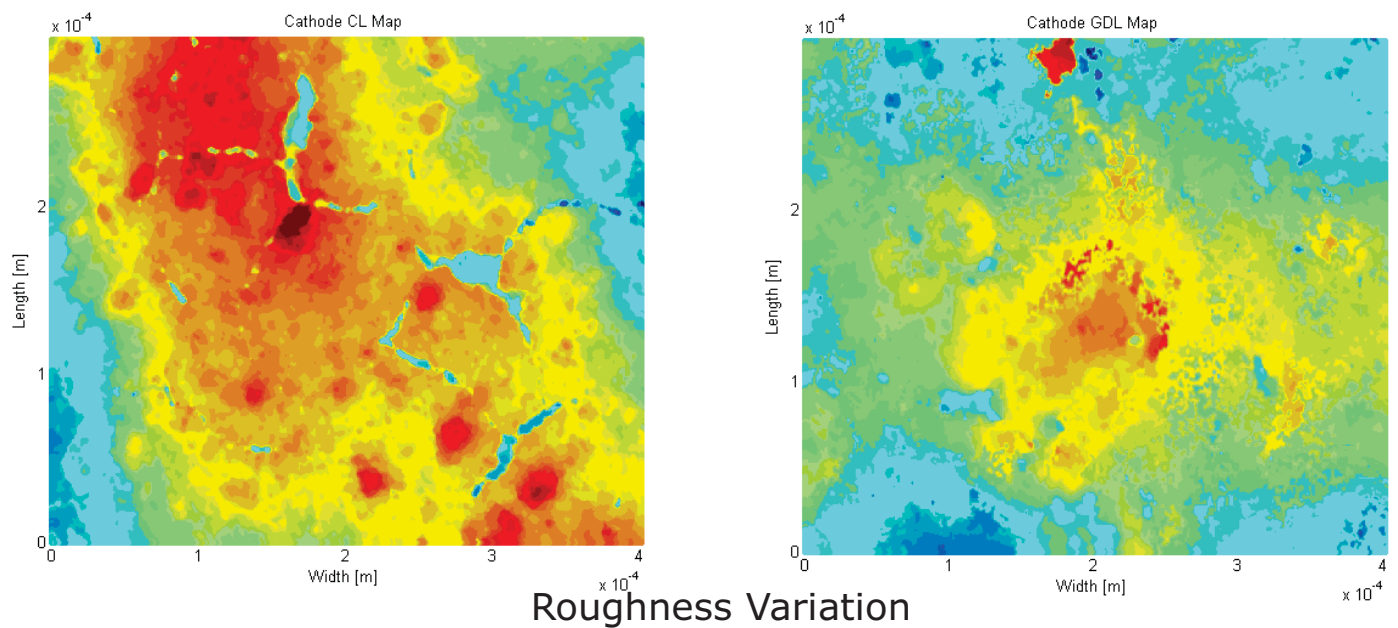

Catalyst layer $=3.2$ to $3.7 \mu \mathrm{m}, \mathrm{GDL}=4.1$ to $6.5 \mu \mathrm{m}$

Figure 19: Surface roughness maps measured on the Catalyst Layer and PTL

From the surface roughness maps, the contact resistance between the surfaces as a function of the applied compression was determined, an example of this is shown in Figure 20 . This contact resistance vs. pressure plot can either be coupled explicitly with a Finite Element style simulation for the prediction of local contact resistance or used as a lookup curve with a 
DE-EE0000466

Ballard Material Products Inc

compression pressure to estimate the contact resistance to be included between components (as is done in this model).

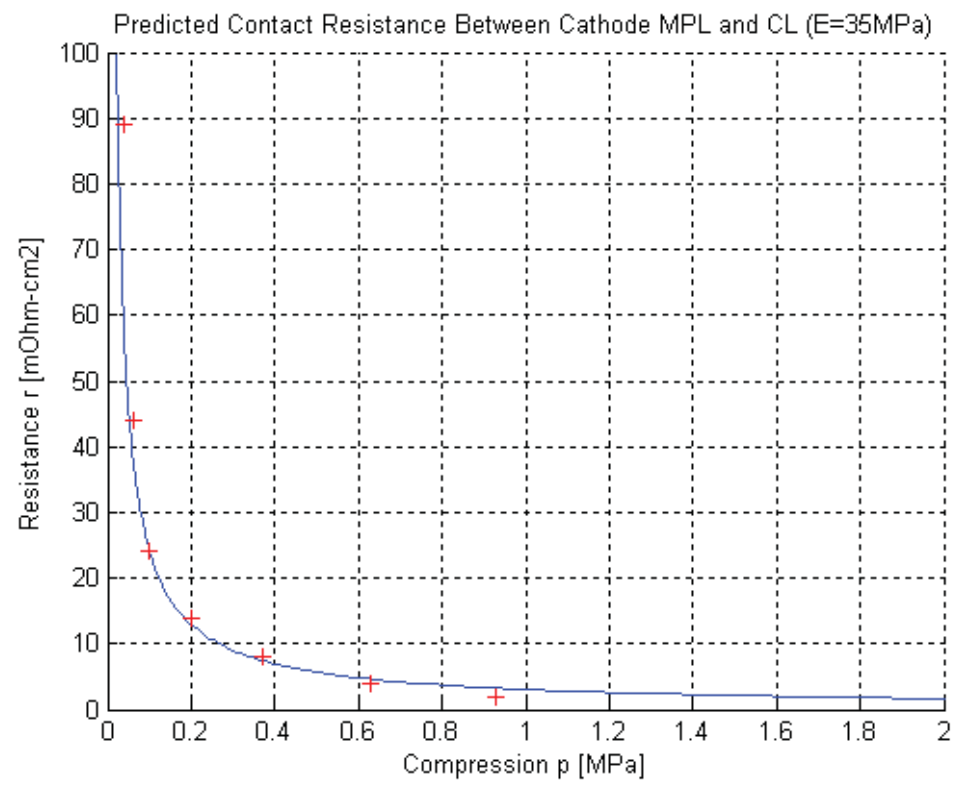

Figure 20: Predicted and Experimental Contact Resistance vs. Compression 
DE-EE0000466

Ballard Material Products Inc

\subsection{Statistical BOT Performance Model}

Based on the derived model in section 7.2.1, an established validation and equation set has been demonstrated. However this validation was generally on a one-off basis (with repeats of the model around experimental variations in order to assess accuracy). However, there are natural distributions in incoming materials, manufacturing processes, and operational conditions due to the systems involved. As a result, it would be expected that any such validation for a model should be completed on a statistical basis as it would be impossible to determine whether a "one-off" validation would be representative of the mean or if the included model relationships accurately can capture the coupling of parameters in order to identify abnormally high or low performing unit cells/MEAs.

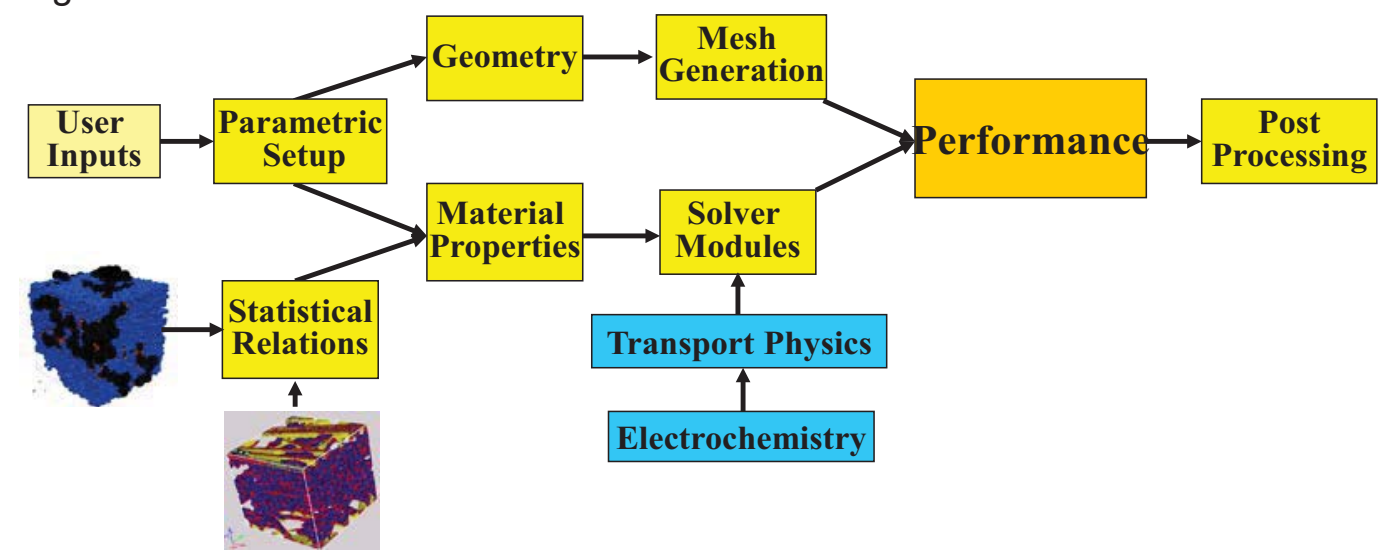

Figure 21: Methodology for the modification of the model into a statistically based code

The original model was modified to follow a module algorithm, shown in Figure 21. This flow path effectively split out the relevant sections of the model and allowed for an additional set of modules to be applied: (1) Statistical User Inputs, and (2) Statistical Relations. The first module allowed the user to assign a mean and standard deviation such that any one of the base input parameters would be randomly determined within that distribution at run time, while the second module allowed for the input of relationships predicted from microstructural based codes which are inherently statistical and generally used to form relations between structural variables and transport coefficients or material properties.

In order to test the model, the inherent sensitivities of various input parameters was tested. Figure 22 shows a selection of these and their overall impact on the model predictions when allowed to vary in isolation. From this figure, it can be seen that a $2.5 \%$ variation in the relevant kinetic parameters that affect the Tafel slope generate as significant an impact as a $10 \%$ variation in the base relationship that governs the protonic conductivity.

In order to assess the validation of the code, a fully coupled set of statistical variations was used. Figure 23 shows the resulting variability over a series of model runs for platinum loading and Figure 24 shows the resulting effect on one of the affected parameters, ECSA. 
DE-EE0000466

Ballard Material Products Inc
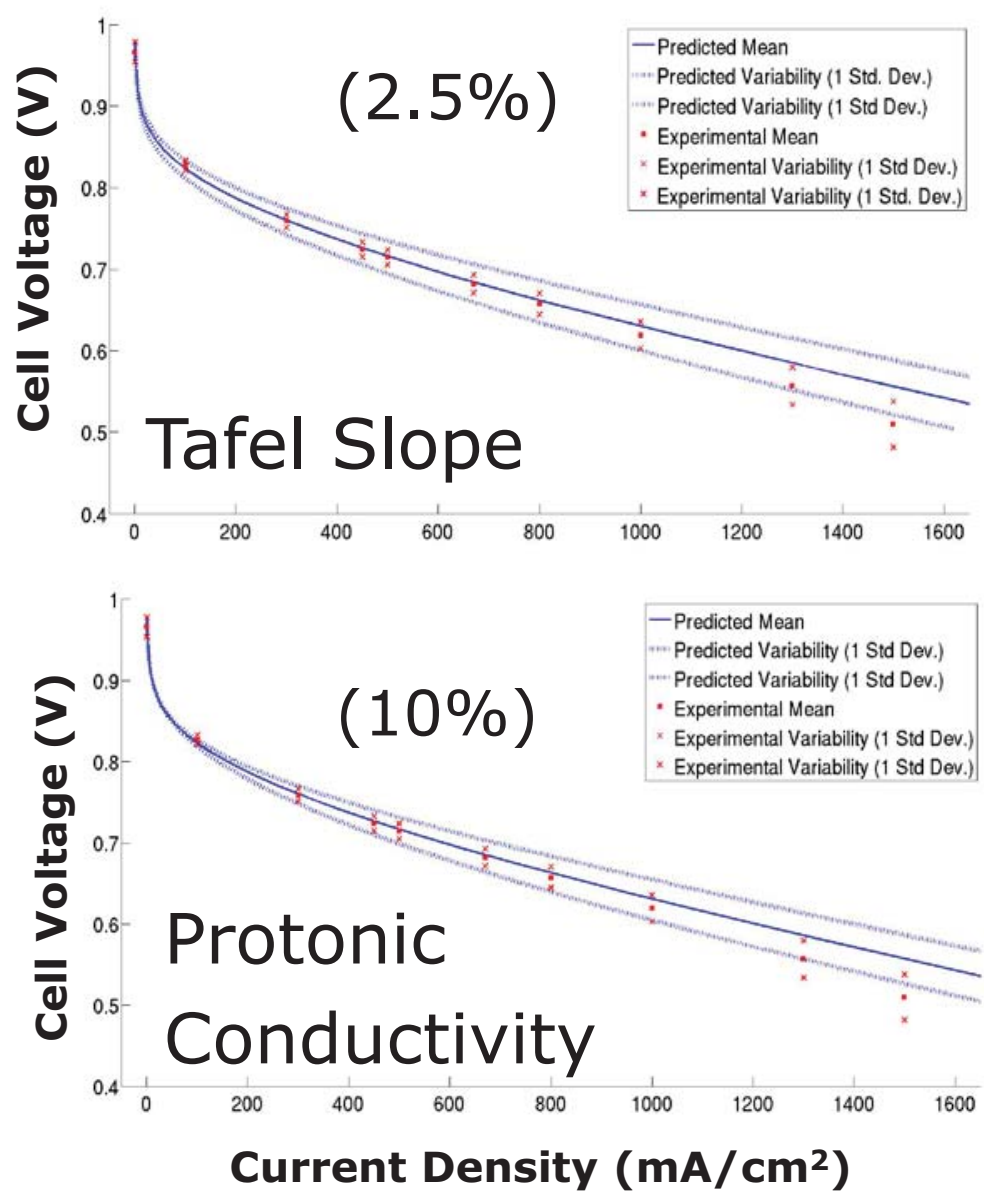

Figure 22: Statistical test variation for variable sensitivity

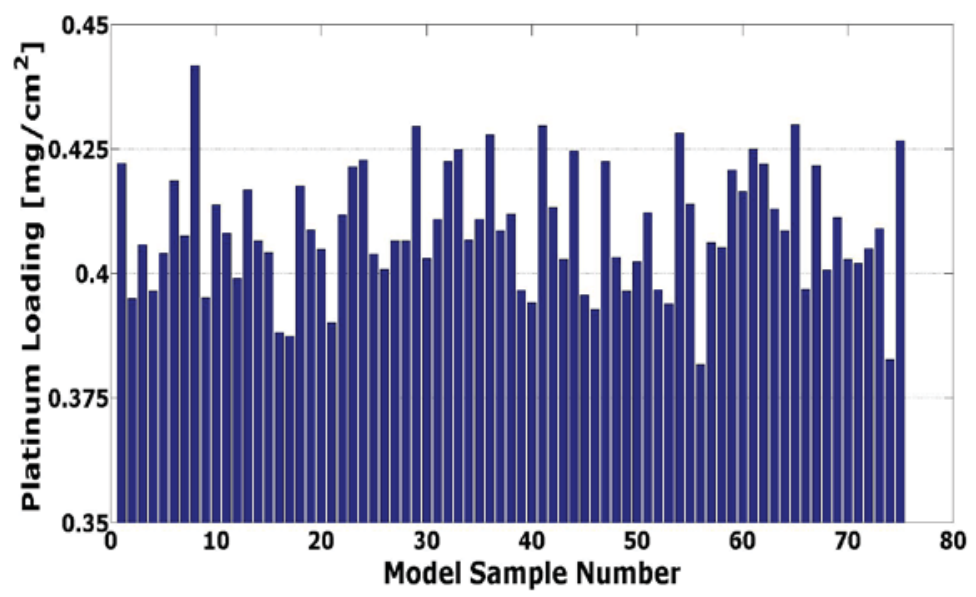

Figure 23: Statistical variation of platinum loading for 75 repeats 
DE-EE0000466

Ballard Material Products Inc

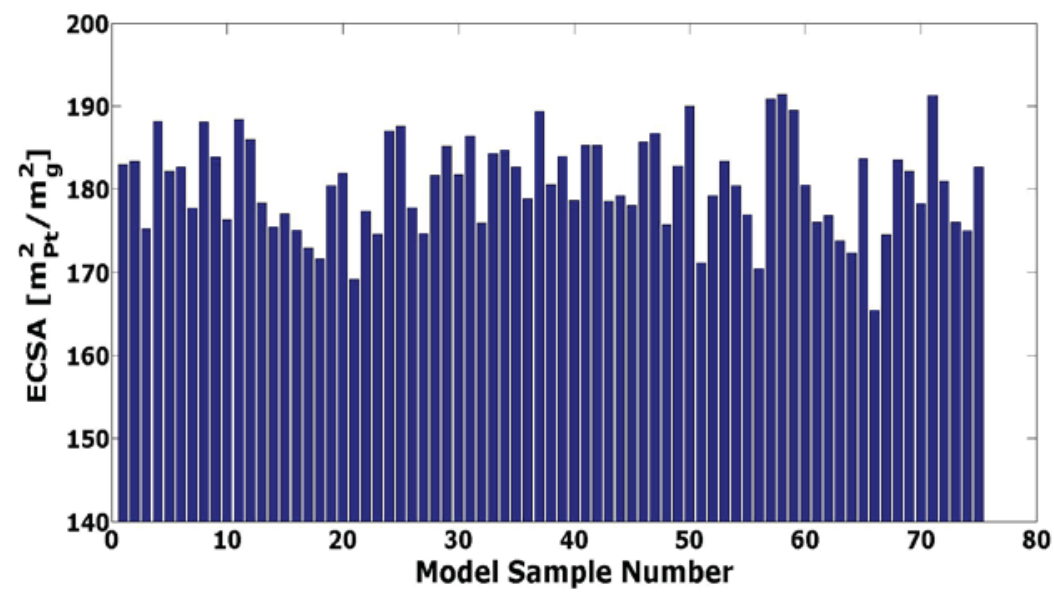

Figure 24: Statistical variation in ECSA as a result of platinum loading variation over 75 repeats

Using the assigned distributions determined from the microstructural codes, incoming manufacturing specifications, and/or process variation analysis, the model was revalidated based on a sample set of "baseline" MEAs. In the first instance of validation, the model with only the single phase module was tested against the experimental data - shown Figure 25 . As seen previously the model, compares well with the experimental data up to a current density of approximately $1 \mathrm{~A} / \mathrm{cm}^{2}$ after which the effect of the liquid water is clear. Further, the polarization curves generated from the simulation show a systematic deviation from the mean value the entire length of the polarization curve which suggests little interaction between variables -- even at higher current densities.

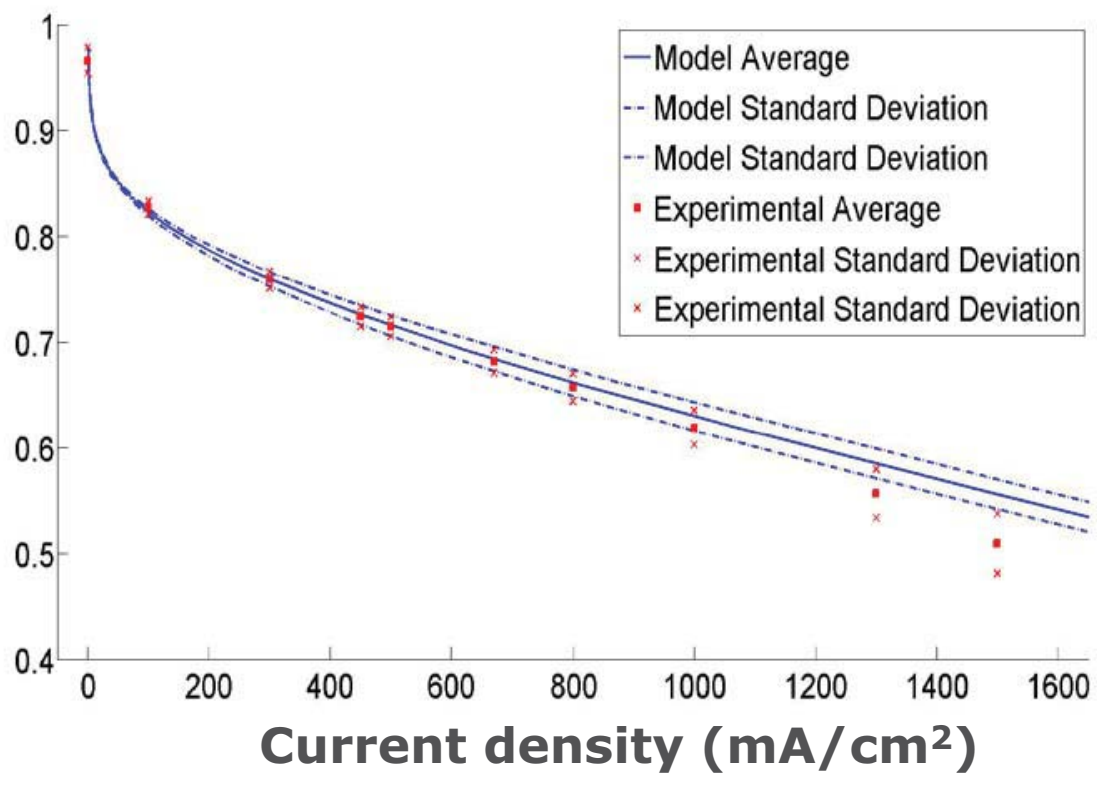

Figure 25: Statistical model validation using the single phase module 
The fully two-phase model was then validated against the experimental data using the same variation in input parameters, Figure 26. The comparisons follow the same prediction and standard deviation seen in the single phase code up to the almost the $1 \mathrm{~A} / \mathrm{cm}^{2}$ point, however at higher current density the two-phase model continues to be a good predictor of the cell performance. Further, where the single phase model showed an unchanging deviation from the mean, the two-phase model in fact shows an interaction between variables such that there are combinations that result in a non-linear coupling that results in lower performing variations/combinations.

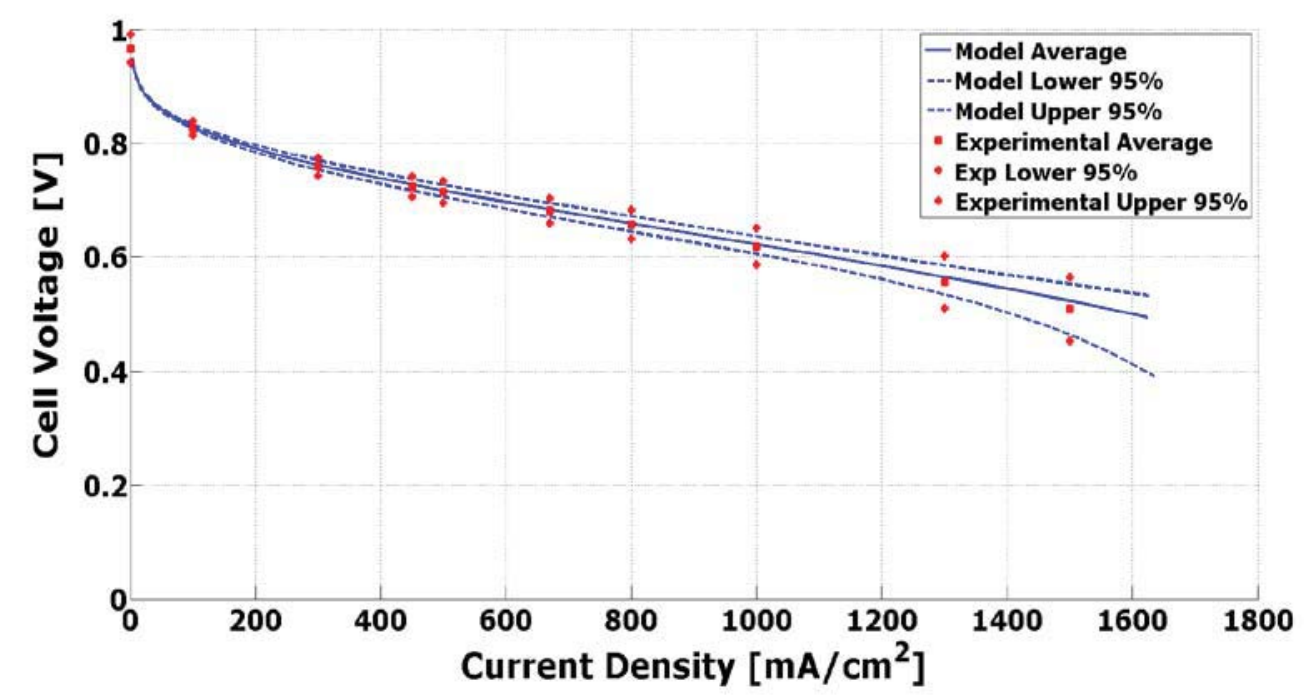

Figure 26: Statistical model validation using the two-phase module 
DE-EE0000466

Ballard Material Products Inc

\subsection{1-D Durability Model}

From section 7.2, the development of a BOT performance model has been described. This model provides the basis upon which the associated durability model has been developed. Much of the key methodology from this stage forward is on the application of key materials aging models that then reflect changes in parameters that alter the behaviour of the included physics in the previous section to assess the "degraded performance". However, the overall degradation predictions are not done only in this strict linear sense as there is in fact a degree of coupling in the change of local conditions due to the aging process itself. This change alters the local conditions which in turn re-affect the aging process - a feedback loop for all intents and purposes.

\subsubsection{Platinum Oxide}

In order to simulate the ORR kinetics, originally the Butler-Volmer model was employed. However, while this model was adequate to reproduce the performance of the cell at beginning of test conditions the model has no representation regarding the surface state of the platinum, which is critical in extending the model to simulate platinum dissolution.

Existing models for the prediction of platinum oxide coverage were assessed. The model by Darling and Meyers which was used to simulate platinum dissolution under H2/N2 based cycling conditions and as such included oxide formation from a water precursor was investigated. However, the accelerated stress test cycling that was intended to be simulated in this model was done in $\mathrm{H} 2 / \mathrm{O} 2$ environments such that oxide could be formed from either gaseous oxygen or liquid/vapour water. This resulted in an inability to leverage the previous work of Darling and Meyers; given this, the work of Wang et al. was considered. Wang et al. published a "Dual Pathway" approach to ORR kinetics, this dual pathway model was built on RDE data in fitting the key parameters of the model and included several steps in the ORR electrochemical reaction. Figure 27 shows a schematic of the proposed ORR reaction pathway and accounts for both an oxide covered platinum surface as well as a hydrated oxide surface that would exist at lower potentials.

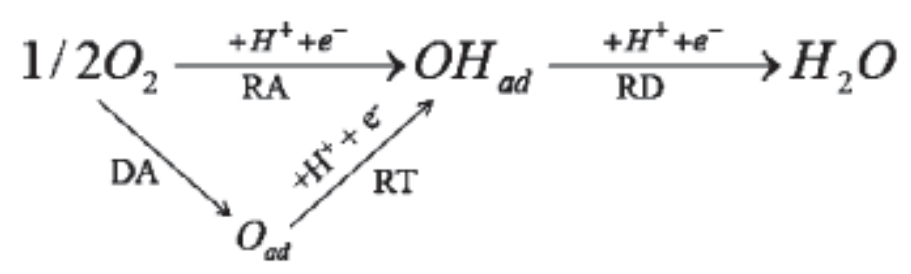

Figure 27: ORR "Dual Pathway" Model as published by Wang et al.

In the early analysis of this model, it was identified that the while the model worked relatively well at reproducing steady-state polarization curves, depending on the parameters used, the model was unable to capture the behaviour related to cyclic voltammetry. Figure 28 shows the results when using an appropriate described implementation of the double trap to simulate a CV sweep. 
DE-EE0000466

Ballard Material Products Inc

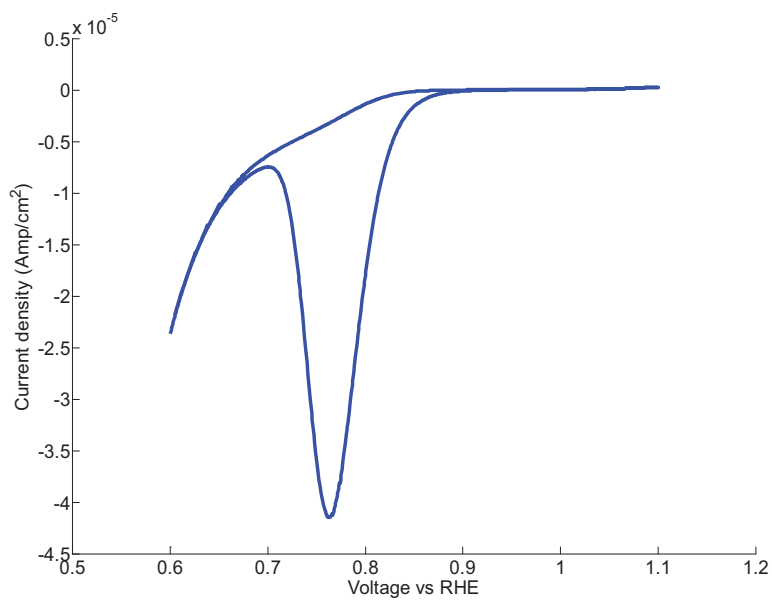

Figure 28: Initial attempt to use the double pathway ORR model to simulate the Cyclic Voltammogram for an MEA

Based on the result for the cyclic voltammogram it was clear that the model was incapable of predicting the oxide coverage that was observed at high potentials, further comparison of the Dual Pathway model predictions to EQCM data clearly illustrated the primary issue with the original model, shown in Figure 29.

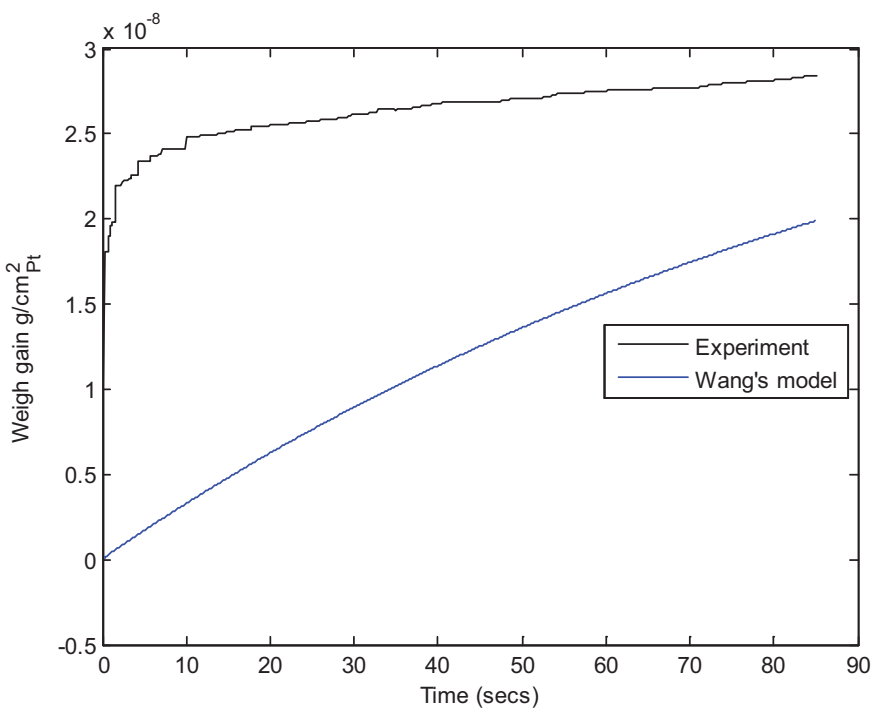

Figure 29: Comparison of the Dual Pathway model to experimental EQCM data for oxide growth

Further, certain aspects of the model did capture behaviour that was desirable. Shown in Figure 30, the Dual Pathway model was run to compare the effect of running between an "Air" and "Nitrogen" state on the oxide. From this figure it is clear that the formation of the oxide along the potential range is affected by the amount of oxygen available in gaseous form; however in the potential range above $0.95 \mathrm{~V}$ the oxide coverage collapses towards $100 \%$ regardless of the amount of gaseous oxygen present suggesting that at high voltages the 
DE-EE0000466

Ballard Material Products Inc

dominant variable for oxide formation is the water species (note that the upper coverage behaviour is still incorrect).

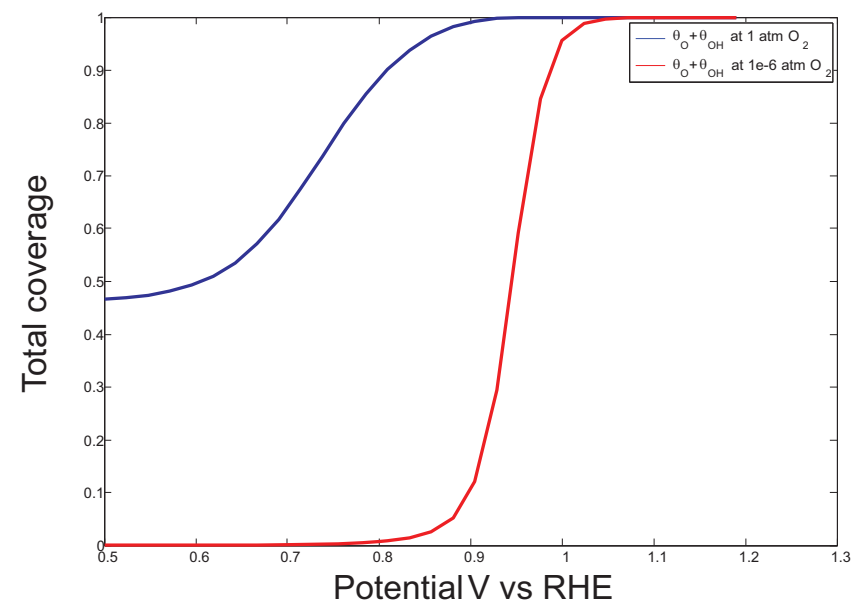

Figure 30: Oxide Coverage predictions using the Dual Pathway model for the ORR

In order to address this issue with relationship between oxide growth and potential, the dual pathway model was modified to re-derive the coverage relationships and base equations.

Besides the current-voltage characteristics the re-derived model was also required to reproduce three experimentally observed characteristics, namely:

1. The oxide coverage in the presence and absence of oxygen

2. Logarithmic growth of oxide during cyclic voltammetry (D.V Heyd and D.A. Harrington 1991)

3. Logarithmic growth of oxide during potential step experiments monitored by EQCM (A. Kongkanand and J.M. Ziegelbauer 2012)

In order to address the behaviour of the oxide growth at high potentials, an additional term was added to describe the logarithmic behaviour such that the equation set took the following form:

$$
\begin{aligned}
& v_{R D}=j^{*} \exp \left(-\Delta G_{R D} / k T\right) *\left(\exp (2 * \beta F \eta / R T) \theta_{O H}-\exp (2 *(1-\beta) F \eta / R T) * \exp \left(\frac{-\omega\left(\theta_{O}+\theta_{O H}\right)}{R T}\right)\right) \\
& v_{R T}=j^{*} \theta_{O} \exp \left(-\Delta G_{R T} / k T\right)-j^{*} \exp \left(-\Delta G_{-R T} / k T\right) * \theta_{O H} \\
& v_{R A}=j^{*}\left(1-\theta_{O}-\theta_{O H}\right) \exp \left(-\Delta G_{R A} / k T\right)-j^{*} \theta_{O H} \exp \left(-\Delta G_{-R A} / k T\right) \\
& v_{D A}=j^{*}\left(1-\theta_{O}-\theta_{O H}\right) \exp \left(-\Delta G_{D A} / k T\right)-j^{*} \theta_{O} \exp \left(-\Delta G_{-D A} / k T\right)
\end{aligned}
$$

In this re-derived set the rate expression $v_{R D}$ is key, a term has been introduced such that there now exists a logarithmic growth term in the rate expression similar to D.V Heyd and D.A. Harrington's (1991) work:

$$
\exp \left(\frac{-\omega\left(\theta_{O}+\theta_{O H}\right)}{R T}\right)
$$


It must be noted that the pre-exponential term is zero order with respect to coverage and the kinetic expression describes a situation where there is logarithmic growth of $\mathrm{PtOH}$ accompanied by a place exchange process. The onset potential of the place exchange process is still a question in the open literature.

It is also valid to think of a mechanism where the logarithmic growth can be attributed to reaction "RT" with a Butler-Volmer type reaction "RD". This set-up would describe a situation where Pt-OH forms through reaction "RD" which logarithmically oxidizes to subsurface PtO. This idea was also analyzed for its kinetic performance under different operating conditions but did not yield satisfactory results.

Using the kinetic model from above, the energy barrier parameters were estimated using RDE data from an experiment in $0.1 \mathrm{M} \mathrm{HClO} 4$ electrolyte. The model fit is shown in Figure 31.

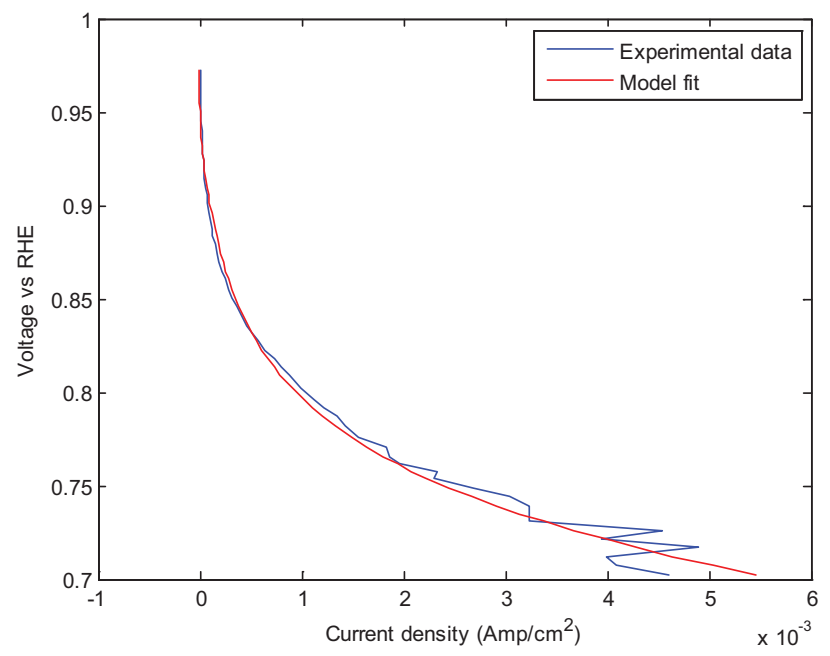

Figure 31: Modified Dual Pathway model fit to RDE data

Using these kinetic parameters, Figure 32 shows the CV response predicted by the model at low oxygen concentration. The current plateau above $0.85 \mathrm{~V}$ is a feature that is seen in CV experiments in liquid electrolyte and the model is able to predict that due to the addition of the logarithmic term in the kinetic model. Similarly Figure 33 shows the predictive capabilities of the model to simulate potential step experiments. Once again the logarithmic growth of oxide coverage is a feature that is seen in EQCM experiments. 
DE-EE0000466

Ballard Material Products Inc

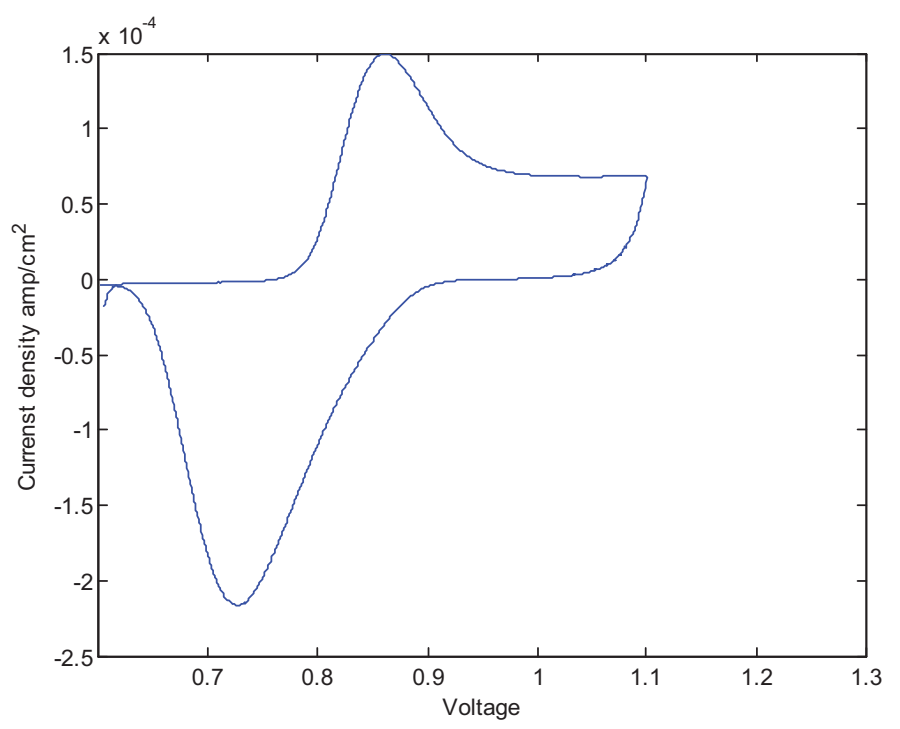

Figure 32: CV predicted from the Modified Dual Pathway model using RDE fit parameters at low oxygen concentration

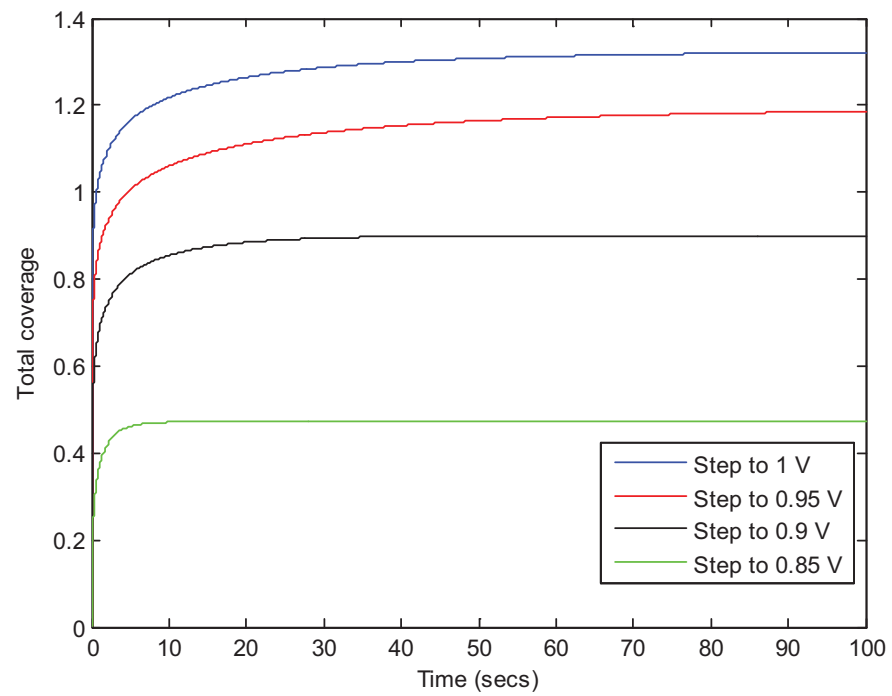

Figure 33: Coverage response to step changes in potential from $0.43 \mathrm{~V}$ to a UPL, similar to EQCM data

Through a further parametric analysis, the relationship between different features of the CV peaks and the key model parameters were determined. Figure 34 and Figure 35 show the energies associated with the RD and RT steps and their associated effect on the oxide formation and reduction peaks. 
DE-EE0000466

Ballard Material Products Inc

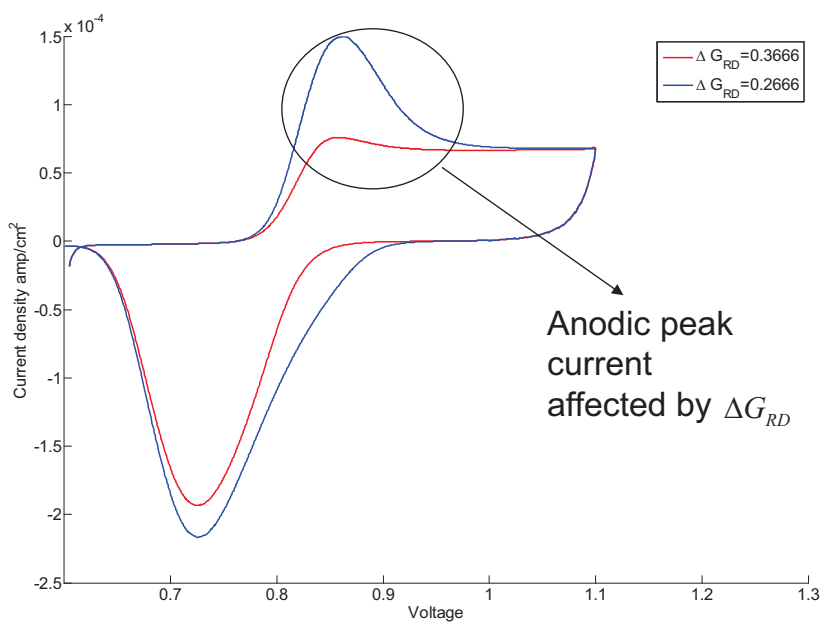

Figure 34: Parametric analysis for the oxide formation peak using the Modified Dual Pathway model.

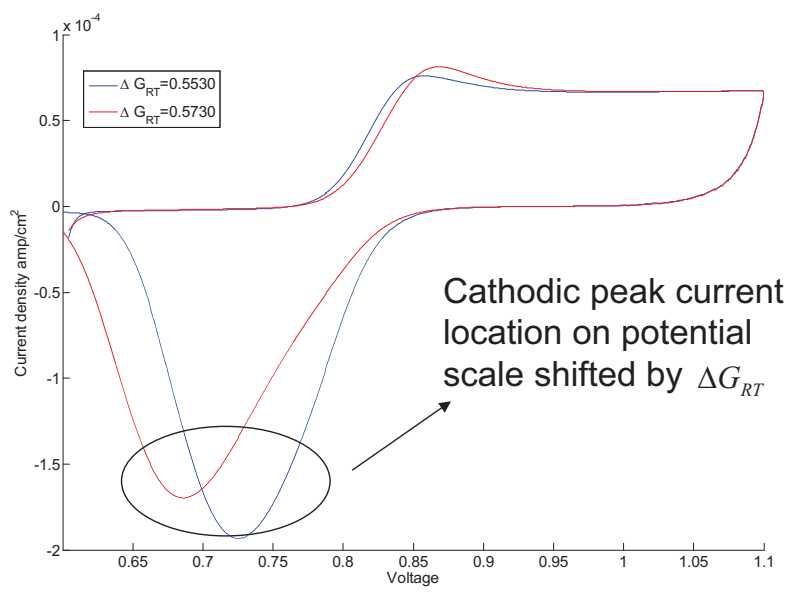

Figure 35: Parametric analysis for the oxide reduction peak using the Modified Dual Pathway model

This parametric study led to the formulation of an additional characteristic parameter for the model, tmax, which is a descriptor related to the available of active platinum sites for the formation of oxide $(\mathrm{PtO})$ or the hydrated oxide $(\mathrm{PtOH})$. Figure 36 shows the effect of using the active site factor and Figure 37 shows the resulting comparison to EQCM data. 
DE-EE0000466

Ballard Material Products Inc

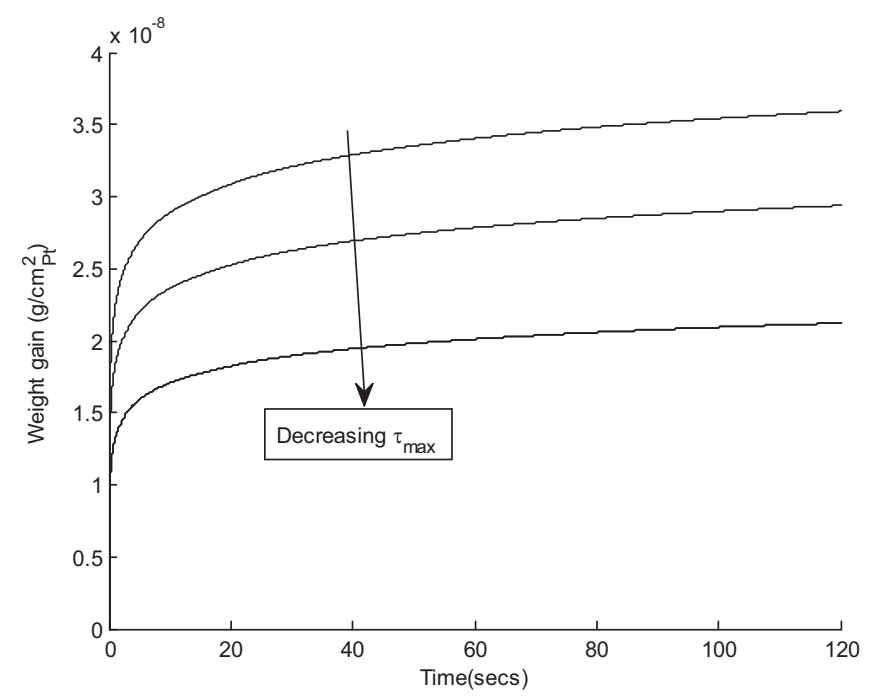

Figure 36: Effect of the active site correction factor based on the surface species

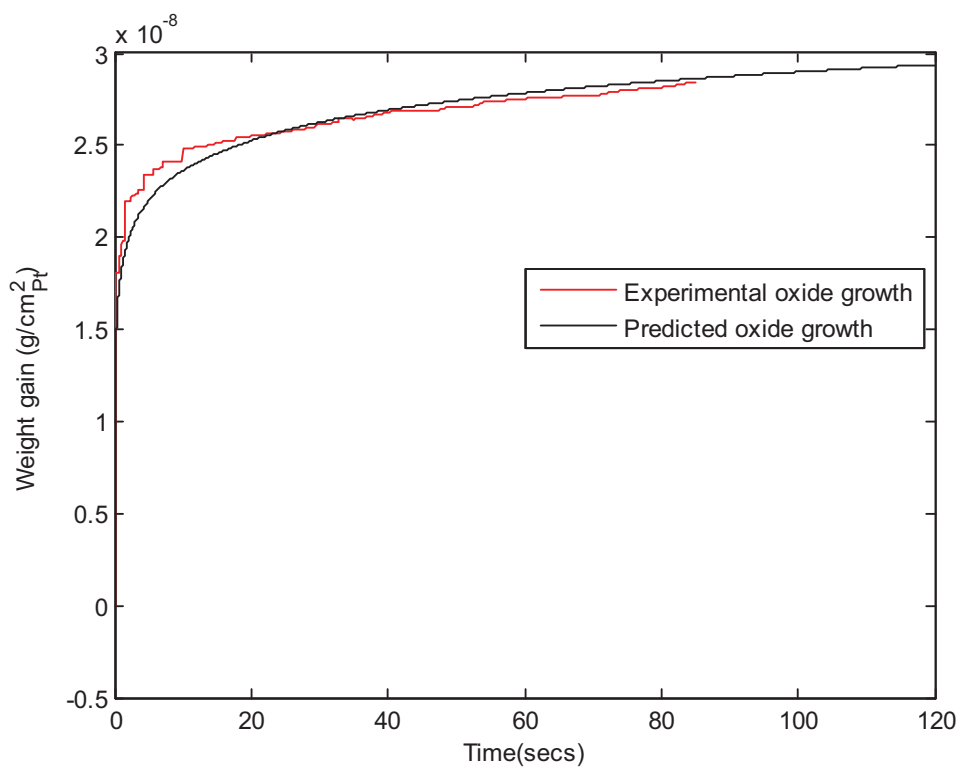

Figure 37: Comparison of the EQCM predictions based on the model reformulation to include a description of the available active sites

Based on the modified oxide growth rate and inclusion of a descriptor for the availability of the active sites, the new predicted CV was found to be significantly a better comparison to the experimentally measured data. However, it was still observed that the lower oxide reduction peak demonstrated a greater magnitude than that observed in the experimental data - shown in Figure 38. This prompted further analysis in which it was found that the local proton concentration affects the lower peak related to the oxide reduction, Figure 39. Interestingly this lower peak shift tends to be observed in fuel cell data and is generally absent in RDE data. 
DE-EE0000466

Ballard Material Products Inc

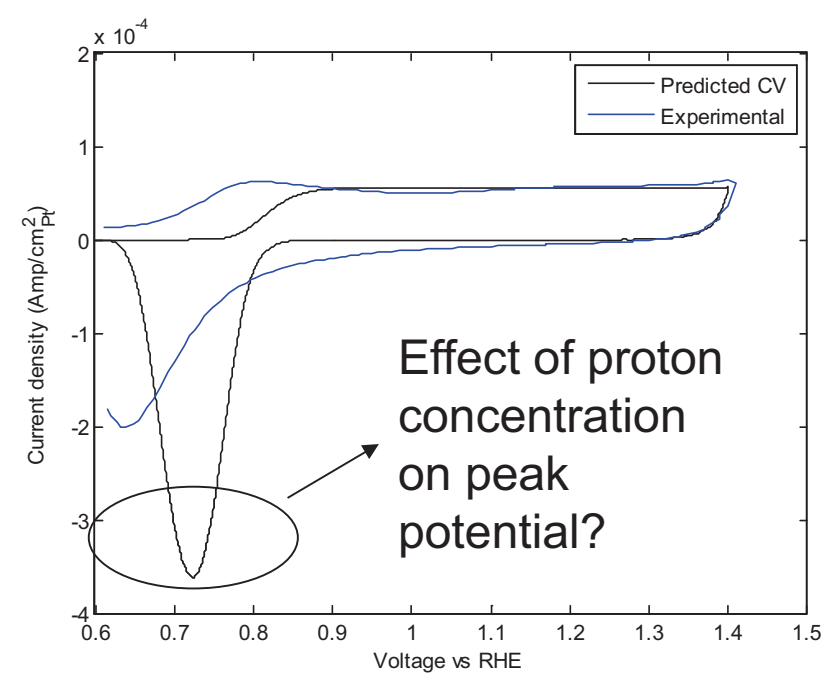

Figure 38: Comparison of Modified Dual Pathway Model with experimental CV data

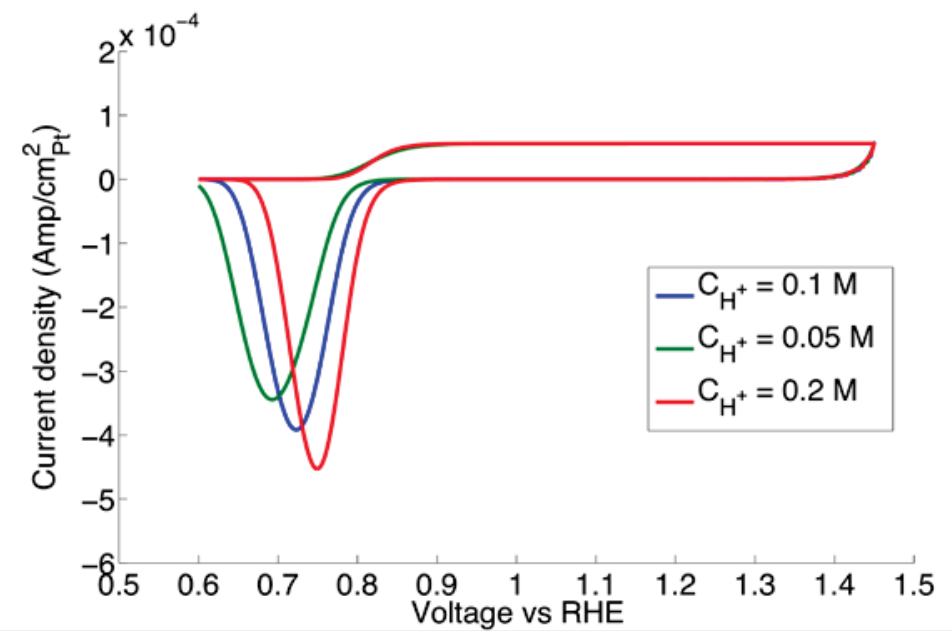

Figure 39: Effect of proton concentration variation on the predicted location and magnitude of the lower CV peak

Based on these modifications, the final comparison of the original Dual Pathway model to the Modified Dual Pathway model shows significant differences in the model predictions and these differences are clearly observed in the various experimental comparisons, both fuel cell data and RDE. The Modified Dual Pathway model provided the basis upon which the platinum dissolution model was implemented. 
DE-EE0000466

Ballard Material Products Inc

\subsection{Platinum Dissolution and Plating}

The process of electrochemical dissolution and plating was based on the mechanism originally proposed by Darling and Meyers and coupled to the platinum oxide model presented in the previous sub-section. Figure 40 shows the general schematic describing the coupling between the platinum dissolution and the platinum oxide multi-step ORR model.

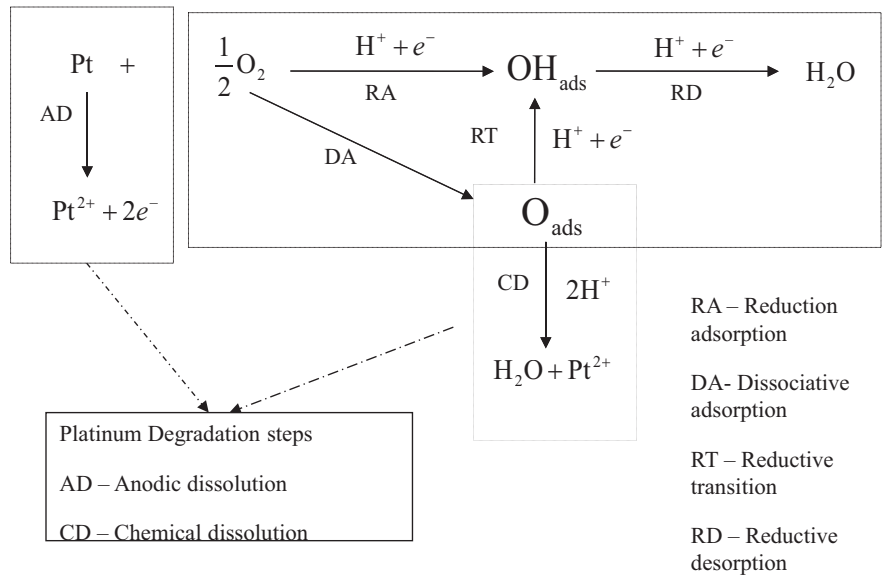

Figure 40: Multi-step ORR pathway with the addition of platinum dissolution

The process for the chemical dissolution is assumed to be negligible relative to the rate of platinum dissolution driven by the potential cycling.

$$
\begin{aligned}
R_{p t, A D} & =k_{p t, A D}\left(1-\theta_{O}-\theta_{O H}\right) \exp \left[\frac{\beta_{A D} n_{A D} F}{R T}\left(\phi_{e}-\phi_{p}-\phi_{e q}\right)\right] \\
& -k_{p t, C P}\left(1-\theta_{O}-\theta_{O H}\right) \exp \left[\frac{\beta_{C P} n_{C P} F}{R T}\left(\phi_{e}-\phi_{p}-\phi_{e q}\right)\right]
\end{aligned}
$$

where, $k_{p t, A D}$ and $k_{p t, C P}$ are the anodic dissolution and cathodic plating rates respectively. $\beta_{A D}$ and $\beta_{C P}$ are the symmetry factors for the "AD" reaction in Figure $40, \mathrm{n}_{\mathrm{AD}}$ and $\mathrm{n}_{\mathrm{CP}}$ represent the number electrons involved in the dissolution/plating step (assumed to be two), and $\varphi_{\text {eq }}$ is the equilibrium potential for the platinum dissolution step.

The equilibrium potential, $\varphi_{\text {eq }}$, is written as follows, with the inclusion of a term to shift the equilibrium potential away from that listed for bulk platinum in an attempt to account for the cohesion platinum atoms at the surface of the particle which are a function of the particle size:

$$
\phi_{e q}=\phi_{e q}^{b u l k}-\frac{E_{A C E} M_{w}^{p t}}{2 \rho_{p t} r_{p t} F}
$$

Where, $E_{A C E}$ is the atomic cohesive energy of the top layer of platinum atoms in the cluster (this is a point of coupling with the molecular dynamics model and in spirit is similar to the surface 
tension concept used by Darling and Meyers), $r_{p t}$ is the radius of the platinum particle, and $\varphi_{\text {eq }}^{\text {bulk }}$ represents the equilibrium dissolution potential observed for nanoparticle or planar platinum electrode dissolution tests.

The addition of platinum dissolution also results in the inclusion of an additional governing equation which is solved in the $\mathrm{aCL}$, pMEM, and $\mathrm{CCL}$ domains. Based on the results from the molecular dynamics simulations and phenomenological concepts of the local potentials, the platinum ions are assumed to be generated from the dissolution reaction as a $\mathrm{Pt}(\mathrm{OH})_{\mathrm{n}}$ complex such that they have an overall neutral charge. As a result, the platinum ions are assumed to move via diffusion (and potentially water drag), with the potential gradient not influencing the transport mechanism, the governing equation takes the following form:

$$
\left(\frac{\partial C_{P t O H}}{\partial t}\right)+\nabla \cdot\left(D_{P t O H}^{e f f} \nabla C_{P t O H}\right)=S_{A D-C P}
$$

Where, the source term is present in the catalyst layers and is related to the process by which the dissolved platinum complex is either consumed due to plating or generated via dissolution. It should be noted that the alternative assumption related to the generation of a platinum ion, which is subsequently dissolved in the local ionomer/water and moves according to its diffusion coefficient and ionic mobility was also investigated/implemented.

Figure 41 shows preliminary data from the large parallel runs being completed for the AST cycling of $0.6-1.1 \mathrm{~V}$. From this test, we see the systematic loss of platinum surface due to the particle dissolution and subsequent growth due to plating. It should be noted that at this stage the model does not account for platinum complexes that are dissolved in the free water which may or may not move out of the cell.

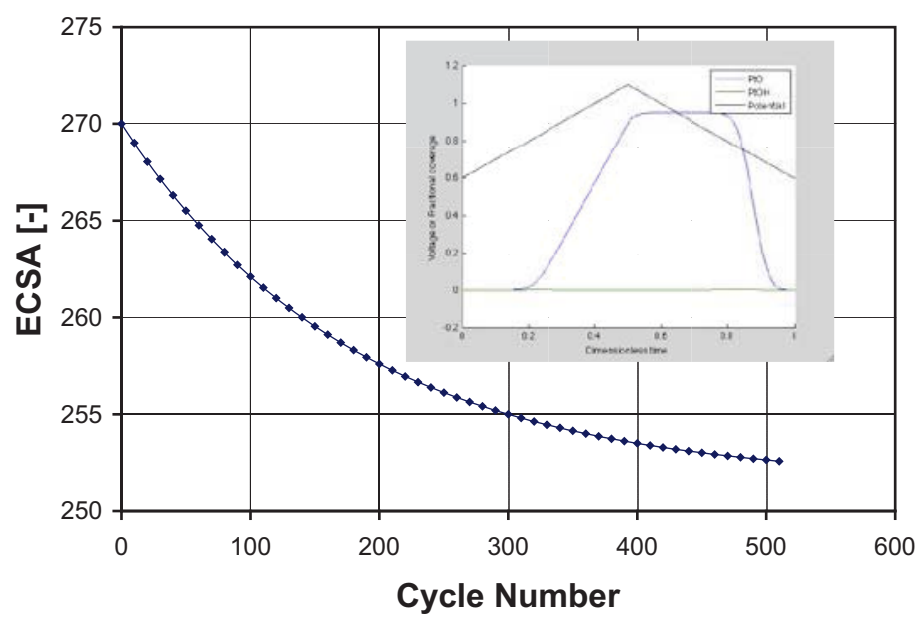

Figure 41: Initial results for the platinum dissolution AST simulations, based on a wave profile from $0.6-1.1 \mathrm{~V}$ and $\sim 500$ cycles 
DE-EE0000466

Ballard Material Products Inc

\subsection{Carbon Surface Oxidation and Carbon Corrosion ${ }^{1}$}

The carbon corrosion process was modelled as two reaction steps: (1) carbon surface oxidation to intermediate carbon-oxygen groups; and (2) a subsequent reaction of the intermediate carbon-oxygen groups to carbon dioxide:

$$
\begin{aligned}
& \mathrm{C}(\mathrm{s})+\mathrm{H}_{2} \mathrm{O} \rightarrow \mathrm{C}-\mathrm{O}_{a d s}+2 \mathrm{H}^{+}+2 e^{-} \\
& \mathrm{C}-\mathrm{O}_{a d s}+\mathrm{H}_{2} \mathrm{O} \rightarrow \mathrm{CO}_{2,(\mathrm{~g})}+2 \mathrm{H}^{+}+2 e^{-}
\end{aligned}
$$

Throughout the remainder of this section these two reaction steps will be referred to as the oxidation and corrosion reactions, respectively.

The reaction rate constants are compared between two different carbons and their dependence on UPL, temperature, and gas relative humidity $(\mathrm{RH})$ were investigated.

The UPL study was utilized to develop a semi-empirical equation that determines the rate constants for the two-step carbon corrosion process. Both the initial surface oxidation and further corrosion to $\mathrm{CO}_{2}$ were assumed to be first order reactions with respect to the normalized carbon surface area, as described by the following equations:

$$
\begin{aligned}
& R_{\text {graphitic }}=\frac{d S_{C, \text { graphite }}}{d t}=-k_{\text {graphite }} S_{C, \text { graphite }} \\
& R_{\text {oxide }}=\frac{d S_{C, \text { oxide }}}{d t}=k_{\text {graphite }} S_{C, \text { graphite }}-k_{\text {oxide }} S_{C, \text { oxide }}
\end{aligned}
$$

Where, $R_{\text {graphite }}$ and $R_{\text {oxide }}$ are the reaction rates, $k_{\text {graphite }}$ and $k_{\text {oxide }}$ are the rate constants, and $\mathrm{S}_{\mathrm{C} \text {,graphite }}$ and $\mathrm{S}_{\mathrm{C} \text {,oxide }}$ are the normalized surface areas of the graphitic and oxidized surfaces, respectively.

Previous testing that used different $\mathrm{Pt} / \mathrm{C}$ ratios has shown that platinum catalyzes carbon oxidation and corrosion; however, since the testing for this project used the same $\mathrm{Pt} / \mathrm{C}$ ratio, an assumption of negligible platinum effect was made to simplify the model. Therefore, until improvements to the model are made to account for these phenomena, this model should only be used to compare catalysts with a similar Pt-C interface.

Integrating equations the previous rate equations in time leads to more useful forms of the rate equations described:

$$
\begin{aligned}
& S_{C, \text { graphite }}=S_{C, \text { graphite }}^{o} \exp \left(-k_{\text {graphite }} t\right) \\
& S_{C, \text { oxide }}=S_{C, \text { graphite }}^{o}\left(\frac{k_{\text {graphite }}}{k_{\text {graphite }}+k_{\text {oxide }}}\right)\left[\exp \left(-k_{\text {graphite }} t\right)-\exp \left(-k_{\text {oxide }} t\right)\right]
\end{aligned}
$$

Where $S^{\circ}$ graphite is the initial graphitic carbon surface area and $t$ is the time in seconds.

\footnotetext{
${ }^{1}$ A. P. Young, V. Colbow, D. Harvey, E. Rogers, and S. Wessel, "A Semi-Empirical Two Step Carbon Corrosion Reaction Model in PEM Fuel Cells", Journal of The Electrochemical Society, 160 (4) F381F388 (2013).
} 
The initial graphitic carbon surface area was calculated by taking the difference between the total surface area obtained from BET gas adsorption measurements and the catalyst metal surface area measured by $\mathrm{CO}$ absorption.

Assumptions:

1. All of the measured carbon surface area is covered with ionomer and contributes to the Double Layer Capacitance measurement.

2. All of the carbon surface is initially graphitic.

The total $\mathrm{Cdl}$ of the carbon was then calculated by multiplying each respective surface area by their specific capacitance:

$$
C_{D L, \text { carbon }}^{\text {total }}=C_{D L, \text { graphite }} S_{C, \text { graphite }}+C_{D L, \text { oxide }} S_{C, \text { oxide }}
$$

Where $C^{\text {total }}{ }_{D L}$,carbon is the total carbon capacitance, $C_{D L \text {,graphite }}$ is the specific capacitance of the graphitized carbon surface, and $\mathrm{C}_{\mathrm{DL} \text {,oxide }}$ is the specific capacitance of the oxidized carbon surface.

Further, the total capacitance of the catalyst was calculated by adding the capacitance of the platinum surface.

$$
C_{D L}^{\text {total }}=C_{D L, \text { carbon }}^{\text {total }}+C_{D L, p t} S_{P t}
$$

Where $C_{D L, p t}$ is the specific capacitance of platinum and $S_{p t}$ is the platinum surface area that is covered with ionomer.

The rate constants, $\mathrm{k}_{\text {graphite }}$ and $\mathrm{k}_{\text {oxide }}$, and specific capacitances, $\mathrm{C}_{\mathrm{DL} \text {,graphite }}, \mathrm{C}_{\mathrm{DL} \text {,oxide }}$, and $\mathrm{C}_{\mathrm{DL}, \mathrm{pt}}$ were considered as unknowns and obtained through a successive fitting process applied to the analysed experimental data. Fitting was accomplished by allowing the fitting parameters to vary in order to minimize the sum of the squared error between model and experiment.

The first parameters determined were $C_{D L}$,graphite and $C_{D L, p t}$, via a fitting of the total capacitance in the 0.9 and 1.0V UPL tests, where negligible surface oxidation and corrosion rates were assumed and as such it was assumed that any change in $C_{D L}{ }^{\text {Total }}$ was solely due to the reduction in the platinum surface area. Of note, this assumption depends on the carbon type and may not be valid for non-graphitic carbons, where a lower UPL would be required to make this assumption. A platinum specific capacitance of $62 \mu \mathrm{F} / \mathrm{cm}^{2}$ was found by via the slope of ECSA versus $C_{D L}{ }^{\text {Total }}$ which was relatively close to $40 \mu \mathrm{F} / \mathrm{cm}^{2}$ which has been reported in literature. This value of the specific platinum capacitance was then held constant for all remaining test cases.

Moving forward, in subsequent samples all the carbon surface area was assigned to be graphitic - consistent with the earlier assumption - which allowed $\mathrm{C}_{\mathrm{DL} \text {,graphite }}$ to be calculated at the beginning of each test. This resulted in an average carbon specific capacitance of $5.4+/-$ $0.2 \mu \mathrm{F} / \mathrm{cm}^{2}$, which was within the 3 to $56 \mu \mathrm{F} / \mathrm{cm}^{2}$ range reported in literature and dependent on the carbon type.

The rate constant and the specific capacitance of the oxidized carbon surface was then fitted using the data set which had the best resolution for the change in $\mathrm{C}_{\mathrm{DL}}{ }^{\text {Total }}$ (which showed both an increase and decrease); the 1.3V UPL. The specific capacitance of oxidized carbon ranged from 45 to $48 \mu \mathrm{F} / \mathrm{cm}^{2}$ and was assumed to be constant for all other tests, leaving only the two rate constants as fitting parameters. 
With the average values for the specific capacitances fixed, the rate constants were fitted for all UPL tests independently. A clear relationship between the rate constants and the UPL for both carbon supports was observed. It was noted that at higher UPLs the difference in oxidation rates was reduced.

A temperature and gas $\mathrm{RH}$ sensitivity study utilizing was then used to correct the model for temperature and relative humidity. The 1.4V UPL tests were used in order to observe faster corrosion responses and minimize the platinum dissolution effects.

The 1.4V UPL oxidation rate constant showed greater response to both temperature and gas $\mathrm{RH}$, with significantly greater rates at high temperature and high $\mathrm{RH}$ compared to the corrosion rate constant. An Arrhenius form was used to normalize the behaviour for the temperature effect and the rate constants were then corrected for the water content of the ionomer using the Springer relationship for Nafion 117. Arrhenius parameters, including activation energy, were calculated before the normalization for water content and are comparable to the $106+/-17$ $\mathrm{kJ} / \mathrm{mol}$ quoted for the thermal combustion energy of carbon used in a $\mathrm{Pt} / \mathrm{C}$ catalyst.

A validation of the model was conducted using the temperature study as shown in Figure 42, comparing the measured loss of carbon to the modeled loss of carbon surface area, on a percentage basis. The agreement between model and data is reasonable with greater differences occurring at lower temperatures. The discrepancy between model and experiment can be speculated to be mainly caused by the simplifying model assumptions.

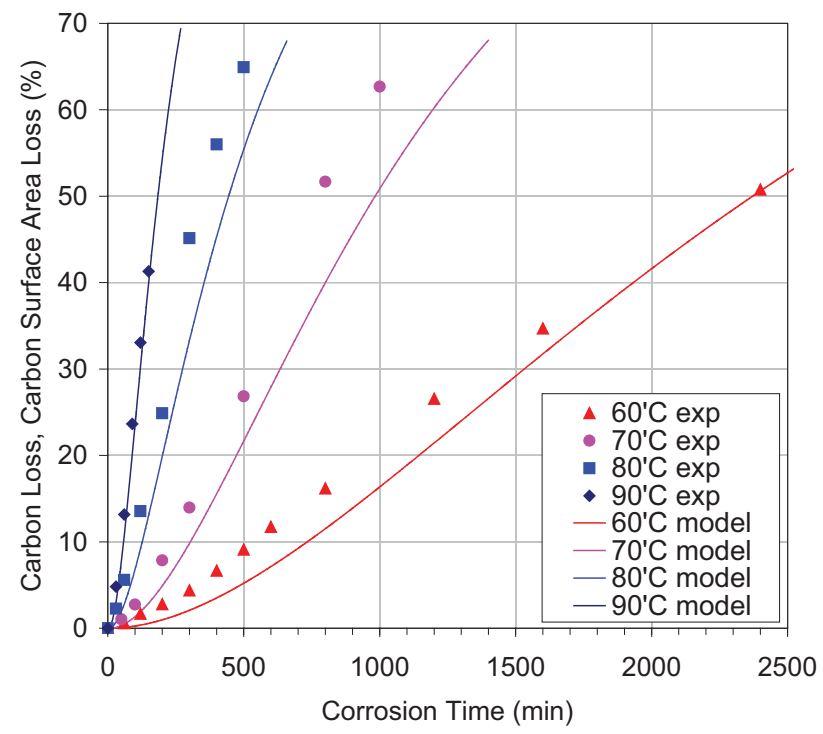

Figure 42: Model validation based on the prediction of the per cent loss of carbon surface area versus the per cent loss of carbon as measured in the gas exhaust

This semi empirical model for the carbon oxidation and corrosion rates is then implemented in the MEA model such that the carbon mass declines over the course of the cycling resulting in a reduction in the overall carbon volume fraction. In order to account for the thickness change, a moving mesh would be required; this has not been implemented at this stage. However, adjusting for the modified carbon volume fraction and re-calculating the new lonomer:(Pt/C) ratio results based on an assumption that the total porosity remains constant - results in an increasing effect of a shifting composition and thus the associated performance loss due to carbon corrosion. 